\title{
Understanding Fundamental Material Degradation Processes in High Temperature Aggressive Chemomechanical Environments
}

\section{Reactor Concepts RD\&D}

Dr. James Stubbins University of Illinois at Urbana-Champaign 


\title{
Understanding Fundamental Material-Degradation Processes in High Temperature Aggressive Chemomechanical Environments
}

\author{
DOE-INL-00091210 \\ University of Illinois at Urbana-Champaign \\ James Stubbins, PI
}

1 September 2009 to 30 August 2013

\section{Final Technical Report}

15 January 2014

Co-Principal Investigators

Andrew Gewirth, Huseyin Sehitoglu, Petros Sofronis, Ian Robertson ${ }^{1}$

\section{Principal Contributors}

Kun Mo, Xiang Chen, Hsiao-Ming Tung, Wei Lv, Gian Franco Lovicu², Garrett Patkay, Mohsen Dadfarnia, Buyang Cao, Jeremy Hatch, Bai Cui, Andrew Gewirth, Ian Robertson ${ }^{1}$, Huseyin Sehitoglu, Petros Sofronis, James Stubbins

University of Illinois at Urbana-Champaign

${ }^{1}$ University of Wisconsin-Madison

${ }^{2}$ University of Pisa 


\section{Understanding Fundamental Material-Degradation Processes in High Temperature Aggressive Chemomechanical Environments}

\section{EXECUTIVE SUMMARY}

The objective of this research project was to address major materials performance and methodology issues for the design and construction of high-temperature and very high-temperature nuclear systems. This work provided a synergy between the development of simplified, but robust, design rules for high-temperature systems and materials testing, along with performance and improvement of these systems. Such systems would have to deal with time-dependent materials properties (creep, creep-fatigue, high-temperature corrosion) in components with complex stress states, long intended service lives, and aggressive operating environments. Because routine mechanical properties data and current high-temperature design methodology does not provide adequate information for long-term, robust system design, this project investigated these issues with the focus on long-term material property degradation analysis. In addition, high-temperature materials testing in relevant corrosive environments (such as low oxygen, partial pressure with substantial carbon activities) has been performed to support further code qualification of existing alloys and the development of emerging alloys. Alloy 617 and Alloy 230 are the two lead alloys for very high temperature applications, and these two alloys were studied extensively in this research effort.

The work in this program concentrated heavily on identifying and quantifying materials degradation mechanisms at high temperatures, including mechanical properties performance, corrosion resistance, and the synergy between the two. The materials design issues center primarily on a "damage mechanisms" approach rather than on the more tradiational approach of quantifying limitations on loads and operational times (e.g. isochronis stress-strain curves). It was found that in the temperature range studied in this program, 800 to $1000^{\circ} \mathrm{C}$, a major issue with materials mechanical properties degration was the evolution of the internal carbide structure. The initial strengthening that the matrix carbide structure provides quickly dissipates and the migration of carbon to the grain boundaries results in significant weakening of the grain boundary strength during mechanical loading. This results in an internal damage mechanism that results in intergranular fracture.

The materials response was also examined after long term aging, up to 10,000 hours at elevated temperature. Long term aged materials also showed the effects of materials degradation, principally through the redistribution of the carbide structure.

Corrosion studies in high oxidizing, low oxidizing, and carburizing atmospheres indicate that Alloy 230 performs better than Alloy 617 in oxidizing conditions, but is more prone to degradation in carburizing atmospheres.

The creep-rupture properties of the two alloys were studied in detail using pressurized tubes. In most cases, the elevated creep response showed a very short period of secondary creep and an extended period of tertiary creep. The pressurized tubes produce a biaxial stress state. The post test microstructure showed that grain boundary "damage," again associated with the carbide structure, was the major controlling feature in the creep-rupture response. 


\section{TABLE OF CONTENTS}

EXECUTIVE SUMMARY. 1

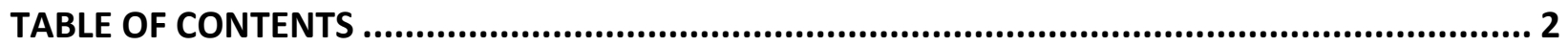

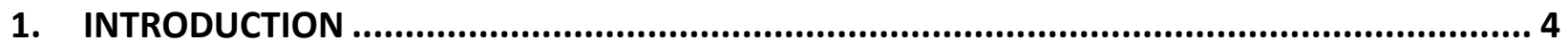

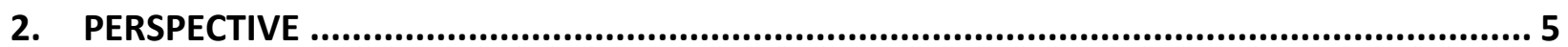

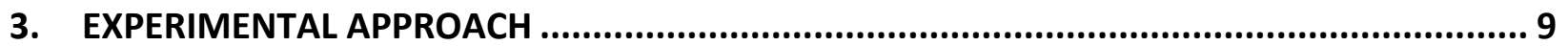

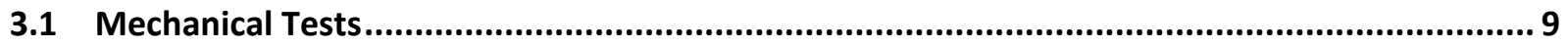

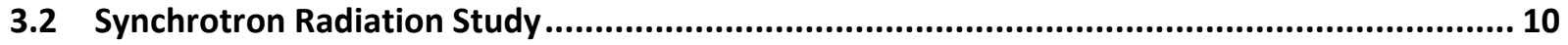

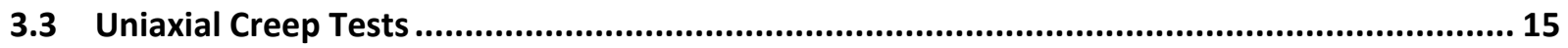

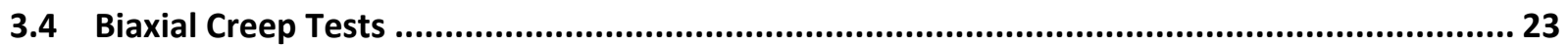

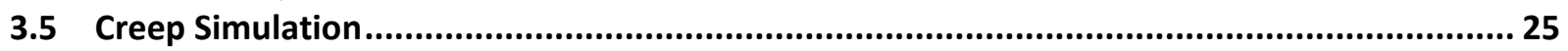

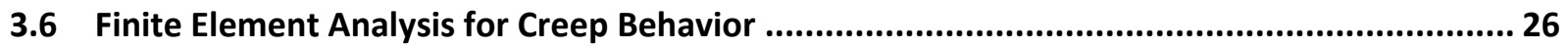

3.7 High Temperature Fatigue Crack Growth of Haynes 230 ..................................................... 27

3.8 Transpassive Behavior of Ni-based Alloys ................................................................................ 30

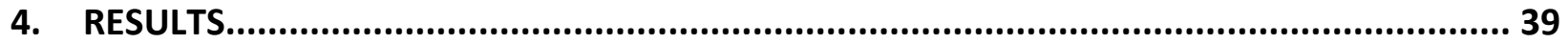

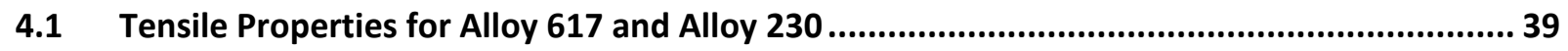

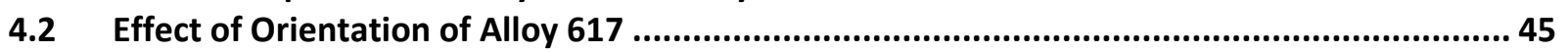

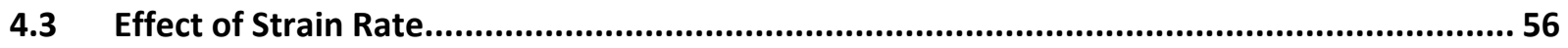

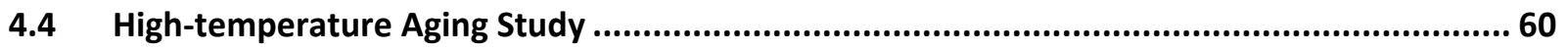

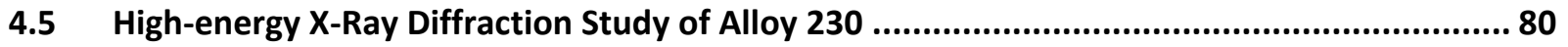

4.6 In-situ Creep Study Using Pressurized Creep Tubes ............................................................. 93

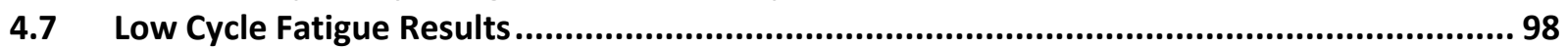

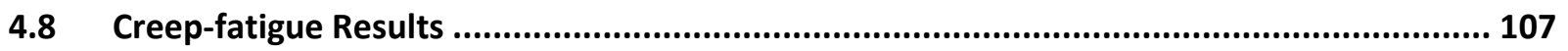

4.9 SEM and EDS Results of Uniaxial Creep and Fatigue Tests............................................... 123

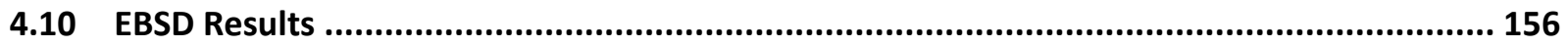

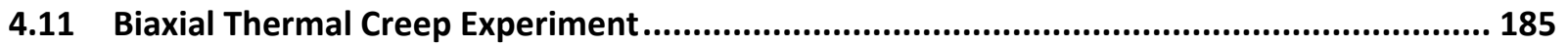

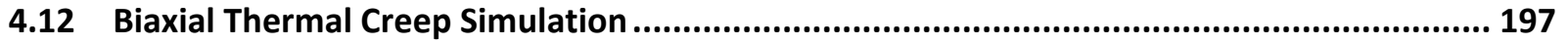

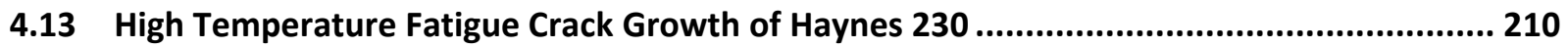

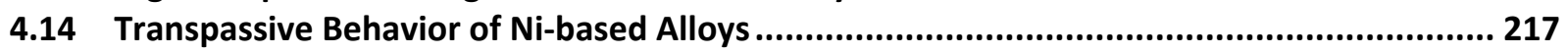

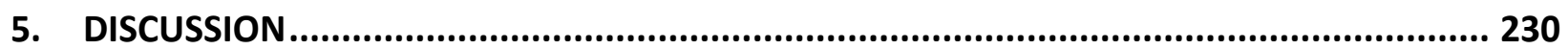

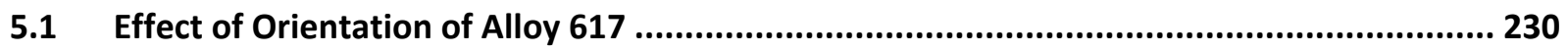

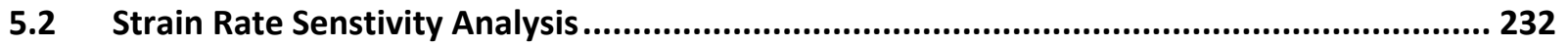

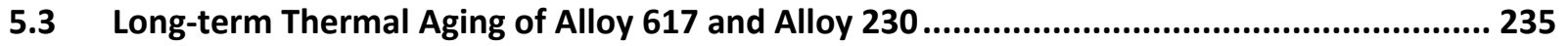

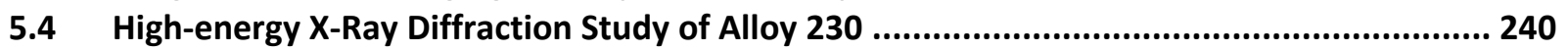

5.5 In-situ Creep Study Using Pressurized Creep Tubes .......................................................... 241

5.6 Creep-fatigue Life Prediction ......................................................................................... 242

5.7 Deformation Mechanism of Creep and Fatigue ............................................................. 254

5.8 Influence of Material Properties on LCF and Creep-Fatigue Life ....................................... 256

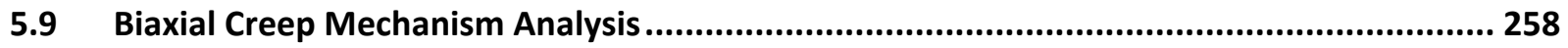

5.10 Evaluation of the Empirical Relation Predicting Long-term Creep Life ............................... 261

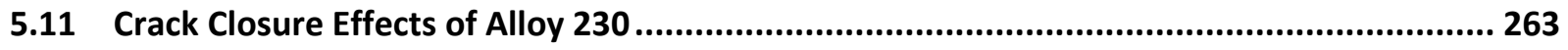

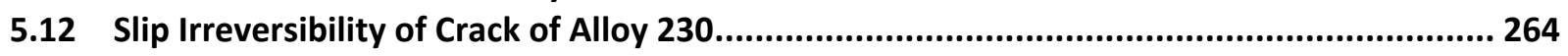

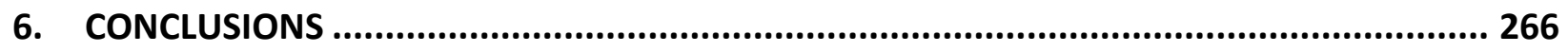


Understanding Fundamental Material-Degradation Processes in High Temperature Aggressive Chemomechanical Environments

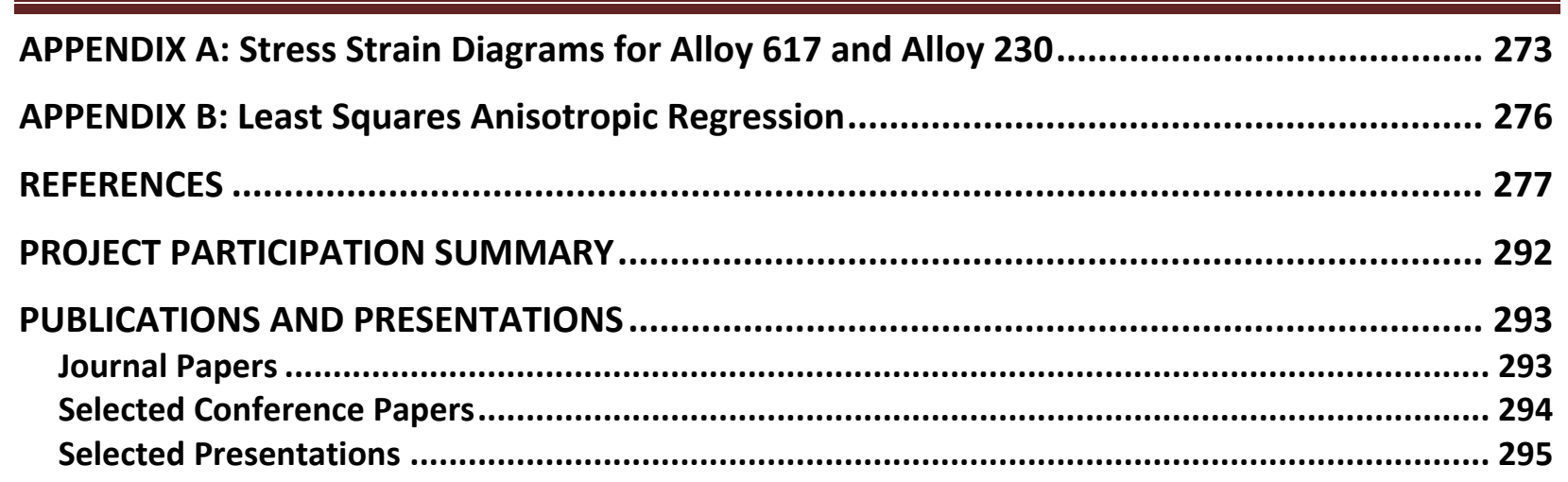




\section{Understanding Fundamental Material-Degradation Processes in High Temperature Aggressive Chemomechanical Environments}

\section{INTRODUCTION}

The objective of this research program was to address major materials performance and design methodology issues for the design and construction of high temperature and very high temperature nuclear systems such as the Very High Temperature Reactor (VHTR). The work provides a synergy between the development of simplified, but robust, design rules for high temperature systems and materials testing, performance, and improvement of these systems. These systems will have to deal with time dependent material properties (creep, creep-fatigue, high temperature corrosion) in components with complex stress states, long intended service lives, and aggressive operating environments. Routine mechanical properties data and current high temperature design methodology do not provide adequate information or approaches for long term, robust system design. This program will address these issues. In addition, high temperature materials testing in relevant corrosive environments (low oxygen partial pressure with substantial carbon activities) will be performed to support further code qualification of existing alloys and the development and qualifications of developmental materials alloys.

The development of materials and design methodologies for high temperature components has been a major challenge, and perhaps the most limiting factor, in the development and advancement of current and future energy systems, such as the Ultra Supercritical Boiler Program, the development of Heat Recovery Steam Generators (HRSG), and high temperature reactor systems, including the Very High Temperature Reactor (VHTR) in the Generation IV nuclear initiative. A balance must be struck between the goal of reaching the highest possible temperature for thermodynamic efficiency reasons and the limitation of materials of construction to withstand a variety of static and dynamic structural loads for extended periods in high temperature service. Nowhere is this balance more important than in the development of high temperature and very high temperature nuclear systems, where the service life of major components should exceed 30, or now possibly 60 years, of continuous service without any compromise on the safe operational limits of the system. Other very high temperature energy systems, for instance jet engine components, have been able to meet this challenge by novel materials processing techniques (e.g. directional solidification or single crystal technology), advanced heat removal technology (e.g. microchannels for blade and vane cooling), and frequent component replacements (e.g. blade and vane service limits of 10,000 hours). However, these high temperature issues are confined to relatively small volumes of materials where highly specialized solutions are possible. Even in non-nuclear applications, high temperature systems for fossil or petrochemical uses, with larger high temperature components, routine replacement of components is common. These options are not available for nuclear service applications which place more severe materials selection and design constraints on system components.

This motivates the dual need for materials which can withstand extended operation in high temperature environments and the need for design methodologies to adequately assess their use. Since these two issues are intimately linked, this proposal addresses an approach for meeting both needs in a coordinated program. In addition, this program was closely coordinated with on-going and planned research efforts at ORNL and INL, in order to maximize the program productivity through well-coordinated Lab-University interactions. 


\section{Understanding Fundamental Material-Degradation Processes in High Temperature Aggressive Chemomechanical Environments}

\section{PERSPECTIVE}

The development and characterization of materials for very high temperature energy conversion applications is a major component of developing reliable, robust energy sources for efficient electricity generation. High temperature materials are also important for the development of thermo-chemical conversion processes which can also be driven at much higher conversion efficiencies. Of particular interest to the US nuclear industry is the development of a very high temperature gas-cooled nuclear reactor which could reach temperatures approaching $1000^{\circ} \mathrm{C}$. At these elevated temperatures, it would be possible to use direct cycle helium turbines to produce electricity at efficiencies of $\sim 60 \%$ thermal to electric conversion. It would also be possible to drive hydrogen production at high efficiencies based on one of a number of thermo-chemical cycles, the most promising of which is the S-I (sulfur-iodine) cycle.

The need to find materials which are useful in systems designed for very high temperatures has been an ongoing quest for all energy conversion sectors, including transportation, chemical processing, and electricity production. Metallic alloys are of considerable interest because of their ability to resist catastrophic fracture, their workability and formability, their availability, and the vast design experience. The ability of metallic alloys to perform at high temperature is based on their melting or softening points, their strength, and their resistance to exposure in corrosive atmospheres. Exposure in extreme environments severely limits the selection of materials for these applications and is also very restrictive in terms of the design limitations on long term service conditions.

In the current program, two of the most promising metallic alloys for high temperature applications, Alloy 617 (Inconel 617) and Alloy 230 (Haynes Alloy 230), were examined to characterize their behavior under extreme conditions similar to those they might encounter in service. Alloy 617 has been studied for similar applications for a number of years, though there is still not sufficient information to fully qualify it for elevated temperature service. Alloy 230 is a relatively new alloy with similar mechanical properties, workability, formability, and physical properties to Alloy 617. It may also offer some advantages for elevated temperature services when compared to Alloy 617. This program was designed to compare the performance of these two alloys under conditions which were characteristic of service conditions in a very high temperature gas-cooled nuclear reactor.

In the period between the 1950's and the early 1980's, there was a very significant advancement in high temperature materials and design methodologies for a variety of applications, mostly for energy systems. This period saw the development of superalloys, refractory alloys, structural ceramics, composites, and other high temperature materials and alloy systems to meet and extend these service needs. It also saw the successful development of several high temperature nuclear systems including gas-cooled reactors, where component temperatures exceeded $650^{\circ} \mathrm{C}$. That success spawned efforts in the US, UK, France, Germany and Japan to develop very high temperature gas cooled reactor systems, typically He-cooled, with anticipated core outlet temperatures in excess of $850^{\circ} \mathrm{C}$ and preferably approaching $1000^{\circ} \mathrm{C}$. Because of the very high coolant temperature, these systems could embrace non-standard energy conversion methodologies which avoided steam-based energy conversion cycles. In many cases, these systems were pursued for their ability to provide process heat to support a number of chemical conversion processes. The US program had a dual focus - "nuclear process heat" (NPH) for chemical conversion applications and "direct cycle helium turbine" (DCHT) technology for electricity production using a Brayton cycle. In addition to the thermodynamic advantages, these 


\section{Understanding Fundamental Material-Degradation Processes in High Temperature Aggressive Chemomechanical Environments}

systems were appealing for their low thermal inertia which provides a long heat-up time-constant under accident conditions resulting in nearly "inherently safe" systems.

The efforts to develop these systems were limited by materials performance issues and by limitations in design methodologies to address the complex, additional materials deformation and degradation issues at high temperatures. Large materials research programs were developed to start to address the need to characterize high temperature materials mechanical and corrosion performance; a substantive review is available [2.1]. Principal additional materials issues at elevated temperature include the effect of time dependent deformation (e.g. creep), low tensile strengths, dynamic materials aging issues (e.g. phase transformations, grain boundary segregation, dissolution of strengthening phases, grain growth, recrystallization), synergetic and adverse interactions between creep and fatigue, dynamic crack propagation (e.g. creep and creep-fatigue crack growth), and others. In addition, for high temperature gas-cooled reactor systems, the coolant, He, is slightly contaminated by minor impurity gases from leaks and other internal and external sources. The composition of these impurity gases (primarily $\mathrm{H}_{2} \mathrm{O}, \mathrm{H}_{2}, \mathrm{CH}_{4}$, $\mathrm{CO}$, and $\mathrm{CO}_{2}$ ) results in an atmosphere which is reducing for most alloying elements, but has a carbon activity greater than or equal to 1 . The reducing, but carburizing, atmosphere results in a highly corrosive environment. Most high temperature alloys, which are designed to form and maintain self-protecting oxide films under exposure to oxidizing atmospheres such as jet engines, cannot form self-protecting barriers, and pick up significant amounts of carbon, resulting in internal carbide formation, and minor amounts of oxygen, resulting in internal oxidation. Both of these effects result in materials degradation which can lead to reduced strength or, more dramatically, crack initiation or enhanced corrosion sites. All of these effects become more problematic as the temperature increases.

In addition to the multiple international programs to understand elevated temperature materials performance issues, major efforts were undertaken to develop useful design guidelines for high temperature components for these systems. In the US, ASME Boiler and Pressure Vessel code case N-47 (also referred to as Code Case 1592) was an attempt to develop a system of design guides for high temperature design. The structure of these ASME B\&PV design rules were developed out of techniques already in use for lower temperature designs where creep (i.e. time-dependent deformation) was not an issue. Most of the developments were and are directly analogous to other design principles in ASME B\&PV Code Division III (for nuclear component design) and VIII (for non-nuclear pressure system design). For example, rules which address limits on stresses to some fraction of the yield strength at low temperatures were transformed into limits on a "quasi-yield strength" from isochronous stress-strain curves (i.e. time dependent stress strain curves, one for each $50^{\circ} \mathrm{F}$ and for various component life times up to 300,000 hours). In addition, relatively severe limits on (monotonic) creep strain were imposed: $1 \%$ total elongation (including strain on loading), $80 \%$ of the stress for tertiary creep and $67 \%$ of the rupture stress (assumes low ductility rupture since the $1 \%$ strain limit would normally be limiting). These design rules have now been incorporated in the ASME B\&PV Code, Section III, Subsection NH.

The development of design rules for high temperature components maintained the standard stress classifications used at low temperatures: Primary Stress (P), Secondary Stress (Q, self-equilibrating stresses), and Peak or Local Stress (F), which in their own right, are often difficult to interpret. This leads to a real dilemma in design since stress states, or their classifications, are not generally known during the initial, major stages of the design process, so 


\section{Understanding Fundamental Material-Degradation Processes in High Temperature Aggressive Chemomechanical Environments}

design under the rules becomes a circular process where a proposed design requires stress analysis leading to (perhaps with much complication) classifications of the stress states which, if not consistent with the design rules, leads to redesign. This becomes extremely cumbersome for complex, high temperature components where stress relaxation, due to creep, can play a major role in redistributing strains and altering stress states.

A problem which is always present in high temperature applications, but which becomes a major concern in very high temperature problems, is the issue of creep damage assessment. Damage reveals itself in two ways, through tertiary creep, or accelerating creep rate under nominally constant stress, and by void initiation and growth. At very high temperature, tertiary creep, due to time related metallurgical changes, appears virtually from the outset of operation and continues throughout the life of the component. This phenomenon cannot be ignored without incurring significant errors in life assessment. Furthermore, contrary to common belief, tertiary creep is not necessarily linked with microstructural damage involving void formation and growth. The latter may evolve according to an entirely different set of rules from tertiary creep and, at the extremely long lives being considered for the VHTR, can lead to failure at low strains. Current design practice, as defined by Section III/NH, does not account for the added awareness of time related evolution of damage mechanisms needed to adequately assess components of the VHTR variety.

A further complication of the past and current versions of Subsection NH is that in order to set reasonable materials stress limits, specific materials data are required for code qualification. The general guidelines are that data can be extrapolated by only a factor of three (x3), so a 300,000 hour (about 40 year) component life requires 100,000 hour data. This data should be available at $50^{\circ} \mathrm{F}$ intervals up to the temperature of interest (i.e. from the non-creep, lower temperature regime up to the highest service temperature for which the material would be qualified), though some exceptions have been made to allow for interpolation of data which is only available at $100^{\circ} \mathrm{F}$ intervals. A further major restriction is that the data should be representative of the design limits (e.g. $1 \%$ total strain, $80 \%$ of the tertiary creep stress or $67 \%$ of the rupture stress), so tests performed for extended periods should aim for these limits, and not higher strain limits or shorter ruptures lives where creep (or isochronous tensile deformation) is controlled by other deformation mechanisms.

Because of these stringent requirements, only five alloys are currently qualified, with one more in the process of being qualified, for elevated temperature service under Section III, Subsection NH (others are qualified under either Section I or Section VIII where other high temperature systems are covered): $316 \mathrm{SS}$ and $304 \mathrm{SS}$ to $816^{\circ} \mathrm{C}$, Alloy $800 \mathrm{H}$ to $760^{\circ} \mathrm{C}, 2 \frac{1}{4} \mathrm{Cr}-$ $1 \mathrm{Mo}$ ("annealed") and $9 \mathrm{Cr}-1 \mathrm{MoV}$ ferritic steels to $650^{\circ} \mathrm{C}$, with Alloy 617 (Inconel 617) still in the qualification process ${ }^{1}$. It is clear that, apart possibly from Inconel 617 , none of the materials would perform adequately for service temperatures approaching $1000^{\circ} \mathrm{C}$. Materials development during the lengthy process of gaining approval of the code case could provide better performing materials. But even then, extensive testing would have to be performed to make a reasonable case for code qualification. The addition of Alloy 230 (Haynes Alloy 230) provides an alternate possibility for very high temperature design applications.

\footnotetext{
${ }^{1}$ Note that the results of the current program on Alloy 617 are a part of a much larger effort by INL and ORNL to code qualify Alloy 617 for high temperature applications.
} 


\section{Understanding Fundamental Material-Degradation Processes in High Temperature Aggressive Chemomechanical Environments}

In fact, the materials qualification procedures, as stringent as they are, do not require direct qualification for a number of extremely important materials performance characteristics. Rather, these performance characteristics must be accounted for in the design process. This leaves very large gaps in the information with which designers must rely upon, especially in areas where (simplified) approximate methods are applied. Examples of these areas include all but the most rudimentary materials properties issues such as the effect of corrosive environments, fatigue and creep-fatigue interactions, creep and creep-fatigue crack initiation and growth, materials aging issues, and dynamic loading conditions which can lead to ratcheting or other incremental materials and structural failure modes. In most cases, these are the most important design issues since local stress at stress concentrators, welds, joints, discontinuities, etc. present much more severe loading conditions as well as more extreme time-dependent dynamic loading conditions than other structural features. The component life will depend much more on these special features than the more major component characteristics, thus the design methodology to handle them must be conservative, but reasonable. Methodologies such as "linear damage summation" (and more complicated off-shoots), the Bree diagram, creep and creep-fatigue crack growth parameters (e.g. $\mathrm{C}^{*}$ ), Larson-Miller parameters, and so-forth have been developed to attempt to deal with these critical issues. These fall far short of a robust design methodology, and apply to various materials systems in different ways.

To summarize, the following highlights several of the most critical and least resolved design and materials issues for high temperature and very high temperature systems. A strong link between materials and design methodologies exists. Sound structural design and analysis is limited by the dearth of materials performance information and by cumbersome and uninformed design methodologies. On the other hand, materials test programs often concentrate on the simplest tests, neglecting more complicated stress, corrosion, and aging issues that are of most interest for setting design limits.

This project helped to address these two issues in concert, rather than separating them into two unlinked research topics, as is typically done. The coordinated program has the advantage that design-specific materials information can be developed alongside simplified design methodologies and materials-specific implications. Contrast this to the evolution of ASME B\&PV Section III, Subsection NH, which has been in the works for more than thirty years and still lacks qualification of structural materials and contains design methodologies which have yet to be tested for the increased temperatures anticipated in future applications. Preliminary work sponsored by ORNL has shown distinct areas in which design rules contained in Section III/NH fail to cope adequately with the special problems of very high temperature applications [2.2]. Work in this program has demonstrated the value of a "damage mechanism" approach where specific materials degradation paths set design limitations. These degradation paths are analyzed based on the underlying physical phenomena controlling materials degradation and failure in extreme conditions. 


\section{Understanding Fundamental Material-Degradation Processes in High Temperature Aggressive Chemomechanical Environments}

\section{EXPERIMENTAL APPROACH}

\subsection{Mechanical Tests}

The prospective design of the IHX for the VHTR requires the thickness of the chosen material to be in the range from 0.2 to $2 \mathrm{~mm}$. [3.1] Considering a compatible dimension, miniature specimens were used to study the alloys' tensile properties. (Fig. 3.1) The Alloy 617 specimens with the same geometry and dimensions have been employed to study grain boundary engineering (GBE) and irradiation effects. [3.2] Another important reason for using miniature tensile specimens is that the material's strength in the plate thickness direction can be readily tested.

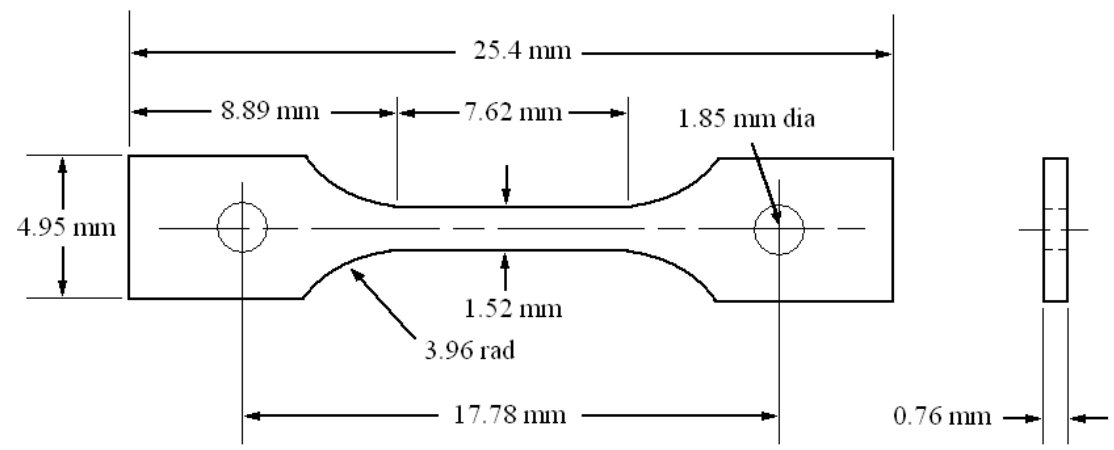

Fig. 3.1: Geometry and dimensions of tensile specimens

Tensile specimens were cut along the long-transverse (LT) direction from the rolling plane. (Fig. 3.2) Tensile tests with a strain rate of $\sim 10^{-3} / \mathrm{s}$ were conducted at temperatures ranging from room temperature (RT) to $1000^{\circ} \mathrm{C}$ using an INSTRON 1331 servo-hydraulic test system equipped with a high temperature furnace. Temperature was maintained for $\sim 40$ minutes after heating to the desired value to gain a stabilized and homogenous heat zone. The temperature variation was $\pm 3^{\circ} \mathrm{C}$ during testing.

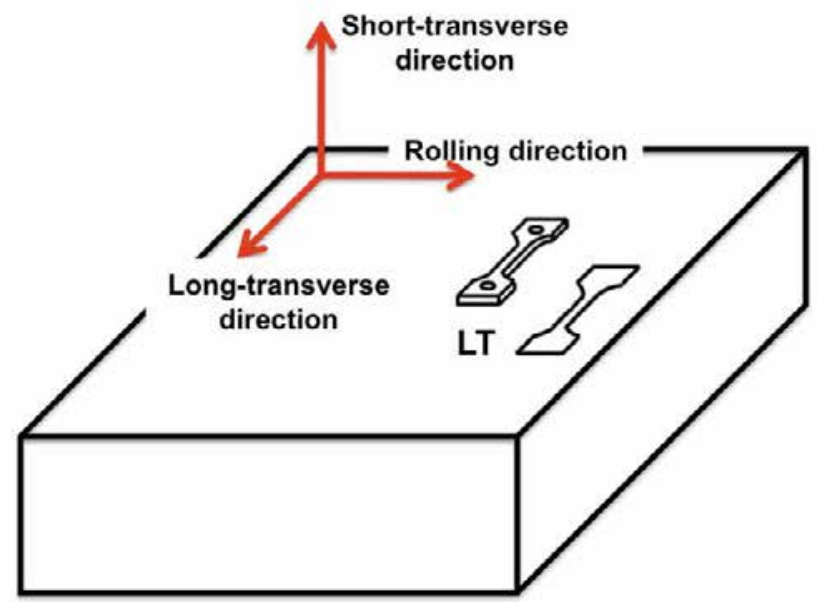

Fig. 3.2: In-plane sampling directions of tensile specimens 


\section{Understanding Fundamental Material-Degradation Processes in High Temperature Aggressive Chemomechanical Environments}

\subsection{Synchrotron Radiation Study}

High-energy X-ray diffraction measurements were carried out at the 1-ID beamline of the Advanced Photon Source (APS) at Argonne National Laboratory (ANL). The experimental setup and its schematic are shown in Figs. 3.3 and 3.4, respectively. Uniaxial tensile tests were performed on an MTS closed-loop servo-hydraulic test frame (Model 858) at a crosshead speed of $0.005 \mathrm{~mm} / \mathrm{s}$, corresponding to an initial specimen strain rate of $\sim 10^{-3} / \mathrm{s}$. Diffraction measurements were conducted continuously with a monochromatic $86 \mathrm{keV}(\lambda=0.0144 \mathrm{~nm})$ $\mathrm{X}$-ray beam every 10 seconds. The incident beamsize was $300 \times 300 \mu^{2}$. Complete DebyeScherrer diffraction rings from the solid-solution austenitic matrix and the carbide from the diffraction volume were recorded using an area detector (GE angio type). An ion chamber and a PIN diode (p-type, intrinsic, n-type diode) embedded within the beamstop were used to measure the initial and transmitted beam intensities, respectively. The distance between the sample and the detector was $1.336 \mathrm{~m}$.

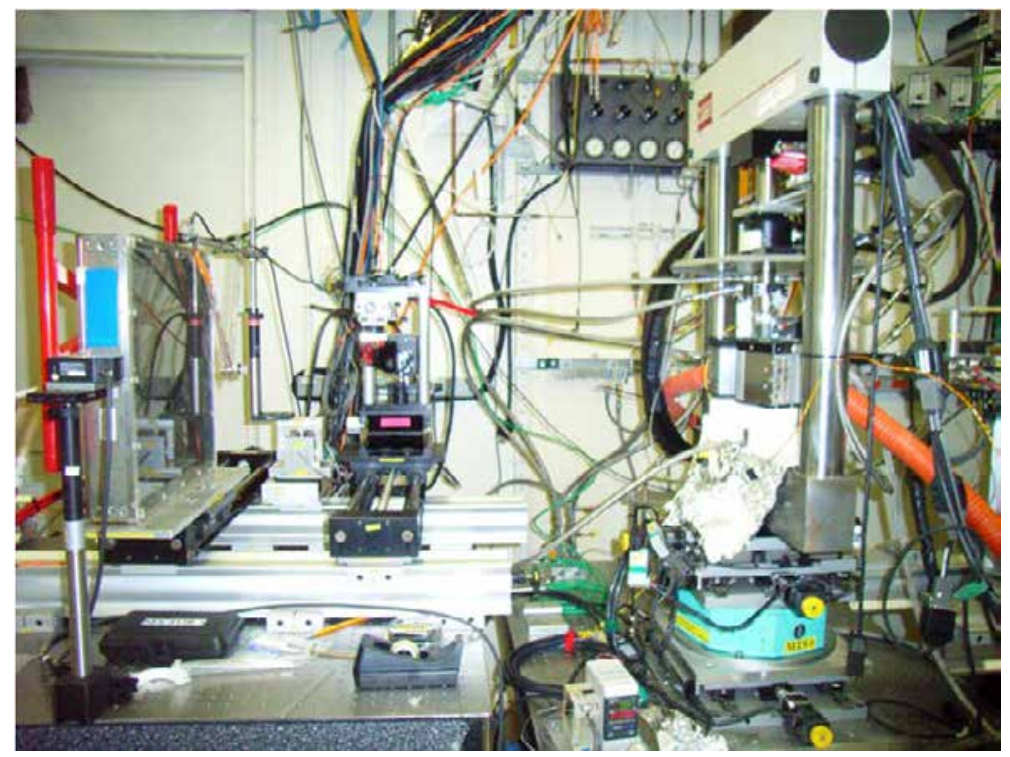

Fig. 3.3: The experimental diffraction setup at APS 1-ID beamline at ANL

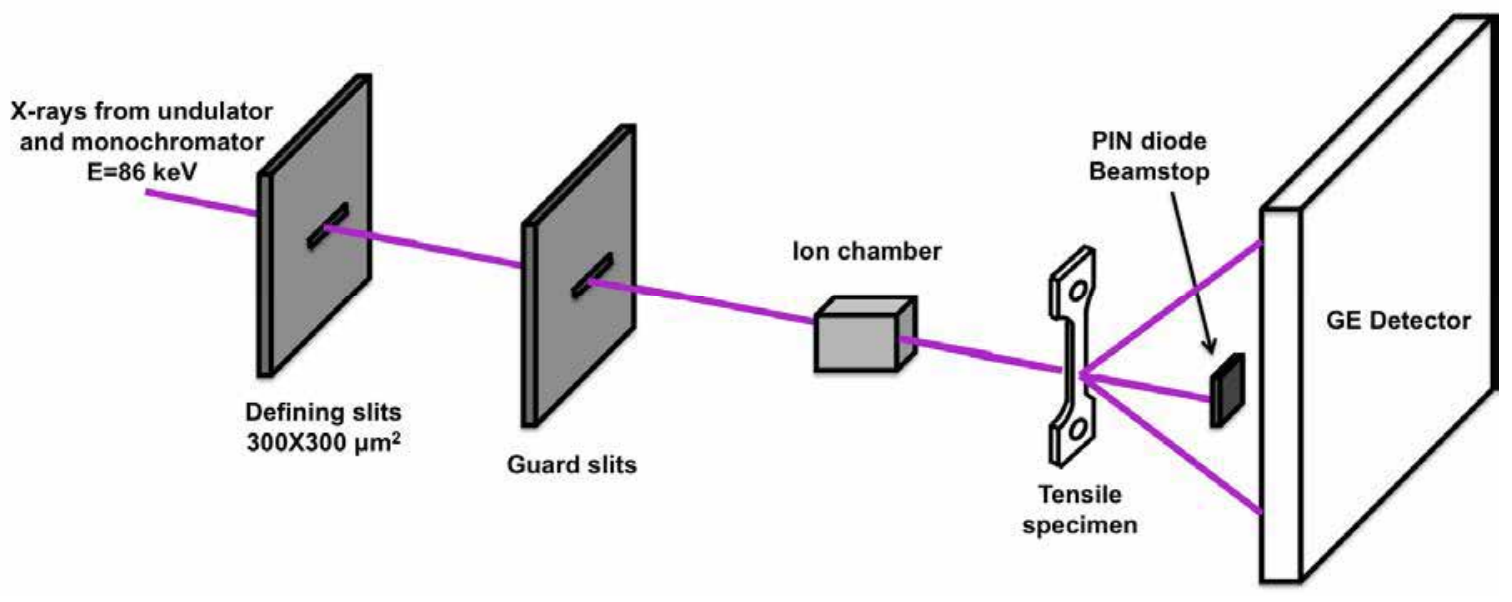

Fig. 3.4: Schematic of the experimental diffraction setup 


\section{Understanding Fundamental Material-Degradation Processes in High Temperature Aggressive Chemomechanical Environments}

Fig. 3.5(a)\&(b) show typical 2D diffraction patterns from an Alloy 230 specimen before and after tensile loading ( $45 \%$ strain), respectively. The Debye rings associated with various radii are reflected by lattice planes with various interplanar spacings, $d_{h k l}$. Before tensile loading, the diffraction pattern was incomplete rings (relatively sporadic with varying intensity for diffracted areas). (Fig. 3.5(a)) After tensile loading with a large strain, the alloy experienced significant plastic deformation. The dislocation density development and subgrain structure (e.g. dislocation cell) formation resulted in increased random crystalline orientations in the alloy, which can be seen in the uniform Debye rings. (Fig. 3.5(b)) Nevertheless, texture formation during plastic deformation caused additional variations of diffraction intensities in the azimuthal direction. The diffraction pattern in Fig. 3.5(b) shows the duplex fiber texture: [111]+[100]. Another important phenomenon that occurs after external loading is the change in shape of the Debye rings. Under the external applied stress, the spherical diffraction rings became ellipsoidal. The change in shape of the diffraction rings is the foundation for measuring lattice strain caused by the macrostresses, or Type I stress. Schematic interpretations of the changes in diffraction patterns for tensile response are shown in Fig. 3.6. The internal stress formed during vertical loading causes the radii of Debye rings to be shortened in the axial direction (Azimuth $(\eta)=90$ and $270^{\circ}$ ) and lengthened in the transversal direction (Azimuth $(\eta)=0$ and $180^{\circ}$ ). To deduce the lattice strain, $\varepsilon$, from the change in radii, an algorithm similar to those found in refs. 3.3, 3.4 3.5, \& 3.6 was applied to the diffraction patterns of Alloy 230. The detailed analysis procedure that was performed is detailed below:

1. Determined the beam center, detector tilt, and sample-to-detector distance using software FIT2D [3.3, 3.4 3.7] according to (200) and (333) reflections of reference material $\mathrm{CeO}_{2}$.

2. Converted the diffraction pattern from polar to Cartesian coordinates through re-binning processing. The converted images of the original diffraction patterns from Alloy 230 (Fig. 3.5) are shown in Fig. 3.7. 36 azimuthal bins were used in the study, corresponding to an angle increment of $10^{\circ}$.

3. Fitted diffraction peaks using a pseudo-Voigt function to find the average center of the peak intensity, $R(\eta)$. (Fig. 3.8) For each reflection, the radius profile changes with applied stresses with an intersection at a single radius. This radius, $R_{0}$, and its corresponding azimuth, $\eta_{0}$, are considered invariant during deformations. (Fig. 3.9) Thus, the lattice strain caused by applied stresses can be calculated by:

$$
\varepsilon(\eta)=\frac{R(\eta)-R_{0}}{R_{0}}
$$

4. Applied the bi-axial strain model to determine the axial lattice strain, $\varepsilon_{11}$, and transversal lattice strain, $\varepsilon_{22}$, in the sample coordinate system. $\left(\varepsilon_{11}=\varepsilon\left(\eta=90\right.\right.$ or $\left.270^{\circ}\right) \& \varepsilon_{22}=\varepsilon(\eta=0$ or $\left.180^{\circ}\right)$ ) (Fig. 3.10) 
Understanding Fundamental Material-Degradation Processes in High Temperature Aggressive Chemomechanical Environments

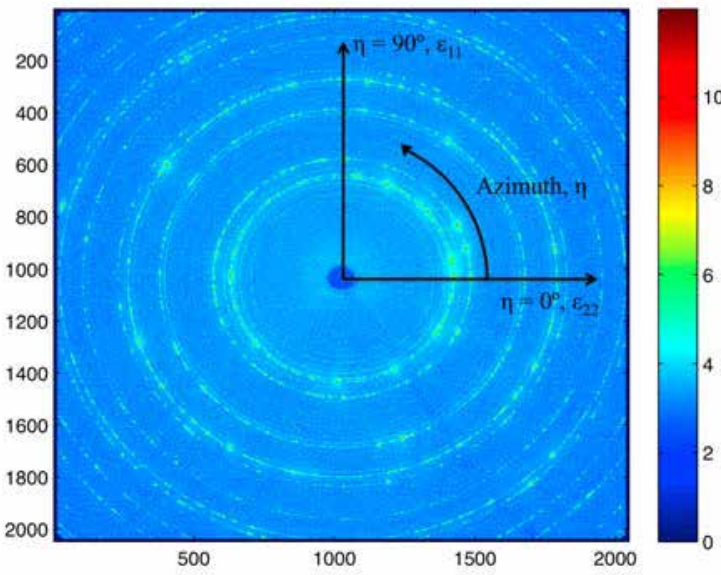

(a)

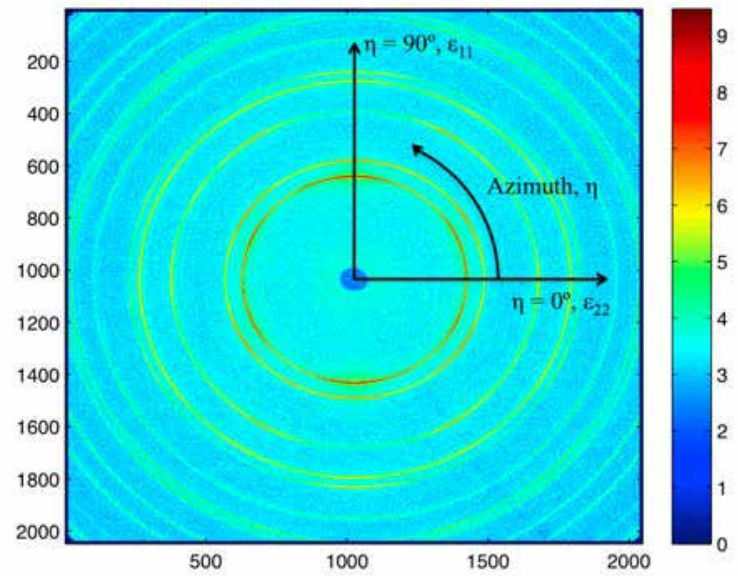

(b)

Fig. 3.5: Diffraction patterns from Alloy 230 (a) before and (b) after deformation

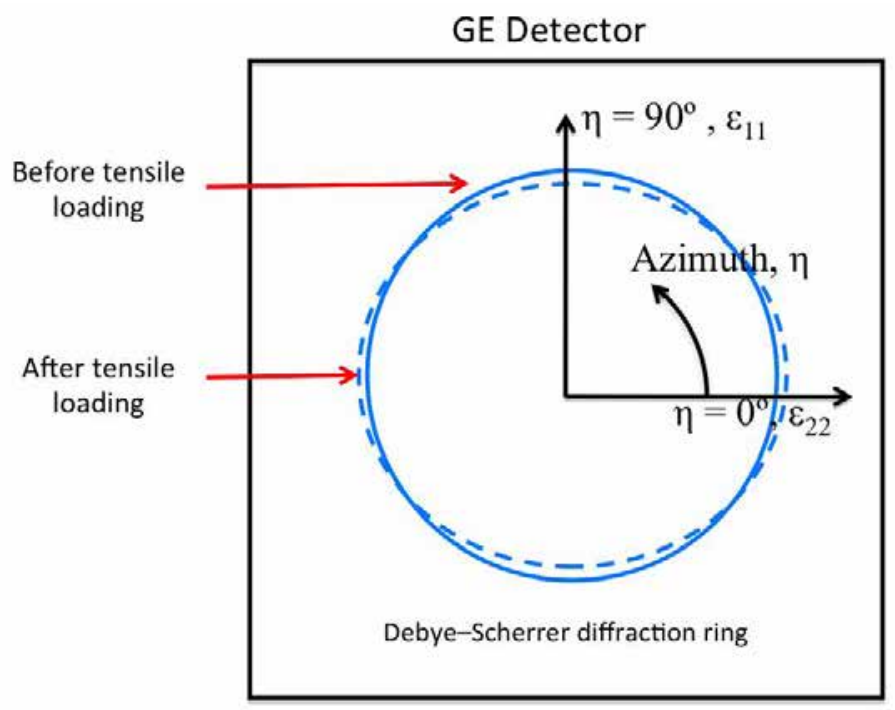

Fig. 3.6: Schematic interpretation of the changes in diffraction patterns for the tensile response 


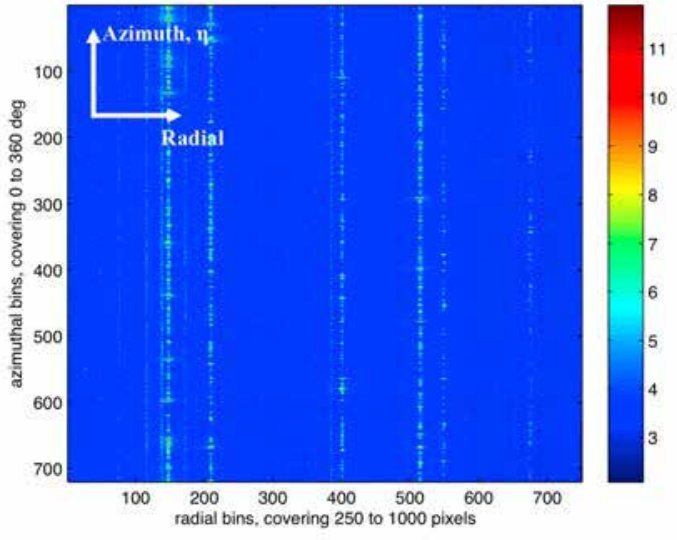

(a)

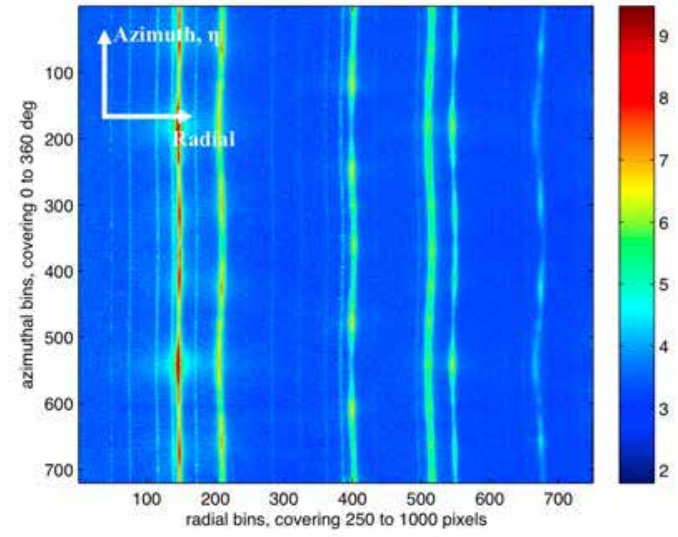

(b)

Fig. 3.7: Converted diffraction patterns from Alloy 230 (a) before and (b) after deformation
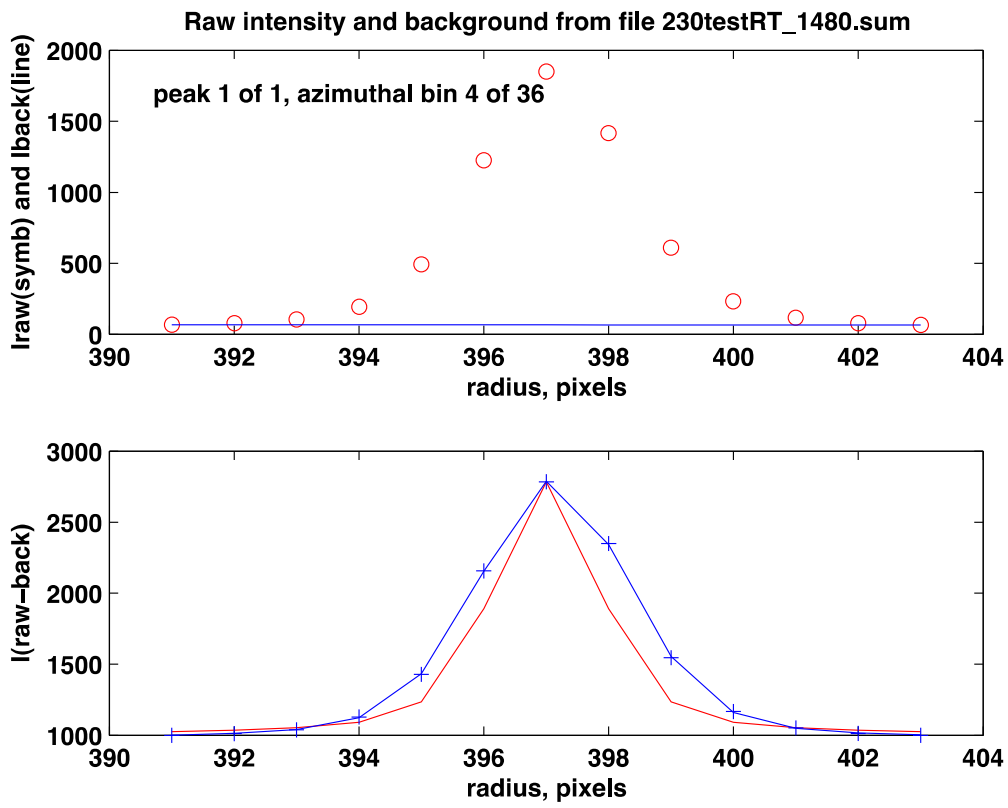

Fig. 3.8: Peak fitting using a pseudo-Voigt function for the average center of the peak intensity 


\section{Understanding Fundamental Material-Degradation Processes in High Temperature}

Aggressive Chemomechanical Environments

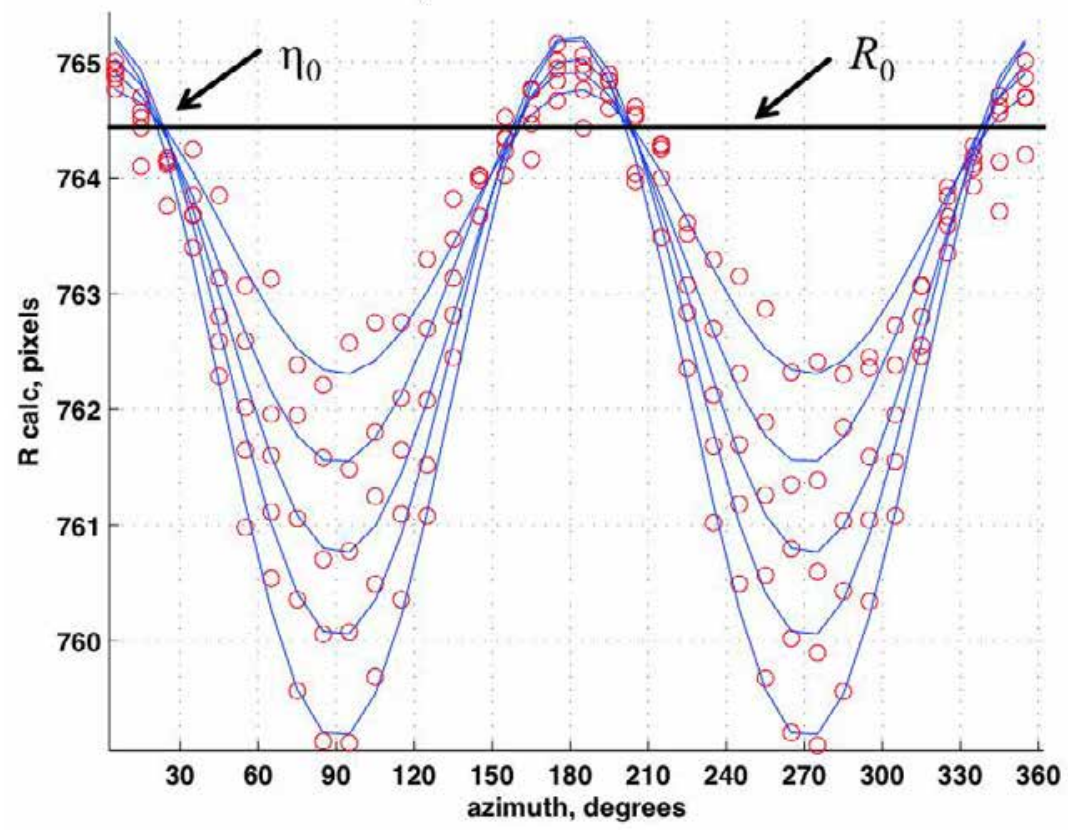

Fig. 3.9: Radius peak position versus azimuthal degree for the (311) reflection as a function of applied stresses for Alloy 230

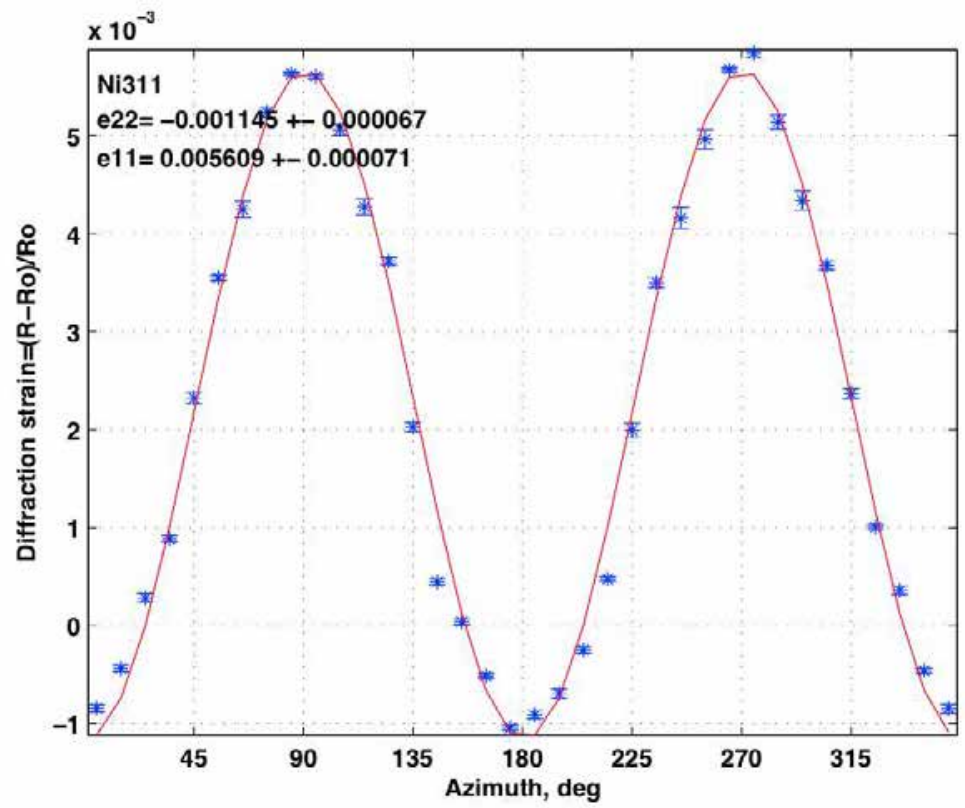

Fig. 3.10: Lattice strain analysis based on a profile fit of diffraction $\operatorname{strain}(\varepsilon)$ versus $\operatorname{Azimuth}(\eta)$ 


\section{Understanding Fundamental Material-Degradation Processes in High Temperature Aggressive Chemomechanical Environments}

\subsection{Uniaxial Creep Tests}

Creep-fatigue tests are usually performed on uniform gage section test specimens. ASTM standard E 2714-09, the Standard Test Method for Creep-Fatigue Testing, specifies the geometry and dimensions of specimens used for creep-fatigue testing [3.8]. In accordance with that standard, two specimen designs for two different test frames were used in this study. Specimens were made from as-received materials in the solution-treatment condition with the long axis aligned with the rolling direction of raw materials. Figs. 3.11 and 3.12 illustrate the detailed schematic of two specimen designs used for creep-fatigue testing.

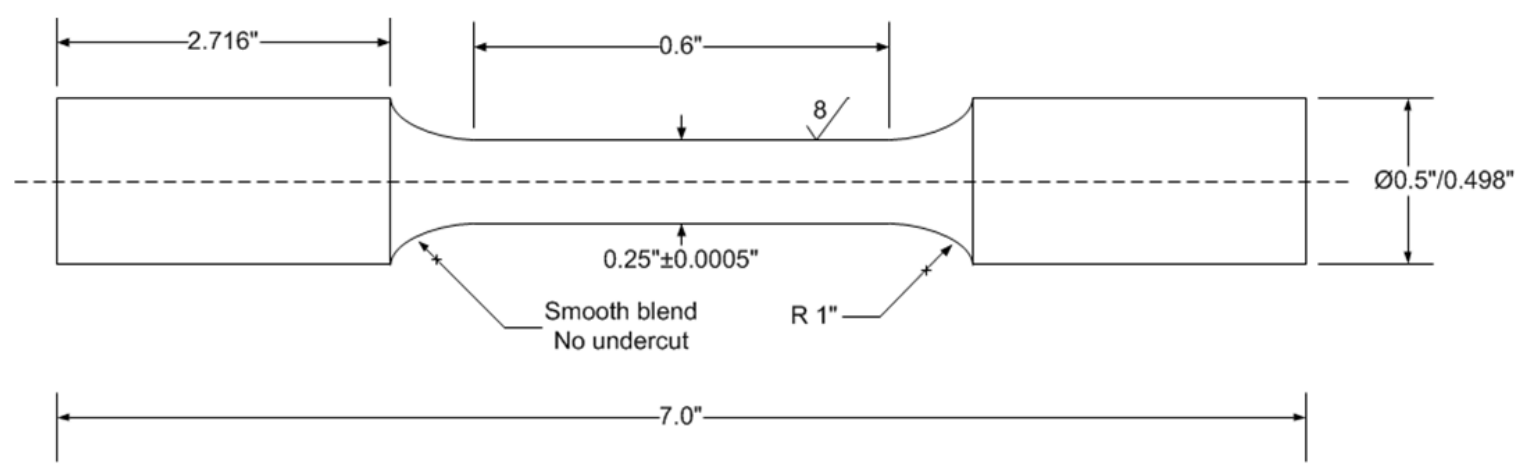

Additional Notes

- Parallelism: $<=0.01 \mathrm{~mm}$

- Concentricity: $<=0.01 \mathrm{~mm}$

- Perpendicularity: $<=0.01 \mathrm{~mm}$

- Final machining and polishing shall eliminate all circumferential scratches.

Final grinding followed by longitudinal mechanical polishing is recommended

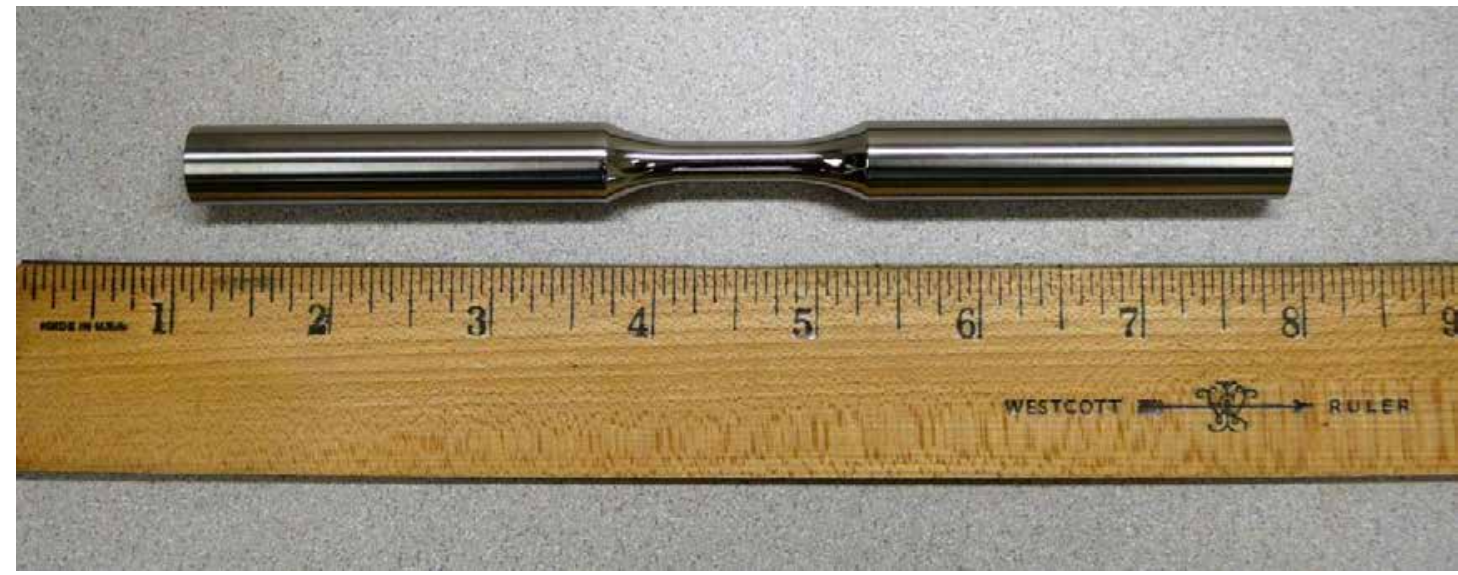

Fig. 3.11: Schematic for the straight-sided creep-fatigue specimen for hydraulic grips 


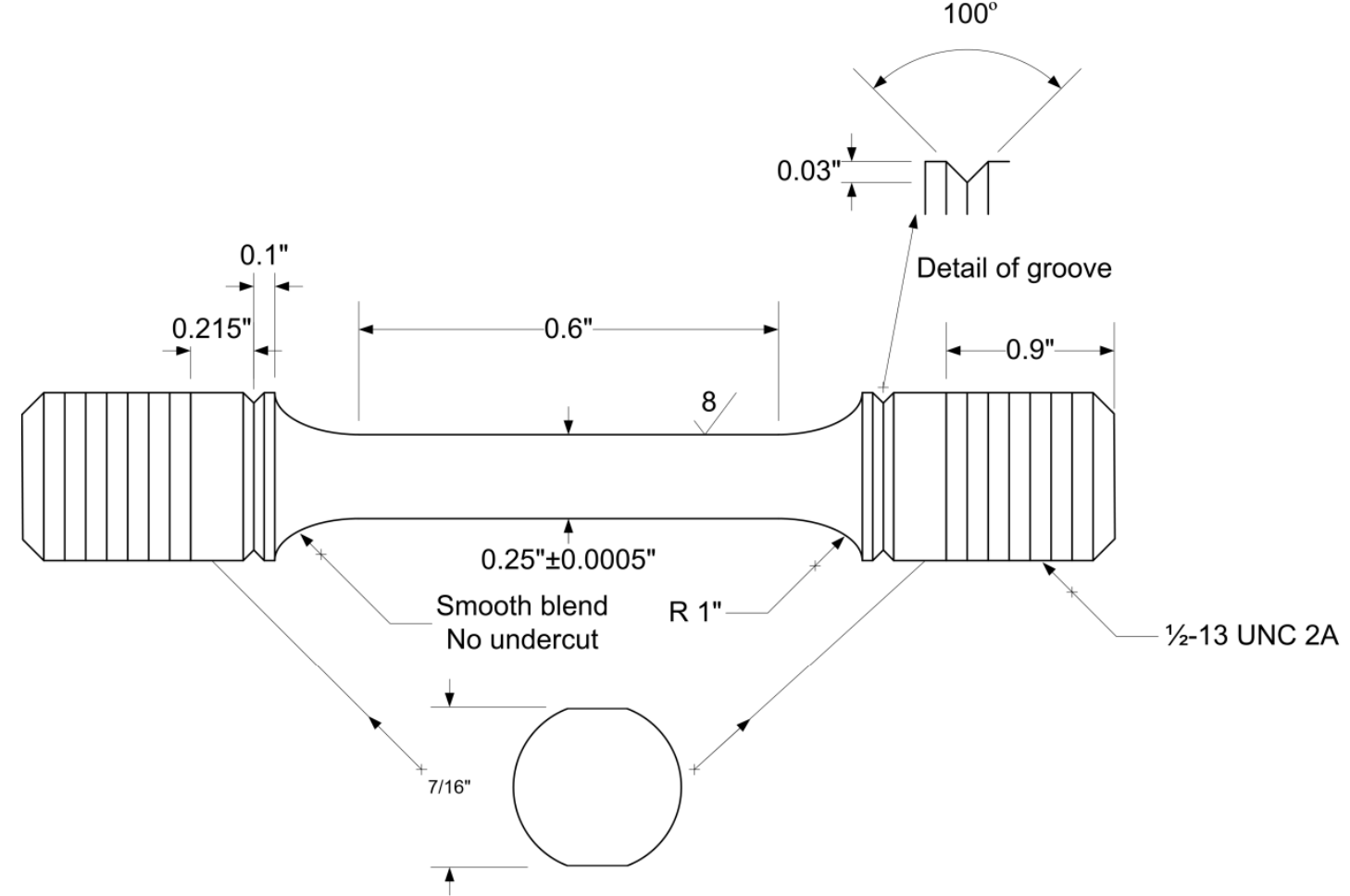

Have two small flat areas only at the unthreaded part 180 deg apart, the distance between the flat surface is $7 / 16 "$ along the diametrical direction.

\section{Additional Notes}

Parallelism: $<=0.01 \mathrm{~mm}$

Concentricity: $<=0.01 \mathrm{~mm}$

Perpendicularity: $<=0.01 \mathrm{~mm}$

Final machining and polishing shall eliminate all circumferential scratches. Final

grinding followed by longitudinal mechanical polishing is recommended

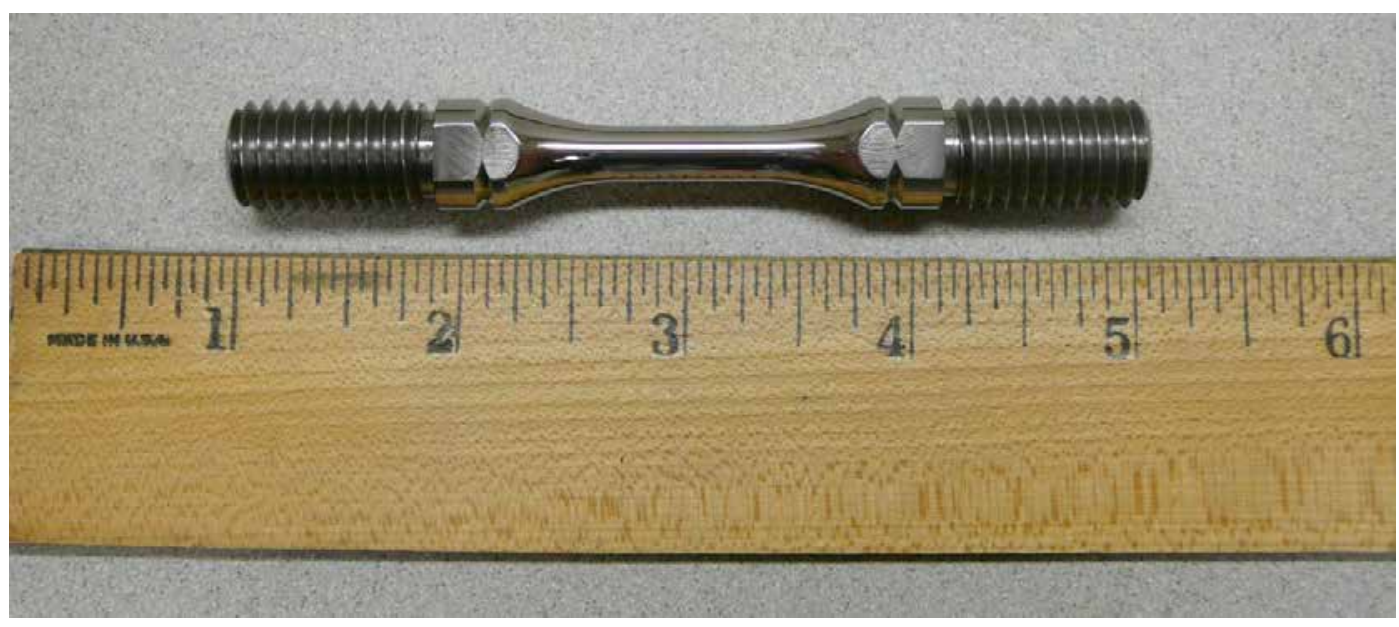

Fig. 3.12: Schematic for the threaded creep-fatigue specimen 


\section{Understanding Fundamental Material-Degradation Processes in High Temperature Aggressive Chemomechanical Environments}

The high temperature creep-fatigue behavior of materials is usually influenced by many different factors, such as temperature, environment, strain rate, total strain range, creep-fatigue waveform, and strain hold time. This study focuses on the effect of total strain range and duration of hold time on the creep-fatigue behavior of materials. In addition, LCF tests at the same strain level as the creep-fatigue tests were done to serve as a benchmark for determining the extent of reduced fatigue life under creep-fatigue tests. To realize these goals, a test matrix as shown in Table 3.1 was proposed. Further, the test matrix was designed to generate enough data for developing modeling for creep-fatigue life predictions. The test temperature was controlled in the following fashion: the specimen was first heated to the target temperature at a heating rate of $10^{\circ} \mathrm{C}$ per minute, and then the specimen was held at that temperature with zero load for about 45 minutes to allow temperatures to stabilize before the commencement of the test. The end-of-test criterion is defined as the attainment of $40 \%$ decrease in the cyclic maximum tensile stress.

Table 3.1: Test matrix for LCF and creep-fatigue testing of Alloy 617 and Alloy 230

\begin{tabular}{|c|c|c|c|c|c|c|c|c|c|}
\hline \multirow{2}{*}{ Material } & \multirow{2}{*}{ Temperature $\left({ }^{\circ} \mathrm{C}\right)$} & \multirow{2}{*}{ Environment } & \multirow{2}{*}{$\dot{\varepsilon}$} & \multicolumn{6}{|c|}{ Total Strain Range and Hold Time } \\
\hline & & & & & $5 \%$ & & $.0 \%$ & & $5 \%$ \\
\hline Alloy 617 & \multirow{2}{*}{850} & \multirow{2}{*}{ Air } & \multirow{2}{*}{$\begin{array}{c}2.5 \mathrm{E}-4 \\
/ \mathrm{s}\end{array}$} & \multirow{2}{*}{$\begin{array}{l}\text { No } \\
\text { Hold }\end{array}$} & \multirow{2}{*}{$\begin{array}{l}3 \mathrm{~min} \\
\text { in } \mathrm{PT}^{*}\end{array}$} & \multirow{2}{*}{$\begin{array}{l}\text { No } \\
\text { Hold }\end{array}$} & \multirow{2}{*}{$\begin{array}{c}3,10,30 \\
\text { min in } \\
\text { PT }^{*}\end{array}$} & \multirow{2}{*}{$\begin{array}{l}\text { No } \\
\text { Hold }\end{array}$} & \multirow{2}{*}{$\begin{array}{l}3 \mathrm{~min} \\
\text { in PT* }\end{array}$} \\
\hline Alloy 230 & & & & & & & & & \\
\hline
\end{tabular}

*PT: Peak tensile strain

Two creep-fatigue test frames were established at ORNL as shown in Figs. 3.13 and 3.14. Both frames used servo-hydraulic power to perform the creep-fatigue test in air. The controller for the frame in Fig. 3.13 was an Instron Model 8500 testing system. The temperature control was accomplished using a three-zone resistance furnace with three S-type thermocouples attached to the gauge section of the specimen with Pt wires, as illustrated in Fig. 3.15. An Epsilon Model 3448 high temperature extensometer was used to measure the axial strain of the specimen. The working principle of the high temperature extensometer is that the central gauge part of the specimen is in direct contact with the ceramic feeler arms of the extensometer (as shown in Fig. 3.16), hence the elongation or contraction of the gauge part can be tracked and measured. In order to attach the extensometer to the specimen, two spring-loaded ceramic wires were used and the extensometer was also supported from a fixture via a glass fiber wire. In this way the extensometer could maintain a relatively stable and horizontal position during the whole duration of the test. Forced air flow was also applied for cooling the extensometer during high temperature creep-fatigue testing to yield a more stable strain reading.

The second set of creep-fatigue test frames used more up-to-date hardware compared with the first one, and provided easier operation and better repeatability. It consisted of MTS 646 hydraulic grips for clamping the specimen ends, an MTS 407 controller for controlling the test waveform, a one-zone resistance furnace, and an MTS 632.53 high temperature extensometer for strain control and measurement. Fig. 3.17 shows the picture of a straight sided specimen installed 


\section{Understanding Fundamental Material-Degradation Processes in High Temperature Aggressive Chemomechanical Environments}

in the second set of creep-fatigue test frames. Since the furnace of the second load frame had one-zone control, one S-type thermal couple was tied to the middle of gauge section of the specimen. The high temperature extensometer was pushed against the specimen by a spring gadget of the extensometer housing so that the ceramic feeler arm of the extensometer was in firm contact with the specimen gauge. Normally a temperature gradient exists inside the furnace at elevated temperatures and it is necessary to know the magnitude of this gradient and make necessary adjustments to the original target temperature $\left(850^{\circ} \mathrm{C}\right)$ to make sure the whole gauge section of the specimen is within the allowable temperature range per ASTM Standard E2714-09. To realize this, one specimen with three S-type thermal couples attached at the upper, middle, and lower parts of the gauge section was used. The reading of the middle thermal couple was used for controlling the temperature, while the readings of the upper and lower thermal couples were used for determining the temperature gradient. Once the temperature gradient inside the furnace at the original target temperature was determined, adjustments were made to the original control temperature. All of the following tests were done with this adjusted temperature and only one S-type thermal couple attached to the middle gauge section of the specimen was used. For the purpose of yielding high quality results, major components of both test frames, such as the load cell, extensometer, and the load frame LVDT sensor, were all calibrated to satisfy the relevant ASTM standards. The alignment of both load frames was also checked with strain-gaged specimens and the alignment fixture was adjusted to ensure that the maximum bending strain was less than $5.0 \%$ of the axial strain. 
Understanding Fundamental Material-Degradation Processes in High Temperature Aggressive Chemomechanical Environments

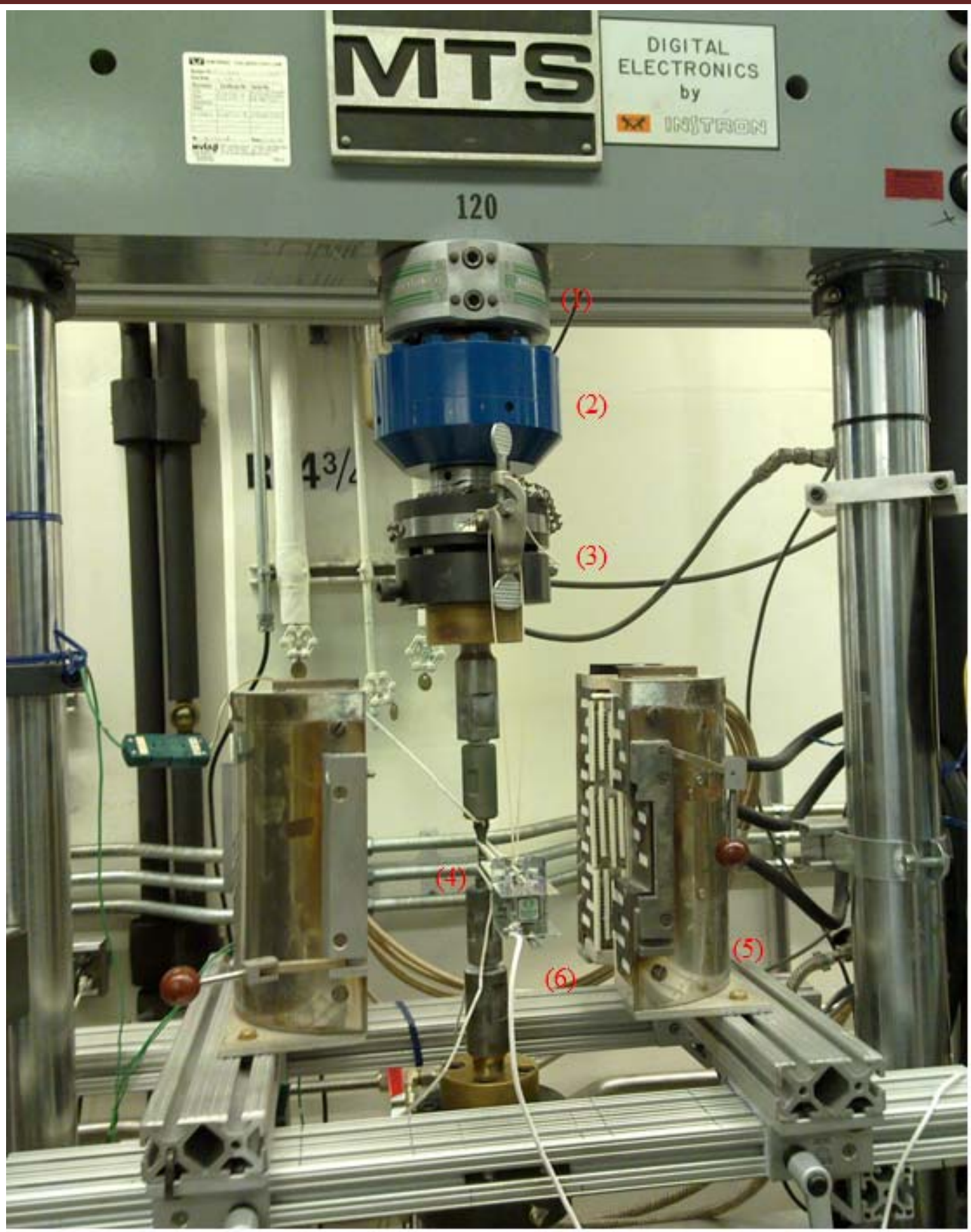

Fig. 3.13: First set of creep-fatigue test frames. Detailed item description: (1) alignment fixture; (2) load cell; (3) specimen grips; (4) specimen; (5) furnace; (6) high temperature extensometer 
Understanding Fundamental Material-Degradation Processes in High Temperature Aggressive Chemomechanical Environments

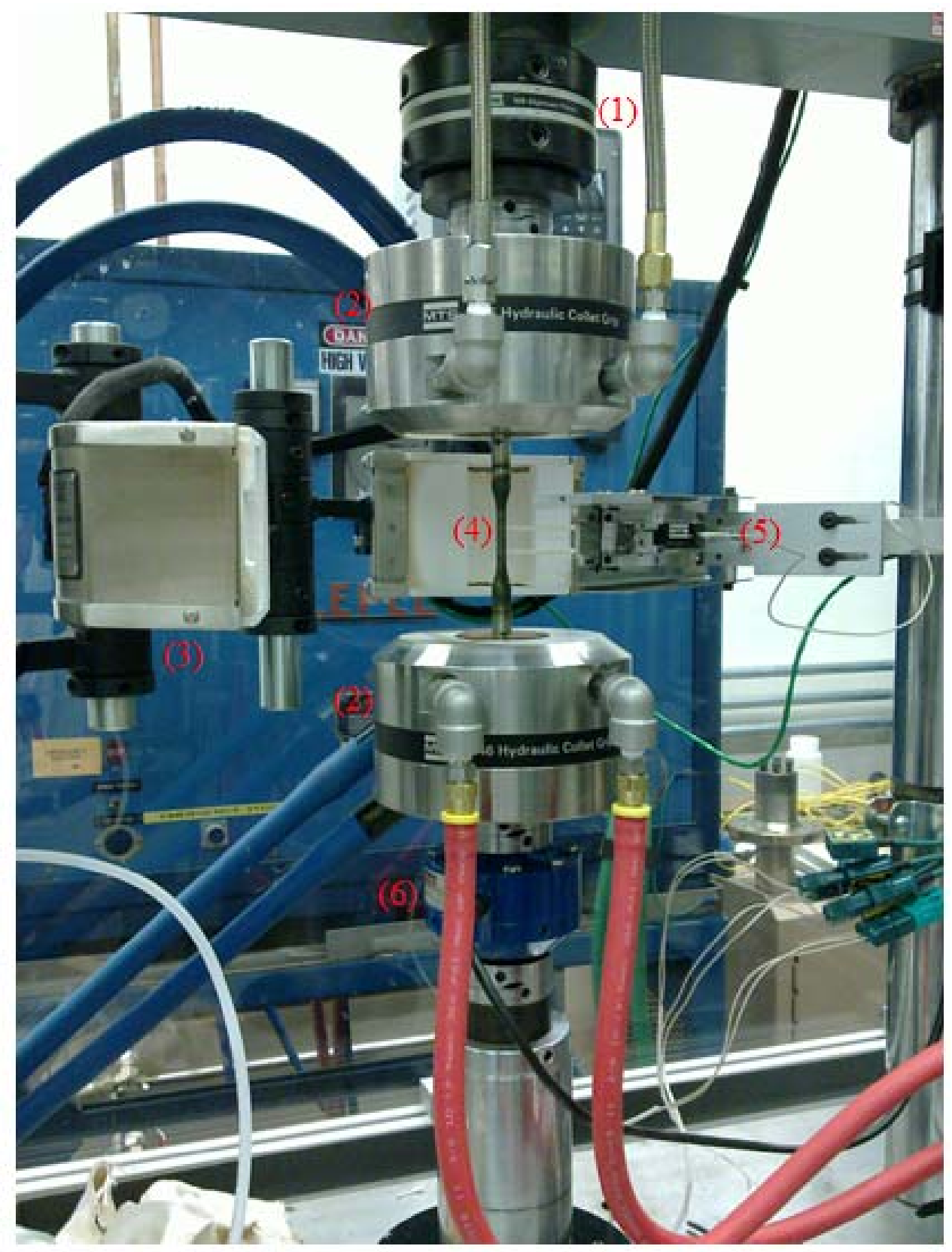

Fig. 3.14: Second set of creep-fatigue test frames. Detailed item description: (1) alignment fixture; (2) hydraulic grips; (3) furnace; (4) specimen; (5) high temperature extensometer; (6) load cell 
Understanding Fundamental Material-Degradation Processes in High Temperature Aggressive Chemomechanical Environments

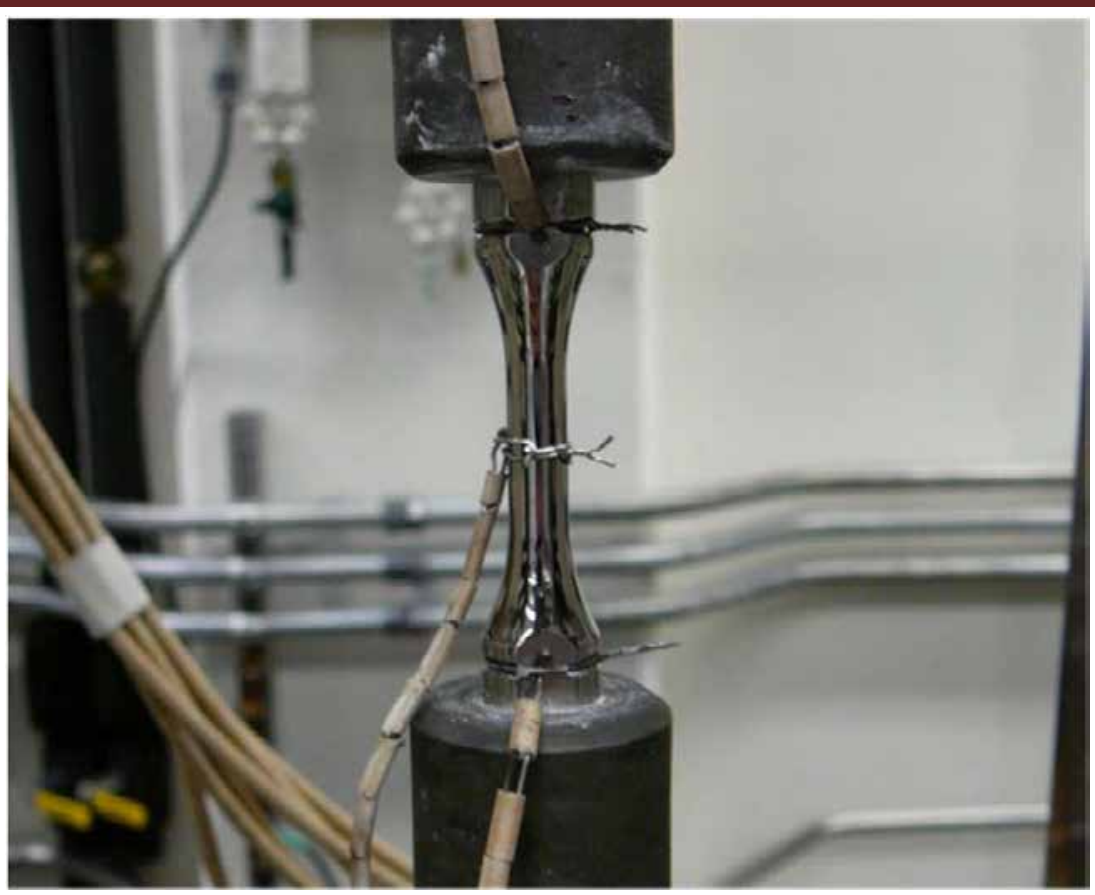

Fig. 3.15: Attachment of three S-type thermal couples to the specimen with Pt wires

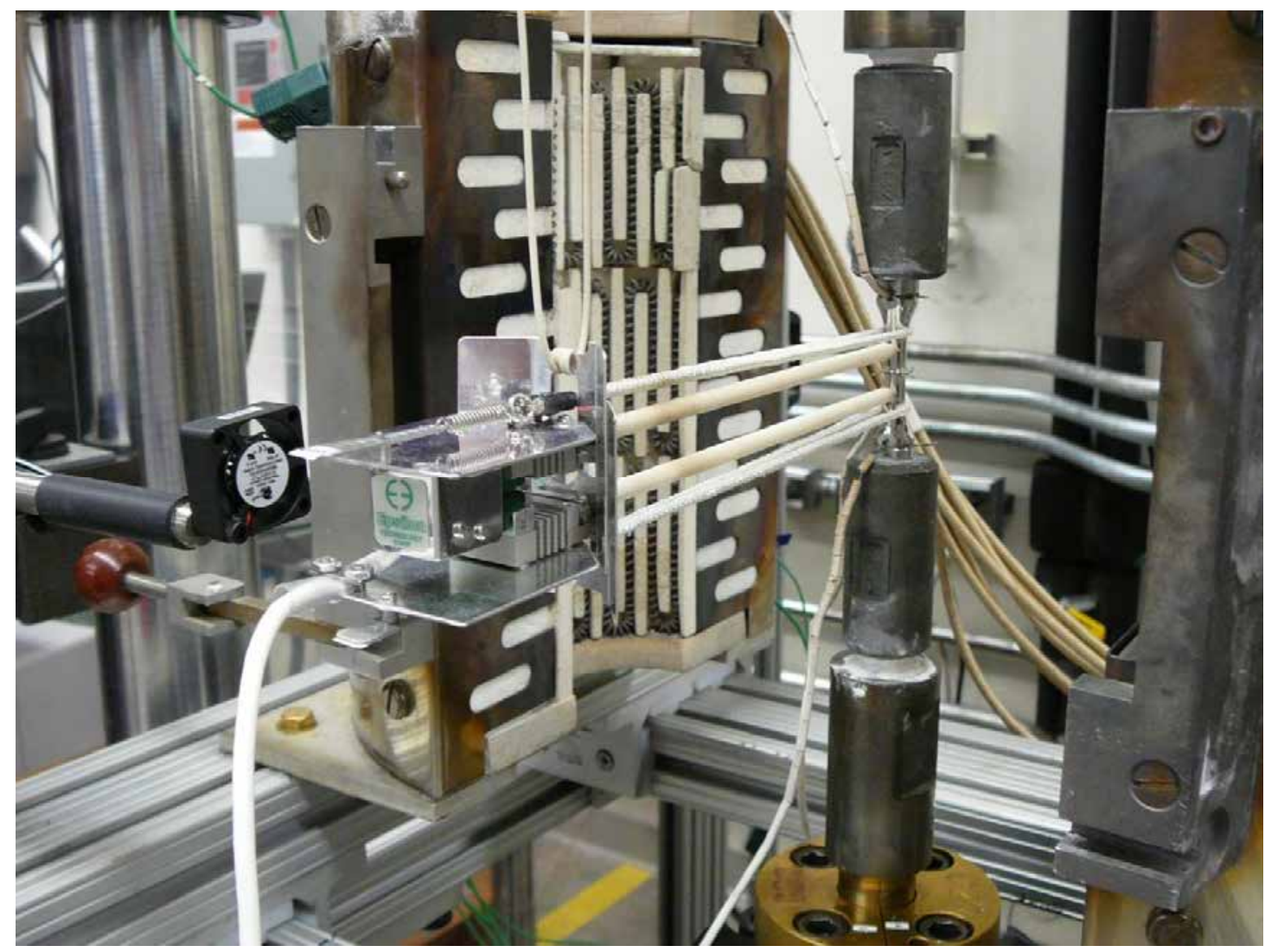

Fig. 3.16: Attachment of an Epsilon Model 3448 high temperature extensometer to the specimen 


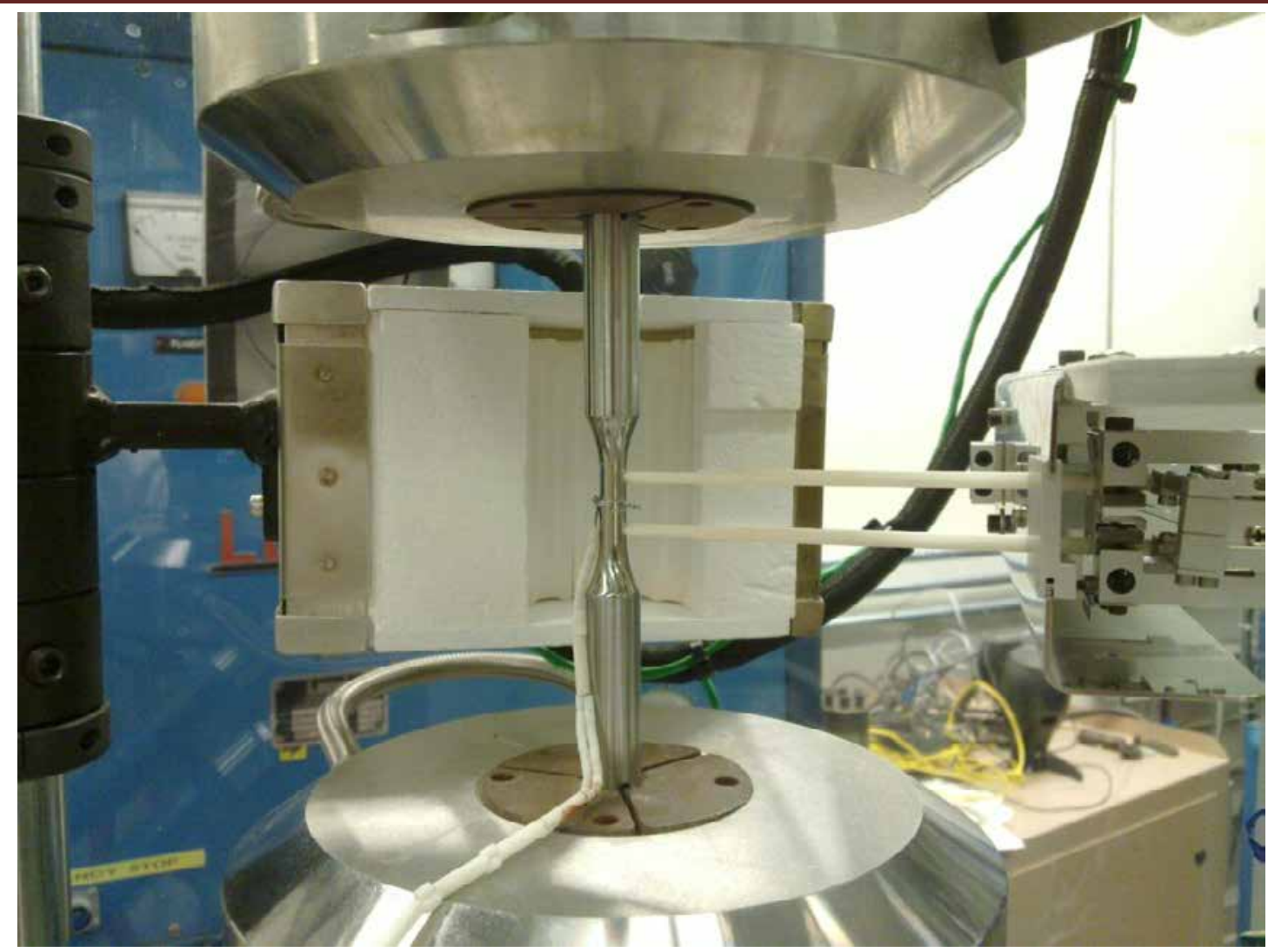

Fig. 3.17: A straight-sided specimen installed in the hydraulic grips with one S-type thermal couple and the high temperature extensometer installed

Post-test specimens with a size of $4 \mathrm{~mm}$ by $4 \mathrm{~mm}$ were first cut into small strips along the longitudinal direction. The strip sample was polished up to $0.05 \mu \mathrm{m}$ finish with alumina suspensions. To remove the polishing damage and reveal the grain structure, polished specimens were etched with a solution consisting of $10 \mathrm{ml}$ nitric acid, $10 \mathrm{ml}$ acetic acid, and $15 \mathrm{ml}$ hydrochloric acid for 1 to 2 minutes. JEOL 6500F at the SHaRE User Facility of ORNL was used for carrying out scanning electron microscope (SEM), energy dispersive spectroscopy (EDS), and electron backscatter diffraction (EBSD) examinations on the specimens in this study. This facility makes use of a field emission gun for the electron source and a Si drift detector for X-ray microanalysis. It is also equipped with a high speed EBSD detector and Orientation Imaging Microscopy (OIM) data collection and analysis software from TSL that can be used for making EBSD analysis. The detailed EBSD data acquisition conditions are summarized in Table 3.2 .

Table 3.2: EBSD data acquisition condition summary

\begin{tabular}{|c|c|c|c|c|}
\hline Sample tilt angle & Electron accelerating voltage & Working distance & Image binning & Scan rate \\
\hline $70 \mathrm{deg}$ & $20 \mathrm{KV}$ & $\sim 20 \mathrm{~mm}$ & $4 \mathrm{x} 4$ & $\sim 15 \mathrm{FPS}$ \\
\hline
\end{tabular}




\section{Understanding Fundamental Material-Degradation Processes in High Temperature Aggressive Chemomechanical Environments}

\subsection{Biaxial Creep Tests}

Tube specimens of Alloy 617 and Alloy 230 were produced by Century Tubes Inc. The composition of the two materials is given in Table 3.3 with certified test report of heat numbers XX58A7UK for Alloy 617 and 8305-8-7171 for Alloy 230.

The specimens used in the study are compact and rely only on internal pressure. The dimensions of the creep specimen and a photograph of the specimen are shown in Fig. 3.18. Using the electron beam welding technique, the specimens were sealed by two end caps with a small hole drilled in one of the two end caps. Before pressurizing the specimen, the specimen was pumped down by a mechanical pump. Then the furnace was heated up to the working temperature and the specimen was put into the middle of the furnace. High purity argon gas was then used to pressurize the specimen. The use of argon can help avoid oxidation of the inner wall. A pressure gauge was used to monitor the pressure in the tube. Fig. 3.19 shows the schematic diagram of the system. After a period of time, the specimen was taken out and then the gas inside the specimen was released. The diameter change of the specimen was measured using a laser scanner, the resolution of which is $0.5 \mu \mathrm{m}$. Fig. 3.20 shows the schematic diagram and photograph of the laser instrument used in the present study. Tables 3.4 and 3.5 list the experimental conditions for Alloy 617 and Alloy 230, respectively. The stresses indicated in Tables 3.4 and 3.5 are the mid-wall effective stresses based on the von Mises criterion, converted by the following equations [3.9]:

$$
\begin{aligned}
& \sigma_{h}=\frac{p R_{i}^{2}}{R_{0}^{2}-R_{i}^{2}}\left[1+\frac{R_{0}^{2}}{R_{m}^{2}}\right] \\
& S=\frac{\sqrt{3} R_{0}^{2}}{R_{0}^{2}+R_{m}^{2}} \sigma_{h}
\end{aligned}
$$

where $\mathrm{R}_{\mathrm{i}}$ : inner radius, $\mathrm{R}_{\mathrm{o}}$ : outside radius, $\mathrm{R}_{\mathrm{m}}$ : mid-wall radius, $\mathrm{p}$ : internal gas pressure, $\sigma_{h}$ : mid-wall hoop stress, and $\mathrm{S}:$ mid-wall effective stress. The diameter strain can be calculated by the following equation:

$$
\varepsilon_{\text {dia }}=\frac{\Delta D}{D_{0}}
$$

where $\mathrm{D}_{0}$ : original diameter, $\Delta D$ : diameter change, and $\varepsilon_{\text {dia }}$ : diameter strain. To examine the microstructure change during the secondary creep, an interrupted test was conducted for Alloy 230 at $950^{\circ} \mathrm{C}$ under the applied stress of $12 \mathrm{MPa}$.

Table 3.3: Chemical composition of Alloy 617 and Alloy 230 in wt\% for tube specimens

\begin{tabular}{|c|c|c|c|c|c|c|c|c|}
\hline Alloy & $\mathrm{Al}$ & $\mathrm{B}$ & $\mathrm{C}$ & $\mathrm{Co}$ & $\mathrm{Cr}$ & $\mathrm{Cu}$ & $\mathrm{Fe}$ & $\mathrm{P}$ \\
\hline 617 & 1.04 & 0.02 & 0.08 & 12.46 & 22.21 & 0.14 & 1.55 & - \\
\hline 230 & 0.37 & 0.03 & 0.11 & 0.19 & 22.39 & - & 0.94 & 0.008 \\
\hline & $\mathrm{Mn}$ & $\mathrm{Mo}$ & $\mathrm{Ni}$ & $\mathrm{S}$ & $\mathrm{Si}$ & $\mathrm{Ti}$ & $\mathrm{W}$ & $\mathrm{La}$ \\
\hline 617 & 0.07 & 8.97 & 53.02 & 0.01 & 0.17 & 0.29 & - & - \\
\hline 230 & 0.5 & 1.36 & $\mathrm{BAL}$ & 0.02 & 0.38 & 0.01 & 14.26 & - \\
\hline
\end{tabular}




\section{Understanding Fundamental Material-Degradation Processes in High Temperature}

Aggressive Chemomechanical Environments

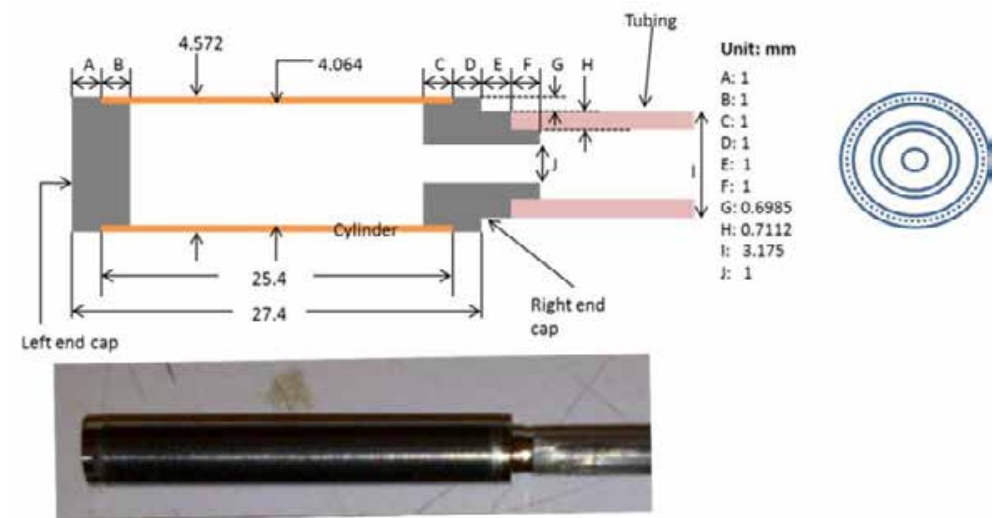

Fig. 3.18: Schematic diagram and photograph of the pressurized creep tub

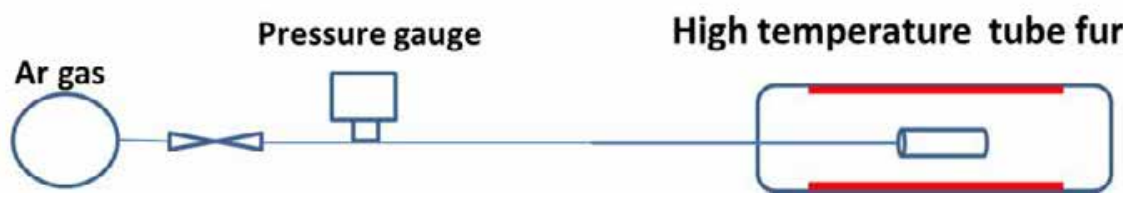

Fig. 3.19: The schematic diagram of creep tube testing performed at high temperatures

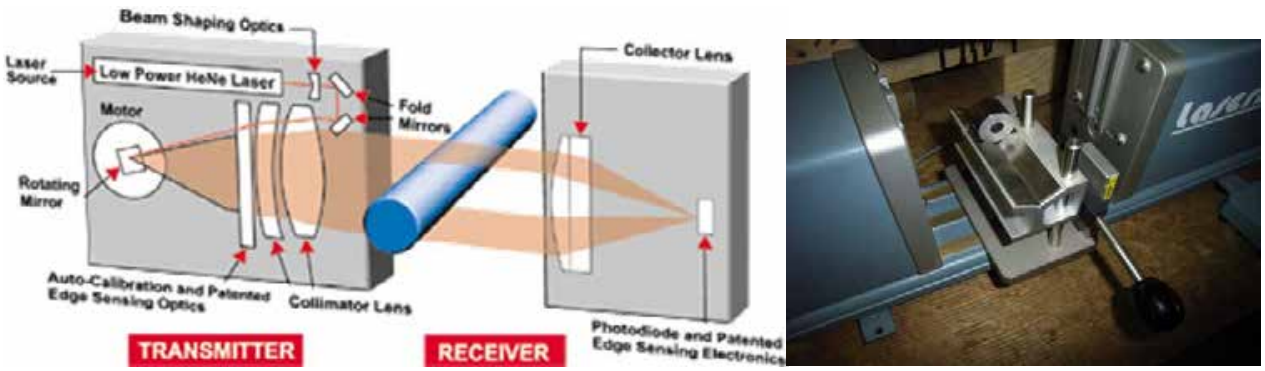

Fig. 3.20: Laser instrument used to measure the diameter change of the tube specimen. The resolution is $0.5 \mu m[3.10]$

Table 3.4: Experimental conditions of Alloy 617

\begin{tabular}{|c|c|c|c|}
\hline Temperature $\left({ }^{\circ} \mathrm{C}\right)$ & Inner Pressure $(\mathrm{MPa})$ & Effective Stress $\sigma_{\text {eff }}(\mathrm{MPa})$ & Rupture Time \\
\hline 900 & $4.10,2.46,2.05$ & $30,18,15$ & up to $1000 \mathrm{hr}$ \\
\hline
\end{tabular}

Table 3.5: Experimental conditions of Alloy 230

\begin{tabular}{|c|c|c|c|}
\hline Temperature $\left({ }^{\circ} \mathrm{C}\right)$ & Inner Pressure $(\mathrm{MPa})$ & Effective Stress $\sigma_{\text {eff }}(\mathrm{MPa})$ & Rupture Time \\
\hline 900 & $4.79,4.10,3.15,2.46$ & $35,30,23,18$ & up to $1000 \mathrm{hr}$ \\
\hline
\end{tabular}




\section{Understanding Fundamental Material-Degradation Processes in High Temperature Aggressive Chemomechanical Environments}

\subsection{Creep Simulation}

The experimental results are central to understanding material properties at certain temperatures and stresses. However, these experimental conditions are limited and cannot directly provide long-term creep life estimations. To understand the creep properties of both Alloy 617 and Alloy 230 comprehensively, a computational method to extend the existing experimental results to a larger range of temperatures and stresses is required. The experimental data of Alloy 617 and Alloy 230 at $950^{\circ} \mathrm{C}$ and $850^{\circ} \mathrm{C}$ was used for this simulation.

To develop a simulation equation, we begin with a description of the time-dependent relationship between stress and strain. For example, consider the experimental data of Alloy 617 at $950^{\circ} \mathrm{C}$ and at a creep time of 30 hours (Fig. 3.21). We can describe its behavior as the sum of two strain components: the first describes a linear stress dependence at low stress levels, and the second describes a stronger stress dependence at higher stress levels.

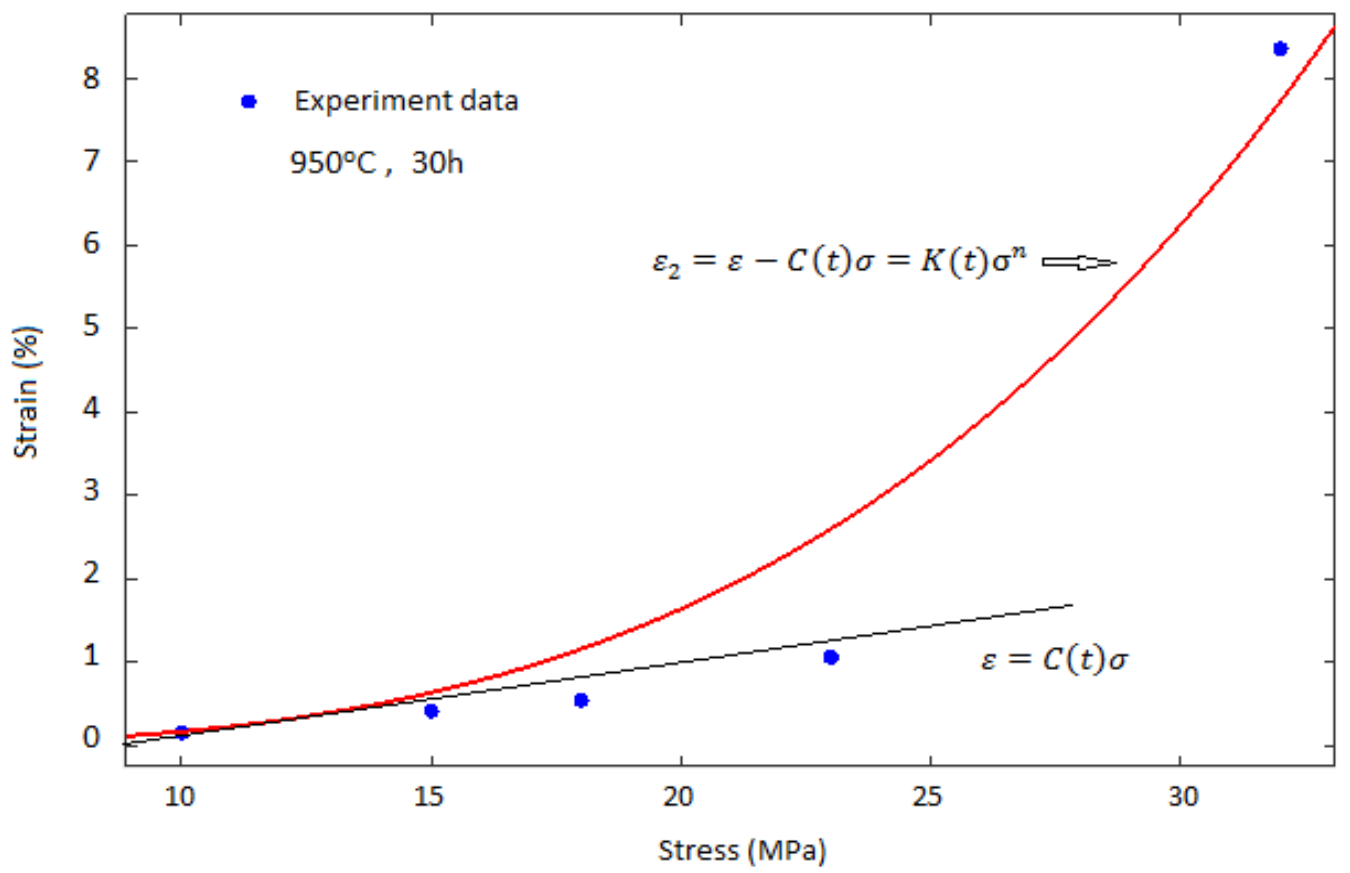

Fig. 3.21: Illustration of the analysis of stress-strain data

The linear portion can be approximated by the relation $\varepsilon=C(t) \sigma$, where $t$ represents time. This fitting line is used to evaluate the slope $C(t)$ and is allowed to intercept the strain axis at nonzero values. The interception value is considered to be a measurement uncertainty. The portion that has a stronger dependence on stress can be represented by $\varepsilon=K(t) \sigma^{n}$. In this relation, $n$ is the stress exponent, which indicates the creep mechanism. Then, the total stress-strain relationship can be represented by:

$\varepsilon=C(t) \sigma+K(t) \sigma^{n}$

From the previous work by Tung [3.11], the value of $n$ for Alloy 617 at $950^{\circ} \mathrm{C}$ is 3.3. The values of $C(t)$ and $K(t)$ are determined from each set of strain-stress data by curve fitting. Table 3.6 provides the $C(t)$ and $K(t)$ for Alloy 617 at $950^{\circ} \mathrm{C}$ for different periods of time. 
Understanding Fundamental Material-Degradation Processes in High Temperature Aggressive Chemomechanical Environments

Table 3.6: Curve-fitting values of $C(t)$ and $K(t)$ for Alloy 617 at $950^{\circ} \mathrm{C}$

\begin{tabular}{|c|c|c|}
\hline Time (h) & $\boldsymbol{C}(\boldsymbol{t})$ & $\boldsymbol{K}(\boldsymbol{t})$ \\
\hline $\mathbf{1 0}$ & $1.316 \mathrm{e}-7$ & $1.81 \mathrm{e}-5$ \\
\hline $\mathbf{3 0}$ & $2.479 \mathrm{e}-8$ & $8.333 \mathrm{e}-5$ \\
\hline $\mathbf{1 0 0}$ & $1.059 \mathrm{e}-9$ & $2.524 \mathrm{e}-4$ \\
\hline $\mathbf{1 6 0}$ & $4.933 \mathrm{e}-10$ & $5.525 \mathrm{e}-4$ \\
\hline $\mathbf{2 5 6}$ & $1.127 \mathrm{e}-10$ & $7.648 \mathrm{e}-4$ \\
\hline
\end{tabular}

As suggested by Swindeman and Pugh [3.12], $C(t)$ and $K(t)$ can be represented as:

$C(t)=A \cosh ^{-1}(1+r t)$

$K(t)=P t^{m}$

$A, r, P$, and $m$ are temperature dependent constants obtained from curve fitting. Finally, the experimental creep data can be represented as:

$\varepsilon=A \sigma \cosh ^{-1}(1+r t)+P \sigma^{n} t^{m}$

\subsection{Finite Element Analysis for Creep Behavior}

A more accurate manner to simulate the creep behavior is to apply a finite element method (FEM). FEM is a numerical technique for finding approximate solutions to boundary value problems. The commercial software, ABAQUS, was employed for the FEM analysis. ABAQUS/CAE provides numerical functions for both modeling and analysis of mechanical components and assemblies, and allows visualization of the finite element analysis result. Creep, a huge nonlinear deformation problem, is especially suitable for simulation by ABAQUS. The ABAQUS model of the creep tube before deformation is shown in Fig. 3.22. 


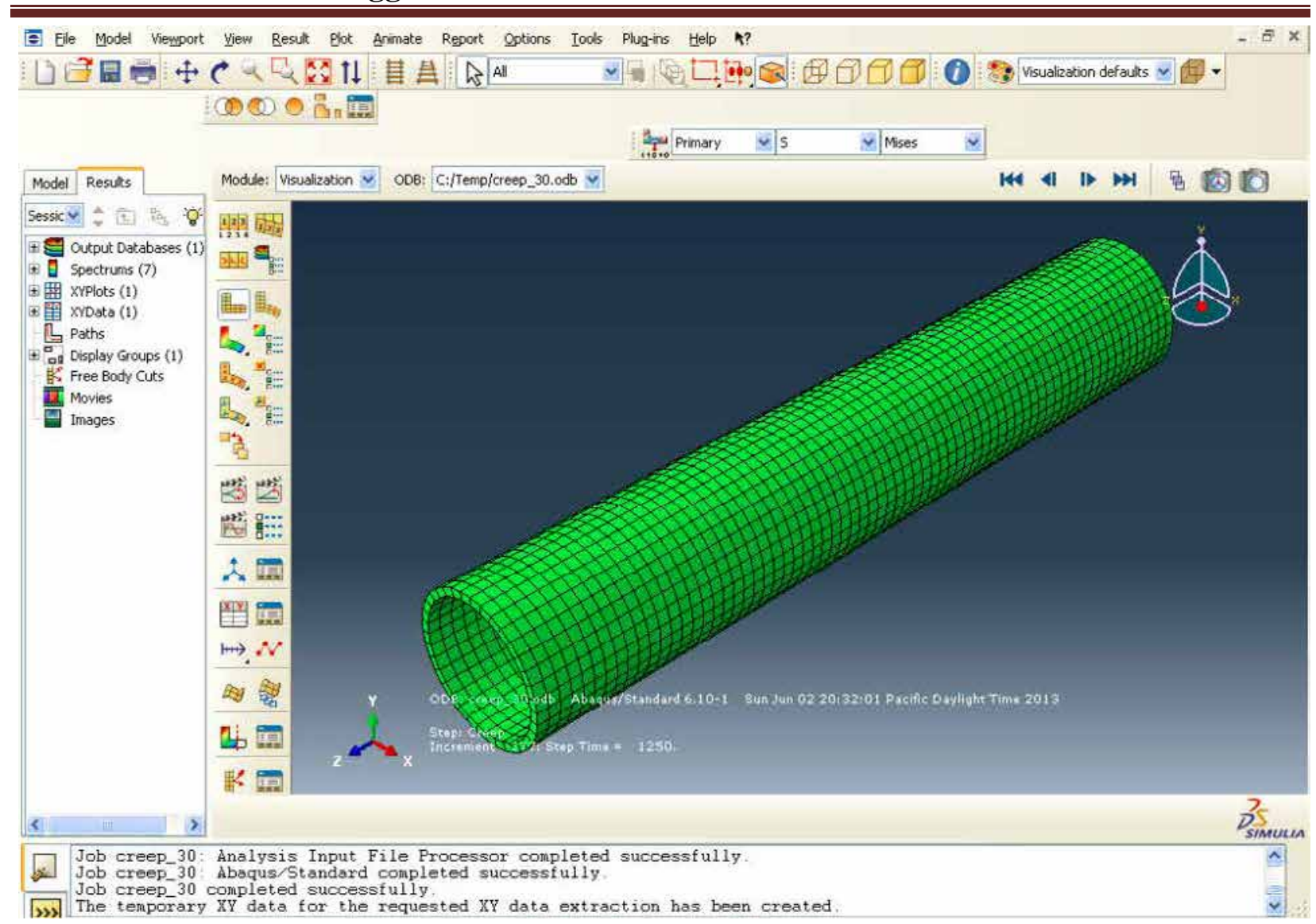

Fig. 3.22: ABAQUS modeling for a pressurized creep tube

ABAQUS provides three basic models for describing creep behavior: the time-hardening, strain-hardening, and hyperbolic-sine models. However, none of these models can precisely describe the three-stage creep development, so the implementation of user subroutines is needed to develop better simulation accuracy. The user subroutines in ABAQUS allow the program to be customized for particular applications and projects, and greatly extend the applicability of ABAQUS. With slight modifications to the constants' values, equation 3.8 is used in the creep subroutine as the constitutive function. The dimensions of the pressurized tubes defined in ABAQUS are of the same size as the ones used in the experiment. Since the dimensions of a sample near two end caps barely changes in our experiment, the two ends are assumed to be fixed in the model as a boundary condition. The subroutine program is attached in the appendix.

\subsection{High Temperature Fatigue Crack Growth of Haynes 230}

The experiments in this study were performed on the commercially available nickel-based superalloy Haynes 230. The chemical composition for the sheet used is shown in Table 3.7. Haynes 230 is a solid-solution strengthened alloy, and the additions of chromium, tungsten, and molybdenum contribute to the high temperature properties of the alloy. The typical microstructure has a wide range of grain sizes and is displayed in the optical photomicrograph in Fig. 3.23. Using the linear intercept method, as outlined in ASTM E-112, the average grain size was found to be about $50 \mu \mathrm{m}$. A large amount of annealing twins were observed in the material, so electron back-scatter diffraction (EBSD) was performed on an area of approximately 


\section{Understanding Fundamental Material-Degradation Processes in High Temperature Aggressive Chemomechanical Environments}

$0.39 \mathrm{~mm}^{2}$ to obtain statistical information. A portion of the EBSD data is shown in Fig. 3.24(a). Fig. 3.24(b) shows a histogram of the coincident lattice notation, CSL, grain boundary types for the entire area scanned. 573 out of the 1249 grain boundaries were described using the CSL criterion and almost two-thirds were $\sum 3$ boundaries.

Table 3.7: Chemical composition (wt $\%$ ) of the Haynes 230 studied

\begin{tabular}{|c|c|c|c|c|c|c|c|c|}
\hline $\mathrm{Al}$ & $\mathrm{B}$ & $\mathrm{C}$ & $\mathrm{Co}$ & $\mathrm{Cr}$ & $\mathrm{Cu}$ & $\mathrm{Fe}$ & $\mathrm{La}$ & $\mathrm{Mn}$ \\
\hline 0.35 & 0.005 & 0.1 & 0.16 & 22.14 & 0.04 & 1.14 & 0.015 & 0.5 \\
\hline $\mathrm{Mo}$ & $\mathrm{Ni}$ & $\mathrm{P}$ & $\mathrm{S}$ & $\mathrm{Si}$ & $\mathrm{Ti}$ & $\mathrm{W}$ & $\mathrm{Zr}$ & \\
\hline 1.25 & $\mathrm{bal}$ & 0.005 & 0.002 & 0.49 & 0.01 & 14.25 & 0.01 & \\
\hline
\end{tabular}

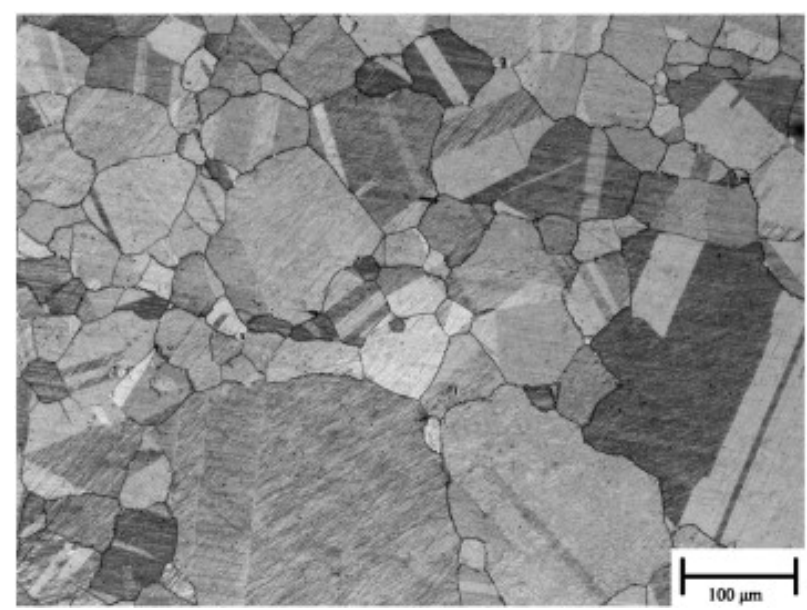

Fig. 3.23: Microstructure of the as-received Haynes 230 material showing annealing twins and a wide range of grain sizes 

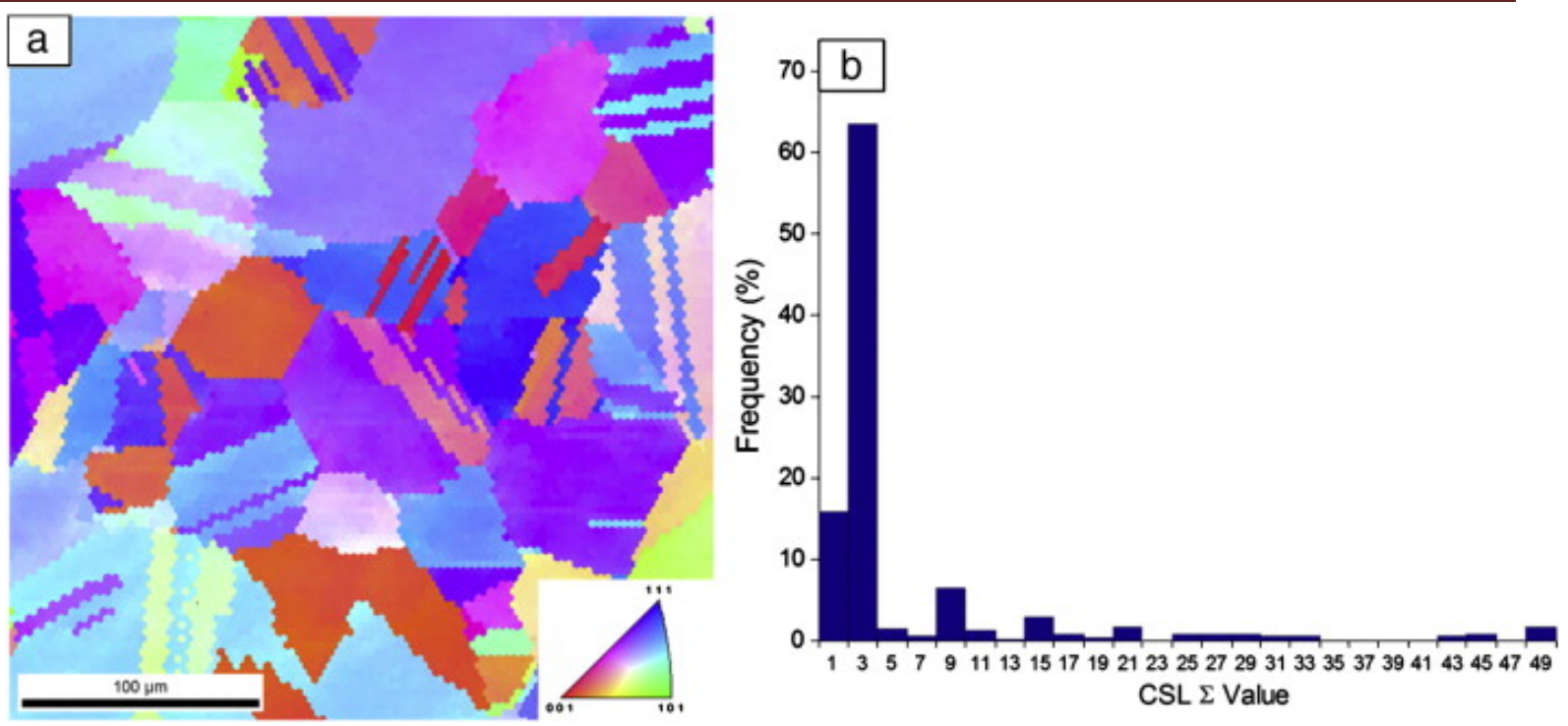

Fig. 3.24: a) section of the grain orientation map from EBSD of Haynes 230; b) CSL boundary frequency for the $0.39 \mathrm{~mm}^{2}$ area scanned, showing a high percentage of $\sum 3$ boundaries

The sheet received was $2.38 \mathrm{~mm}$ (3/32") thick, and single-edge notch "dogbone" specimens were electrical discharge machined (EDM) with a gauge length of $25.00 \mathrm{~mm}$ and a width of $4.00 \mathrm{~mm}$. A $0.5 \mathrm{~mm}$ deep notch was EDM in the center of the gauge length to facilitate pre-cracking during the fatigue crack growth experiments.

In order to prepare the specimens for DIC, two separate procedures were required that depended on the temperature condition. For the room temperature experiment, the specimens were mechanically polished to a mirror finish using abrasive paper up to P2400 grit size. Black paint was then airbrushed onto the specimen surface to create a speckled pattern. The high temperature (HT) experiment required a different preparation, since at $900{ }^{\circ} \mathrm{C}$ the sample glowed. The front of the sample was painted white with a thin layer of very high temperature paint. The paint was then cured using a three-step process, exposing it to a maximum temperature of $315^{\circ} \mathrm{C}$. A layer of black paint was then airbrushed onto the specimen, as was done with the room temperature experiment. The white paint was required for the HT sample to reduce the brightness that comes with the glowing and to prevent the speckle pattern from changing due to oxidation.

A servo-hydraulic load frame was used during the experiments. The HT specimen was heated using induction heating. A type-K thermocouple was spot welded onto the middle of the back of the specimen, approximately $2 \mathrm{~mm}$ above the notch plane. While at zero load, the specimen was heated at a rate of $75^{\circ} \mathrm{C}$ per minute until the sample reached $900^{\circ} \mathrm{C}$. The temperature was kept within $\pm 3^{\circ} \mathrm{C}$ for the entirety of the experiment. An IMI 202FT digital camera was used to capture images during the fatigue crack growth experiments. The camera resolution was $1600 \times 1200$ pixels, the maximum frame rate was $15 \mathrm{fps}$, and an adjustable lens with a $12 \times$ magnification range and $2 \times$ adapter was used to achieve different magnifications. A magnification of $2.5 \times$ was used to capture the images. The specimens were loaded in fatigue at a load ratio, $\mathrm{R}$, of 0.05 and a frequency of $3 \mathrm{~Hz}$. The $\mathrm{RT}$ specimens were fatigue loaded with a 


\section{Understanding Fundamental Material-Degradation Processes in High Temperature Aggressive Chemomechanical Environments}

stress range of $270 \mathrm{MPa}$. The stress range had to be reduced due to the lower yield stress at $900^{\circ}$ $\mathrm{C}$, and the HT specimen was fatigue loaded with a stress range of 104.5 MPa. A computer program controlled the servo-hydraulic load frame and captured images and their corresponding loads measured by a $7.5 \mathrm{kN}$ load cell during the test. Once a crack was visually identified, measurement cycles were periodically run at a frequency of $0.25 \mathrm{~Hz}$ in order to capture a greater number of images per cycle to provide an in-depth analysis of the fatigue cycles.

A commercially available image correlation program was used to perform DIC analysis. For a description of the digital image correlation technique, see [3.13] and [3.14]. For the crack closure analysis, the first image of each measurement cycle, captured at minimum load, was used as the reference image for that cycle. When studying slip irreversibility, the first image of the experiment, again captured at minimum load, was used as the reference image since strains due to the crack were not yet present. The full field of displacements, both behind and ahead of the crack tip, was used for correlations. The displacements were used in the least-squares regression, as briefly described in the Appendix, in order to determine the effective stress intensity factor ranges and crack closure levels. At each correlated point, the horizontal $(\Delta x)$ and vertical $(\Delta y)$ displacements were calculated and differentiated to obtain the strains assuming a small strain approximation.

For transmission electron microscopy (TEM), $1 \mathrm{~mm}$ thick discs were sectioned with a low-speed diamond saw parallel to the loading axis from the failed specimens, and then mechanically ground and polished down to $0.15 \mathrm{~mm}$ foil thickness. Large electron transparent areas were obtained in these foils by conventional twin jet polishing using a solution consisting of $5 \%$ perchloric acid in ethanol at $-20^{\circ} \mathrm{C}$ and $15 \mathrm{~V}$. The TEM was operated at a nominal voltage of $200 \mathrm{kV}$ and a double-tilt specimen holder was employed for imaging under two-beam conditions.

\subsection{Transpassive Behavior of Ni-based Alloys}

Very high temperature reactors (VHTRs) with the dual capacity of electricity generation and hydrogen production are promising candidates for future nuclear generators [3.15]. Two routes for hydrogen generation currently under consideration are electrochemical or thermochemical water splitting [3.16]. Thermochemical water splitting is preferred because it only requires heat [3.16]. The sulfur-iodine cycle is a promising candidate because it can efficiently produce hydrogen at temperatures achievable with VHTRs (between 900 and $1000^{\circ} \mathrm{C}$ ) [3.16]. Inconel 617 $(\mathrm{Ni}-\mathrm{Cr}-\mathrm{Co}-\mathrm{Mo})$ and Haynes $230(\mathrm{Ni}-\mathrm{Cr}-\mathrm{W}-\mathrm{Mo})$ are solid-solution strengthened Ni-based alloys developed as high temperature and corrosion resistant materials and are currently under consideration for use in components of next generation reactors [3.15-3.19]. The effect of aging at high temperature on the alloys' electrochemical behavior has important implications for use in VHTRs.

$\mathrm{Ni}, \mathrm{Cr}$, and $\mathrm{Ni}-\mathrm{Cr}$ alloys are known to undergo transpassive dissolution, the electron-mediated formation of soluble species from an oxide layer, at sufficiently anodic potentials [3.20-3.25]. Ring-disk polarization and AC impedance [3.20-3.23], as well as in situ X-ray photoelectron spectroscopy (XPS) and X-ray absorption near edge spectroscopy (XANES) of binary (Ni-Cr) alloys, show that the main products of transpassive dissolution are soluble $\mathrm{Cr}(\mathrm{VI})$ species [3.26]. 


\section{Understanding Fundamental Material-Degradation Processes in High Temperature Aggressive Chemomechanical Environments}

Raman spectroscopy and surface-enhanced Raman spectroscopy (SERS) have been used to investigate the effect of pressurized water reactors on Ni alloys (alloy 600 and alloy 690) in which a $\mathrm{Cr}$ (III) rich phase was determined to be $\alpha-\mathrm{Cr}_{2} \mathrm{O}_{3}$ [3.27-3.32]. Devine's group has used in situ surface-enhanced Raman Spectroscopy with electrodeposited gold to investigate the passive layer that forms on austenitic nickel samples exposed to $\mathrm{Cl}^{-}$in acidic and basic solutions. In $\mathrm{HCl}$ solutions they found the passive layer of alloy $\mathrm{C} 22$ (Ni-Cr-Mo-W) to be composed of a combination of $\mathrm{MoO}_{3}, \mathrm{MoO}_{2}$, and amorphous $\mathrm{Cr}$ (III) oxide species at low potentials, with evidence for $\mathrm{Cr}_{2} \mathrm{O}_{3}$ at transpassive potentials [3.33].

Although in situ techniques have been used to characterize the passive layer in ambient conditions and with high temperature and pressure solutions, the effect of heat treatment has not been fully investigated [3.15, 3.18-3.19, 3.34-3.39]. It is unknown whether heat aging changes the distribution of surface species that form during transpassive dissolution in sulfuric acid. Herein we demonstrate the use of Shell Isolated Surface Enhanced Raman Spectroscopy (SHINERS) to interrogate the speciation of surface species that form in the anodic potentials for Haynes 617 and Haynes 230 that have been aged at high temperature in air and compare to the as-received alloy. The SHINERS technique allows examination of the electrode surface without possible complications arising from deposition of a second metal, such as Au. It also allows for potential cycling through a wide potential range.

All solutions were prepared from ultrapure water (Milli-Q UVplus, Millipore Inc., 18.2 $\mathrm{M} \Omega \mathrm{cm}^{-1}$ ) and high purity $\mathrm{H}_{2} \mathrm{SO}_{4}$ (Optima, Fisher Scientific). Electrochemical experiments were performed using a $\mathrm{CHI} 760 \mathrm{D}$ potentiostat ( $\mathrm{CH}$ Instruments) with a two component cell, $\mathrm{Pt}$ gauze counter electrode, and "no leak" $\mathrm{Ag} / \mathrm{AgCl}$ reference electrode filled with $3 \mathrm{M} \mathrm{KCl}$ (Cypress). Working electrodes were held in place with Kel-F collets and rotated with a modulated speed rotator (Pine Research Instrumentation). Plates of Alloy 617 and Alloy 230, synthesized by hot working and solution treatment, were supplied by Haynes International [3.15]. The composition of the as-received alloys is listed in Table 3.8 as described in Mo et al. [3.15]. Disks (2-3 mm thick and 5-10 $\mathrm{mm}$ in diameter) were cut from the plates. Heat treated samples were aged in laboratory air at temperatures of 900 and $1000^{\circ} \mathrm{C}$ for $3000 \mathrm{~h}$. These disks were used as the working electrodes and were polished to mirror finish with $0.3 \mu \mathrm{m} \mathrm{Al}_{2} \mathrm{O}_{3}$, sonicated in ultrapure water, then rinsed with copious amounts of ultrapure water before use in experiments.

Table 3.8: Elemental composition of alloys in average weight percent from Mo et al. [3.15].

\begin{tabular}{lllllllll}
\hline Alloy & $\mathrm{Al}$ & $\mathrm{B}$ & $\mathrm{C}$ & $\mathrm{Co}$ & $\mathrm{Cr}$ & $\mathrm{Cu}$ & $\mathrm{Fe}$ & $\mathrm{La}$ \\
\hline 617 & 1.03 & $<0.002$ & 0.08 & 12.2 & 22.1 & 0.017 & 1.104 & - \\
230 & 0.38 & 0.004 & 0.11 & 0.29 & 21.7 & 0.04 & 1.4 & 0.014 \\
& $\mathrm{Mn}$ & $\mathrm{Mo}$ & $\mathrm{Ni}$ & $\mathrm{P}$ & $\mathrm{S}$ & $\mathrm{Si}$ & $\mathrm{Ti}$ & $\mathrm{W}$ \\
\cline { 2 - 8 } & $\mathbf{0}$ & & & \\
217 & 0.064 & 9.46 & 52.86 & 0.002 & $<0.002$ & 0.05 & 0.39 & - \\
230 & 0.46 & 1.75 & $\mathrm{BAL}$ & 0.005 & $<0.002$ & 0.4 & $<0.01$ & 13.89 \\
\hline
\end{tabular}




\section{Understanding Fundamental Material-Degradation Processes in High Temperature}

Aggressive Chemomechanical Environments

SHINERS experiments were performed using methods described previously [3.40-3.42]. In particular, an aqueous colloid of $\mathrm{SiO}_{2}$ capped $\mathrm{Au}$ was synthesized as discussed in Li et al. [3.40, 3.41] with Au diameter of $18 \mathrm{~nm}$ and shell diameter of 1-2 nm. The colloid was drop cast onto the polished surface and dried under a stream of Ar. SHINERS measurements were performed at room temperature using an in situ cell described previously [3.43] with a $\mathrm{Au}$ wire counter electrode and $\mathrm{Ag} / \mathrm{AgCl}$ reference electrode. The excitation line was a $\mathrm{HeNe}$ laser (632.8) impinging the surface at a $45^{\circ}$ angle. Scattered light was collected with an air cooled CCD. The typical acquisition time for each spectrum was $30 \mathrm{~s}$.

XPS experiments were performed with an Axis ULTRA spectrometer (Kratos Analytical). A monochromatic Al X-ray source was used with a $150 \mathrm{~W}$ source power, a $1000 \mathrm{meV}$ step energy, and a $100 \mathrm{~ms}$ dwell time for two sweeps. Data were calibrated to a C $1 \mathrm{~s}$ peak. 


\section{Understanding Fundamental Material-Degradation Processes in High Temperature Aggressive Chemomechanical Environments}

\subsection{Material Characterization}

\subsubsection{Tensile specimens}

Alloy 617 and Alloy 230 were supplied by Haynes International as 1 inch and 1.5 inch thick plates, and each was hot worked and solution treated at $1177^{\circ} \mathrm{C}$ for 37 minutes and $1191^{\circ} \mathrm{C}$ for 3 hours, respectively. (Heat Number: 861758808 for Alloy 617 and 830587801 for Alloy 230) The chemical composition is shown in Table 3.8.

Microstructure analysis was performed using a JEOL 2100 Cryo TEM, JEOL 2010 LaB6 TEM, and JEOL 7000F SEM coupled with EBSD capabilities. The TEM samples were prepared using electropolishing methods with a solution of $10 \%$ acetic acid in methanol at $-45^{\circ} \mathrm{C}$ and $22 \mathrm{~V}$. The SEM samples were etched for $\sim 3$ minutes using $10 \mathrm{ml}$ acetic acid, $15 \mathrm{ml} \mathrm{HCl}$, and $10 \mathrm{ml}$ $\mathrm{HNO}_{3}$. The EBSD samples were polished with $\mathrm{SiC}$ abrasive paper down to 600 grit followed by $1 \mu \mathrm{m}$ and $0.3 \mu \mathrm{m}$ alumina solutions. Finally, the polishing process was completed with vibratory polishing with $0.02 \mu \mathrm{m}$ colloidal silica. The intrinsic particles in Alloy 617 are $\mathrm{Cr}$ rich $\mathrm{M}_{23} \mathrm{C}_{6}$, Mo rich $\mathrm{M}_{6} \mathrm{C}$, and a small amount of $\mathrm{Ti}(\mathrm{C}, \mathrm{N})$. [3.44] Similar to Alloy 617, Alloy 230 has intrinsic $\mathrm{W}$ rich $\mathrm{M}_{6} \mathrm{C}$. [3.45] Fig. 3.25(a) \& (b) show the microstructure of Alloy 617 and Alloy 230 , respectively. The inclusion particles in both alloys are induced from the hot rolling process and cannot be re-dissolved during solid solution annealing. Similar to the particles in a hot rolling steel, these carbides in Alloy 617 and Alloy 230 are strung as bands in the transverse planes and flattened in the rolling planes. Additionally, these carbide particles in Alloy 617 are not evenly distributed in the material, but instead spread as clusters in various areas. (Fig. 3.25(a)) Comparatively, the inclusion particles in Alloy 230 distribute more homogenously. (Fig. $3.25(\mathrm{~b}))$

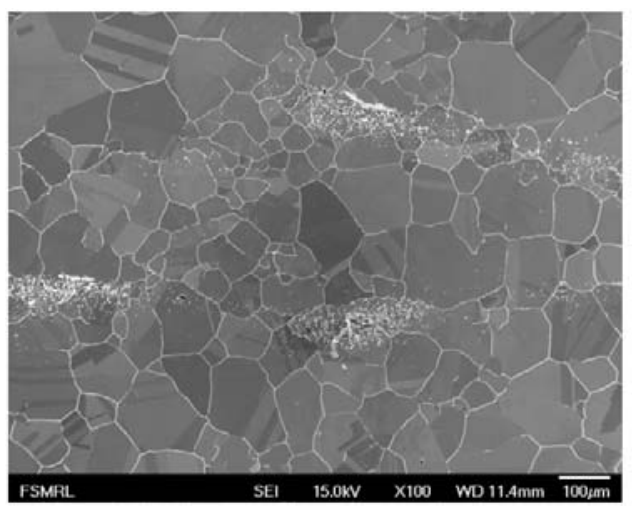

(a)

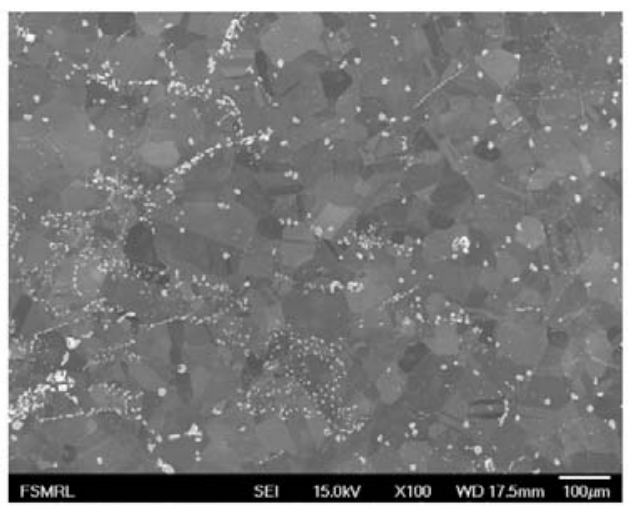

(b)

Fig. 3.25: Microstructure of the materials in the as-received condition: (a) Alloy 617; (b) Alloy 230

In addition to the large inclusion particles, many intergranular precipitates were observed in both alloys. (Fig. 3.26 and Fig. 3.27) In Alloy 617, these second phase particles are present as a thin film precipitating on grain boundaries and connected to large inclusion particles. (Fig. 3.26) In Alloy 230, this intergranular particle is dendritic, but it also connects to large inclusion particles. (Fig. 3.27) Due to a small volume fraction, these intergranular particles have little impact on both alloys' mechanical properties. 


\section{Understanding Fundamental Material-Degradation Processes in High Temperature}

Aggressive Chemomechanical Environments

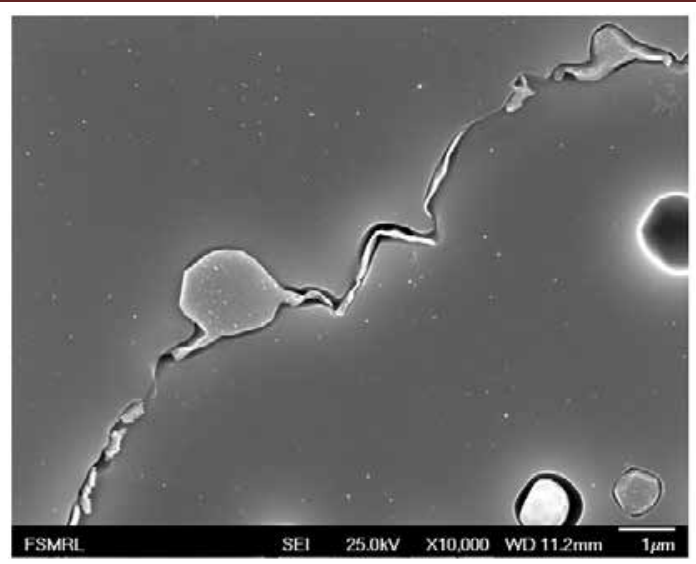

(a)

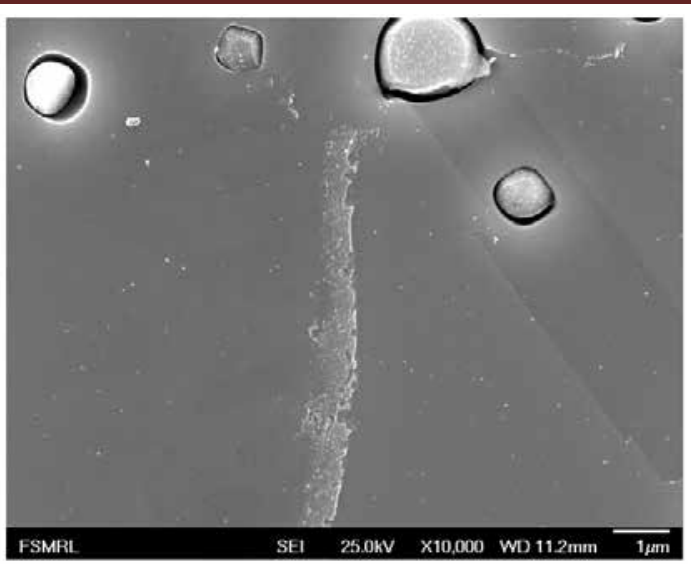

(b)

Fig. 3.26: Intrinsic particles in Alloy 617 in the as-received condition: (a) inclusion particles; (b) intergranular precipitates

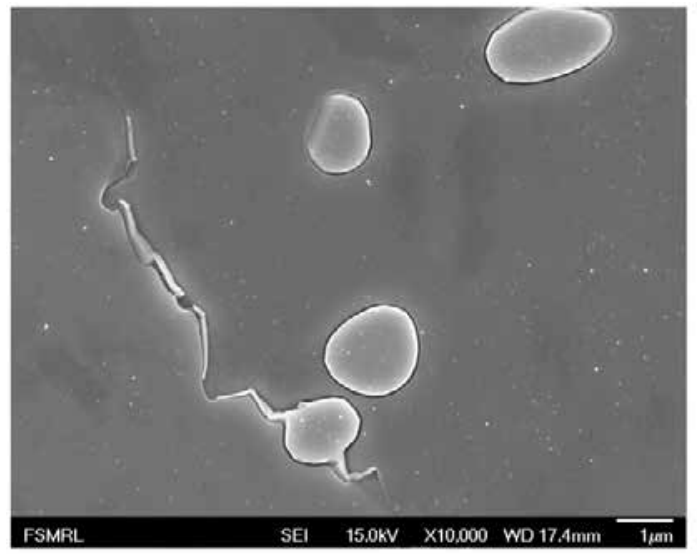

(a)

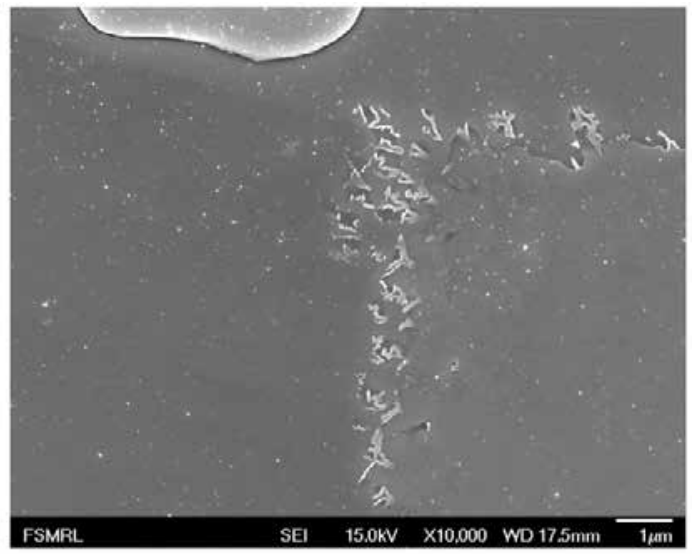

(b)

Fig. 3.27: Intrinsic particles in Alloy 230 in the as-received condition: (a) inclusion particles; (b) intergranular precipitates

The distribution of grain size in Alloy 617 is duplex: small grains with size ranging from 10 to $30 \mu \mathrm{m}$ in the inclusion rich areas and large grains with size ranging from 40 to $100 \mu \mathrm{m}$ in the inclusion free areas. During solid solution annealing, the inclusion particles exert a pinning pressure on grain boundaries and impede their movement/immigration (Zener pinning) so that the crystal growth is much slower in the inclusion rich areas. Hence, small grains are obtained in the inclusion rich areas after annealing, whereas coarse grains are found in the inclusion free areas. (Fig. 3.25(a) and Fig. 3.28(a)) Nevertheless, particle distribution in Alloy 230 is more homogenous. This results in moderate grain size variation after solid solution annealing. The average grain size is $\sim 70 \mu \mathrm{m}$ in Alloy 230 .

From EBSD analysis, $\sim 60 \%$ and $\sim 53 \%$ of the grain boundaries are twin boundaries (or coincidence site lattice (CSL) $\sum 3$ grain boundaries) for Alloy 617 and Alloy 230, respectively. The twin boundaries are shown as red lines in Fig. 3.28. 


\section{Understanding Fundamental Material-Degradation Processes in High Temperature}

Aggressive Chemomechanical Environments

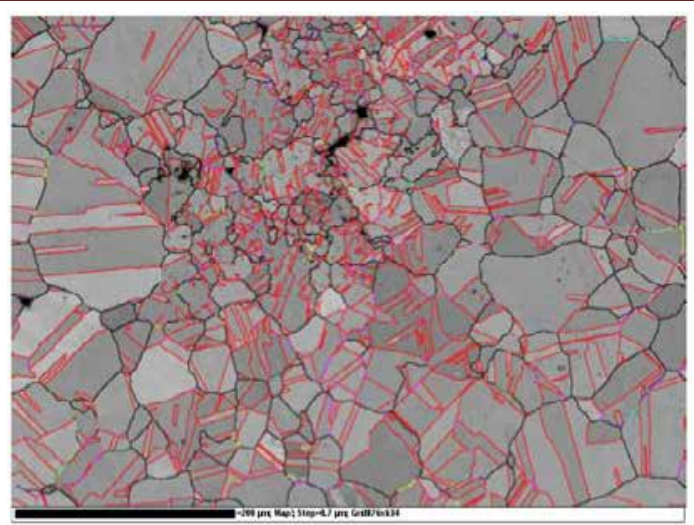

(a)

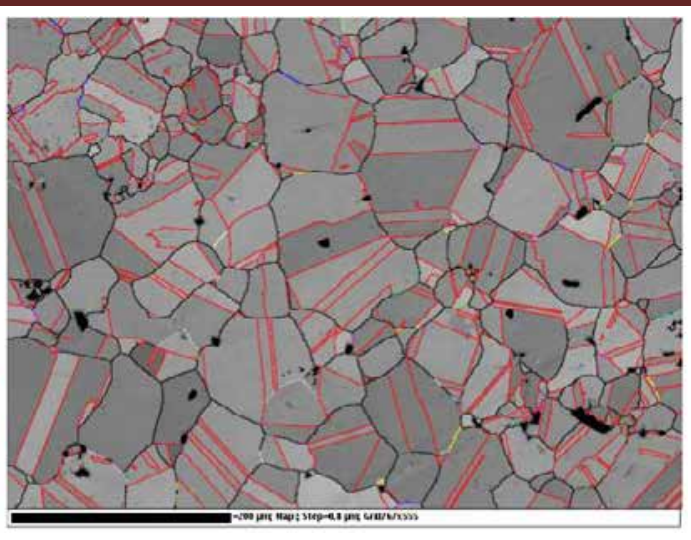

(b)

Fig. 3.28: EBSD and CSL boundary analysis of the materials in the as-received condition: (a) Alloy 617; (b) Alloy 230

\subsubsection{Creep tube specimens}

To characterize the corroded layer, microstructural analysis was carried out in the Frederick Seitz Materials Research Laboratory (FS-MRL). For creep tests, the cross-sectional surface was cut using a low-speed diamond saw machine, and then polished and etched using $15 \mathrm{ml} \mathrm{HCl,} 10 \mathrm{ml}$ acetic, and $10 \mathrm{ml} \mathrm{HNO}_{3}$. The etching time for Alloy 617 and Alloy 230 was about 180 and $270 \mathrm{~s}$, respectively. The specimen surface was examined by SEM. Before the creep tests, the as-received cross-sectional view and side view (longitudinal direction) of the metallic surface for both alloys was also examined. This is shown in Fig. 3.29 and 3.30 for Alloy 617 and Alloy 230, respectively. 
Understanding Fundamental Material-Degradation Processes in High Temperature Aggressive Chemomechanical Environments

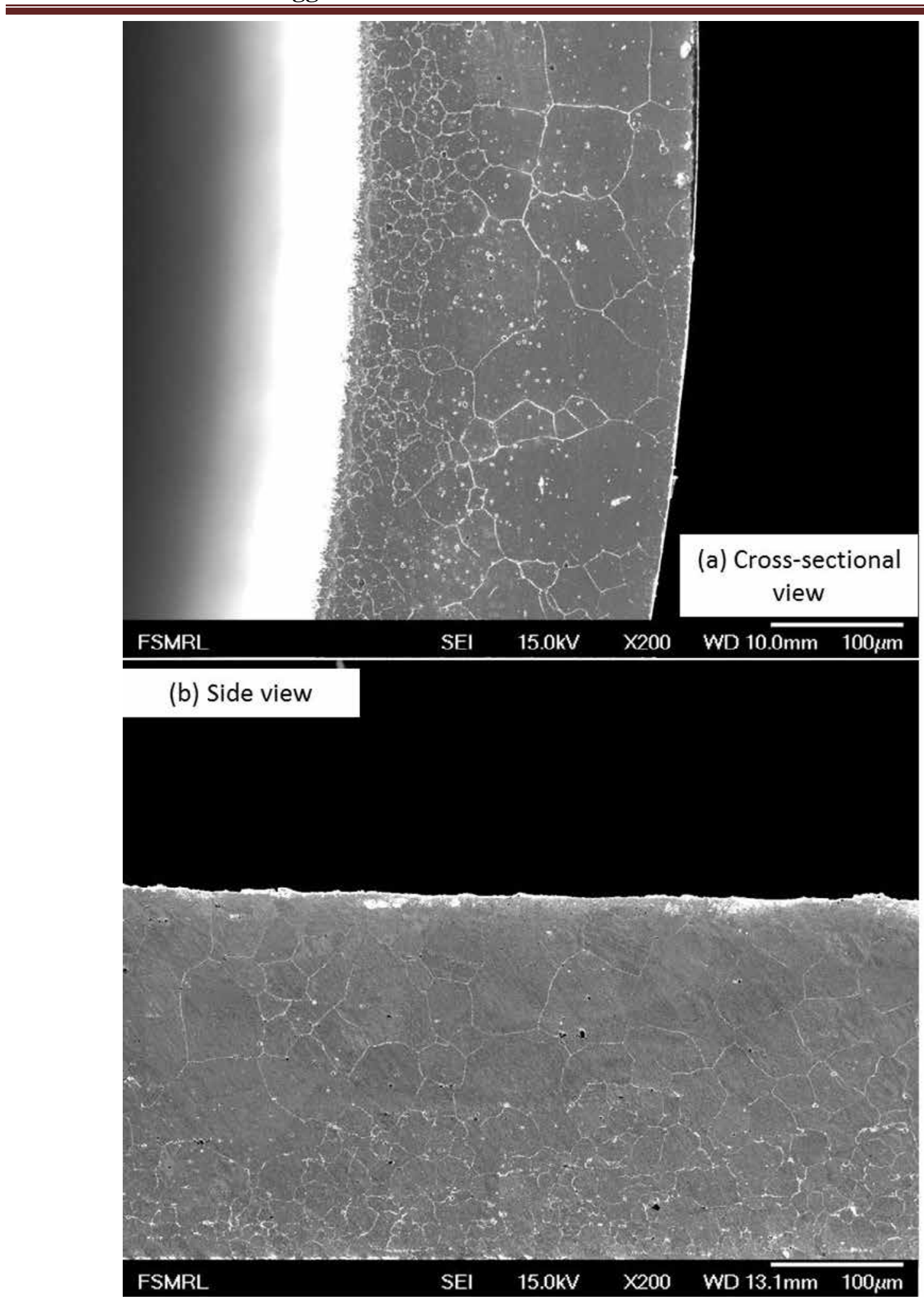

Fig. 3.29: Microstructures of as-received Alloy 617 with (a) cross-sectional view and (b) side view 


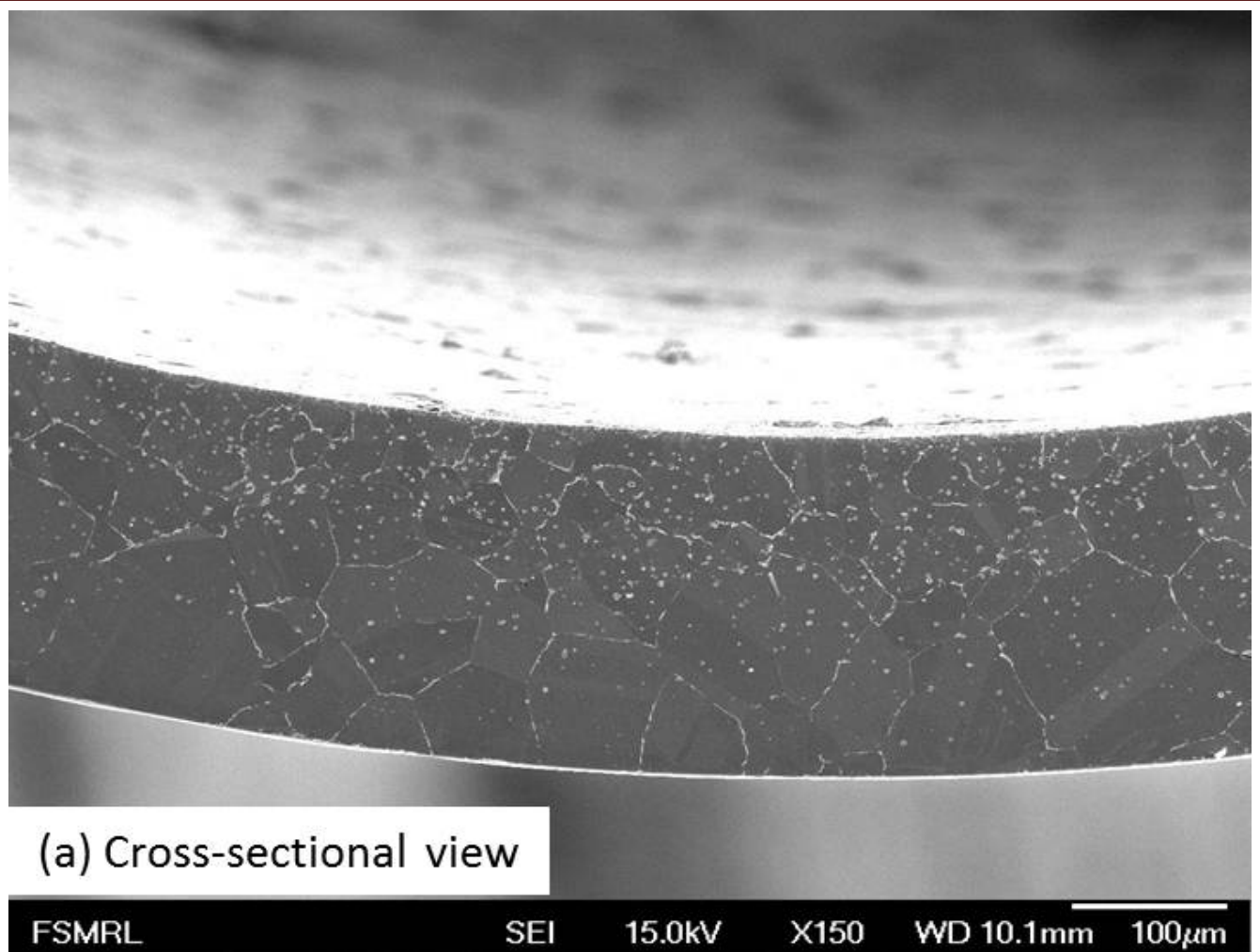

(b) Side view

FSMRL

SEI $15.0 \mathrm{kV}$

Fig. 3.30: Microstructures of as-received Alloy 230 with (a) cross-sectional view and (b) side view 


\section{Understanding Fundamental Material-Degradation Processes in High Temperature}

Aggressive Chemomechanical Environments

\subsubsection{Long-term aged specimens}

The original Alloy 617 was provided by Haynes international, Inc. in a 1 inch plate, which was hot worked and solution heated at $1177^{\circ} \mathrm{C}$ for 37 minutes (Heat Number: 861758808). The original Alloy 230 used for aging was also supplied by Haynes international, Inc. in a 1.5 inch plate with hot work and solution heat processing at $1191{ }^{\circ} \mathrm{C}$ for 3 hours (heat Number: 830587801). Then these materials were aged in VULCANTM 3-350 furnaces at $900^{\circ} \mathrm{C}$ and $1000^{\circ} \mathrm{C}$ for 10,000 hours, respectively. 


\section{Understanding Fundamental Material-Degradation Processes in High Temperature Aggressive Chemomechanical Environments}

\section{RESULTS}

\subsection{Tensile Properties for Alloy 617 and Alloy 230}

Fig. 4.1(a)\&(b) show the strain-stress diagram of Alloy 617 and Alloy 230, respectively. Generally, the alloys can withstand high stresses during tensile testing at temperatures up to $700^{\circ} \mathrm{C}$. Above $800^{\circ} \mathrm{C}$, the effect of strain hardening is significantly weakened. The stress rapidly decreases once it reaches the yield point. Particularly at $900^{\circ} \mathrm{C}$ and $1000^{\circ} \mathrm{C}$, strain hardening can hardly be observed. Fig. 4.2 shows the measured tensile properties for Alloy 617 and Alloy 230. The strengths at various temperatures are analogous for both alloys. From RT to $800^{\circ} \mathrm{C}$, both alloys maintained a relatively high yield strength (Y.S. $>250 \mathrm{MPa}$ ) with a slight decline with increasing temperature. Similarly, the decrease in ultimate tensile strength (U.T.S.) from RT to $700^{\circ} \mathrm{C}$ is moderate. At higher temperatures $\left(>900^{\circ} \mathrm{C}\right.$ for Y.S. $\&>800^{\circ} \mathrm{C}$ for U.T.S), both alloys were unable to withstand a large stress. (Fig. 4.1) Hence, a notable loss of strength was found for these temperatures. (Fig. 4.2)

It should be noted that the tensile responses for Alloy 617 using miniature specimens are analogous to properties found during experiments using standard size specimens [4.1, 4.2], indicating little, if any, size effects.

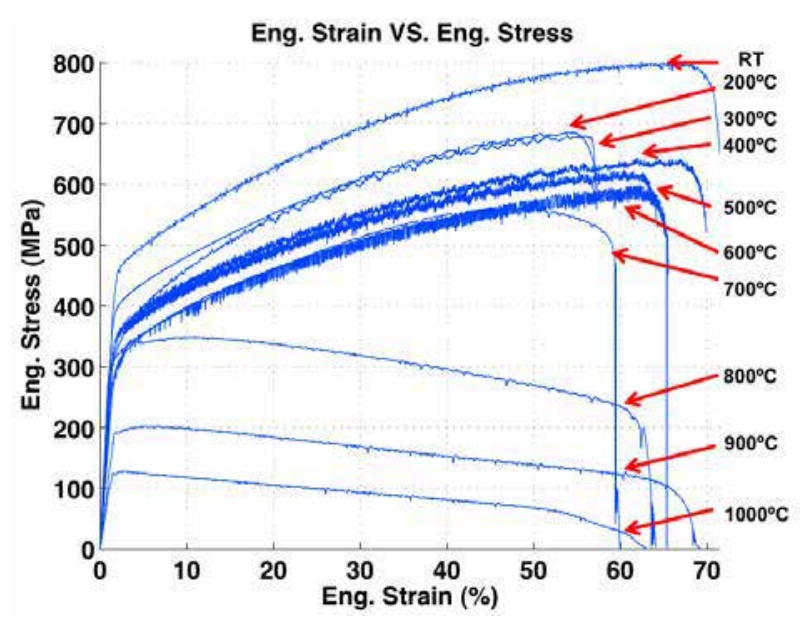

(a)

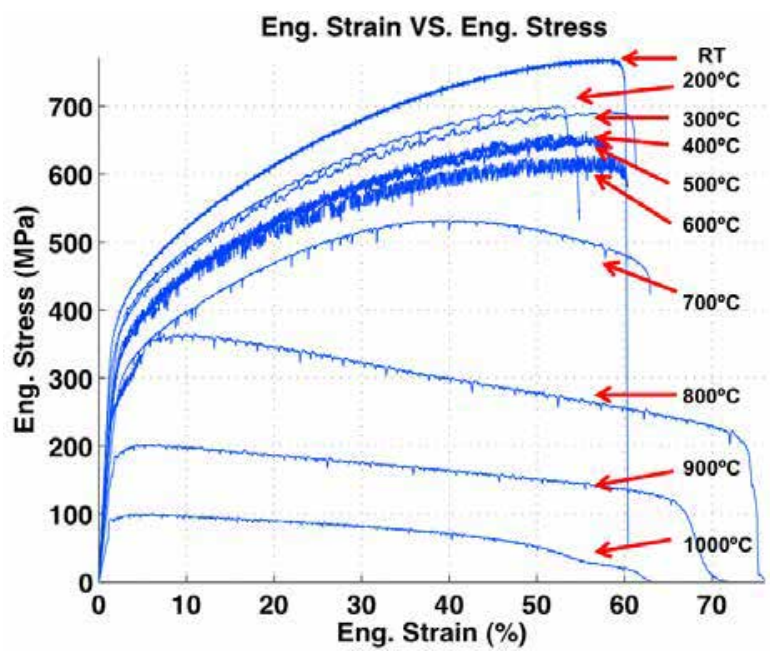

(b)

Fig. 4.1: Strain-stress diagrams of (a) Alloy 617; (b) Alloy 230 


\section{Understanding Fundamental Material-Degradation Processes in High Temperature Aggressive Chemomechanical Environments}

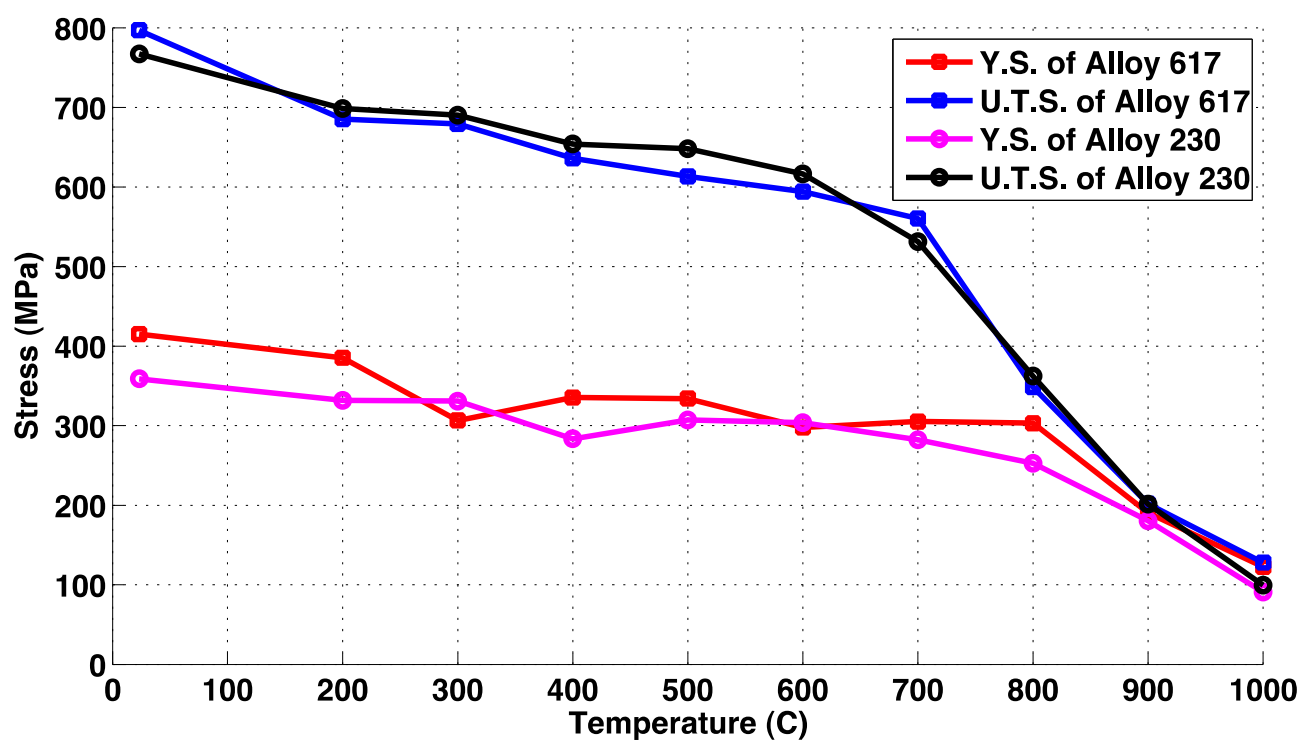

Fig. 4.2: Tensile properties (yield strength (Y.S.) and ultimate tensile strength (U.T.S.)) of Alloy 617 and Alloy 230 at various temperatures

\subsubsection{Tensile response from room temperature to $700^{\circ} \mathrm{C}$}

At room temperature, the strain-stress curve is smooth and continuous without apparent serrations. With increasing testing temperature, the strain-stress curve becomes serrated and unstable. (Fig. 4.1) These serrated strain-stress curves can be described by the Portevin-LeChatelier effect. During plastic deformations, dislocations become locked by solute atoms (e.g. interstitial carbon atoms) and can only be released when sufficient external stress is applied. This "drag and release" process is displayed by the serrated flow in a strain-stress curve. Since the flow stress can temporarily increase during external loading, this phenomenon is called dynamic strain aging, which suggests that aging occurs during deformation. [4.3] For both alloys, the dynamic strain aging is evident at temperatures ranging from 300 to $600^{\circ} \mathrm{C}$. (Fig. 4.1 ) Fig. 4.3 shows the flow stresses at selected temperatures for both alloys. The amplitude for the stress oscillation is found to be slightly higher for Alloy 230 than Alloy 617 (Table 4.1). For both alloys, the amplitude of serrations is found to increase with studied temperature due to the higher atom diffusibility attained at higher temperatures. 


\section{Understanding Fundamental Material-Degradation Processes in High Temperature Aggressive Chemomechanical Environments}

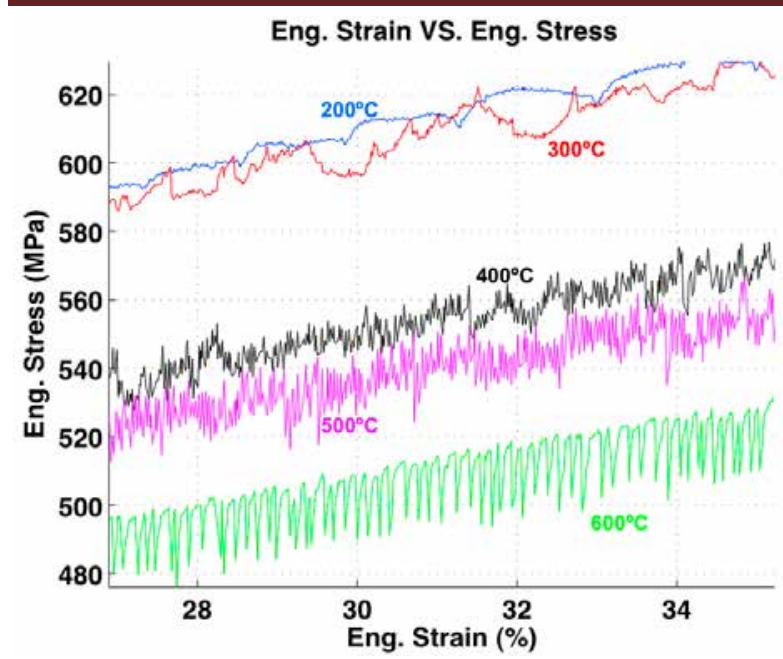

(a)

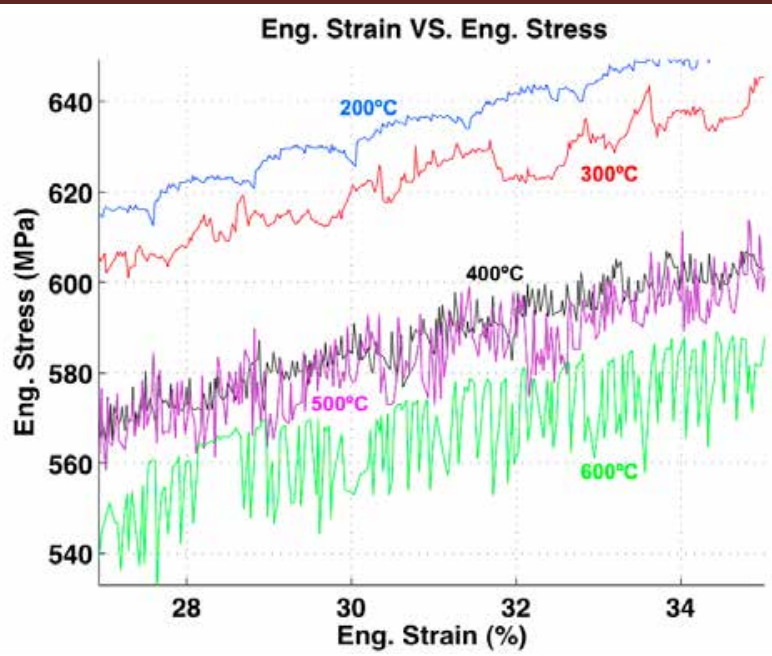

(b)

Fig. 4.3: Portevin-LeChatelier effect on (a) Alloy 617; (b) Alloy 230

Table 4.1: Amplitude of flow stress oscillations of Alloy 617 and Alloy 230 (in MPa)

\begin{tabular}{ccccc}
\hline Temperature $\left({ }^{\circ} \mathbf{C}\right)$ & $\mathbf{3 0 0}$ & $\mathbf{4 0 0}$ & $\mathbf{5 0 0}$ & $\mathbf{6 0 0}$ \\
\hline Alloy $\mathbf{6 1 7}$ & 8 & 10 & 15 & 18 \\
Alloy 230 & 8 & 10 & 16 & 20 \\
\hline
\end{tabular}

\subsubsection{Tensile response from $800^{\circ} \mathrm{C}$ to $1000^{\circ} \mathrm{C}$}

The previous analysis of tensile response at intermediate temperatures cannot be applied to higher temperature regions $\left(>800^{\circ} \mathrm{C}\right)$ because of the involvement of additional deformation mechanisms and the accompanying loss of strain hardening. For both alloys, $800^{\circ} \mathrm{C}$ can be considered the equicohesive temperature. [4.4] Above this temperature, grain boundaries are unable to impede dislocation movement. Hence, matrix grains and grain boundaries have comparable strength. High-temperature creep induced by thermal assisted dislocation glide and climb becomes a major deformation mechanism. The changes in deformation behavior at different temperatures are evaluated from observations of fracture micromechanisms. (Fig. 4.4) During tensile testing at temperatures below $800^{\circ} \mathrm{C}$, dislocations in the alloys do not have sufficient mobility to climb over carbide particles. Instead, they pile up on the interphase boundaries between matrix and carbide particles. (Fig. 4.5) Nevertheless, as the number of dislocations increases, sufficiently large stresses are generated and placed on the particles. Then, the particles begin to crack and produce voids during plastic deformation. (Fig. 4.4(a)) The coalescence of the crack voids finally results in pre-mature failure during testing. This can be seen in the strain-stress diagram for temperatures up to $600^{\circ} \mathrm{C}$ (Fig. 4.1 ), which shows that elongation is small beyond the maximum stress (U.T.S) (i.e. little necking occurs). In contrast, dislocations attained a high thermal-activated mobility at elevated temperatures $\left(>700^{\circ} \mathrm{C}\right.$ for Alloy 617 and Alloy 230).

At these high temperatures, particle pinning is not as effective and efficient as at low temperatures because many dislocations with enhanced mobility are able to bypass the particles 


\section{Understanding Fundamental Material-Degradation Processes in High Temperature Aggressive Chemomechanical Environments}

by dislocation creep or cross-slip. Furthermore, dislocation boundaries (incidental dislocation boundaries (IDB's) in the small scale or geometrically necessary boundaries (GNB's) in the large scale) can transform into low-angle grain boundaries during high-temperature deformation. This process of recrystallization releases the strained areas closed to the interphase boundaries, and thus decreases the stresses that are placed on the particles. As a result, few particle crack voids were observed at temperatures above $700^{\circ} \mathrm{C}$ for both alloys. Instead, intergranular fracture initiated by triple junction cracks became the major fracture mechanism. (Fig. 4.4(b)) Intergranular fracture is a combined result of grain boundary sliding (controlled by dislocation creep) and grain boundary cavitation. Cavity formation was found to depend on the locally applied stresses and grain boundary surface energy that related to the coherence of the boundary. The cavity formation can be described by the critical radius for stable cavity development, $r_{c}$, where $r_{c}=2 \gamma_{s} / \sigma, \gamma_{s}$ is the grain boundary surface energy, and $\sigma$ is the local tensile stress normal to the grain boundary. [4.5] Due to a relatively high strain rate and a corresponding high stress level, grain boundary cavities formed and coalesced to produce grain boundary cracks. Failure finally occurred at wedge cracks located at grain boundary triple points. (Fig. 4.4(b))

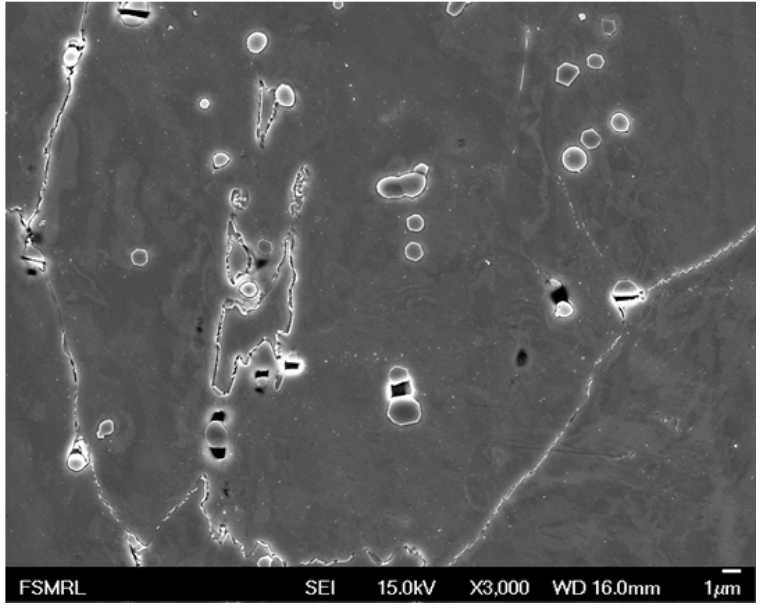

(a)

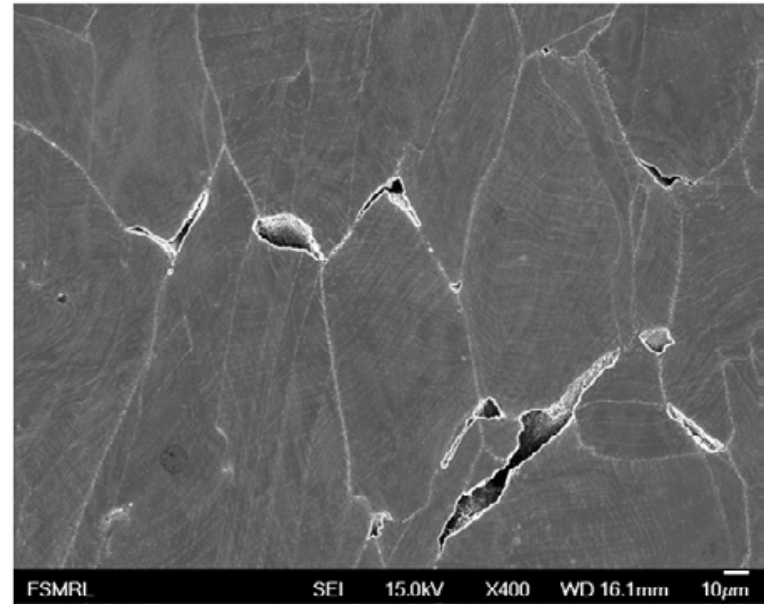

(b)

Fig. 4.4: Fracture micromechanisms: (a) particle cracks at $200^{\circ} \mathrm{C}$ (b) triple point cracks at $800^{\circ} \mathrm{C}$

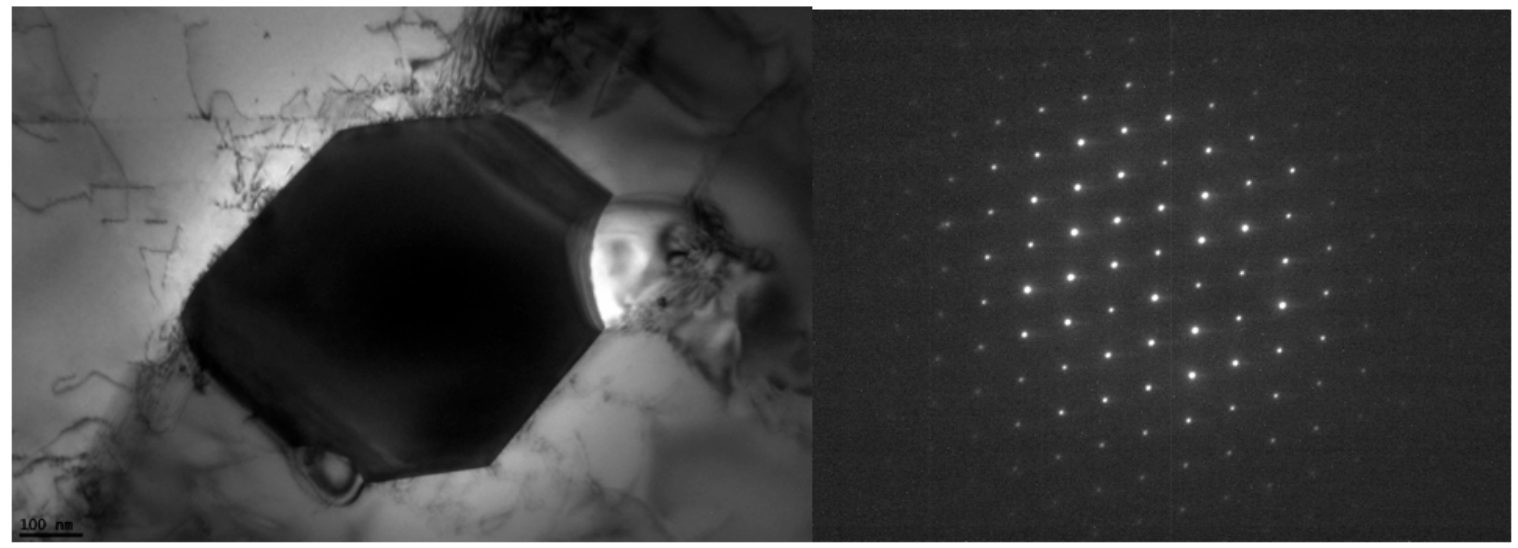

(a)

(b)

Fig. 4.5: $\mathrm{M}_{23} \mathrm{C}_{6}$ in Alloy 617 with dislocations: (a) bright field image; (b) diffraction pattern $(\mathrm{B}=[011])$ 


\section{Understanding Fundamental Material-Degradation Processes in High Temperature Aggressive Chemomechanical Environments}

Another important phenomenon that occurs during high-temperature deformation is dynamic recrystallization (DRX). Fig. 4.6 shows the SEM and EBSD analyses of a tensile specimen after testing at $800^{\circ} \mathrm{C}$. In order to differentiate between the recrystallized grains and inclusions and precipitates, both alloy matrix grains and carbides were scanned, recognized, and mapped by EBSD. (Fig. 4.6(b)\&(c)) A very small number of recrystallized grains with a size of $\sim 1 \mu \mathrm{m}$ or smaller were found in grain boundaries near a triple point crack. No apparent recrystallization was seen in other areas. This indicates that dynamic recrystallization rarely occurs during plastic deformation at $800^{\circ} \mathrm{C}$.

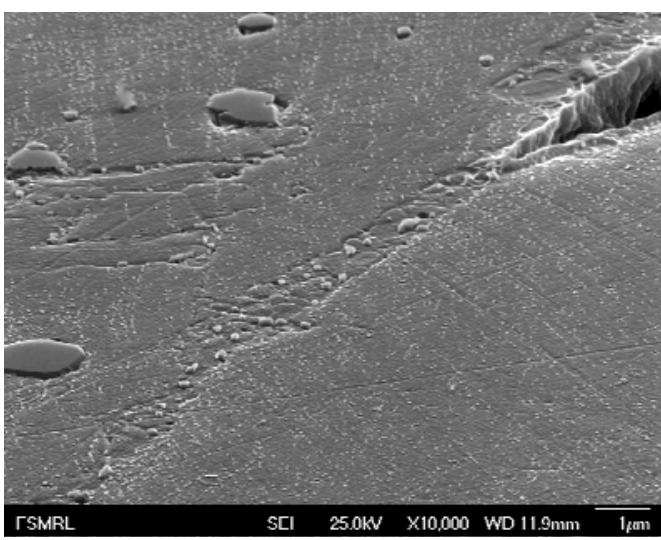

(a)

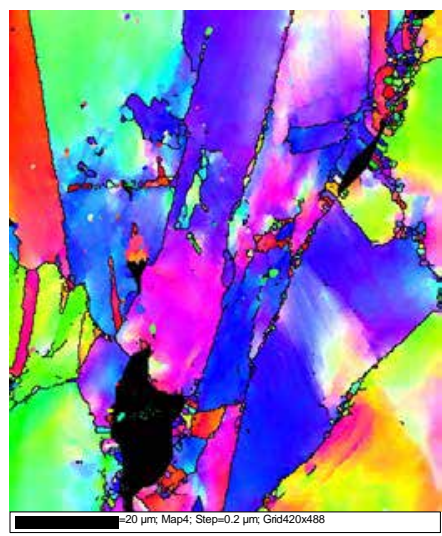

(b)

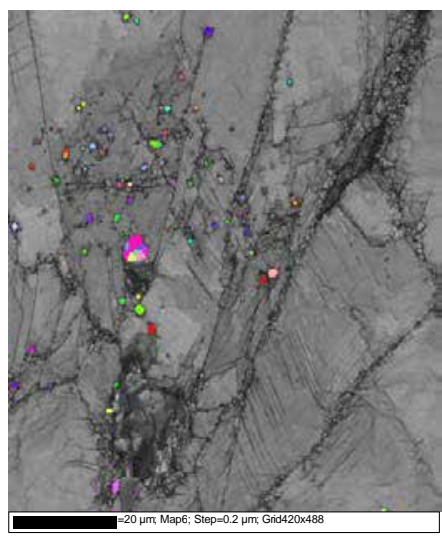

(c)

Fig. 4.6: DRX in the tensile test at $800^{\circ} \mathrm{C}$ : (a) SEM image of a grain boundary with a crack; (b) EBSD mapping showing the crystal orientations; (c) EBSD sampling of the inclusion particles $\left(\mathrm{M}_{23} \mathrm{C}_{6}\right)$

Fig. 4.7 shows dynamic recrystallization during tensile testing at $1000^{\circ} \mathrm{C}$. Due to a higher temperature, both the number and the size of recrystallized grains are much larger compared to those at $800^{\circ} \mathrm{C}$. The recrystallized grains possess various crystal orientations, whereas the adjacent large grains attain a developed texture (e.g. $<111>$ ) after plastic deformation. Additionally, a great number of twin boundaries are generated during dynamic recrystallization due to low stacking fault energy (SFE) in Alloy 617. [4.6] Compared to a large fraction of recrystallized grains in the necking area (Fig. 4.7(b)), dynamic recrystallization that occurs $2 \mathrm{~mm}$ beyond the necking area is less intense (Fig. 4.7(a)) with a smaller number of recrystallized grains. This detail indicates the factor of strain on dynamic recrystallization: higher strain in the necking area promotes dynamic recrystallization. Fig 4.7(b)\&(c) show a great number of fine grains that nucleated in an inclusion-rich area. The high volume fraction of carbide particles and small grain sizes strengthen this area by impeding dislocation movement. Hence, higher dislocation density and more subgrain structures are developed in an inclusion-rich area. During high-temperature deformation, subgrain structures can easily form new grains by the transformation of subgrain boundaries into low-angle grain boundaries. [4.7] In addition to the inclusion rich areas, original high-angle grain boundaries are another preferential region for dynamic recrystallization (the "original" is used to differentiate the boundaries that existed before dynamic recrystallization from the boundaries formed by recrystallization).

During plastic deformation, a large number of original twin boundaries lose their coherence and develop into high $\sum$ or high angle boundaries. However, a very small number of 


\section{Understanding Fundamental Material-Degradation Processes in High Temperature Aggressive Chemomechanical Environments}

recrystallized grains are found in these boundaries. This indicates that different DRX kinetics exist for different types of grain boundaries. Finally, although the strain was over 0.5 when the specimen ruptured, the recrystallized region was much smaller than the un-recrystallized area. This indicates that steady state recrystallized microstructure was not achieved.
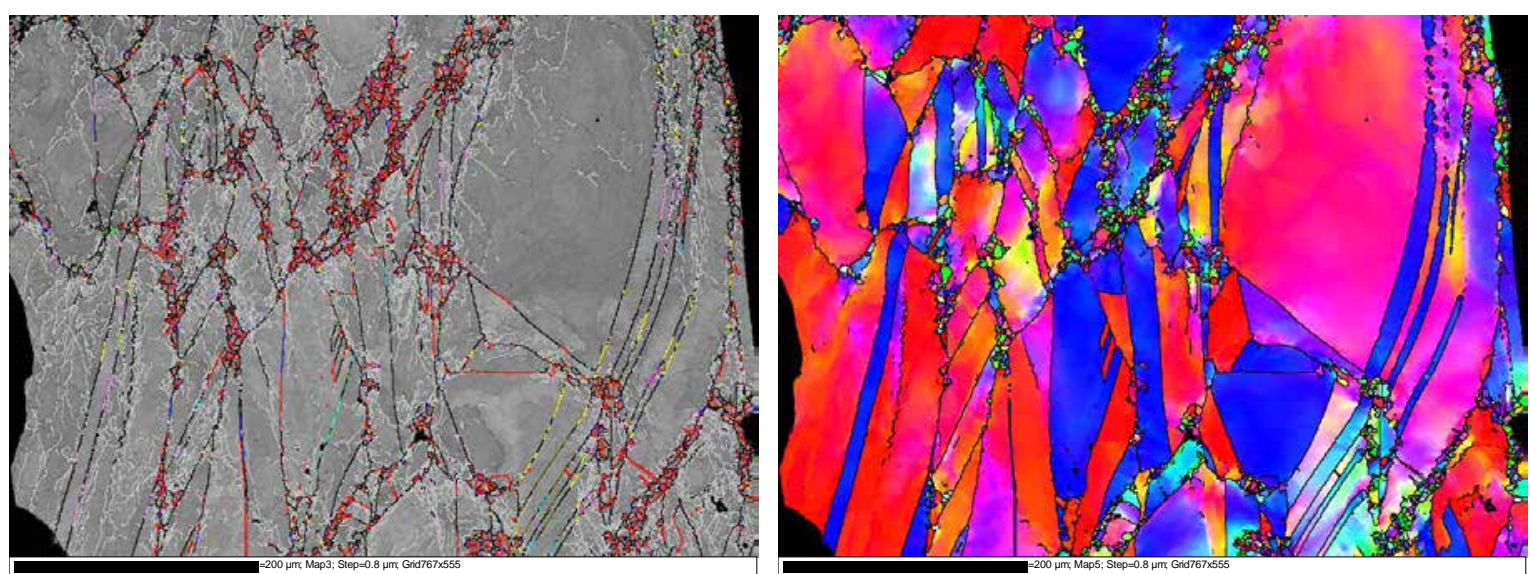

(a)
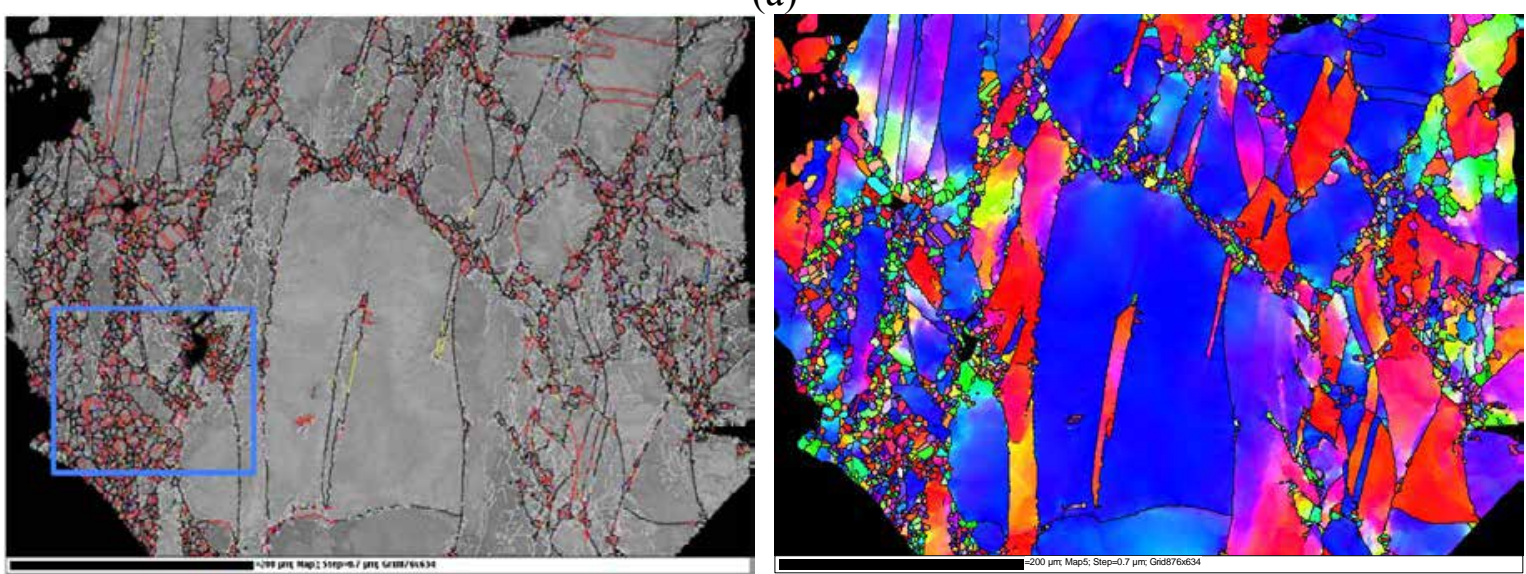

(b)

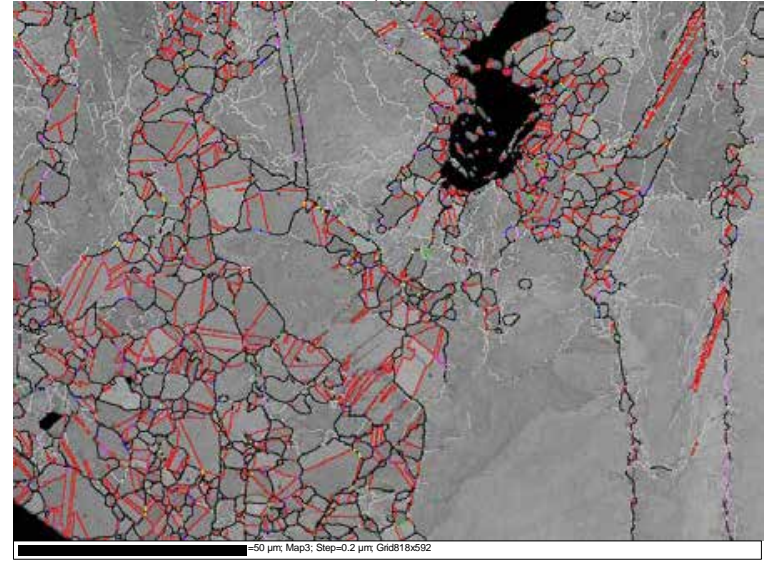

(c)

Fig. 4.7: DRX in the tensile test at $1000^{\circ} \mathrm{C}$ : (a) $2 \mathrm{~mm}$ from the necking area; (b) necking area; (c) enlarged image of the selected area seen in (b) 


\section{Understanding Fundamental Material-Degradation Processes in High Temperature Aggressive Chemomechanical Environments}

\subsection{Effect of Orientation of Alloy 617}

Although numerous studies have been conducted on the creep and corrosion resistance of Alloy 617 and Alloy 230, little research has focused on the evaluation and characterization of the orientation effect on each alloy. The effect of orientation on mechanical properties is significant in terms of engineering design and safety, because the loss of strength in one direction can impact the integrity of required component shapes and cause localized failure. This effect can be exacerbated for components of the VHTR due to its harsh environment and long-term operation. [4.8] For instance, the recently proposed PCHE for use in the VHTR may induce a potential issue with respect to the mechanical directionality of the material. [4.9] Because of the block-shaped design of the PCHE, high-temperature material damages (e.g. creep or creep-fatigue) will not only occur along the gas flow channels, but they will also endanger the transverse direction of the heat exchanger. Unfortunately, the transverse/thickness direction usually possesses the weakest mechanical properties, so failure will be more likely to occur in this direction. Other components with special geometry in the VHTR, such as the thick wall tubes under high internal pressure [4.4], can also suffer the adverse effect of orientation with dramatically lowered strength in the transverse directions. It is therefore necessary for the engineering design of reactor components to measure the mechanical properties of a material in the transverse direction and clarify the source of anisotropy.

In order to study the effect of orientation, tensile testing on the plate form of Alloy 617 with various sampling directions was conducted at temperatures up to $1000^{\circ} \mathrm{C}$. The specimens were sampled from different orientations with respect to the long-transverse direction. (Fig. 4.8) Previous studies indicate that little or no deviation of mechanical properties exists between the rolling (longitude) and the long-transverse direction of Alloy 617. [4.10] Thus, the long-transverse direction was employed as a reference to study the mechanical anisotropy and associated microstructure gradients in Alloy 617.

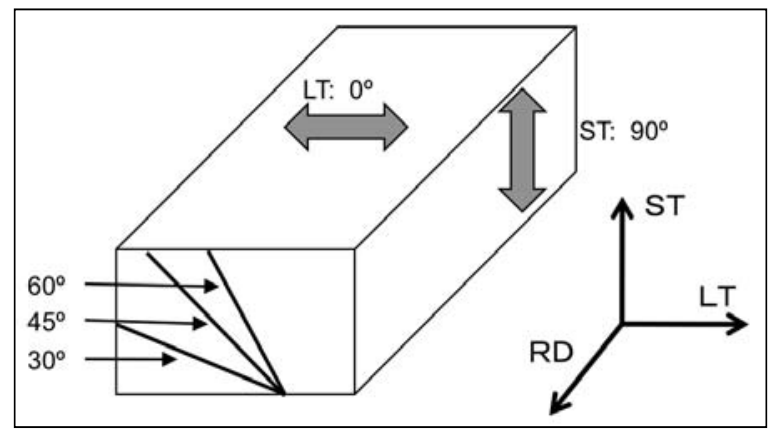

Fig. 4.8: In-plane sampling directions of tensile specimens

\subsubsection{Mechanical anisotropy}

The results of tensile tests on Alloy 617 are presented in Fig. 4.9. The best tensile properties were observed from specimens in the long-transverse direction. Specimens in the other testing directions presented a comparatively lower strength. First, for specimens at $0^{\circ}$ and $30^{\circ}$ angles to the long-transverse direction, the difference in yield strength is $\sim 60 \mathrm{MPa}$ from room temperature to $800^{\circ} \mathrm{C}$. The difference becomes much smaller for temperatures higher than $800^{\circ} \mathrm{C}$. Between $30^{\circ}$ and $45^{\circ}$ angles, the loss of yield strength is more moderate: $\sim 20 \mathrm{MPa}$ on average from RT to 


\section{Understanding Fundamental Material-Degradation Processes in High Temperature Aggressive Chemomechanical Environments}

$800^{\circ} \mathrm{C}$. Finally, yield strength does not show a noticeable change for angles of $45^{\circ}$ to $90^{\circ}$. Unlike the changes in yield strength, the ultimate tensile strength decreases at an approximately constant value from one angle to another. In other words, Alloy 617 shows a linear loss in tensile strength from the long-transverse $\left(0^{\circ}\right)$ to the short-transverse $\left(90^{\circ}\right)$ direction. It should also be noted that the loss in tensile strength is generally greater than the loss in yield strength.

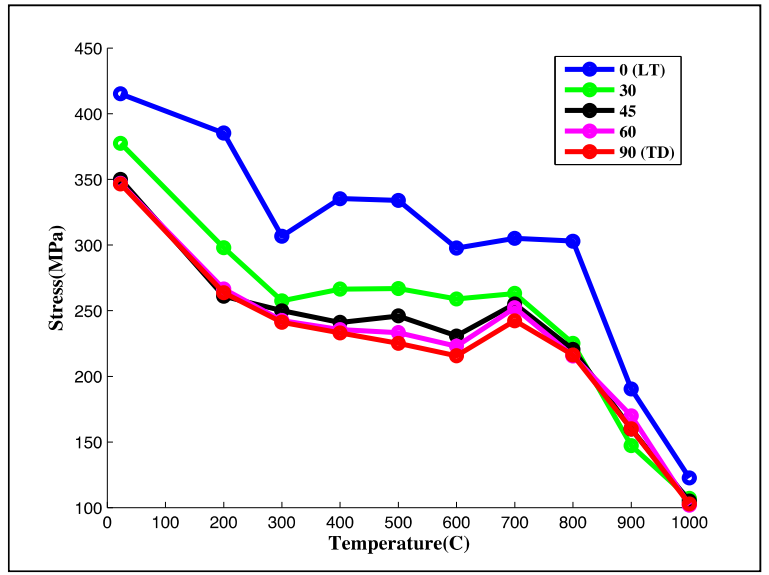

(a)

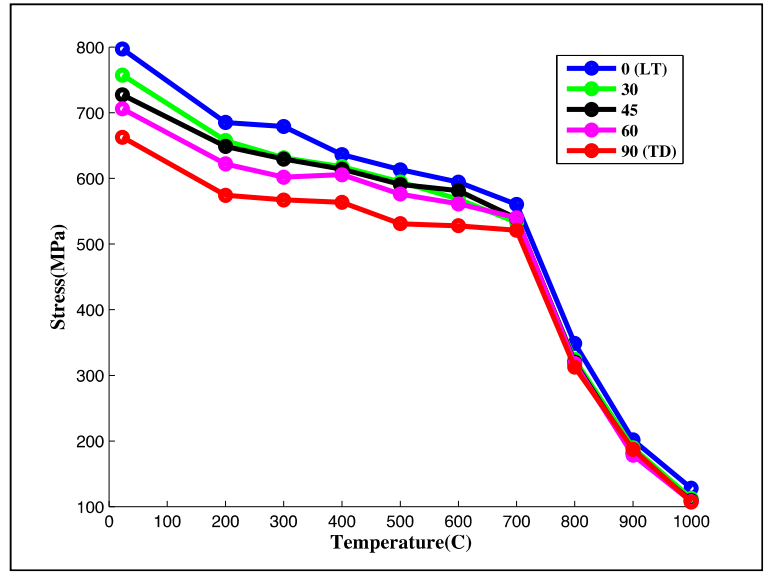

(b)

Fig. 4.9: Tensile properties versus temperature for various angles with the long-transverse direction: (a) Y.S.; (b) U.T.S.

Additionally, the temperature dependence of mechanical anisotropy is pronounced for Alloy 617. The difference in yield strength between the long-transverse and short-transverse directions is reduced from $\sim 70 \mathrm{MPa}$ at room temperature to $\sim 20 \mathrm{MPa}$ at $1000^{\circ} \mathrm{C}$. In tensile strength, the difference decreases from $\sim 135 \mathrm{MPa}$ at room temperature to $\sim 20 \mathrm{MPa}$ at $1000^{\circ} \mathrm{C}$. Note that the difference in magnitude of strength becomes much smaller for temperatures higher than $800^{\circ} \mathrm{C}$. (Fig. 4.9) Nevertheless, it is difficult to conclude that the effect of orientation diminishes as the temperature increases, because the absolute values of both the yield and tensile strength have already decreased. Therefore, a simple comparison of absolute strength values is insufficient to evaluate the anisotropy, since a small stress difference may be caused by a large fraction of strength reduction. Thus, in order to evaluate the strength as a fraction, the long-transverse strengths are employed as references. Hence, the relative Y.S. and U.T.S. can be expressed by equations 4.1 and 4.2 , respectively.

$$
\begin{aligned}
& \text { Relative Y.S. }=\frac{\text { Y.S. for a specific angle }}{\text { Y.S. in long }- \text { transverse direction }\left(\theta=0^{\circ}\right)}=\frac{\text { Y.S. }(\theta, \mathrm{T})}{\mathrm{Y} . \mathrm{S} .\left(\theta=0^{\circ}, \mathrm{T}\right)} \\
& \text { Relative U.T.S. }=\frac{\text { U.T.S. for a specific angle }}{\text { U.T.S. in long }- \text { transverse direction }\left(\theta=0^{\circ}\right)}=\frac{\text { U.T.S. }(\theta, \mathrm{T})}{\text { U.T.S. }\left(\theta=0^{\circ}, \mathrm{T}\right)}
\end{aligned}
$$

In the above equations, $\theta$ represents the angle from the long-transverse direction $\left(\theta=0^{\circ}(\mathrm{LT})\right.$, $30^{\circ}, 45^{\circ}, 60^{\circ}$ or $\left.90^{\circ}(\mathrm{ST})\right)$ and $\mathrm{T}$ is the studied temperature. Additionally, the mean value of the relative Y.S. and U.T.S. for all studied temperatures can be defined as: 


$$
\begin{aligned}
\overline{\text { Relative Y.S. }}= & \frac{\text { Mean value of Y.S. for a specific angle for all studied temperatures }}{\text { Mean value of Y.S. in long - transverse direction }\left(\theta=0^{\circ}\right) \text { for all studied temperatures }} \\
& =\frac{\sum_{\mathrm{T}=\mathrm{RT}}^{1000^{\circ} \mathrm{C}} \mathrm{Y} . \mathrm{S} .(\theta, \mathrm{T})}{\sum_{\mathrm{T}=\mathrm{RT}}^{1000^{\circ} \mathrm{C}} \mathrm{Y} . \mathrm{S} .\left(\theta=0^{\circ}, \mathrm{T}\right)} \\
& =\frac{\sum_{\mathrm{T}=\mathrm{RT}}^{1000^{\circ} \mathrm{C}} \mathrm{U} . \mathrm{T} . \mathrm{S} .(\theta, \mathrm{T})}{\sum_{\mathrm{T}=\mathrm{RT}}^{\text {Mean valive U.T.S.S. }\left(\theta=0^{\circ}, \mathrm{T}\right)}}
\end{aligned}
$$

The mean values of relative strength are calculated to study the overall effect of orientation for the entire range of studied temperatures. Meanwhile, these mean values serve as standards of comparison for the relative Y.S. and U.T.S. at different temperatures.

Fig. 4.10 shows the results of the analysis of relative strength. Similar to the changes in absolute strength, the relative yield strength decreases rapidly once the sample orientation is not parallel to the rolling planes. The relative yield strength maintains a small value for angles between $45^{\circ}$ and $90^{\circ}$. The relative tensile strength exhibits a linear relation with respect to the orientation with the long-transverse direction. These results agree well with the analysis of absolute strength at lower temperatures. However, the difference in relative strength between the long-transverse direction and the other studied angles at high temperatures does not diminish as observed for the trend in absolute strength. The loss in yield strength in the short-transverse direction is $\sim 17 \%$ at both room temperature and $1000^{\circ} \mathrm{C}$, slightly less than the mean value of $22 \%$ ('AVG' in Fig. 4.10(a)). The loss in tensile strength in the short-transverse direction at room temperature, $300^{\circ} \mathrm{C}$, and $1000^{\circ} \mathrm{C}$ has a similar value of $\sim 17 \%$, which is also close to the mean value ('AVG' in Fig. 4.10(b)). Therefore, even though the difference in absolute strength between the long-transverse and short-transverse directions is much smaller at high temperatures, the difference in relative strengths is comparable to the value at low temperatures. This indicates that the effect of orientation on the mechanical properties at high temperatures is analogous to that at low temperatures. 


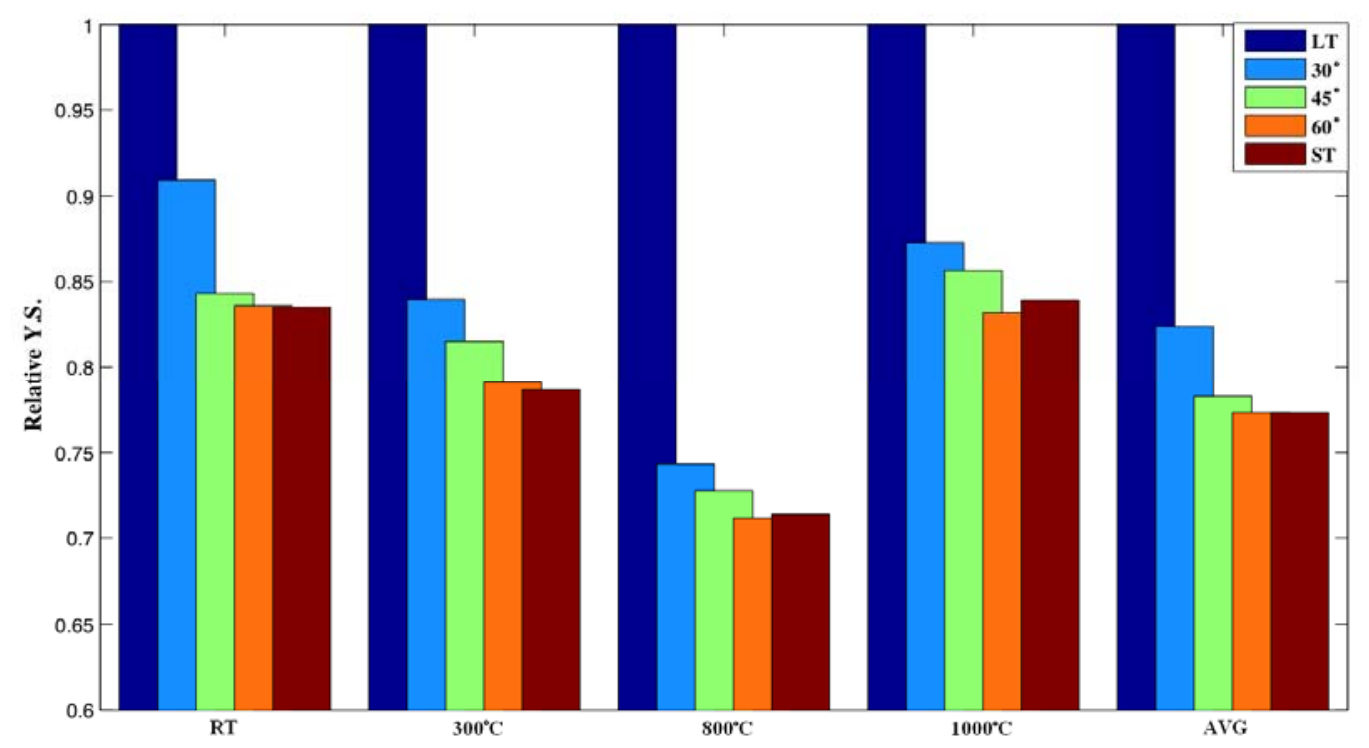

(a)

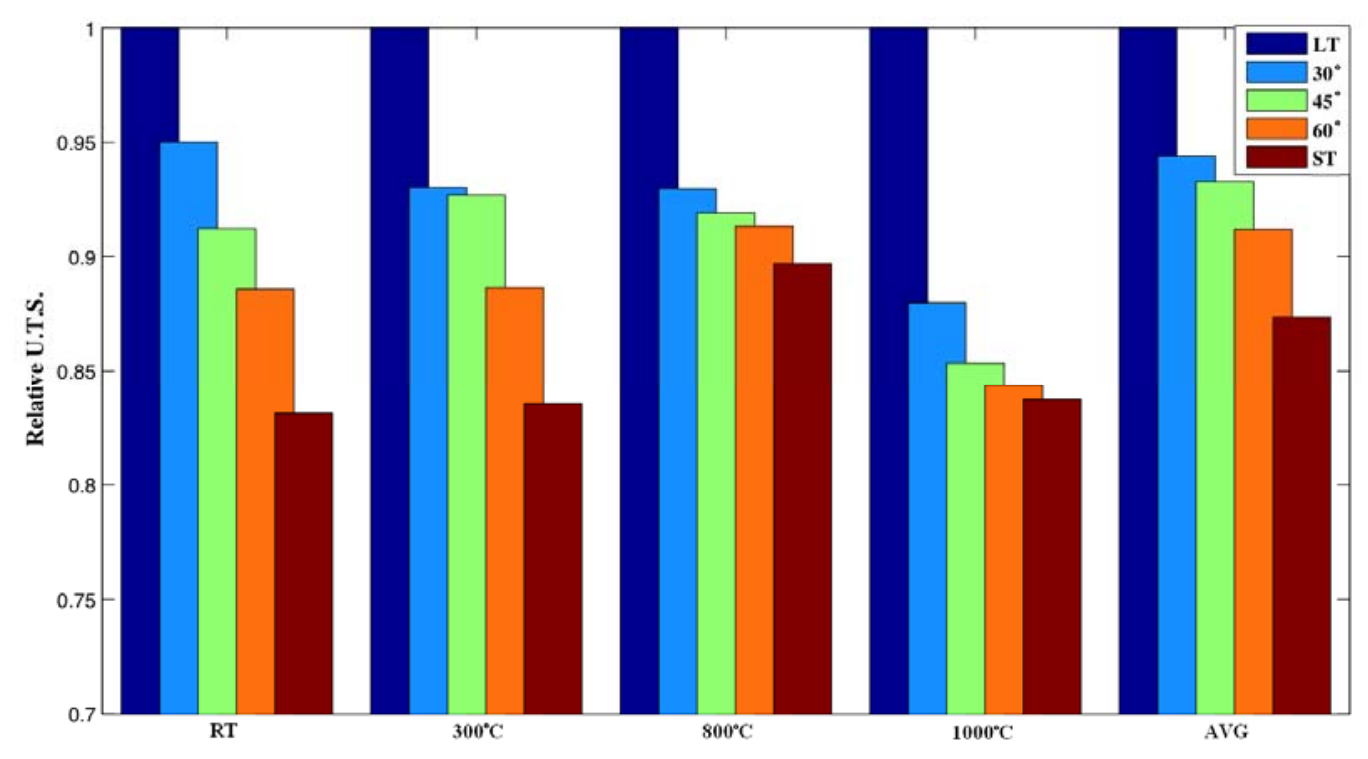

(b)

Fig. 4.10: Relative strengths of Alloy 617: (a) relative Y.S.; (b) relative U.T.S.

\subsubsection{Microstructural anisotropy}

The triplanar montage generated from SEM images exhibits the microstructure gradients in the three principle directions. (Fig. 4.11) A great number of intrinsic carbide inclusions, composed of coarse $\mathrm{M}_{23} \mathrm{C}_{6}$ type carbides with a small amount of $\mathrm{Ti}(\mathrm{C}, \mathrm{N})$, are presented as white spots in 


\section{Understanding Fundamental Material-Degradation Processes in High Temperature Aggressive Chemomechanical Environments}

Fig. 4.11. These carbides are shown as bands on the transverse plane (TP) and longitudinal plane (LP) and grouped as clusters on the rolling plane (RP).

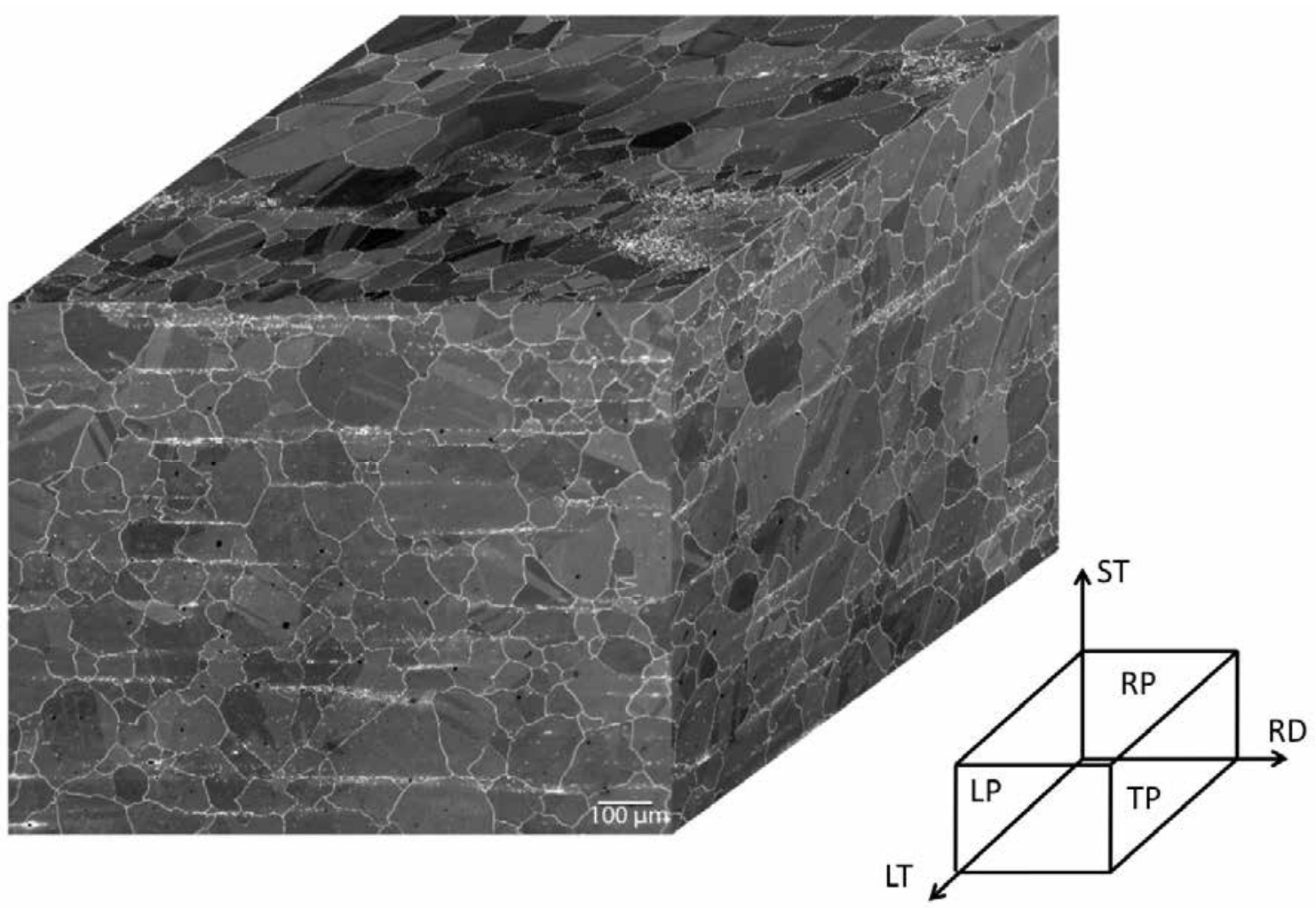

Fig. 4.11: Triplanar montage illustrating the grain structure and inclusions in the as-received condition. The rolling direction (RD), short transverse (ST) direction, long transverse (LT) direction, rolling plane (RP), transverse plane (TP), and longitudinal plane (LP) are noted.

The EBSD mappings for the rolling and transverse plane are shown in Fig. 4.12(a)\&(b), respectively. Similar to the band-shaped distribution of inclusion particles (Fig. 4.11), the alignment of crystals in the transverse plane appears to be parallel to the rolling plane. Most small grains are located near carbide bands. The lamellar-shaped inclusions and the associated fiber-shaped grain distributions are known as mechanical fibering. [4.4, 4.5] The EBSD analysis also shows that both coarse and fine grains are randomly oriented, indicating no intrinsic texture in the as-received Alloy 617. (Fig. 4.13) 


\section{Understanding Fundamental Material-Degradation Processes in High Temperature Aggressive Chemomechanical Environments}

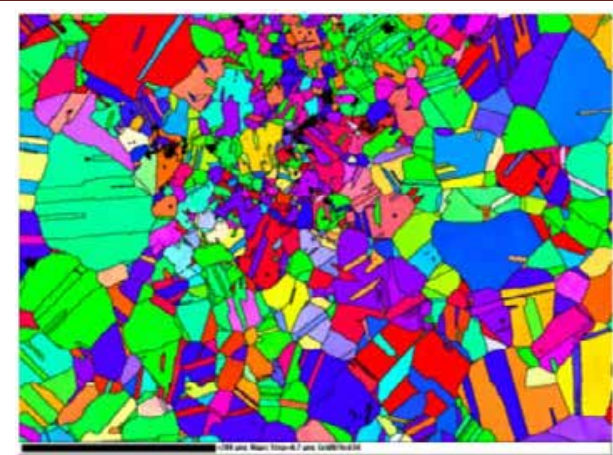

(a)

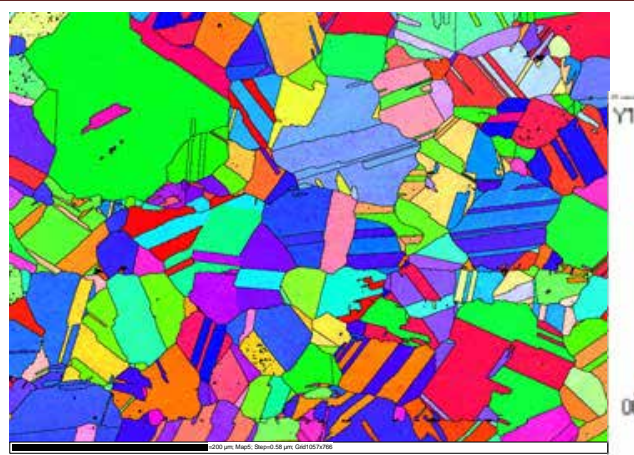

(b)

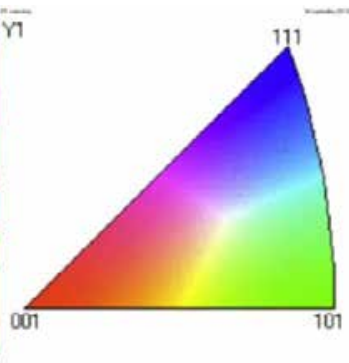

(c)

Fig. 4.12: EBSD analysis of Alloy 617 in the as-received condition: (a) rolling plane; (b) transverse plane; (c) legend
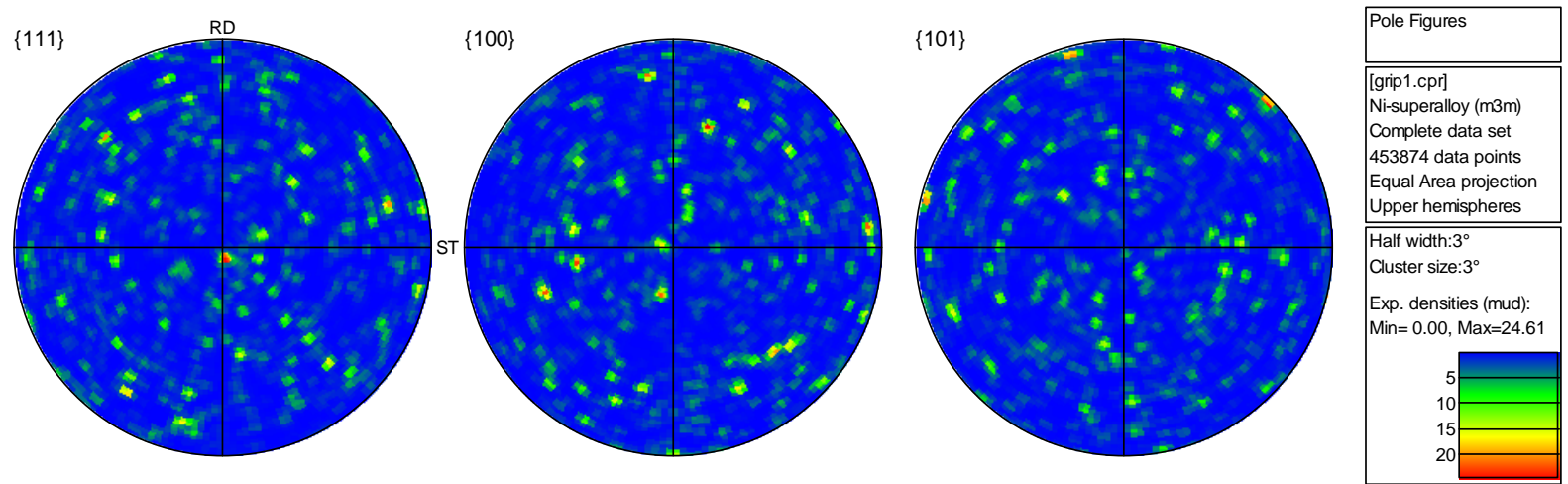

Fig. 4.13: Texture analysis for Alloy 617 in the as-received condition

Fig. 4.14 and Fig. 4.15 show the results of EBSD analyses for the long- and short-transverse specimens, respectively, that have been tensile tested at room temperature. The sampling area for each specimen is $\sim 0.5 \mathrm{~mm}$ from the rupture edge. The crystallography in Fig. 4.14(a) and Fig. 4.15(a) shows that the dominant textures after plastic deformation are primary $<111>$ and secondary $<001>$ parallel to the tension direction (refer to the legend in Fig. 4.12(c)). A direct observation of the texture development in specimens comes from an intensity mapping in the pole figures (Fig. 4.16). The long-transverse specimen showed a developed duplex texture: $<111>+<001>$ (Fig. 4.16(a)). In contrast, many more random crystal orientations were observed in the short-transverse specimen, indicating that the material was not significantly deformed before rupture (Fig. 4.16(b)). 


\section{Understanding Fundamental Material-Degradation Processes in High Temperature}

Aggressive Chemomechanical Environments

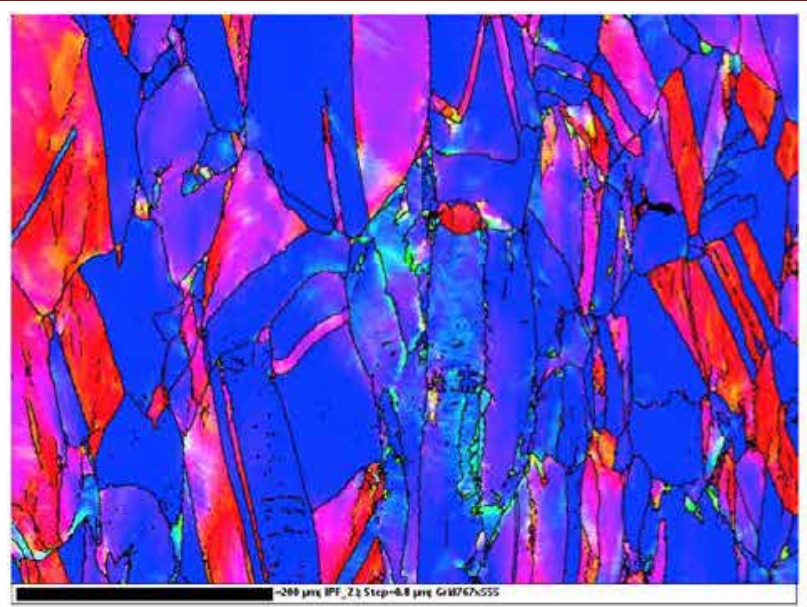

(a)

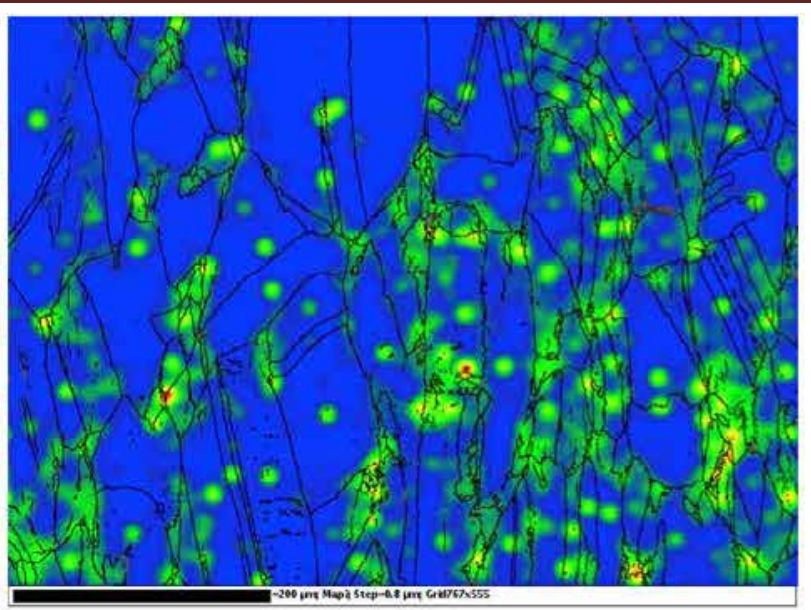

(b)

Fig. 4.14: EBSD analysis for the long-transverse specimen after tensile testing at room temperature: (a) crystal orientation mapping; (b) strain contouring

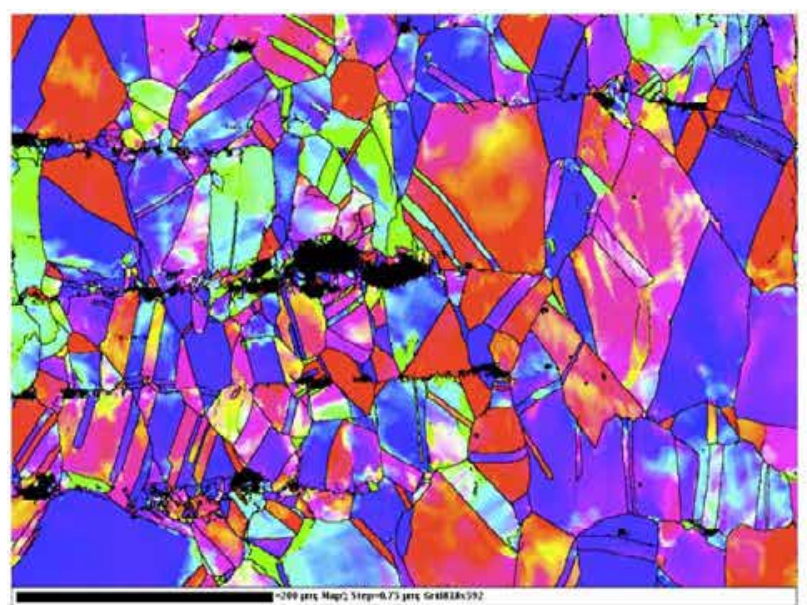

(a)

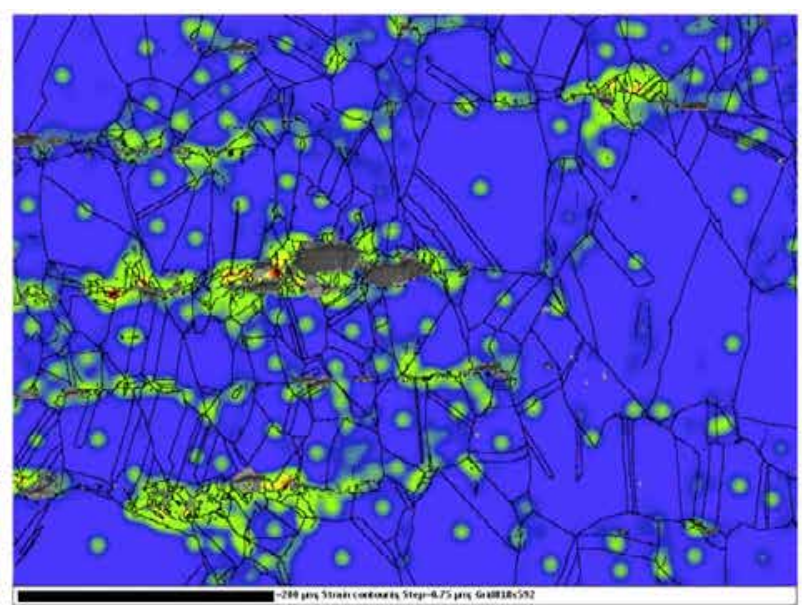

(b)

Fig. 4.15: EBSD analysis for the short-transverse specimen after tensile testing at room temperature: (a) crystal orientation mapping; (b) strain contouring 
Understanding Fundamental Material-Degradation Processes in High Temperature Aggressive Chemomechanical Environments
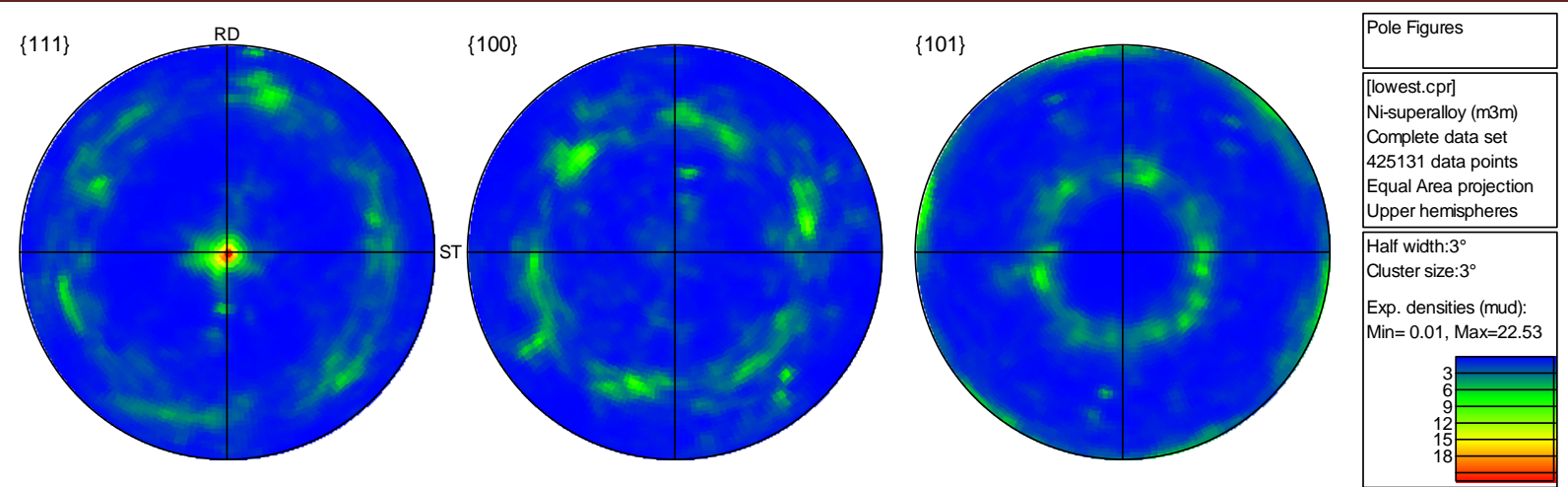

(a)
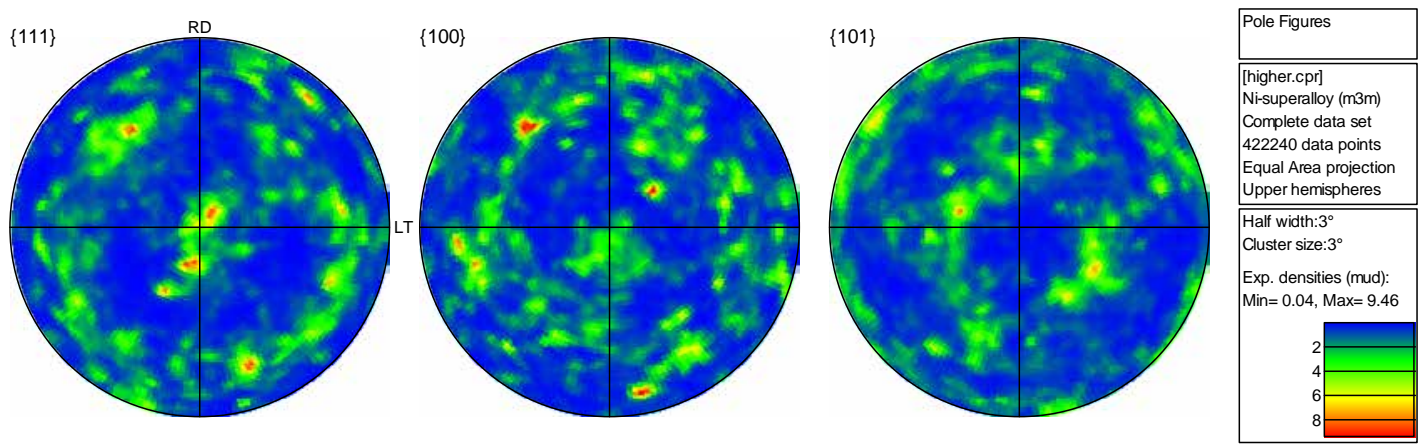

(b)

Fig. 4.16: Texture development after tensile testing at room temperature: (a) long-transverse direction; (b) short-transverse direction

Beyond texture analysis, a more direct method, strain contouring, was employed to study orientation effects on deformation processes of Alloy 617. Based on the measurement of the maximum misorientation in a grain, strain contouring provides an estimate of the amount of strain in individual grains of a material. Detailed procedures for strain contouring can be found in [4.11]. The results of strain contouring for the long- and short-transverse specimens are shown in Fig. 4.14(b) and Fig. 4.15(b), respectively. The intensities of highly strained areas are represented by the red and green portions of the maps, with red signifying a greater intensity. Note that the highly strained areas are located close to the inclusion rich areas for both specimens. For the short-transverse specimen, the highly strained area is close to the large cracks generated by the rupture of the inclusion particles, and thus appears perpendicular to the loading direction. (Fig. 4.15(b)) However for the long-transverse specimen, the highly strained area distributes parallel to the loading direction, because the inclusion rich area was slightly elongated along the loading direction during plastic deformation. (Fig. 4.14(b))

Following thermal aging for a specimen, microstructural analysis can provide additional information to understand the effects of orientation. Alloy 617 develops $\mathrm{M}_{23} \mathrm{C}_{6}$ type precipitates during high-temperature aging. These carbide particles nucleate, diffuse, and coarsen with aging before they finally stabilize in favorable nucleation sites (e.g. interphase and high-angle grain boundaries). [3.15, 4.12] (Detailed discussion of this aging effect will be given in the next chapter.) SEM images show that the grain boundaries of Alloy 617 become decorated with 


\section{Understanding Fundamental Material-Degradation Processes in High Temperature Aggressive Chemomechanical Environments}

continuous intergranular carbide "chains" following thermal aging. (Fig. 4.17) These chains were broken at grain boundaries during plastic deformation. Fig. 4.17 shows a specimen of Alloy 617 aged for 300 hours at $1000^{\circ} \mathrm{C}$ before undergoing a tensile test at room temperature. The tension direction is parallel to the short-transverse direction (perpendicular to the inclusion bands in the picture). During the test, the intergranular carbide chains became disconnected in areas lying between two inclusion bands, indicating that the plastic deformation essentially localized in these areas. The disconnection of the carbide chains and corresponding grain boundary elongation confirm the EBSD microstructural analyses for the short-transverse specimen. These phenomena also reveal the fact that the only strongly deformed areas are the areas between inclusion bands, and the inclusion bands constrain the plastic flow and result in highly strained areas (with large misorientation relative to a whole grain) close to inclusion particles.

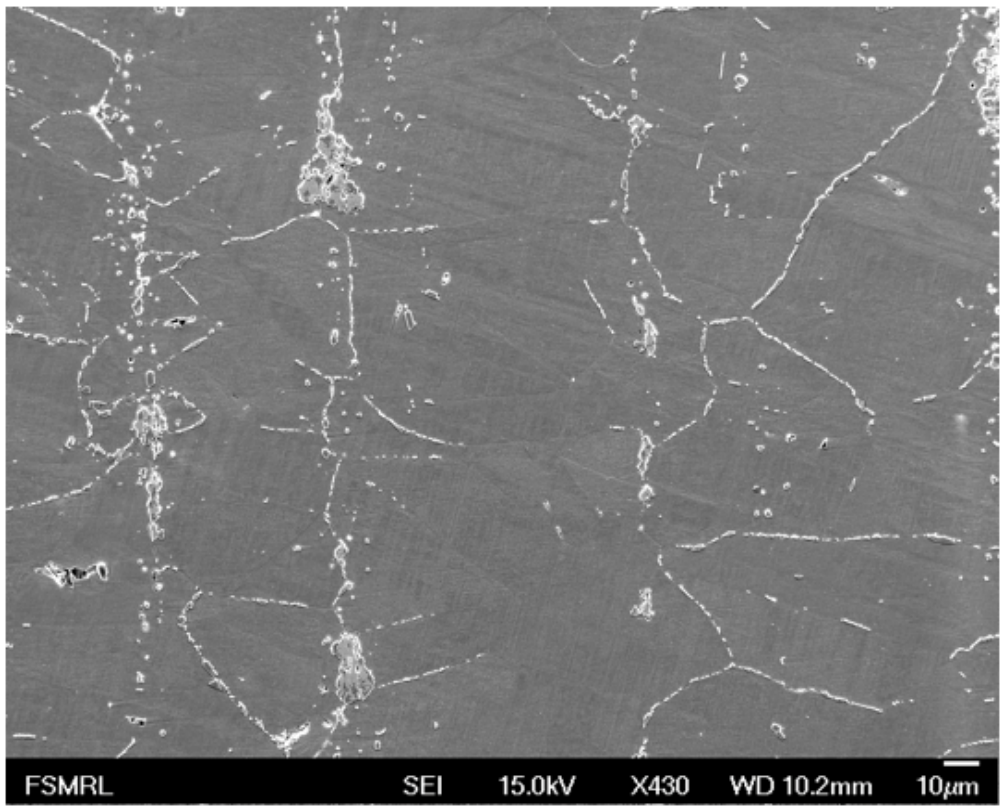

Fig. 4.17: SEM micrograph of a fractured sample of Alloy 617 aged at $1000^{\circ} \mathrm{C}$ for 300 hours

Above $800^{\circ} \mathrm{C}$, the flow stress is found to rapidly decrease once the yield stress is reached. Strain hardening is weakened due to the enhanced dislocation mobility. Additional deformation mechanisms, including dislocation creep and dynamic recrystallization (DRX) are also induced at high temperatures. The effect of orientation on the deformation process for high temperatures is therefore different from that for low temperatures. At a high temperature (e.g. $\left.1000^{\circ} \mathrm{C}\right)$, strains near the inclusion particles can be released by additional deformation mechanisms (e.g. dynamic recrystallization). However, the effect of orientation is not subsequently weakened according to the analysis of relative strengths. (Fig. 4.10) Fig. 4.18 shows the EBSD mapping of a short-transverse specimen after a tensile test at $1000^{\circ} \mathrm{C}$. Compared to a large fraction of recrystallized grains found in the long-transverse specimen (Fig. 4.7), recrystallized grains in the short-transverse specimen developed into another feature: a smaller number of grains with larger grain size. Nevertheless, similar to the long-transverse specimen, many recrystallized grains were found in the area close to the inclusion particles for the same reason given in section 4.1.1. 


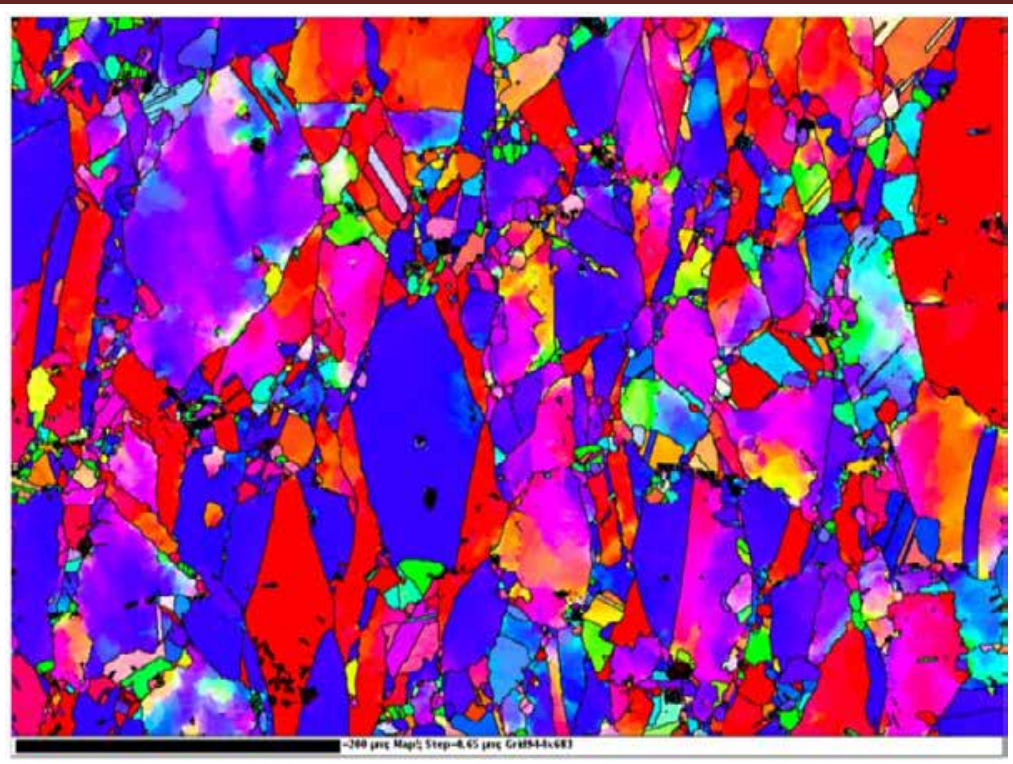

Fig. 4.18: Dynamic recrystallizations in a short-transverse specimen after tensile testing at $1000^{\circ} \mathrm{C}$

\subsubsection{Fractography}

Fractography provides a direct approach to study the various fracture processes of specimens at various angles. Fig. 4.19 shows the fractography of specimens following tensile testing at room temperature. For the specimens at $0^{\circ}$ and $30^{\circ}$ angles from the long-transverse direction, each individual grain can be clearly indentified in the fracture surface, indicating an approximate intergranular fracture. (Fig. 4.19(a)\&(b)) For the specimen at $45^{\circ}$, "dimples" with slightly elongated troughs arise due to microvoids generated by the shear stress acting on the carbide particles. (Fig. 4.19(c)) "Dimples" with equiaxed microvoids can be found in the specimens at $60^{\circ}$ and $90^{\circ}$ (the short-transverse direction). (Fig. 4.19(d)\&(e)) These microvoids are formed in association with fractured inclusion particles and rapidly expand under approximately uniaxial stresses. The results show that the impact of mechanical fibering is relatively small for low angles $\left(0^{\circ}\right.$ and $\left.30^{\circ}\right)$, but becomes significant for higher angles $\left(45^{\circ}, 60^{\circ}\right.$ and $\left.90^{\circ}\right)$. The performed fractography analysis agrees well with mechanical tests and other microstructure analyses. 
Understanding Fundamental Material-Degradation Processes in High Temperature Aggressive Chemomechanical Environments

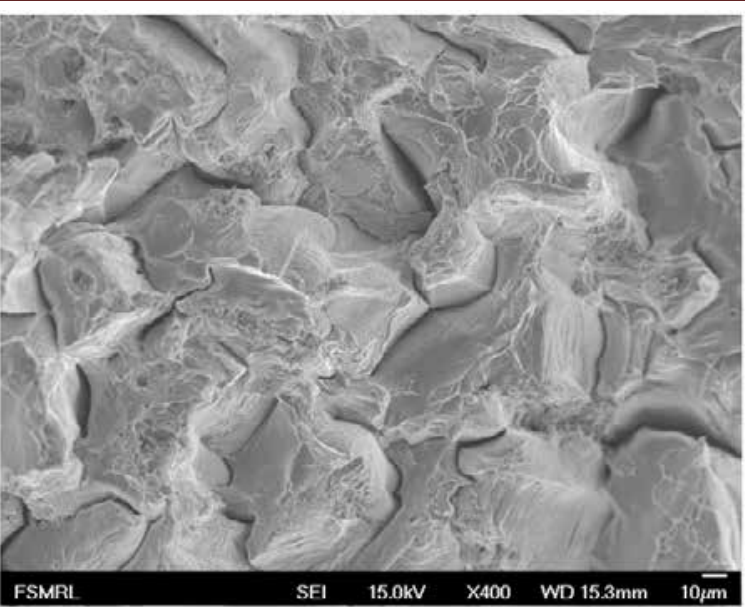

(a)

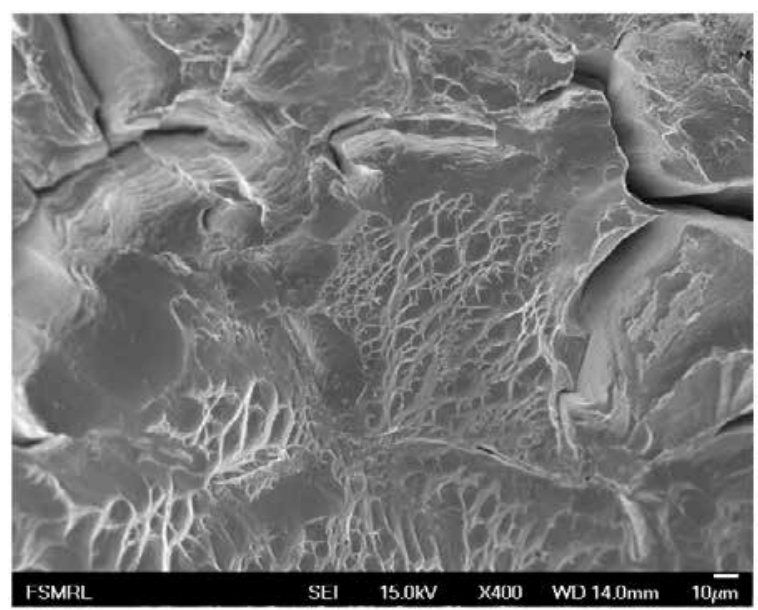

(c)

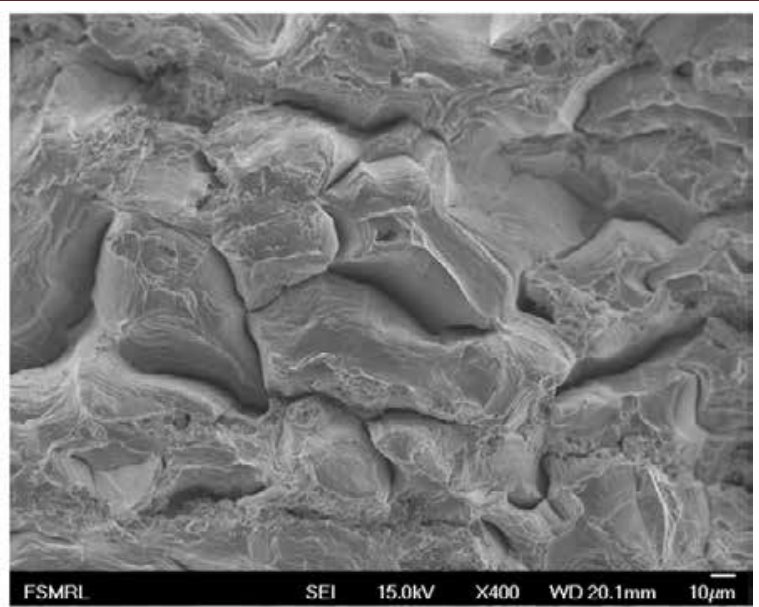

(b)

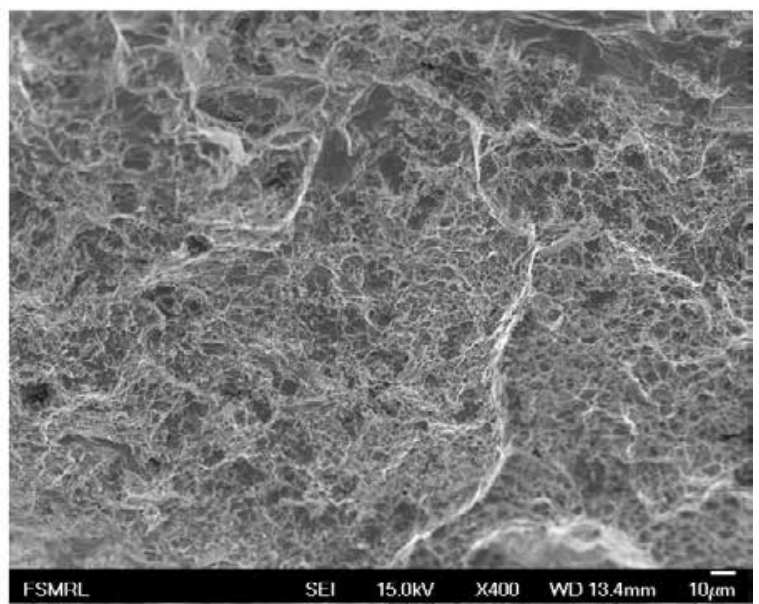

(d)

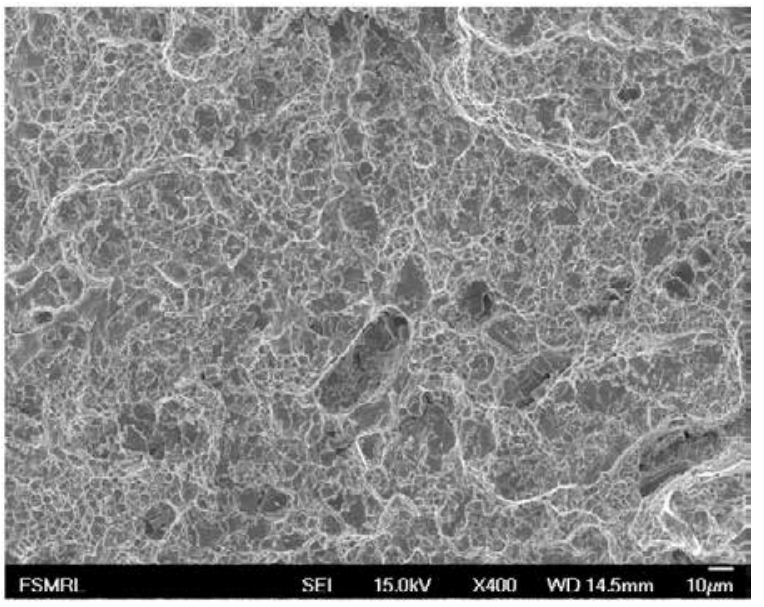

(e)

Fig. 4.19: Fractography after tensile testing at room temperature: (a) $0^{\circ}$ (long-transverse direction); (b) $30^{\circ}$; (c) $45^{\circ}$; (d) $60^{\circ}$; (e) $90^{\circ}$ (short-transverse direction) 


\section{Understanding Fundamental Material-Degradation Processes in High Temperature Aggressive Chemomechanical Environments}

\subsection{Effect of Strain Rate}

The strain rate applied to a material during a tensile test can have a significant impact on flow stress. The strain rate is defined as $\dot{\varepsilon}=d \varepsilon / d t$ and is expressed in units of $\mathrm{s}^{-1}$. For most metallic materials, a higher strain rate generally results in a higher flow stress. In other words, increasing the strain rate in mechanical tests can increase the yield stress, and vice versa. [4.5] This strain rate dependence of flow stress is controlled by the applied temperature. Generally, metallic materials are more sensitive to strain rate at higher temperatures. Since Alloy 617 and Alloy 230 are expected to be used at temperatures up to $1000^{\circ} \mathrm{C}$, the effect of strain rate must be well examined before any practical application can take place.

The true stress $\left(\sigma_{t}\right)$ and true strain $\left(\varepsilon_{t}\right)$ can be found in terms of engineering stress $\left(\sigma_{e}\right)$ and engineering strain $\left(\varepsilon_{e}\right)$ by:

$\sigma_{t}=\sigma_{e}\left(1+\varepsilon_{e}\right)$

$\varepsilon_{t}=\ln \left(1+\varepsilon_{e}\right)$

The true strain rate, $\dot{\varepsilon}_{t}$, is the rate of change in true strain $\left(\varepsilon_{t}\right)$ with respect to time $(t)$, and is defined by:

$\dot{\varepsilon}_{t}=\frac{d \varepsilon_{t}}{d t}=\frac{d\left[\ln \left(L / L_{0}\right)\right]}{d t}=\frac{d L / d t}{L}=\frac{v}{L}$

In equation $4.5, v$ is the crosshead velocity, $L$ is the instantaneous gage length, and $L_{0}$ is the initial gage length. Most tensile tests are conducted at a constant engineering strain rate $\left(\dot{\varepsilon}_{e}\right)$, so it is more convenient to relate the true strain rate and engineering strain rate by:

$\dot{\varepsilon}_{t}=\frac{v}{L}=\frac{d \varepsilon_{e} / d t}{\left(1+\varepsilon_{e}\right)}=\frac{\dot{\varepsilon_{e}}}{1+\varepsilon_{e}}$

When the applied engineering strain is small (e.g. $\varepsilon_{e}=0.002$ ), the difference between $\dot{\varepsilon}_{e}$ and $\dot{\varepsilon}_{t}$ is negligible. Hence, $\dot{\varepsilon}_{t}$ can be replaced by $\dot{\varepsilon}_{e}$ when studying the effect of strain rate on the yield stress. In the following discussion, the general form $\dot{\varepsilon}$ is used to replace $\dot{\varepsilon}_{e}$ and $\dot{\varepsilon}_{t}$.

The relationship between flow stress and strain rate at a constant strain and temperature is given as:

$\sigma_{t}=C(\dot{\varepsilon})^{m}$

In equation $4.7, m$ is the strain-rate sensitivity and $C$ is a fitting constant. This equation can be rewritten as:

$\frac{\sigma_{1}}{\sigma_{2}}=\left(\frac{\dot{\varepsilon_{1}}}{\dot{\varepsilon_{2}}}\right)^{m}$

In equation 4.8, $\sigma_{1}$ and $\sigma_{2}$ are the flow stresses during testing at strain rates of $\dot{\varepsilon}_{1}$ and $\dot{\varepsilon}_{2}$, respectively. Thus, $m$ can be obtained:

$m=\frac{\log \left(\frac{\sigma_{1}}{\sigma_{2}}\right)}{\log \left(\frac{\dot{\varepsilon_{1}}}{\dot{\varepsilon_{2}}}\right)}$ 


\section{Understanding Fundamental Material-Degradation Processes in High Temperature Aggressive Chemomechanical Environments}

In order to obtain a sufficient data set to study the strain-rate sensitivity, tensile tests for Alloy 617 and Alloy 230 were conducted at two different strain rates: $0.01 \mathrm{~s}^{-1}$ and $0.05 \mathrm{~s}^{-1}$, in addition to previous tests completed at $0.001 \mathrm{~s}^{-1}$. Fig. 4.20 shows the strain-stress diagrams of Alloy 617 for three different temperatures: $200^{\circ} \mathrm{C}, 800^{\circ} \mathrm{C}$, and $1000^{\circ} \mathrm{C}$, representing low, high, and very high temperatures. At $200^{\circ} \mathrm{C}$, the flow stress was found to be insensitive to applied strain rates. (Fig. 4.20(a)) Nevertheless, the flow stress substantially increased at 800 and $1000^{\circ} \mathrm{C}$ while increasing the applied strain rate. This relationship shows the temperature dependence of the effect of strain rate for Alloy 617.

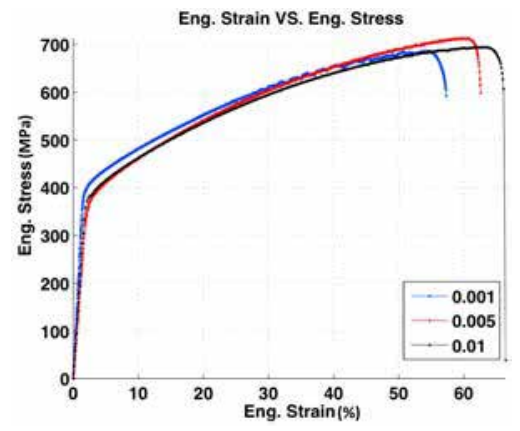

(a)

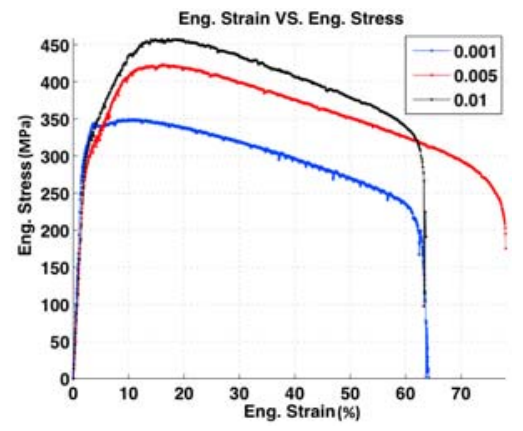

(b)

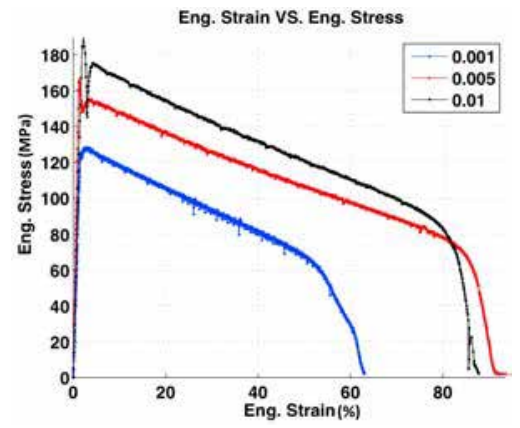

(c)

Fig. 4.20: Engineering strain-stress diagram for Alloy 617 at: (a) $200^{\circ} \mathrm{C}$; (b) $800^{\circ} \mathrm{C}$; (c) $1000^{\circ} \mathrm{C}$

The complete results for the tensile tests are given in APPENDIX A. The value of $m$ is determined by measuring the slope of a plot of $\log (\dot{\varepsilon})$ versus $\log (\sigma)$. (Fig. 4.21) The measured strain-rate sensitivities for $0.2 \%$ flow stress for Alloy 617 and Alloy 230 are given in Tables 4.2 and 4.3, respectively. For Alloy 617, the value of $m$ is small $(<0.1)$ from room temperature to $800^{\circ} \mathrm{C}$. A notable increase in $m$ occurs at 900 and $1000^{\circ} \mathrm{C}$ with a value exceeding 0.17 . For Alloy 230 , the value of $m$ is close to 0 for low temperatures. The only exception occurs at room temperature with a value $m$ of $\sim 0.15$. Similar to the Alloy 617 , the strain-rate sensitivities for Alloy 230 are much higher at 900 and $1000^{\circ} \mathrm{C}$. 


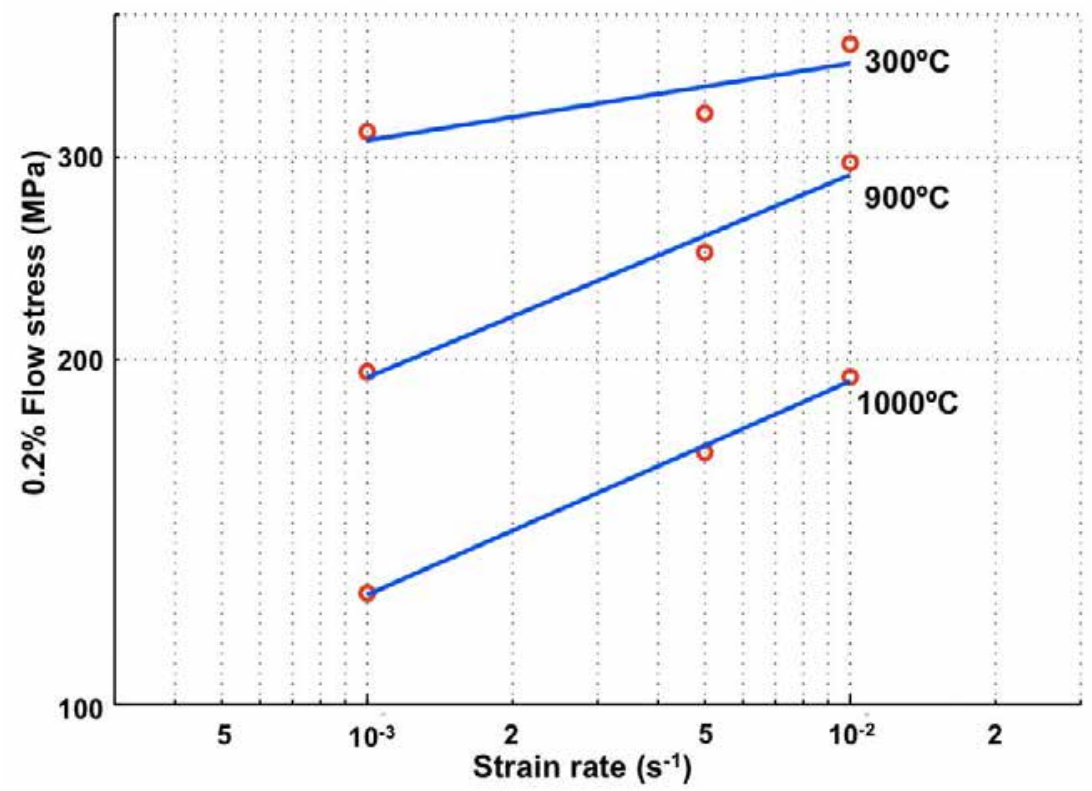

Fig. 4.21: 0.2\% flow stress versus strain rate for Alloy 617 .

Table 4.2: $0.2 \%$ flow stresses for Alloy 617 at temperatures from RT to $1000^{\circ} \mathrm{C}$

\begin{tabular}{ccccc}
\hline Temperature $\left({ }^{\mathbf{0}} \mathbf{C}\right)$ & $\dot{\varepsilon}_{t}=0.001$ & $\dot{\varepsilon}_{t}=0.005$ & $\dot{\varepsilon}_{t}=0.01$ & $m$ \\
\hline $\mathbf{R T}$ & 460 & 470 & 500 & 0.031 \\
$\mathbf{2 0 0}$ & 385 & 374 & 380 & -0.00785 \\
$\mathbf{3 0 0}$ & 316 & 328 & 377 & 0.06 \\
$\mathbf{4 0 0}$ & 339 & 340 & 341 & 0.003 \\
$\mathbf{5 0 0}$ & 328 & 305 & 367 & 0.03 \\
$\mathbf{6 0 0}$ & 303 & 306 & 344 & 0.047 \\
$\mathbf{7 0 0}$ & 298 & 323 & 373 & 0.088 \\
$\mathbf{8 0 0}$ & 283 & 285 & 300 & 0.022 \\
$\mathbf{9 0 0}$ & 195 & 248 & 297 & 0.1772 \\
$\mathbf{1 0 0 0}$ & 125 & 166 & 193 & 0.1866 \\
\hline
\end{tabular}

Table 4.3: $0.2 \%$ flow stresses for Alloy 230 at temperatures from RT to $1000^{\circ} \mathrm{C}$

\begin{tabular}{ccccc}
\hline Temperature $\left({ }^{\circ} \mathbf{C}\right)$ & $\dot{\varepsilon}_{t}=0.001$ & $\dot{\varepsilon}_{t}=0.005$ & $\dot{\varepsilon}_{t}=0.01$ & $m$ \\
\hline $\mathbf{R T}$ & 367 & 470 & 515 & 0.1483 \\
$\mathbf{2 0 0}$ & 350 & 397 & 360 & 0.023 \\
$\mathbf{3 0 0}$ & 336 & 330 & 352 & 0.014 \\
$\mathbf{4 0 0}$ & 305 & 380 & 350 & 0.07 \\
$\mathbf{5 0 0}$ & 336 & 340 & 305 & -0.03 \\
$\mathbf{6 0 0}$ & 315 & 335 & 316 & 0.007 \\
$\mathbf{7 0 0}$ & 289 & 292 & 297 & 0.01 \\
$\mathbf{8 0 0}$ & 250 & 270 & 230 & -0.02 \\
$\mathbf{9 0 0}$ & 190 & 255 & 250 & 0.1304 \\
$\mathbf{1 0 0 0}$ & 93 & 150 & 205 & 0.34 \\
\hline
\end{tabular}




\section{Understanding Fundamental Material-Degradation Processes in High Temperature Aggressive Chemomechanical Environments}

As discussed in section 3.1, the flow stress rapidly decreases once it reaches the yield point at high temperatures $\left(800-1000^{\circ} \mathrm{C}\right)$. The true strain-stress diagram shows that the plastic flow becomes stabilized with an approximately constant flow stress during the plastic regime. (Fig. $4.22 \& 4.23)$ This constant flow stress characterized by strain independence was found to be sensitive to the applied temperature and strain rate. Thus, the strain-rate sensitivities for the constant flow stress can be interpreted from the true strain-stress diagrams (Fig. $4.22 \& 4.23$ ). The results of the analysis are shown in Tables 4.4 and 4.5 for Alloy 617 and Alloy 230, respectively.

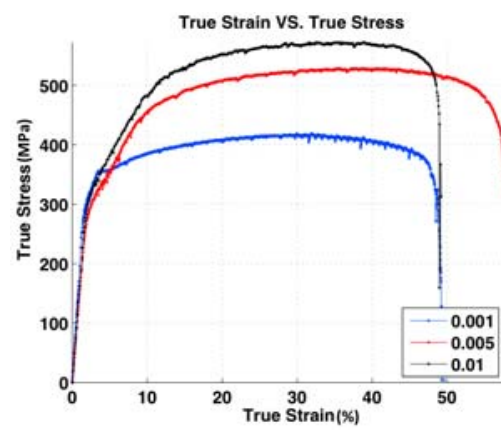

(a)

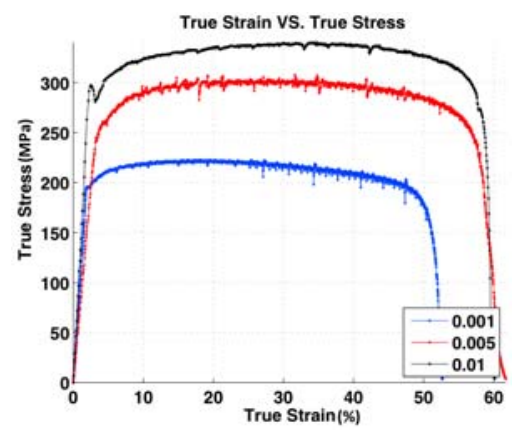

(b)

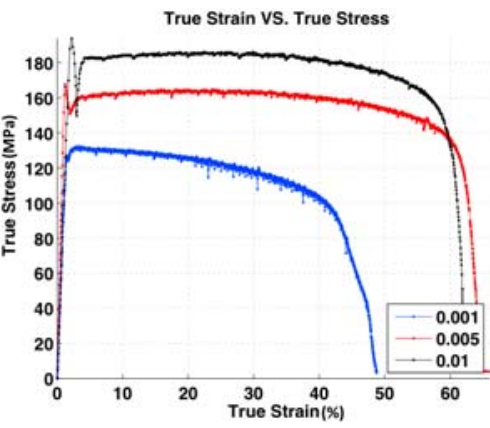

(c)

Fig. 4.22: True strain-stress curves for Alloy 617 at temperature: (a) $800^{\circ} \mathrm{C}$; (b) $900^{\circ} \mathrm{C}$; (c) $1000^{\circ} \mathrm{C}$

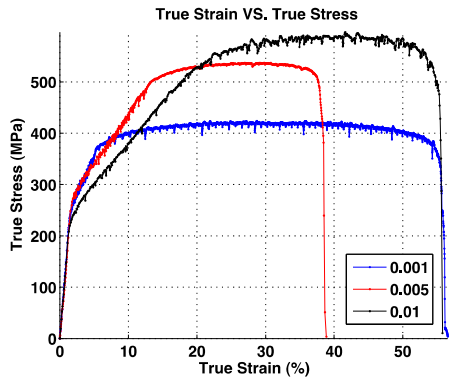

(a)

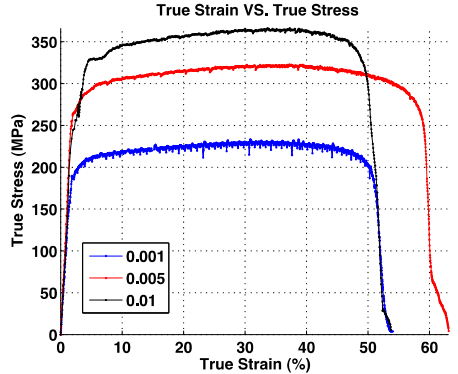

(b)

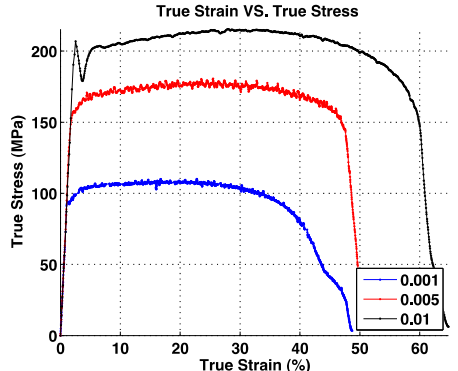

(c)

Fig. 4.23: True strain-stress curves for Alloy 230 at temperature: (a) $800^{\circ} \mathrm{C}$; (b) $900^{\circ} \mathrm{C}$; (c) $1000^{\circ} \mathrm{C}$

Table 4.4: Constant flow stress for Alloy 617 at temperatures from 800 to $1000^{\circ} \mathrm{C}$

\begin{tabular}{ccccc}
\hline Temperature $\left({ }^{\mathbf{0}} \mathbf{C}\right)$ & $\dot{\varepsilon}_{t}=0.001$ & $\dot{\varepsilon}_{t}=0.005$ & $\dot{\varepsilon}_{t}=0.01$ & $m$ \\
\hline $\mathbf{8 0 0}$ & 413 & 523 & 565 & 0.138 \\
$\mathbf{9 0 0}$ & 220 & 300 & 336 & 0.1855 \\
$\mathbf{1 0 0 0}$ & 131 & 163 & 184 & 0.146 \\
\hline
\end{tabular}

Table 4.5: Constant flow stress for Alloy 230 at temperatures from 800 to $1000^{\circ} \mathrm{C}$

\begin{tabular}{ccccc}
\hline Temperature $\left({ }^{\mathbf{0}} \mathbf{C}\right)$ & $\dot{\varepsilon}_{t}=0.001$ & $\dot{\varepsilon}_{t}=0.005$ & $\dot{\varepsilon}_{t}=0.01$ & $m$ \\
\hline $\mathbf{8 0 0}$ & 417 & 535 & 586 & 0.149 \\
$\mathbf{9 0 0}$ & 227 & 320 & 364 & 0.2065 \\
$\mathbf{1 0 0 0}$ & 108 & 176 & 214 & 0.298 \\
\hline
\end{tabular}




\section{Understanding Fundamental Material-Degradation Processes in High Temperature Aggressive Chemomechanical Environments}

\subsection{High-temperature Aging Study}

Aging experiments can provide essential information for predicting material degradation during long-term, high-temperature applications. During long-term aging, the microstructure evolution, including grain growth, phase transformation, and precipitate ripening, significantly influences the material's mechanical properties [4.13]. For nickel alloys, precipitation and coarsening of secondary phases make up the dominant features of microstructure evolution during high-temperature aging. Since the type, size, and coherence of precipitates depend on the composition of the material, the microstructure development during aging is unique for different alloys. In order to gain a better understanding of the high-temperature degradation process of the candidate materials for the IHX and provide practical data for its engineering design, long-term (up to 3000 hours) aging experiments were carried out to investigate the microstructural evolution for Alloy 617 and Alloy 230 at $900^{\circ} \mathrm{C}$ and $1000^{\circ} \mathrm{C}$. Microstructural analysis showed a process of carbide particle precipitation, growth, and maturation for both alloys. The corresponding changes in mechanical properties (hardness and tensile properties) on post-aging samples were also examined. The results agreed well with the expected microstructural development.

\subsubsection{Microstructural characteristics}

High-temperature thermal aging of metallic materials often causes a microstructural evolution characterized by phase transformation, precipitate development, crystallite growth, etc. Phase transformation usually requires relatively high temperatures and fundamentally changes material properties. Similarly, significant grain growth only occurs at very high temperatures and results in decreased material strength. Both phase transformation and grain growth generally occur during heat treatment in order to achieve a required nature of material and should be avoided in practical engineering applications.

Another typical microstructural development in thermal aging is nucleation and growth of second phase particles. Modern metallurgy often employs many different elements into commercial alloys in order to improve various material properties (e.g. alloying $\mathrm{Al}$ and $\mathrm{Cr}$ for corrosion resistance or $\mathrm{W}$ and Mo for high-temperature resistance). The alloying process requires a solution treatment: heating the alloy into the single-phase field followed by a rapid quench. Thus, although the concentration of solute elements becomes supersaturated, and the phase diagram predicts mixtures with two or more phases, the alloy presents only a single phase due to the quench and a slow diffusivity of heterogeneous elements at low temperatures. Nevertheless, this insufficiency of atomic diffusion breaks down when the alloy is heated to higher temperatures below the solvus temperature. Significant thermal-enhanced diffusivity of the solute results in the precipitation of second-phase particles within matrix grains or at their respective grain boundaries. Depending on various equilibrium/thermal conditions, the precipitation can happen at a relatively wide range of temperatures and often involves the relevant temperature region for engineering applications.

The development of second-phase particles is often described as a process of nucleation, growth, and coarsening. After the alloy is heated up to an aging temperature, the supersaturated elements begin to diffuse and coalesce into clusters. Then the fine, second-phase particles begin nucleating within the matrix grains or in their respective grain boundaries. Meanwhile, the newly formed particles grow at the expense of smaller particles. Once an equilibrium condition is achieved, the volume faction of the second-phase particles no longer increases, and the 


\section{Understanding Fundamental Material-Degradation Processes in High Temperature Aggressive Chemomechanical Environments}

nucleation process is finished. Nevertheless, the particle growth continues in order to reduce the total free energy of the system by decreasing the total amount of interfacial area between phases. This process is known as Oswald ripening. The growth of the particles can last for a very long time with a decreasing coarsening rate until a relatively stable microstructure is achieved. This steady state condition can be considered the coarsening stage of thermal aging.

For Alloy 617 and Alloy 230, four types of particles were found in previous studies after certain heat treatments. [4.14] These particles are: $\mathrm{M}_{23} \mathrm{C}_{6}, \mathrm{M}_{6} \mathrm{C}, \mathrm{Ti}(\mathrm{N}, \mathrm{C})$, and $\gamma^{\prime}\left(\mathrm{Ni}_{3} \mathrm{Al}\right)$. The former two types of particles are significant in the present study not only due to their prevalence over other types of particles in the materials, but also because they appear as precipitates during high-temperature $\left(>900^{\circ} \mathrm{C}\right)$ aging (particularly the $\mathrm{M}_{23} \mathrm{C}_{6}$ type carbide). Due to the low fraction of titanium in both alloys $(0.39 \%$ in Alloy $617 \&<0.01 \%$ in Alloy 230 ), the amount of Ti (N,C) is so small that its impact on mechanical properties is almost negligible. $\mathrm{Ti}(\mathrm{N}, \mathrm{C})$ can be detected in Alloy 617 by the observation of the color orange in optical microscopy, but it can hardly be found in Alloy 230 because of an even lower fraction of titanium. The typical dimensions of Ti $(\mathrm{N}, \mathrm{C})$ in the alloys is $10-100 \mu \mathrm{m} . \gamma^{\prime}$ is the typical strengthening phase extensively found in nickel-based alloys. Many nickel-based superalloys (e.g. Inconel 718 and Haynes 282) are strengthened by additional aging treatments to obtain $\gamma^{\prime}$. However, both Alloy 617 and Alloy 230 are solid-solution strengthening alloys that are not purposed to employ $\gamma^{\prime}$ strengthening, although very small amounts of $\gamma^{\prime}$ have been reported in some aging studies of Alloy 617. [4.13] Moreover, $\gamma^{\prime}$ has never been found at temperatures higher than $900^{\circ} \mathrm{C}$ in either alloy (also not found in the present study). The following discussion will focus on the $\mathrm{M}_{23} \mathrm{C}_{6}$ and $\mathrm{M}_{6} \mathrm{C}$ carbides.

\section{The $M_{23} C_{6}$ type carbide:}

The $\mathrm{M}_{23} \mathrm{C}_{6}$ carbide was found in both Alloy 617 and Alloy 230 over the course of the study. This carbide has a complex, cubic structure with a large lattice parameter $(>1 \mathrm{~nm})$. The structure of the $\mathrm{M}_{23} \mathrm{C}_{6}$ belongs to the space group $\mathrm{Fm} \overline{3} \mathrm{~m}$, and the basic crystal structure is face-centered cubic (FCC). Fig. 4.24 shows the typical structure of the $\mathrm{M}_{23} \mathrm{C}_{6}$ carbide. It should be noted that although $\mathrm{M}_{23} \mathrm{C}_{6}$ is a chromium rich carbide, the rest of the composition of $\mathrm{M}$ can be made up of different metallic atoms, depending on the alloying elements in the material. In other words, partial contents of $\mathrm{Cr}$ in the original form of $\mathrm{M}_{23} \mathrm{C}_{6}$ (i.e. $\mathrm{Cr}_{23} \mathrm{C}_{6}$ ) can be substituted with $\mathrm{Ni}, \mathrm{Fe}$, $\mathrm{Mn}, \mathrm{Mo}, \mathrm{W}$, etc. when these elements have been alloyed in the material. Hence, the lattice parameter of $\mathrm{M}_{23} \mathrm{C}_{6}$ is not a fixed value, but instead falls in a range of 1.048-1.08 nm for Alloy 617. [4.15] 


\section{Understanding Fundamental Material-Degradation Processes in High Temperature}

Aggressive Chemomechanical Environments

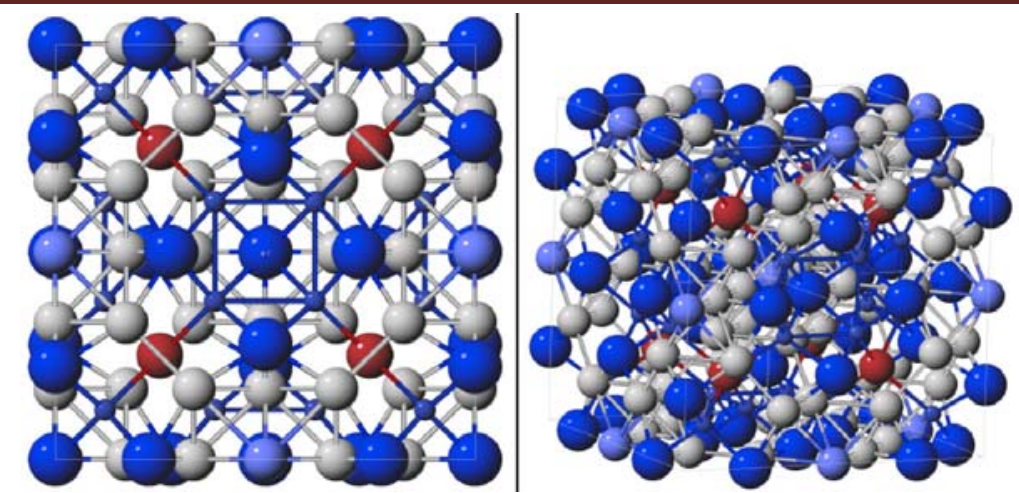

Fig. 4.24: Typical structure of $\mathrm{M}_{23} \mathrm{C}_{6}\left(\mathrm{Cr}_{12} \mathrm{Ni}_{8} \mathrm{Fe}_{2} \mathrm{Mn}_{1} \mathrm{C}_{6}\right)$. In the diagram, large blue spheres $=\mathrm{C}$ atoms; small blue spheres $=\mathrm{Ni}$ atoms; red spheres $=\mathrm{Fe}$ atoms; light blue spheres $=\mathrm{Mn}$ atoms; and grey spheres $=\mathrm{Cr}$ atoms. (Pictures provided by the Center for Computational Materials Science of the United States

Naval Research Laboratory). [4.16]

\section{The $M_{6} C$ type carbide:}

The $\mathrm{M}_{6} \mathrm{C}$ carbide was also found in both Alloy 617 and Alloy 230 during experimentation. Similar to the $\mathrm{M}_{23} \mathrm{C}_{6}$ carbide, the $\mathrm{M}_{6} \mathrm{C}$ carbide also has a complex, cubic structure and a large lattice parameter. The structure of this carbide belongs to the space group $\mathrm{Fd} \overline{3} \mathrm{~m}$, and the basic crystal structure is diamond cubic. Fig. 4.25 shows the typical structure of $\mathrm{M}_{6} \mathrm{C}$. Depending on the material composition, the contents of $\mathrm{M}$ in $\mathrm{M}_{6} \mathrm{C}$ can be rich in Fe, Mo, Co, W, etc. The $\mathrm{M}_{6} \mathrm{C}$ carbides in Alloy 617 and Alloy 230 were found to be rich in Mo and W, respectively. The lattice constant of the $\mathrm{M}_{6} \mathrm{C}$ carbide is within a range of 1.085-1.175 nm for Alloy 617. [4.15] Compared to the $\mathrm{M}_{23} \mathrm{C}_{6}$ carbide, $\mathrm{M}_{6} \mathrm{C}$ has a better thermal stability at high temperatures (800 to $\sim 1200^{\circ} \mathrm{C}$ ). This property can be seen at equilibrium phases calculated by Thermocalc $\mathbb{R}[4.14$, 4.17], showing that the $\mathrm{M}_{23} \mathrm{C}_{6}$ carbide is stable at temperatures up to $780^{\circ} \mathrm{C}$, but transforms into $\mathrm{M}_{6} \mathrm{C}$ at higher temperatures (up to $\sim 1200^{\circ} \mathrm{C}$, the solid solution temperature).
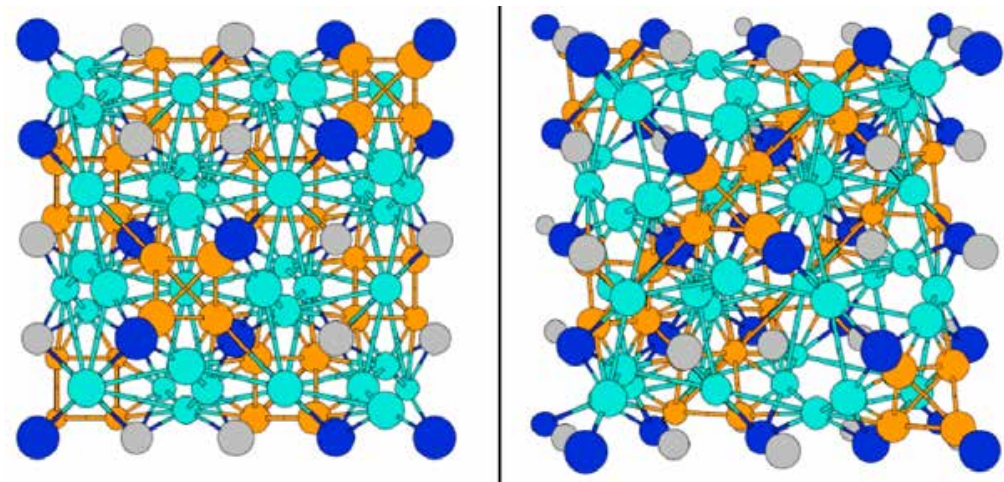

Fig. 4.25: Typical structure of $\mathrm{M}_{6} \mathrm{C}\left(\mathrm{W}_{3} \mathrm{Fe}_{2} \mathrm{CrC}_{6}\right)$. In the diagram, cyan spheres $=\mathrm{W}$ atoms; blue spheres $=$ $\mathrm{C}$ atoms; orange spheres $=\mathrm{Fe}$ atoms; grey spheres $=\mathrm{Cr}$ atoms $[4.16]$

Both $\mathrm{M}_{23} \mathrm{C}_{6}$ and $\mathrm{M}_{6} \mathrm{C}$ particles have been found in various alloys with moderate to high chromium content, including nickel-based alloys [3.15] and low carbon steels [4.18, 4.19]. Conventionally, both types of particles are believed to both add and detract from material performance. During high-temperature deformations, these particles can inhibit grain-boundary sliding (Zener pinning) and increase the rupture strength of the material. However, premature 


\section{Understanding Fundamental Material-Degradation Processes in High Temperature Aggressive Chemomechanical Environments}

failures can also be initiated by the fracture or decohesion of the particles. [4.20] Both $\mathrm{M}_{23} \mathrm{C}_{6}$ and $\mathrm{M}_{6} \mathrm{C}$ type carbides have a high thermal stability at high temperatures in Alloy 617 and Alloy 230. The particles formed during heat treatment can withstand a temperature up to $1000^{\circ} \mathrm{C}$ (even higher for $\mathrm{M}_{6} \mathrm{C}$ ). The thermal stability can be seen in calculated TTT diagrams [4.15] and equilibrium phase diagrams [4.14, 4.17]. It should be noted that $\mathrm{M}_{23} \mathrm{C}_{6}$ and $\mathrm{M}_{6} \mathrm{C}$ carbides can react and transform according to the following chemical equation:

$$
\mathrm{M}_{6} \mathrm{C}+\mathrm{M}^{\prime} \leftrightarrow \mathrm{M}_{23} \mathrm{C}_{6}+\mathrm{M}^{\prime \prime} \text {, }
$$

where, $\mathrm{M}^{\prime}$ and $\mathrm{M}^{\prime \prime}$ are substitute elements (e.g. Cr, Mo, Co, Fe, etc.). In Alloy 617 and Alloy 230 , the lattice constants for $\mathrm{M}_{23} \mathrm{C}_{6}$ and $\mathrm{M}_{6} \mathrm{C}$ carbides are comparable, both of which are $\sim 1.08$ $\mathrm{nm}$, or about three times the lattice constant of the $\gamma$ matrix $(\sim 0.36 \mathrm{~nm})$. Therefore, these particles can develop a cube-on-cube orientation with the host matrix (i.e. (100) carbide // (100) matrix, [001] carbide // [001] matrix). (Fig. 4.26) This semi-coherent structure is more pronounced for $\mathrm{M}_{23} \mathrm{C}_{6}$ than $\mathrm{M}_{6} \mathrm{C}$ because of a slightly larger lattice misfit of the $\mathrm{M}_{6} \mathrm{C}$ to the host matrix that often results in a loss of coherence of interphase boundaries (IPBs).

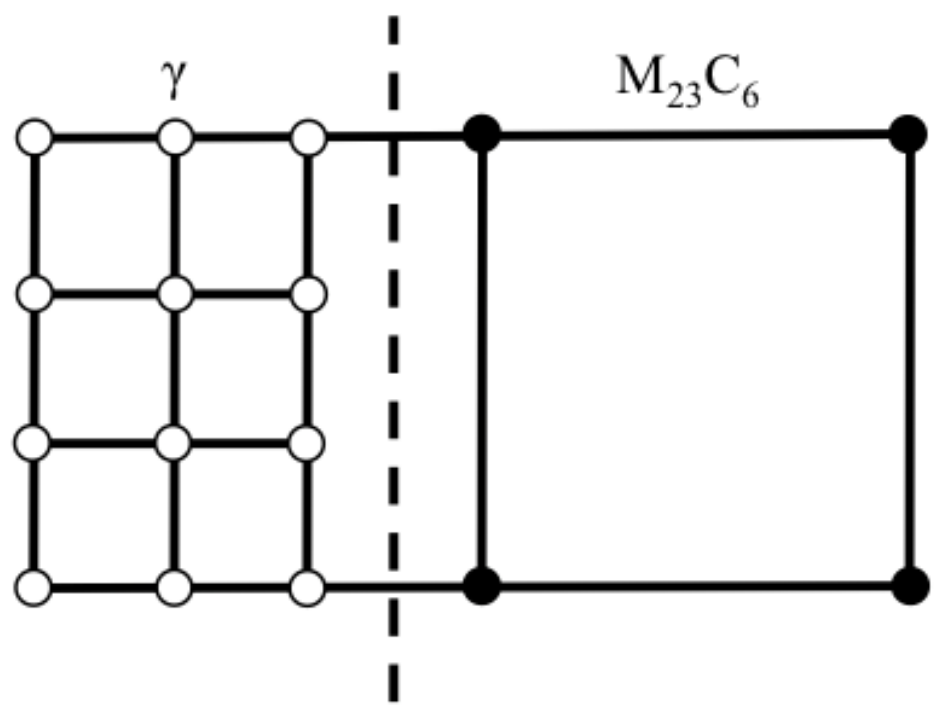

Fig. 4.26: Cube-on-cube structure of the $\gamma$ matrix and the $\mathrm{M}_{23} \mathrm{C}_{6}$ carbide. The dashed line shows the partial coherent interphase boundaries.

\subsubsection{Microstructure during various aging conditions}

The microstructural development during thermal aging inevitably changes a material's mechanical properties. A typical example is the time-dependence of material strength. Material strength increases with time during the early aging stage and then decreases with further aging. The changes in material strength involve different dislocation-particle interaction mechanisms related to the nature of particles (e.g. type, coherency, dimension, density, etc.) in alloys.

Fig. 4.27 shows two typical strengthening mechanisms based on dislocation-particle interaction. The first strengthening mechanism is dislocation cutting. (Fig. 4.27(a)) Some small and highly coherent particles (e.g. GP-I, GP-II, and $\theta^{\prime}$ in aged Al-Li alloys) are found to be deformable: dislocations are able to cut though them when sufficient stress is applied. The equation governing this strengthening contribution was found to be: 


$$
\tau=C G \varepsilon^{3 / 2}(f r / b)^{1 / 2}
$$

where $\tau=$ stress required for dislocation passing the particle

$\varepsilon=$ misfit strain (proportional to difference in lattice parameter of the two phases)

$r=$ particle radius

$f=$ volume fraction of precipitated second phase

$G=$ shear modulus

$b=$ Burgers vector

As discussed in the previous section, the volume fraction of second phase particles increases during the early stage of thermal aging until an equilibrium volume fraction is achieved. The large second-phase particles continue to coarsen at the expense of smaller particles during further aging. With particle growth, the spacing between particles and the misfit strain in particle-matrix interface both increase. When the misfit strain is sufficiently large, the particle-matrix interface loses coherency, and dislocations are unable to cut through particles. Similarly, when the increased particle separation is higher than a critical value, dislocation cutting cannot occur. For both situations, a dislocation will bow/loop around particles and require a larger stress to further its movement. This dislocation-particle interaction is also referred to as Orowan looping. (Fig. 4.27(b)) The required stress for a dislocation to pass particles is analogous to the stress given for the activation of Frank-Read sources:

$\tau=\frac{G b}{L-2 r}$

where, $L$ is distance between pinning points (particles).

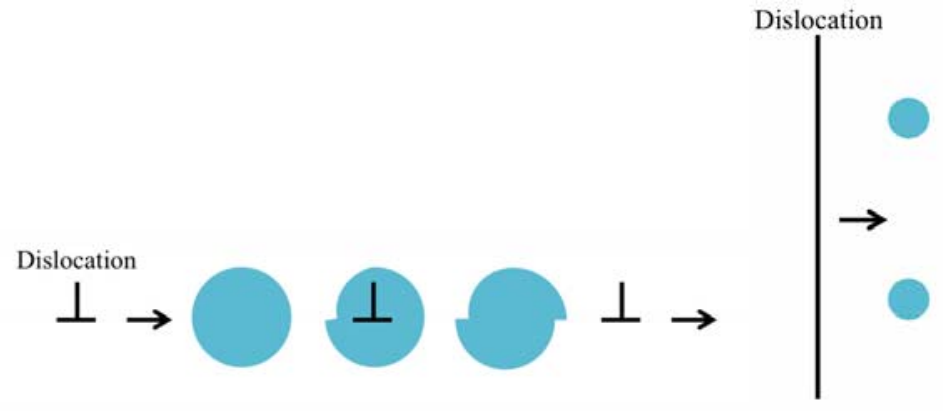

(a)

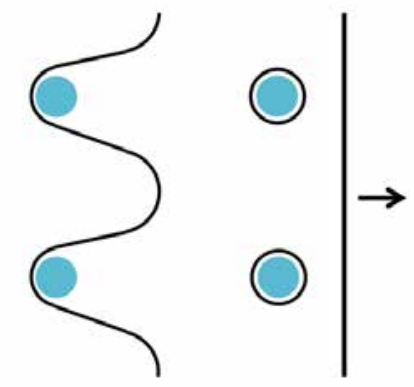

(b)

Fig. 4.27: Schematic representation of two strengthening mechanisms by dislocation-particle interaction:

(a) particle cutting; (b) Orowan looping

Beyond particle hardening, the aging process influences another important strengthening mechanism: solid solution strengthening. The solid solution elements can generate either asymmetrical or symmetrical stress fields that result in tetragonal and spherical distortion, respectively. The strengthening caused by the asymmetrical stress field is rather significant. A typical example can be found in carbon atoms present in martensite. In contrast, the capacity of 


\section{Understanding Fundamental Material-Degradation Processes in High Temperature Aggressive Chemomechanical Environments}

the symmetrical stress field to impede dislocation movement is limited. The increase in yield strength can be estimated by:

$\tau=\frac{G \varepsilon^{3 / 2} c^{1 / 2}}{700}$

where $\varepsilon$ is the misfit strain (proportional to size difference between the solute and solvent atoms) and $c$ is the solute concentration (expressed as atomic fraction). For nickel-based alloys, the solid solution elements often possess a symmetrical stress field [4.21].

Incorporating all introduced strengthening components, an overview of material strength evolution during thermal aging is schematically drawn in Fig. 4.28. The changes in overall strength are contributed to three components: dislocation cutting, dislocation looping, and solid solution strengthening. At the onset of thermal aging, the alloy experiences the greatest potential for solid solution strengthening due to the maximum amount of supersaturated elements present in the host matrix. During the early aging stage, small and highly coherent particles precipitate within the matrix grains and their respective grain boundaries at the expense of supersaturated solute. This provides an additional strengthening component, dislocation cutting, to the alloy. Meanwhile, the strengthening component caused by solid solution decreases along with increasing precipitate strengthening. However, the loss of strengthening by the decrease of solute levels can be more than compensated by the precipitation hardening mechanisms. With further aging, the equilibrium condition is achieved, and the associated precipitation process is finished. As a result, the strengthening contributed by the solid solution mechanism decreases to a minimum value (point 'A' in Fig. 4.28). With particle growth, the dominant mechanism of dislocation-particle interaction gradually changes from cutting to bowing. This transition can be characterized by the critical particle radius, $r_{c}$, which is typically within the range of 5-30 nm. Therefore, before particles develop to the critical size $\left(r<r_{c}\right)$, dislocation cutting is the dominant mechanism. With thermal aging and associated particle growth, the particle size will finally exceed the critical value $\left(r>r_{c}\right)$, and dislocation bowing then becomes the dominant mechanism. Point 'B' in Fig. 4.28 indicates the moment when the particle radius reached the critical radius $\left(r=r_{c}\right)$. The peak strength of the alloy obtained during aging is the result of the overall response for all strengthening components. (point ' $\mathrm{C}$ ' in Fig. 4.28) With regard to Fig. 4.28, the alloy before aging to the moment of ' $\mathrm{C}$ ' is considered the 'underaged' condition. Conversely, the alloy is considered to be 'overaging' after progressing past the moment of ' $\mathrm{C}$ '.

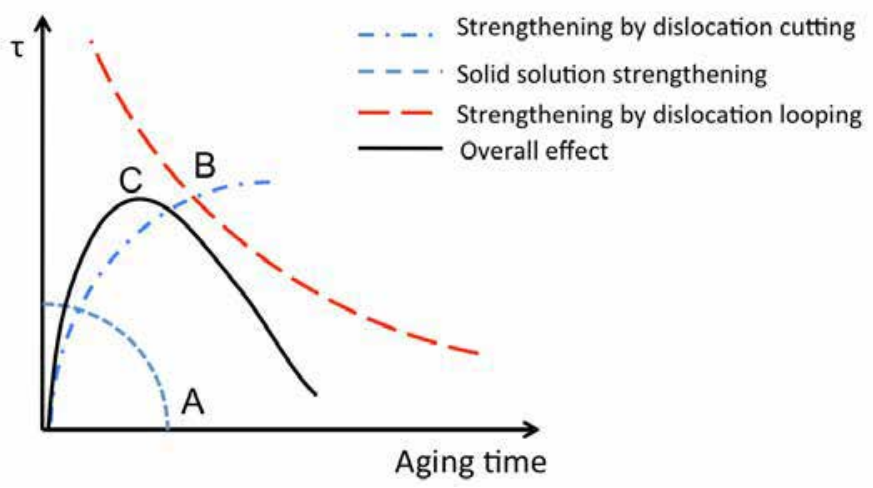

Fig. 4.28: Overall aging effect [4.5] 


\section{Understanding Fundamental Material-Degradation Processes in High Temperature}

Aggressive Chemomechanical Environments

\subsubsection{Microstructure in the as-received condition}

The microstructure of Alloy 617 and Alloy 230 in the as-received condition was introduced in the previous chapter. The typical microstructural characteristics consist of large strengthening particles within the grains and their respective grain boundaries. (Fig. 3.25) These intrinsic particles in Alloy 617 are $\mathrm{Cr}$ rich $\mathrm{M}_{23} \mathrm{C}_{6}$, Mo rich $\mathrm{M}_{6} \mathrm{C}$, and a small amount of Ti(C,N). [3.44] In Alloy 230, the intrinsic particles are $\mathrm{W}$ rich $\mathrm{M}_{6} \mathrm{C}$. [3.45] These particles in both alloys are strung as bands in the transverse planes and flattened in the rolling planes. The effect of orientation on mechanical properties caused by this microstructural gradient was discussed in section 4.2 . These large carbide particles do not exhibit any apparent orientation relation or coherency with the host matrix grains from TEM examinations. (Fig. 4.29) However, the small intergranular particles (primarily $\mathrm{M}_{23} \mathrm{C}_{6}$ type) in the alloys have a cube-on-cube relation with the host matrix. (Fig. 4.30) The interphase boundaries between the particles and matrix are partly coherent in this cube-on-cube structure. (Fig. 4.26) Compared to large particles, these small intergranular particles do not have a significant impact on the alloys' mechanical properties because of their small volume fraction and good coherency with the host matrix.

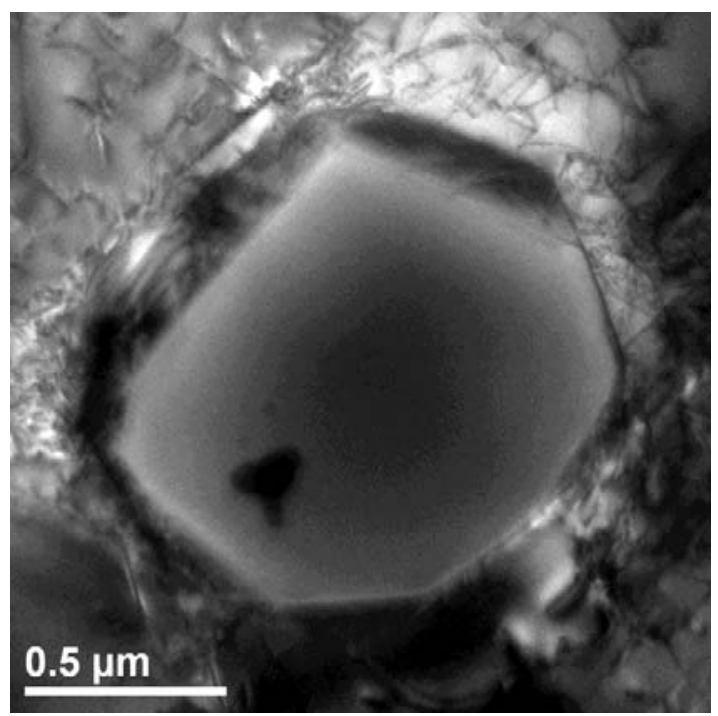

(a)

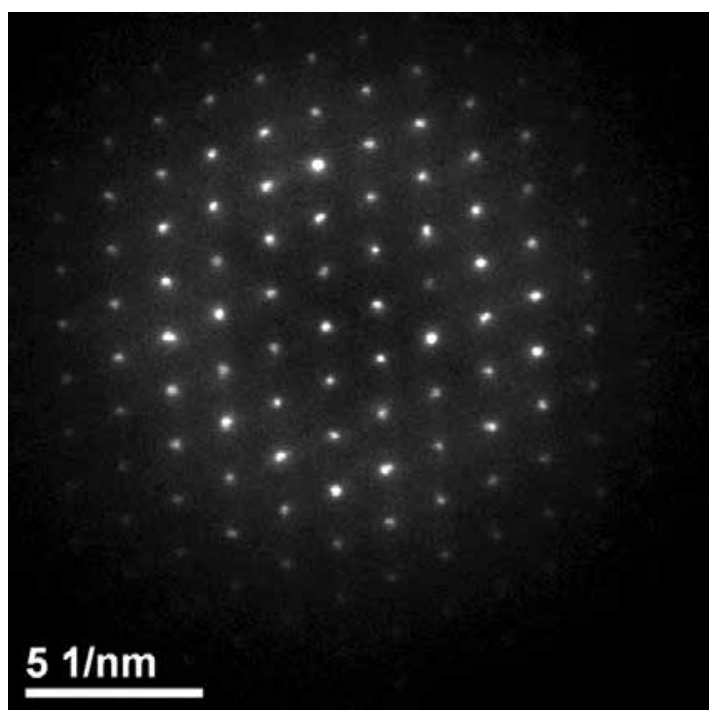

(b)

Fig. 4.29: Large, incoherent $\mathrm{M}_{23} \mathrm{C}_{6}$ carbide in Alloy 617 (a) bright field image; (b) diffraction pattern (zone axis [011]) 


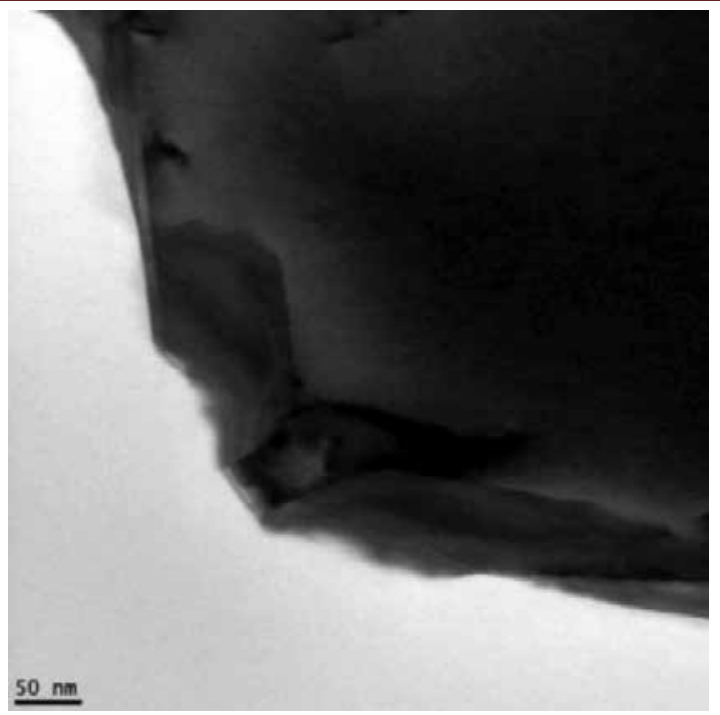

(a)

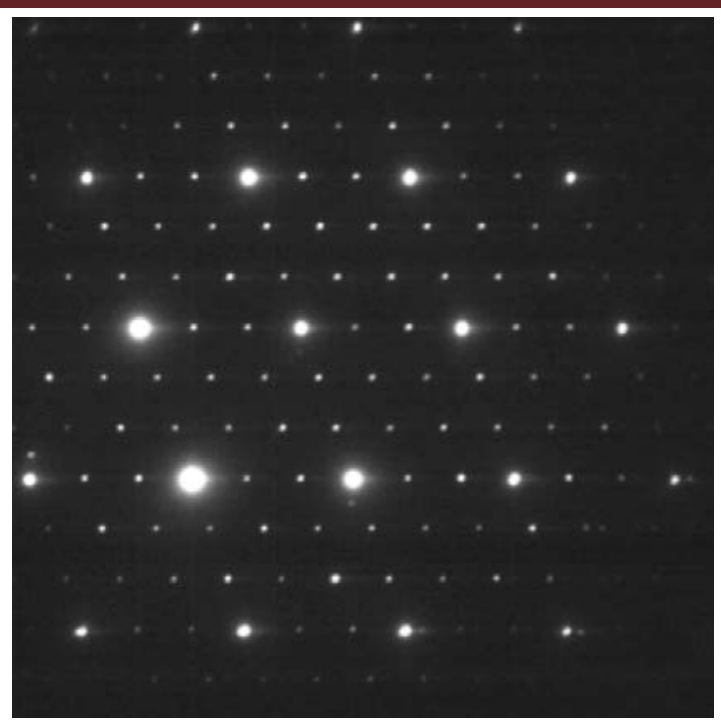

(b)

Fig. 4.30: Intergranular $\mathrm{M}_{23} \mathrm{C}_{6}$ carbides in Alloy 617 (a) bright field image; (b) diffraction pattern (zone axis [011])

The SEM and EBSD analyses show a duplex grain size distribution in Alloy 617: small grains with sizes ranging from 10 to $30 \mu \mathrm{m}$ in the inclusion rich areas, and large grains with sizes ranging from 40 to $100 \mu \mathrm{m}$ in the inclusion free areas where grains grow during annealing without Zener pinning. (Fig. 3.25(a)) The grain size in Alloy 230 did not exhibit a great variety. The average grain size measured in Alloy 230 was $\sim 70 \mu \mathrm{m}$. Additionally, both alloys possess a high fraction of twin boundaries ( $\sum 3$ grain boundaries), which are $\sim 60 \%$ and $\sim 53 \%$ of the total grain boundaries for Alloy 617 and Alloy 230, respectively.

\subsubsection{Microstructure after short-term aging}

Fig. 4.31 shows the microstructural characteristics of Alloy 617 aged at $900^{\circ} \mathrm{C}$ for 30 hours. The dominant feature during short-term aging was found to be the fine and ordered precipitates nucleating on the $\{111\}$ planes. (Fig. 4.31(a)) These intragranular precipitates were the $\mathrm{M}_{23} \mathrm{C}_{6}$ type carbides with the cube-on-cube orientation relations with the $\gamma$ matrix. (Fig. 4.32) Depending on the nucleation site, the size of the fine precipitates ranged from $\sim 10 \mathrm{~nm}$ (within the matrix grain, Fig. 4.31(a) \& Fig. 4.32) to $100 \mathrm{~nm}$ (near different boundaries, Fig. 4.31(b-d))

The characteristics of intergranular precipitates in Alloy 617 after short-term aging were found to differ from those of the as-received condition. First, the shape of intergranular particles was transformed from a film shape to an ellipsoid, along with a considerable thickness increase. (Fig. 4.31(b)) Second, the twin boundaries, which were free of particles in the as-received condition, were subjected to precipitation of long, needle-shaped particles. (Fig. 4.31(c)) Third, some intergranular particles lost their semi-coherency to the host matrix due to the size increase. Fig. 4.33 shows an intergranular carbide that developed a new orientation relation with the matrix: $[-114]_{\mathrm{M}} / /[011]_{\mathrm{p}}$. Alloy 230 experiences a similar process of microstructure development during short-term aging. Fig. 4.34 shows $\mathrm{M}_{23} \mathrm{C}_{6}$ carbides that precipitated within the matrix grains as well as the twin, grain, and interphase boundaries. 
Understanding Fundamental Material-Degradation Processes in High Temperature Aggressive Chemomechanical Environments

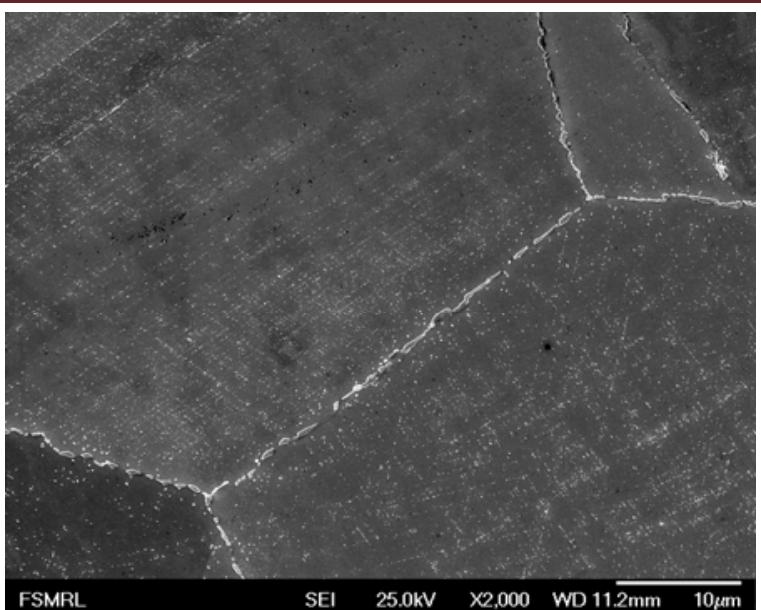

(a)

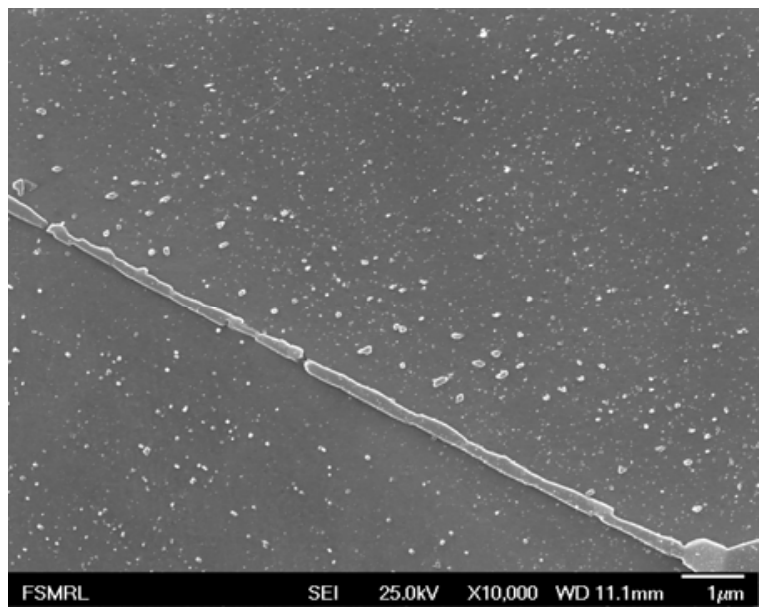

(c)

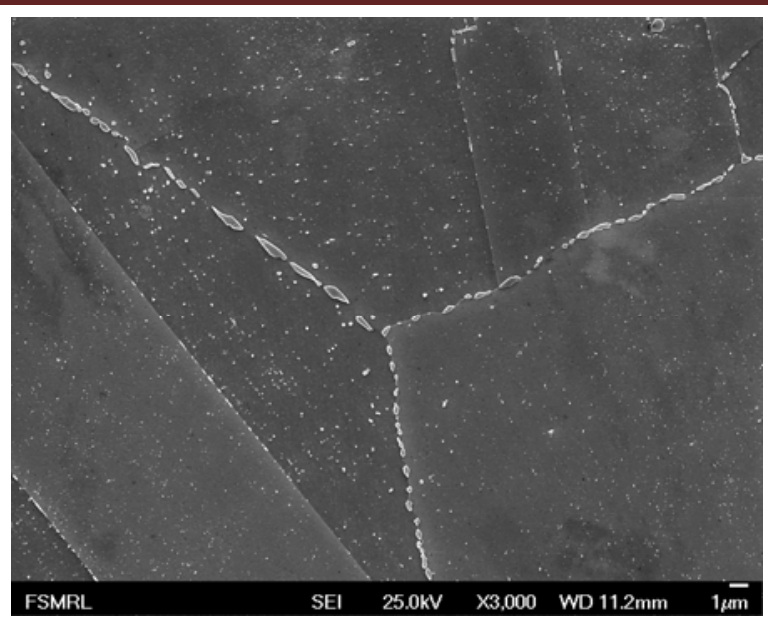

(b)

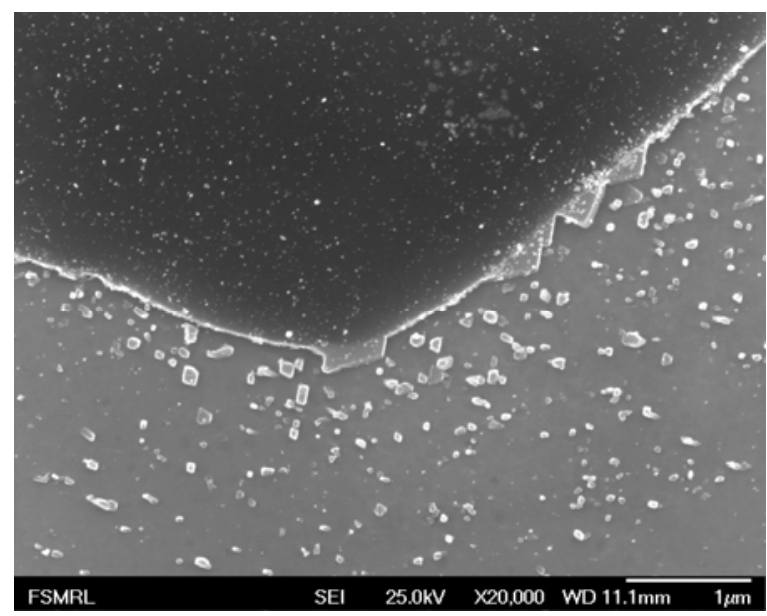

(d)

Fig. 4.31: Microstructure characteristics of Alloy 617 aged at $900^{\circ} \mathrm{C}$ for 30 hours: (a) fine ordered precipitates within matrix grains; (b) particle nucleation and growth on grain boundaries; (c) particle nucleation and growth on a twin boundary; (d) particle growth on interphase boundaries 
Understanding Fundamental Material-Degradation Processes in High Temperature Aggressive Chemomechanical Environments

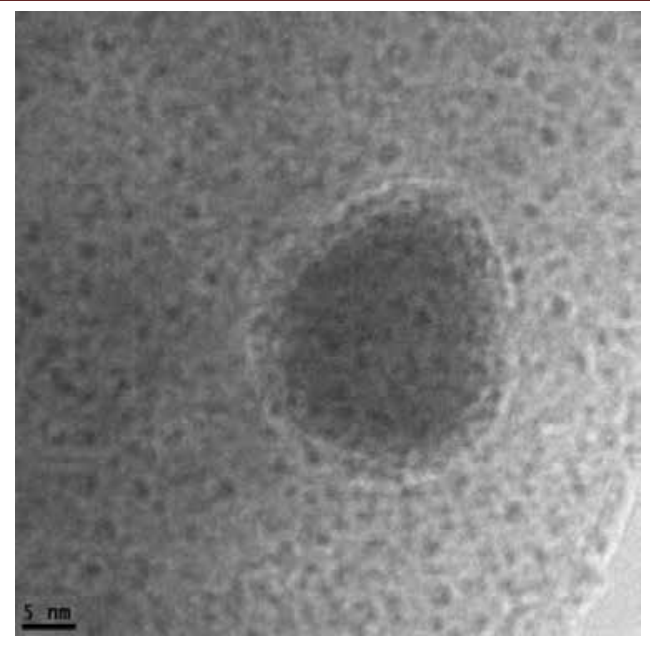

(a)

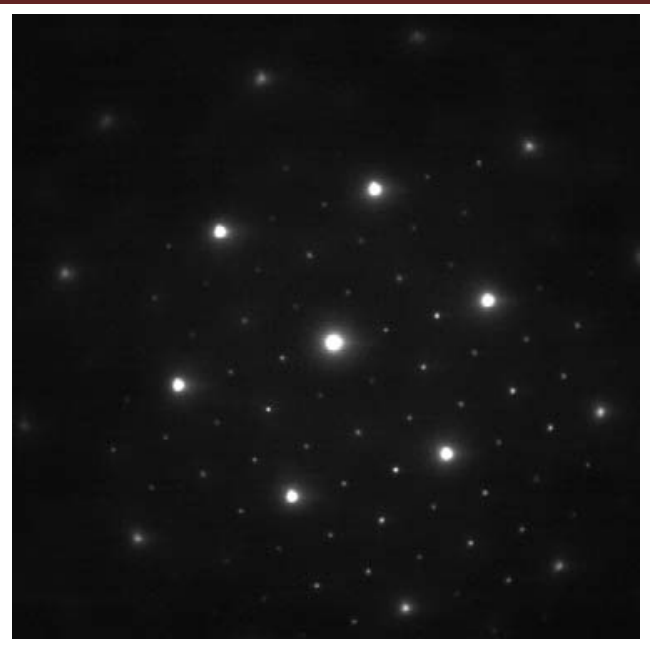

(b)

Fig. 4.32: TEM analysis of small precipitates of Alloy 617 aged at $900^{\circ} \mathrm{C}$ for 30 hours (zone axis [111])

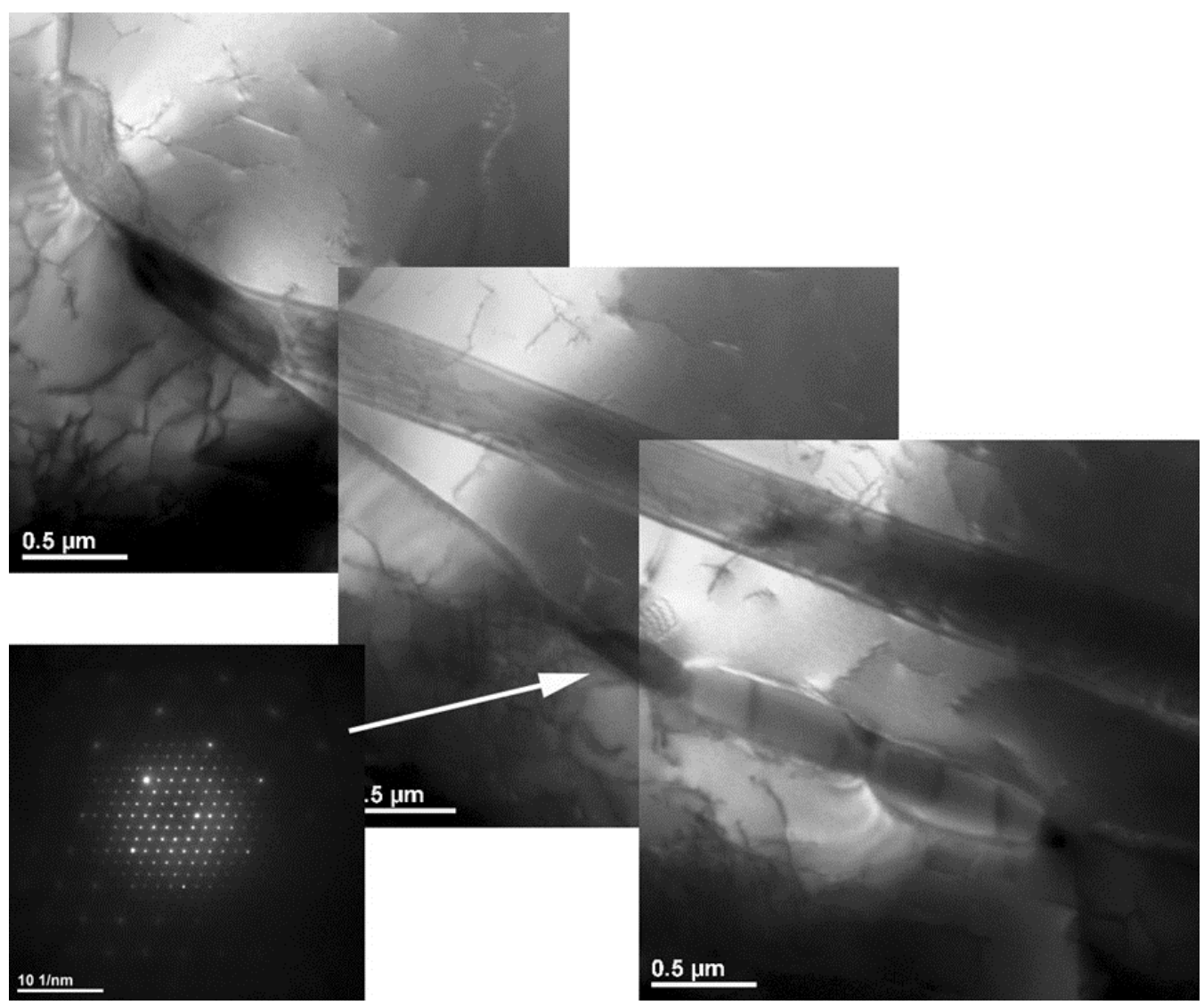

Fig. 4.33: TEM analysis of Alloy 617 aged at $1000^{\circ} \mathrm{C}$ for 300 hours; an intergranular precipitate (P) with an orientation relation with the matrix $(\mathrm{M}):[-114]_{\mathrm{M}} / /[011]_{\mathrm{P}}$ 


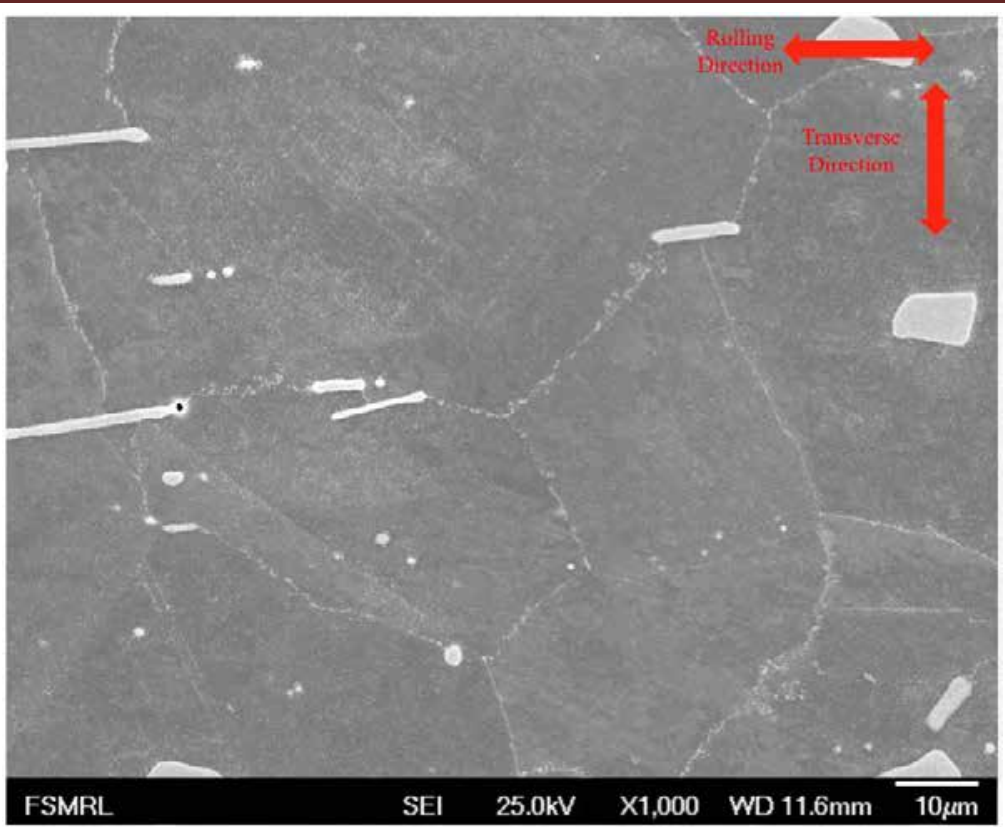

Fig. 4.34: Microstructure characteristics of Alloy 230 aged at $900^{\circ} \mathrm{C}$ for 30 hours

\subsubsection{Microstructure after long-term aging}

After long-term ( $>1000$ hours) aging, most of the fine precipitates that nucleated within matrix grains had diffused and grown within grain boundaries and interphase boundaries. (Fig. 4.35 and Fig. 4.36) In contrast to the high particle density in these boundaries, few precipitates were found on twin boundaries. The intergranular particles experienced significant coarsening during long-term aging. In both alloys, most particles increased to a size exceeding $1 \mu \mathrm{m}^{2}$ after aging at $1000^{\circ} \mathrm{C}$ for 3000 hours. Despite considerable coarsening, most large intergranular precipitates in each alloy were found to still maintain semi-coherency (cube-on-cube orientation relation) with the $\gamma$ matrix from TEM analysis (Fig. 4.37 and Fig. 4.38).

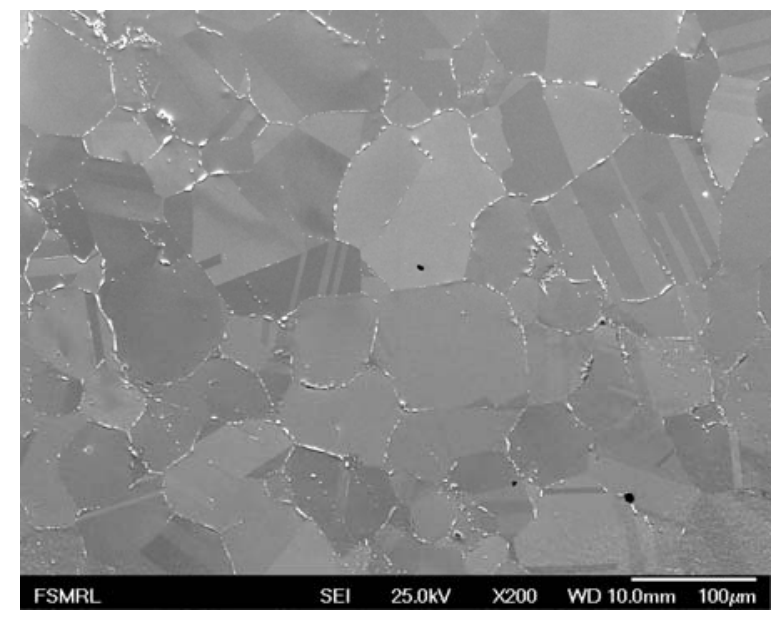

(a)

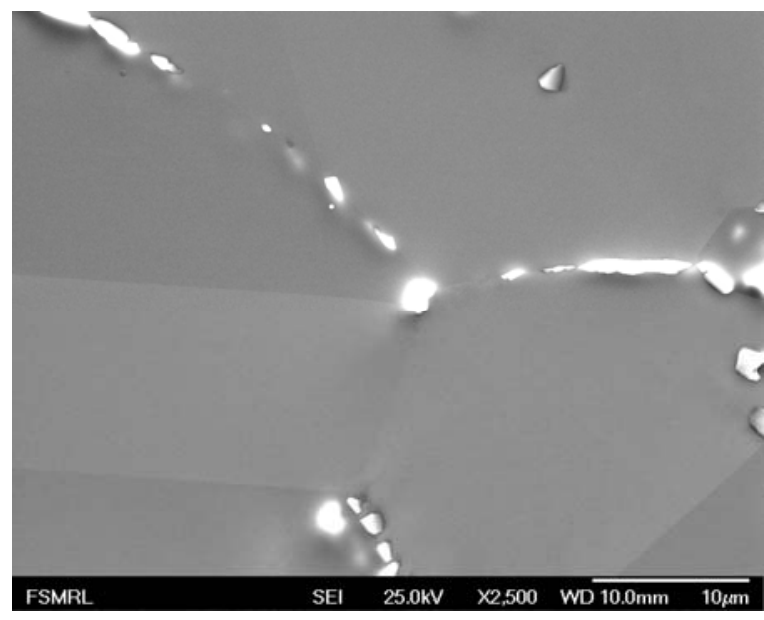

(b)

Fig. 4.35: Microstructure of Alloy 617 aged at $1000^{\circ} \mathrm{C}$ for 3000 hours: (a) overview; (b) intergranular precipitates 
Understanding Fundamental Material-Degradation Processes in High Temperature Aggressive Chemomechanical Environments

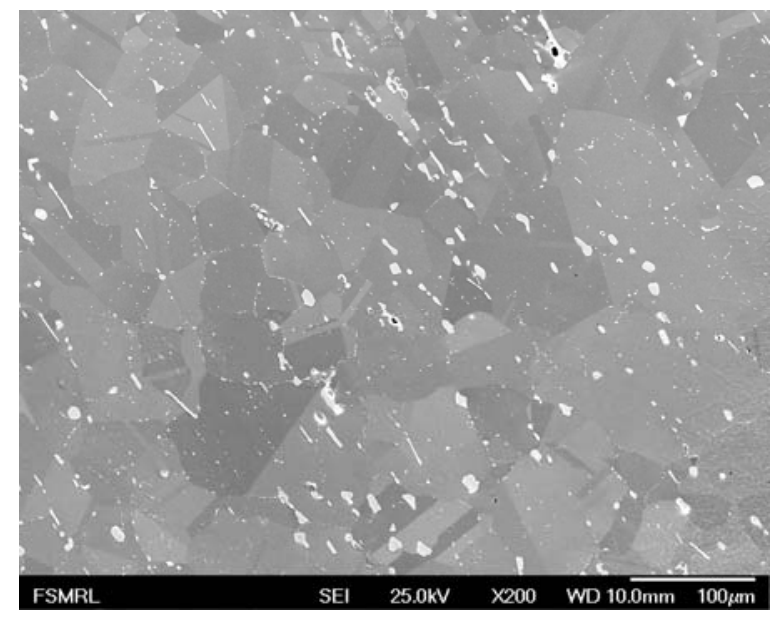

(a)

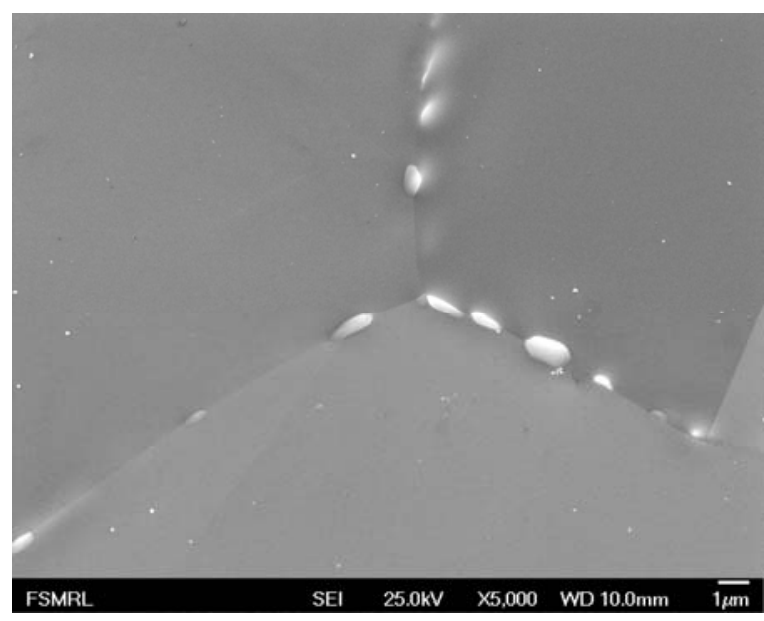

(b)

Fig. 4.36: Microstructure of Alloy 230 aged at $1000^{\circ} \mathrm{C}$ for 1000 hours: (a) overview; (b) intergranular precipitates
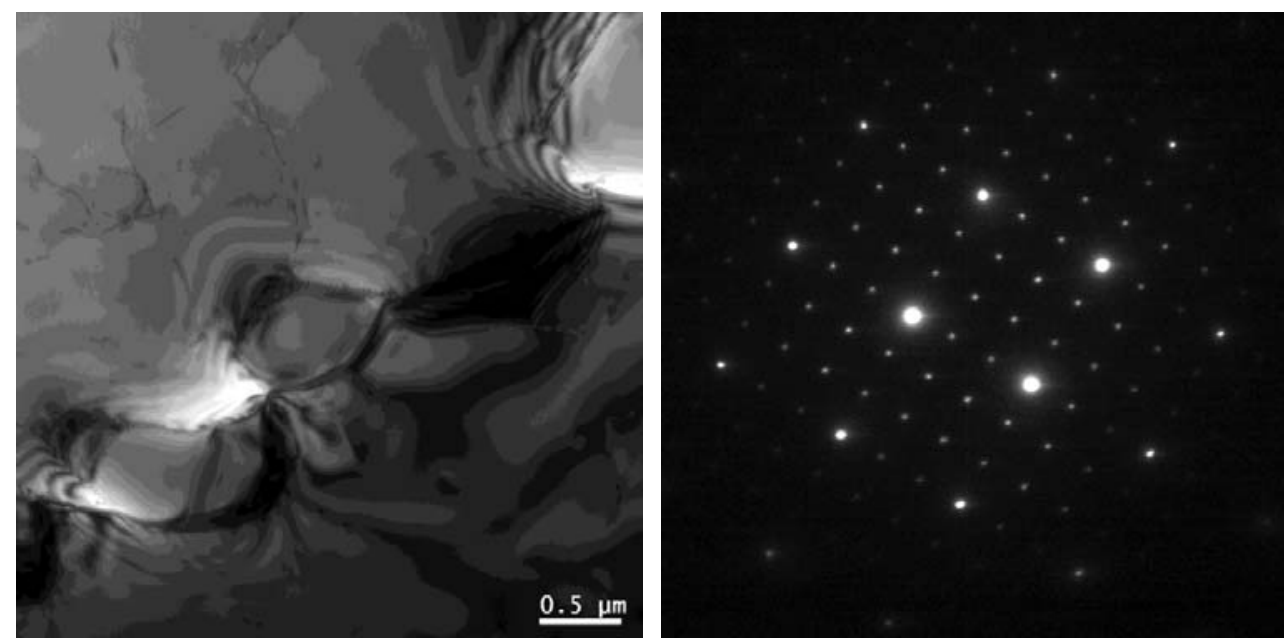

Fig. 4.37: TEM analysis of Alloy 617 aged at $1000^{\circ} \mathrm{C}$ for 1000 hours (zone axis [001]) 

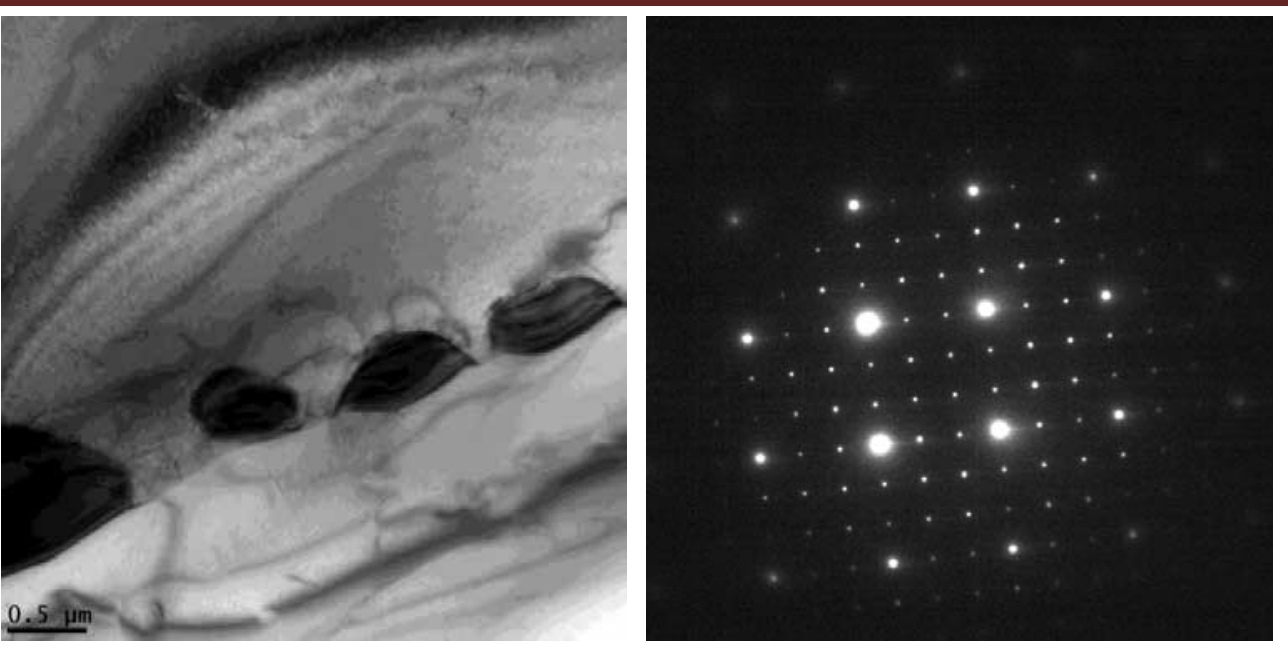

Fig. 4.38: TEM analysis of Alloy 230 aged at $1000^{\circ} \mathrm{C}$ for 1000 hours (zone axis [001])

During thermal aging of Alloy 617, no significant changes in grain boundary character distribution (GBCD) and grain size distribution were found in either alloy. Fig. 4.39 shows the EBSD analysis for Alloy 617 aged at $900^{\circ} \mathrm{C}$ for 1000 hours. The high fraction of twin boundaries and the duplex grain size distribution were preserved after long-term aging. Similar results were observed in Alloy 230, showing a remarkable thermal stability for both alloys. In addition, no topologically close-packed phase (TCP) (e.g. $\operatorname{sigma}(\sigma), \operatorname{mu}(\mu)$, or chi $(\chi))$ was observed during the aging experiment.

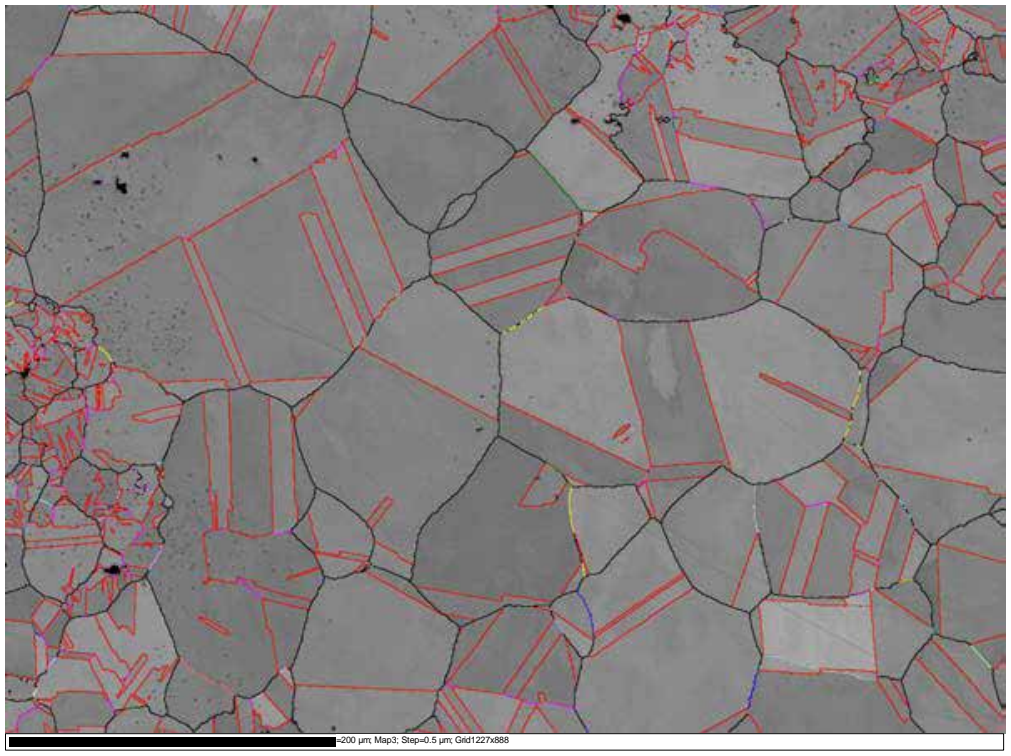

Fig. 4.39: EBSD and CSL boundary analysis for Alloy 617 aged at $900^{\circ} \mathrm{C}$ for 1000 hours 


\section{Understanding Fundamental Material-Degradation Processes in High Temperature Aggressive Chemomechanical Environments}

\subsubsection{Particle growth during thermal aging}

In order to study the kinetics of carbide growth, the process of particle coarsening in grain boundaries was analyzed by measuring the particle volume increase. More than 150 intergranular particles were measured from SEM images from each aging condition. Fig. 4.40 shows an example for measuring the length and width of intergranular particles. The results of the measurements are shown in Fig. 4.41. For both alloys, the intergranular particles became much larger during aging at $1000^{\circ} \mathrm{C}$ compared to $900^{\circ} \mathrm{C}$. This indicates a strong temperature dependence for intergranular particle coarsening. Additionally, a different coarsening rate of intergranular particles in Alloy 617 and Alloy 230 was observed. In nearly every aging condition and despite comparable initial dimensions, the length and width of Alloy 617 particles were found to be twice that of Alloy 230 particles, showing a much higher coarsening rate in Alloy 617.

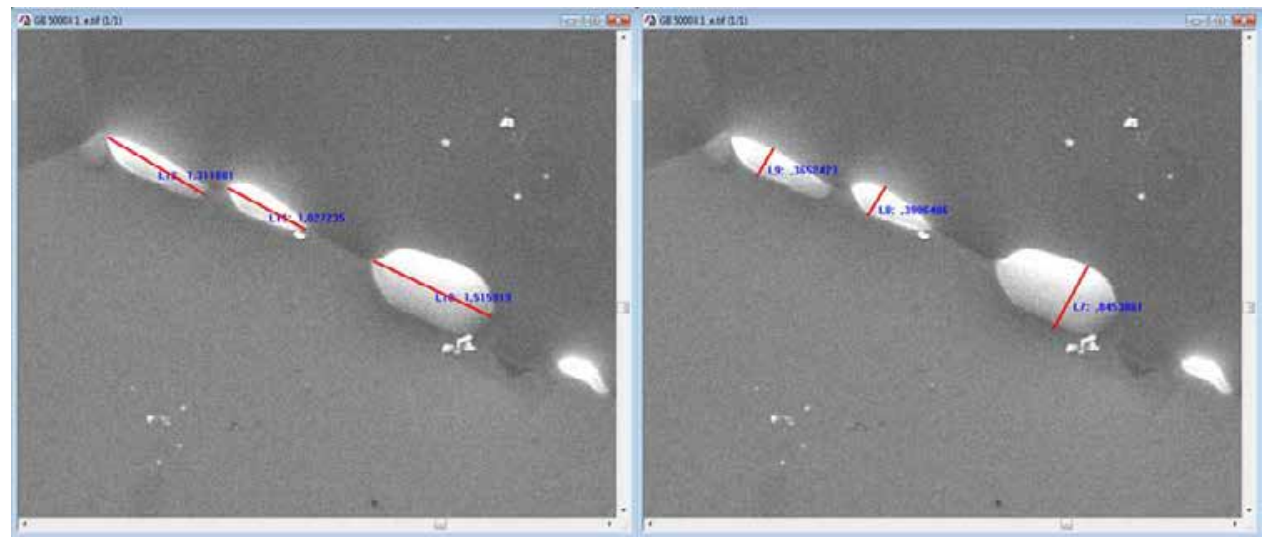

(a)

(b)

Fig. 4.40: Example of the dimension measurements of intergranular precipitates: (a) length; (b) width

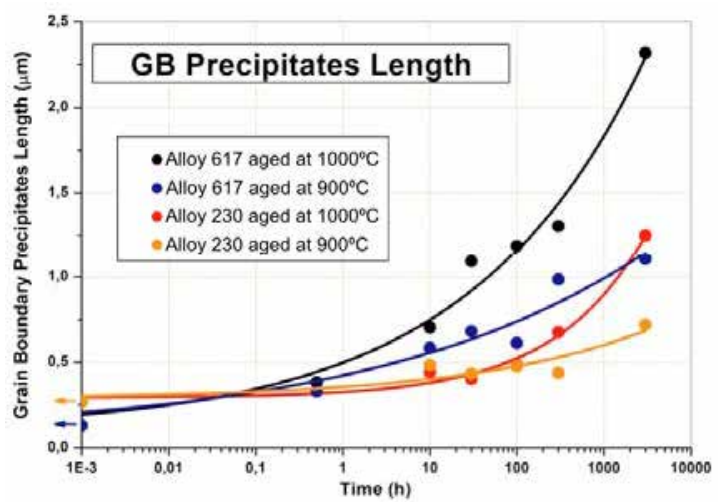

(a)

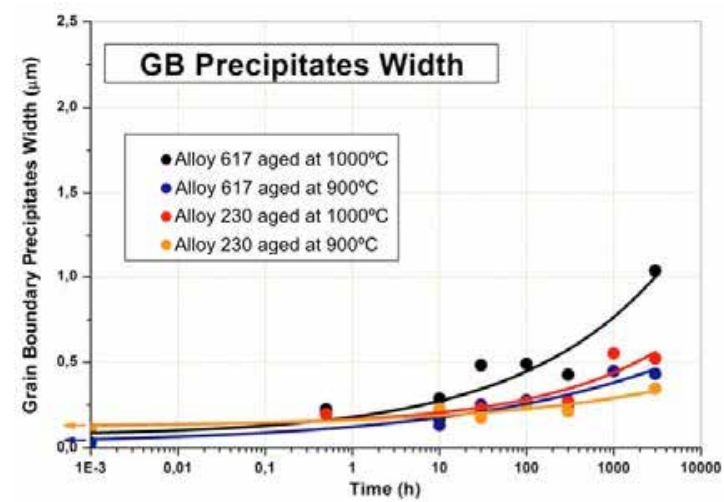

(b)

Fig. 4.41: Dimensions of intergranular precipitates in Alloy 617 and Alloy 230: (a) length; (b) width 


\section{Understanding Fundamental Material-Degradation Processes in High Temperature Aggressive Chemomechanical Environments}

Based on the measurements of the width and length of the particles, 3D analysis for the volume increase is conducted. The basic assumption is that the particle shape is ellipsoidal during long-term aging, which is reasonable from SEM observations. Thus, the particle volume can be calculated by:

$V(t)=\frac{4}{3} r^{2} h$

where $V$ is the volume of second-phase particles as a function of time, and $r$ and $h$ are the radius and height of ellipsoid particles, corresponding to the measured length (Fig. 4.41(a)) and width (Fig. 4.41(b)), respectively. Thus, the volume development for the particles can be fitted using an adapted Avrami equation:

$Y=1-\exp \left(-K t^{n}\right)$

where $Y$ is the particle volume fraction in a function of time and is defined by:

$Y=\frac{V(t)}{V_{\text {mat }}}$

$V_{\text {mat }}$ is the volume of the matured second-phase particles, and $K$ and $n$ are the fitting constants. Because the intergranular particles are considered to be fully developed after aging for 3000 hours [3.44], and little volume will be obtained by an extended aging time, $V(3000 \mathrm{~h})$ can be assumed to be equal to $V_{\text {mat }}$. Fig. 4.42 shows a fit curve for intergranular particle volume development in Alloy 617 aged at $900^{\circ} \mathrm{C}$.

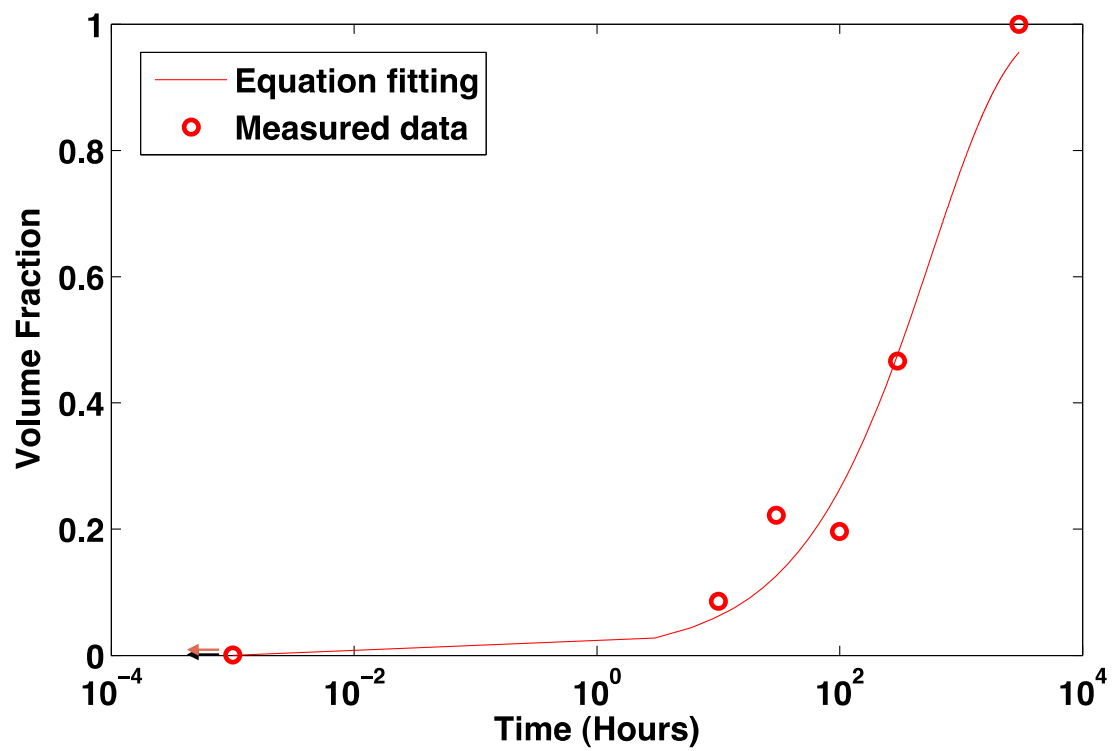

Fig. 4.42: Fitting curve for intergranular particle volume development in Alloy 617 aged at $900^{\circ} \mathrm{C}$

The process of particle volume development can be seen in the 3D analysis shown in Fig. 4.43. The particle volume rapidly increases during the early stage of aging ( $t<300$ hours), and then decelerated coarsening follows after an extended aging time ( $t>300$ hours). The tendency of particle growth and its temperature dependence can be quantitatively studied though analysis of the particle-coarsening rate (i.e. $d Y / d t$ ). (Fig. 4.44) For each alloy, intergranular particles 


\section{Understanding Fundamental Material-Degradation Processes in High Temperature Aggressive Chemomechanical Environments}

obtained a large coarsening rate during the early stage of aging. Nevertheless, the changes in $d Y / d t$ over time are controlled by different kinetics for 900 and $1000^{\circ} \mathrm{C}$. For the situation at $900^{\circ} \mathrm{C}$, despite a high coarsening rate at the beginning of aging, the value decreases dramatically with aging time. This suggests a decline in driving force over aging time. In contrast, the particle coarsening rate during aging at $1000^{\circ} \mathrm{C}$ increased for $\sim 400$ hours and then decreased moderately during further aging. The highest coarsening rate was found to be $\sim 7 \times 10^{-4} / \mathrm{h}$.

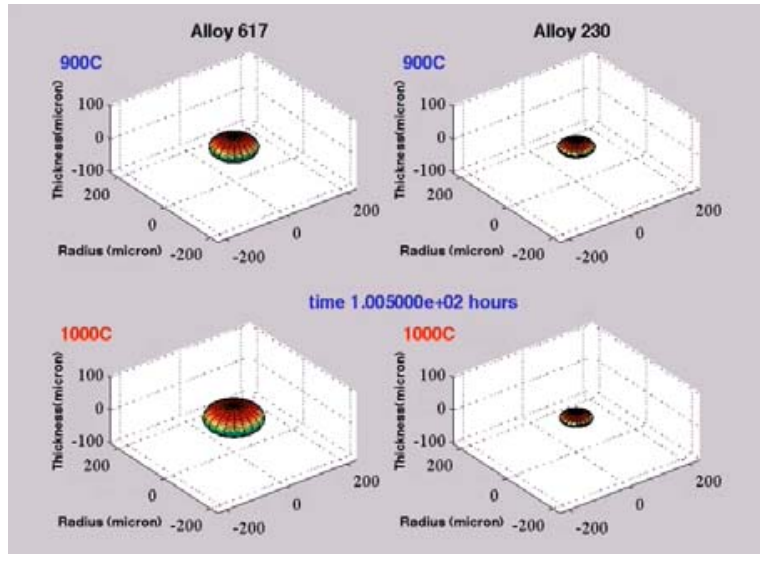

(a)

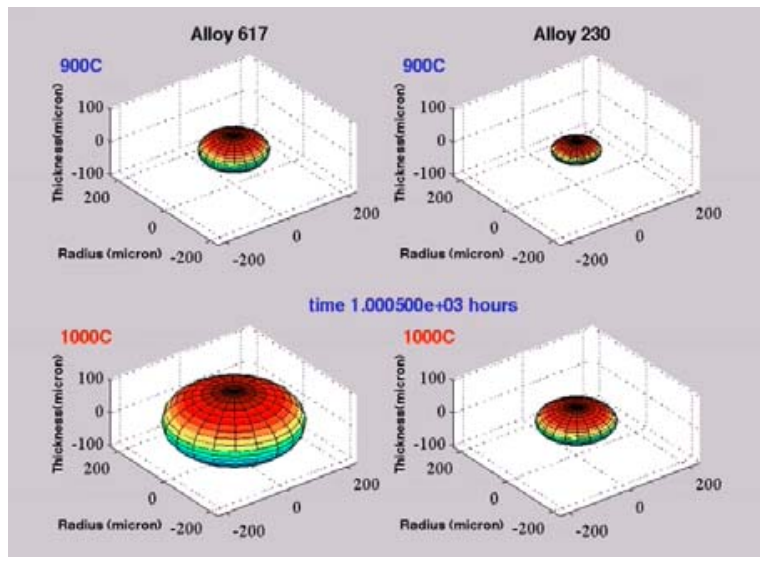

(c)

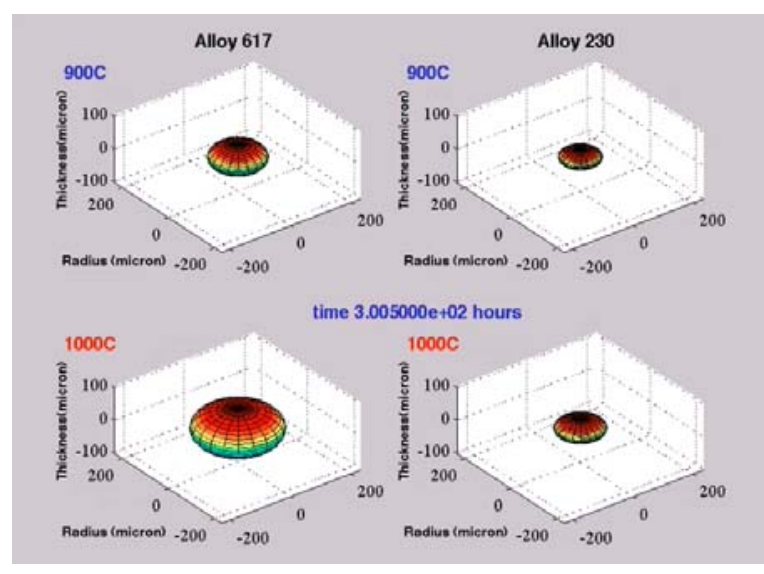

(b)

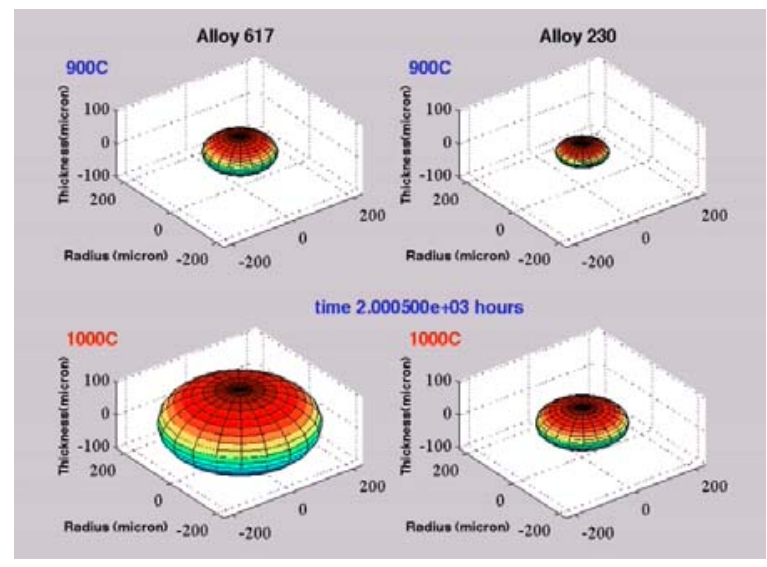

(d)

Fig. 4.43: 3D images showing intergranular particle volume development after: (a) 100 hours; (b) 300 hours; (c) 1000 hours; (d) 2000 hours 


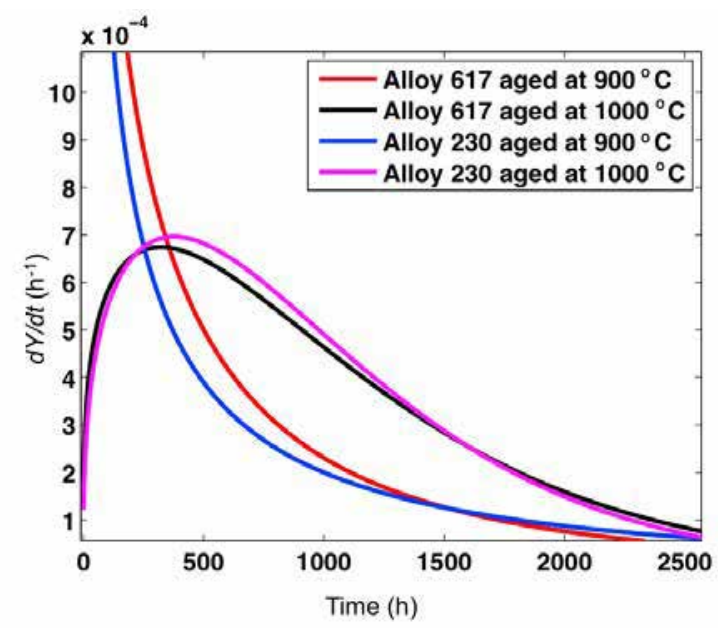

Fig. 4.44: Particle coarsening rate for Alloy 617 and Alloy 230 aged at 900 and $1000^{\circ} \mathrm{C}$

\subsubsection{Mechanical properties after thermal aging}

Hardness and tensile tests were performed on Alloy 617 and Alloy 230 in conditions ranging from as-received to after 3000 hours aging. The tensile tests were conducted at a strain rate of $\sim 10^{-3}$ /s using an INSTRON 1331 servohydraulic test system equipped with a high-temperature furnace. The detailed procedures for tensile testing have been given in section 3.1. The hardness of both alloys was measured using a Wilson ${ }^{\circledR}$ Rockwell $^{\circledR}$ Model 523 hardness tester.

In order to measure the overall response from the rolling and transverse planes, the hardness tests were conducted in each plane direction. The hardness for each aging condition is obtained by averaging the measurement results. Fig. 4.45 shows the measured hardness of Alloy 617 and Alloy 230. Both alloys experienced a similar hardness development during aging: the peak hardness for both alloys is achieved during early stage aging ( $<\sim 10$ hours) and followed by a continuous softening upon extended aging time. A typical hardening process can be seen in the Alloy 617 sample aged at $900^{\circ} \mathrm{C}$ : the material's hardness increased from $\sim 90$ to $\sim 94 \mathrm{HRB}$ in 10 hours and then gradually decreased over time. The hardening for Alloy 617 at $1000^{\circ} \mathrm{C}$ is moderate with a slight hardness increase from $\sim 90$ to $91 \mathrm{HRB}$. For each temperature, the hardness of Alloy 617 decreases to a similar value of $~ 88$ HRB after aging for 3000 hours. This value is lower than the hardness in the as-received condition. Compared to Alloy 617, Alloy 230 experiences a longer hardening effect during aging, especially for the sample aged at $900^{\circ} \mathrm{C}$. Furthermore, the hardness decline in Alloy 230 is less than that of Alloy 617 aged at the same conditions. 


\section{Understanding Fundamental Material-Degradation Processes in High Temperature Aggressive Chemomechanical Environments}

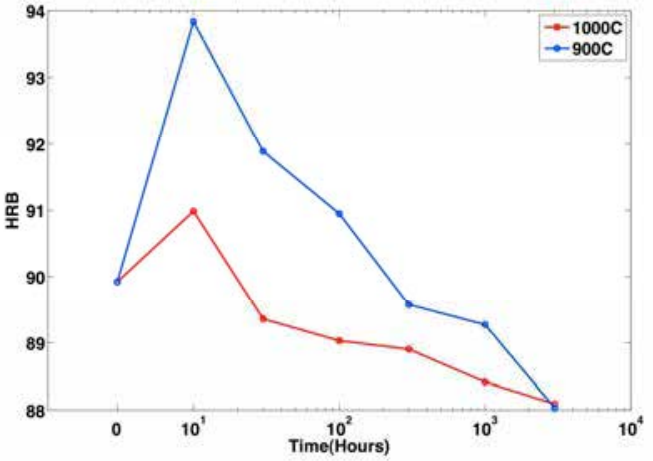

(a)

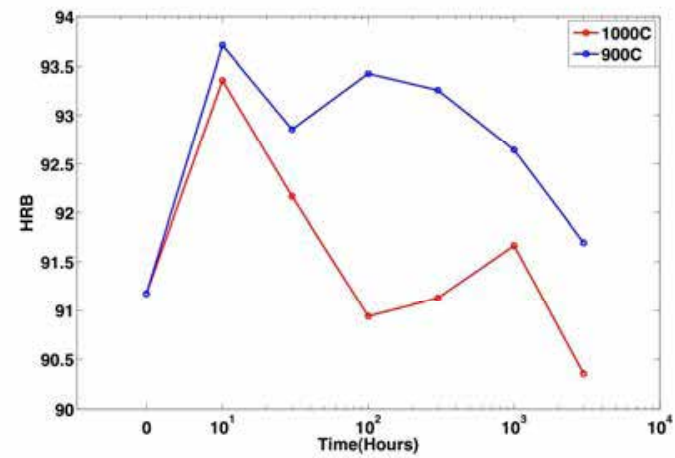

(b)

Fig. 4.45: Hardness of the as-received and aged: (a) Alloy 617; (b) Alloy 230

The results of tensile tests for the as-received and aged alloys are shown in Figs. 4.46 and 4.47. Agreeing with the hardness measurements, the measured tensile properties show a typical aging process characterized by a short-term strengthening and a long-term softening. The only exception lies in the Alloy 617 sample aged at $1000^{\circ} \mathrm{C}$, where no apparent strengthening is observed. (Fig. 4.46) For both alloys, the changes in tensile properties during aging are controlled by the studied temperature. Generally, both alloys aged at $900^{\circ} \mathrm{C}$ attain higher yield and tensile strengths with a longer hardening time compared to samples aged at $1000^{\circ} \mathrm{C}$. This suggests a more intense diffusion process at higher temperatures, so precipitate hardening can only occur for a short time.

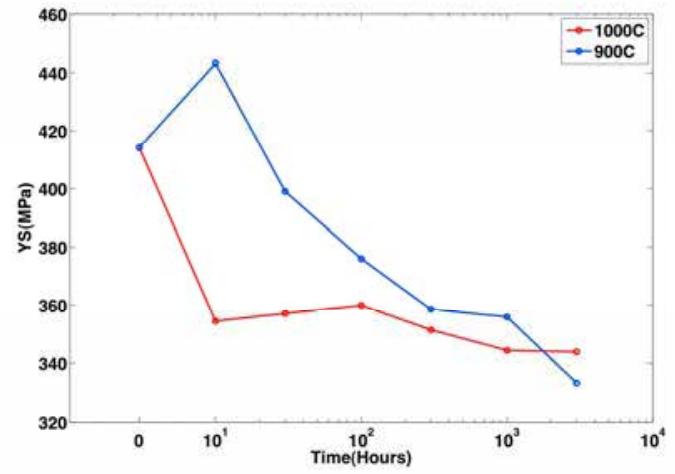

(a)

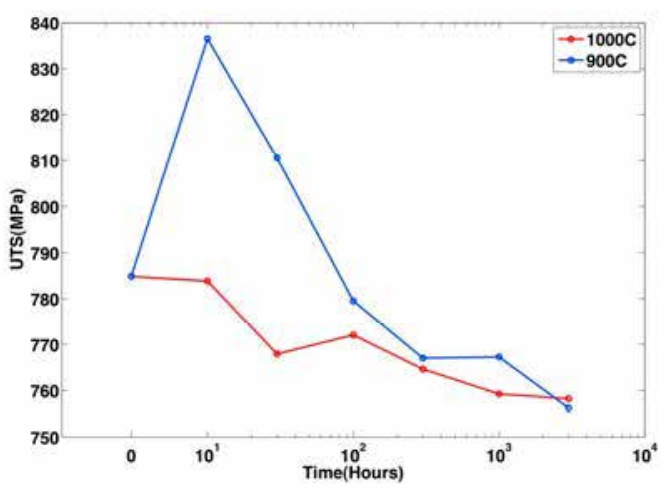

(b)

Fig. 4.46: Tensile properties for the as-received and aged Alloy 617: (a) Y.S.; (b) U.T.S. 


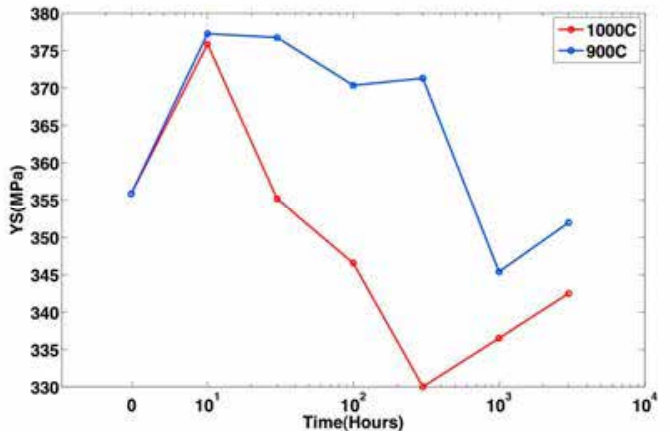

(a)

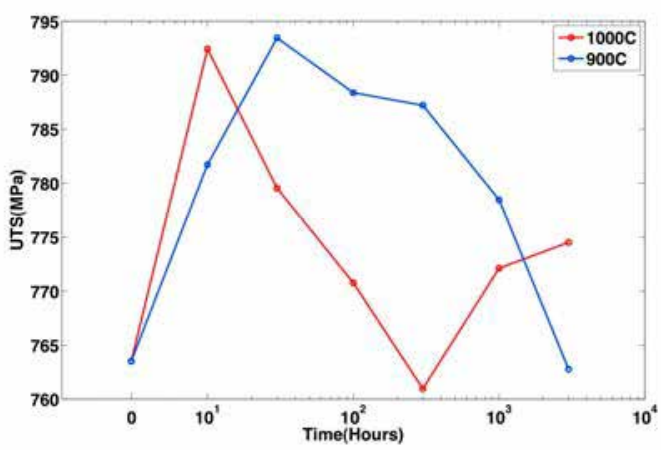

(b)

Fig. 4.47: Tensile properties for the as-received and aged Alloy 230: (a) Y.S.; (b) U.T.S.

Beyond studying mechanical properties at room temperature, it is also important to investigate the aging effect on tensile responses at high temperatures, particularly for the $800-1000^{\circ} \mathrm{C}$ temperature range at which the VHTR will operate. Fig. 4.48 shows a comparison of tensile properties for the as-received and 3000 hours aged (at $1000^{\circ} \mathrm{C}$ ) Alloy 617. Both yield and tensile strengths for various temperatures are found to decrease after aging for 3000 hours. This softening effect can be quantified by calculating the relative strength reduction by:

Y.S. reduction $=\frac{\text { Y.S. }(T, t=0)-\text { Y.S. }(T, t=3000 h)}{\text { Y.S. }(T, t=0)}$

U.T.S. reduction $=\frac{\text { U.T.S. }(T, t=0)-\text { U.T.S. }(T, t=3000 h)}{\text { U.T.S. }(T, t=0)}$,

where $T$ is the studied temperature $\left(\mathrm{RT}\right.$ to $1000^{\circ} \mathrm{C}$ ) during tensile testing. The results of strength reduction are summarized in Table 4.6. The softening effect after long-term aging is much more significant for the Y.S. compared to the U.T.S. The reduction in Y.S. at all studied temperatures (except $700^{\circ} \mathrm{C}$ ) is higher than $15 \%$ with an average value over $20 \%$. In contrast, the mean reduction in U.T.S. is only $\sim 5 \%$. It can be noted that the loss in U.T.S. is temperature sensitive: high strength loss occurs at temperatures higher than $800^{\circ} \mathrm{C}$, while the strength loss is very small at lower temperatures (RT to $700^{\circ} \mathrm{C}$ ). 


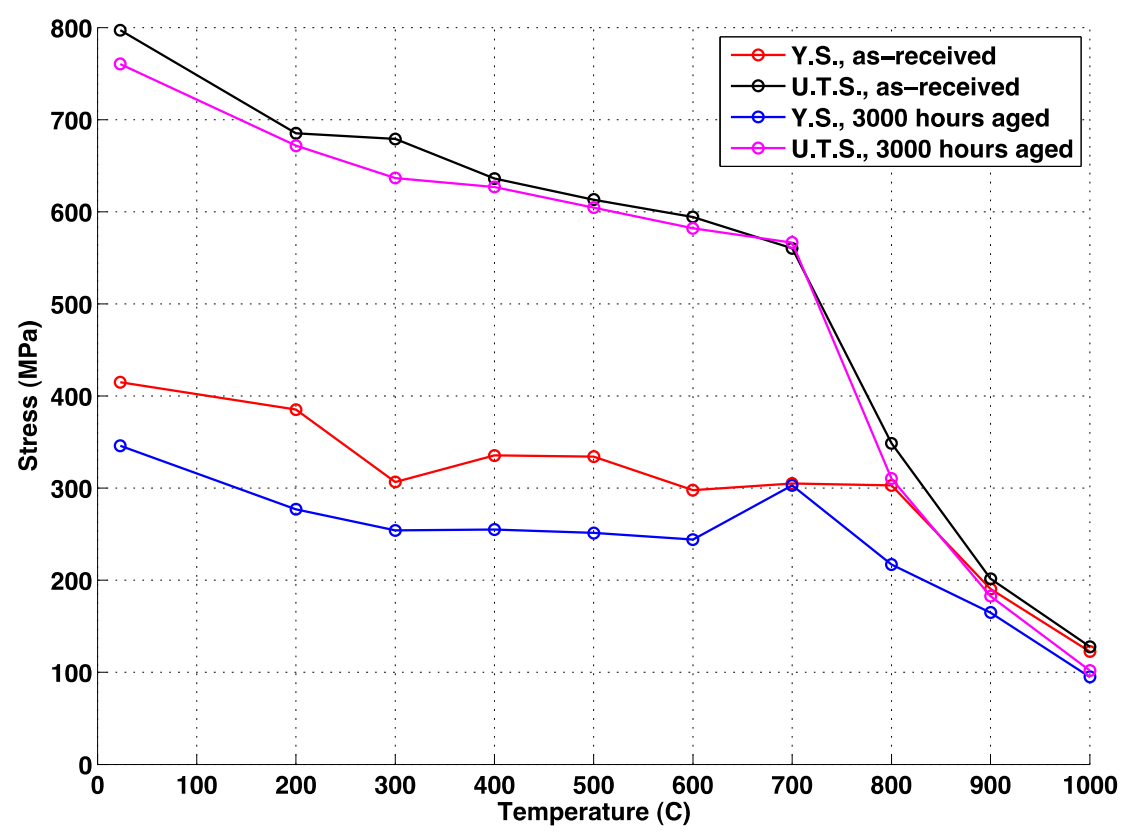

Fig. 4.48: Tensile properties at various temperatures of the as-received and long-term aged $\left(\right.$ at $\left.1000^{\circ} \mathrm{C}\right)$ Alloy 617

Table 4.6: Aging effect on tensile properties of Alloy 617 for various temperatures. The samples were aged for 3000 hours at $1000^{\circ} \mathrm{C}$.

\begin{tabular}{ccccccccccc}
\hline Temperature ( $\left.{ }^{\circ} \mathbf{C}\right)$ & $\mathbf{R T}$ & $\mathbf{2 0 0}$ & $\mathbf{3 0 0}$ & $\mathbf{4 0 0}$ & $\mathbf{5 0 0}$ & $\mathbf{6 0 0}$ & $\mathbf{7 0 0}$ & $\mathbf{8 0 0}$ & $\mathbf{9 0 0}$ & $\mathbf{1 0 0 0}$ \\
\hline $\begin{array}{c}\text { Y.S. in AR } \\
\text { condition } \\
\text { (MPa) }\end{array}$ & 415 & 385 & 306 & 335 & 334 & 297 & 305 & 303 & 190 & 122 \\
$\begin{array}{c}\text { U.T.S. in AR } \\
\text { condition (MPa) }\end{array}$ & 797 & 685 & 679 & 636 & 613 & 594 & 560 & 348 & 201 & 127 \\
$\begin{array}{c}\text { Y.S. after aging } \\
\text { (MPa) }\end{array}$ & 346 & 277 & 254 & 255 & 251 & 244 & 303 & 217 & 165 & 95 \\
$\begin{array}{c}\text { Reduction (\%) } \\
\text { U.T.S. after aging }\end{array}$ & 16.7 & 28.0 & 17.0 & 23.9 & 24.8 & 17.8 & 0.7 & 28.4 & 13.2 & 22.1 \\
$\begin{array}{c}\text { (MPa) } \\
\text { Reduction (\%) }\end{array}$ & 460 & 672 & 637 & 627 & 605 & 582 & 567 & 310 & 183 & 102 \\
\hline
\end{tabular}




\subsection{High-energy X-Ray Diffraction Study of Alloy 230}

\subsubsection{Materials}

Similar to the anisotropy study of Alloy 617 given in section 4.2, the long and short-transverse directions were used to fabricate two different types of tensile specimens. Fig. 4.49 shows a schematic of the studied specimens and their representative microstructures. The large, lamellar-shaped particles seen in the SEM images are tungsten rich $\mathrm{M}_{6} \mathrm{C}$ type carbides. The volume fraction of the tungsten carbide is $\sim 6 \%$. A detailed discussion of the microstructure of Alloy 230 can be found in Chapters $3 \& 4$.

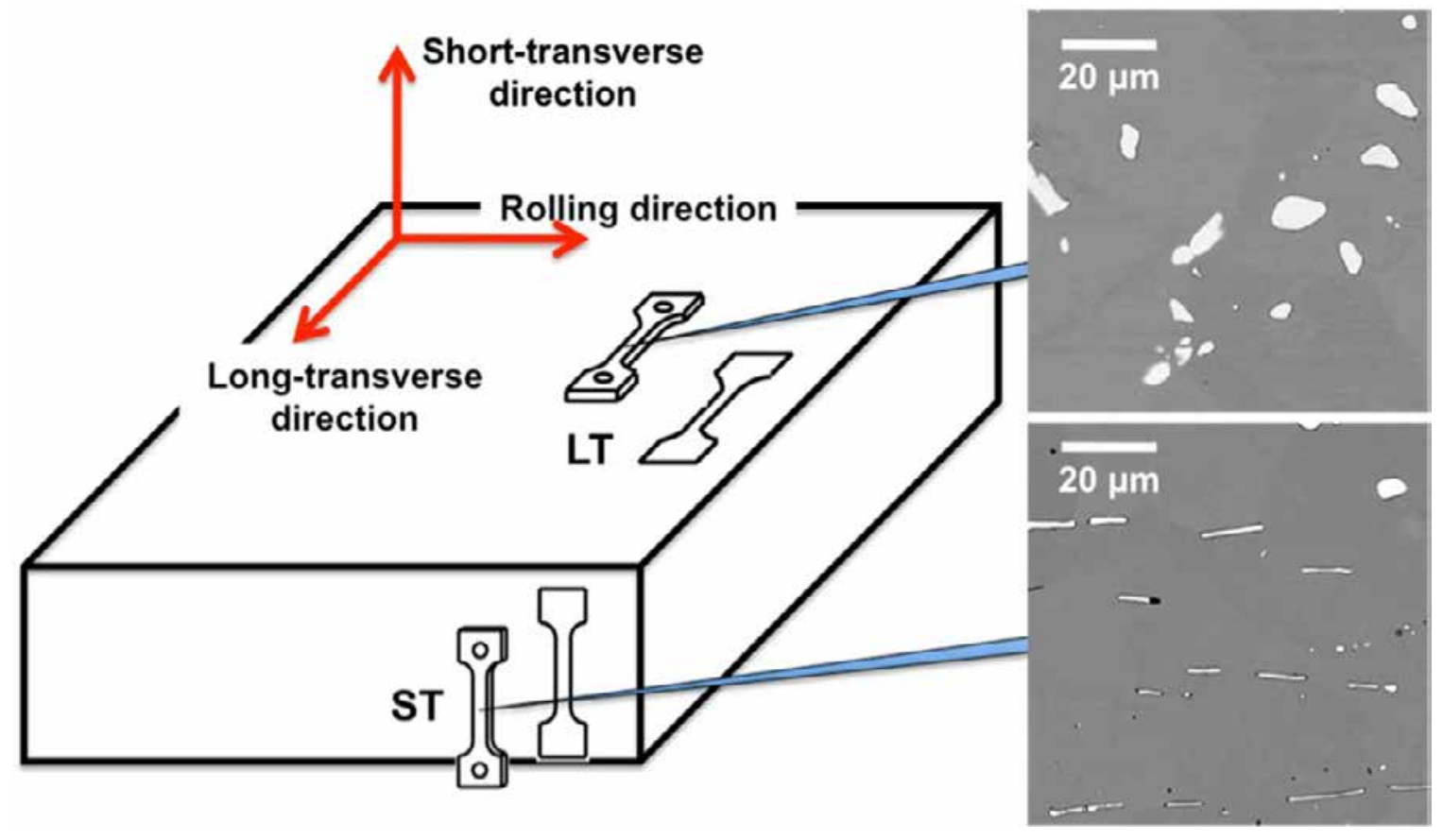

Fig. 4.49: In-plane sampling directions and microstructures of tensile specimens

Fig. 4.50 shows a representative X-ray diffraction pattern of Alloy 230 with phase and peak identifications. Despite low fractions of $\mathrm{M}_{6} \mathrm{C}$ carbides, their diffraction rings can be clearly identified by a distinctive lower intensity compared to the reflections from the $\gamma$ matrix. The most intense reflections for the carbide are (331), (422), (333), (440), and (822). The measured lattice constants for the $\gamma$ matrix and the $\mathrm{M}_{6} \mathrm{C}$ carbide are $3.58 \AA$ and $10.99 \AA$, respectively. 


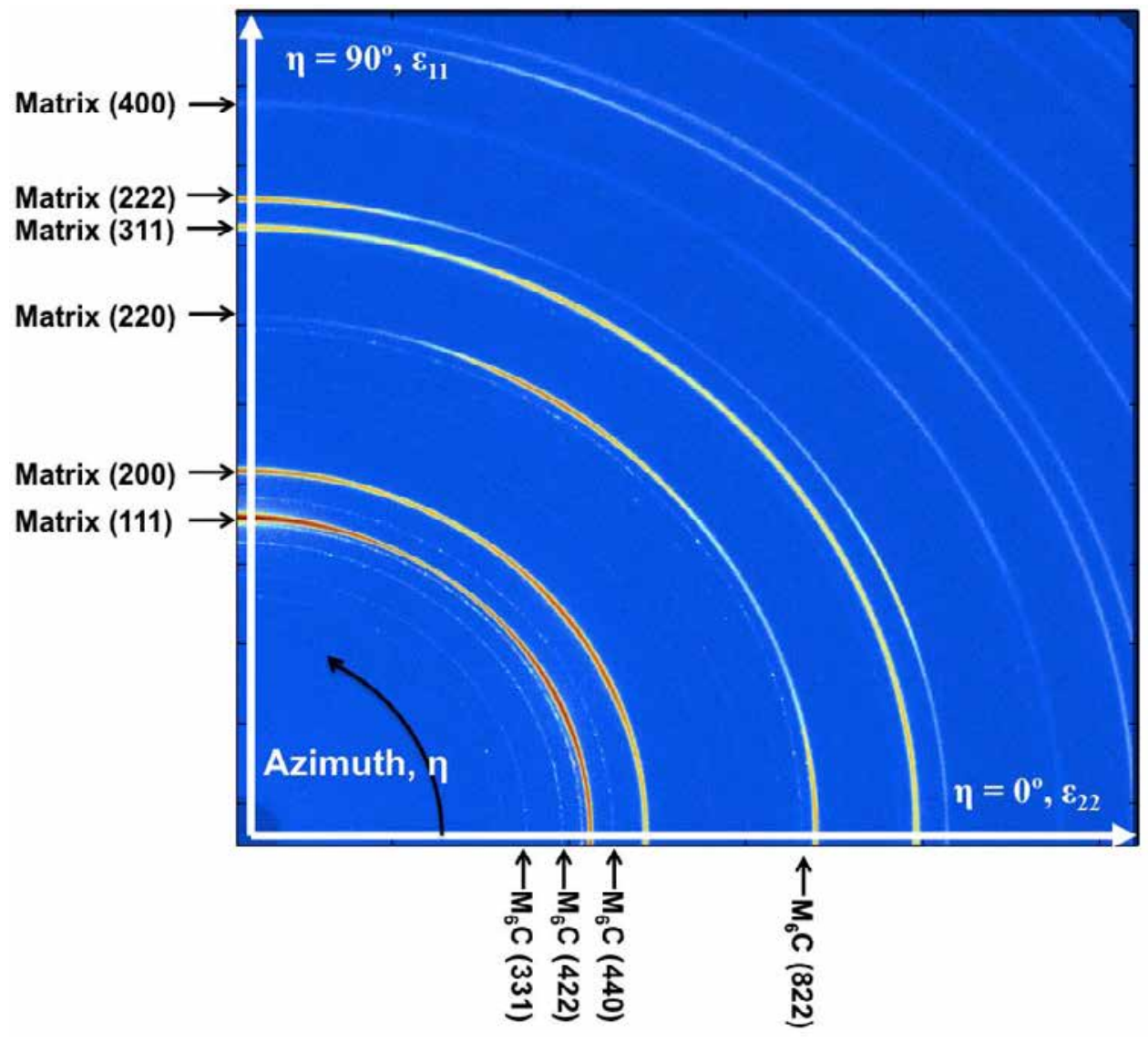

Fig. 4.50: Representative X-ray diffraction pattern (quarter of image plate) for Alloy 230. All Debye rings are identified as belonging to $\gamma$ matrix and $\mathrm{M}_{6} \mathrm{C}$ carbides

\subsubsection{Mechanical tests}

In order to have a large diffraction volume to measure the materials' bulk responses, the specimens were scanned for 10 different axial positions periodically during tensile testing. (Fig. 4.51) The diffraction volume for one single exposure was $300 \times 300 \times 760 \mu \mathrm{m}^{3}$. So, the total diffraction volume obtained from 10 scans was $\sim 68.4 \times 10^{7} \mu \mathrm{m}^{3}$, which provides adequate statistics to extract the bulk response from the material. 


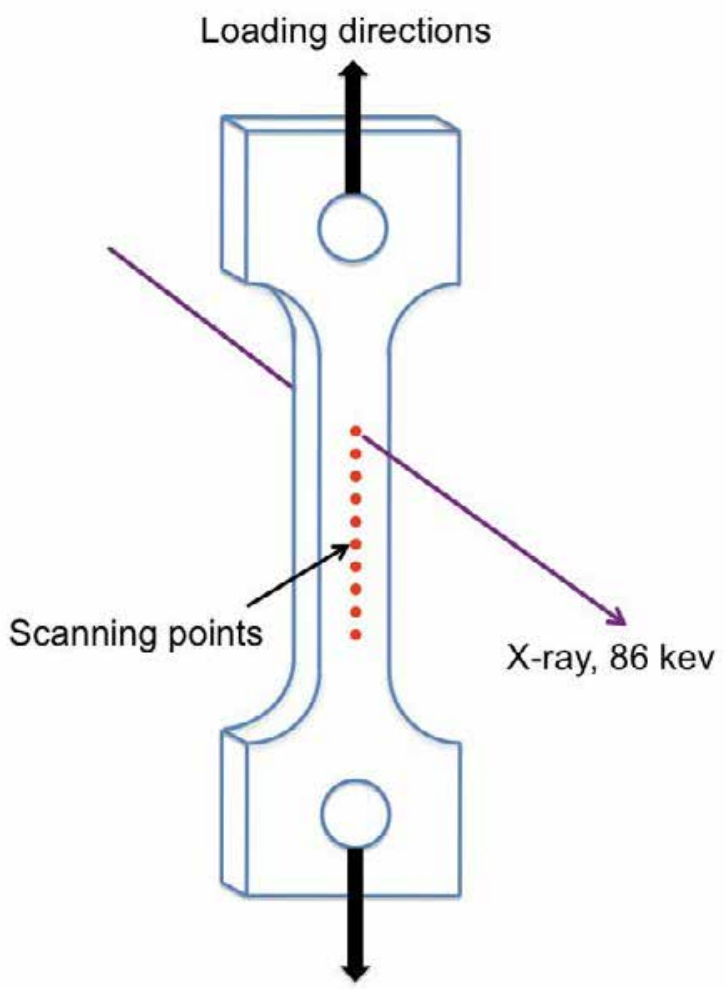

Fig. 4.51: Schematic of axial-scanning measurements

The engineering strain-stress diagram for uniaxial tensile testing during in-situ XRD measurement is shown in Fig. 4.52(a). Both long-transverse (LT) and short-transverse (ST) specimens exhibited a typical tensile response under loading. However, similar to the anisotropy study on Alloy 617, considerable differences in tensile properties were found in two sampling directions. The $0.2 \%$ yield strength was equal to $\sim 380$ and $300 \mathrm{MPa}$ for LT and ST specimens, respectively. Even larger divergence was found in the tensile strength in the LT and ST directions, which are 576 and $839 \mathrm{MPa}$, respectively. Along with decreased U.T.S., the elongation for the ST specimen was $17 \%$, which is much lower than the value for the LT specimen of 59\%. Both specimens failed immediately when stress reached ultimate tensile strength, so necking was not apparent for either specimen.

Fig. 4.52(b) represents the true strain-stress diagrams for short- and long-transverse specimens after having been converted from engineering strain and stress. The true fracture stress for the LT specimen is $\sim 1327 \mathrm{MPa}$, or more than twice the value for the ST specimen of $\sim 668 \mathrm{MPa}$. The bulk Young's modulus for both specimens is $\sim 211 \mathrm{GPa}$, which is the same as the value listed in the material profile. [4.22] The summarized tensile properties are shown in Table 4.7 . 


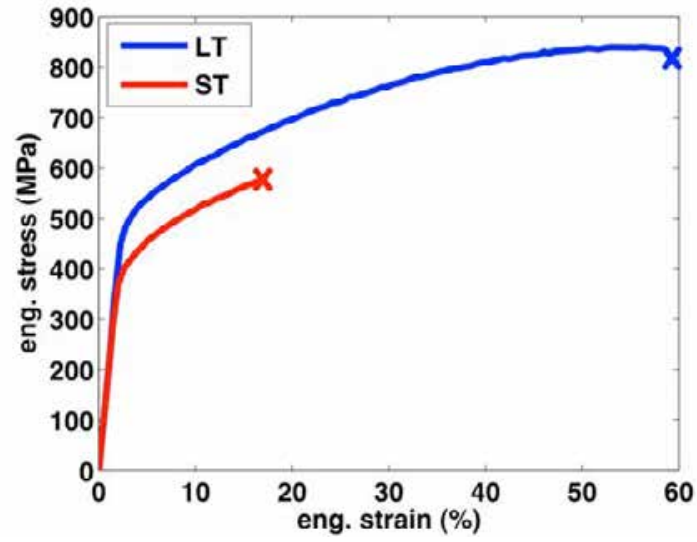

(a)

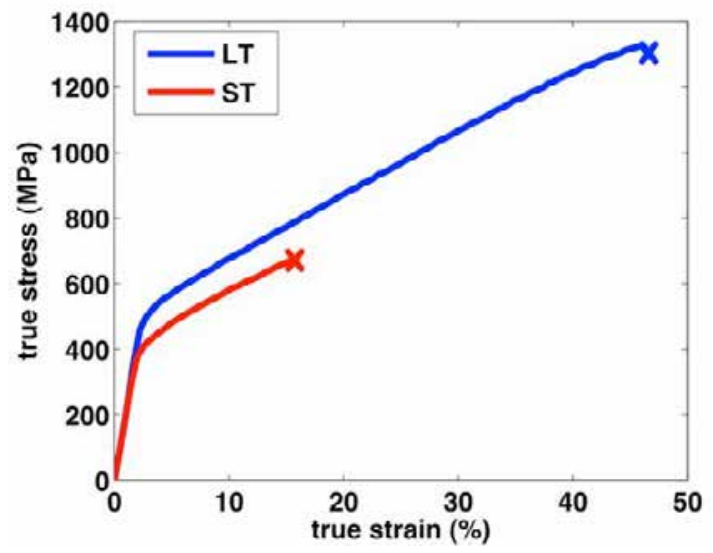

(b)

Fig. 4.52: Macroscopic strain-stress diagrams for Alloy 230 tensile specimens in the long transverse (LT) and short transverse (ST) directions: (a) engineering stress versus engineering strain; (b) true stress versus true strain

Table 4.7: Mechanical properties for the long- and short-transverse specimens of Alloy 230

\begin{tabular}{ccccc}
\hline Direction/Properties & $\begin{array}{c}\mathbf{0 . 2 \%} \text { Y.S. } \\
\text { (MPa) }\end{array}$ & $\begin{array}{c}\text { UTS } \\
\text { (MPa) }\end{array}$ & $\begin{array}{c}\text { Elongation } \\
\text { (\%) }\end{array}$ & $\begin{array}{c}\text { Fractured } \\
\text { stress (MPa) }\end{array}$ \\
\hline Long-transverse & 380 & 839 & 59 & 1327 \\
Short-transverse & 300 & 576 & 17 & 668 \\
\hline
\end{tabular}

\subsubsection{Elastic constant determination}

An important question must be answered before further discussion takes place: how are the measured $h k l$-specific strains interpreted to attain internal stress? The typical expression for the relation of the strain components, $\varepsilon_{i j}$, and stress components, $\sigma_{i j}$, is:

$\sigma_{i j}=C_{i j k l} \varepsilon_{k l}$

or:

$\varepsilon_{i j}=S_{i j k l} \sigma_{k l}$

In these equations, $C_{i j k l}$ is the fourth-rank stiffness tensor and $S_{i j k l}$ is the fourth-rank compliance tensor. For a given material, the values of $C$ and $S$ for single crystal differ from polycrystalline because there are significant interactions between grains in the polycrystalline structure. Therefore, a bar is often added to $C$ and $S$ to distinguish the stiffness and compliance for macroscopic polycrystalline from the corresponding values for a single crystal:

$\sigma=\bar{C} \varepsilon \quad \& \quad \varepsilon=\bar{S} \sigma$ 


\section{Understanding Fundamental Material-Degradation Processes in High Temperature Aggressive Chemomechanical Environments}

In the case of an isotropic polycrystalline material with cubic crystal structure, $\bar{C}$ can be expressed by two independent elastic constants, i.e. Young's modulus, $E$, and Poisson's ratio, $v$. The equation 4.21 can then be simplified to:

$$
\sigma_{i j}=\frac{E}{(1+v)}\left[\varepsilon_{i j}+\frac{v}{(1+2 v)}\left(\varepsilon_{11}+\varepsilon_{22}+\varepsilon_{33}\right)\right]
$$

In this relation, $i, j=1,2,3, \ldots$ indicate the components relative to chosen axes [4.23]. For an anisotropic system (e.g. a single crystal), $E$ and $v$ depend on the loading direction, which is defined by $h k l$ in a cubic system. Although the polycrystalline is generally treated as isotropic, the material is composed of aggregated monocrystals with anisotropic properties. Additionally, the elastic behavior in the polycrystalline involves an interaction between grains. Therefore, precise interpretation from lattice strains to internal stresses (the question posed at the beginning of this section) will essentially require a complete solution of the influence of elastic anisotropy for every grain and their respective interactions. Since this solution is extremely difficult to obtain, various models have been proposed for modeling the interaction between crystallites based on single crystal behavior. [4.24, 4.23, 4.25] The most widely used models are: Reuss's, Voigt's, and Kröner's. These models were initially introduced to predict the overall response (e.g. bulk, shear and Young's moduli, and Poisson's ratio) of polycrystalline materials based on their elastic constants for monocrystals. Nevertheless, the monocrystals are elastic anisotropic, and hence, the $h k l$-specific response for polycrystalline aggregates can also be simulated using these models.

\section{Voigt Model:}

The basic assumption of the Voigt model is that all grains in a polycrystalline aggregate experience the same uniform strain. Thus, the elastic properties can be calculated by averaging the stiffness constants $(C)$ over all crystallites. For cubic materials (e.g. fcc or bcc), $E$ and $v$ for polycrystalline aggregate can be obtained by calculating the bulk modulus, $K$, and the shear modulus, $\mu$ :

$$
\begin{aligned}
& K=\frac{1}{3}\left(C_{11}+2 C_{12}\right) \\
& \mu=\frac{1}{5}\left(C_{11}-C_{12}+3 C_{44}\right)
\end{aligned}
$$

Then, $E$ and $v$ can be given as:

$$
\begin{aligned}
& E=2(1+v) \mu \\
& v=\frac{3 K-2 \mu}{2(3 K+\mu)}
\end{aligned}
$$

One should note that the obtained values for $E$ and $v$ in the Voigt Model are independent of the crystallographic direction, $h k l$. 


\section{Understanding Fundamental Material-Degradation Processes in High Temperature Aggressive Chemomechanical Environments}

\section{Reuss Model:}

The basic assumption of the Reuss model is that all grains in a polycrystalline aggregate experience the same amount of stress. For each crystallite, the lattice strain developed by the applied stress is dependent on the elastic anisotropy of a single crystal. In this hypothesis, elastic constants for the polycrystalline are $h k l$-specific and can be computed by:

$$
\begin{aligned}
& E(h k l)=\frac{1}{S_{11}-2 S_{0} A_{h k l}} \\
& \nu(h k l)=-\frac{S_{12}+S_{0} A_{h k l}}{S_{11}-2 S_{0} A_{h k l}}
\end{aligned}
$$

where,

$$
\begin{aligned}
& S_{0}=S_{11}-S_{12}-\frac{1}{2 S_{44}} \\
& A_{h k l}=\frac{h^{2} k^{2}+h^{2} l^{2}+l^{2} k^{2}}{\left(h^{2}+k^{2}+l^{2}\right)^{2}}
\end{aligned}
$$

$S_{0}$ is a factor related to the amount of elastic anisotropy for a material (Note: the Zener anisotropy factor is $\left.2\left(S_{11}-S_{12}\right) / S_{44}\right)$. $A_{h k l}$ and $3 A_{h k l}$ are known as the anisotropy factor [4.23] and orientation parameter [4.26], respectively.

\section{Kröner Model:}

Both of the models proposed by Voigt and Reuss are based on idealized situations where strain or stress is identical over all elements of the aggregate. In contrast, Kroner's model considers the stress and strain individually according to the Esheby's 'self-consistent approach.' [4.27] In this self-consistent model, each grain is treated as an inclusion particle embedded in a homogenous matrix, and its strain-stress relations can be expressed as:

$$
\sigma_{i j}=\left(C_{i j k l}+r_{j j k l}(\Omega)\right) \varepsilon_{k l}
$$

or:

$$
\varepsilon_{i j}=\left(S_{i j k l}+t_{j j k l}(\Omega)\right) \sigma_{k l},
$$

In these formulas, $C_{i j k l}$ and $S_{i j k l}$ are the stiffness and compliance tensors, respectively, of a single crystal of volume $\Omega$, and $r_{i j k l}$ and $t_{i j k l}$ are tensors that describe the interaction between grains. Following integration of equation 4.31 or 4.32 over all orientations, the macroscopic average values for the bulk material (denoted by subscript B) can be obtained:

$\bar{\sigma}_{i j}=\left(C_{i j k l}\right)_{B} \bar{\varepsilon}_{k l}$

or:

$\bar{\varepsilon}_{i j}=\left(S_{i j k l}\right)_{B} \bar{\sigma}_{k l}$ 


\section{Understanding Fundamental Material-Degradation Processes in High Temperature Aggressive Chemomechanical Environments}

And thus,

$\int_{D} r_{i j k l} d D=\int_{D} t_{i j k l} d D=0$

In this equation, $D$ is the total volume of the body for analysis. The relations of $r_{i j k l}, t_{i j k l}, C_{i j k l}, S_{i j k l}$, $\left(C_{i j k l}\right)_{B}$ and $\left(S_{i j k l}\right)_{B}$ can be determined and are dependent on the shape of grains and their crystal structure (e.g. cubic or hexagonal structures). Therefore, $E(h k l)$ and $v(h k l)$ can be obtained through an iterative computation. [4.24, 4.23, 4.25, 4.26] More details concerning the computation can be found in APPENDIX B.

Several examples are provided herein to clarify the difference between these models. The four selected materials are $\mathrm{Cu}, \mathrm{Al}, \mathrm{W}$, and $\mathrm{Nb}$, whose values of single-crystal elastic constants are given in Table 4.8. Based on the single-crystal elastic constants, Young's moduli for the polycrystalline metals are simulated by the models of Voigt, Reuss, and Kröner, and are shown in 3D (Fig. 4.53) and 2D (Fig. 4.54) plots. As previously discussed, the orientation dependence of Young's modulus for polycrystalline materials is different in each of the three models. The simulated Young's moduli using Voigt's model are independent of $h k l$. In sharp contrast, the Young's moduli calculated by Reuss's model show strong orientation dependence, which is particularly clear for the difference between the [111] and [100] directions. Kröner's model, in general, yields values close to the average of the Voigt and Reuss limits. Beyond the comparison between these models, the impact of the Zener anisotropy factor is notable in the model results that yield different geometries in 3D contouring (Fig. 4.53) and different curves in 2D plots (Fig. 4.54). For $\mathrm{Cu}$ with an anisotropy factor of 3.2, the Young's modulus is highest in the [111] direction and decreased to the minimum in the [100] direction according to both Reuss's and Kröner's models. For a material with an anisotropy factor close to 1 (e.g. Al and W), the orientation dependence of Young's modulus is insignificant. In the case of $\mathrm{Nb}$, the maximum Young's modulus appears in the [100] direction, and the value decreases to the minimum in the [111] direction because the anisotropy factor of $\mathrm{Nb}$ is $0.5<1$.

Among these models, Kröner's model is considered to be the most realistic for calculating overall and $h k l$-specific responses for polycrystals. The calculated results are close to experimentally determined values. [4.25] Therefore, Kröner's model will be employed for the lattice strain/internal stress conversion for the $\gamma$ matrix of Alloy 230 in the following discussion.

Table 4.8: Values of single-crystal elastic constants for selected materials

\begin{tabular}{|c|c|c|c|c|c|}
\hline Material & $\begin{array}{c}\text { Crystal } \\
\text { Structure }\end{array}$ & $C_{11}$ (GPa) & $C_{12}(\mathrm{GPa})$ & $C_{44}(\mathrm{GPa})$ & $2\left(C_{44}\right) /\left(C_{11}-C_{12}\right)$ \\
\hline $\mathrm{Cu}$ & FCC & 168.4 & 121.4 & 75.4 & 3.2 \\
\hline Al & FCC & 107.3 & 60.9 & 28.3 & 1.2 \\
\hline $\mathbf{W}$ & $\mathrm{BCC}$ & 522.4 & 204.4 & 160.8 & 1 \\
\hline Nb & $\mathrm{BCC}$ & 240.2 & 125.6 & 28.2 & 0.5 \\
\hline
\end{tabular}


Young $s$ modulus (Model of Voigt)
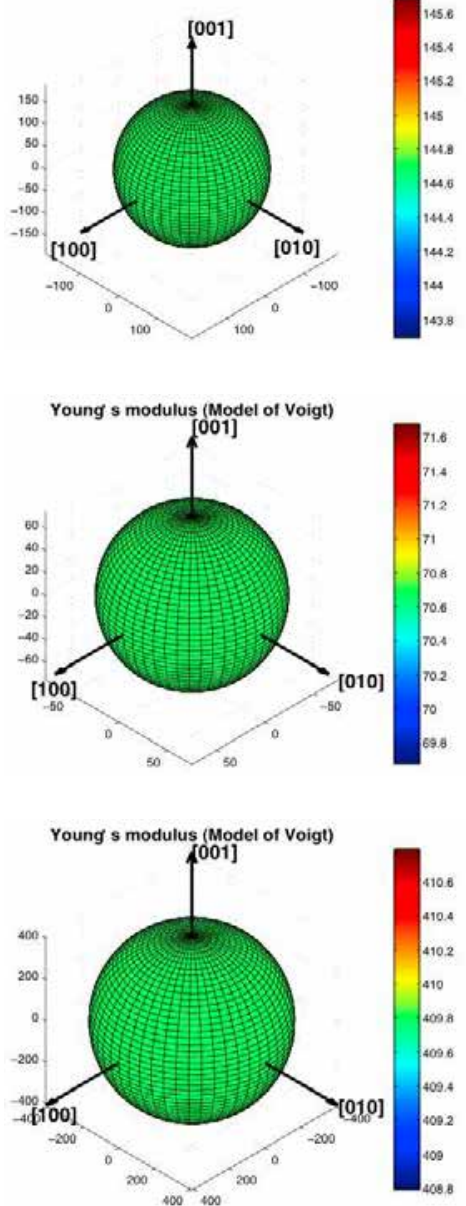

Young s modulus (Model of Voigt)
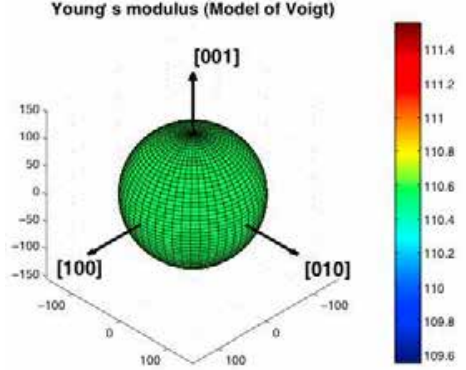

Young $s$ modulus (Model of Reuss)

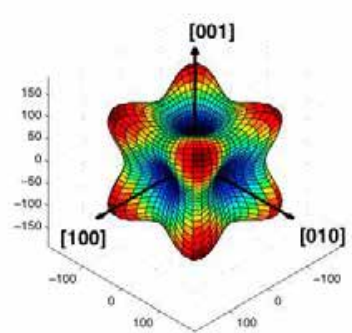

(a)

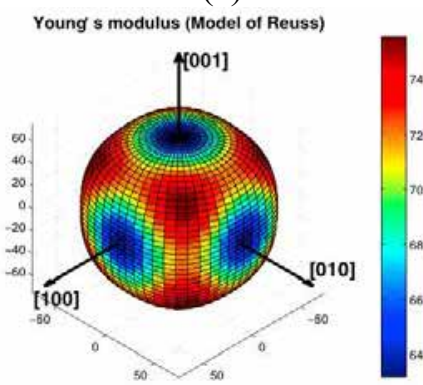

(b)

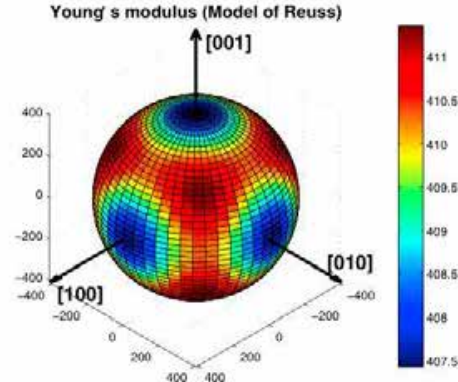

(c)

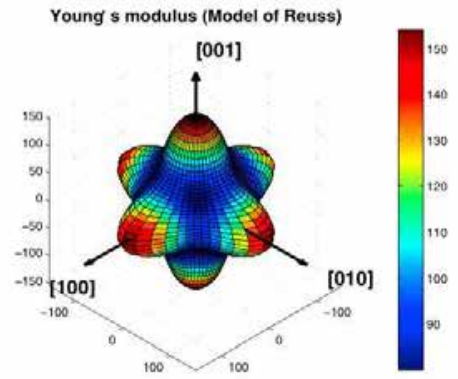

Young s modulus (Model of Kroner)
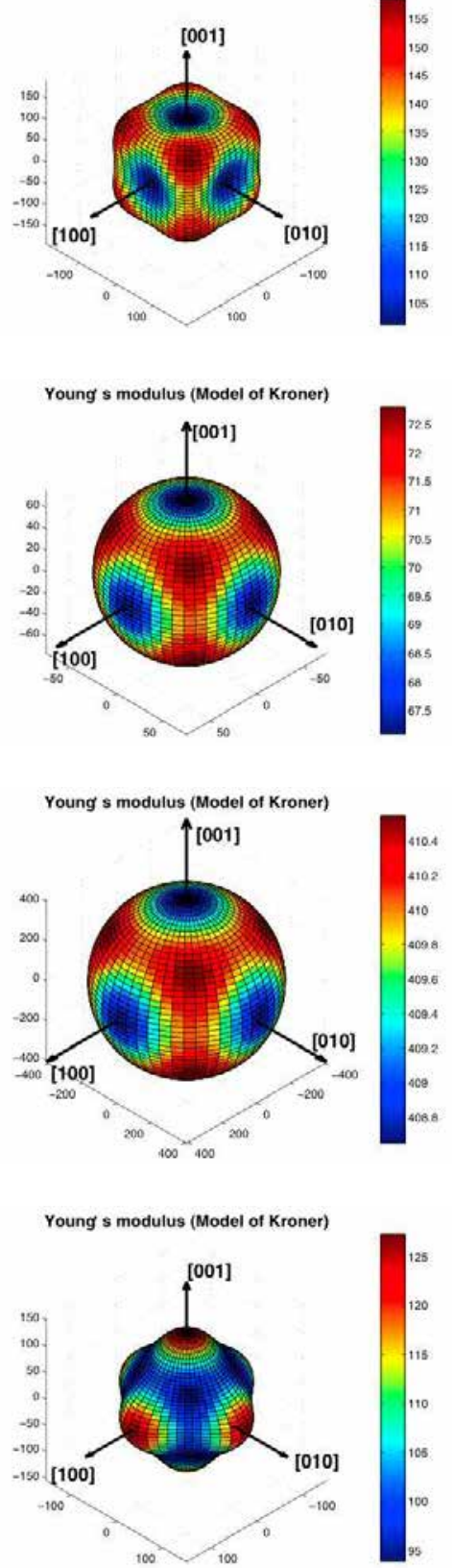

(d)

Fig. 4.53: Orientation dependence of Young's modulus (unit: GPA) for polycrystalline aggregate: (a) $\mathrm{Cu}$; (b) Al; (c) W; (d) Nb 


\section{Understanding Fundamental Material-Degradation Processes in High Temperature Aggressive Chemomechanical Environments}

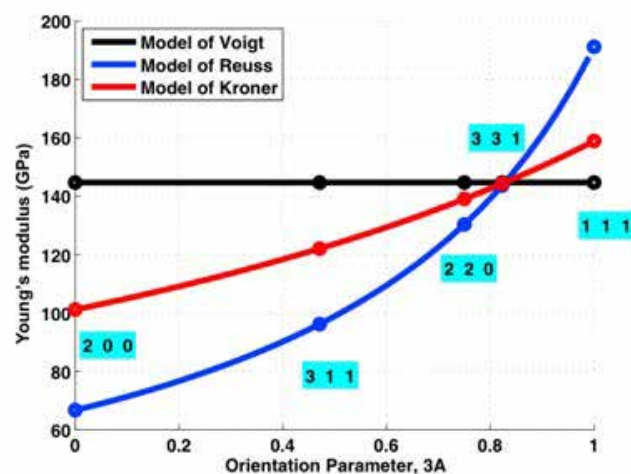

(a)

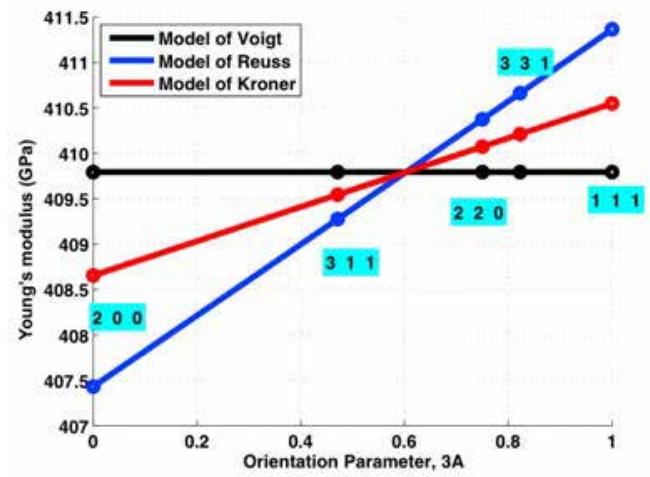

(c)

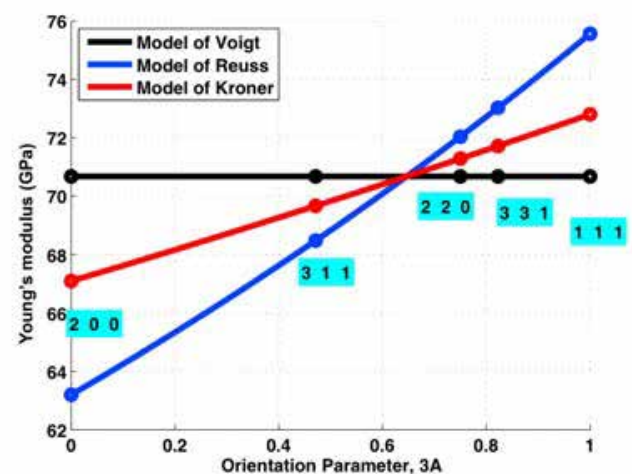

(b)

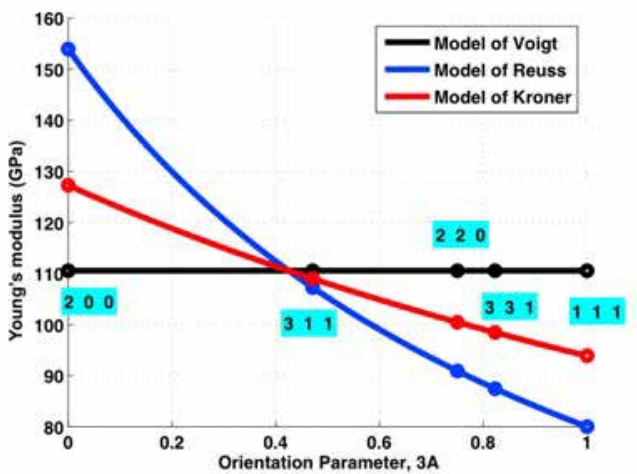

(d)

Fig. 4.54: Elastic anisotropy of Young's modulus: (a) $\mathrm{Cu}$; (b) Al; (c) W; (d) Nb

\subsubsection{Elastic response of the austenitic matrix}

Fig. 4.55 shows the lattice strain development for different reflections of the $\gamma$ matrix in the long-transverse (LT) Alloy 230 specimen. The results from a single scanned position (Fig. 4.51) provide sufficient information for analyzing the elastic anisotropy. During tensile loading, the (222) reflection shows the stiffest strain-stress loading curves, a result of the highest Young's modulus in the [111] orientation. For the same reason, the (200) reflection shows the more compliant behavior compared to the other reflections. The lower lattice strain in the transversal direction $\left(\varepsilon_{22}\right)$ relative to the axial direction $\left(\varepsilon_{11}\right)$ is the result of the Poisson effect, which can be expressed as: $\varepsilon_{22}=-v \varepsilon_{11}$ ( $v$ is the Poisson's ratio, typically $\sim 0.2-0.5$ for metals). This elastic anisotropy of the $\gamma$ matrix is analogous for both alloys, with each showing an almost linear lattice strain development to the applied stress during both elastic and plastic deformations. Fig. 4.56 shows the lattice strain development for different reflections of the $\gamma$ matrix in the short-transverse (ST) Alloy 230 specimen. In spite of an earlier rupture, the anisotropic loading behavior for the ST specimen during in-situ tensile testing is analogous to the LT specimen. 


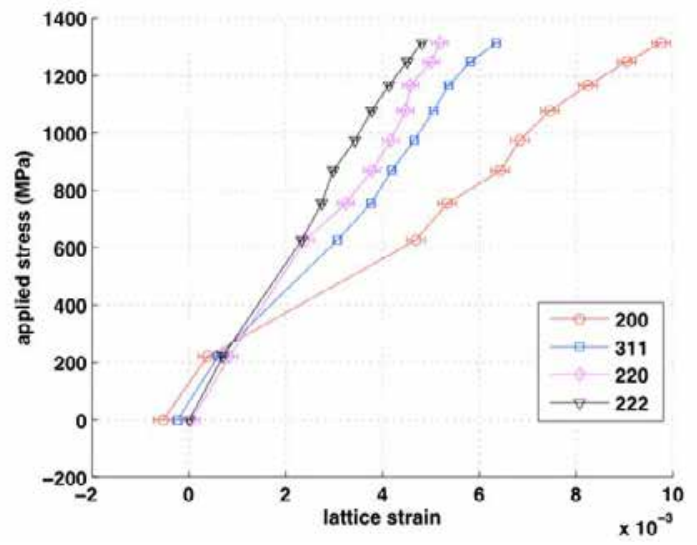

(a)

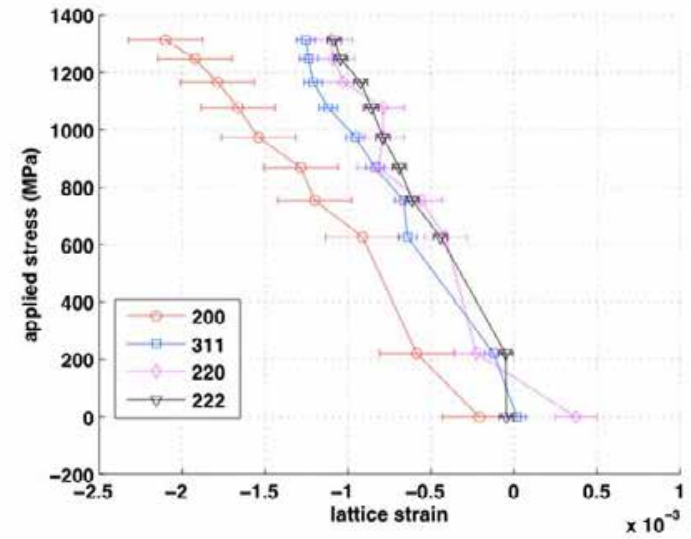

(b)

Fig. 4.55: Lattice strain generated during in-situ tensile tests in the long-transverse Alloy 230 specimens:

(a) axial direction, $\varepsilon_{11}$; (b) transversal direction, $\varepsilon_{22}$

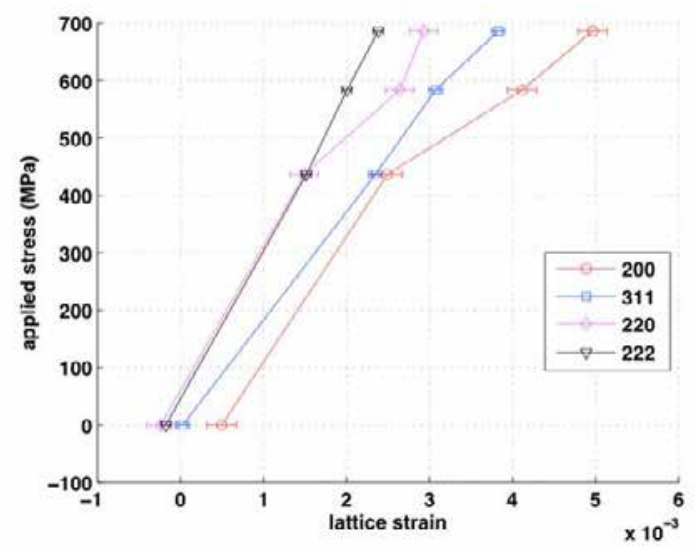

(a)

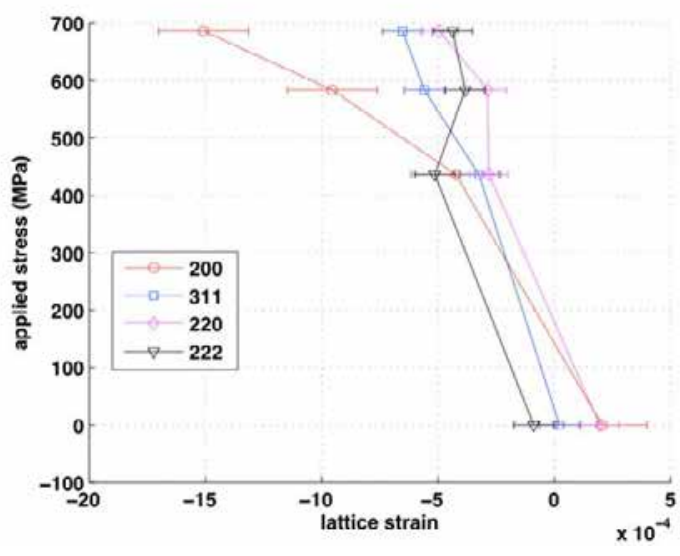

(b)

Fig. 4.56: Lattice strain generated during in-situ tensile tests in the short-transverse Alloy 230 specimens:

(a) axial direction, $\varepsilon_{11} ;$ (b) transversal direction, $\varepsilon_{22}$

Since the single crystal data for both alloys is not available presently, the elastic constants for single crystal nickel were primarily used in this study. $C_{11}, C_{12}$ and $C_{44}$ for single crystal nickel are 250,151, and $123 \mathrm{GPa}$, respectively, and the Zener anisotropy factor is 2.5 . [4.28] Fig. 4.57 and 4.58 show the results for $E(h k l)$ calculated by Kröner's model with a comparison to the Voigt and Reuss limits. The orientation dependence of Young's modulus for Ni is typical for FCC alloys with a high anisotropy factor, which is analogous to modeling results of $\mathrm{Cu}$ in Fig. 4.53(a) and 4.54(a). 


\section{Understanding Fundamental Material-Degradation Processes in High Temperature Aggressive Chemomechanical Environments}
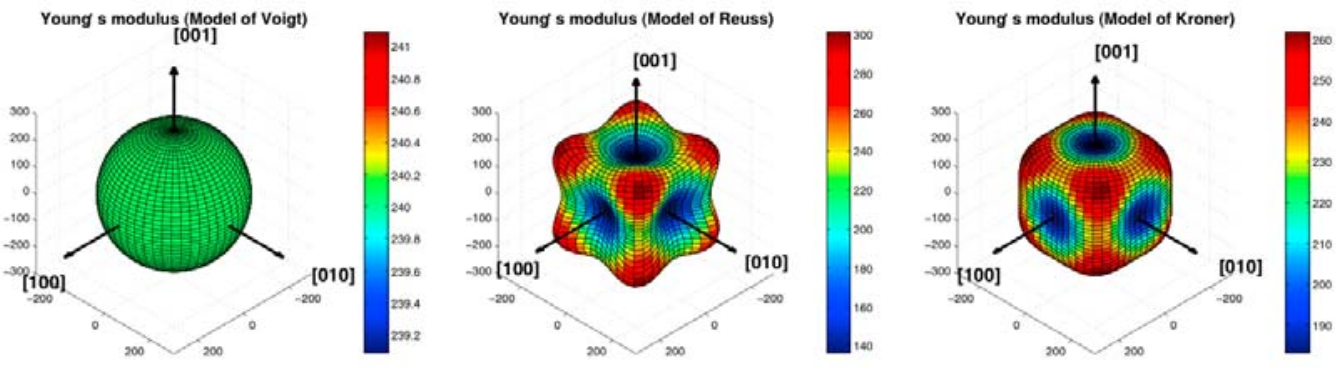

Fig. 4.57: Orientation dependence of Young's modulus (unit: GPA) for Ni polycrystal

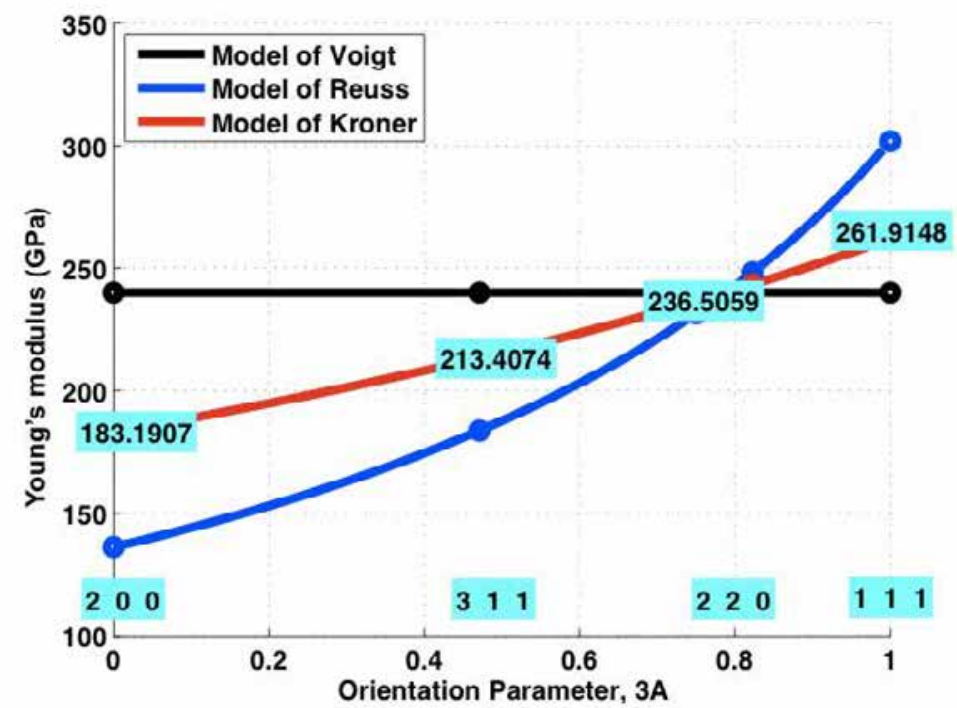

Fig. 4.58: Elastic anisotropy of Young's modulus for Ni

Based on the modeled $E(h k l)$, the measured lattice strains for different reflections can be converted into internal stresses according to equation 4.22. Therefore, the axial and transversal internal stresses (represented by $\sigma_{11}$ and $\sigma_{22}$, respectively), can be expressed as:

$$
\begin{aligned}
& \sigma_{11}=\frac{E}{1+v} \varepsilon_{11}+\frac{v E}{(1+v)(1-2 v)}\left(\varepsilon_{11}+\varepsilon_{22}+\varepsilon_{33}\right) \\
& \sigma_{22}=\sigma_{33}=\frac{E}{1+v} \varepsilon_{22}+\frac{v E}{(1+v)(1-2 v)}\left(\varepsilon_{11}+\varepsilon_{22}+\varepsilon_{33}\right)
\end{aligned}
$$

Since the tensile tests are uniaxial, $\varepsilon_{22}$ can be considered to be equal to $\varepsilon_{33}$ and $v=-\varepsilon_{22} / \varepsilon_{11}$. If we assume that the $h k l$-specific $v$ and $E$ are constant during deformations, equation 4.36 can be simplified to:

$$
\sigma_{11}=E \varepsilon_{11}
$$

In this equation, $E$ and $\varepsilon_{11}$ are obtained from modeling and XRD measurements, respectively.

Fig. 4.59 shows the results for internal stress development in the long-transverse specimen with a comparison to true stress. (Note that XRD measurements from all ten scanned positions are employed to obtain sufficient statistics to extract the average response from the bulk material.) 


\section{Understanding Fundamental Material-Degradation Processes in High Temperature Aggressive Chemomechanical Environments}

The internal stresses measured from (311), (220), and (222) reflections are in good agreement with the true stresses. The internal stresses measured from the (200) reflection are slightly higher than the true stresses, which could be caused by the slightly overestimated $E(111)$ determined by modeling. Generally, the XRD measured lattice strains with modeled Young's moduli provided satisfactory results for the internal stresses, whose values are analogues to the true stresses from the mechanical measurements.

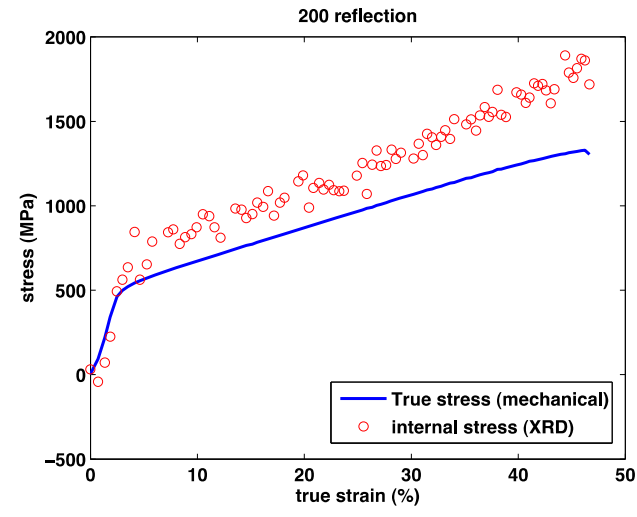

(a)

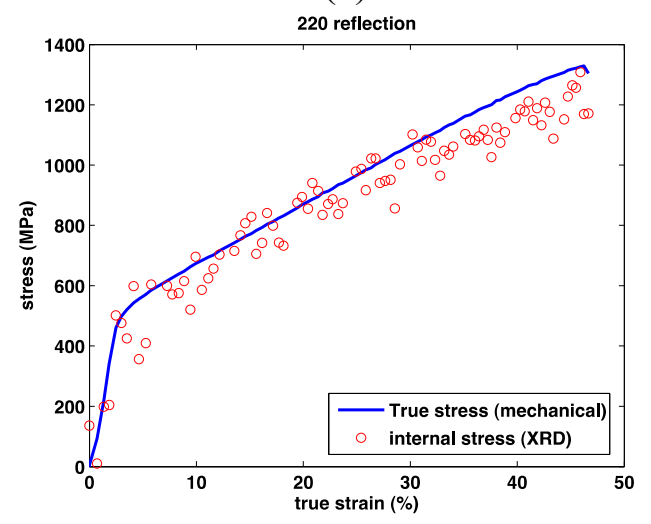

(c)

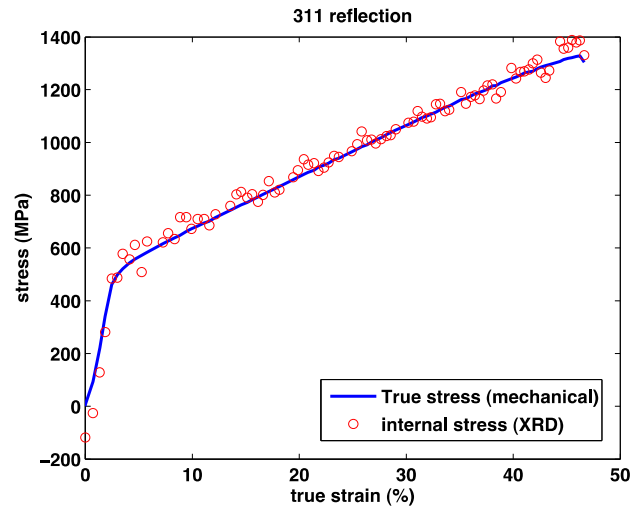

(b)

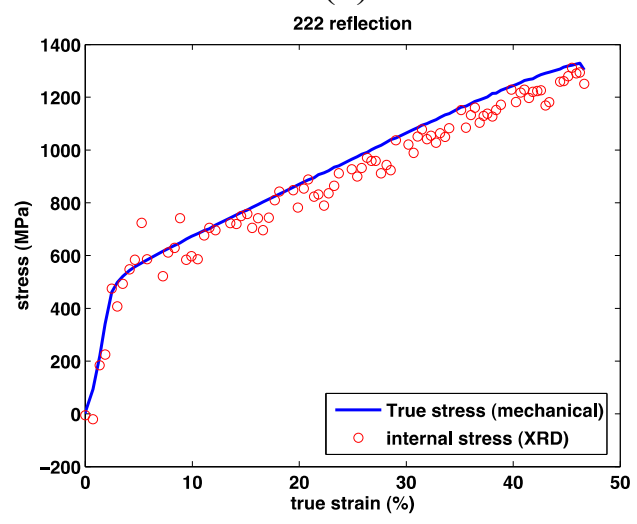

(d)

Fig. 4.59: The results of internal stress in contrast to the true-stress

One should note that the measured internal stresses obtained from the (311) reflection possess the least amount of data scattering and provide the best fit to the true stresses. (Fig. 4.59(b)) Previous studies confirm that (311) reflection is the suitable representation for characterization of macroscopic stresses and strains for FCC metals. [4.29] Therefore, the (311) reflection from the $\gamma$ matrix was selected for further discussion in the following section.

\subsubsection{Particle participation during loading}

Among all measurable reflections, the (422) reflection for the $\mathrm{M}_{6} \mathrm{C}$ carbide is selected for the strain-stress analysis, not only because of its relatively high intensity, but also due to its reciprocal space location around which there exists no intense reflection from the $\gamma$ matrix. In fact, unlike the high anisotropy of the $\gamma$ matrix, the elastic constants for the $\mathrm{M}_{6} \mathrm{C}$ carbide are nearly orientation $(h k l)$ independent. $\mathrm{Li}$ et al. modeled three typical types of $\mathrm{M}_{6} \mathrm{C}$ carbides: 


\section{Understanding Fundamental Material-Degradation Processes in High Temperature Aggressive Chemomechanical Environments}

$\mathrm{Fe}_{3} \mathrm{~W}_{3} \mathrm{C}, \mathrm{Co}_{3} \mathrm{~W}_{3} \mathrm{C}$, and $\mathrm{Ni}_{3} \mathrm{~W}_{3} \mathrm{C}$, and obtained their respective Zener anisotropy factors, which are 0.9725, 0.8740, and 0.9248. [4.30] (Note that $\mathrm{Ni}_{3} \mathrm{~W}_{3} \mathrm{C}$ is close to the $\mathrm{M}_{6} \mathrm{C}$ carbide used in this study.) Since the anisotropy factor for $\mathrm{M}_{6} \mathrm{C}$ carbides is close to 1 , the carbide values of Young's moduli for various $(h k l)$ reflections are essentially equal.

Fig. 4.60(a)\&(b) show the lattice strain development for both the $\gamma$ matrix and the $\mathrm{M}_{6} \mathrm{C}$ carbide, respectively. The lattice strain for the $\gamma$ matrix responds almost linearly to the external applied stress with a slightly decreased slope once the material yields. In contrast, the lattice strain evolution for the $\mathrm{M}_{6} \mathrm{C}$ carbide is not linear during deformations and can be essentially divided into three stages: (1) the linear lattice strain development during elastic deformation, (2) the accelerated straining during the alloys' early yielding, and (3) the gradually decreased lattice strain during plastic deformation. This process is typical for the carbides in the long-transverse specimen, but not apparent in the short-transverse specimen. This difference can be seen in the individual analyses of strain development for the long-transverse and short-transverse specimens in Figs. 4.61(a) and 4.61(b), respectively. The lattice strain of the carbide in the short-transverse specimen increases moderately without a dramatic loading behavior during early yielding. Nevertheless, it should be noted that there is a maximum lattice strain for the carbide for both specimens, which occurs immediately after the alloy yields. The value of this critical lattice strain was found to be $\sim 0.0047$, corresponding to an internal stress of $\sim 1344 \mathrm{MPa}$ (the Young's modulus of $\mathrm{M}_{6} \mathrm{C}$ carbide is $\sim 286 \mathrm{GPa}$ [4.31]). The short-transverse specimen fails immediately once the carbide achieves this critical internal stress, while the long-transverse specimen conducts further plastic deformation with decreasing carbide lattice strain until failure occurs. (Fig. 4.60(a))

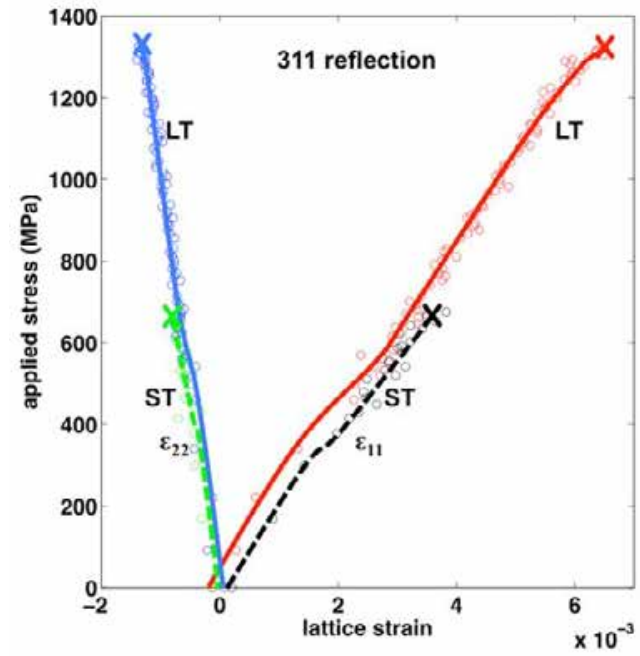

(a)

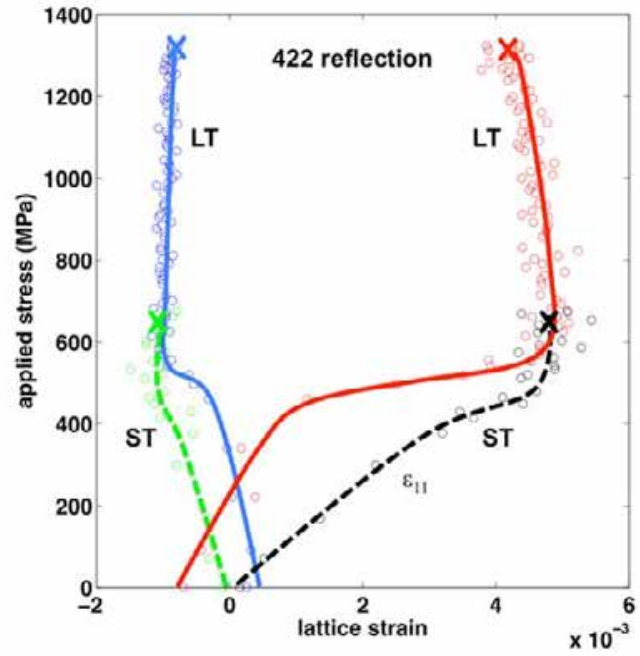

(b)

Fig. 4.60: Applied stress versus lattice strain ( $\varepsilon_{22}$ : lattice strain perpendicular to applied stress and $\varepsilon_{11}$ : lattice strain parallel to applied stress) for (a) the $\gamma$ matrix ((311) reflection), and (b) the $\mathrm{M}_{6} \mathrm{C}((422)$ reflection). Note: The solid and dashed lines are curves fitted though the data points to show the trend. 


\section{Understanding Fundamental Material-Degradation Processes in High Temperature Aggressive Chemomechanical Environments}

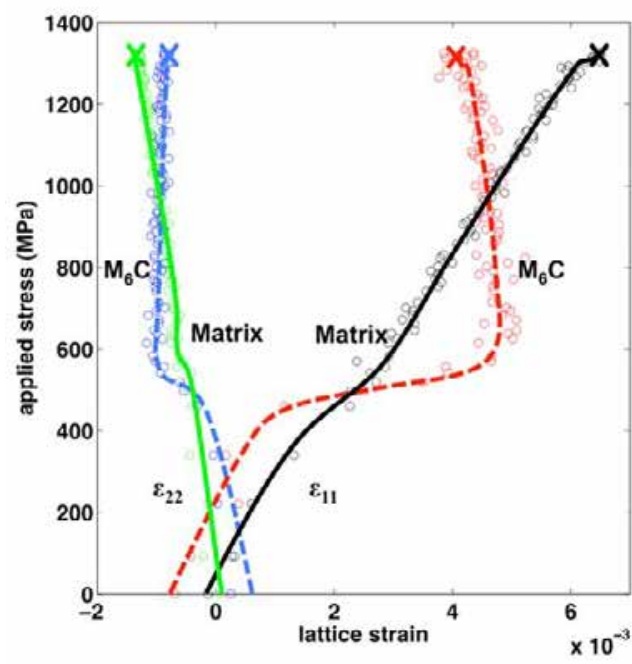

(a)

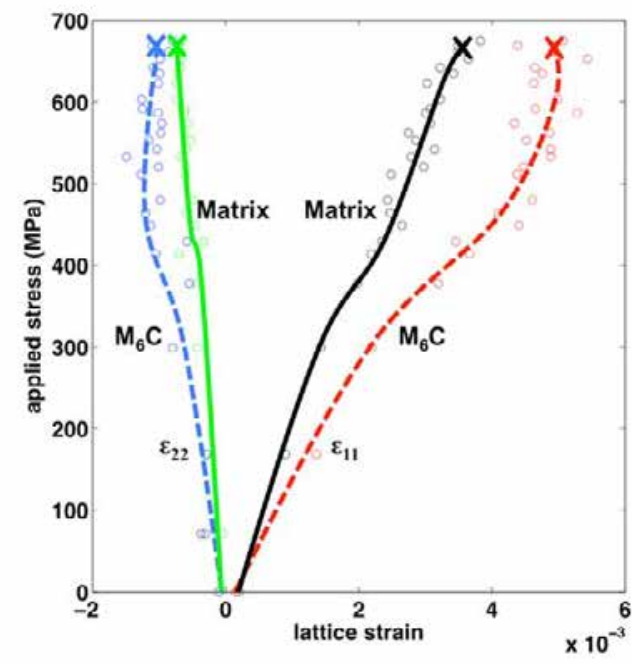

(b)

Fig. 4.61: Applied stress versus lattice strain ( $\varepsilon_{22}$ : lattice strain perpendicular to applied stress and $\varepsilon_{11}$ :

lattice strain parallel to the applied stress) for the $\gamma$ matrix ((311) reflection) and the $\mathrm{M}_{6} \mathrm{C}((422)$ reflection) in (a) long-transverse, and (b) short-transverse directions. Note: The solid and dashed lines are curves fitted though the data points to show the trend.

\subsection{In-situ Creep Study Using Pressurized Creep Tubes}

\subsubsection{Methods}

A typical diffraction pattern from a pressurized creep tube is shown in Fig. 4.62. The enlarged image, sampled from a part of the diffraction pattern (in red square of Fig. 4.62), shows that each reflection forms two Debye-Scherrer diffraction rings that are generated from the two sides of the tube. Fig. 4.63 shows the procedures to extract the diameter of the pressurized tubes from diffraction patterns. The basic steps for the creep strain analysis are: 1) measure the distance, $a$, between two diffraction rings for a single reflection (e.g. (311) reflection); 2) calculate the diameter of the tube, $D$, based on a tangent angle relation with $a$; and 3) evaluate the creep strain by comparing the value of the diameter before and after various stages of creep deformation. (Fig. 4.63(a)) However, due to elastic anisotropy, the distance between two diffraction rings is not a constant value at various azimuths. Therefore, a polar re-binning process turned pixel intensity data into radius/angle $(\mathrm{R} / \mu)$ with respect to the pattern center was performed. This was followed by a peak fitting in $\mathrm{R}$ to determine the center of each diffraction peak in pixels. (Fig. 4.63(b)) Then, the distance of two diffraction rings, which is also the mean distance between two diffraction peaks for azimuth from 0 to $360^{\circ}$, can be calculated by:

$\bar{a}=0.2 \mathrm{~mm} \times \frac{1}{n} \sum_{n=1}^{n}(P 1(\eta)-P 2(\eta))$

In this equation, $n$ is the number of bins used during the polar re-binning process $(n=36$ in this study) and $P 1$ and $P 2$ are radii in pixels of fitted peak 1 and peak 2, respectively. The distance 
between two diffraction rings has a factor of $0.2 \mathrm{~mm}$, which is the dimension of one pixel in the GE detector. Then, we can obtain the diameter of the tube, $D$, by the expression:

$D=a / \tan (2 \theta)$

Relative changes in the tube diameter during the experiment can be calculated with respect to the initial state denoted by subscript 0 and can be converted to the mid-wall hoop strain by use of a factor of 1.063:

$\varepsilon=1.063 \frac{D^{*}-D_{0}}{D_{0}}$

In the above formula, $D_{0}$ is the diameter obtained from equation 4.40 for the initial state, and $D^{*}$ is the diameter during creep. The assumption imperative for equation 4.41 is that the material is incompressible and deforms uniformly during creep [4.32]. More details for converting tube diameter into strain can be found in ref. 4.33. Finally, since $\tan (2 \theta)$ is nearly constant during the measurement, $D_{0}$ and $D^{*}$ in equation 4.41 can be replaced with their values from equation 4.40 , to yield the much simpler equation:

$$
\varepsilon=1.063 \frac{\bar{a}^{*}-\bar{a}_{0}}{\bar{a}_{0}}
$$

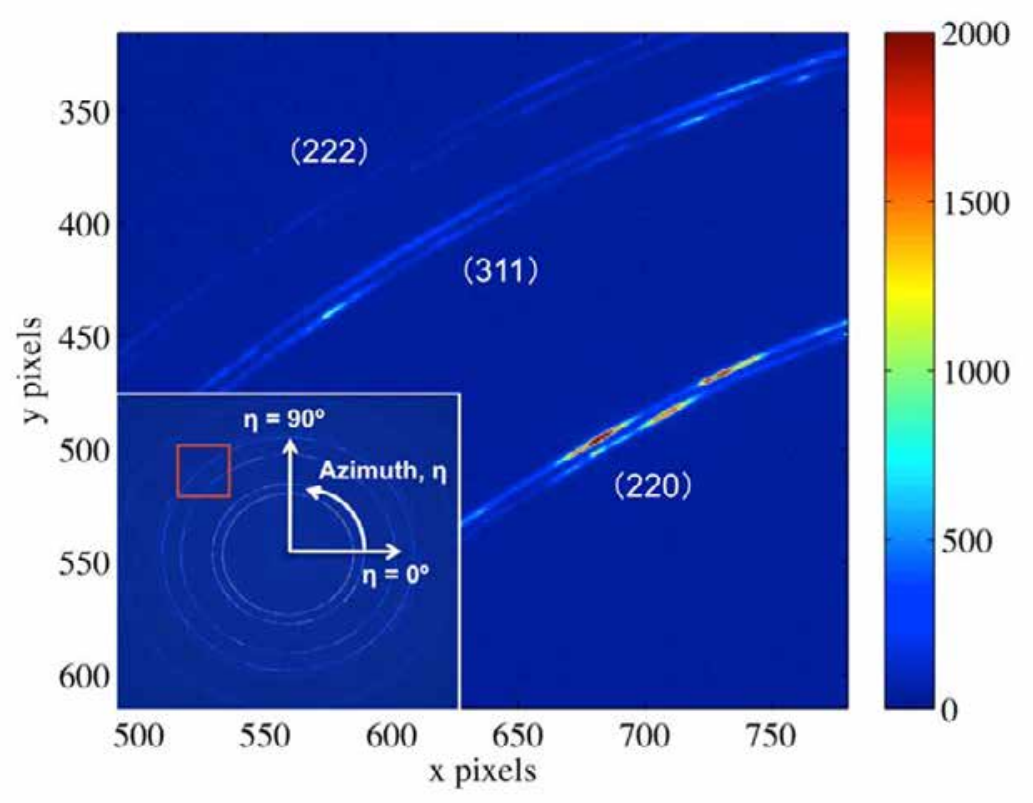

Fig. 4.62: Representative diffraction patterns for a pressurized creep tube 


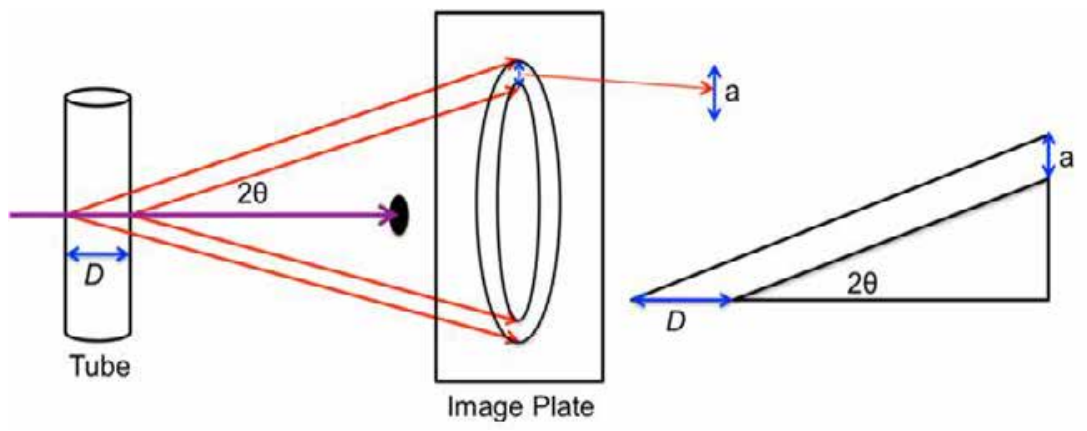

(a)

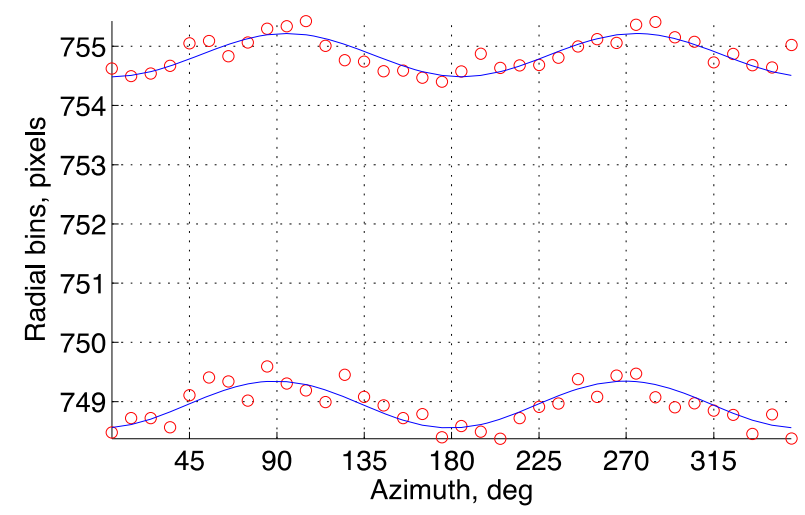

(b)

Fig. 4.63: Diameter measurements for the pressurized creep tube: (a) Schematic representations of the measurement; (b) radius peak position versus azimuth for dual (311) diffraction rings (in red circles) and the fitting on the radius profile for lattice strain analysis (in blue lines)

\subsubsection{Results}

Based on equation 4.42, the macroscopic creep strain can be found directly by using the distance between two diffraction rings obtained in equation 4.39. Fig. 4.64 shows the results from analyzing the (311) reflection. Using this approach, a typical creep curve was obtained from the analysis of the time dependence of the separation of two (311) reflections. The secondary creep behavior, characterized by a constant strain rate, can be seen from the starting point to $\sim 200$ minutes. Primary creep cannot be clearly identified due to rapid deformations resulting from the high temperatures and large applied stresses in the experiment. Following secondary creep, tertiary creep was observed with an increased strain rate. Failure occurred at $\sim 5$ hours.

In addition to creep strain measurements, X-ray data allows for an assessment of the microstructure development. The shape of a diffraction peak changes during material deformation, a phenomenon which is called "X-ray line broadening" [4.25]. The diffraction peak broadening, demonstrated by the full width at half maximum (FWHM), is determined by the distribution of randomly oriented lattice plane distances (or lattice parameters) if the instrumentation factors are constant [4.26]. For plastic deformation, the typical origins for changing the FWHM are crystalline size and micro distortion/strain. Fig. 4.64(a) shows the time-dependence of the macroscopic creep strain and the relative width of the (311) reflection. During the early creep stage, the FWHM of the (311) reflection decreases in contrast to the fact 


\section{Understanding Fundamental Material-Degradation Processes in High Temperature Aggressive Chemomechanical Environments}

that the macroscopic creep strain increases. As the creep progresses, the FWHM reaches a static level during the secondary creep stage. After $\sim 2$ hours at a static level, the FWHM increases until the tube fracture occurs.

Another method used to explore the microstructure development is to measure lattice strain development deduced from the shifts of diffraction peaks. The procedures used to analyze microscopic lattice strain are similar to the ones used in the in-situ tensile studies described in section 3.2. Due to a similar stress state for both sides of the creep tube, both Debye rings reflected from two sides were used for analysis in order to employ a longer X-ray path length $(\sim 0.5 \mathrm{~mm})$, and thus obtain more diffraction volume $\left(\sim 0.046 \mathrm{~mm}^{2}\right)$, over which the average lattice strains were measured. The results from the lattice strain (radial direction) analysis are shown in Fig. 4.64(b) in comparison to the measured macroscopic creep strain. Compared to the creep strain development, the deviation of lattice strain is small, on the range of $10^{-4}$. The average measured lattice strain, $\varepsilon$, is $6.5 \times 10^{-4}$, corresponding to an internal radial stress of $\sim 126 \mathrm{MPa}\left(E^{311}\right.$ is $\sim 193 \mathrm{GPa}$ ). The measured radial stress is slightly lower than the expected hoop stress of 150 $\mathrm{MPa}$ following the thin-wall assumption. It should be noted that an increasing lattice strain occurred at the moment of $\sim 180$ minutes after initializing the creep test. The average strain is $\sim 5.4 \times 10^{-4}$ (corresponding to $105 \mathrm{MPa}$ internal stress) before this transient moment, and it increases to a mean strain of $7.2 \times 10^{-4}$ (corresponding to $139 \mathrm{MPa}$ internal stress) by the end of the test. 


\section{Understanding Fundamental Material-Degradation Processes in High Temperature}

Aggressive Chemomechanical Environments

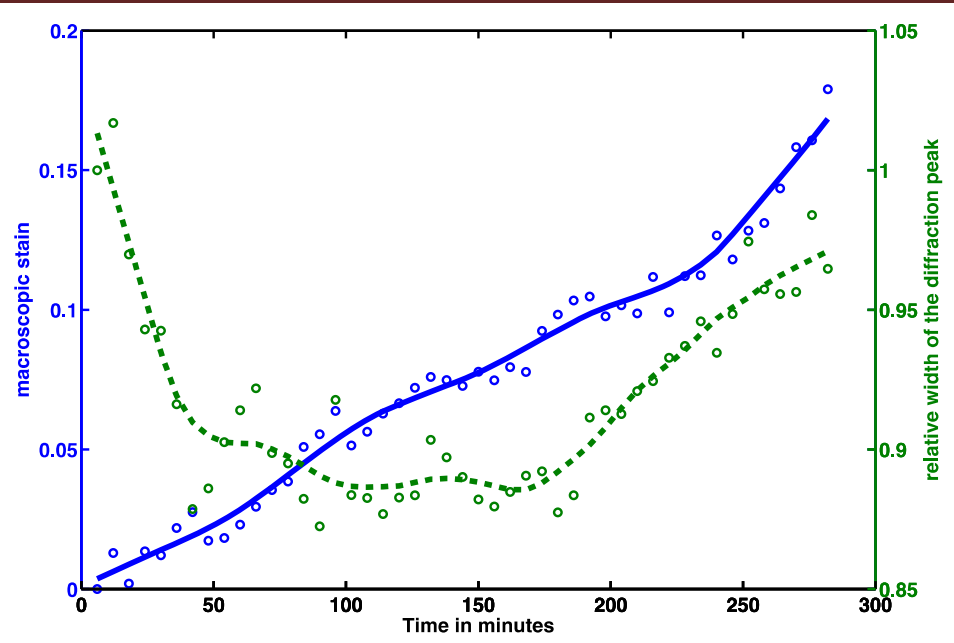

(a)

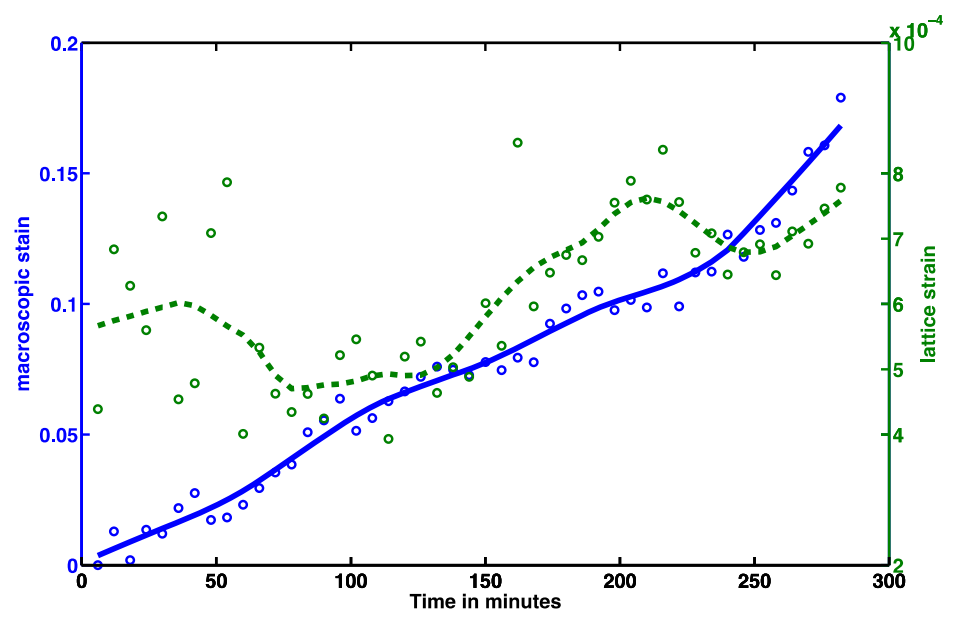

(b)

Fig. 4.64: Results of creep analysis: (a) time-dependence of the macroscopic creep strain (in blue) and the relative width of the (311) reflection (in green); (b) time-dependence of the macroscopic creep strain (in blue) and the lattice strain (in green). The solid and dashed lines are curves fitted though the data points to show the trend. 


\subsection{Low Cycle Fatigue Results}

\subsubsection{LCF life}

The LCF life of both alloys is compared in this section. Based on ASTM Creep-Fatigue Test Standard E2714-09 [4.34], a few options are available for defining the failure criterion. Two failure criteria are adopted here for comparison purposes, namely a $40 \%$ decrease in the cyclic maximum tensile stress and a $20 \%$ reduction in the ratio of peak tensile to peak compressive stress (referred to as stress ratio hereafter) after the stress ratio apparently deviates from its initial trend. The second failure criterion based on the stress ratio allows changes in peak stresses because of cyclic work hardening or softening to be differentiated from changes due to crack formation and propagation [4.35, 4.36]. In Fig. 4.65, LCF test results of Alloy 617 and Alloy 230 based on two different criteria at different total strain levels are compared. The LCF life from both criteria is quite similar to each other, which indicates both criteria can be used as the failure criterion. Under all test conditions, Alloy 230 performed slightly better than Alloy 617.

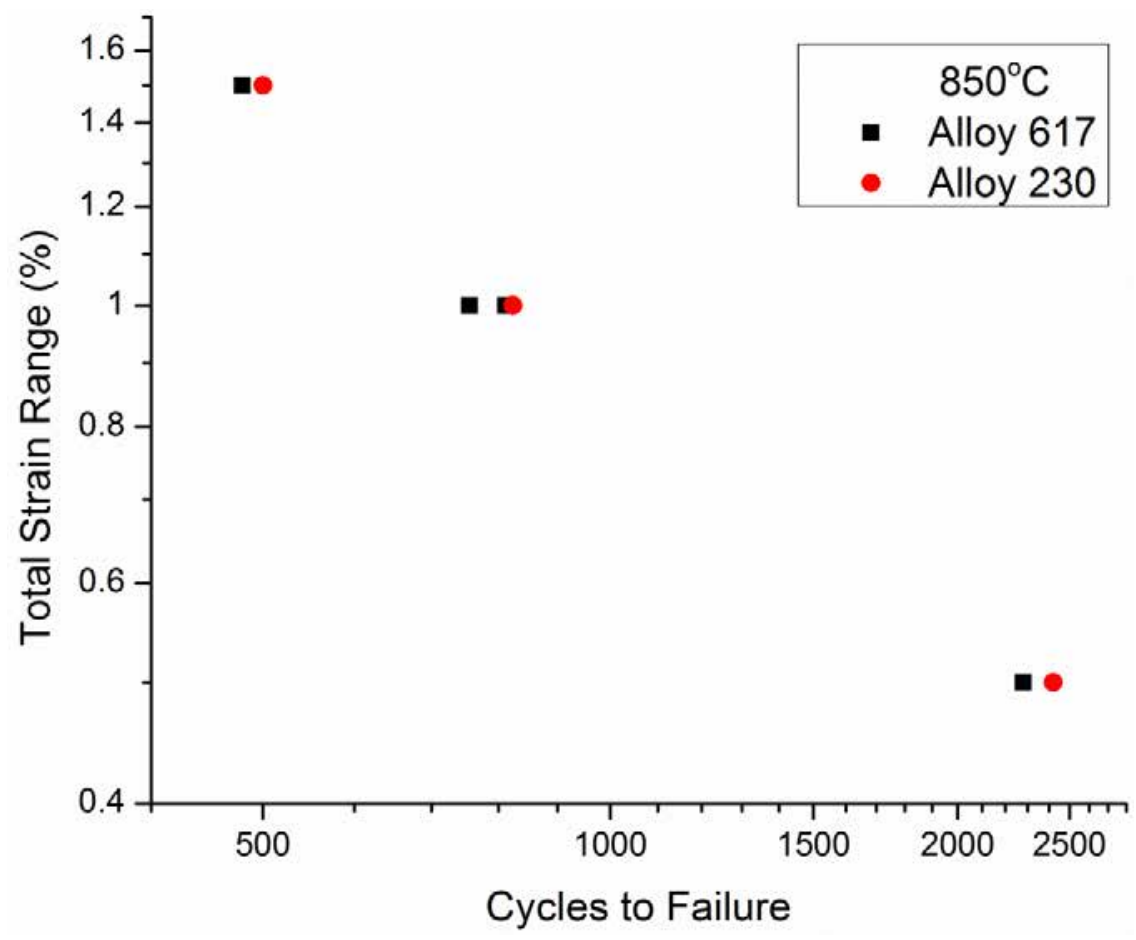

(a) 


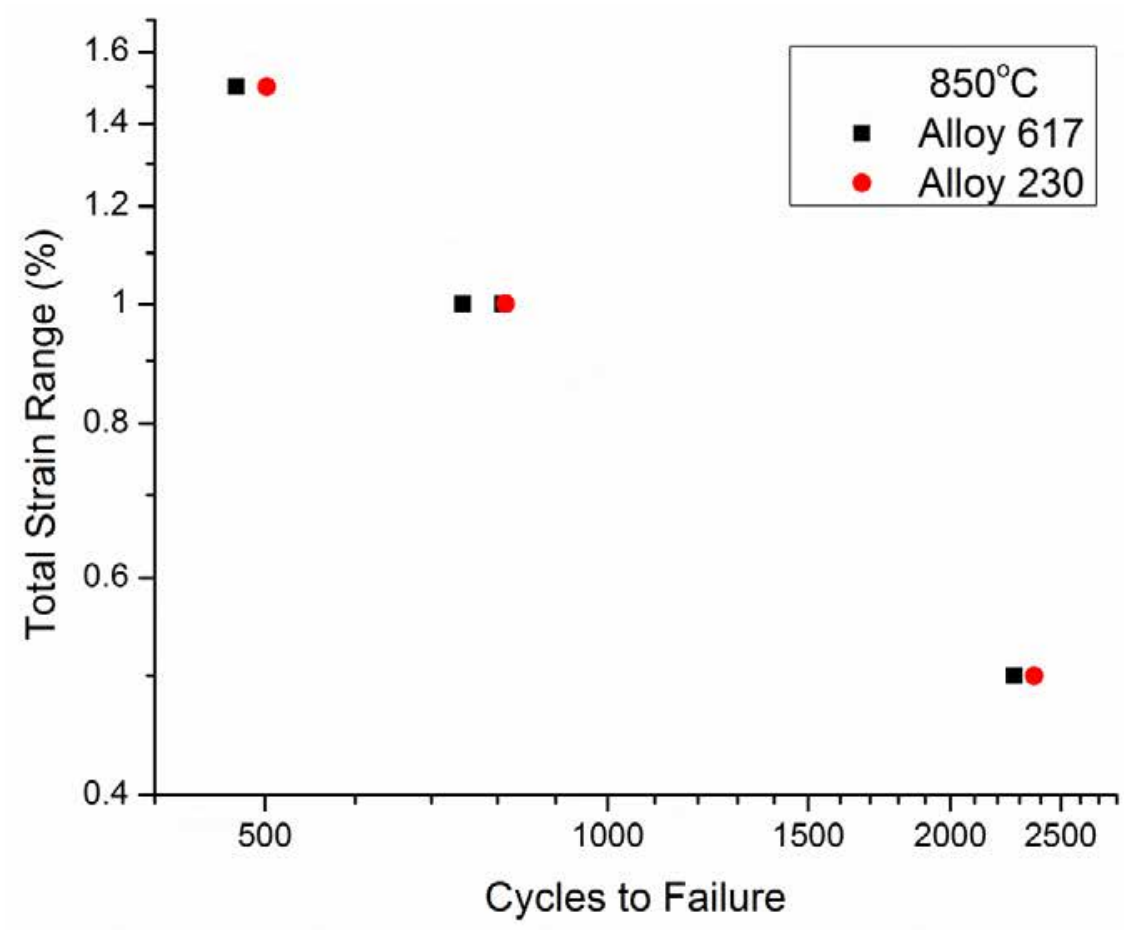

(b)

Fig. 4.65: LCF life of Alloy 617 and Alloy 230 tested at $850^{\circ} \mathrm{C}$. The failure criterion was based on a $40 \%$ maximum tensile stress decrease in (a), and on a $20 \%$ stress ratio drop in (b).

\subsubsection{Materials cyclic fatigue behavior}

Fig. 4.66 shows the cyclic maximum stress and stress ratio of Alloy 617 and Alloy 230 at different total strain levels. Both materials displayed similar cyclic behavior, namely cyclic hardening during the initial few cycles followed by steady state cyclic softening and final accelerated crack growth manifested via fast load drop. Since the as-received material was in the solution-treatment condition, the dislocation density should initially be low. As a result of repeating cyclic deformation, dislocation slip and multiplication occurred rapidly, causing significant strain hardening $[4.5,4.37,4.38]$. At some point, the newly-formed dislocation structure stabilized for that material and for the magnitude of the cyclic strain imposed during the test. The ensuing cyclic softening phenomenon may be attributed to the dislocation recovery process occurring during strain cycling [4.39]. The dislocation recovery is a thermal-driven process during which dislocations can annihilate or rearrange themselves, causing polygonization. The elevated test temperature created a favorable condition for the recovery of dislocations.

Under the same test conditions, the cyclic maximum stress and cyclic stress ratio curves presented different behaviors. Namely, changes in the maximum tensile stress due to initial cyclic work hardening and following steady state cyclic softening were not seen in the stress ratio. The stress ratio remained relatively constant for most of the LCF test. Once macrocrack initiation started, manifested by a negative deviation from the linear plot of maximum stress versus cycles [4.40], the cyclic stress ratio curve also significantly dropped. The difference between the cyclic maximum stress and the stress ratio can be explained by the following 


\section{Understanding Fundamental Material-Degradation Processes in High Temperature Aggressive Chemomechanical Environments}

argument: cyclic work hardening or softening would produce an approximately equal effect on peak tensile and compressive stress, while crack initiation and growth would selectively reduce the net section peak tensile stress [4.41]. Therefore, cyclic work hardening or softening shouldn't cause as significant of changes in stress ratio as cracking would. It is worth noting that the cycles to macrocrack initiation accounted for the major part of the LCF life for both materials, meaning that both materials had good resistance to crack initiation and the fraction of the LCF life consumed in the formation of macrocrack decreased with increasing total strain range. This is because cyclic stress correlates with total strain range in LCF tests and smaller cyclic stress associated with a lower total strain range shifted the LCF test towards the direction of a high cycle fatigue (HCF) test, in which most of the material fatigue life is usually spent in crack initiation rather than crack propagation [4.42].

In the related study of Smith and Yates, LCF tests were conducted on Alloy 617, and provided the same observations [4.38]. Despite the similarity between these two materials, Fig. 4.66 also reveals that higher flow stress was needed to deform Alloy 230 than Alloy 617 to the same strain level. The higher strength of Alloy 230 may contribute to its slightly superior fatigue properties over Alloy 617 [4.43]. The major differences among tests at different total strain ranges are that for the small total strain range test, e.g. $0.5 \%$. The load drop during the steady state cyclic softening stage was less than its counterpart at the higher total strain range tests, i.e. $1.0 \%$ and $1.5 \%$, and the maximum stress scattering at the steady state cyclic softening stage was more obvious for the small total strain range test. The mid-life hysteresis loops, illustrated in Fig. 4.67, demonstrate the smaller plastic strain range for Alloy 230 than Alloy 617 at various strain levels. In addition, the mid-life plastic strain amplitude, $\Delta \varepsilon_{p} / 2$, versus the number of reversals to failure, $2 N_{f}$, is plotted in Fig. 4.68. The data points reveal a good match with the straight linear fitting line in the double logarithmic plot. The linear relationship between $\log \left(\Delta \varepsilon_{p} / 2\right)$ and $\log \left(2 N_{f}\right)$ conforms to the theory first noted by Coffin $[4.44,4.45,4.46]$ :

$\Delta \varepsilon_{p} / 2=\varepsilon_{f}^{\prime}\left(2 N_{f}\right)^{-c}$

where $\varepsilon_{f}^{\prime}$ is the fatigue ductility coefficient, the failure strain for a single reversal, and $c$ - the fatigue ductility exponent - equals the negative of the slope of the straight line in Fig. 4.68. 
Understanding Fundamental Material-Degradation Processes in High Temperature Aggressive Chemomechanical Environments
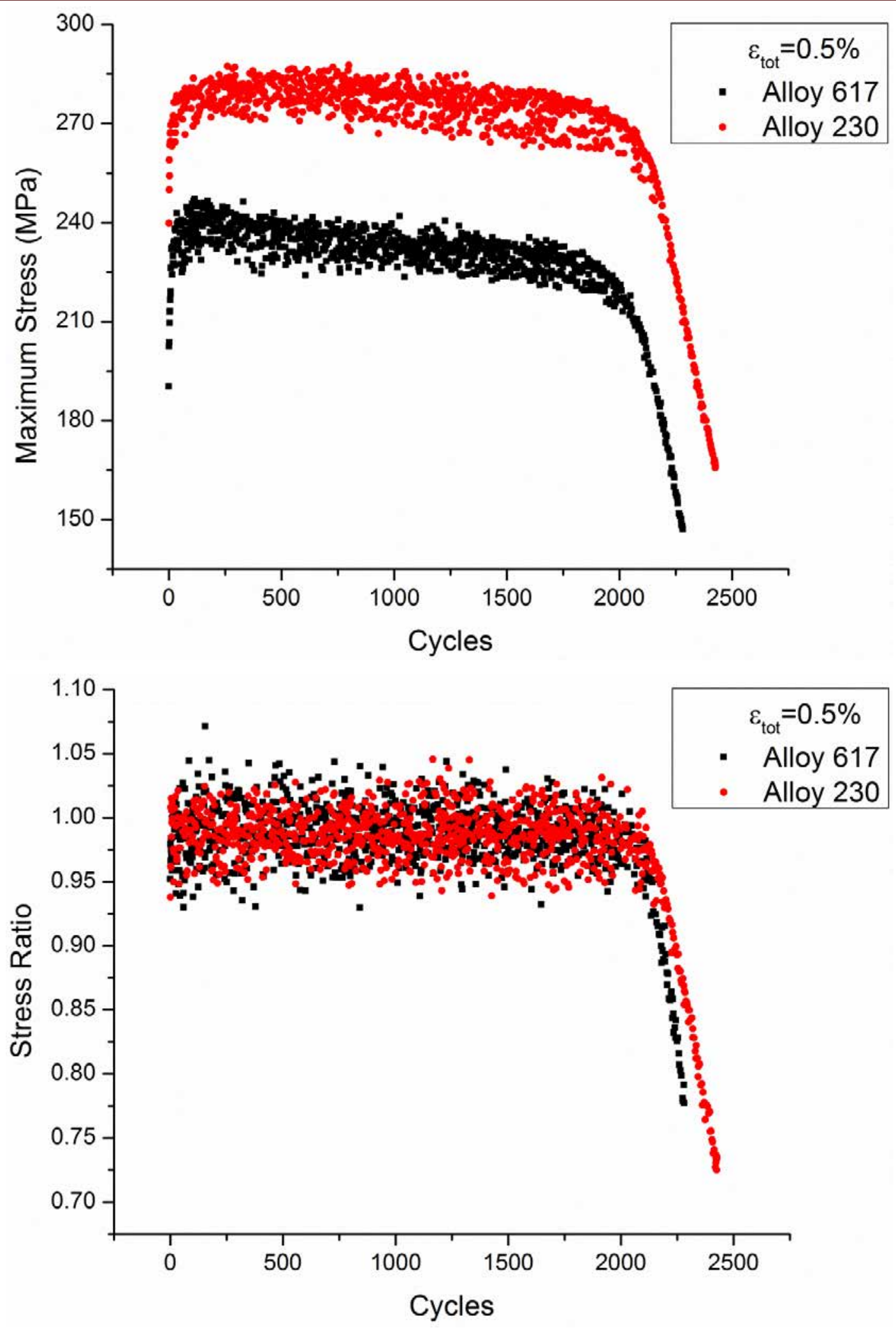

Fig. 4.66: LCF cyclic maximum stress and stress ratio of Alloy 617 and Alloy 230 
Understanding Fundamental Material-Degradation Processes in High Temperature Aggressive Chemomechanical Environments
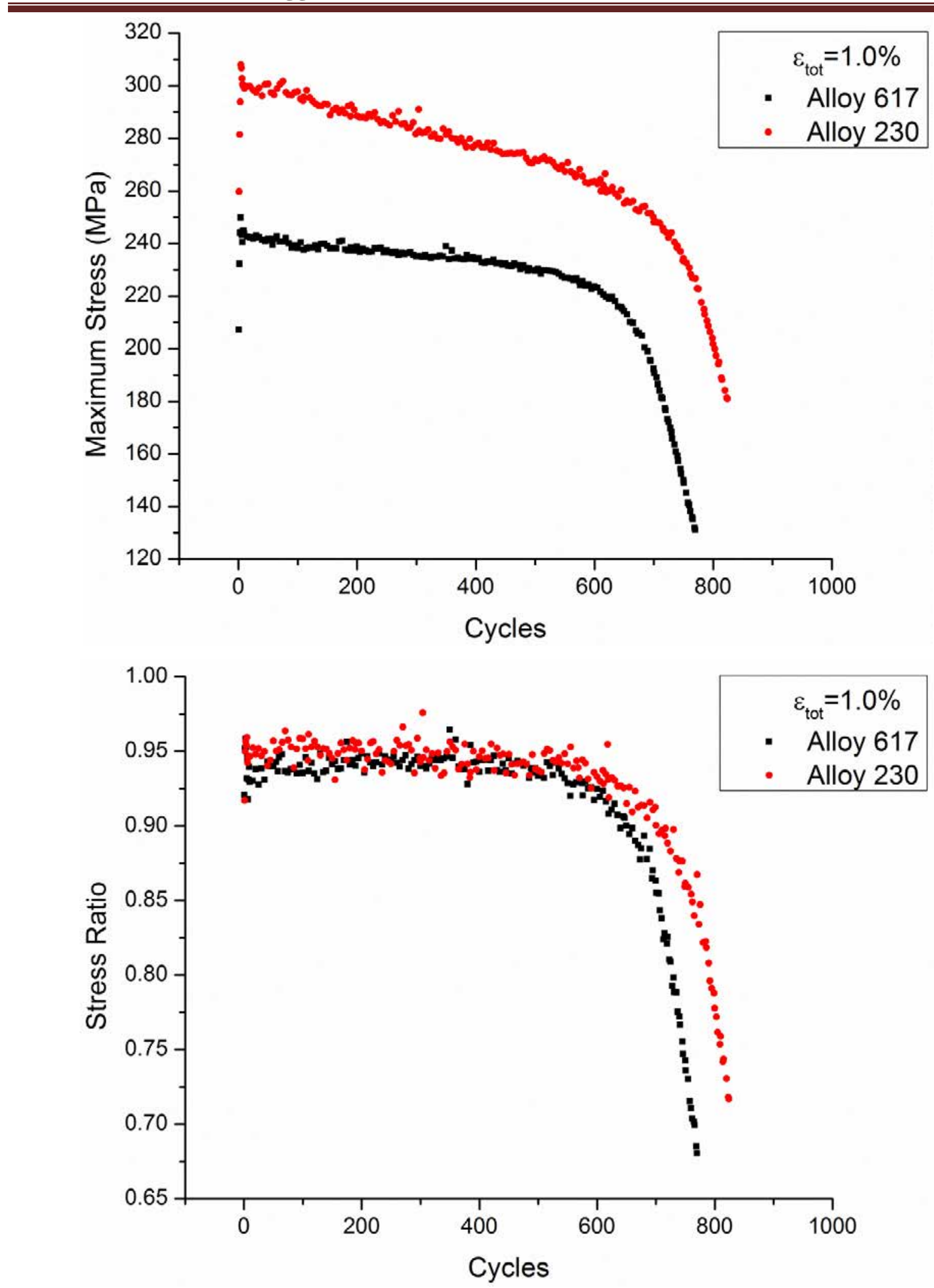

Fig. 4.66 Cont. 
Understanding Fundamental Material-Degradation Processes in High Temperature Aggressive Chemomechanical Environments
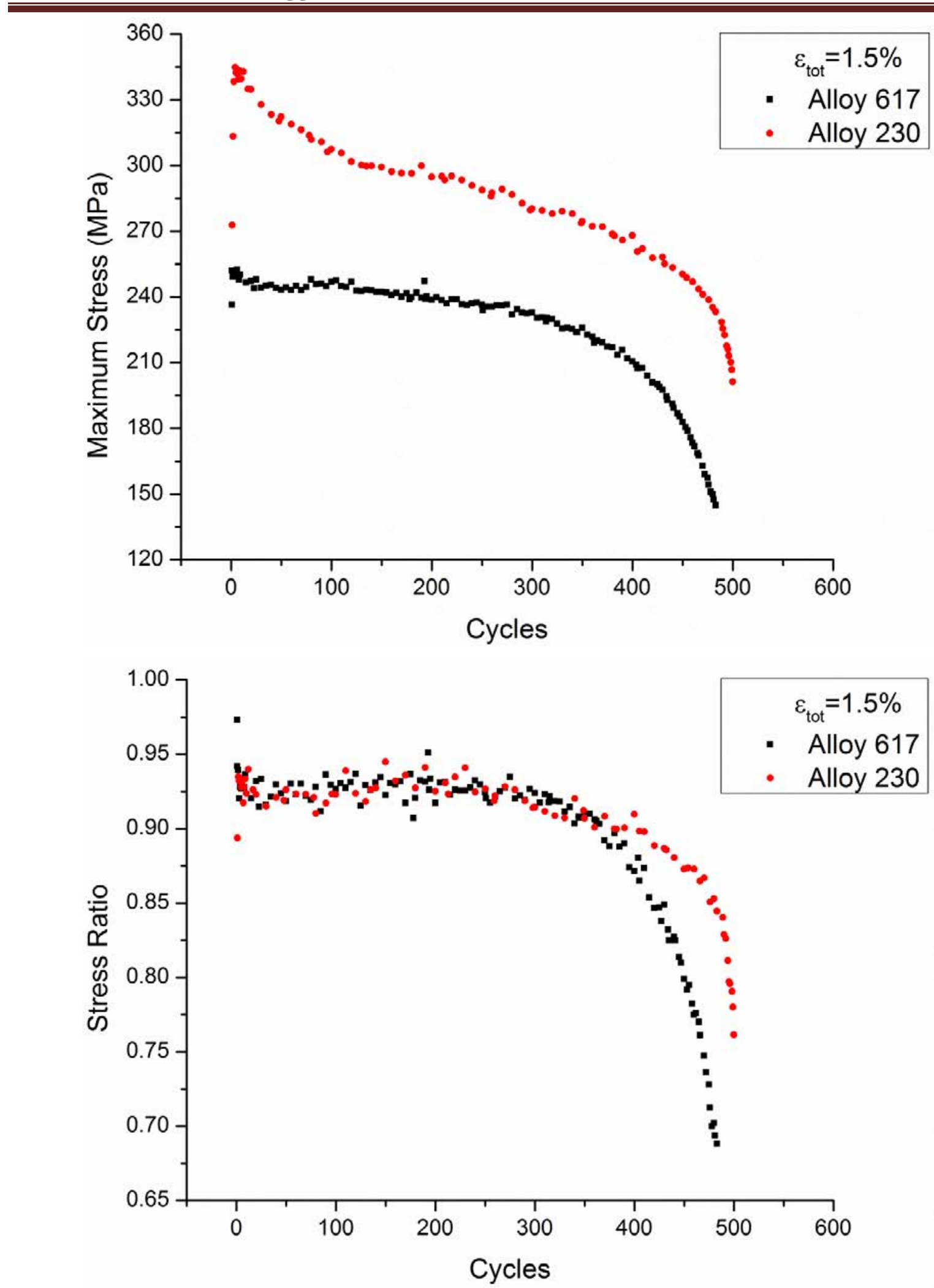

Fig. 4.66 Cont. 
Understanding Fundamental Material-Degradation Processes in High Temperature Aggressive Chemomechanical Environments
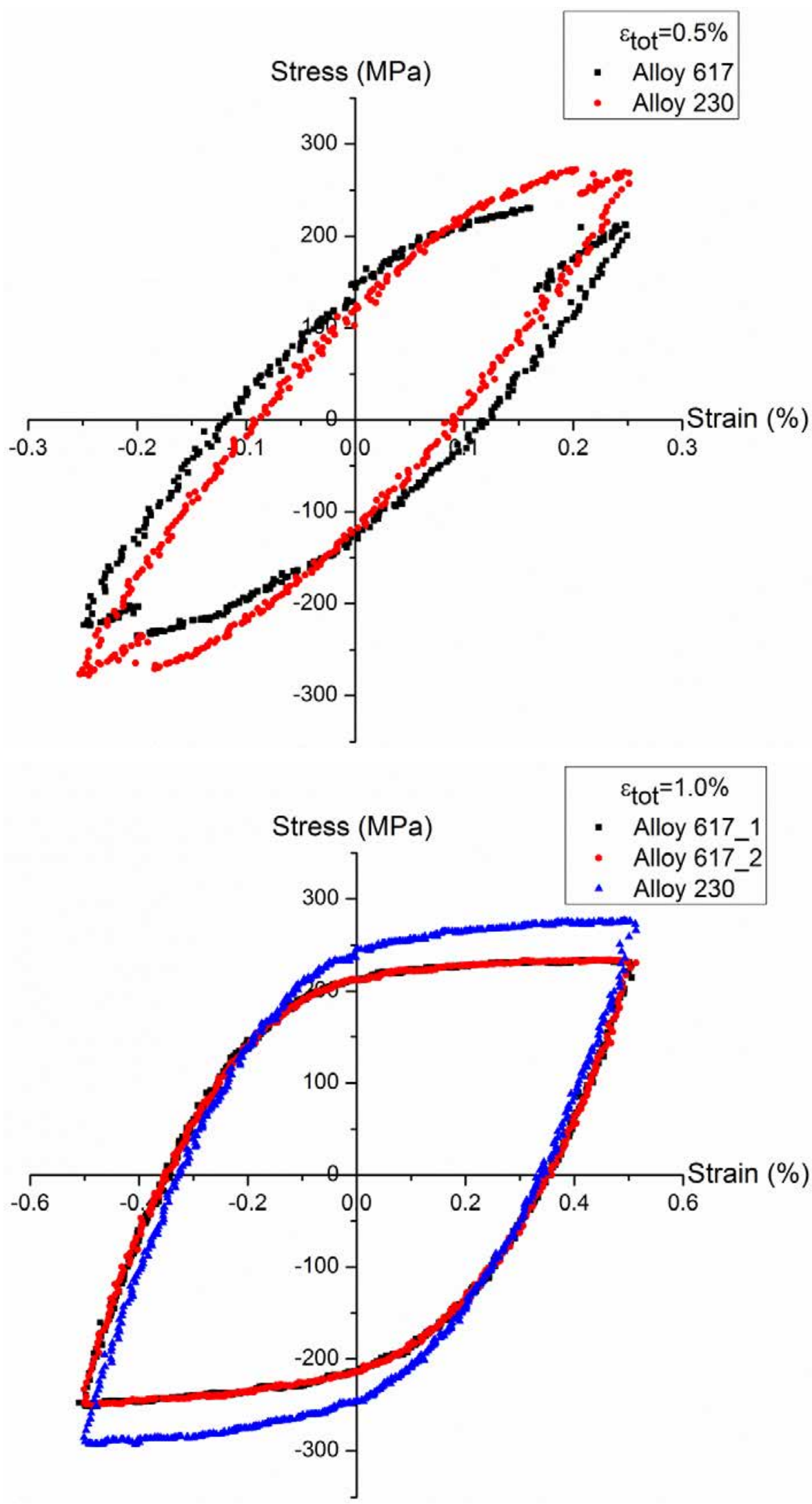

Fig. 4.67: LCF mid-life hysteresis loops of Alloy 617 and Alloy 230 
Understanding Fundamental Material-Degradation Processes in High Temperature Aggressive Chemomechanical Environments

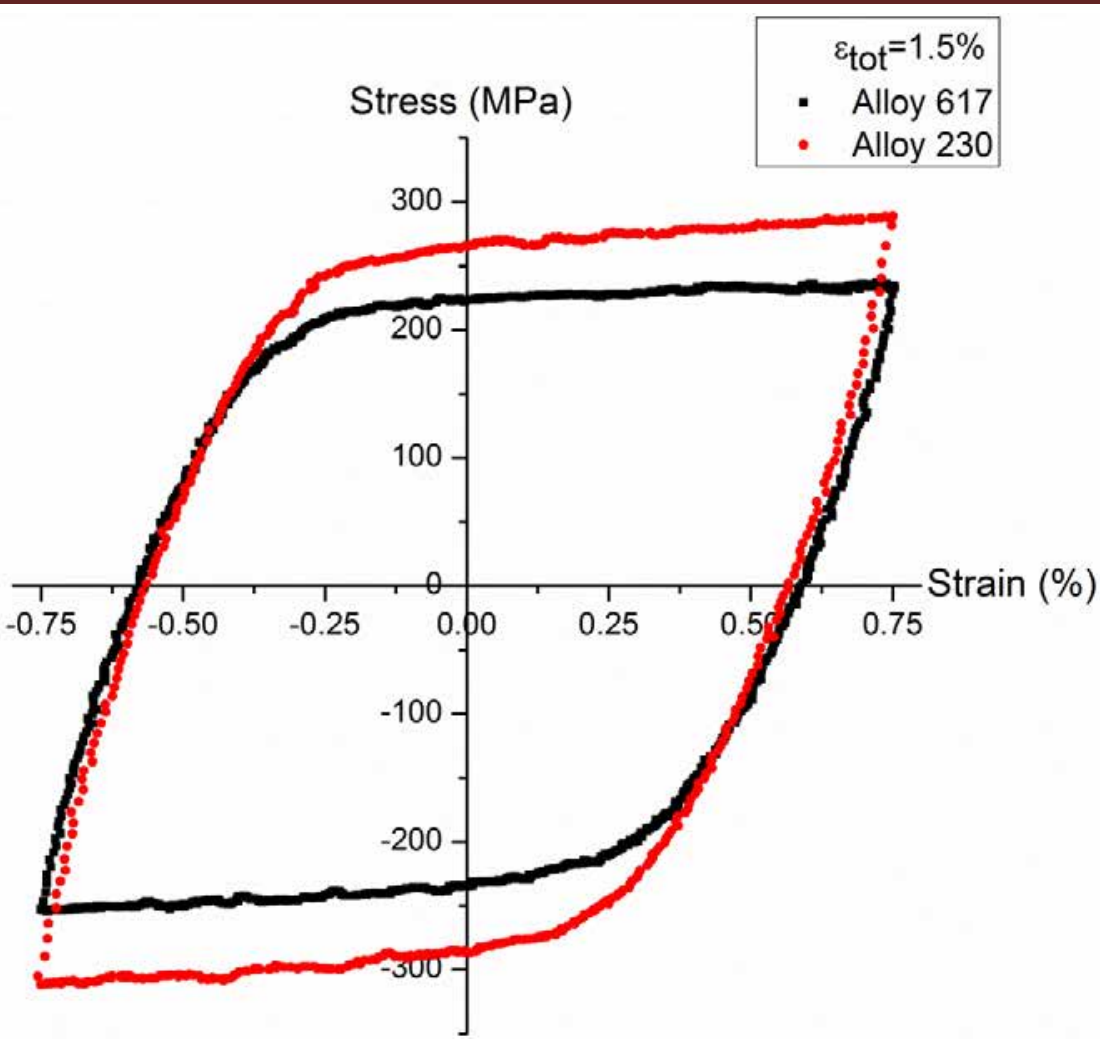

Fig. 4.67 Cont.

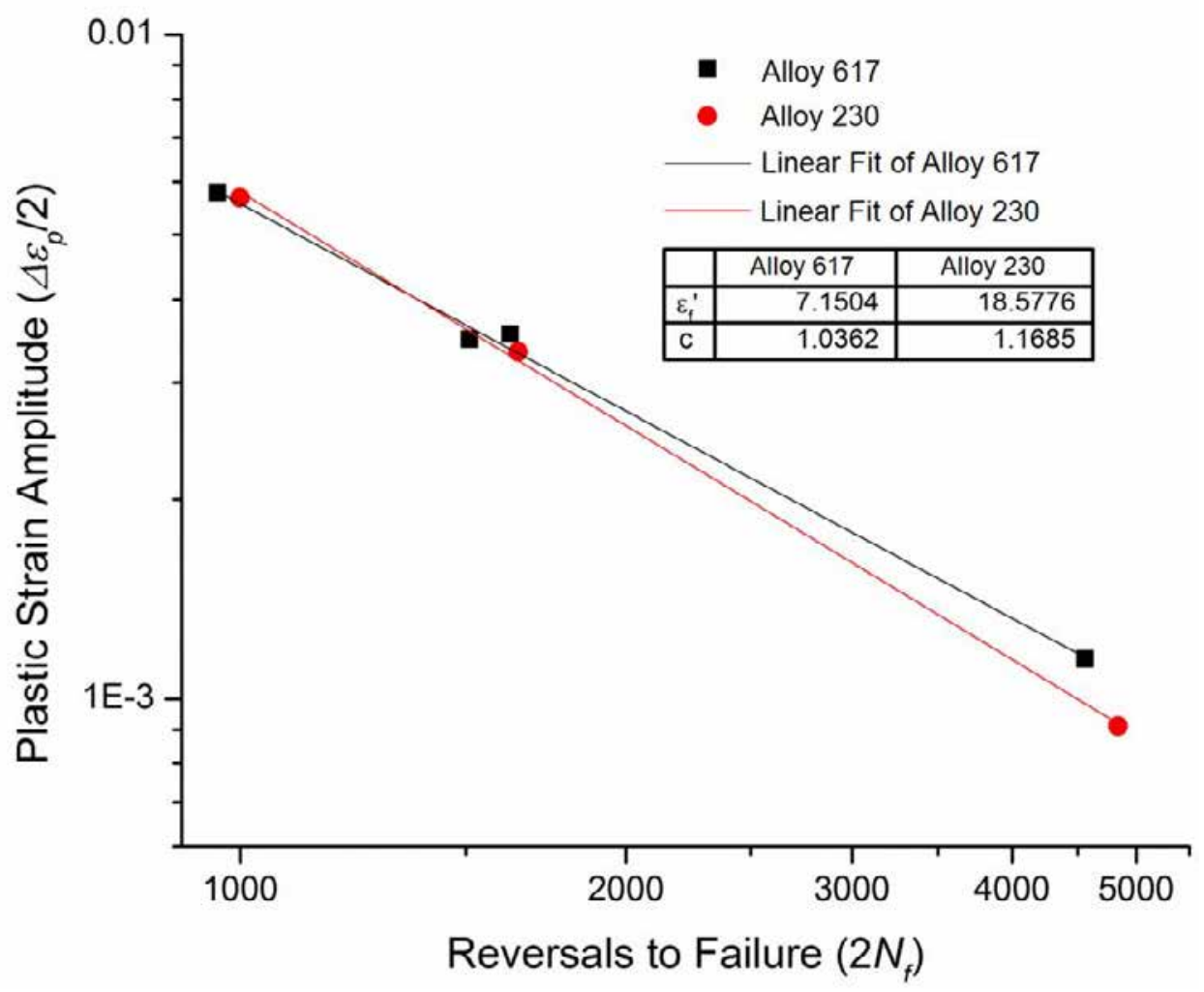

Fig. 4.68: Mid-life plastic strain amplitude versus reversals to failure 


\section{Understanding Fundamental Material-Degradation Processes in High Temperature Aggressive Chemomechanical Environments}

\subsubsection{Dynamic strain aging}

Dynamic strain aging (DSA), also known as the Portevin-LeChatelier effect, describes the interaction between impurity atoms and the dislocation motion during deformation. DSA typically appears as serrated plastic flow in a uniaxial tensile test due to the locking and releasing of the dislocations by the solute atoms [4.2]. In fatigue testing, DSA was manifested as a sudden load drop with an accompanying shock wave sound. Fig. 4.69 illustrates the DSA phenomena present during the LCF tests of Alloy 617 and Alloy 230 at various total strain levels. The total strain range can influence the extent of DSA phenomena. When the test total strain range $\geq$ $1.0 \%$, DSA usually does not last for more than 10 cycles, while for the smallest total strain range test, $0.5 \%$, DSA could last for hundreds of cycles before the serrated flow disappeared. This could explain the existence of the low cyclic softening rate and the large maximum stress scattering in the $0.5 \%$ total strain range LCF test as described in section 4.7.2. It is known that if other conditions remain the same, small strain fatigue favors the planar slip character of dislocations, while large strain fatigue promotes wavy slip, or nonplanar dislocation arrangements [4.47]. DSA normally tends to favor planar slip character, thus affecting the LCF test at $0.5 \%$ total strain range to a greater extent [4.48].

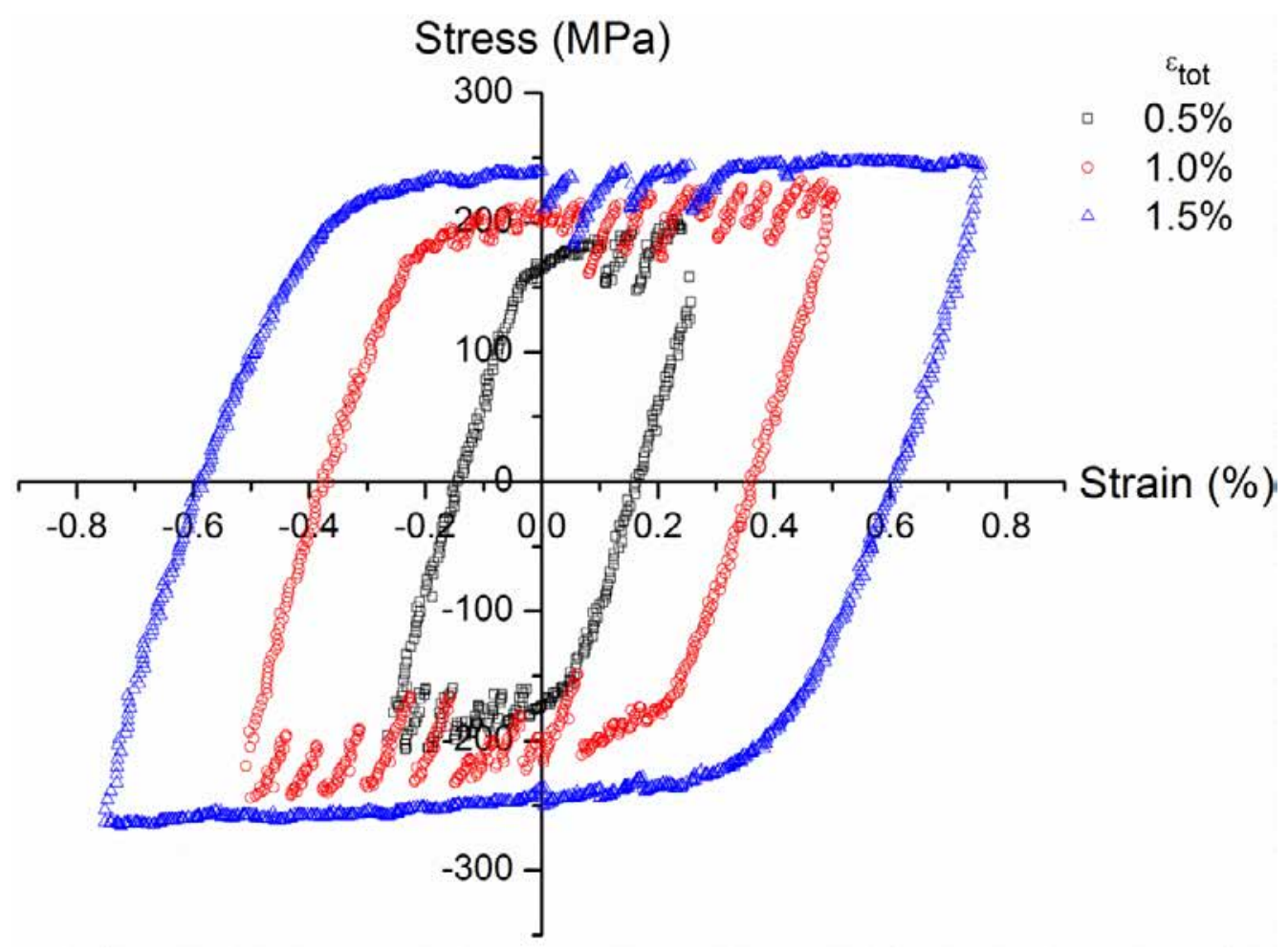

(a) 


\section{Understanding Fundamental Material-Degradation Processes in High Temperature Aggressive Chemomechanical Environments}

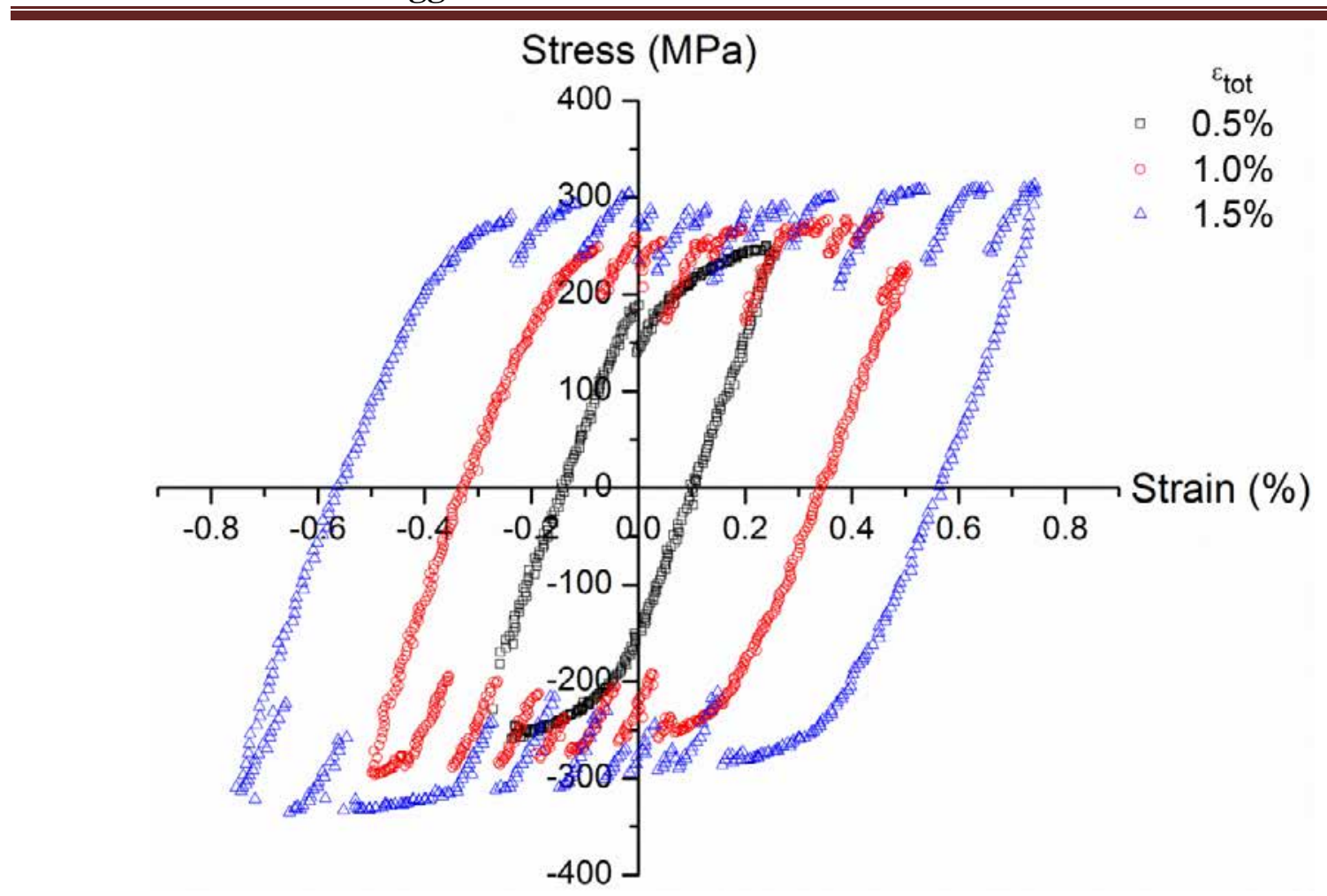

(b)

Fig. 4.69: Second cycle hysteresis loops showing DSA of (a) Alloy 617 and (b) Alloy 230

\subsection{Creep-fatigue Results}

\subsubsection{Creep-fatigue life}

The cycles to failure at different total strain ranges with various durations of the dwell time at maximum strain level are shown in Fig. 4.70. Compared with the LCF results, the cycles to failure for both materials became reduced at the creep-fatigue test conditions. Again, no apparent differences were found in the cyclic creep-fatigue life for the two failure standards described previously. Numeric data of LCF and the creep-fatigue life of Alloy 617 and 230 based on a 40\% cyclic maximum tensile strength drop is shown in Table 4.9. Similar to the LCF results, Alloy 230 exhibited a longer creep-fatigue life than Alloy 617, especially for the creep-fatigue tests with the smallest total strain range (i.e. $0.5 \%$ ) and the longer dwell time (i.e. 10 and 30 minutes at $1.0 \%$ total strain range). The greatest fatigue life reduction was found in the creep-fatigue test of Alloy 617 with $0.5 \%$ total strain range with a 3 minute dwell time at peak tensile strain (i.e. $40 \%$ reduction in the fatigue life compared with the LCF test at the same conditions).

In the related works of Meurer et al. [4.49] and Totemeier [4.36], it was observed that the largest fatigue life reductions occurred when the strain hold period was introduced into low total strain range LCF tests of Alloy 617. It is clear from Fig. 4.70 that longer hold times at the maximum tensile strain cause a further decrease in both materials' creep-fatigue lives. In previous studies, some authors discovered a saturation effect of the hold time at peak strain on 


\section{Understanding Fundamental Material-Degradation Processes in High Temperature Aggressive Chemomechanical Environments}

the reduction of the fatigue life in Alloy 617 [4.49, 4.36] and stainless steel 304 [4.50]. Fig. 4.71 can be indicative of such a saturation effect, although creep-fatigue tests at hold times longer than 30 minutes are needed to verify this.

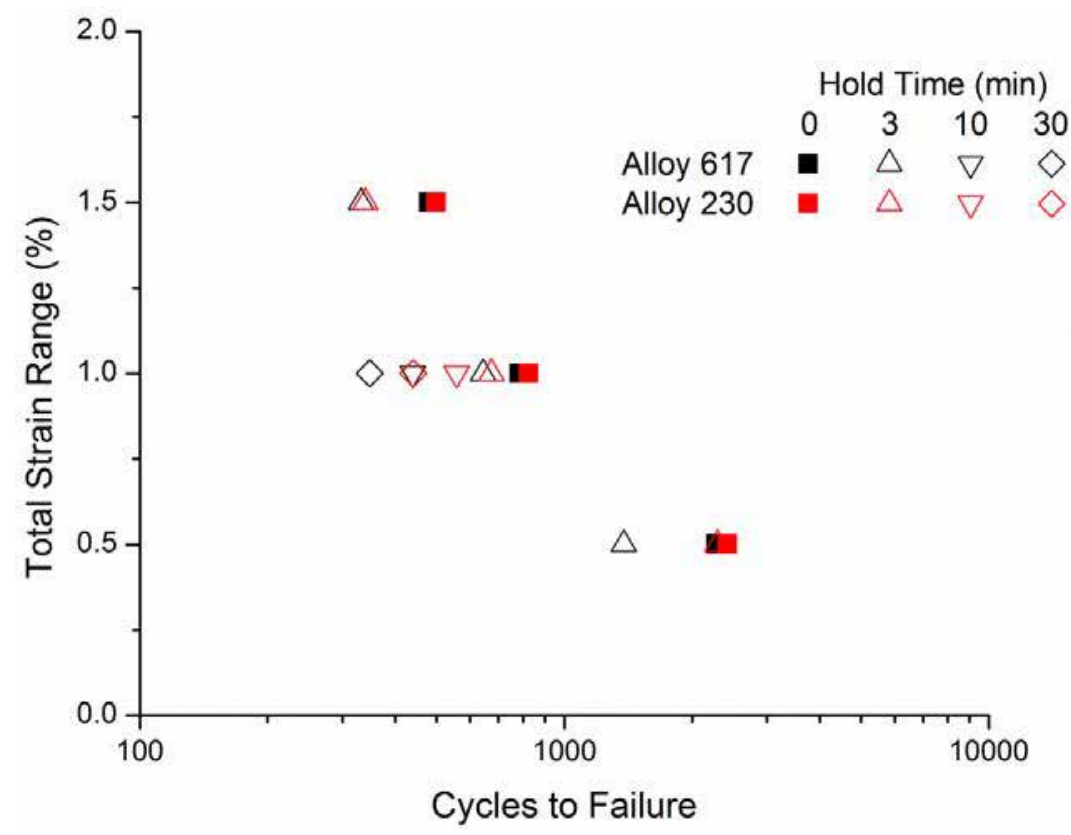

(a)

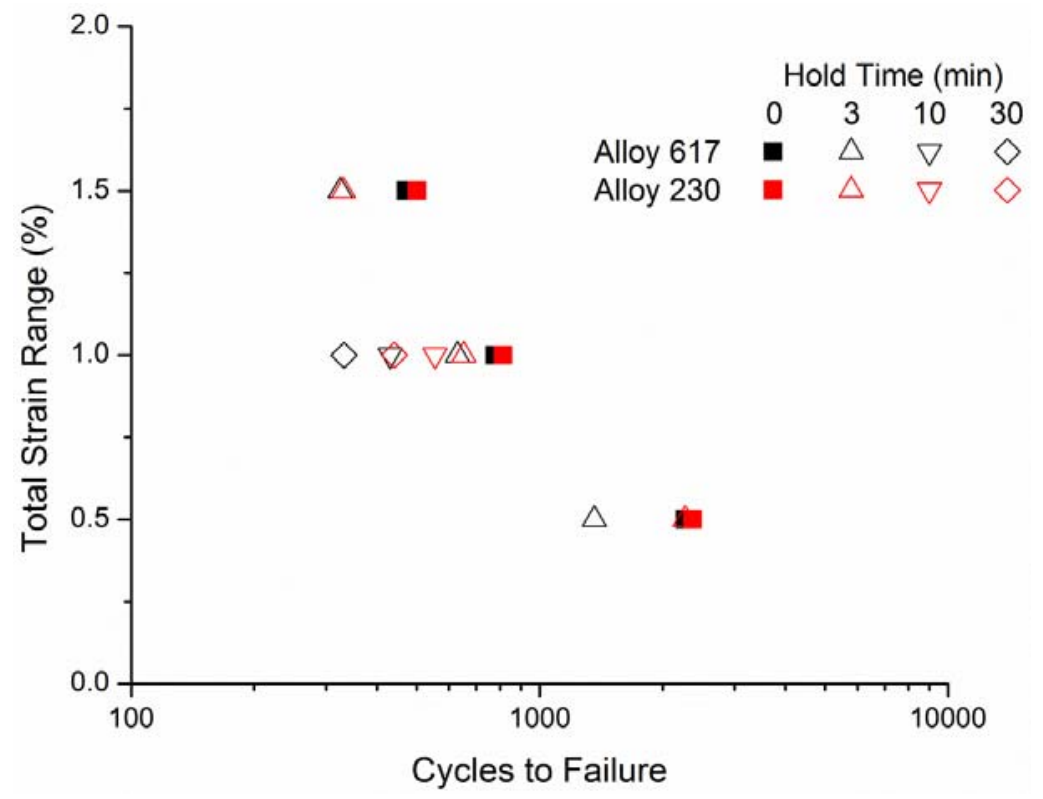

(b)

Fig. 4.70: Creep-Fatigue life of Alloy 617 and Alloy 230 at different total strain ranges and different hold times at peak tensile strain. Failure criterion was based on a $40 \%$ maximum tensile stress decrease in (a) and a $20 \%$ stress ratio drop in (b) 


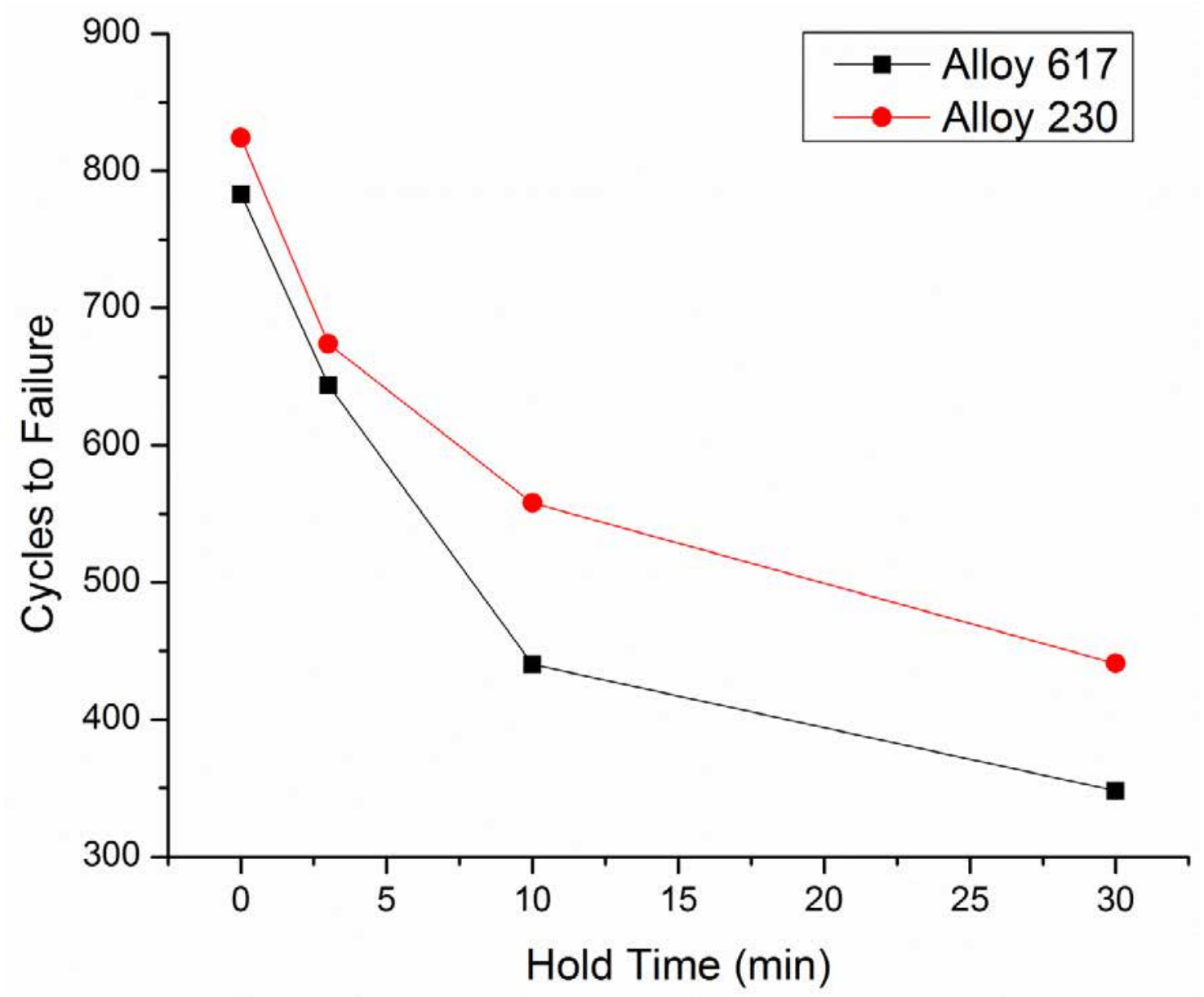

Fig. 4.71: Influence of hold time at peak tensile strain on the creep-fatigue life. $\varepsilon_{\text {tot }}=1.0 \%$

Table 4.9: LCF and creep-fatigue life of Alloy 617 and 230 based on $40 \%$ cyclic maximum tensile strength drop

\begin{tabular}{|c|c|c|c|c|c|c|c|c|}
\hline \multirow{3}{*}{ Material } & \multicolumn{9}{|c|}{ Total Strain Range and Hold Time } & \multicolumn{3}{c|}{$1.0 \%$} \\
\cline { 2 - 9 } & \multicolumn{2}{|c|}{$0.5 \%$} & \multicolumn{6}{c|}{$1.5 \%$} \\
\cline { 2 - 9 } & $0 \mathrm{~min}$ & $3 \mathrm{~min}$ & $0 \mathrm{~min}$ & $3 \mathrm{~min}$ & $10 \mathrm{~min}$ & $30 \mathrm{~min}$ & $0 \mathrm{~min}$ & $3 \mathrm{~min}$ \\
\hline Alloy 617 & 2280 & 1380 & 755,812 & 644 & 440 & 348 & 480 & 332 \\
\hline Alloy 230 & 2420 & 2295 & 824 & 674 & 558 & 441 & 500 & 340 \\
\hline
\end{tabular}

\subsubsection{Materials cyclic creep-fatigue behavior}

In Fig. 4.72, the cyclic maximum stress and stress ratio of Alloy 617 and Alloy 230 under creep-fatigue tests are illustrated. Compared with the cyclic maximum stress of Alloy 617 and Alloy 230 under LCF tests as shown in Fig. 4.66, the cyclic stress behavior of both alloys under creep-fatigue tests at a total strain range of $0.5 \%$ and $1.0 \%$ revealed different characteristics. 


\section{Understanding Fundamental Material-Degradation Processes in High Temperature Aggressive Chemomechanical Environments}

There was an additional transition stage, manifested by the rapid maximum stress drop rate which eventually tailed off to a steady state value, between the cyclic hardening phase and the steady state cyclic softening phase. As mentioned before, the cyclic softening phenomenon may be due to dislocation recovery, which is a thermal-active process. The strain hold period allowed more time for dislocation rearrangement and annihilation. Hence there was an additional transition stage of accelerated maximum stress drop. The mid-life hysteresis loops of both materials under creep-fatigue testing are shown in Fig. 4.73. Again, Alloy 230 showed a smaller plastic stain range than Alloy 617 for all the test conditions as in the LCF tests. 
Understanding Fundamental Material-Degradation Processes in High Temperature Aggressive Chemomechanical Environments

$\varepsilon_{\text {tot }}=0.5 \%$, dwell time $=3 \mathrm{~min}$

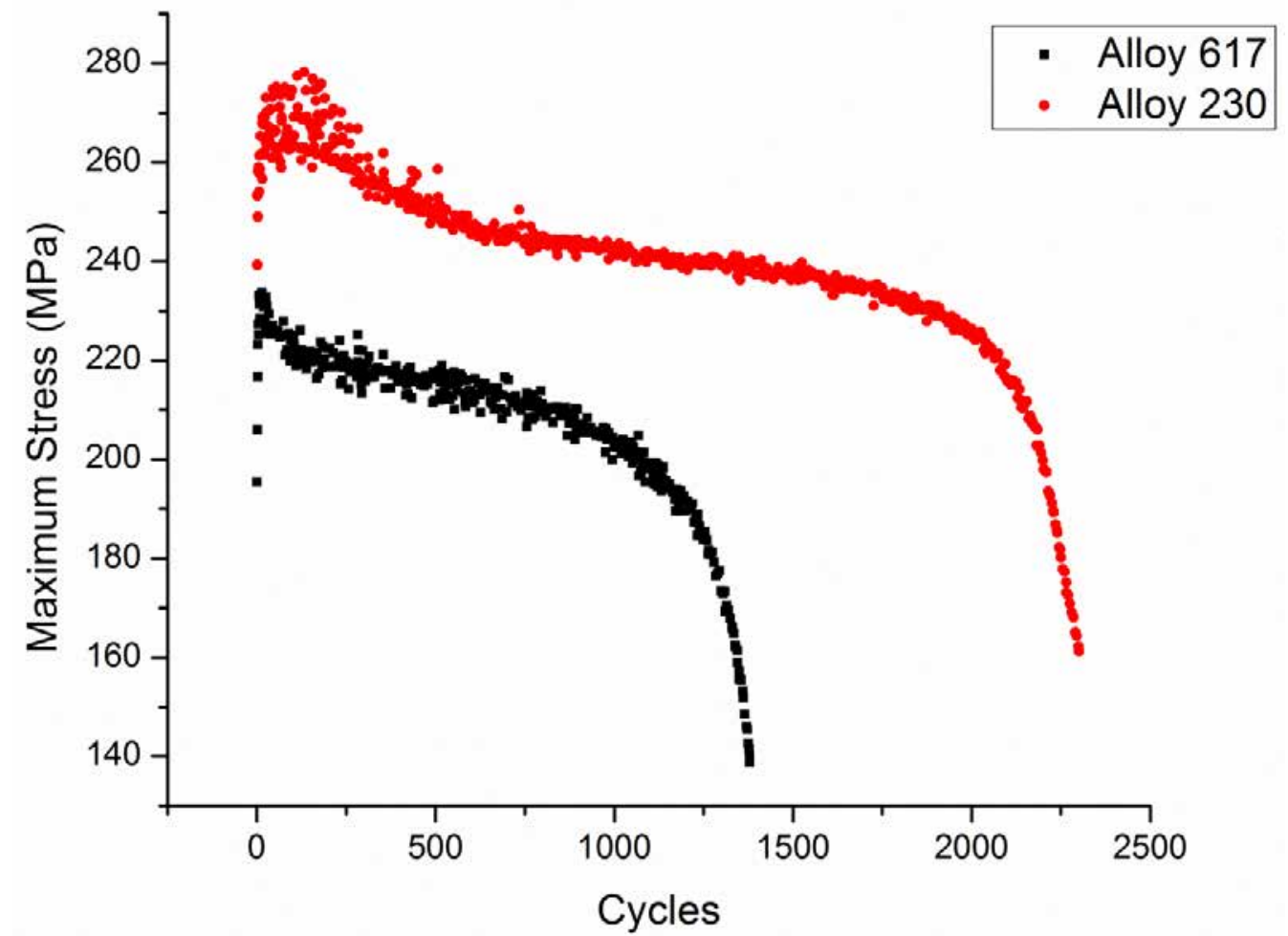

$\varepsilon_{\text {tot }}=0.5 \%$, dwell time $=3 \mathrm{~min}$

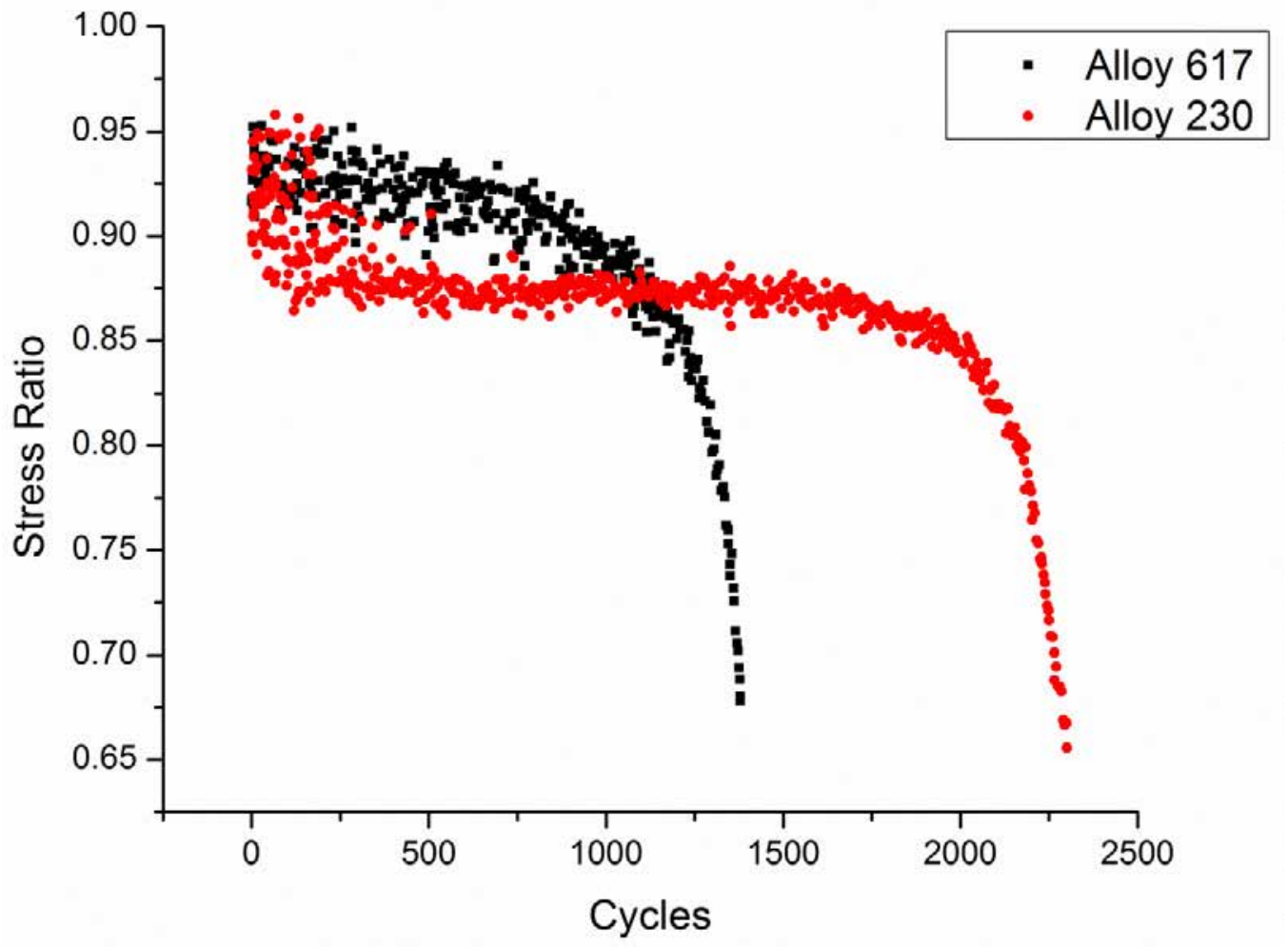

Fig. 4.72: Creep-fatigue cyclic maximum stress and stress ratio of Alloy 617 and Alloy 230 
Understanding Fundamental Material-Degradation Processes in High Temperature Aggressive Chemomechanical Environments
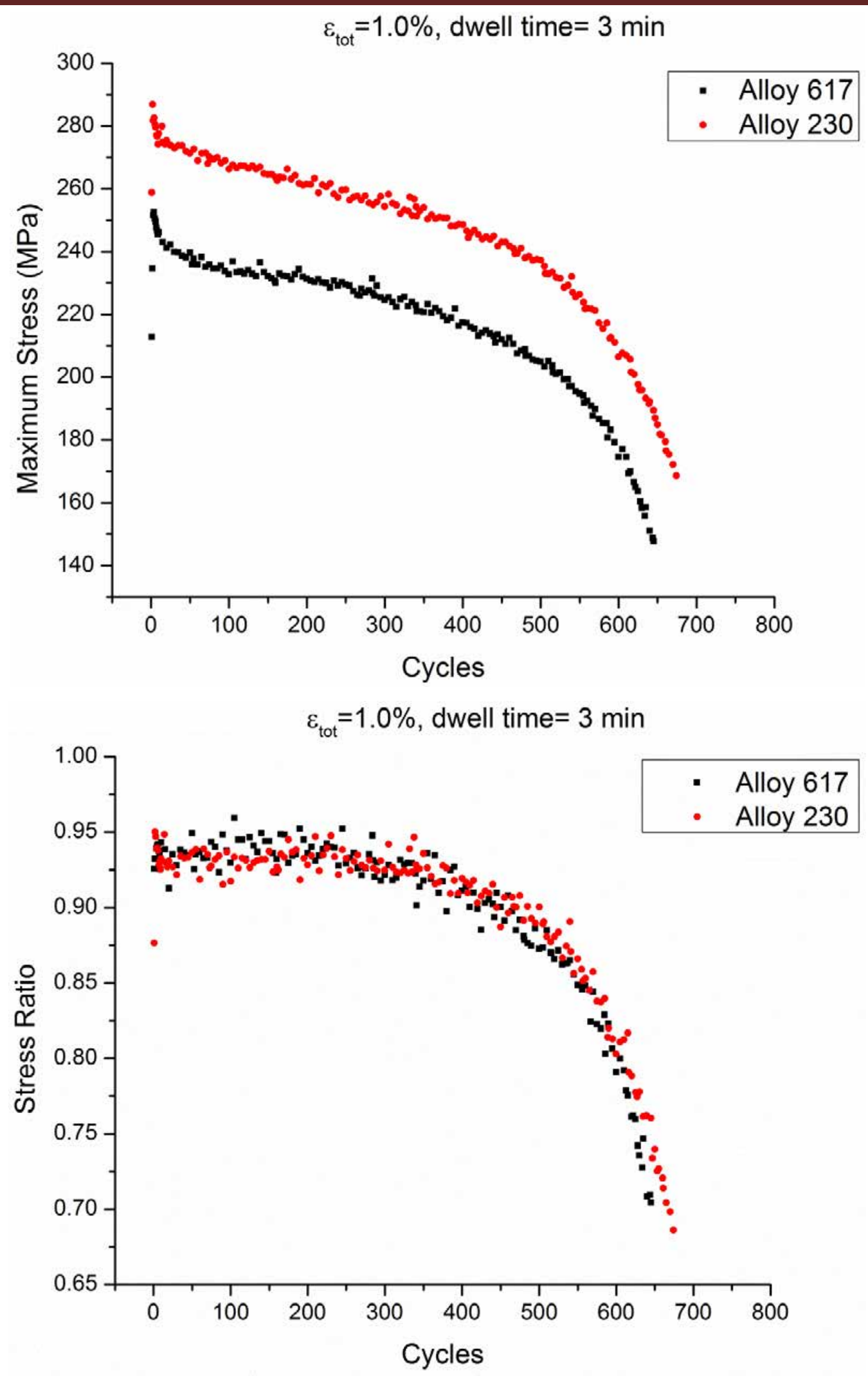

Fig. 4.72 Cont. 


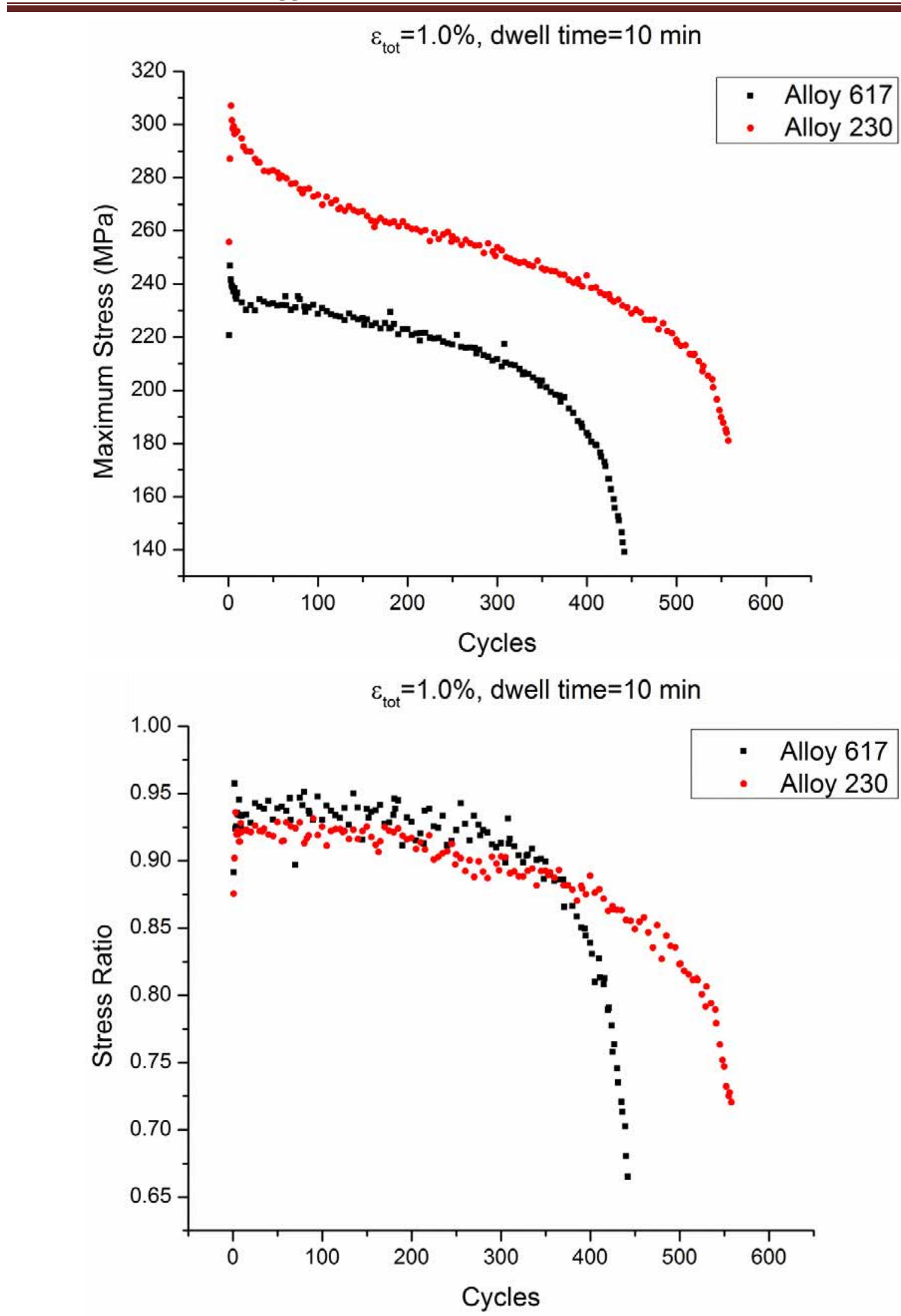

Fig. 4.72 Cont. 
$\varepsilon_{\text {tot }}=1.0 \%$, dwell time $=30 \mathrm{~min}$

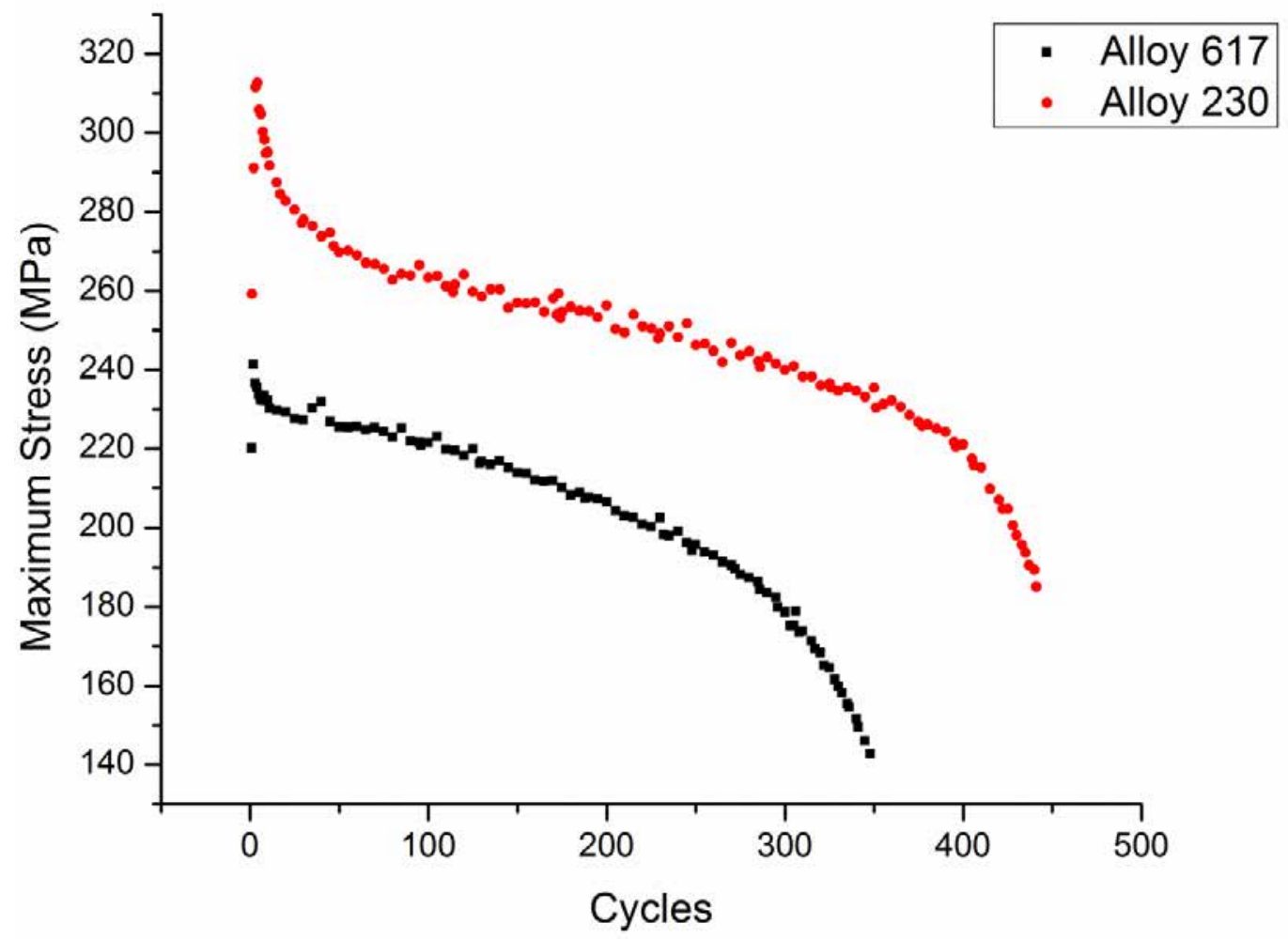

$\varepsilon_{\text {tot }}=1.0 \%$, dwell time $=30 \mathrm{~min}$

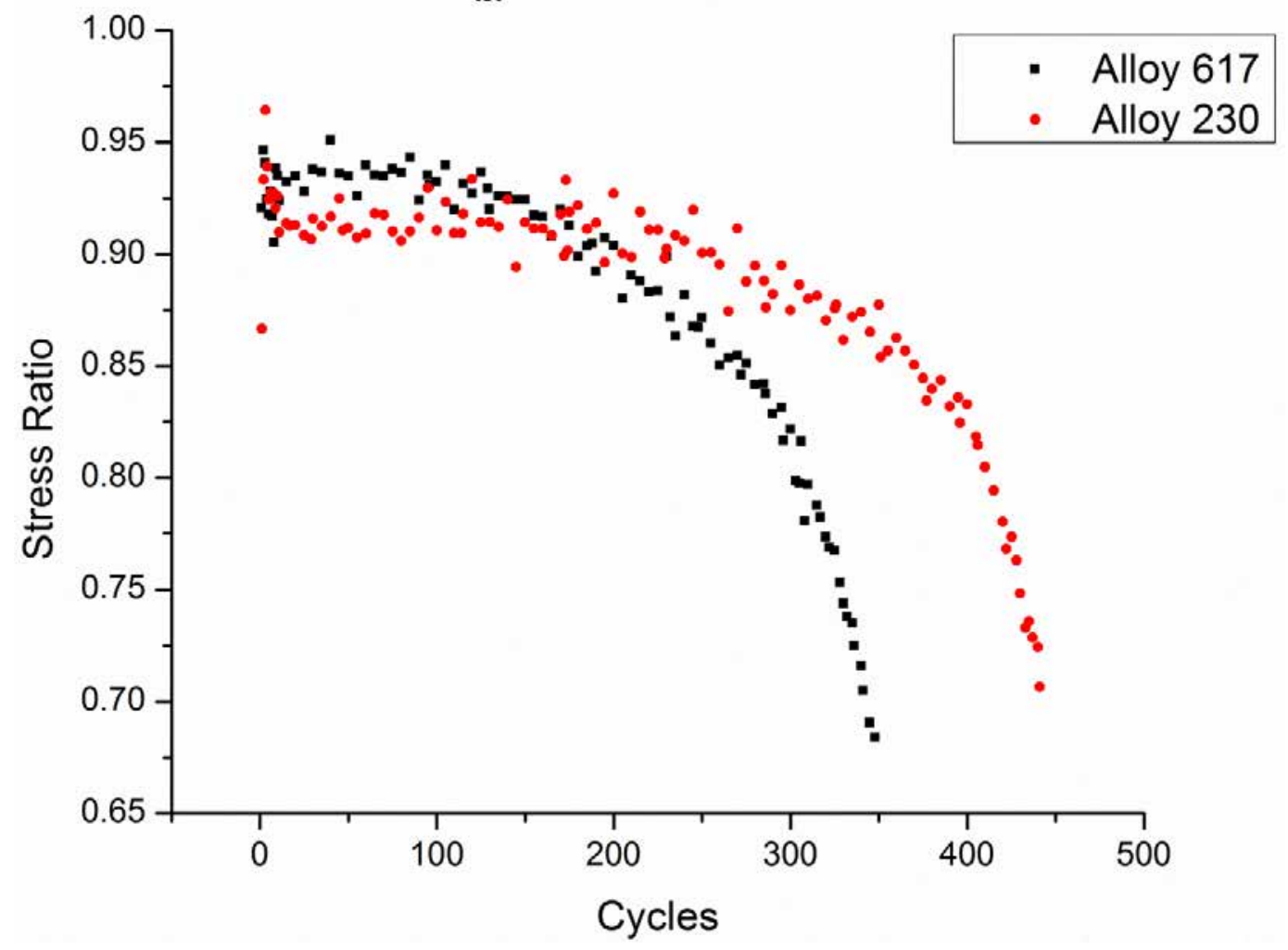

Fig. 4.72 Cont. 
Understanding Fundamental Material-Degradation Processes in High Temperature Aggressive Chemomechanical Environments

$\varepsilon_{\text {tot }}=1.5 \%$, dwell time $=3 \mathrm{~min}$
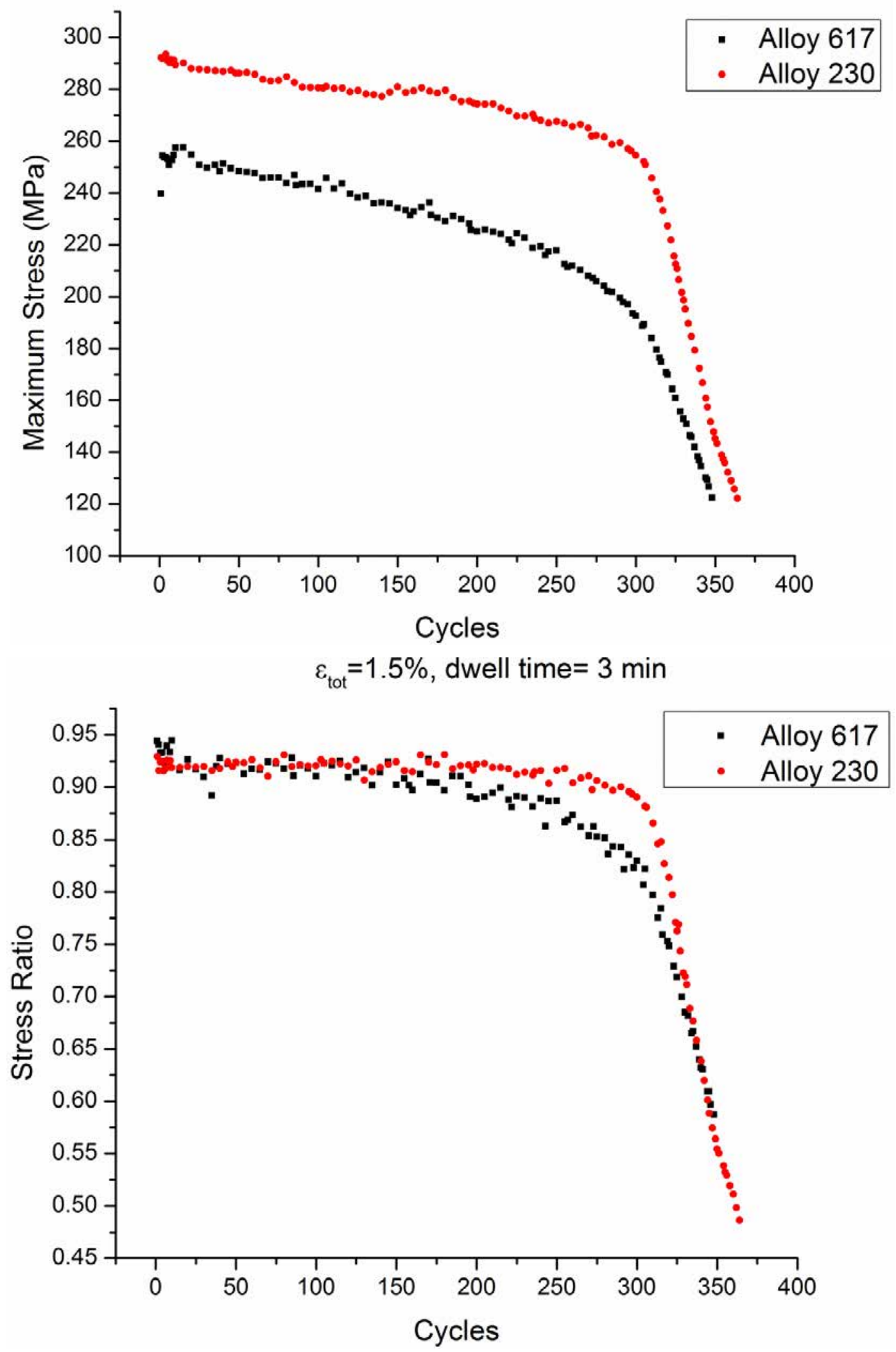

Fig. 4.72 Cont. 

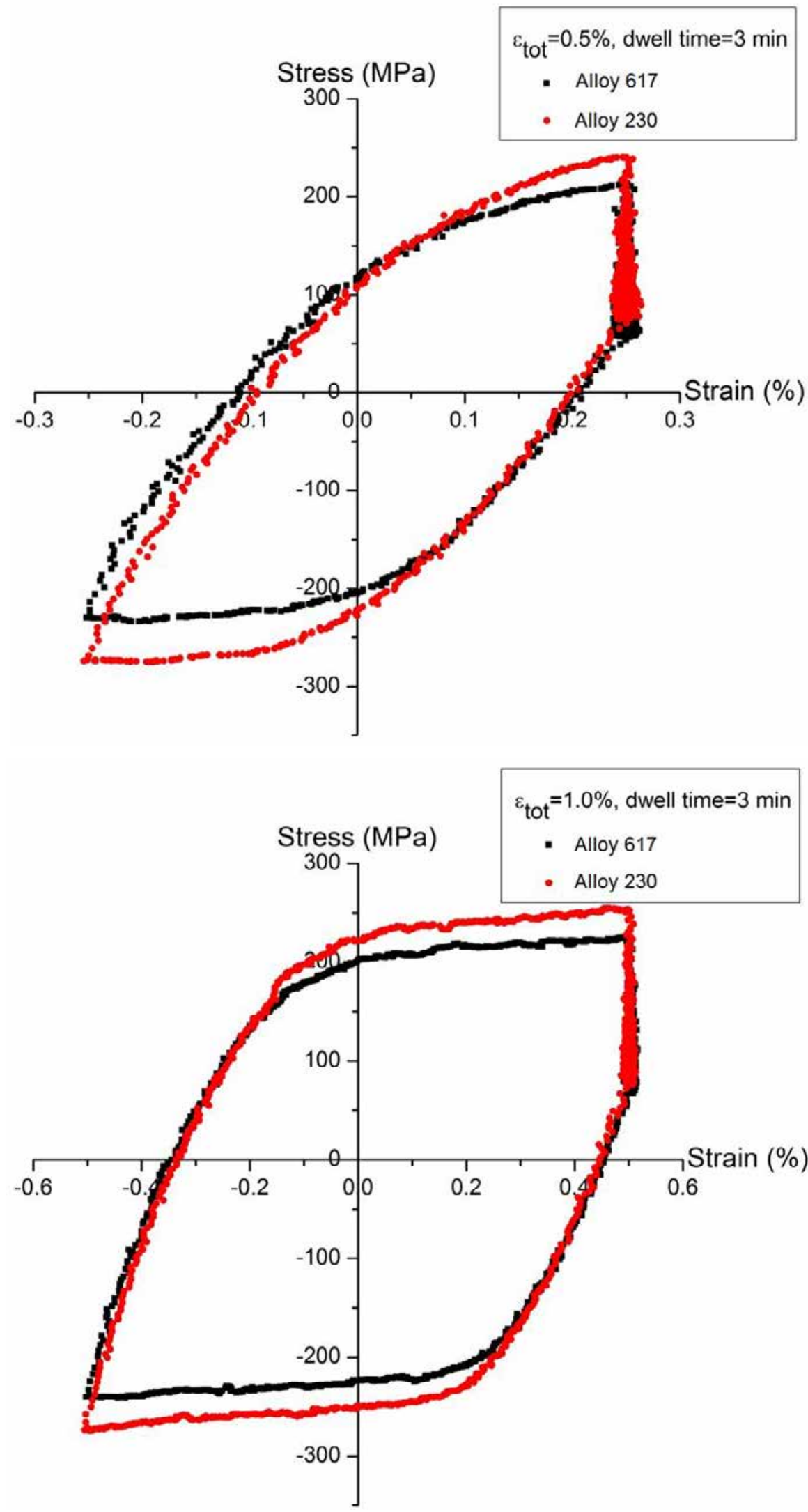

Fig. 4.73: Creep-fatigue mid-life hysteresis loops of Alloy 617 and Alloy 230 
Understanding Fundamental Material-Degradation Processes in High Temperature Aggressive Chemomechanical Environments
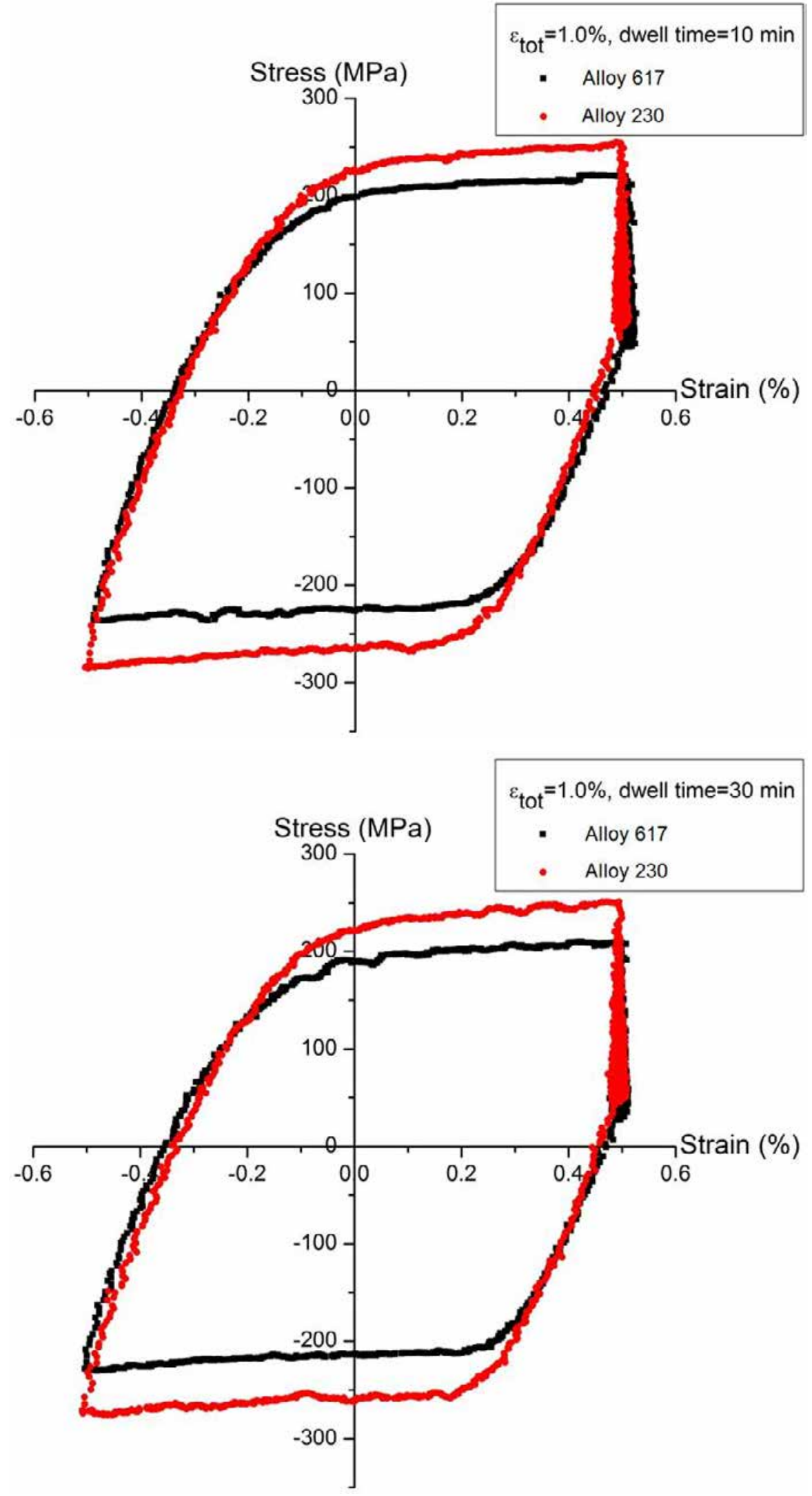

Fig. 4.73 Cont. 


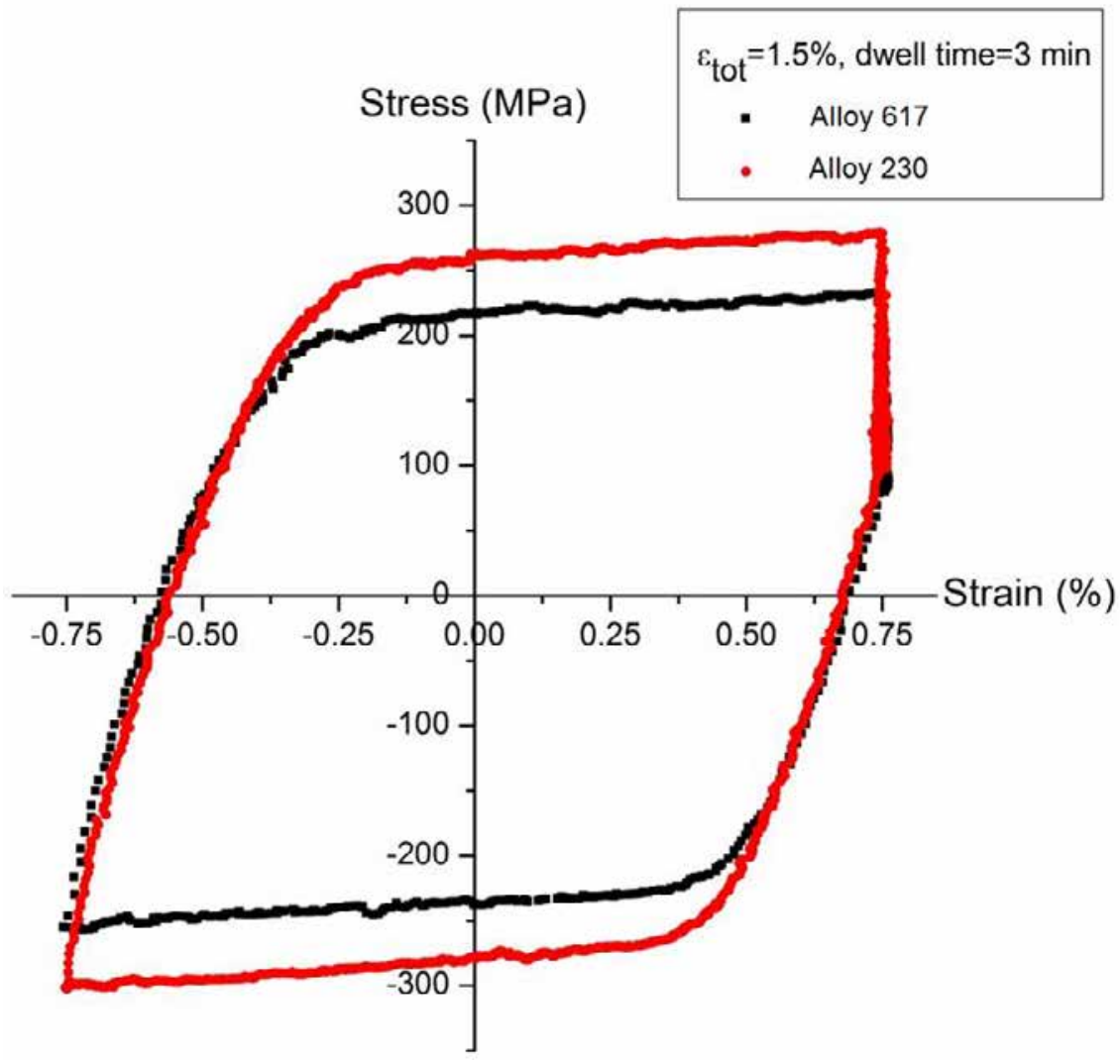

\subsubsection{Stress relaxation during the strain hold period}

The major differences between the mid-life hysteresis loops under LCF tests and creep-fatigue tests involved the appearances of stress relaxation behavior during the peak tensile strain hold period in creep-fatigue tests. During the stress relaxation period, in order to maintain the total strain constant, the stress was reduced to convert elastic strain to inelastic strain. Therefore, stress relaxation is a form of creep [4.50, 4.51]. In Fig. 4.74, the stress relaxation behavior at different test conditions is displayed. Regardless of the test conditions, in the first few seconds, a very rapid drop in the stress occurs, which may be as high as $50 \%$ of the initial stress level. During the remaining period, relatively small additional stress relaxation occurs. For the test with a longer hold time, i.e. a 30 minute hold time at $1.0 \%$ total strain range, a quasi-steady-state stress relaxation rate was achieved. 

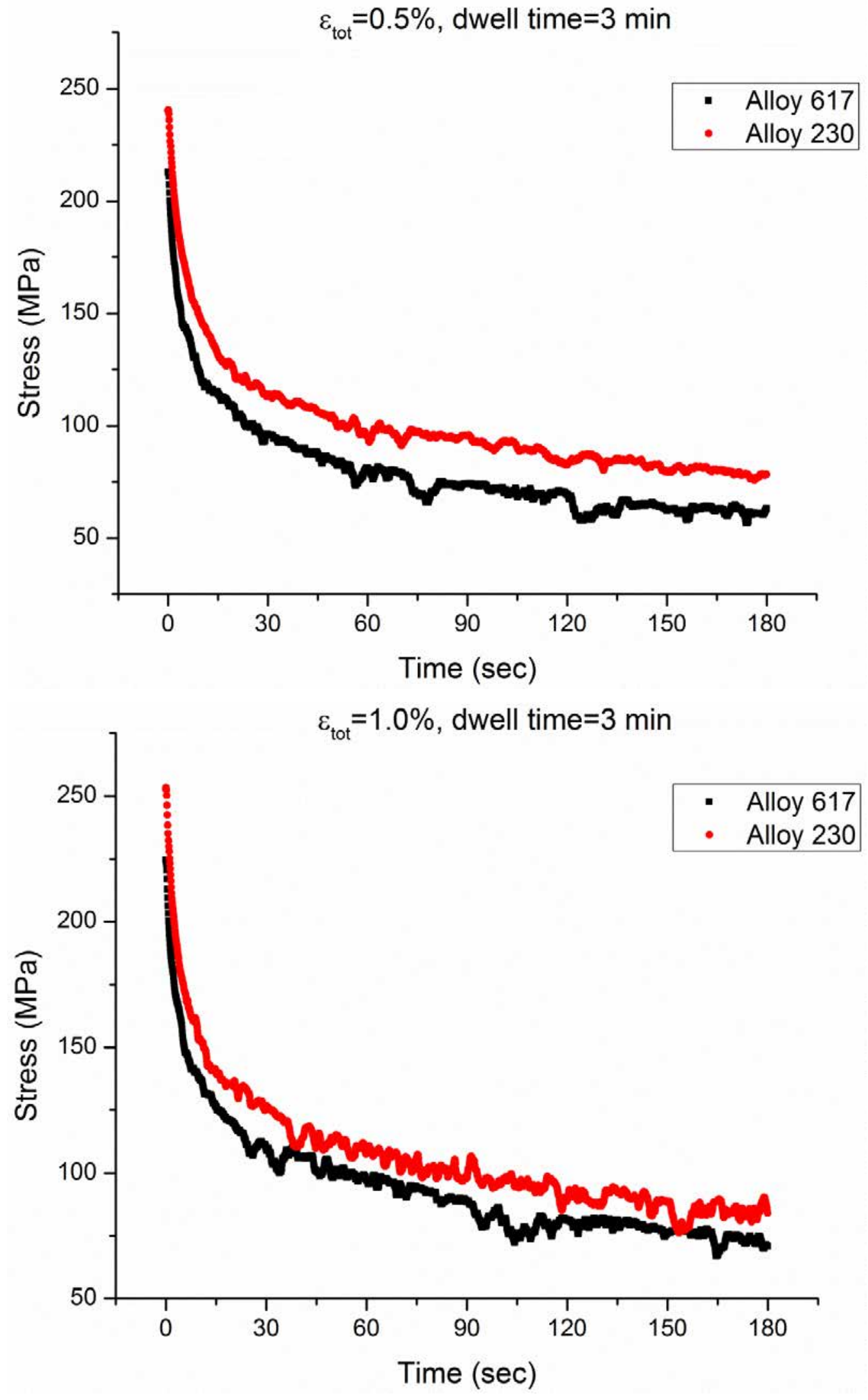

Fig. 4.74: Stress relaxation behavior during peak tensile strain hold period 
Understanding Fundamental Material-Degradation Processes in High Temperature Aggressive Chemomechanical Environments
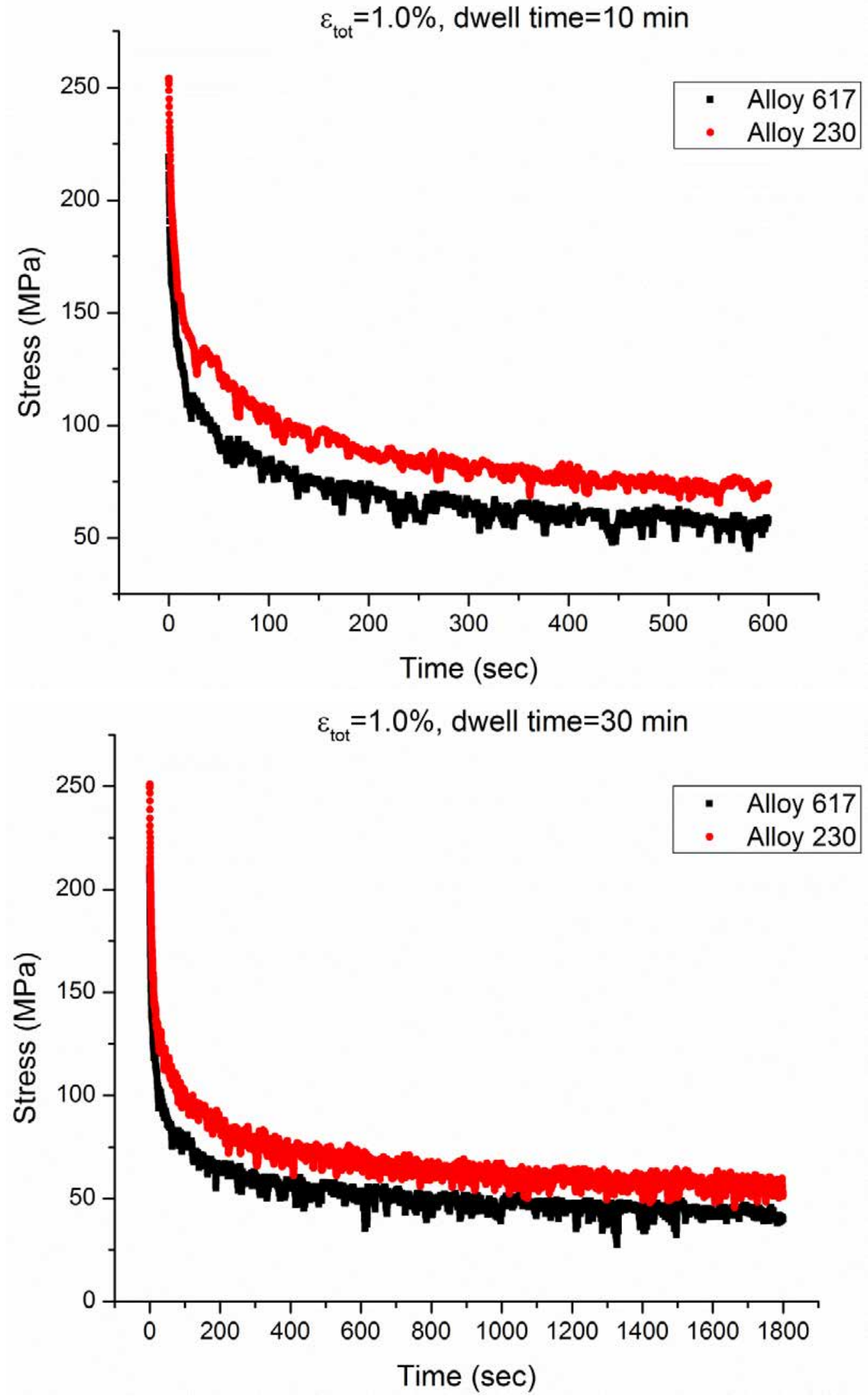

Fig. 4.74 Cont. 


\section{Understanding Fundamental Material-Degradation Processes in High Temperature}

Aggressive Chemomechanical Environments

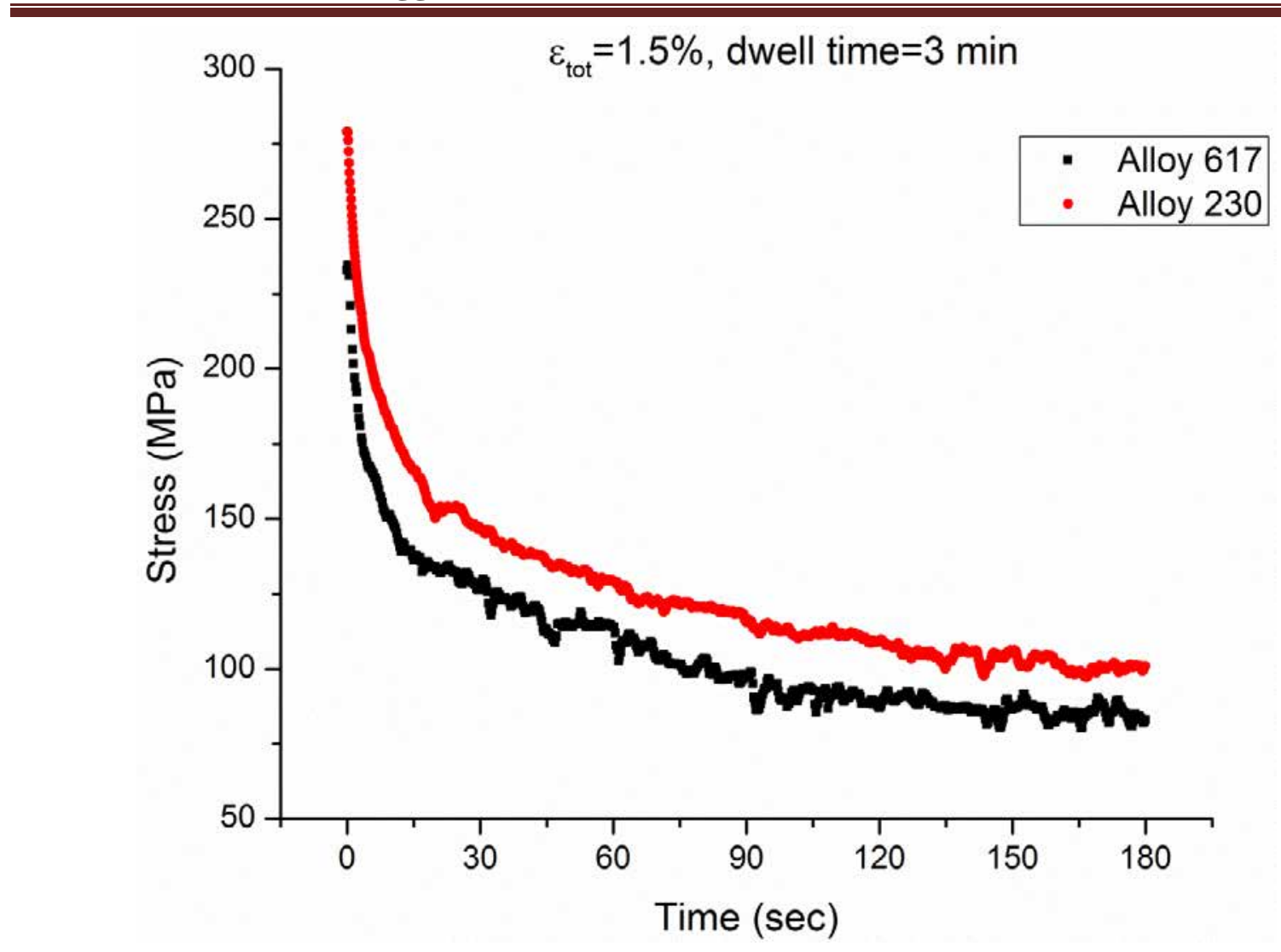

\subsubsection{Dynamic strain aging}

Similar to LCF tests, DSA also occurred in the creep-fatigue tests. Fig. 4.75 illustrates the second cycle hysteresis loops of both materials in the creep-fatigue tests with a 3 minute strain dwell time, showing serrated plastic flow. The same effect of the total strain range on the number of cycles during which DSA took place likewise applied to all creep-fatigue tests. 


\section{Understanding Fundamental Material-Degradation Processes in High Temperature}

Aggressive Chemomechanical Environments

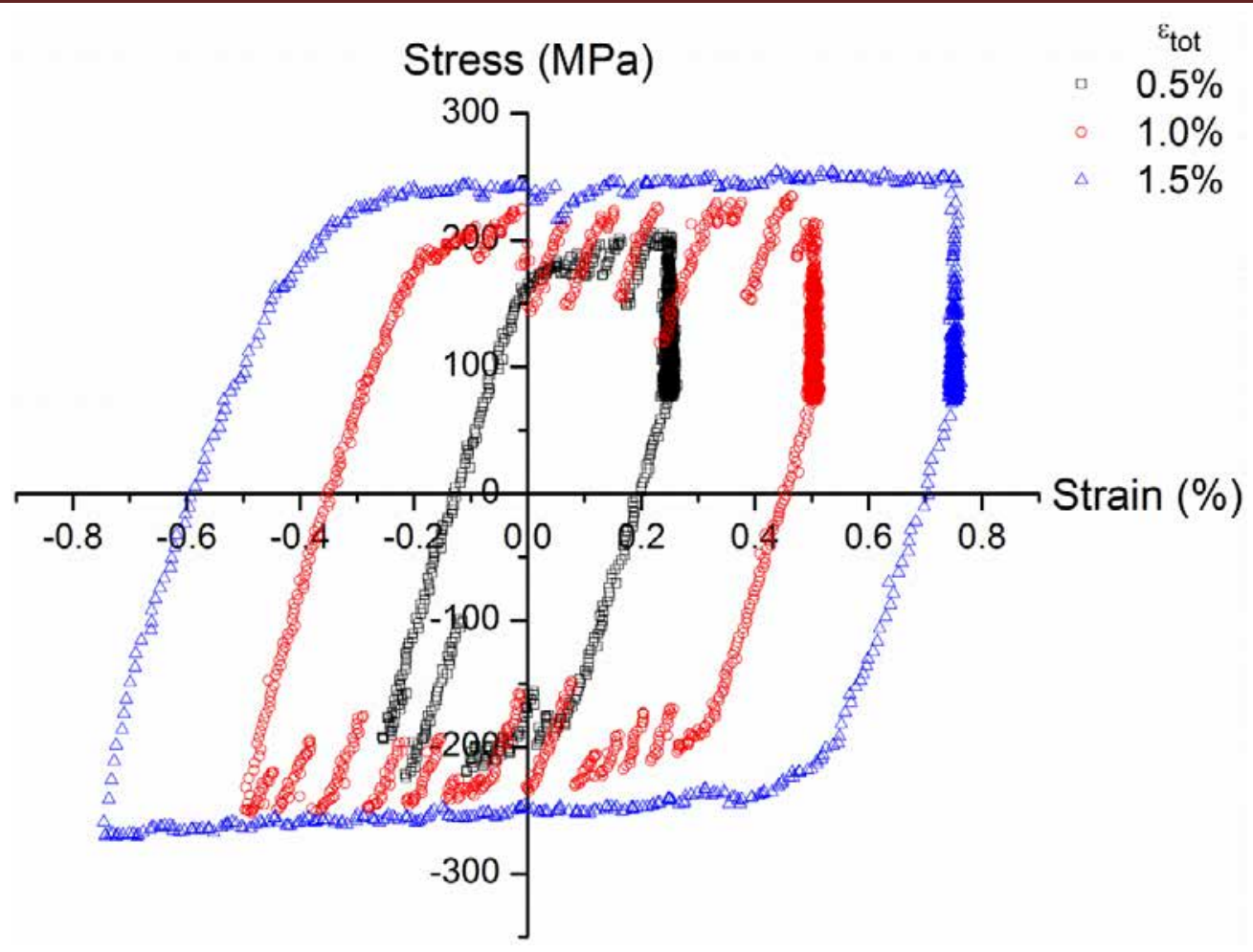

(a)

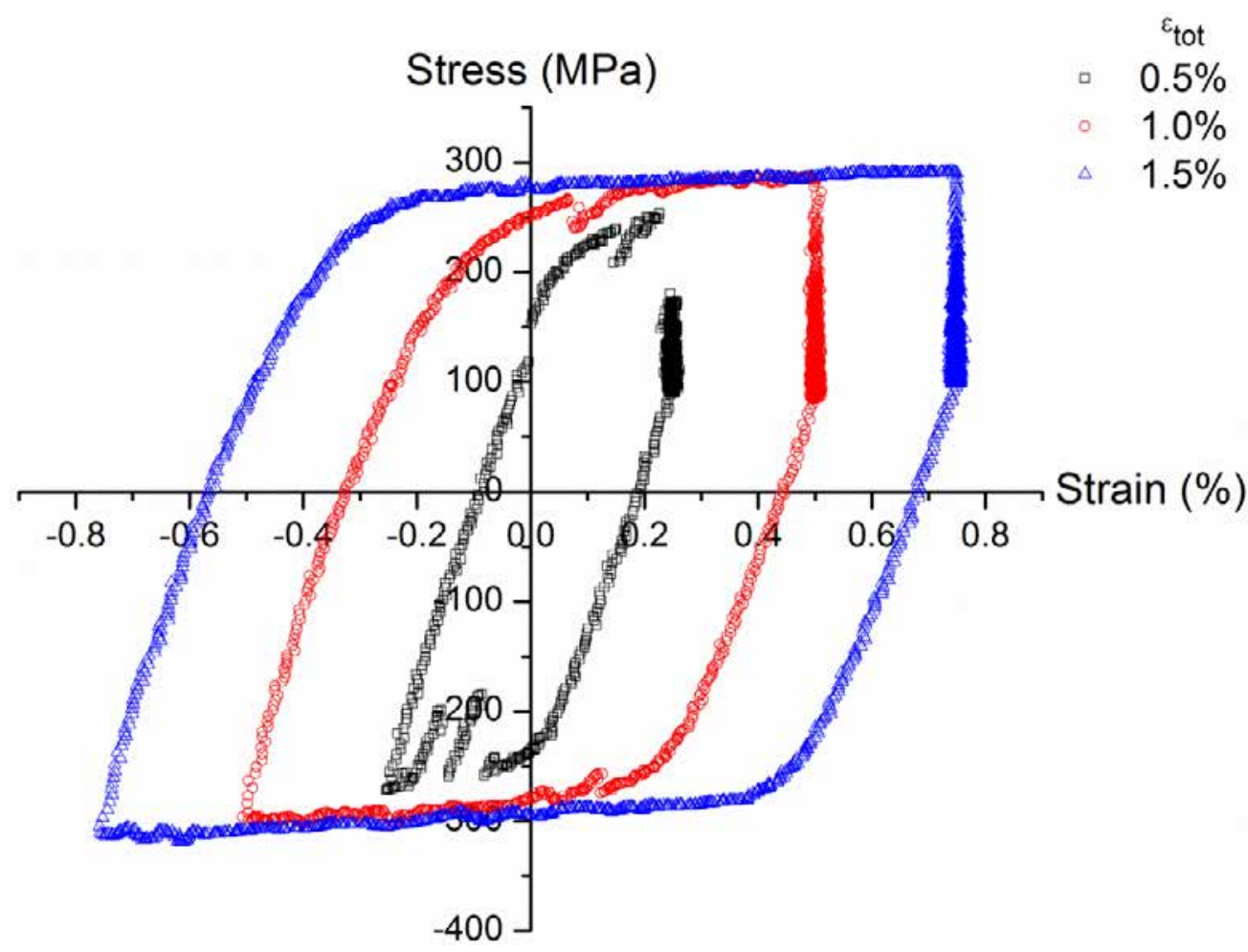

(b)

Fig. 4.75: Second cycle hysteresis loops showing DSA of (a) Alloy 617 and (b) Alloy 230. Peak tensile strain dwell time: 3 minutes 


\subsection{SEM and EDS Results of Uniaxial Creep and Fatigue Tests}

Microstructure analyses of all the specimens were done on the rolling plane or longitudinal plane of the materials as shown in Fig. 4.76 to avoid any influence of specimen orientation on microstructural properties. In all the following microanalysis figures of LCF and creep-fatigue tested specimens, the stress direction is parallel to the vertical direction of the reading plane.

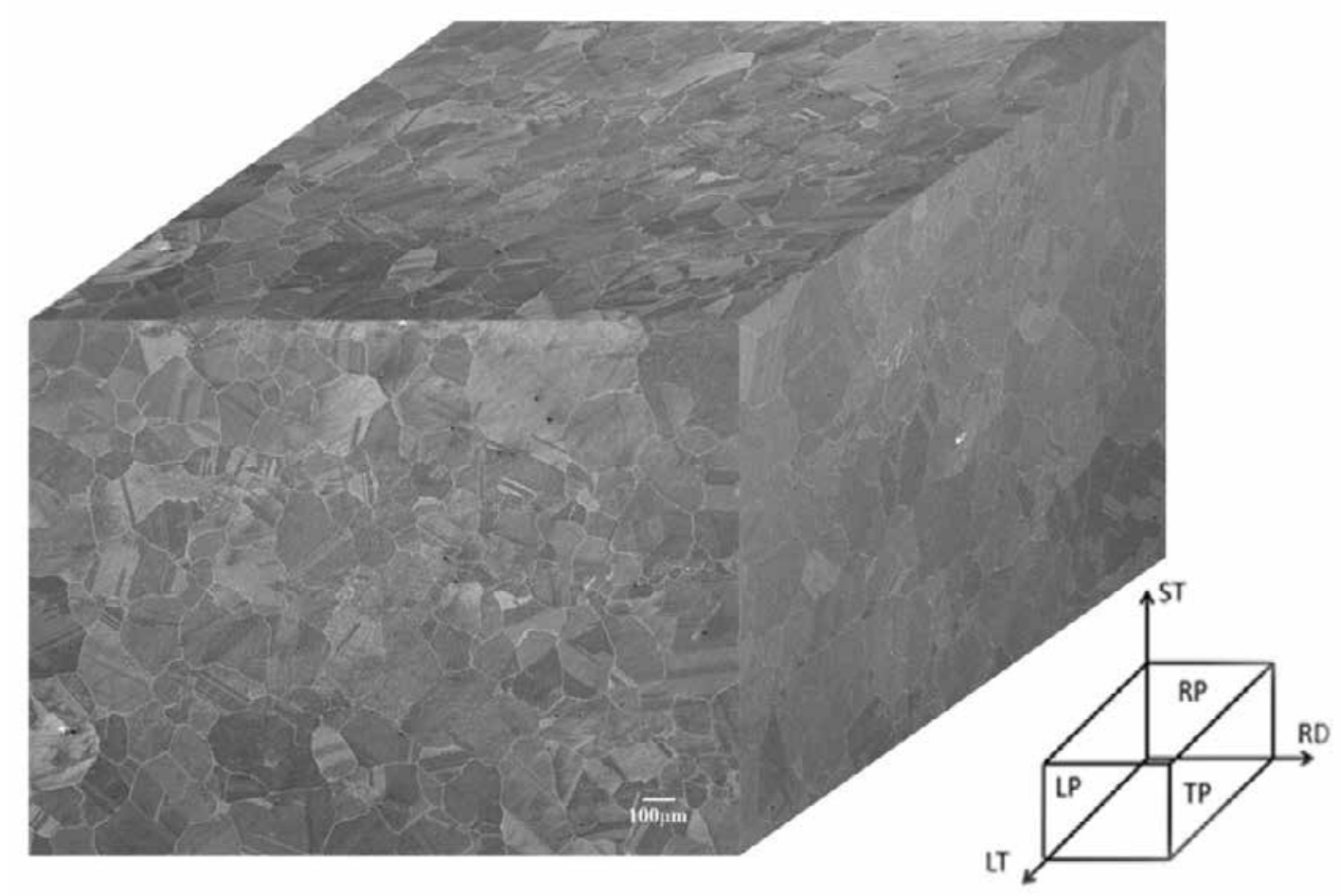

(a) 


\section{Understanding Fundamental Material-Degradation Processes in High Temperature Aggressive Chemomechanical Environments}

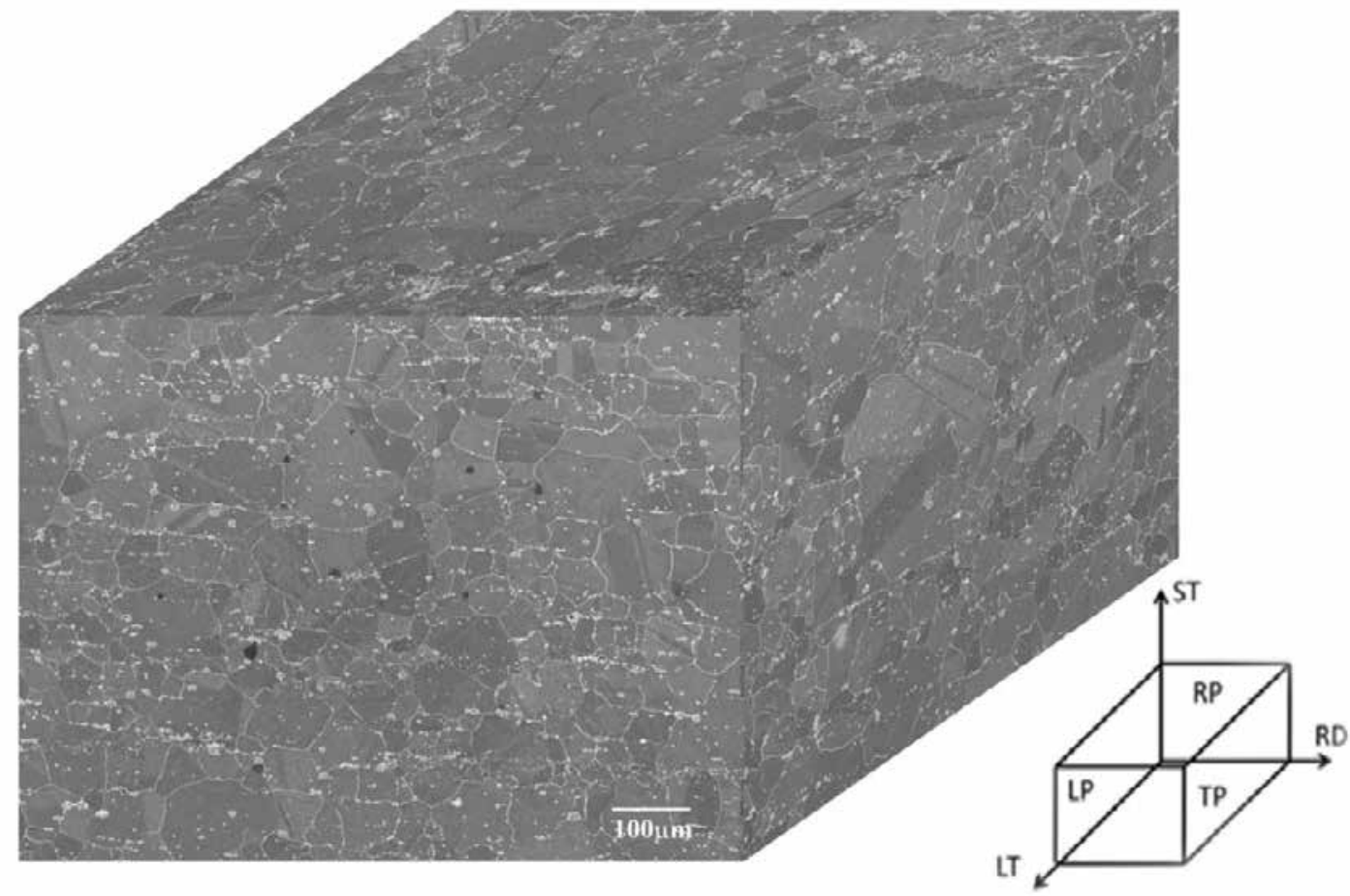

(b)

Fig. 4.76: 3D montage microstructure of as-received (a) Alloy 617 and (b) Alloy 230. The rolling direction (RD), short transverse (ST) direction, long transverse (LT) direction, rolling plane (RP), transverse plane (TP), and longitudinal plane (LP) are noted.

\subsubsection{As-received and aged materials}

In this section, the microstructures of the as-received specimens and specimens aged at $850^{\circ} \mathrm{C}$ for 45 minutes are studied. The thermal aging conditions mimicked the high temperature baking period during which materials were held at $850^{\circ} \mathrm{C}$ before the start of a test in order to stabilize the testing temperature. The microstructures of aged specimens were investigated in order to determine possible microstructural changes when materials were held at $850^{\circ} \mathrm{C}$. At elevated temperatures, precipitation reactions are possible for both Alloy 617 and Alloy 230, and thus the microstructure of materials before the commencement of the test could be quite different from that of the as-received materials due to the 45 minute hold time at $850^{\circ} \mathrm{C}$.

Fig. 4.76 shows the 3D microstructure of the as-received Alloy 617 and Alloy 230 at low magnification. For both materials, equiaxed grain structures and plenary annealing twinning boundaries were observed. After solution treatment there were still many precipitates undissolved and distributed inside the grain and on GBs of both materials. This was more obvious for Alloy 230, in which precipitate clusters were clearly visible and aligned along the rolling direction. The morphology and composition of these precipitates in Alloy 617 and Alloy 230 are shown in Fig. 4.77 and Fig. 4.78, respectively. For Alloy 617, relatively large black precipitates composed of $\mathrm{Ti}(\mathrm{CN})$ were observed. The small intragranular precipitates and GB continuous thin plate-like precipitates that were observed were exclusively $\mathrm{Cr}$-enriched $\mathrm{M}_{23} \mathrm{C}_{6}$. In regards to Alloy 230, the large precipitate clusters consisted of $\mathrm{W}$-rich $\mathrm{M}_{6} \mathrm{C}$ and $\mathrm{Cr}$-rich $\mathrm{M}_{23} \mathrm{C}_{6}$, while $\mathrm{GB}$ thin-plate-like precipitates consisted of $\mathrm{Cr}$-rich $\mathrm{M}_{23} \mathrm{C}_{6}$. No precipitates were 


\section{Understanding Fundamental Material-Degradation Processes in High Temperature Aggressive Chemomechanical Environments}

found on the twinning boundaries of either material. In addition, earlier research works reported the existence of coarse carbide stringers in the as-received Alloy 617 (as shown in Fig. 4.11), which caused the sample to fracture easily in tensile tests and had a negative impact on the material's ductility $[4.52,4.36,4.53,4.14,4.54]$. This microstructure feature was not observed in this work.
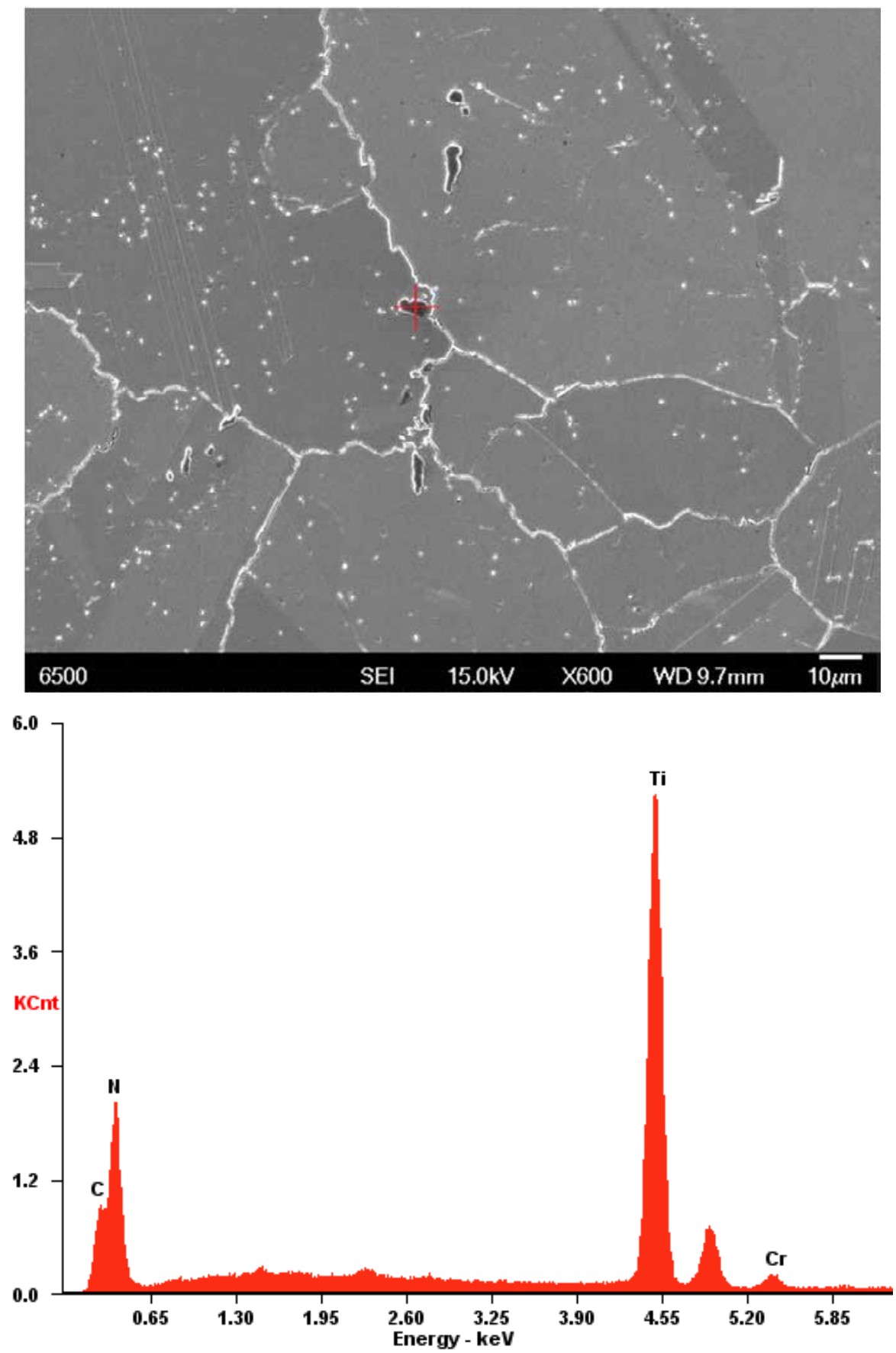

(a)

Fig. 4.77: The morphology and composition of precipitates in the as-received Alloy 617. The EDS spectrum was taken from the highlighted point in the SEM figure. (a) $\mathrm{Ti}(\mathrm{CN})$; (b) intragranular Cr-rich $\mathrm{M}_{23} \mathrm{C}_{6}$ precipitate; (c) $\mathrm{GB}$ Cr-rich $\mathrm{M}_{23} \mathrm{C}_{6}$ precipitate 
Understanding Fundamental Material-Degradation Processes in High Temperature Aggressive Chemomechanical Environments
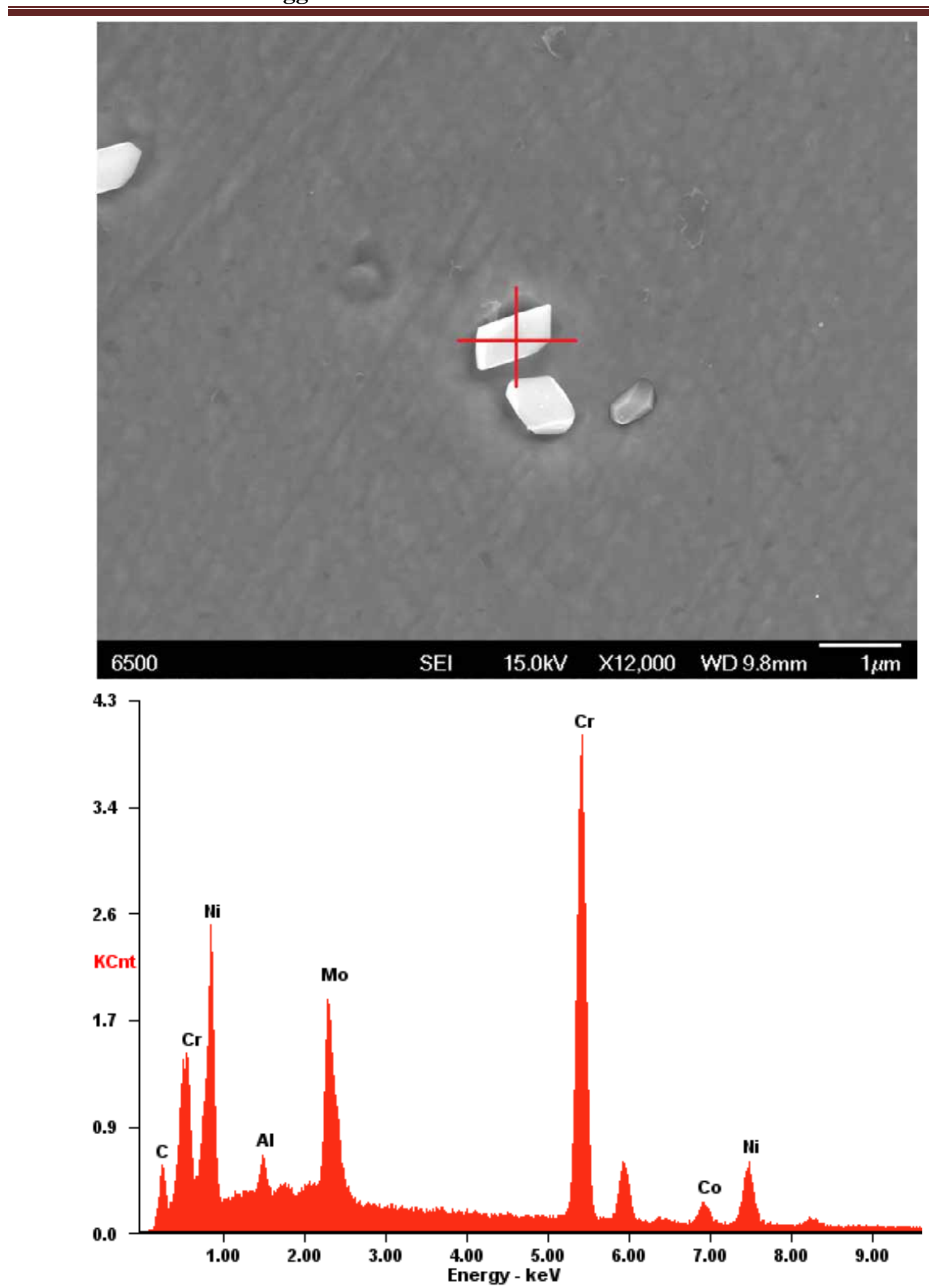

(b)

Fig. 4.77 Cont. 
Understanding Fundamental Material-Degradation Processes in High Temperature Aggressive Chemomechanical Environments
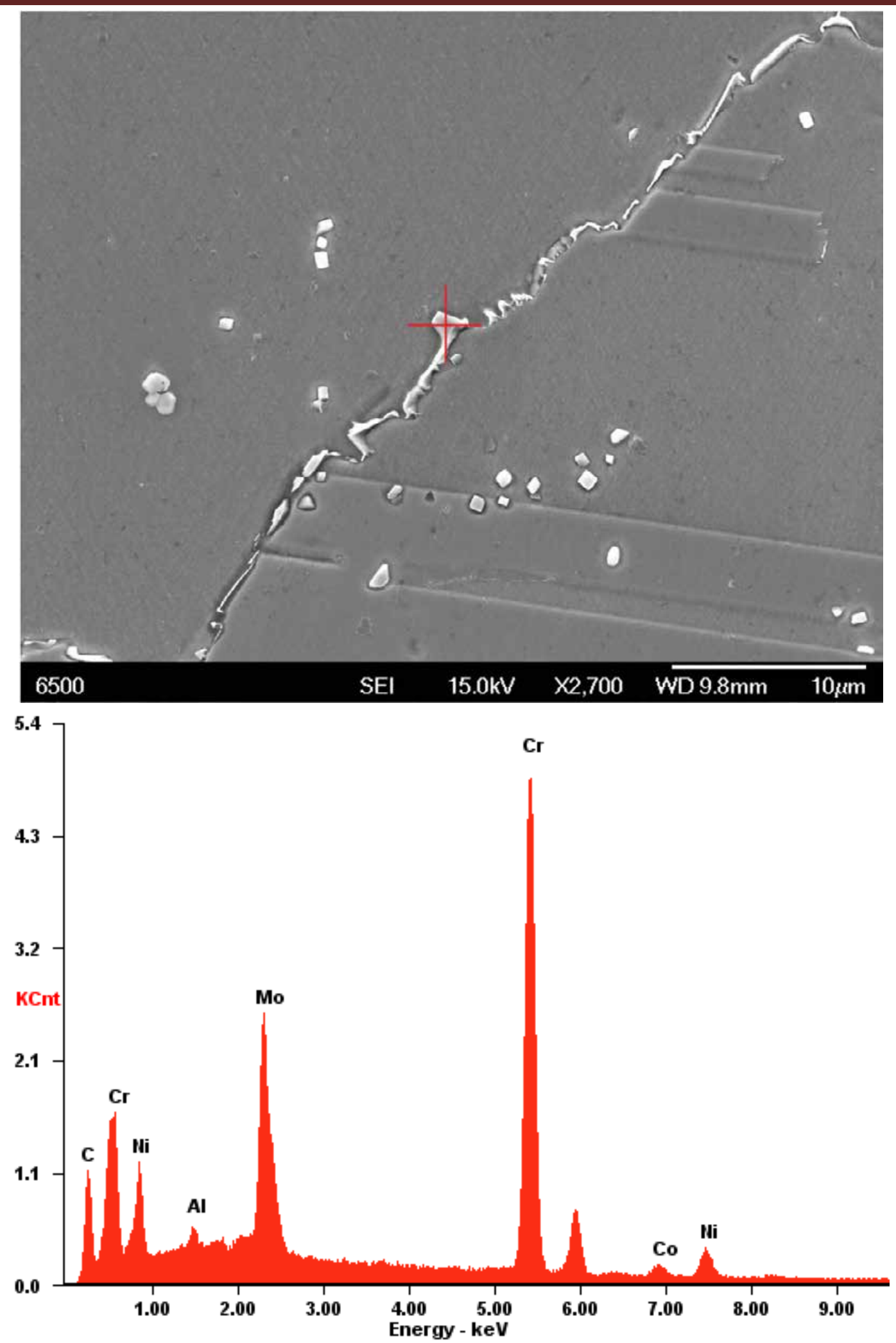

(c)

Fig. 4.77 Cont. 

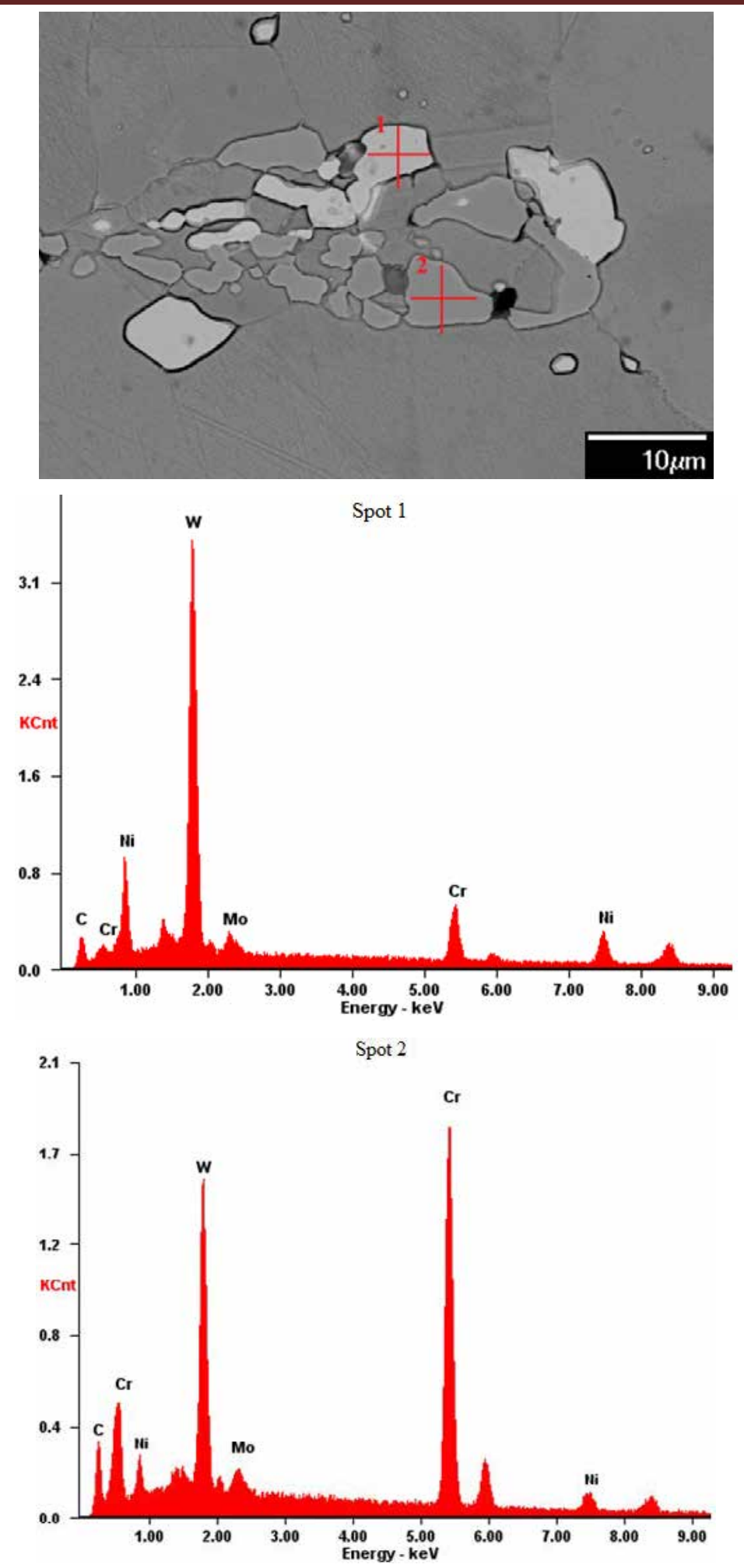

(a)

Fig. 4.78: The morphology and composition of precipitates in the as-received Alloy 230. The EDS spectrum was taken from the highlighted point in the SEM figure. (a) spot 1: W-rich $\mathrm{M}_{6} \mathrm{C}$ and spot 2:

Cr-rich $\mathrm{M}_{23} \mathrm{C}_{6}$; (b) $\mathrm{GB}$ Cr-rich $\mathrm{M}_{23} \mathrm{C}_{6}$ precipitate 
Understanding Fundamental Material-Degradation Processes in High Temperature Aggressive Chemomechanical Environments
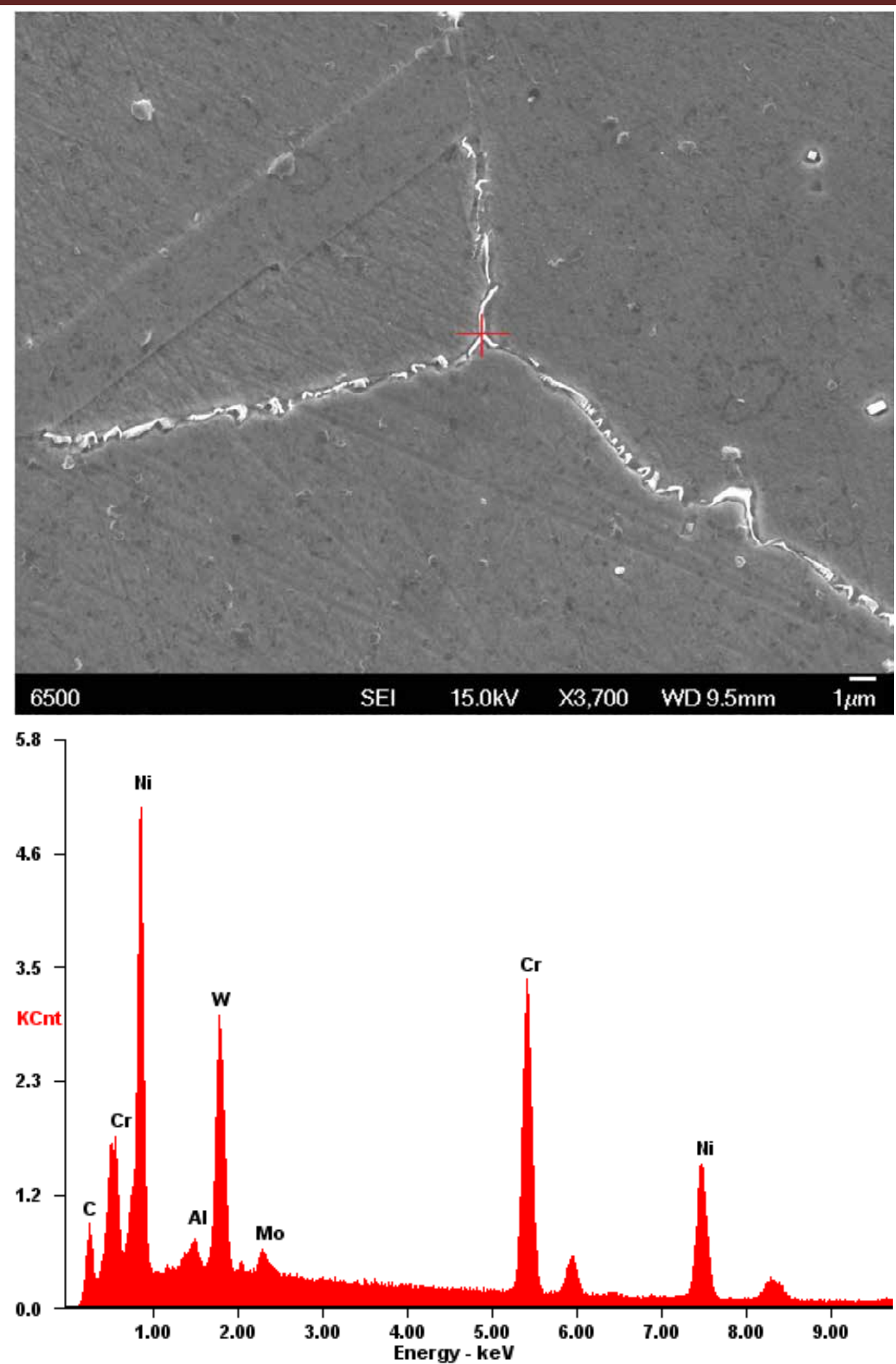

(b)

Fig. 4.78 Cont. 


\section{Understanding Fundamental Material-Degradation Processes in High Temperature Aggressive Chemomechanical Environments}

After thermal aging treatment at $850^{\circ} \mathrm{C}$ for 45 minutes, microstructure changes that were different in each of the materials became evident. Cr-rich $\mathrm{M}_{23} \mathrm{C}_{6}$ precipitates formed around the original $\mathrm{Ti}(\mathrm{CN}), \mathrm{GB}$, and intragranular $\mathrm{M}_{23} \mathrm{C}_{6}$ precipitates in Alloy 617, as shown in Fig. 4.79. The smaller, newly formed, carbides surrounded the original intragranular $\mathrm{M}_{23} \mathrm{C}_{6}$ precipitates and created a sunburst pattern. It was proposed by Mankins et al. that the growth of original intragranular carbides as the alloy cools from the annealing temperature during the solution treatment generated dislocations on which smaller carbides could precipitate when the alloy was subsequently exposed to elevated temperatures [3.44]. In many cases, the newly formed plate-shaped intragranular $\mathrm{M}_{23} \mathrm{C}_{6}$ precipitate particles, shown in Fig. 4.80, aligned along specific crystallographic planes or directions of the crystal matrix resembling a typical Widmanstätten structure [4.55].

Unlike the as-received Alloy 617 specimens, precipitation on twinning boundaries was found as needle-shaped $\mathrm{M}_{23} \mathrm{C}_{6}$ precipitate particles on coherent twinning boundaries, and "zipper" form $\mathrm{M}_{23} \mathrm{C}_{6}$ precipitates formed at twin ends, as illustrated in Fig. 4.81. The twin ends were incoherent twin interfaces upon which lath-like precipitates formed. Based on the work of Singhal et al., the formation mechanism of such lath-like precipitates involved precipitation on Shockley partial dislocation, which bowed out from the incoherent twinning boundaries [4.56]. The precipitate morphology in Alloy 230 after aging treatment is presented in Fig. 4.82. Similar to Alloy 617, Cr-rich $\mathrm{M}_{23} \mathrm{C}_{6}$ aggregated around the original intragranular precipitates in aged Alloy 230, and twinning boundaries also became sites for new carbide formation. However, GB cellular type precipitates were found, and from other studies were shown to initiate premature rupture failures [4.57]. The cellular precipitate was mainly $\mathrm{Cr}$-rich $\mathrm{M}_{23} \mathrm{C}_{6}$, as shown in Fig. 4.83. In addition, in some cases, precipitation free zones formed on both sides of a GB decorated with cellular precipitates, such as in Fig. 4.82(b). Concentration of plastic deformation in these zones was possible, and had been shown to lead to crack initiation in a wrought Ni-based superalloy [4.40, 4.47].

Driver et al. investigated the effect of the precipitation free zone width on the elevated temperature fatigue properties of a $\mathrm{Nb}$-stabilized austenitic stainless steel and found that materials without precipitation free zones had improved fatigue resistance over materials with precipitation free zones, but if precipitation free zones were experienced in the material, an optimum precipitation free zone width of about $1 \mu \mathrm{m}$ existed for maximization of the fatigue resistance [4.58]. Fig. 4.84 illustrates the similar occurrence of Widmanstätten structure precipitates in aged Alloy 230. It is worth noting that not every GB developed cellular type precipitates in Alloy 230, and some GB precipitates still had a thin plate shape, as shown in Fig. 4.85. This signifies that GB character, such as misorientation, can influence the morphology of a GB precipitate, which will be stressed further in the EBSD analysis. 
Understanding Fundamental Material-Degradation Processes in High Temperature Aggressive Chemomechanical Environments

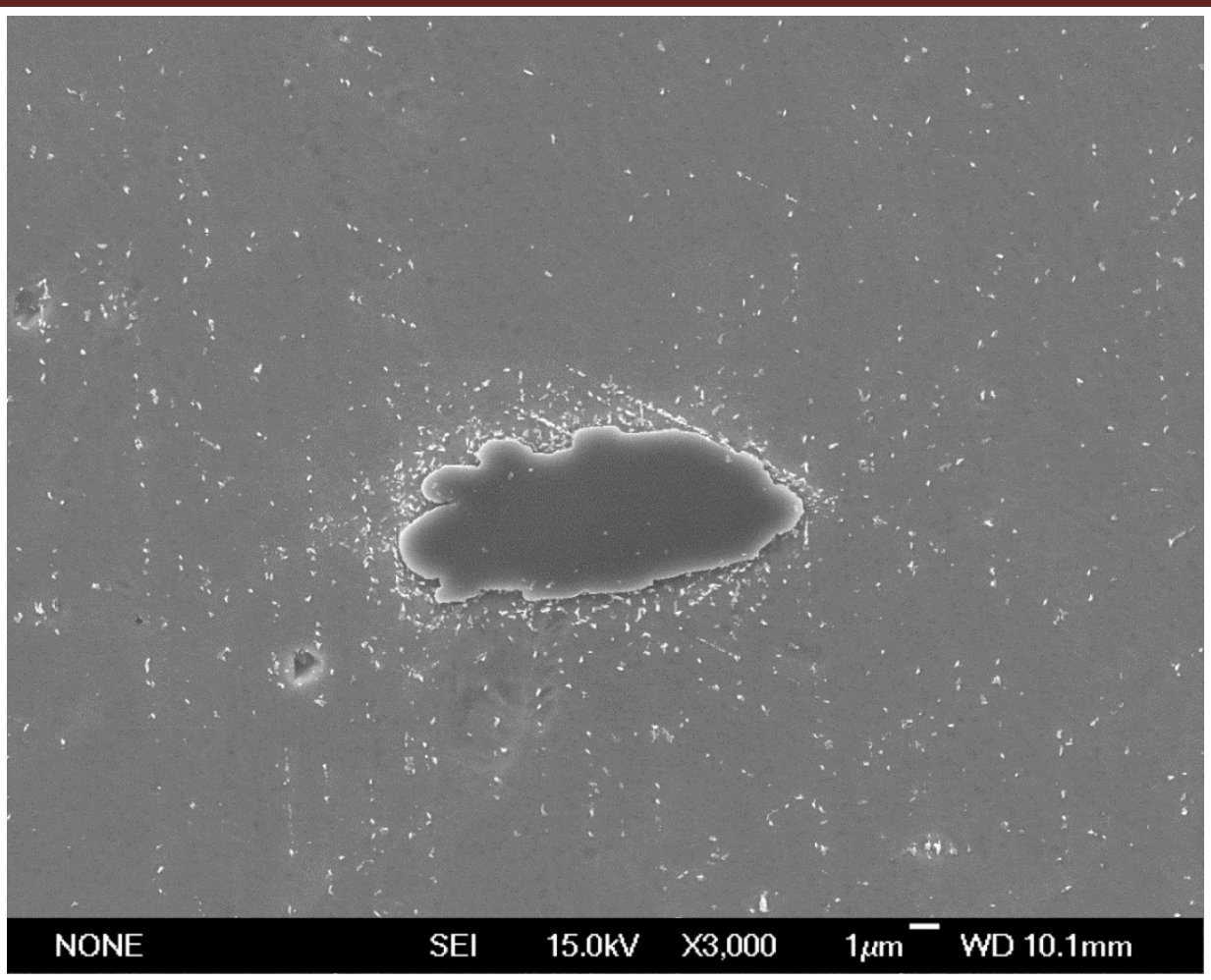

(a)

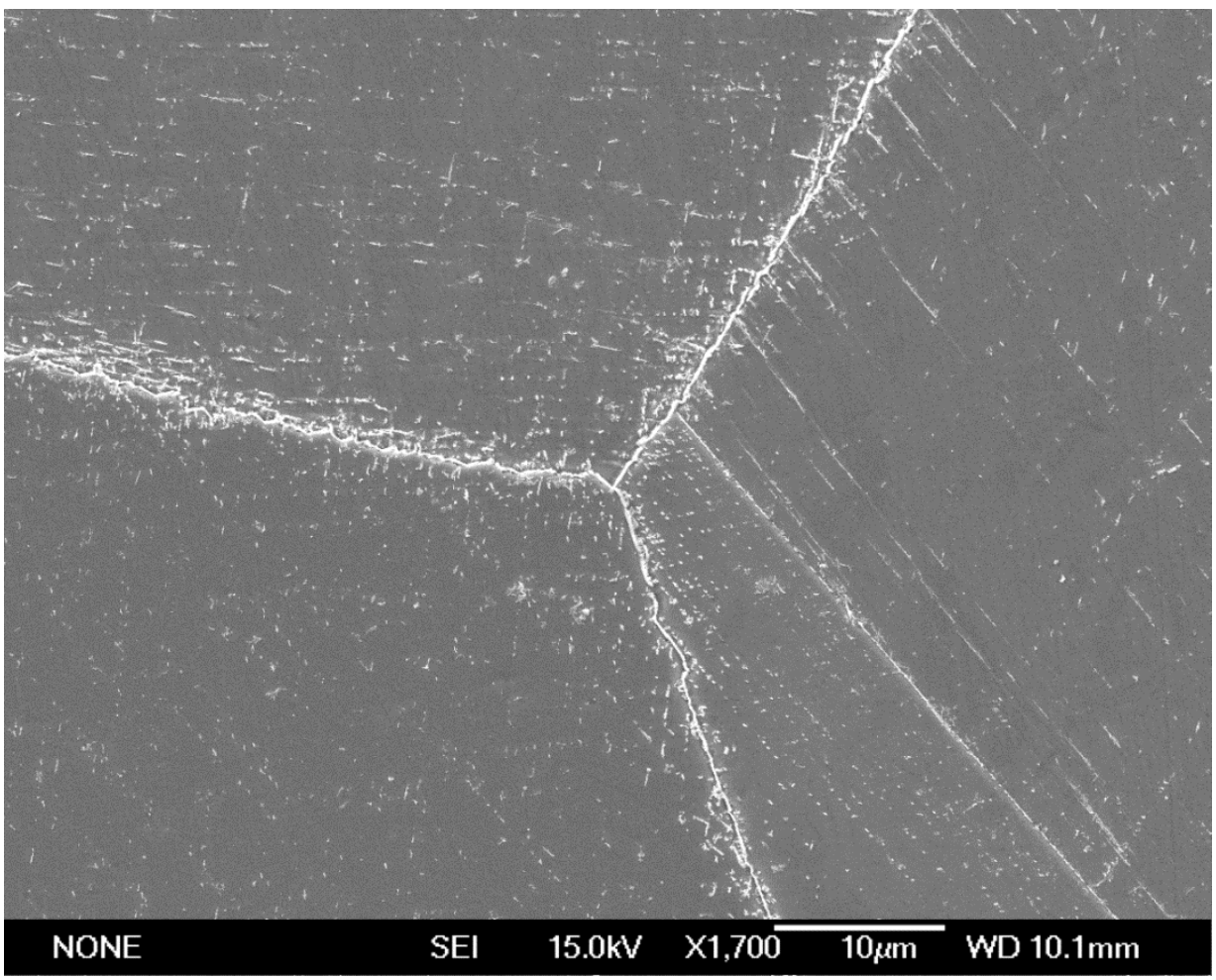

(b)

Fig. 4.79: Cr-rich $\mathrm{M}_{23} \mathrm{C}_{6}$ formed around original (a) Ti(CN), (b) GB precipitates, and (c) intragranular precipitates in Alloy 617 after aging treatment. 
Understanding Fundamental Material-Degradation Processes in High Temperature Aggressive Chemomechanical Environments

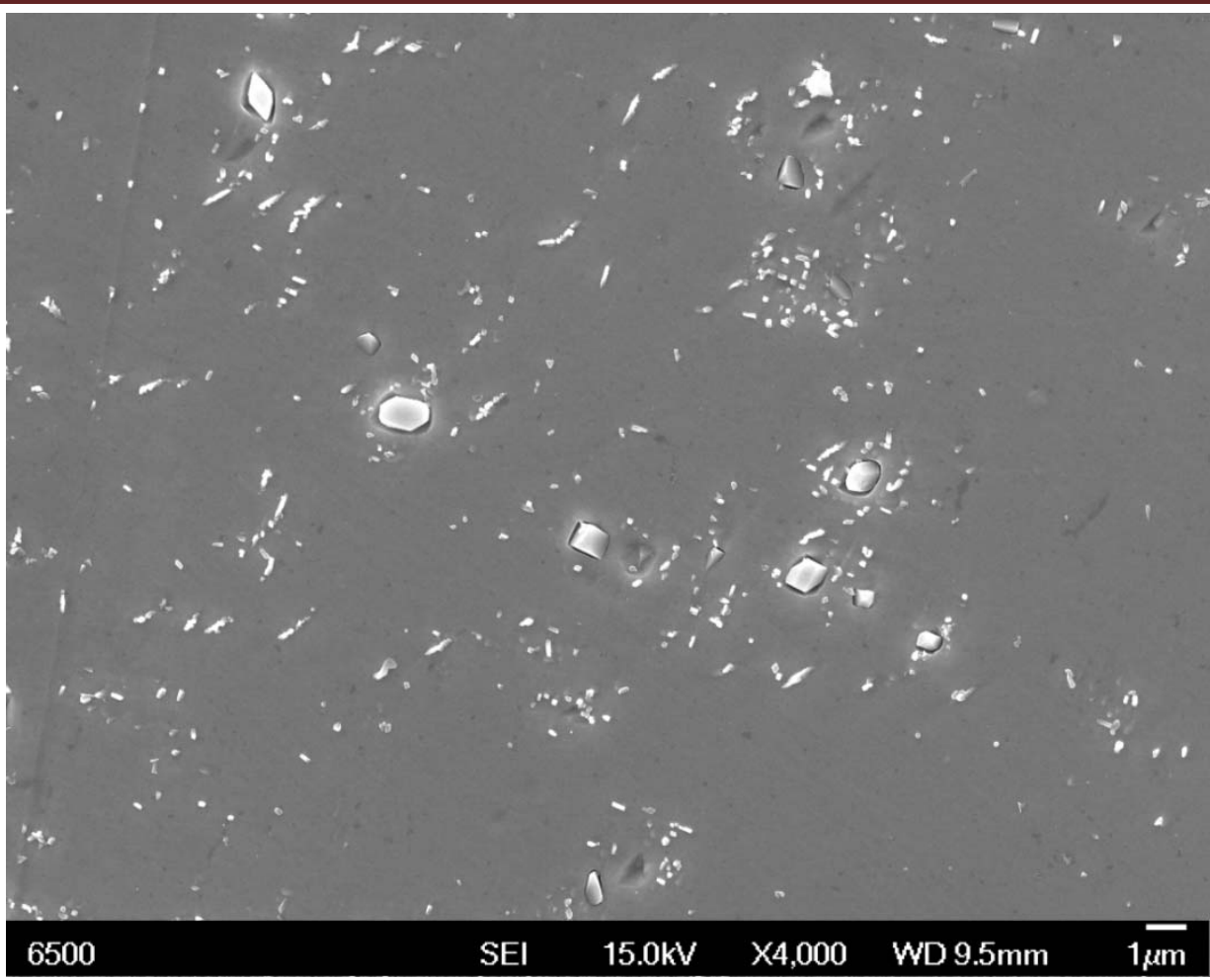

(c)

Fig. 4.79 Cont.

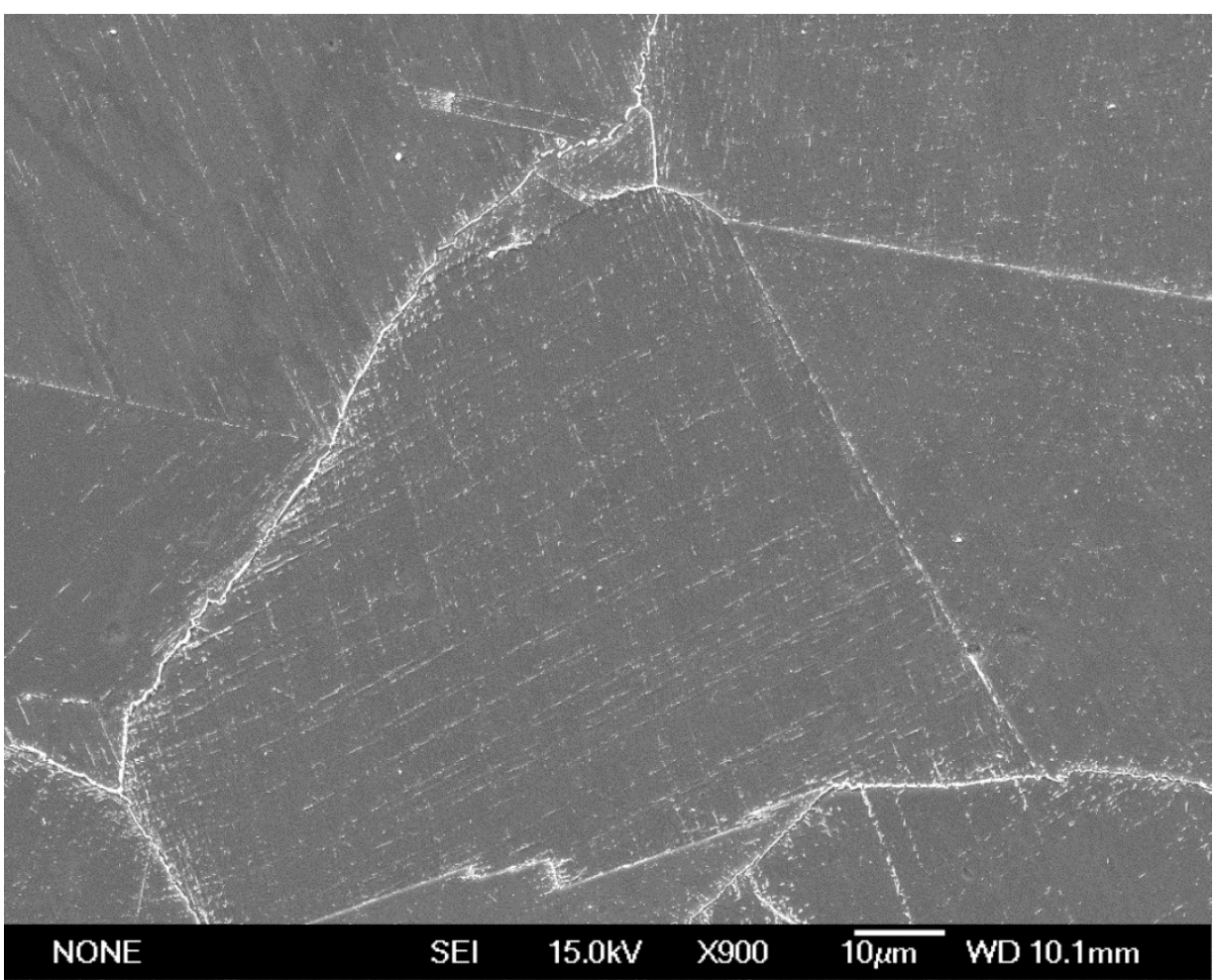

Fig. 4.80: Newly-formed intragranular precipitate particles resembled Widmanstätten structure in the aged Alloy 617 specimens. 
Understanding Fundamental Material-Degradation Processes in High Temperature Aggressive Chemomechanical Environments

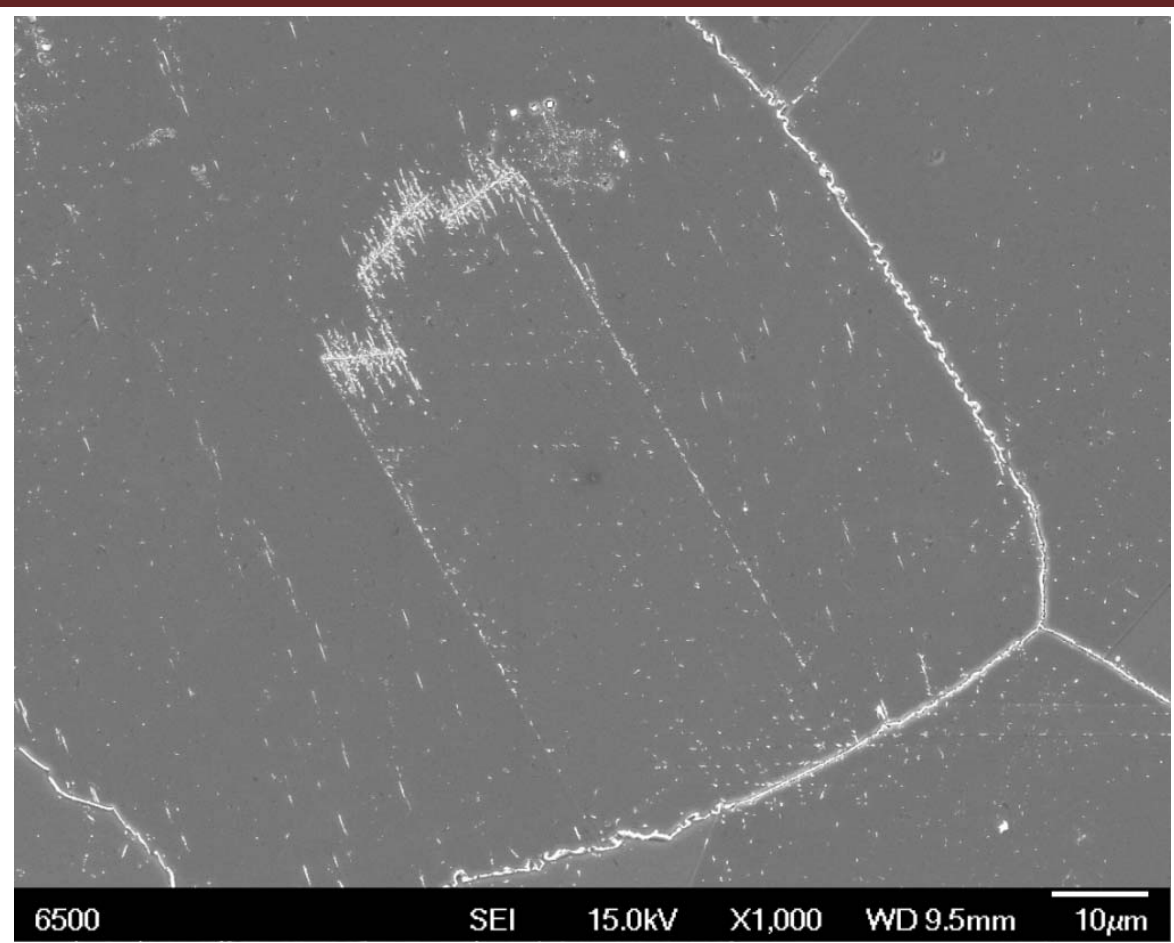

Fig. 4.81: Precipitation morphology on coherent and incoherent twinning boundaries of aged Alloy 617 specimens

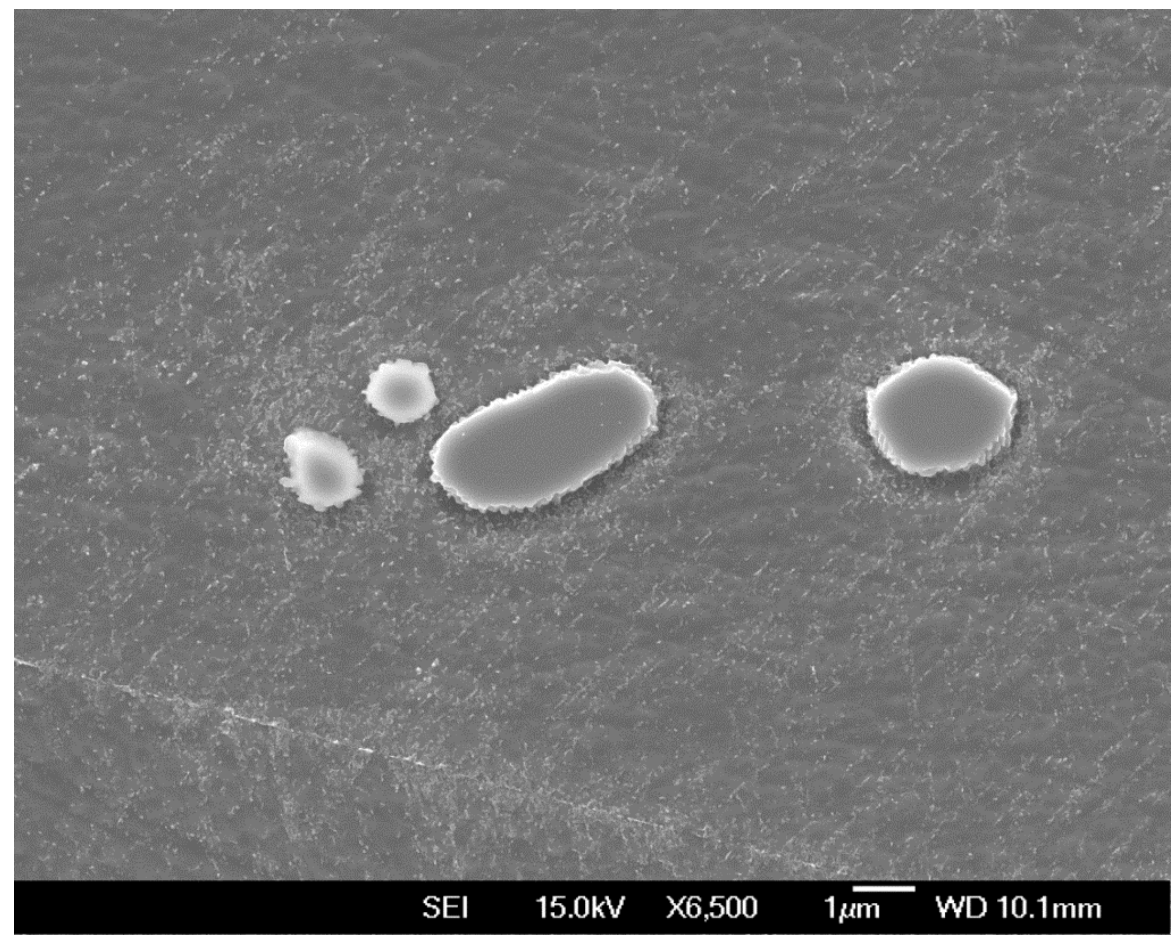

(a)

Fig. 4.82: Precipitate morphology in Alloy 230 after aging treatment. (a) Cr-rich $\mathrm{M}_{23} \mathrm{C}_{6}$ formed around original intragranular precipitate; (b) GB cellular type precipitate; (c) precipitation on twinning boundaries 
Understanding Fundamental Material-Degradation Processes in High Temperature Aggressive Chemomechanical Environments

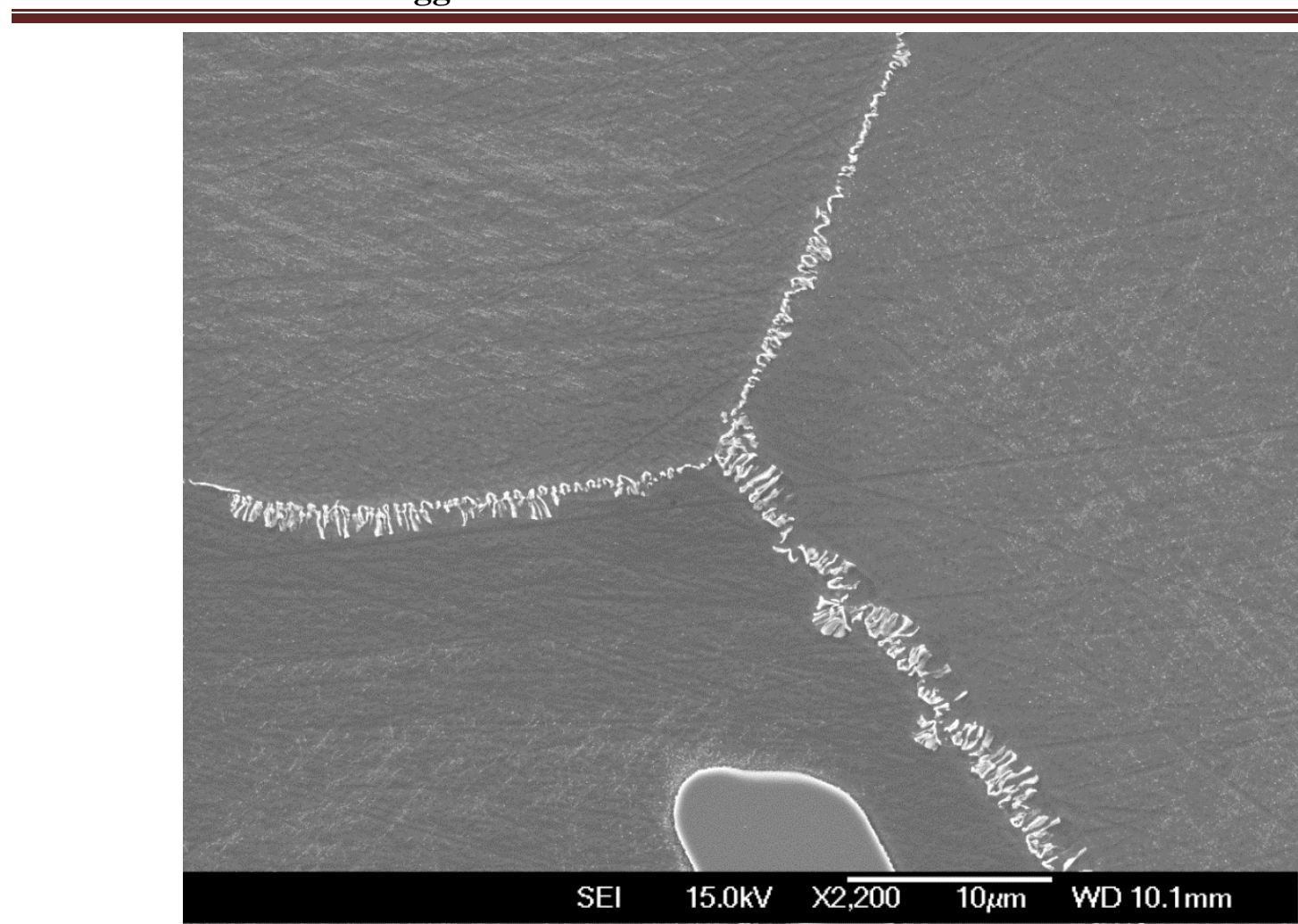

(b)

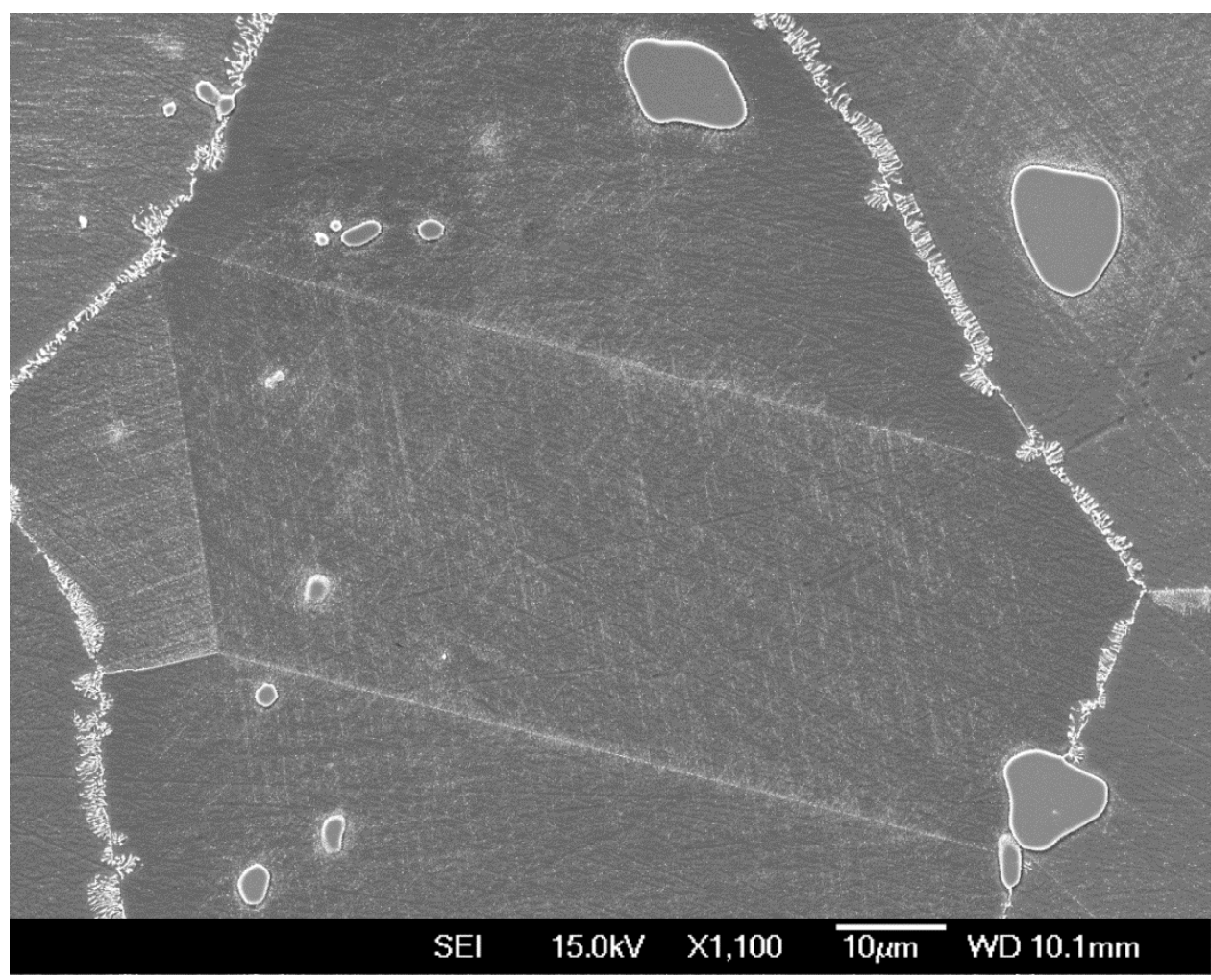

(c)

Fig. 4.82 Cont. 
Understanding Fundamental Material-Degradation Processes in High Temperature Aggressive Chemomechanical Environments

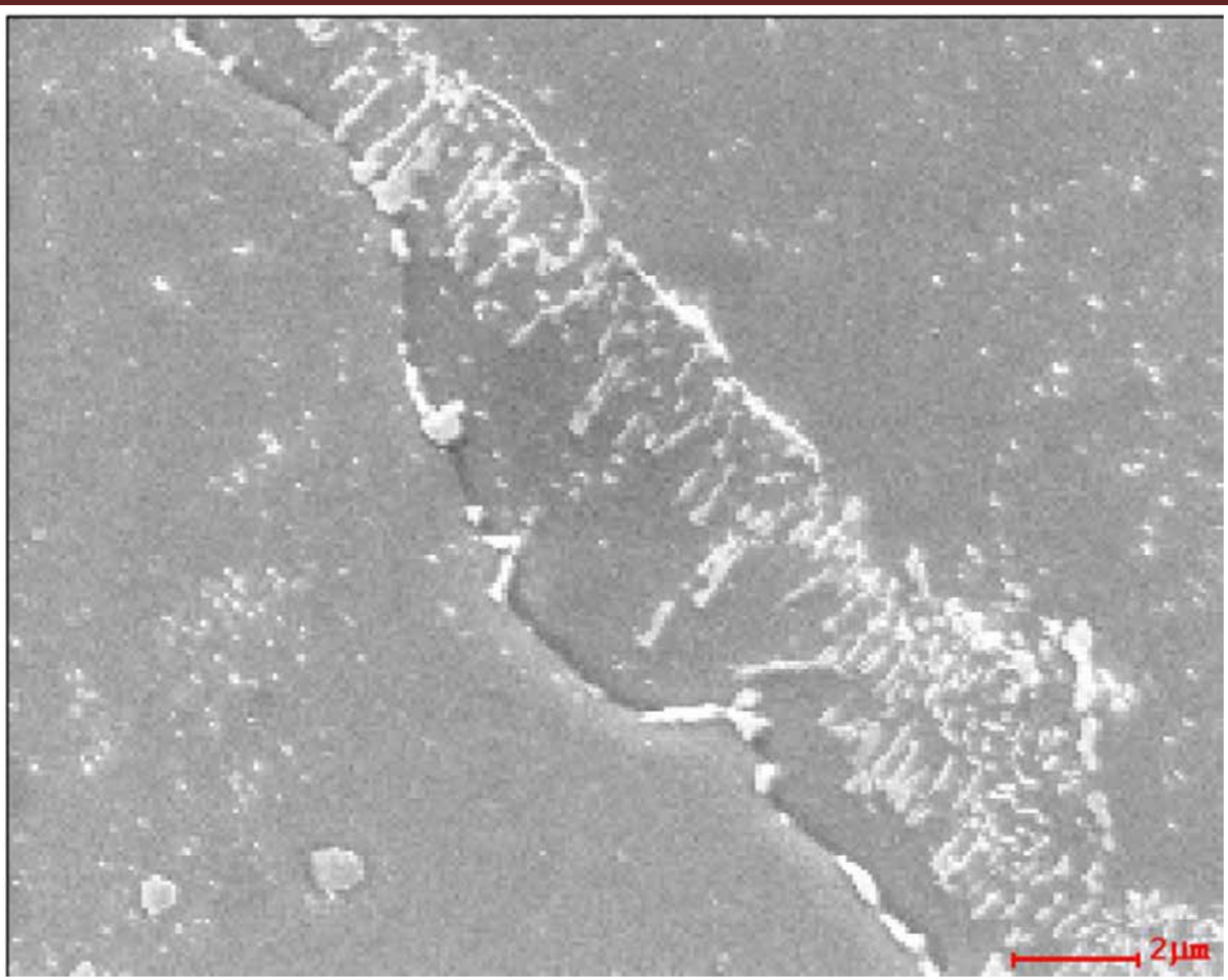

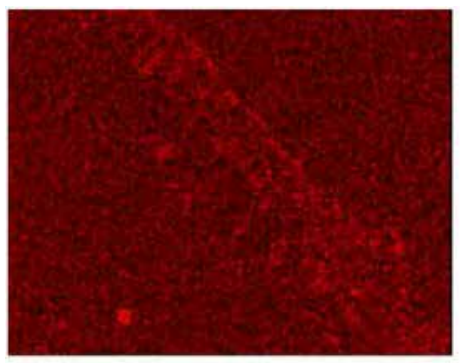

C

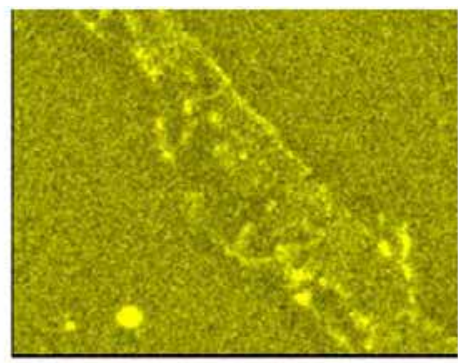

$\mathrm{Cr}$

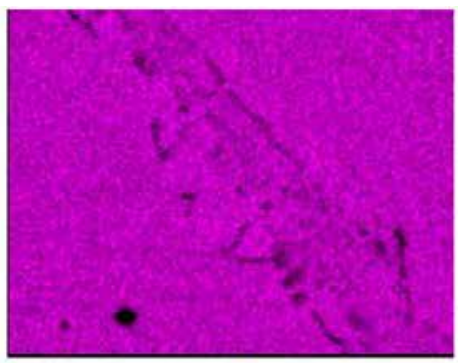

Ni

Fig. 4.83: EDS mapping showing that GB cellular precipitates in Alloy 230 were mainly Cr-rich carbides 
Understanding Fundamental Material-Degradation Processes in High Temperature Aggressive Chemomechanical Environments

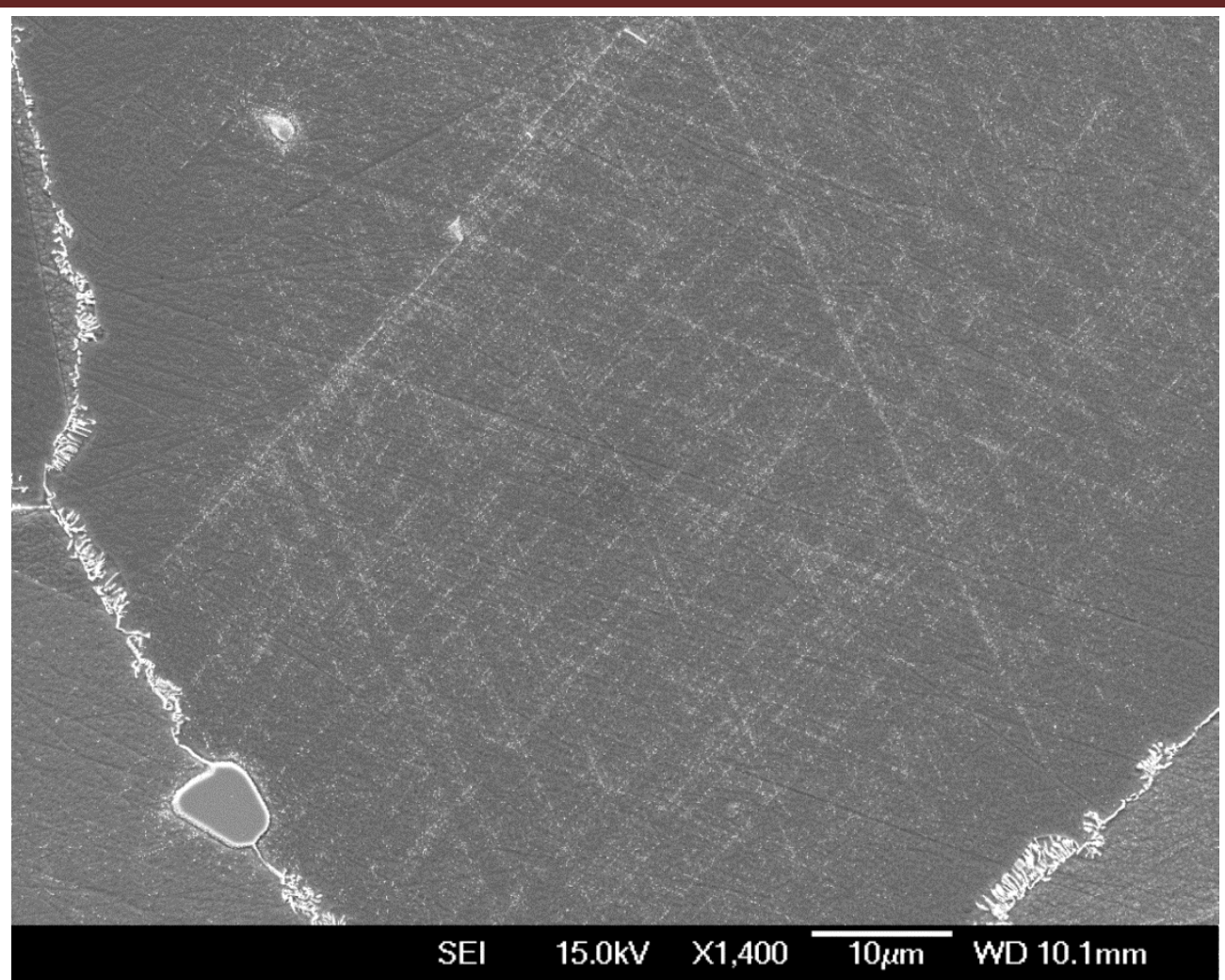

Fig. 4.84: Intragranular Widmanstätten structure precipitate in aged Alloy 230

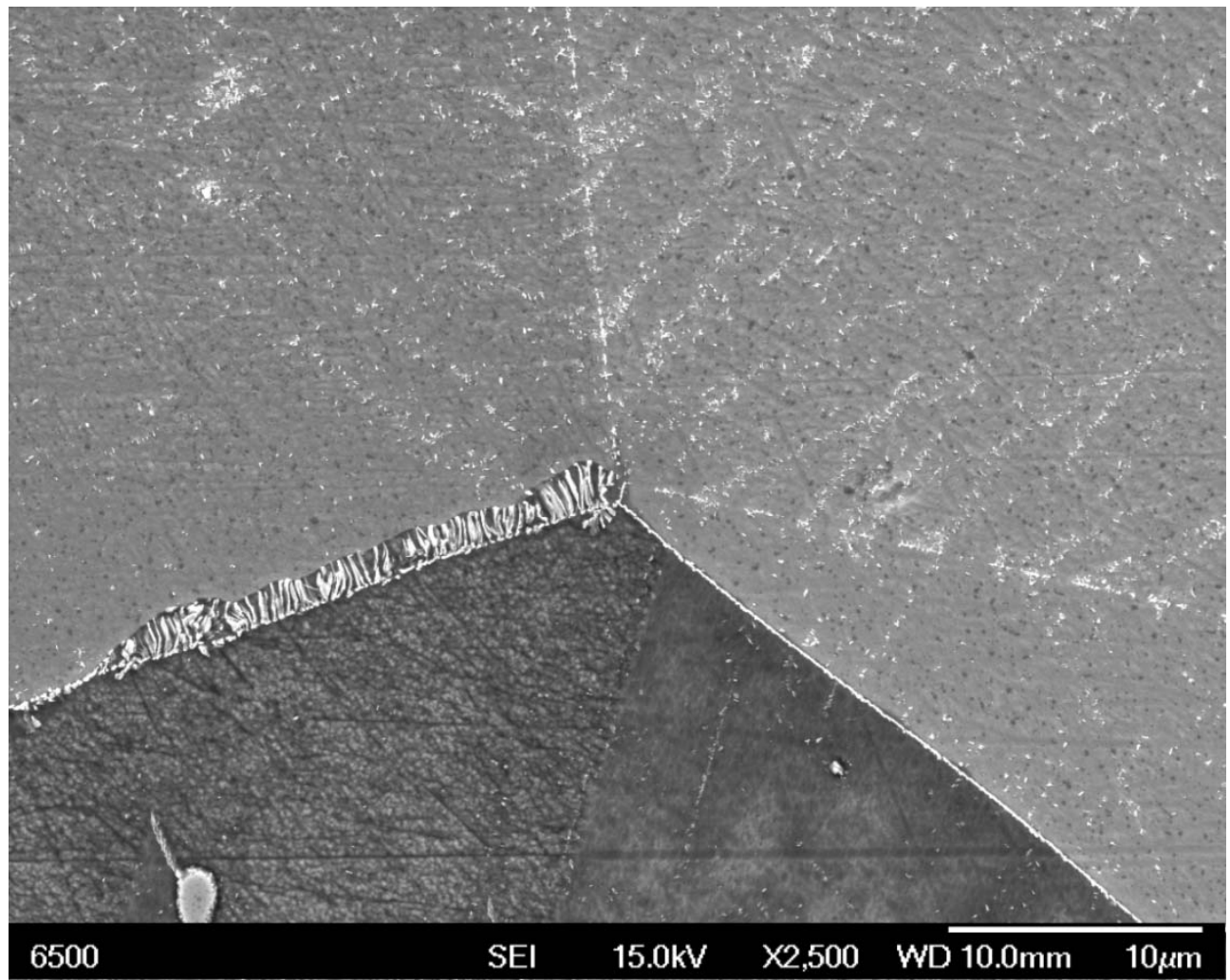

Fig. 4.85: Influence of GB character on precipitate morphology in aged Alloy 230 


\section{Understanding Fundamental Material-Degradation Processes in High Temperature}

Aggressive Chemomechanical Environments

\subsubsection{LCF-tested materials}

The microstructural changes that occurred in both materials after LCF testing are highlighted in this section. Regardless of the chosen total stain range during the LCF tests, classic transgranular crack initiation and propagation were observed for Alloy 617 specimens, with one example shown in Fig. 4.86 (The determination of the cracking mode in LCF and creep-fatigue tests was checked with inverse pole figures from EBSD analysis, which will be addressed later). The transgranular cracking was perpendicular to the principle stress axis. Such a fatigue fracture mode is called a Stage II fracture, and is usually in conjunction with the wavy slip character of the material [4.47]. In Ni-based superalloys, the temperature and strain rate conditions necessary to attain wavy slip have been determined quantitatively. Under the current test conditions ( $\mathrm{T}=850$ ${ }^{\circ} \mathrm{C}, \dot{\varepsilon}=2.5 \mathrm{E}-4 / \mathrm{s}$ ), wavy slip was expected [4.59]. Because of the elevated temperature and air environment, oxidation on the specimen surface and crack opening was visible after the LCF tests, as shown in Fig. 4.87(a). Based on EDS analysis, the top gray scale in Fig. 4.87(a) was enriched with $\mathrm{Cr}$, and the dark, finger-shaped region was enriched with Al. On occasion, voids developed at the interface between the surface oxide and the metal substrate, as illustrated in Fig. 4.87(b). These voids might become a site for early crack initiation. As presented in Fig. 4.88, a new precipitate phase, Mo-rich $\mathrm{M}_{6} \mathrm{C}$, was found at the $\mathrm{GB}$ region of Alloy 617 after all LCF tests. Rao et al. claim that there is an incubation time for $\mathrm{M}_{6} \mathrm{C}$ precipitate formation in Alloy 617 [4.35]. Moreover, plastic deformation during LCF tests may promote dissociation of Cr-rich $\mathrm{M}_{23} \mathrm{C}_{6}$ and the formation of Mo-rich $\mathrm{M}_{6} \mathrm{C}$, as suggested in the work of Jo et al [4.60]. Thus, the $\mathrm{M}_{6} \mathrm{C}$ precipitate was absent in the as-received and 45-minute aged specimen, since the material had not remained long enough at the elevated temperature, and no plastic deformation was experienced by the material. Inside the material, Fig. 4.89 shows the fracture of $\mathrm{Ti}(\mathrm{CN})$ and the void formation associated with the GB and intragranular precipitates. The void formation was observed in Alloy 617 specimens only when the test total strain range was $1.0 \%$ or $1.5 \%$. The GB carbides also coarsened, and their distribution on the GBs became discontinuous, as seen in Fig. 4.89(b). The frequency of these voids was still scarce, and they were not able to coalesce to form cracks, hence these defects should not influence material performance for short term LCF tests. Nevertheless, for materials subject to long-term loads at high temperatures, these defects cannot be ignored. 
Understanding Fundamental Material-Degradation Processes in High Temperature Aggressive Chemomechanical Environments

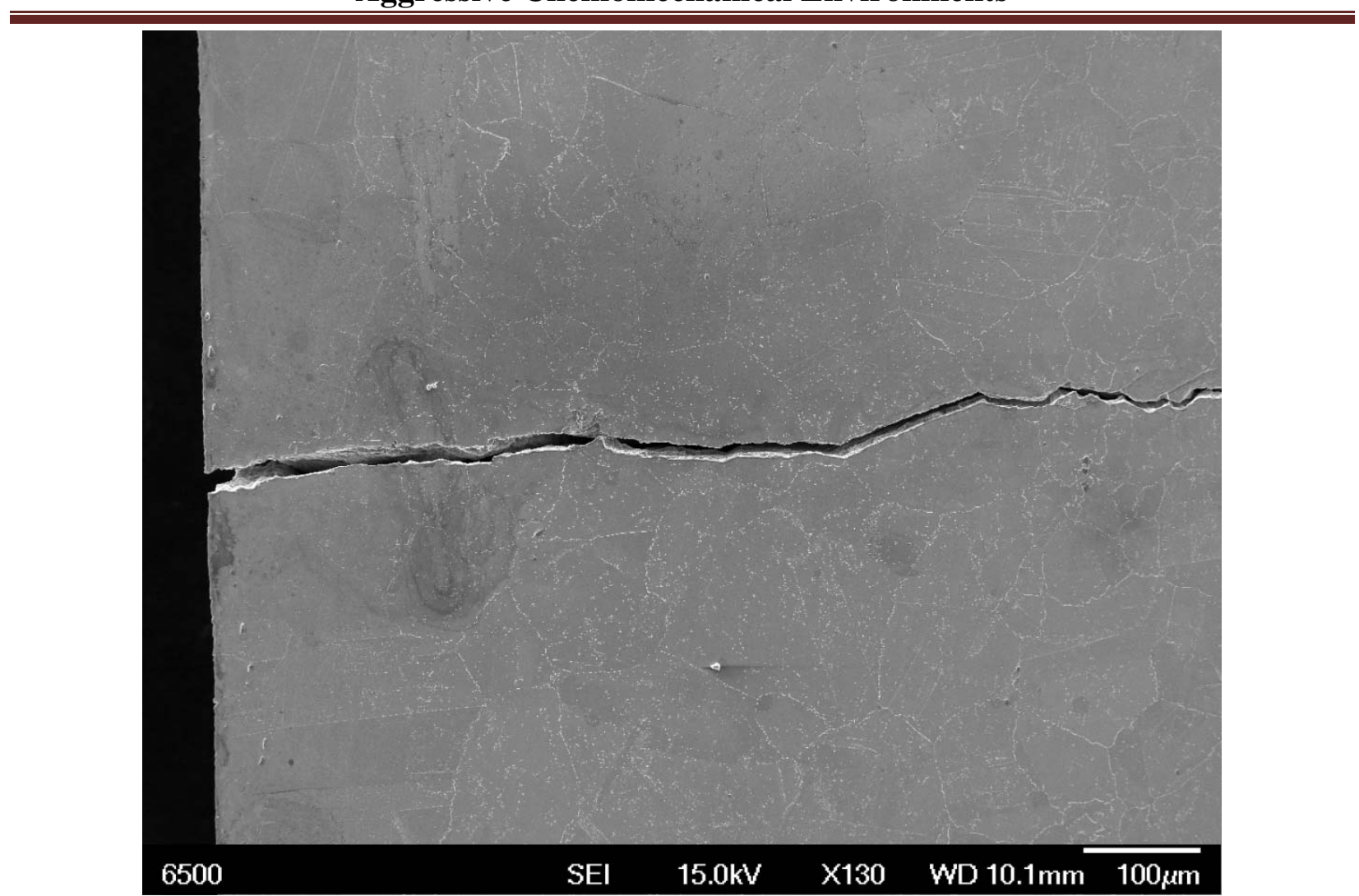

Fig. 4.86: Transgranular crack initiation and propagation in Alloy 617 during LCF tests. $\varepsilon_{\text {tot }}=0.5 \%$ 
Understanding Fundamental Material-Degradation Processes in High Temperature Aggressive Chemomechanical Environments

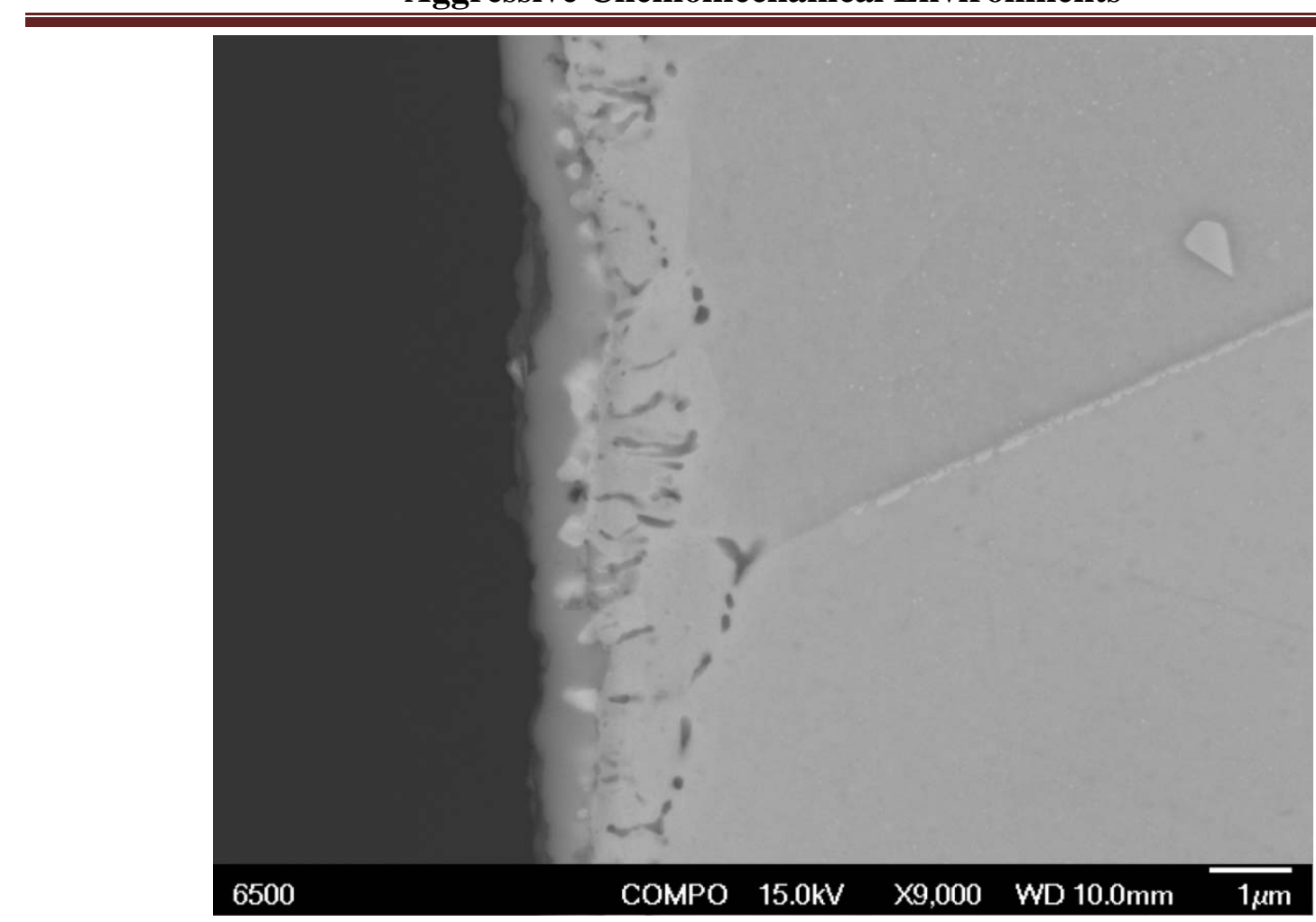

(a)

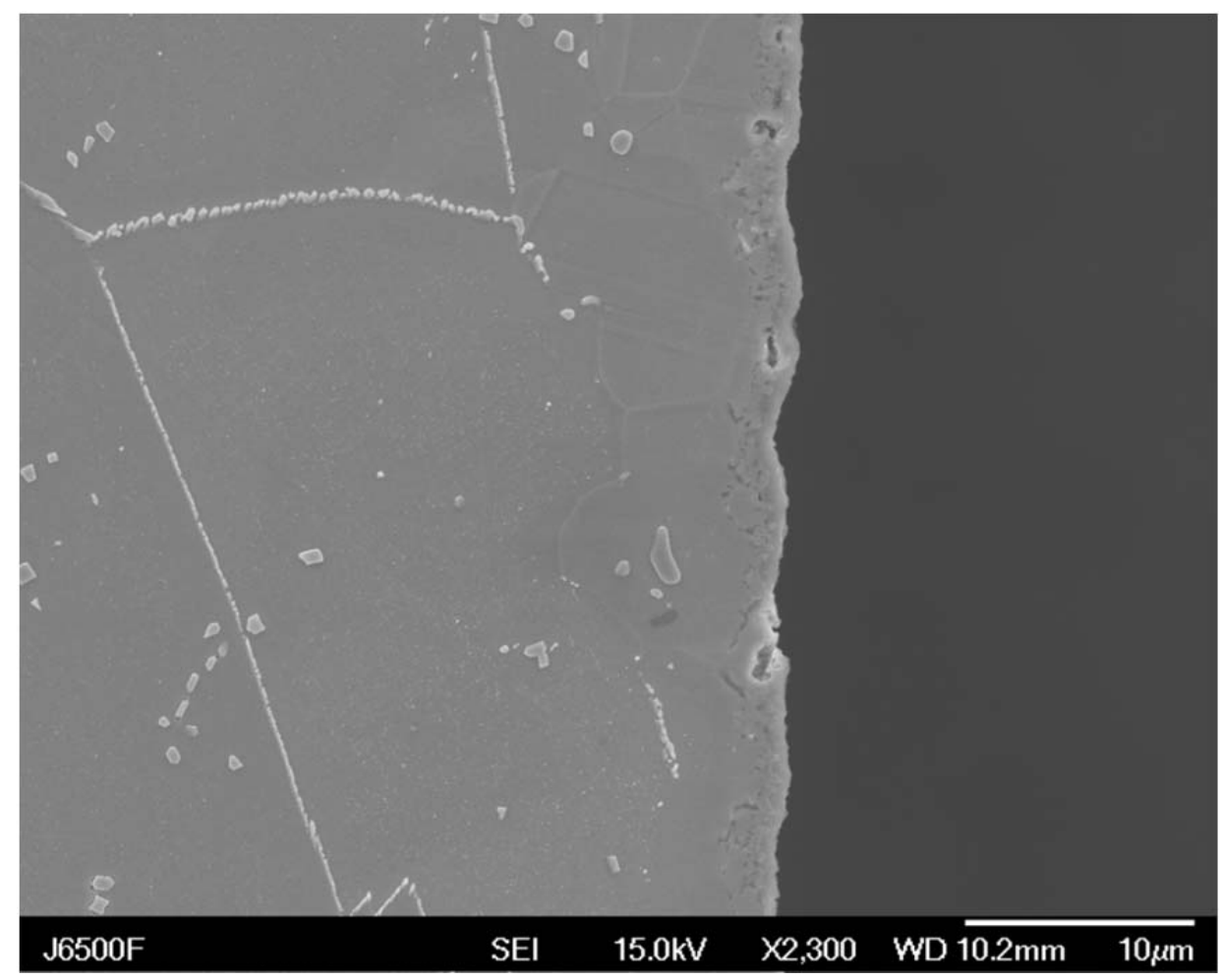

(b)

Fig. 4.87: (a) Oxidation on Alloy 617 after LCF tests; (b) voids developed at the interface of the surface oxide and metal substrate. $\varepsilon_{\text {tot }}=1.0 \%$ 
Understanding Fundamental Material-Degradation Processes in High Temperature Aggressive Chemomechanical Environments
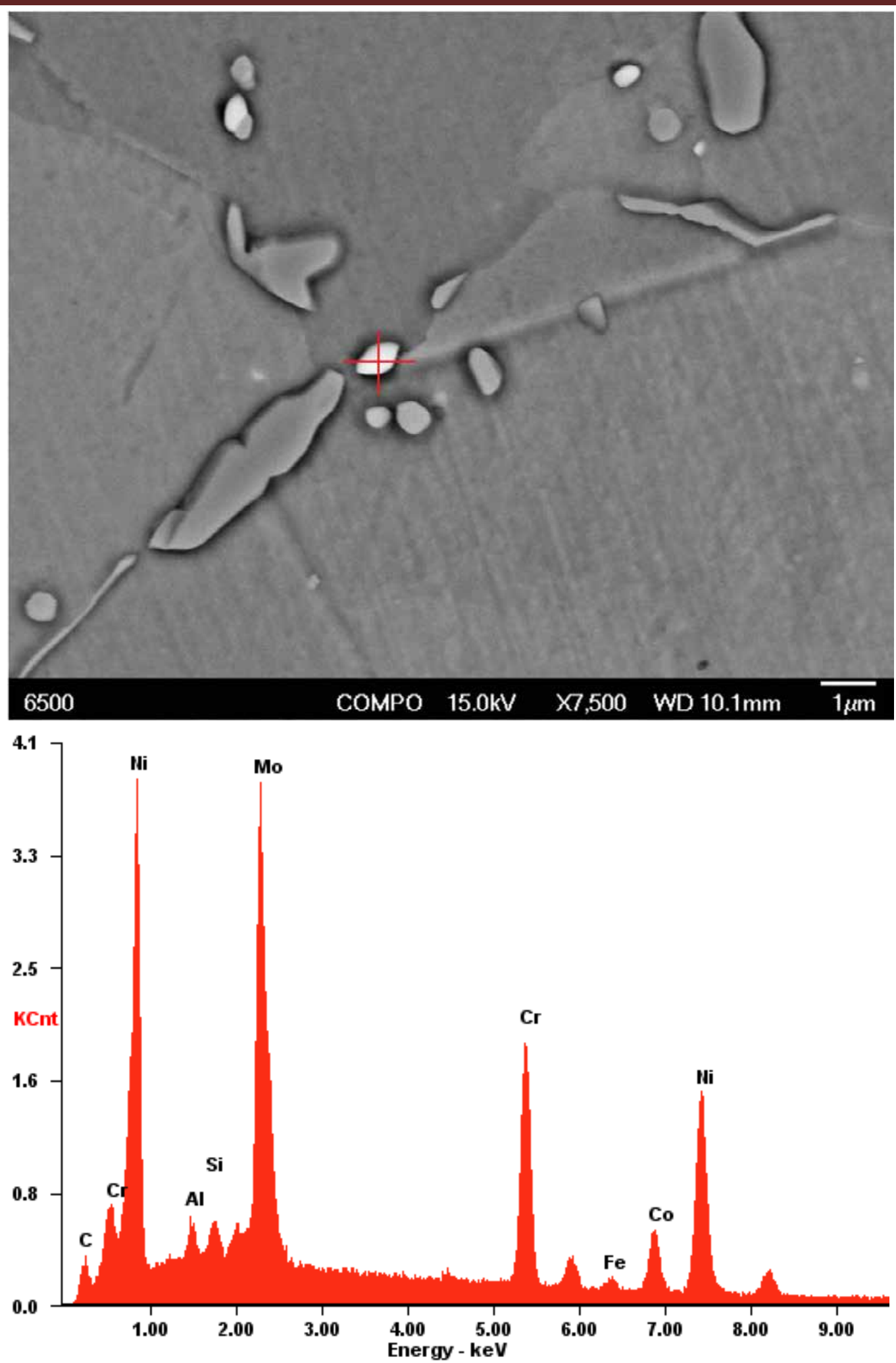

Fig. 4.88: Mo-rich $\mathrm{M}_{6} \mathrm{C}$ formed at the GB region of Alloy 617 after the LCF test. The EDS spectrum was taken at the marked point. $\varepsilon_{\text {tot }}=0.5 \%$ 
Understanding Fundamental Material-Degradation Processes in High Temperature Aggressive Chemomechanical Environments

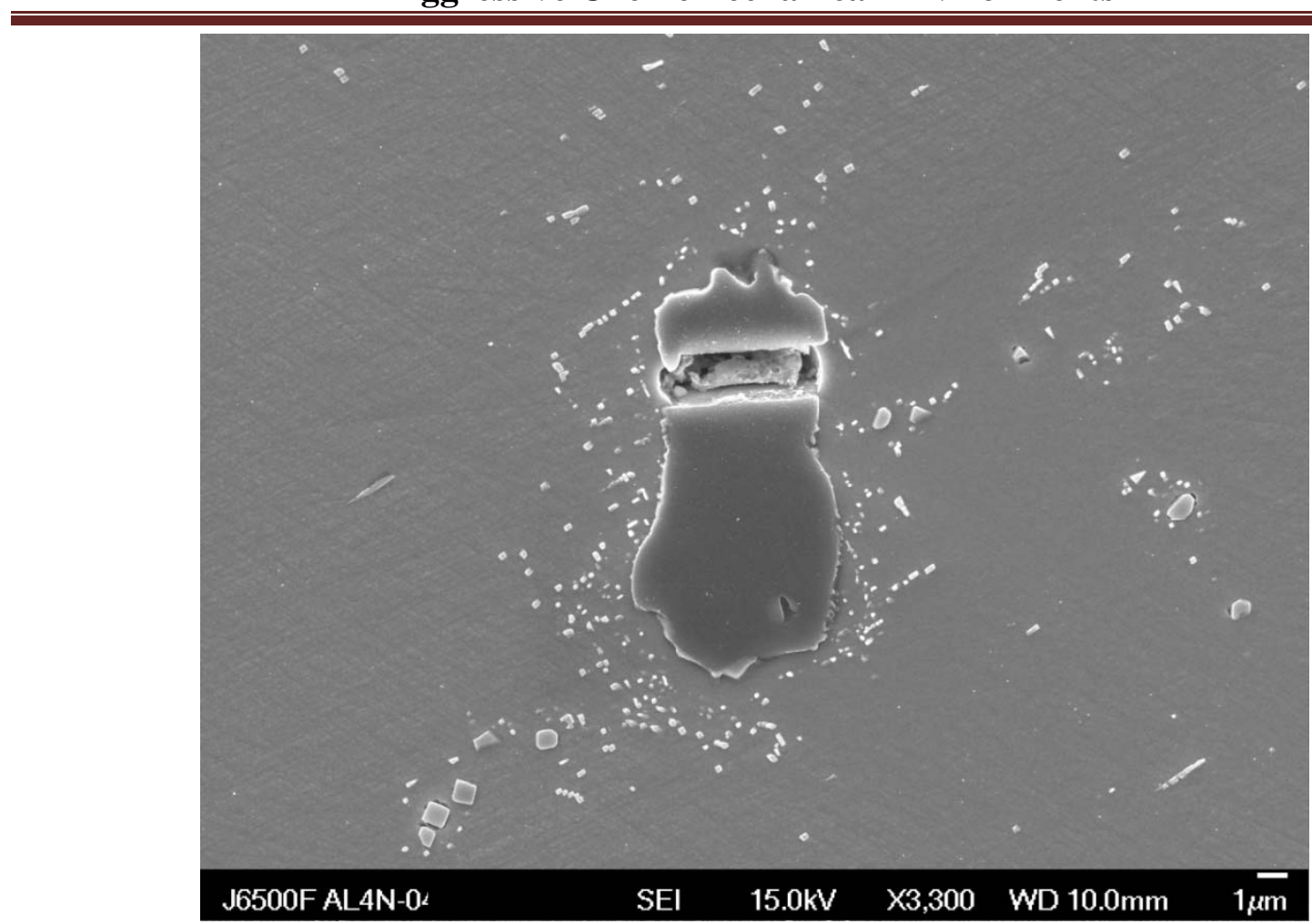

(a)

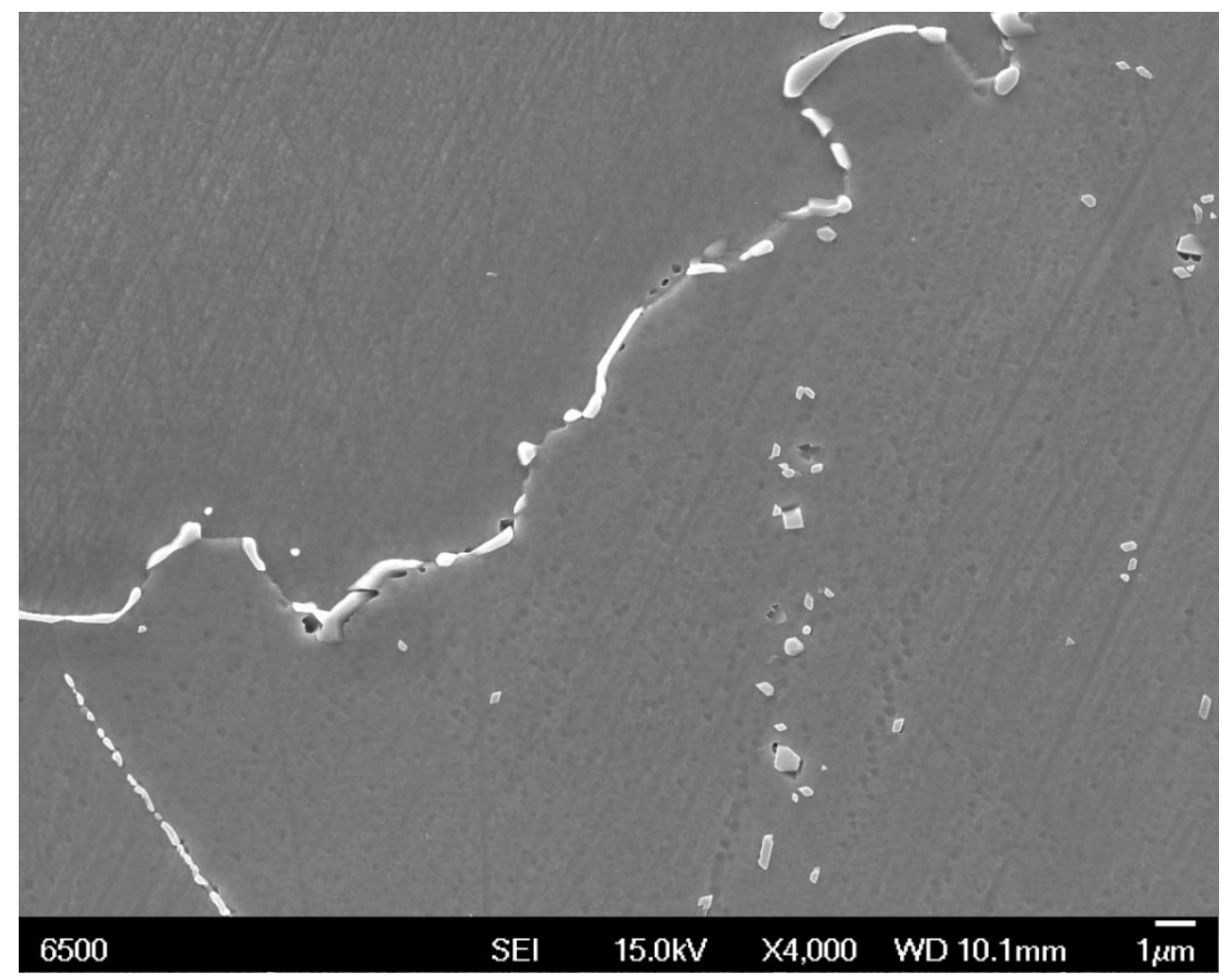

(b)

Fig. 4.89: (a) Fraction of $\operatorname{Ti}(\mathrm{CN})$ in Alloy 617 after the LCF test. $\varepsilon_{\text {tot }}=1.0 \%$; (b) void formation associated with GB and intragranular precipitates. $\varepsilon_{\text {tot }}=1.5 \%$ 


\section{Understanding Fundamental Material-Degradation Processes in High Temperature Aggressive Chemomechanical Environments}

Similar to Alloy 617, transgranular crack initiation and propagation were observed in Alloy 230 specimens for all the LCF tests. One example is exemplified in Fig. 4.90. During the LCF tests, a thin oxide layer enriched with $\mathrm{Cr}$ and $\mathrm{Al}$ developed on the specimen surface, as seen in Fig. 4.91. It was found that for the LCF test with the largest total strain range, $1.5 \%$, in some cases, the crack path would follow GBs. For instance, in Fig. 4.92, the red arrow points out the crack path along the GB. GB cellular precipitates were found in Alloy 230 after exposure to the elevated temperature. The formation of cells of $\mathrm{M}_{23} \mathrm{C}_{6}$ lamellae results in a significant decrease in the high temperature ductility of Ni-based alloys [4.61]. Moreover, precipitation free zones close to the GB cellular precipitates could further enhance the local plasticity concentration. Therefore, the growth of cracks along GBs is possible, especially with the aid of high stress levels at the $1.5 \%$ total strain range LCF test.

Fig. 4.93 presents void formation under cyclic deformation inside the material as a result of fracture of the intragranular precipitates or rupture at the interface between the intragranular precipitates and the matrix. The carbide precipitates were usually brittle, and hence inductive to rupture under high stress. The interface of the matrix and precipitates was also a possible region for strain concentration, due to the strain field across the phase boundary. In contrast to the 45-minute aged Alloy 230 specimens, where GB cellular precipitates were found everywhere in the material, the LCF-tested Alloy 230 specimens showed that GB cellular precipitates could only exist at some distance away from the surface region. Fig. 4.94 depicts this feature, with the red vertical line defining the boundary below which the cellular precipitates could be found. In the work of Burke et al., the lack of cellular precipitation near the surface region was attributed to the oxygen penetration through the surface region [4.62]. Oxygen penetration along the surface-connected GB inhibited GB migration, which was a necessary process for the cellular precipitation to occur. The GB migration associated with the cellular precipitation formation will be addressed further in later sections. 
Understanding Fundamental Material-Degradation Processes in High Temperature Aggressive Chemomechanical Environments

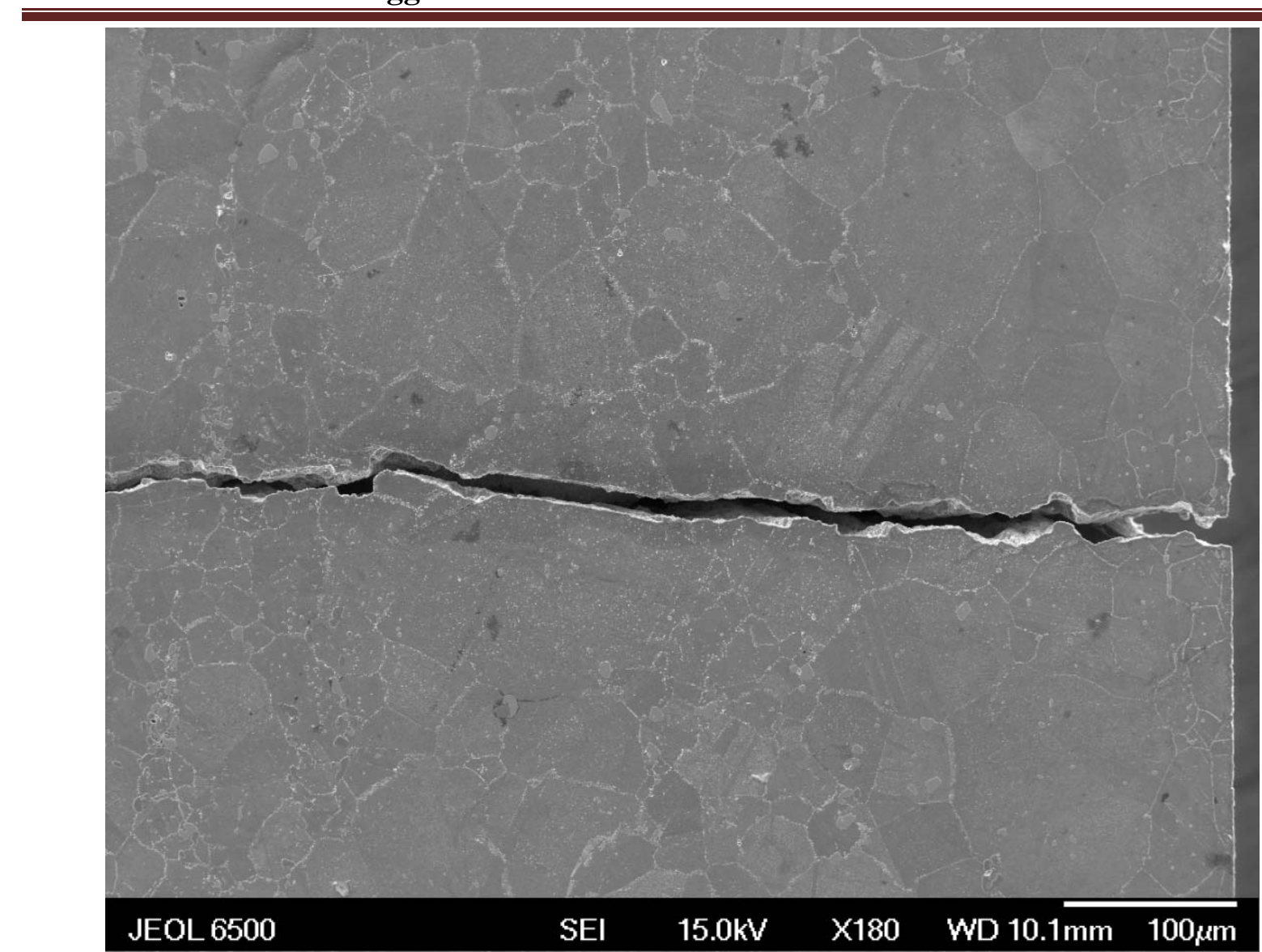

Fig. 4.90: Transgranular crack initiation and propagation in Alloy 230 during LCF tests. $\varepsilon_{\text {tot }}=0.5 \%$ 
Understanding Fundamental Material-Degradation Processes in High Temperature Aggressive Chemomechanical Environments
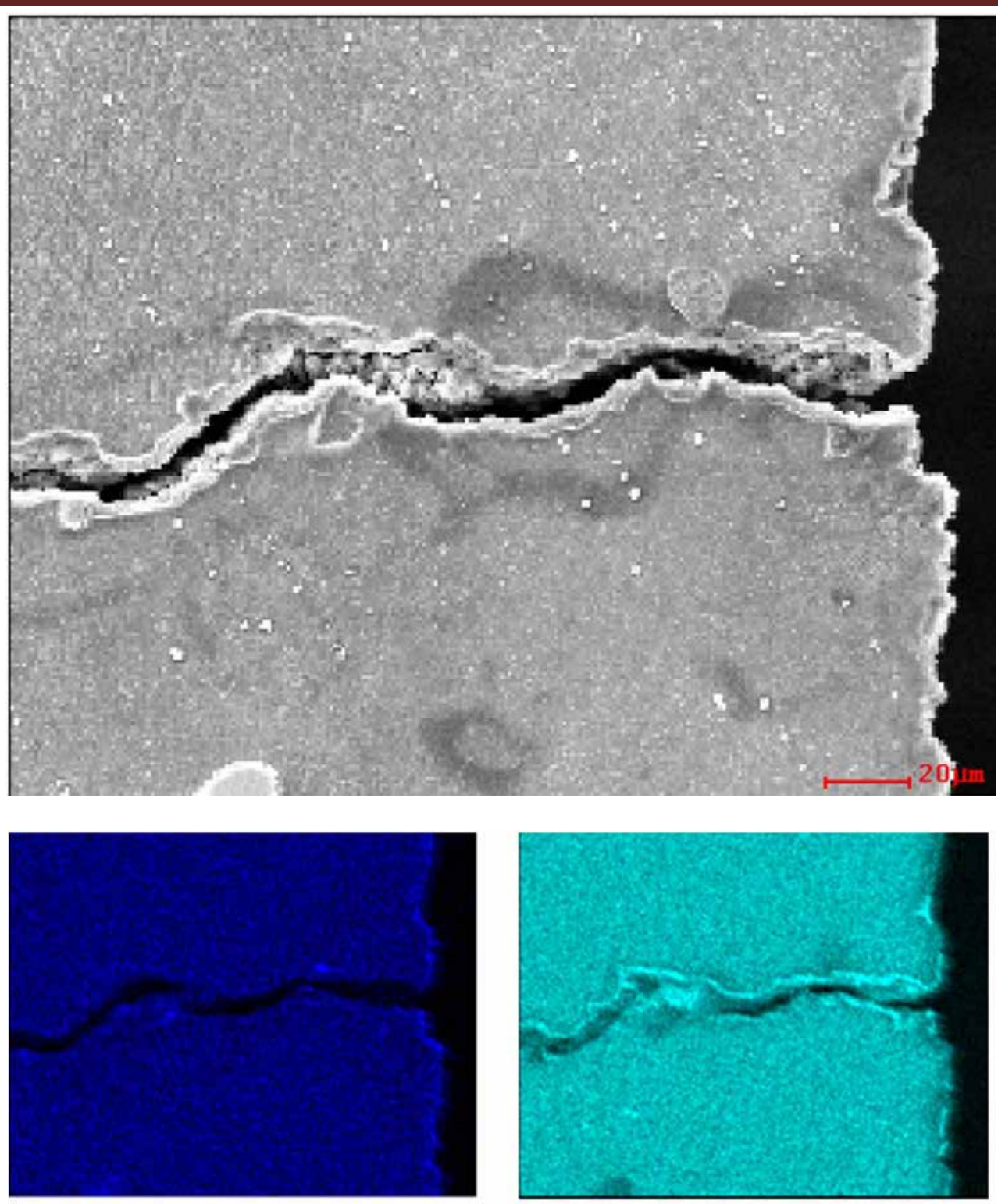

Al

$\mathrm{Cr}$

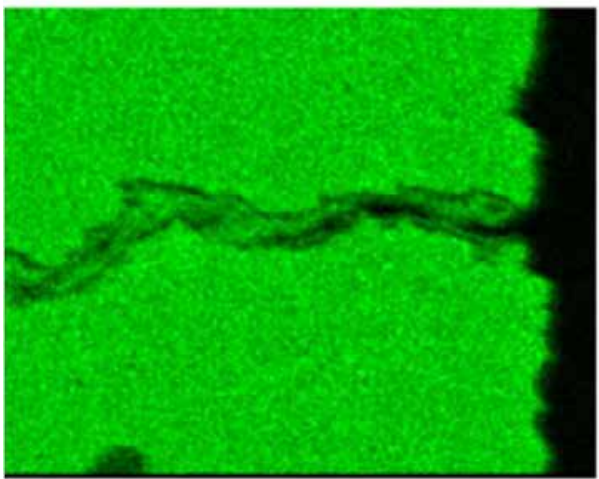

Ni
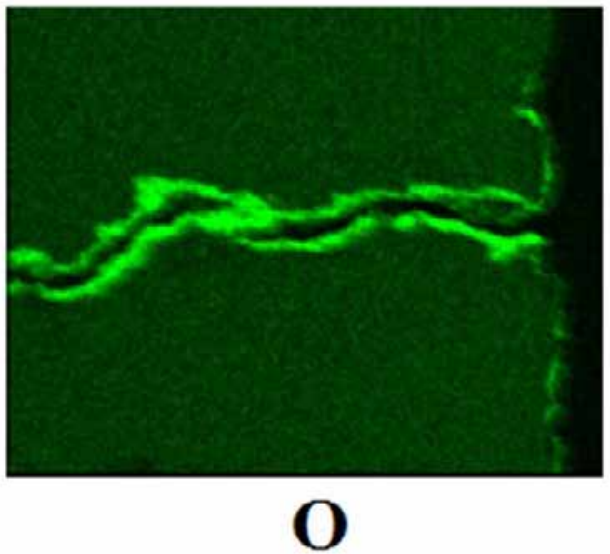

Fig. 4.91: Surface oxidation on a LCF tested Alloy 230 specimen. $\varepsilon_{\text {tot }}=1.0 \%$ 
Understanding Fundamental Material-Degradation Processes in High Temperature Aggressive Chemomechanical Environments

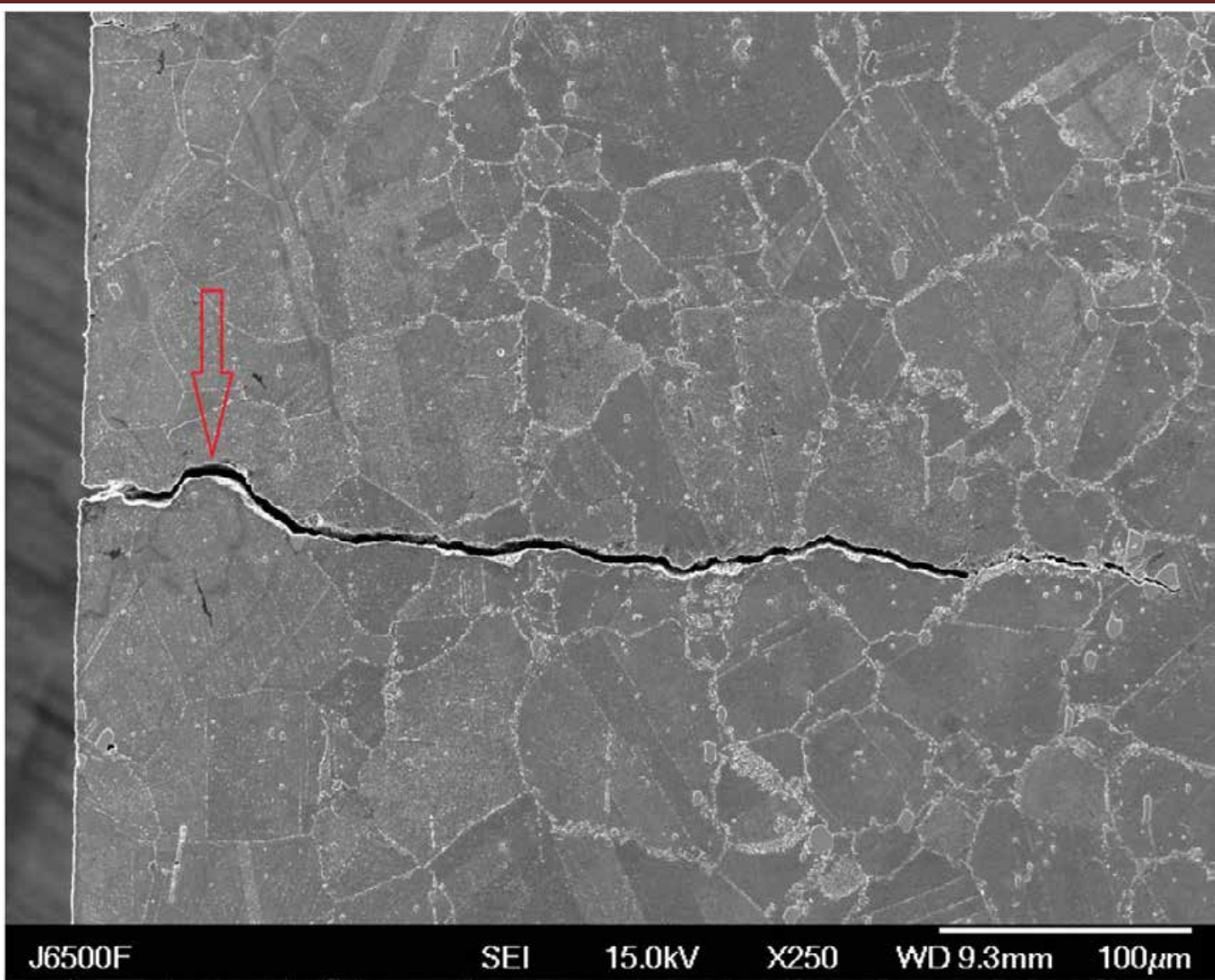

Fig. 4.92: The transgranular crack path could sometimes follow the GB in the $1.5 \%$ total strain range LCF tested Alloy 230. 


\section{Understanding Fundamental Material-Degradation Processes in High Temperature}

Aggressive Chemomechanical Environments

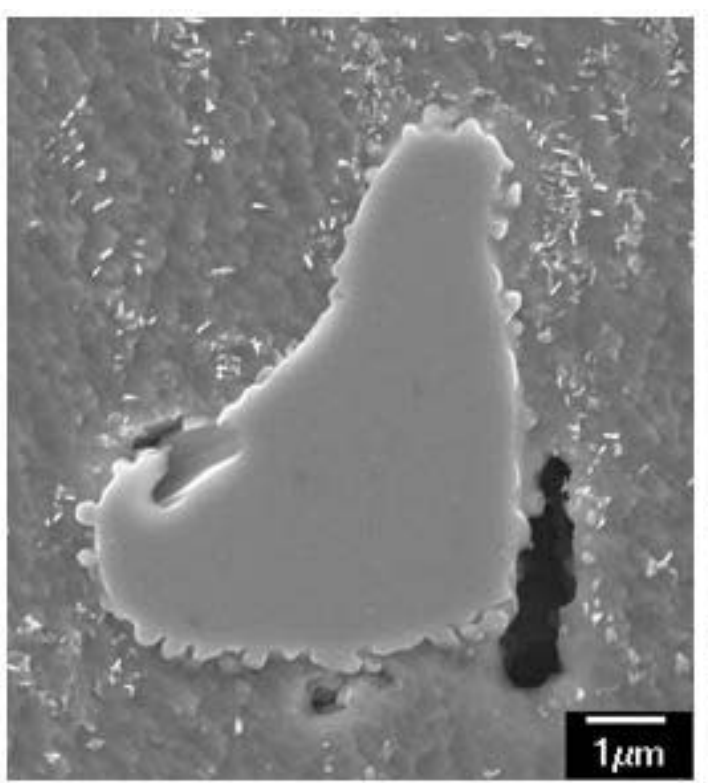

(a)

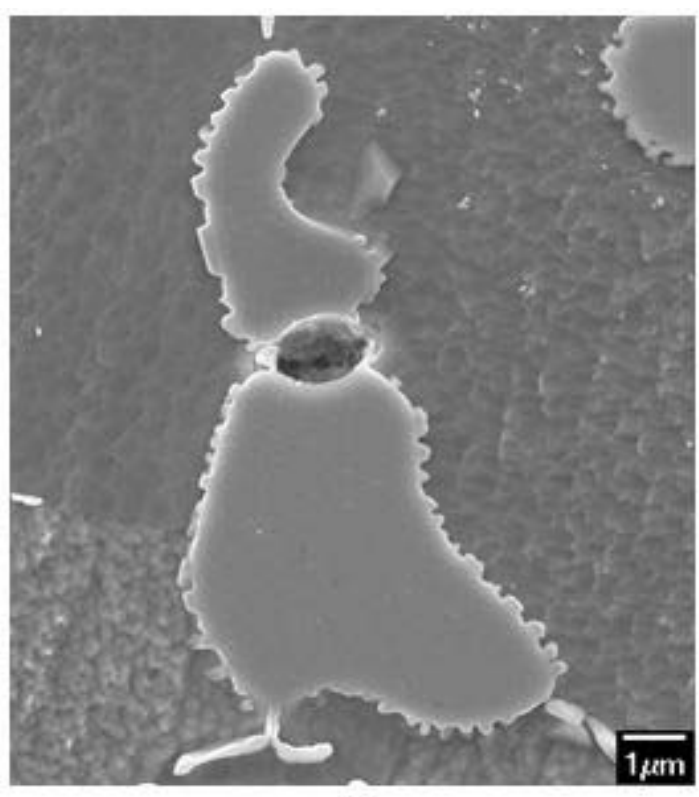

(b)

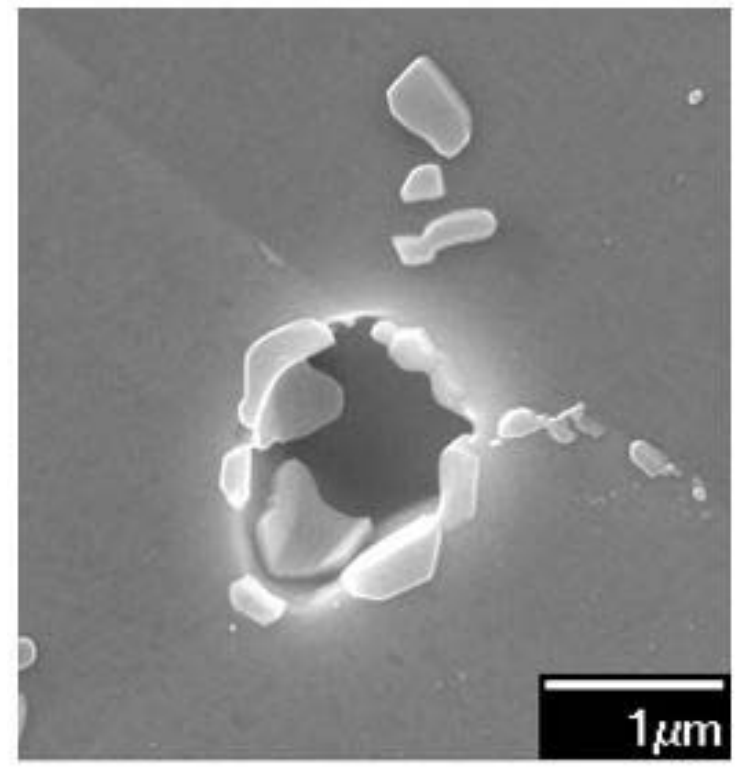

(c)

Fig. 4.93: Void development in Alloy 230 due to (a) rupture at the interface of the matrix and the large intragranular precipitate, (b) fracture of the large intragranular precipitate itself, and (c) fracture of small intragranular precipitates. $\varepsilon_{\text {tot }}=1.0 \%$ 


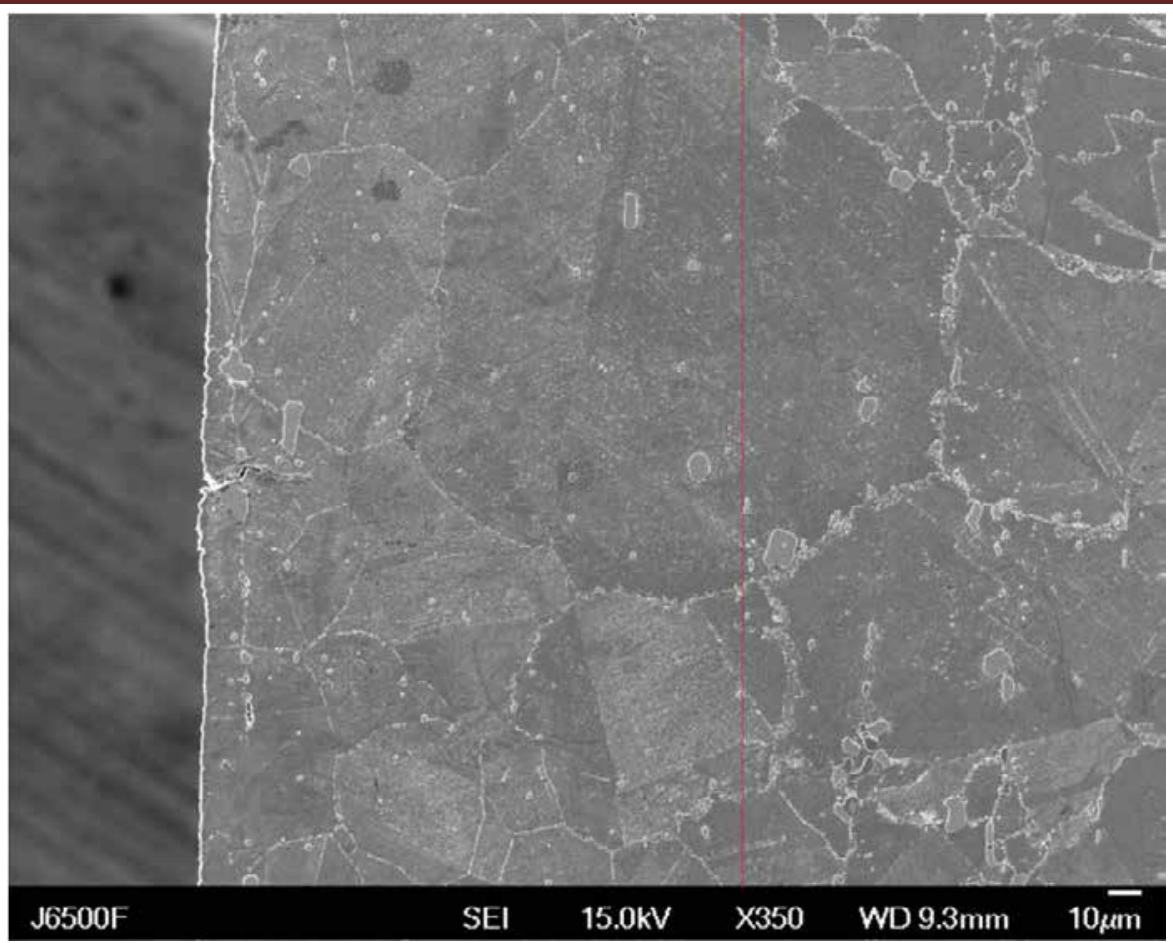

Fig. 4.94: Disappearance of GB cellular precipitates near the surface region of Alloy 230. $\varepsilon_{\text {tot }}=1.5 \%$

\subsubsection{Creep-fatigue tested materials}

Microstructures that lead to a reduced fatigue life of Alloy 617 and Alloy 230 in creep-fatigue tests are studied in this section. For the creep-fatigue tests of Alloy 617 with a 3 minute dwell time at peak tensile strain, cracks initiated and propagated mostly in an intergranular manner, as exemplified in Fig. 4.95(a) for the 0.5\% total strain range.

Meanwhile, cracks initiated intergranularly, but still propagated transgranularly, for the larger total strain range tests, i.e. $1.0 \%$ and $1.5 \%$, as shown in Fig. 4.95(b). In general, intergranular cracking occurs at a faster rate than transgranular cracking [4.47], which may explain why the combination of $0.5 \%$ total strain range with a 3 minute hold time at maximum tensile strain resulted in a greater fatigue life reduction than the other two creep-fatigue tests with larger total strain ranges. Moreover, the further decrease in fatigue life of Alloy 617 when a longer dwell time was incorporated into the $1.0 \%$ total strain range creep-fatigue test was accompanied by a gradual increase in the intergranular cracking component of the crack growth mode. Comparing Fig. 4.95(c) with Fig. 4.95(b) clearly shows this trend. In Fig. 4.95(b), the surface crack initiated on the GB of the outmost surface grain and then grew transgranularly, while in Fig. 4.95(c), the crack propagated through three grains along their GB before converting into the transgranular crack growth mode.

Due to the longer test time of a creep-fatigue test than a LCF test, time-dependent phenomenons such as oxidation could play a large role in the deformation processes of materials. Oxidation occurred on the surface-connected GBs in Alloy 617, and the oxidized GBs became preferential sites for surface cracking. Fig. 4.96 illustrates an example of surface cracking due to 


\section{Understanding Fundamental Material-Degradation Processes in High Temperature Aggressive Chemomechanical Environments}

oxidized GBs. The oxidation structure, similar to the case in LCF tests, was composed of an outer layer of chromium oxide with finger-shaped aluminum oxide beneath. Kim et al. discovered a similar oxidation structure in Alloy 617 after the material was exposed to a high temperature impure helium environment. They believed that the oxidation structure was responsible for the low temperature embrittlement of the material as a result of cracking along the oxidized surface GB [4.63]. Additional damages resulting from creep-fatigue tests include intergranular cracks in the interior of Alloy 617 specimens. These intergranular cracks, presented in Fig. 4.97, were usually aligned at 45 degrees to the axial stress or perpendicular to the axial stress. GBs at 45 degrees to the tensile axis exist on planes of maximum shear stress, where GB sliding would tend to open cracks around the GB carbides. GBs oriented perpendicular to the tensile axis experienced the greatest normal force during the creep-fatigue experiments, which also tended to open cracks [4.64]. The interior intergranular cracks demonstrated that additional creep damage results from the maximum tensile strain hold period.

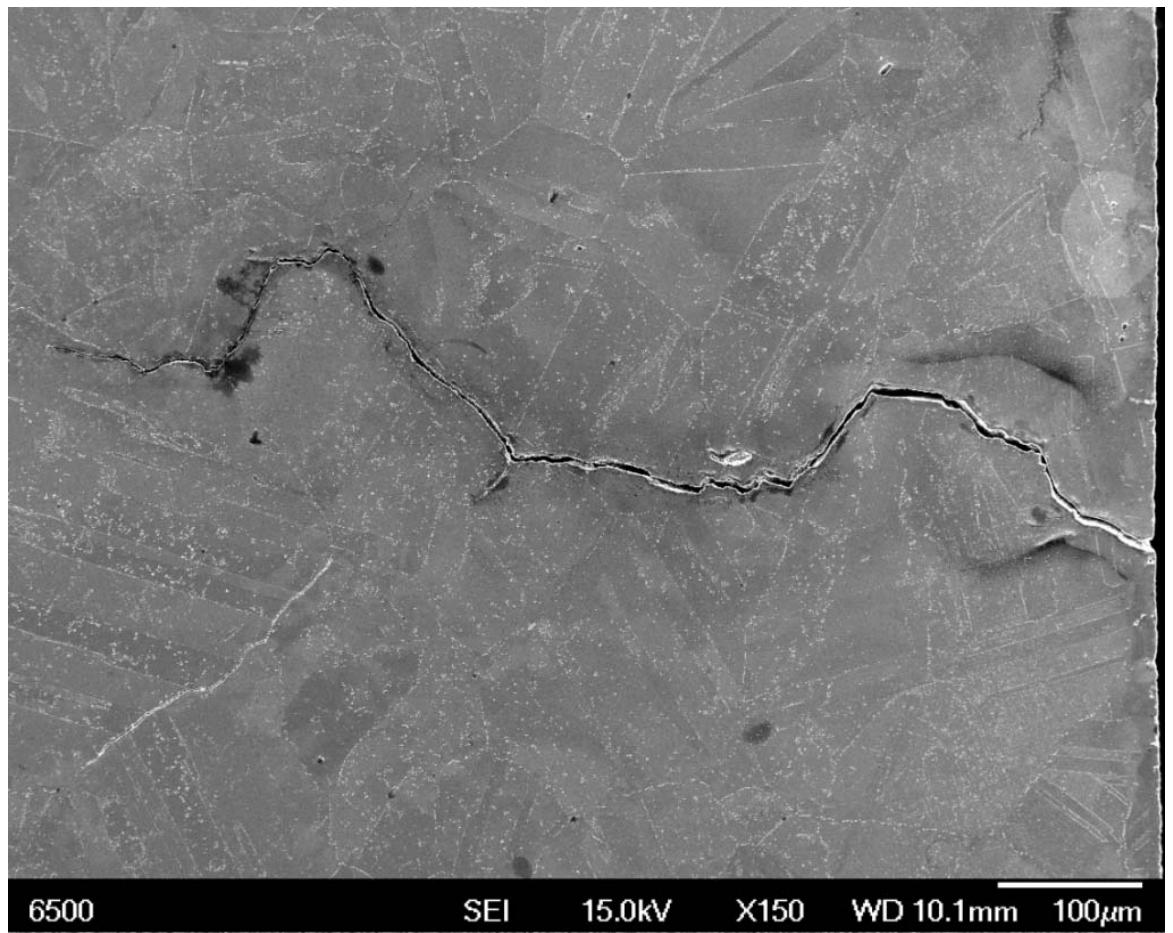

(a)

Fig. 4.95: Crack morphology in creep-fatigue tested Alloy 617. Test conditions: (a) $\varepsilon$ tot $=0.5 \%$, dwell time $=3$ minutes; (b) $\varepsilon_{\text {tot }}=1.5 \%$, dwell time $=3$ minutes; (c) $\varepsilon_{\text {tot }}=1.0 \%$, dwell time $=30$ minutes 
Understanding Fundamental Material-Degradation Processes in High Temperature Aggressive Chemomechanical Environments

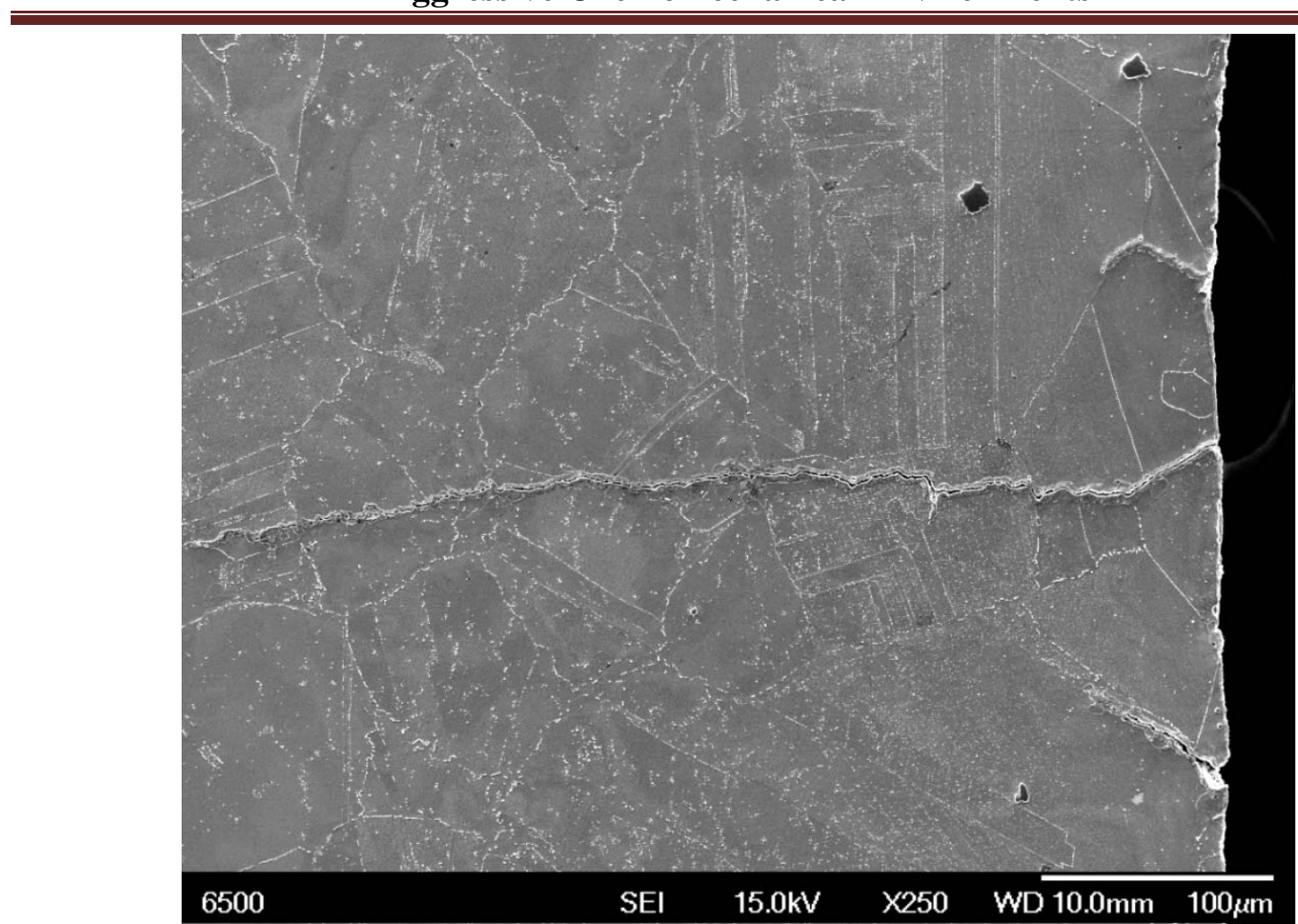

(b)

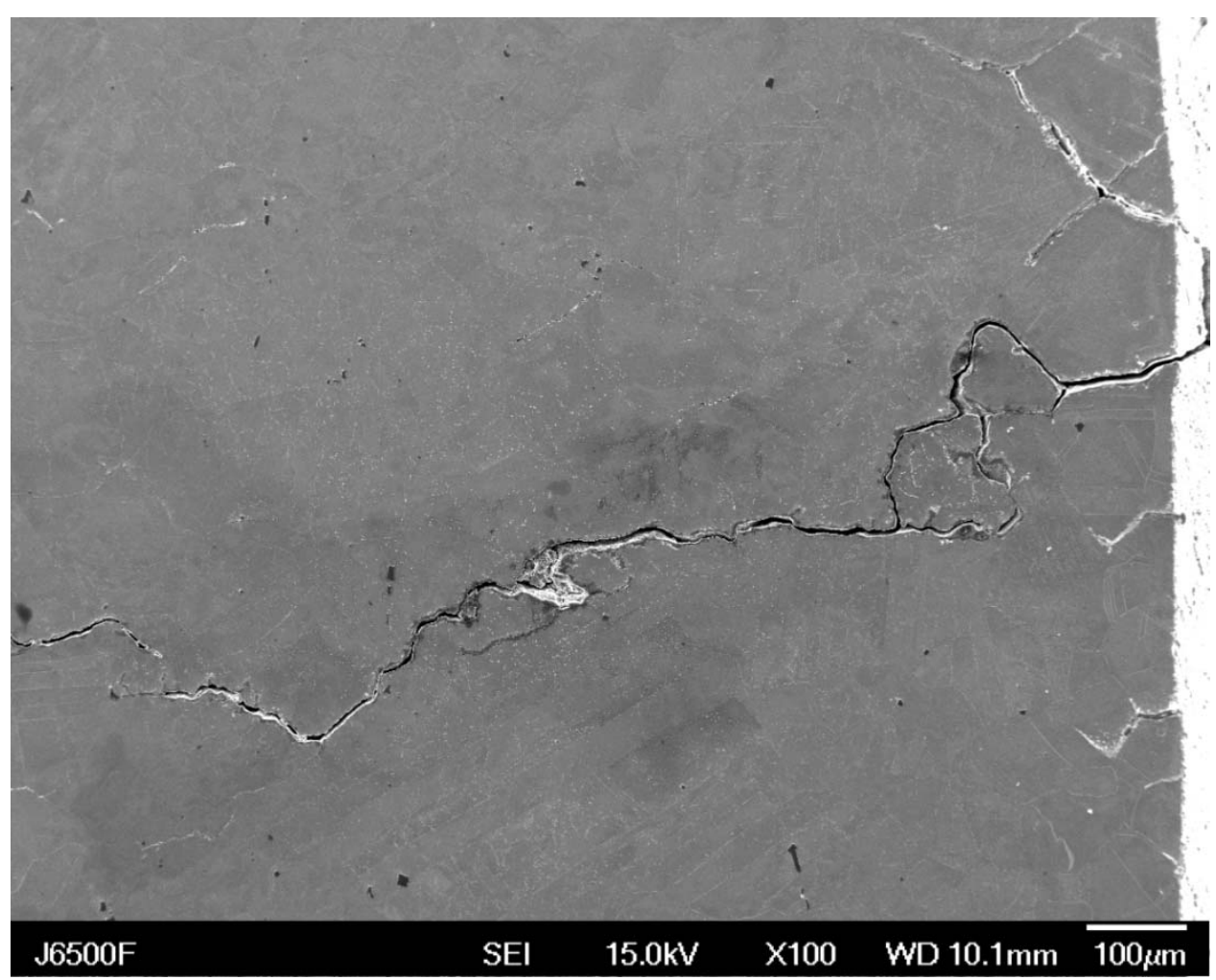

(c)

Fig. 4.95 Cont. 


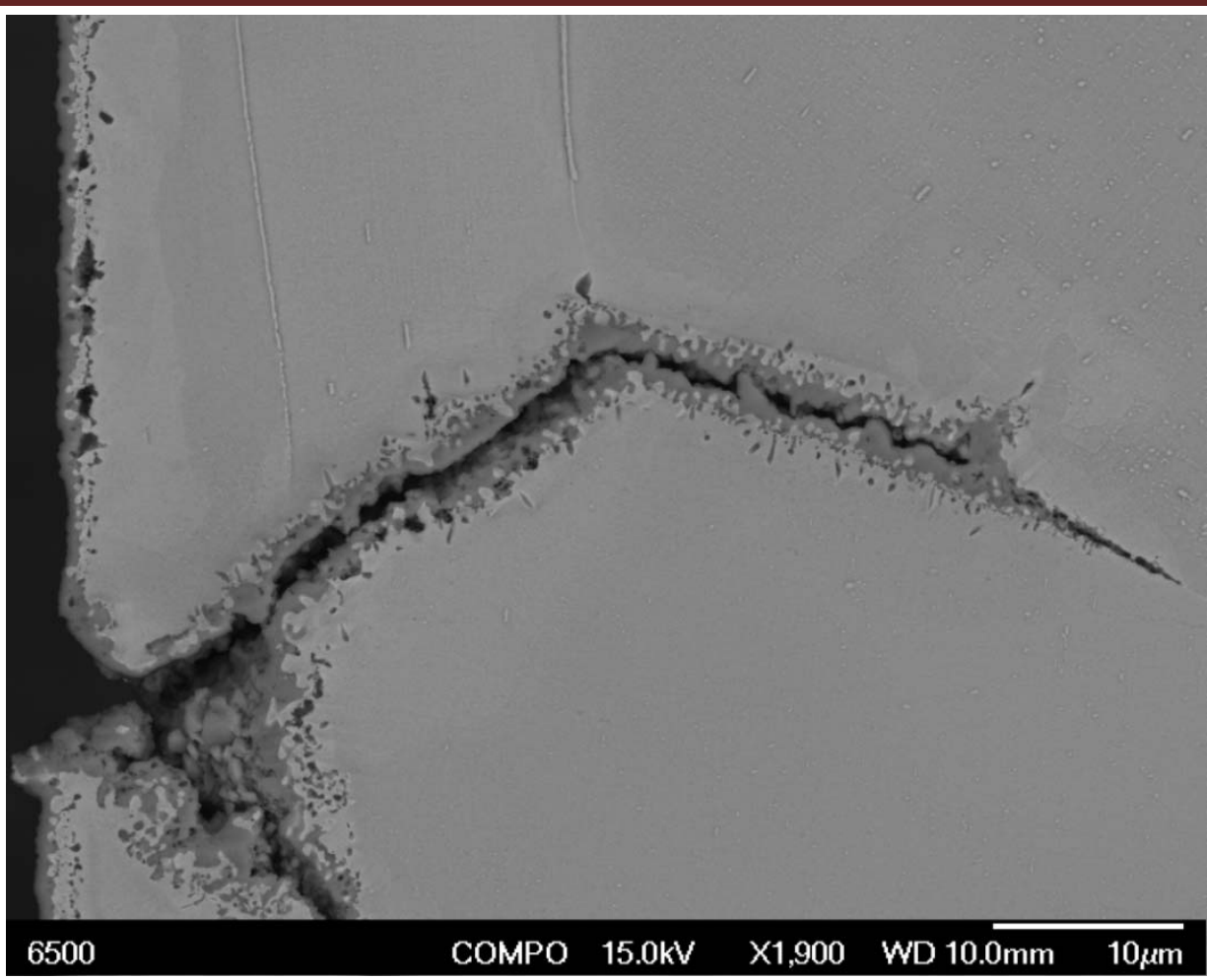

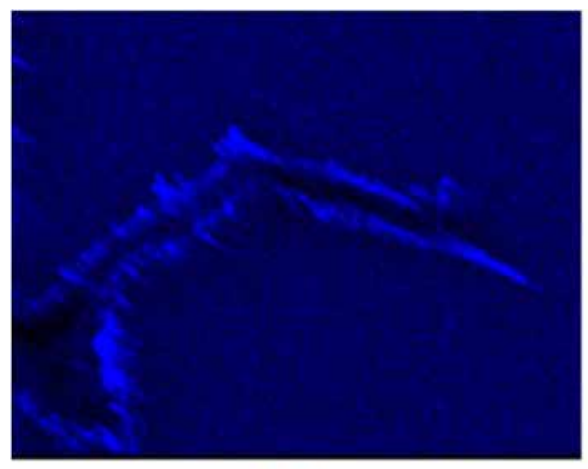

Al

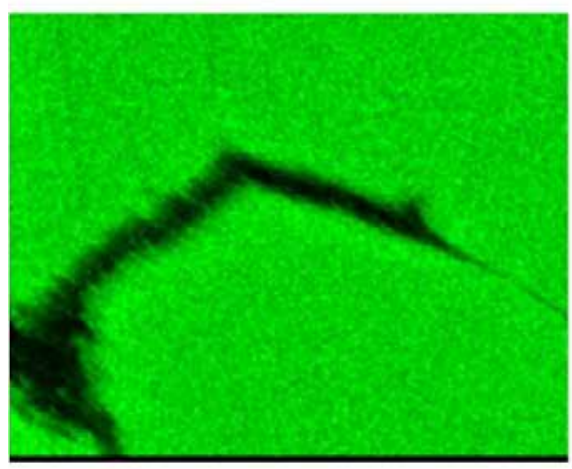

Ni

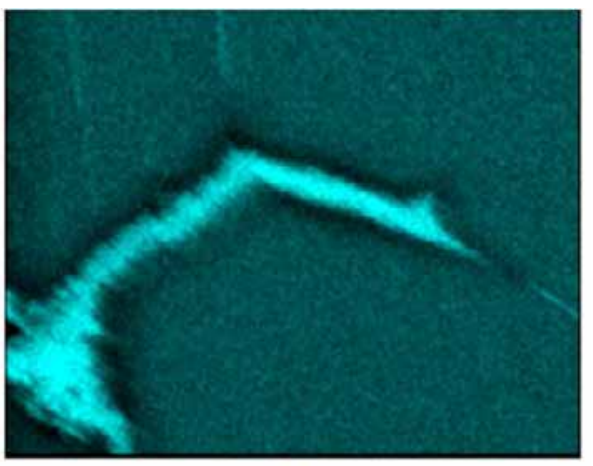

$\mathrm{Cr}$

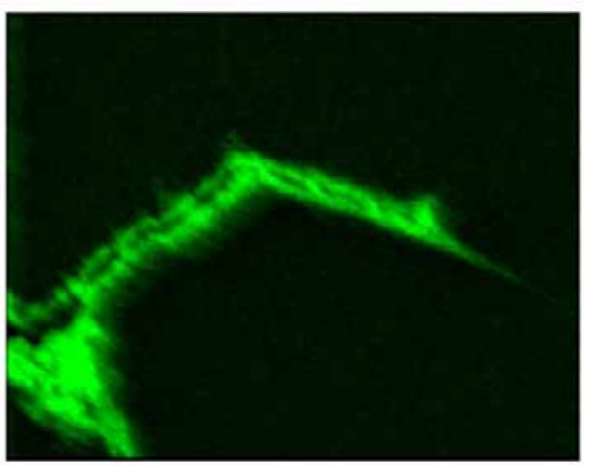

$\mathbf{O}$

Fig. 4.96: Crack initiation on surface-connected GBs in Alloy 617. Oxidation along the surface-connected GB was apparent, with finger-shaped aluminum oxide underneath the surface chromium oxide. Test conditions: $\varepsilon_{\text {tot }}=0.5 \%$, dwell time $=3$ minutes 


\section{Understanding Fundamental Material-Degradation Processes in High Temperature}

Aggressive Chemomechanical Environments

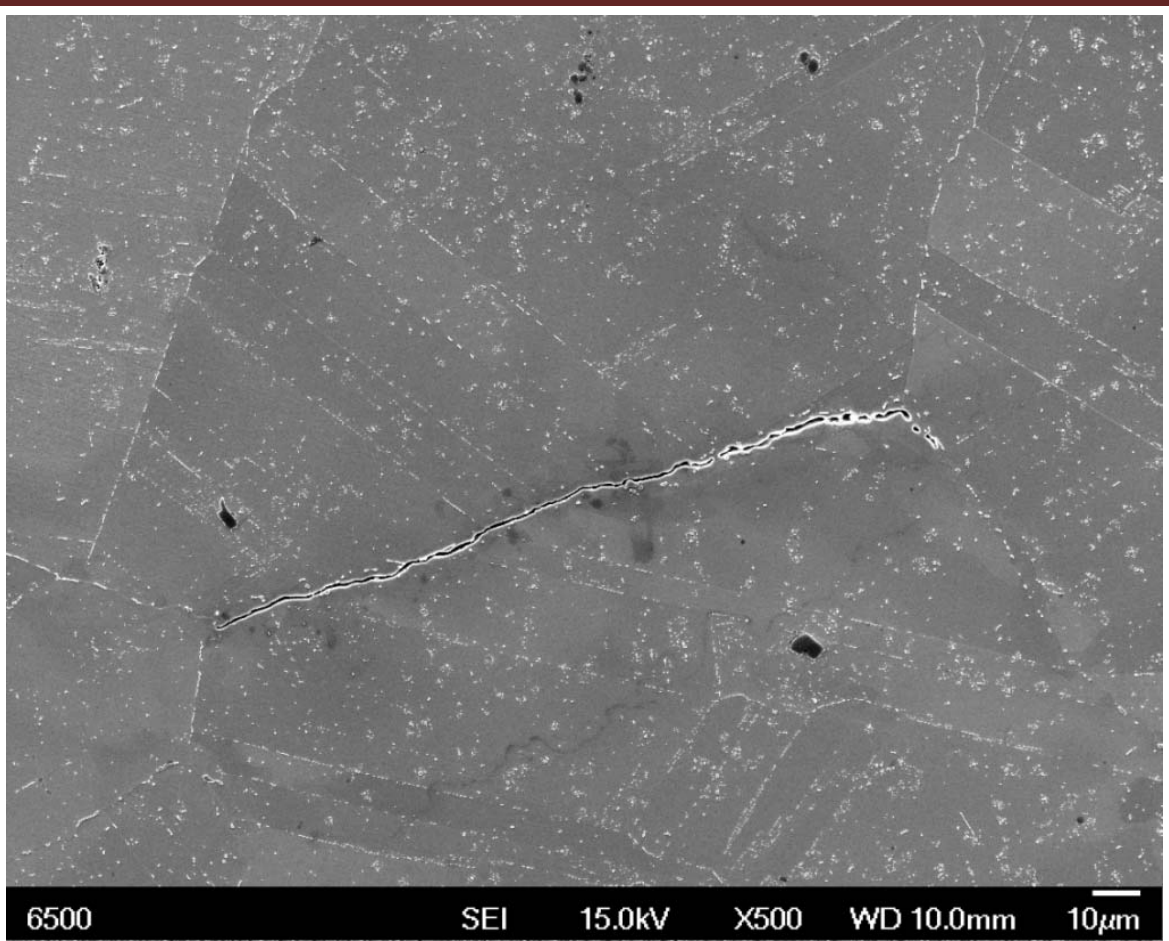

(a)

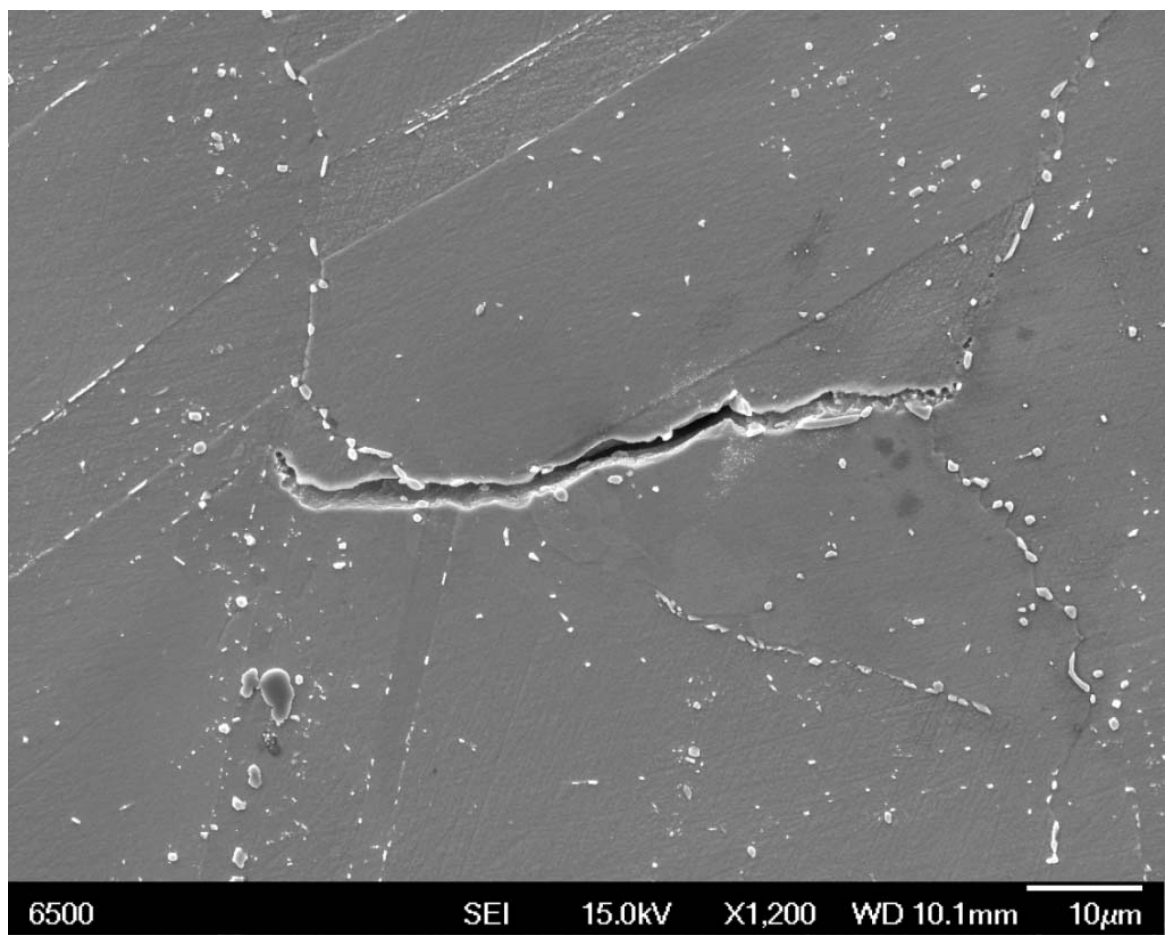

(b)

Fig. 4.97: Intergranular crack development inside the creep-fatigue tested Alloy 617. In many cases, the crack opening was either 45 degrees to the stress direction or perpendicular to the stress direction. Test conditions: (a) $\varepsilon_{\text {tot }}=0.5 \%$, dwell time $=3$ minutes; (b) $\varepsilon_{\text {tot }}=1.0 \%$, dwell time $=10$ minutes 


\section{Understanding Fundamental Material-Degradation Processes in High Temperature Aggressive Chemomechanical Environments}

In comparison with Alloy 617, the slightly better creep-fatigue performance of Alloy 230 could be attributed to its better resistance to intergranular cracking. For instance, except for the $1.5 \%$ total strain range creep-fatigue test, transgranular crack initiation and propagation was observed for all the creep-fatigue tests with 3 minute dwell times, as shown in Fig. 4.98(a). For the creep-fatigue test of Alloy 230 at $1.5 \%$ total strain range with a 3 minute hold time, the cracking morphology exemplified in Fig. 4.98(b) indicates the intergranular initiation and transgranular propagation. The change in the crack initiation mode may be attributed to the synergy of the high stress level at $1.5 \%$ total strain range and the GB embrittlement due to the occurrence of GB cellular precipitates in Alloy 230. For creep-fatigue tests of Alloy 230 at 1.0\% total strain range with varied peak tensile strain hold periods, the crack initiation and propagation modes were always transgranular, except for the 30 minute strain hold time creep-fatigue test, in which crack initiation morphology reveals intergranular fractures due to rising creep damage. Fig. 4.98(c) depicts this cracking morphology in Alloy 230. In comparison with the cracking morphology of Alloy 617 at the same test conditions, as shown in Fig. 4.95(c), the cracks propagated through fewer grains intergranularly in Alloy 230, indicating a better resistance of Alloy 230 to intergranular cracking. Nonetheless, interior GB cracks were observed in all creep-fatigue tested Alloy 230 specimens, as shown in Fig. 4.99. Similar to the case of Alloy 617, the interior GB cracks in Alloy 230 aligned either at an angle of 45 degrees to the axial stress or perpendicular to the stress direction. In addition, cellular precipitate lamellae with accompanying precipitation free zones on both sides of the GB were found in the vicinity of interior GB cracks. Strain concentration in the precipitation free zones and a reduced GB ductility due to the cellular carbide precipitates jointly caused the GBs containing cellular precipitates to be the preferred sites for intergranular cracking. 


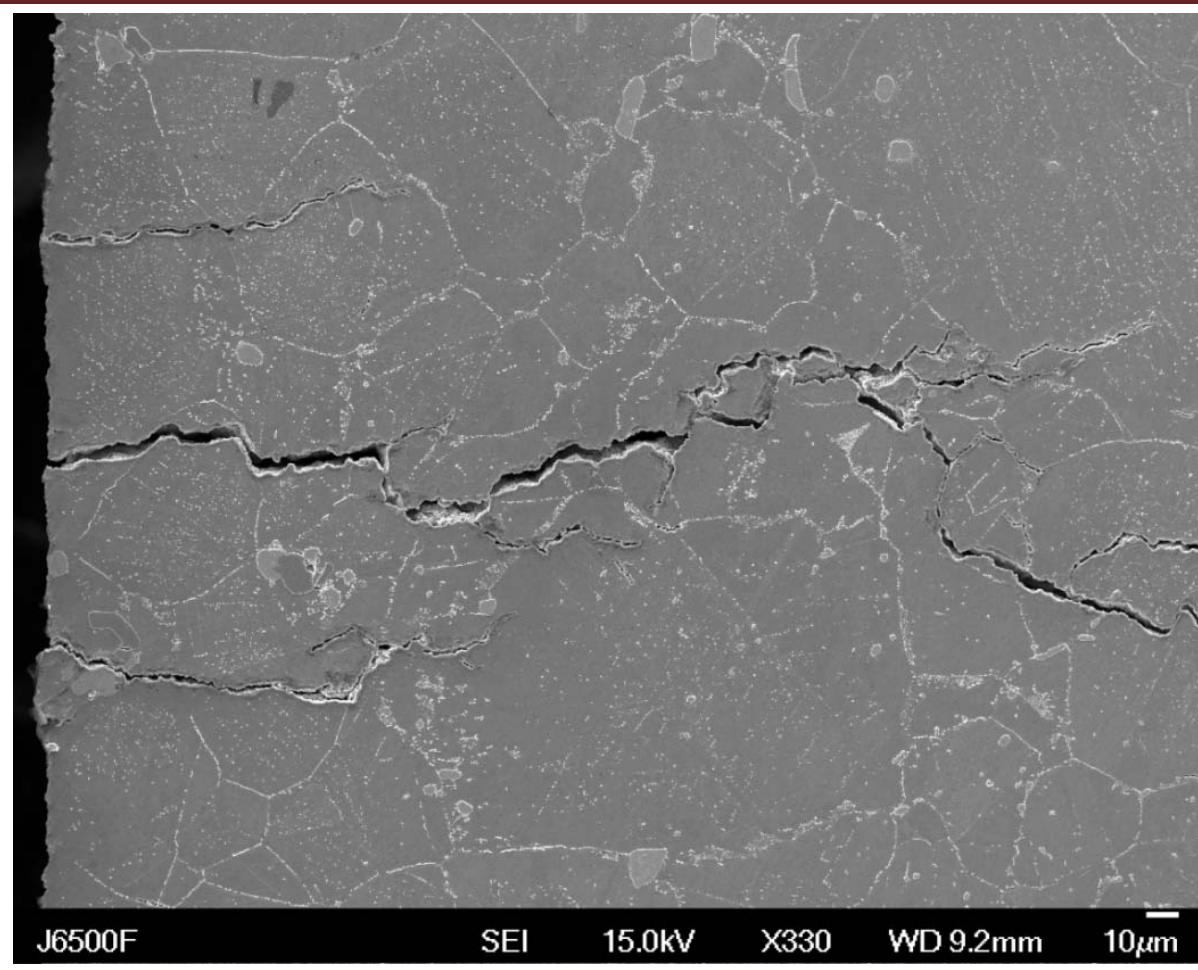

(a)

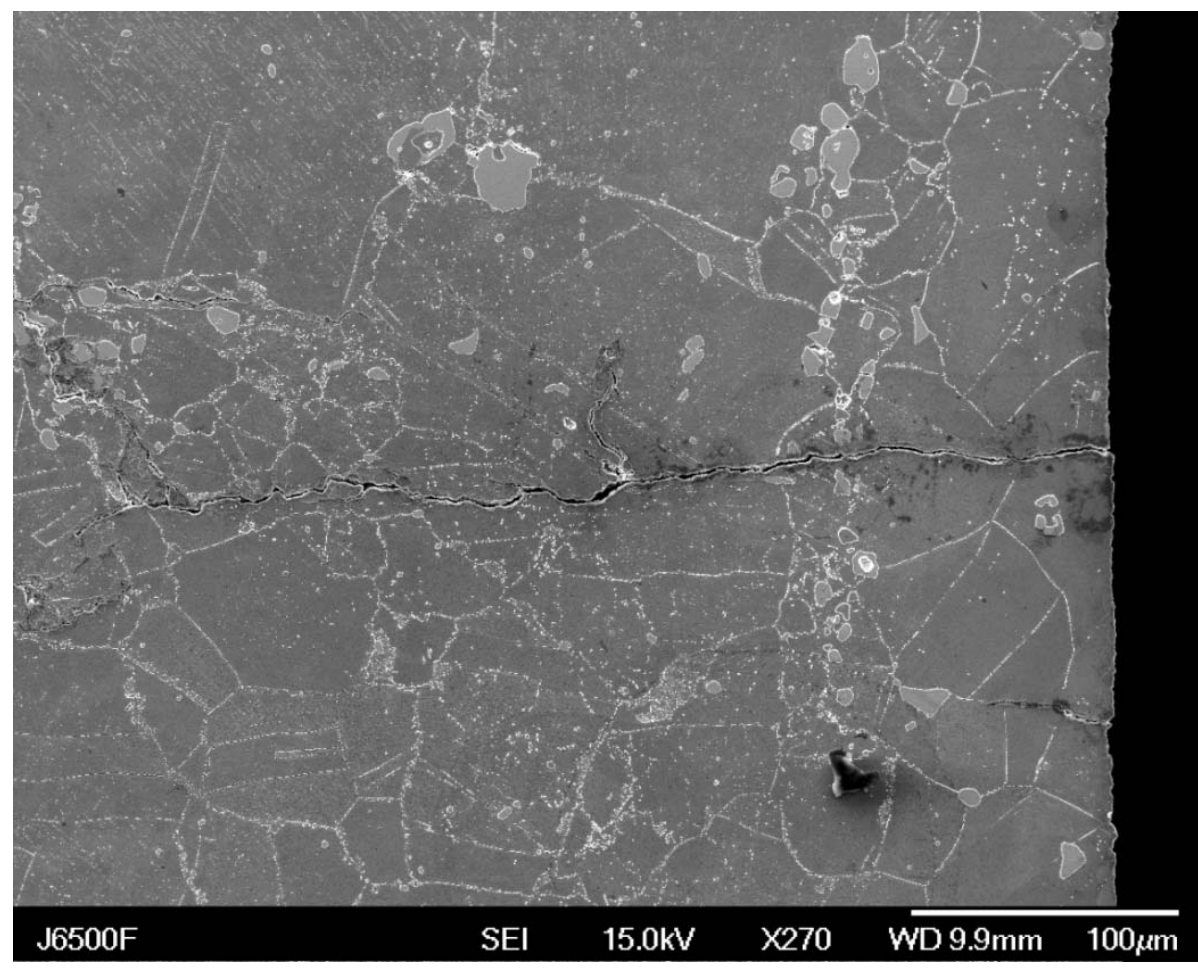

(b)

Fig. 4.98: Crack morphology in creep-fatigue tested Alloy 230. Test conditions: (a) $\varepsilon$ tot $=0.5 \%$, dwell time $=3$ minutes; (b) $\varepsilon_{\text {tot }}=1.5 \%$, dwell time $=3$ minutes; (c) $\varepsilon_{\text {tot }}=1.0 \%$, dwell time $=30$ minutes 
Understanding Fundamental Material-Degradation Processes in High Temperature Aggressive Chemomechanical Environments

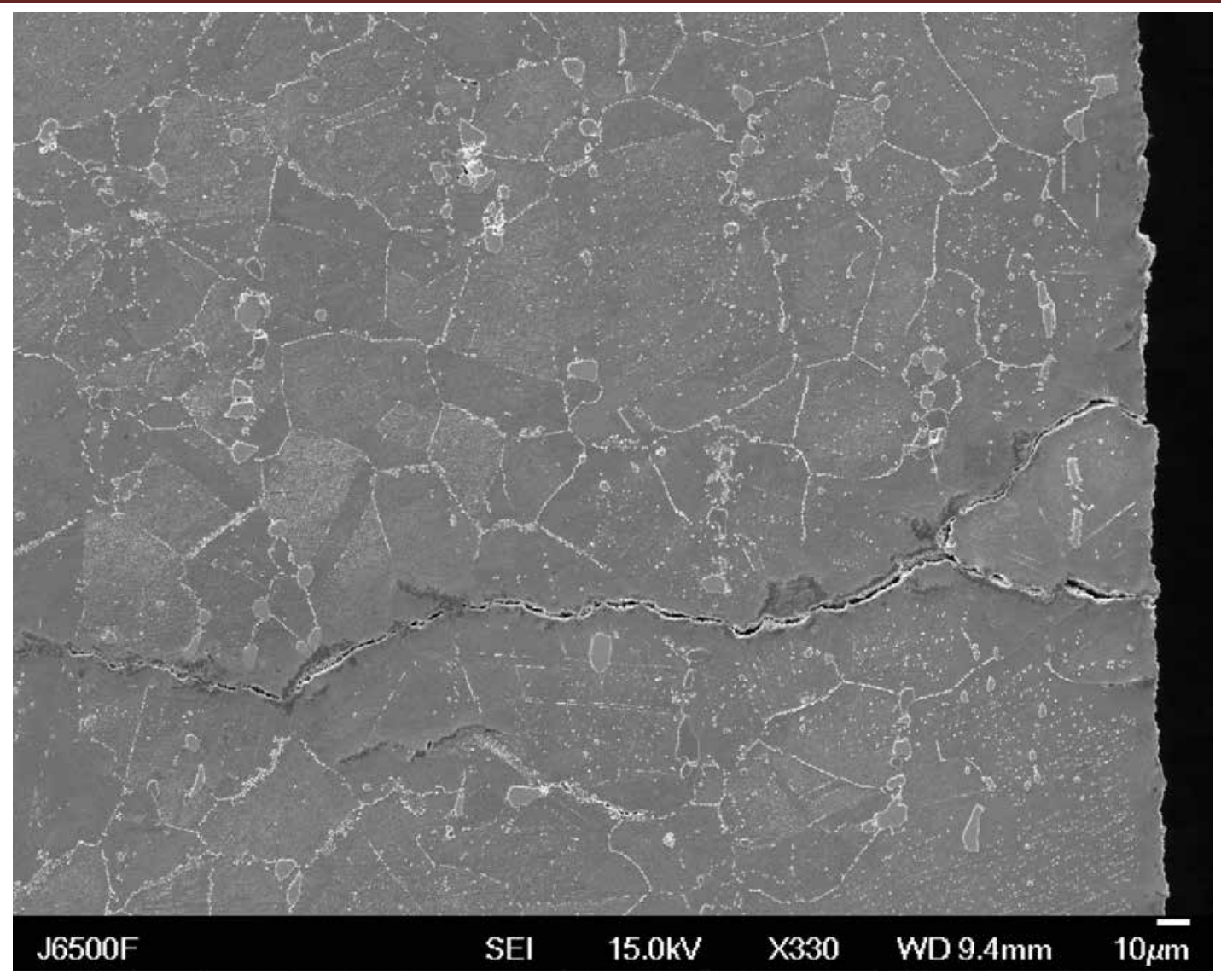

(c)

Fig. 4.98 Cont. 


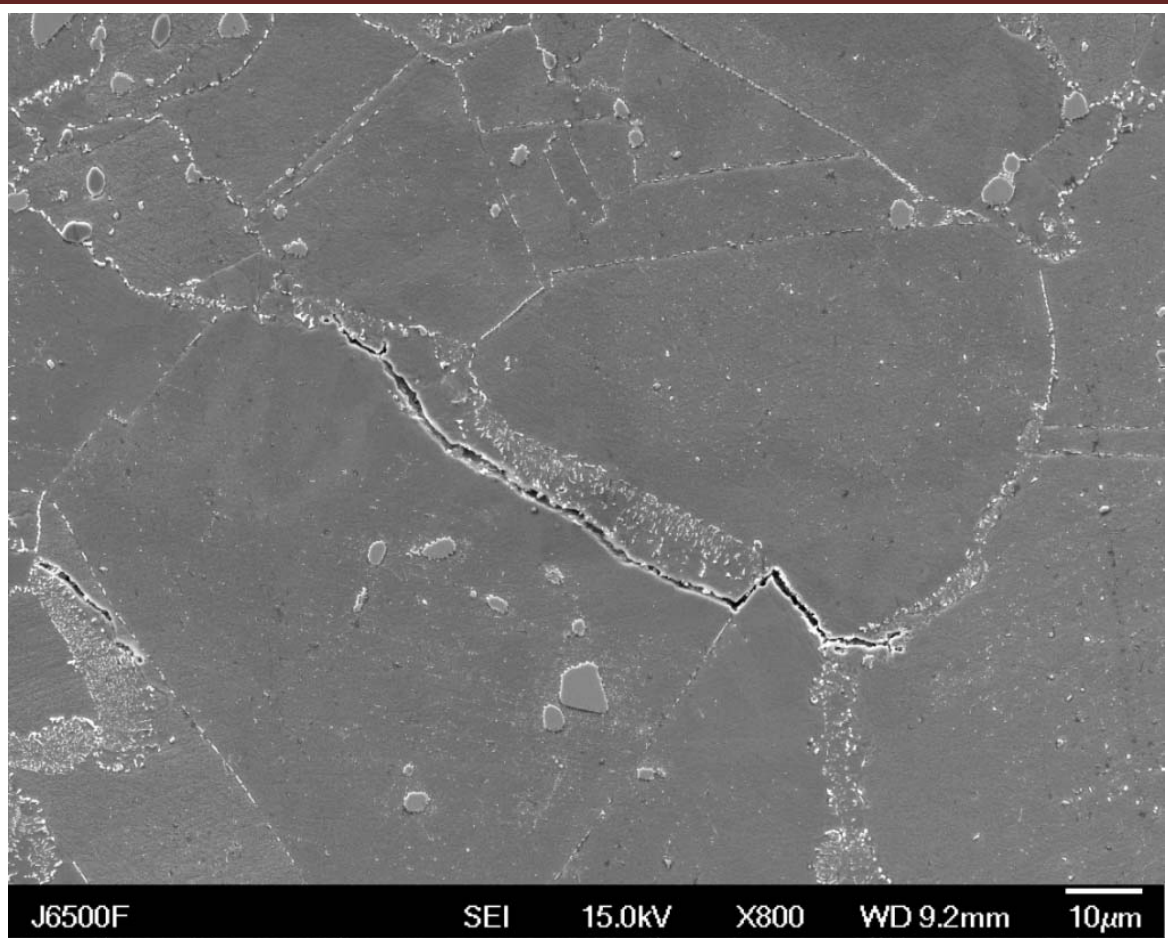

(a)

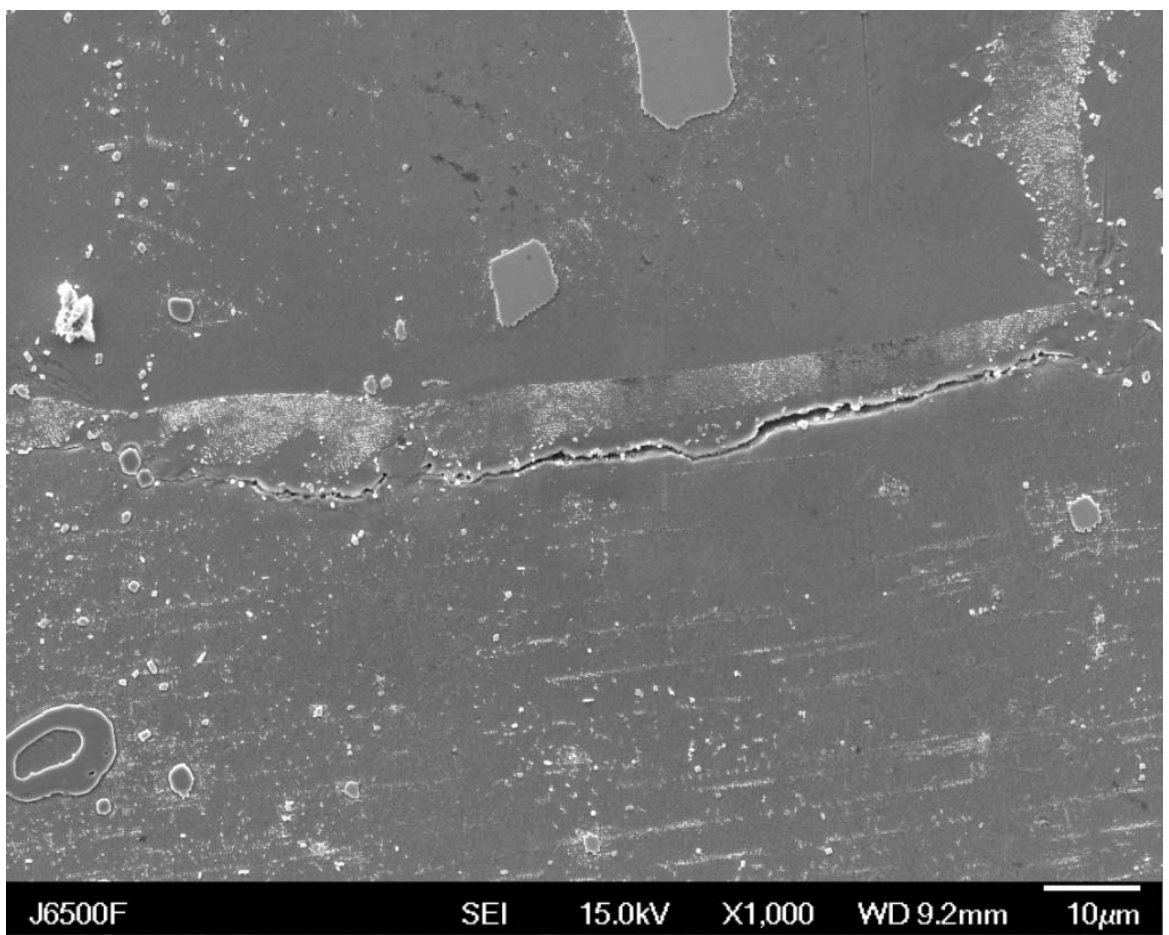

(b)

Fig. 4.99: Intergranular crack development inside creep-fatigue tested Alloy 230. In many cases, the crack opening was either at 45 degrees to the stress direction or perpendicular to the stress direction. Test conditions: (a) $\varepsilon_{\text {tot }}=1.5 \%$, dwell time $=3$ minutes; (b) $\varepsilon_{\text {tot }}=1.0 \%$, dwell time $=3$ minutes 


\section{Understanding Fundamental Material-Degradation Processes in High Temperature Aggressive Chemomechanical Environments}

\subsection{EBSD Results}

To further investigate microstructures that might influence the LCF and creep-fatigue test results, EBSD analysis was performed on both the as-received and experimentally-used specimens. Additional microstructure results, inaccessible from conventional microanalysis techniques, can be derived from the EBSD analysis, and a direct link between the material microstructure and crystallographic information can be found. Therefore, this section is divided into two subsections. In the first subsection, microstructural features which might influence the LCF and creep-fatigue properties of both alloys are identified. EBSD studies of these features on a relatively large scale were carried out to yield statistically significant results. The second subsection describes the EBSD scans performed on relatively small areas so that microstructural features derived from conventional microanalysis techniques, such as SEM, could be correlated with EBSD-based results.

\subsubsection{Statistical EBSD analysis}

Microstructural features which might influence the LCF and creep-fatigue properties of both alloys include grain size, the twinning boundary fraction of high angle grain boundaries (HAGBs), the coherent twinning boundary fraction of the total twinning boundaries, the fraction of $\sum \leq 29$ coincidence site lattice (CSL) boundaries among all boundaries, the length and distribution of low angle grain boundaries (LAGBs), and the existence of any texture. The end results of these microstructural features in Alloy 617, listed in Table 4.10, were extracted from EBSD scans done on an area of $1100 \mu \mathrm{m} \times 1100 \mu \mathrm{m}$ with a step size of $1.6 \mu \mathrm{m}$. The average grain size for the as-received Alloy 617 specimens was about $81.8 \mu \mathrm{m}$. The 45 -minute aging treatment at $850^{\circ} \mathrm{C}$, and most other test conditions, didn't affect the average grain size of Alloy 617. However, for both the LCF creep-fatigue tests of Alloy 617 with $1.5 \%$ total strain range, a reduction in the average grain size was apparent, and was found to be due to recrystallization of smaller grains. The elevated temperature and large total strain range favored dynamic recrystallization (DRX). The DRX in Alloy 617 was also observed for elevated temperature $\left(\geq 800^{\circ} \mathrm{C}\right)$ tensile tests, especially near strain concentrated regions [4.65].

Table 4.10: Statistical EBSD analysis for Alloy 617

\begin{tabular}{|c|c|c|c|c|c|c|c|c|c|c|}
\hline & \multicolumn{10}{|c|}{ Alloy 617} \\
\hline & Raw & Aged & $0.5 \%$ & $1.0 \%$ & $1.5 \%$ & $\begin{array}{c}0.5 \%+3 \\
\text { min }\end{array}$ & $\begin{array}{c}1.0 \%+3 \\
\text { min }\end{array}$ & $\begin{array}{c}1.5 \%+3 \\
\text { min }\end{array}$ & $\begin{array}{c}1.0 \%+10 \\
\text { min }\end{array}$ & $\begin{array}{c}1.0 \%+30 \\
\text { min }\end{array}$ \\
\hline Grain size $(\mu \mathrm{m})$ & 81.8 & 76.9 & 97.5 & 100.6 & 42.3 & 81.0 & 71.5 & 33.2 & 65.2 & 74.2 \\
\hline Twinning (\%) & 64.3 & 63.6 & 71.1 & 68.3 & 50.3 & 69.0 & 60.7 & 35.1 & 57.4 & 61.7 \\
\hline $\begin{array}{c}\text { Coherent } \\
\text { Twinning (\%) }\end{array}$ & 46.8 & 52.9 & 62.9 & 67.1 & 64.1 & 63.8 & 62.8 & 49.3 & 53.4 & 56.7 \\
\hline CSL fraction (\%) & 66.7 & 66.5 & \multicolumn{7}{|c|}{$\mathrm{N} / \mathrm{A}$} & \multicolumn{1}{|c|}{} \\
\hline LAGB & $\begin{array}{c}1.8 \\
\mathrm{~mm}\end{array}$ & $\begin{array}{c}2.5 \\
\mathrm{~mm}\end{array}$ & $\begin{array}{c}2.2 \\
\mathrm{~mm}\end{array}$ & $\begin{array}{c}6.0 \\
\mathrm{~mm}\end{array}$ & $\begin{array}{c}4.9 \\
\mathrm{~cm}\end{array}$ & $8.2 \mathrm{~mm}$ & $2.2 \mathrm{~cm}$ & $8.0 \mathrm{~cm}$ & $2.6 \mathrm{~cm}$ & $1.5 \mathrm{~cm}$ \\
\hline Texture $(\mathrm{Y} / \mathrm{N})$ & $\mathrm{N}$ & $\mathrm{N}$ & $\mathrm{N}$ & $\mathrm{N}$ & $\mathrm{N}$ & $\mathrm{N}$ & $\mathrm{N}$ & $\mathrm{N}$ & $\mathrm{N}$ & $\mathrm{N}$ \\
\hline
\end{tabular}

*During grain size calculation, coherent twinning boundaries were not counted as GBs, since they were generally immobile and not a constituent of the intergranular transport network. 


\section{Understanding Fundamental Material-Degradation Processes in High Temperature Aggressive Chemomechanical Environments}

In contrast to random HAGBs, the boundary structure of twinning boundaries has a high degree of atomic matching, a low mobility, and a low GB energy. Thus, twinning boundaries are believed to possess desirable properties, such as resistance to cracking and corrosion $[4.54,4.6]$. Characterization of the twinning boundary percentage in the as-received Alloy 617 specimens showed that over $60 \%$ of HAGBs were twinning boundaries. Similar to the case of grain size of Alloy 617, the twinning boundary fraction remained constant except for the LCF and creep-fatigue tests with $1.5 \%$ total strain range. Large strain induces grain rotations that result in twinning boundaries progressively losing their special character [4.7]. The change of twinning boundary distribution inside the material is shown in Fig. 4.100.

In contrast to parallel boundary lines pertaining to annealing twinning boundaries in the aged specimens, twinning boundaries from the creep-fatigue tested specimens at $1.5 \%$ total strain range seemed curved and discontinuous due to grain rotations. Moreover, twinning boundaries were further divided into coherent and incoherent twinning boundaries, since coherent twinning boundaries are considered to have more special properties than incoherent twinning boundaries [4.66]. Coherent twinning boundaries require the boundary plane to be aligned with the twinning plane, while incoherent twinning boundaries don't parallel the twinning plane [4.55]. In OIM analysis software, the plane trace of the boundary plane was checked against that of the twinning plane; if alignment was confirmed, the twinning boundary was considered to be coherent, and vice versa. After application of this procedure, the ratio of coherent twinning boundaries to all twinning boundaries in Alloy 617 was determined to be about 50-60\%. Thermal treatment and mechanical testing did not alter this value, as presented in Table 4.10.

Fig. 4.101(a)\&(b) show the configuration of the coherent and incoherent twinning boundaries in a thermal-aged specimen and a creep-fatigue tested specimen, respectively. The total fraction of twinning boundaries changed between these two specimens, while the percentage of coherent twinning boundaries among the total twinning boundaries didn't change. A twinning boundary is a subgroup of a broader category of special boundaries called CSL boundaries, in which repeated units are formed from points where the two misoriented lattices happen to coincide. CSL boundaries also fall into the category of low-energy boundaries, and possess desirable properties [4.67]. The type of CSL boundary is usually represented by a parameter $\sum$ which describes the degree of fit between the structures of the two grains. Mathematically, $\sum$ is calculated as the reciprocal of the ratio of coincidence sites to the total number of sites. For instance, an $\sum 1$ CSL boundary represents LAGBs with a GB misorientation angle less than 15 degrees. An $\sum 3$ CSL boundary represents twinning boundaries typically formed by a 60 degree rotation around the $<111>$ crystal direction in FCC materials. In reality, the boundary of two grains usually does not exactly satisfy the misorientation condition of CSL boundaries due to local atomic relaxation and/or the inclusion of dislocations into the boundary. Therefore, some maximum allowable deviation from the perfect CSL configuration needs to be defined. One widely used criterion, the Brandon criterion, defines the maximum allowable misorientation $\Delta \theta$ from the exact CSL by:

$$
\Delta \theta=\Delta \theta_{m} \Sigma^{-1 / 2}
$$

$\Delta \theta_{\mathrm{m}}$ is the maximum misorientation angle for LAGBs (typically 15 degrees [4.6]). In recent years, a more restrictive geometric criterion for special CSL boundaries was proposed by Palumbo et al., who claimed that the fraction of CSL boundaries with special properties based on the Palumbo criterion was higher than its counterpart based on the Brandon criterion [4.68], 


\section{Understanding Fundamental Material-Degradation Processes in High Temperature Aggressive Chemomechanical Environments}

making the Palumbo criterion more suitable for defining CSL boundaries. The form of the Palumbo criterion is given by:

$$
\Delta \theta=\Delta \theta_{m} \Sigma^{-5 / 6}
$$

In this study, the Palumbo criterion was adopted, and the fraction of CSL boundaries with $\sum \leq 29$ was calculated for the as-received and aged specimens. As shown in Table 4.10, the fraction of CSL boundaries in Alloy 617 was about 70\%, which was well above the threshold value of $50 \%$ known for producing a noticeable difference in the intergranular corrosion resistance [4.69]. It is worth noting that the calculation of fractions of CSL boundaries in tested Alloy 617 specimens was omitted, since many LAGBs were created during the deformation of the material. These LAGBs, belonging to the $\sum 1$ CSL boundaries, do not qualify as special boundaries for two reasons. First, most of the LAGBs spread within the material without forming complete grains. Second, the LAGBs formed due to dislocation segregation, plasticity, and other means, and were representative of the material deformation behavior [4.70].

While the total fraction of CSL boundaries is important, perhaps even more important is a concept called grain boundary character distribution, which describes the arrangement of random HAGB and CSL boundaries. An optimized grain boundary character distribution should produce a more discontinuous and random HAGB network and a high frequency of CSL boundaries [4.71]. Fig. 4.102(a) illustrates how the random HAGBs (depicted by dark lines) and $\sum \leq 29 \mathrm{CSL}$ boundaries (depicted by light grey lines) were arranged inside an aged Alloy 617 specimen. The CSL boundaries can interrupt the random HAGB network to some extent, as shown in Fig. 4.102(b) after removal of the CSL boundaries in Fig. 4.102(a). However, the interruption was limited - namely, if a defect starts from the end of one GB on the left side of Fig. 4.102(b), it can always reach the end of another GB on the right side of the same image, meaning there is a contoured, but still continuous, GB path for potential defect propagation. As mentioned before, generation of LAGBs can be used to characterize materials deformation behavior.

In the wake of plastic deformation, large numbers of dislocations were created. These dislocations tended to reorganize themselves into dislocation cells which eventually coalesced to form subgrain boundaries [4.72]. As the deformation continued, the misorientation angle of the subgrain boundary gradually increased. When the misorientation angle was larger than 2-5 degrees, the subgrain boundary became a LAGB. To accommodate the deformation, more dislocations must be introduced, which results in a growing wall of dislocations. Therefore, LAGBs can essentially be considered to be an array of dislocations. The length of total LAGBs in Alloy 617 under different test conditions is listed in Table 4.10, with corresponding LAGB distribution maps shown in Fig. 4.103(a)-(f). Beginning with the aged specimen as shown in Fig. 4.103(a), except for those pre-existing LAGBs, no new LAGBs were created, due to the lack of deformation. The same results were observed in the LCF tests at $0.5 \%$ total strain range. With the total strain range increased to $1.0 \%$ for the LCF test, some LAGBs started to form near the original random HAGBs, as shown in Fig. 4.103(b). This situation was exaggerated for the LCF test at $1.5 \%$ total strain range as more LAGBs generated near random HAGBs, as shown in Fig. 4.103(c).

Fig. 4.104 indicates that DRX can occur at some LAGB-concentrated sites. Deformation in the LAGB-concentrated zones transformed dislocation walls into GBs in a continuous way, which resulted in the formation of new grains [4.73]. This explains why, as shown in Table 4.10, the grain size decreased for the LCF and creep-fatigue tests with $1.5 \%$ total strain range. 


\section{Understanding Fundamental Material-Degradation Processes in High Temperature Aggressive Chemomechanical Environments}

Comparing LCF tests and creep-fatigue tests at the same total strain range, more LAGBs were generated for creep-fatigue tests, as shown in Table 4.10 and Fig. 4.103(d)\&(e). Elastic strain was converted to inelastic strain, i.e. creep strain, during the strain hold period, which resulted in more inelastic deformation of the material. In addition, the segregation of LAGBs to the random HAGBs became more apparent. The influence of the length of the strain hold period at $1.0 \%$ total strain range on LAGBs seemed more complicated. When the strain hold period increased from 3 minutes to 10 minutes, more LAGBs formed due to the increased creep deformation during the longer hold time creep-fatigue test. However, when the strain hold period increased from 10 minutes to 30 minutes, less LAGBs formed, and the average grain size slightly increased, as exemplified in Table 4.10 and Fig. 4.103(f). This may be attributed to the competing dynamic recovery process during which extended dwell times at elevated temperatures cause dislocation annihilation and polygonization, and thus less formation of LAGBs.

It is well known that crystal orientation texture has a strong influence on the mechanical properties of materials. Therefore, texture investigations were conducted for all the test conditions of Alloy 617. No texture development was found for Alloy 617 either before or after mechanical testing. For instance, Fig. 4.105 shows the Inverse Pole Figure (IPF) of the aged and creep-fatigue tested Alloy 617 specimens. The grains of both specimens did not show a preferred orientation, but rather a strong orientation gradient inside the grain can be observed for the creep-fatigue tested specimen as a result of the grain rotation due to the plastic deformation during the tests. 


\section{Understanding Fundamental Material-Degradation Processes in High Temperature}

Aggressive Chemomechanical Environments

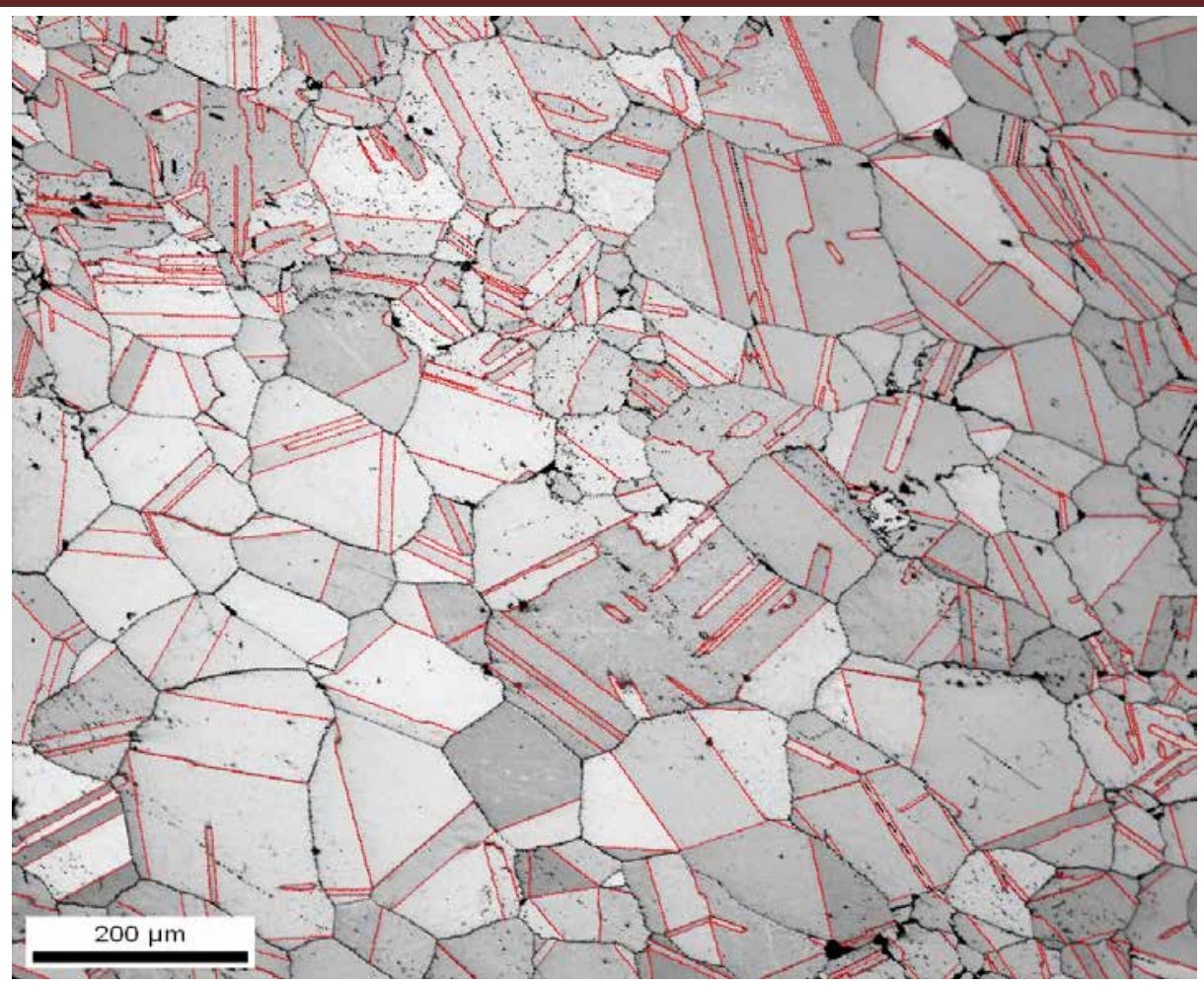

(a)

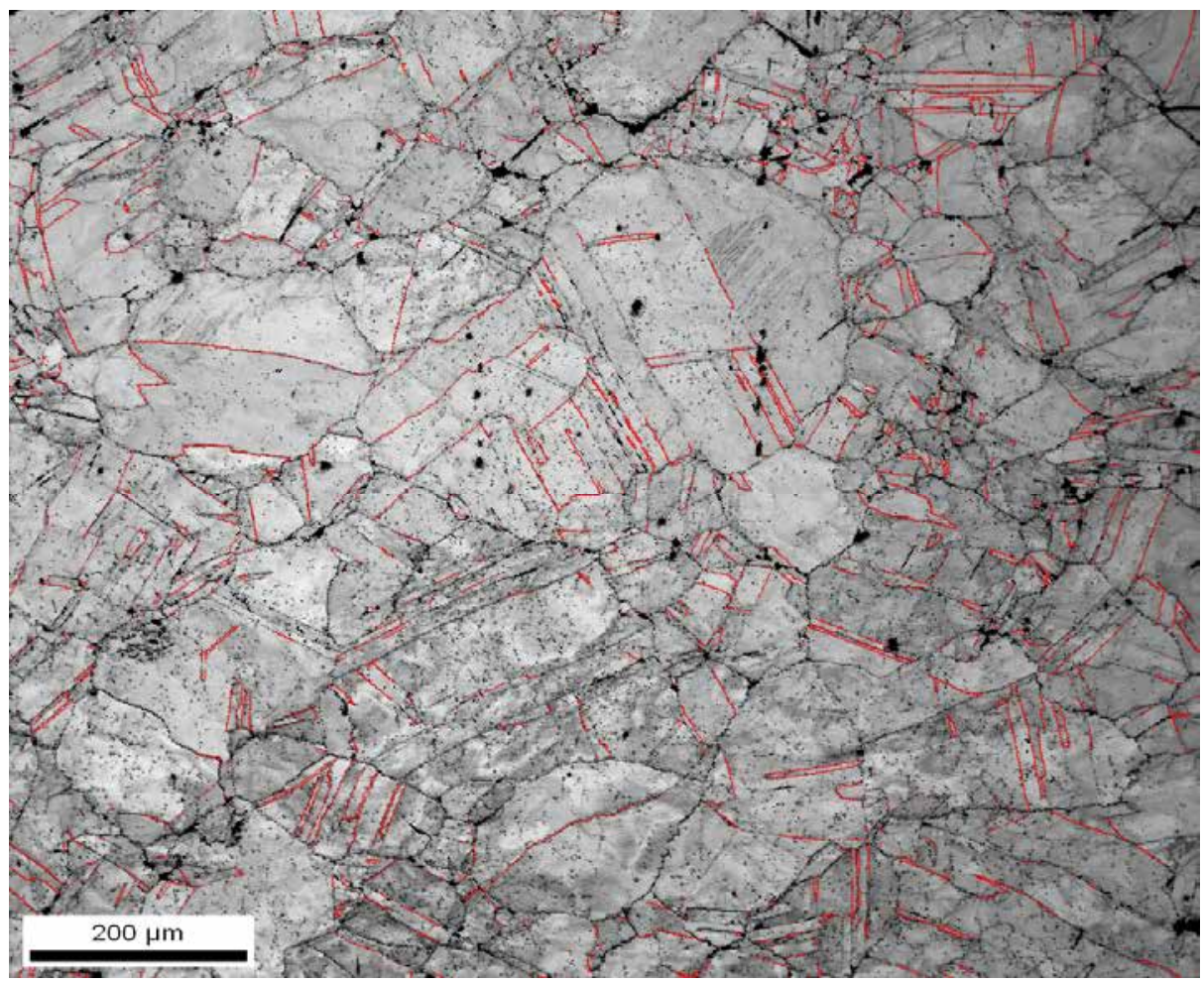

(b)

Fig. 4.100: Twinning boundaries highlighted by red lines in Alloy 617. Test conditions: (a) aged; (b) $\varepsilon_{\text {tot }}=1.5 \%$, dwell time $=3$ minutes 


\section{Understanding Fundamental Material-Degradation Processes in High Temperature Aggressive Chemomechanical Environments}

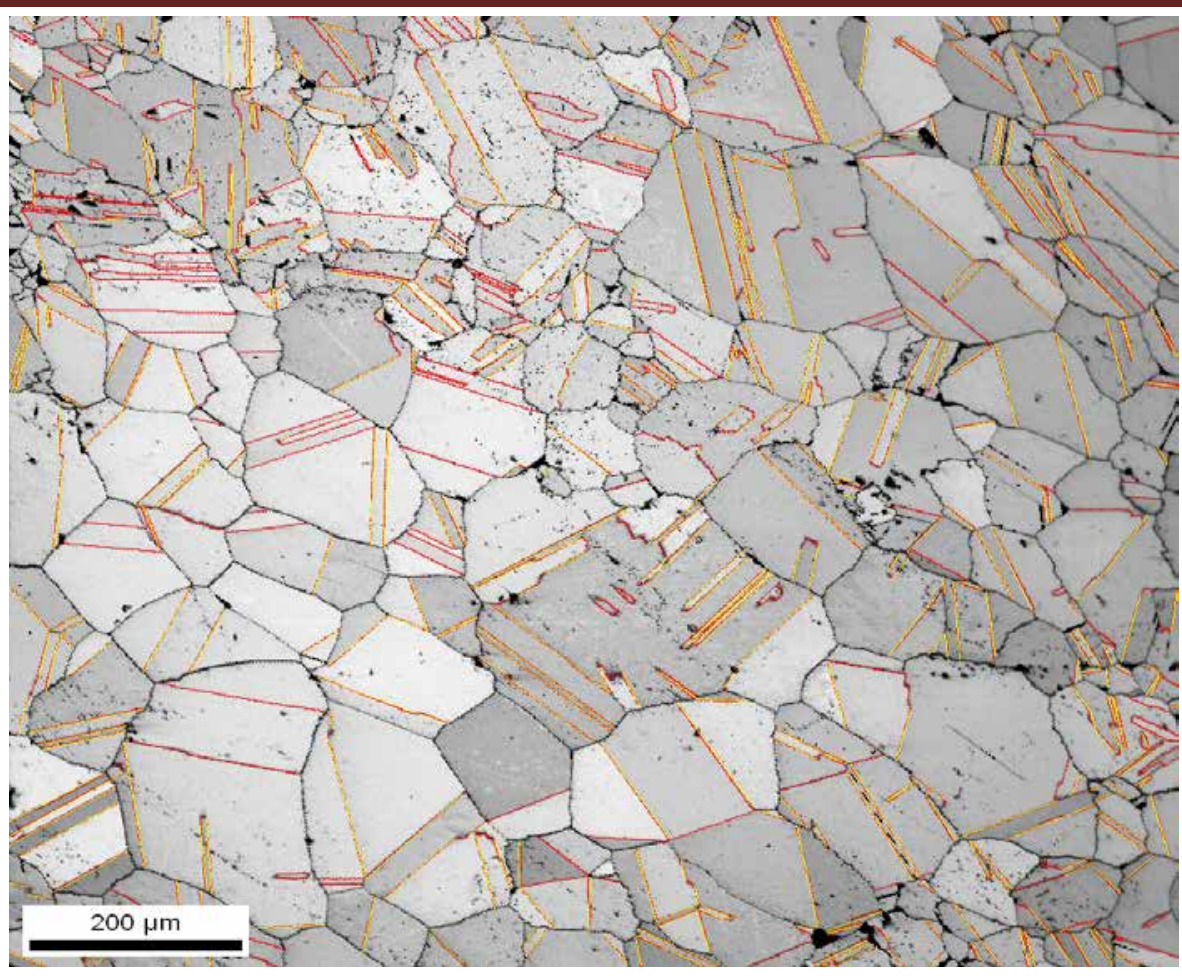

(a)

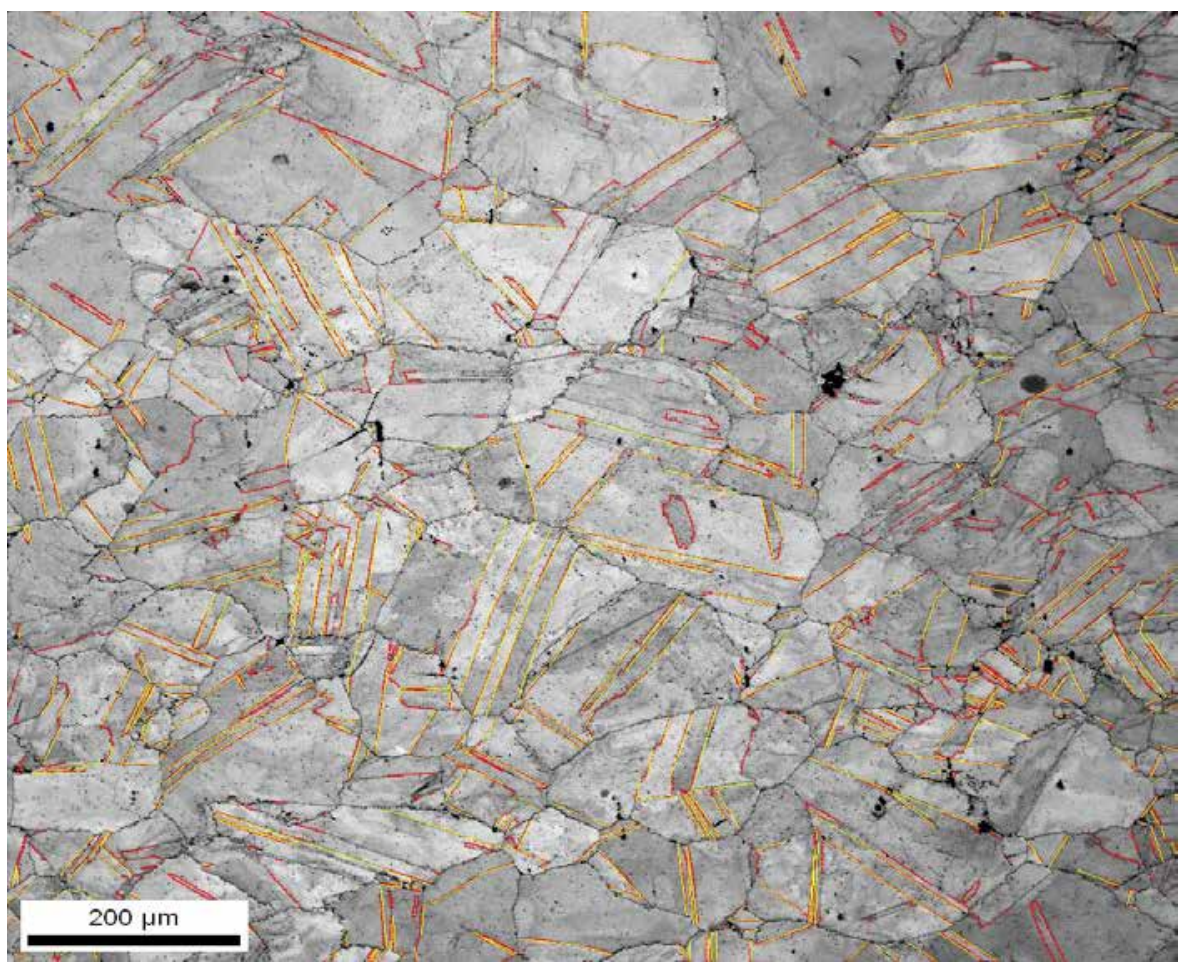

(b)

Fig. 4.101: Yellow lines highlight coherent twinning boundaries and red lines highlight both coherent and incoherent twinning boundaries in Alloy 617. Test condition: (a) aged; (b) $\varepsilon_{\text {tot }}=1.5 \%$ 


\section{Understanding Fundamental Material-Degradation Processes in High Temperature}

Aggressive Chemomechanical Environments

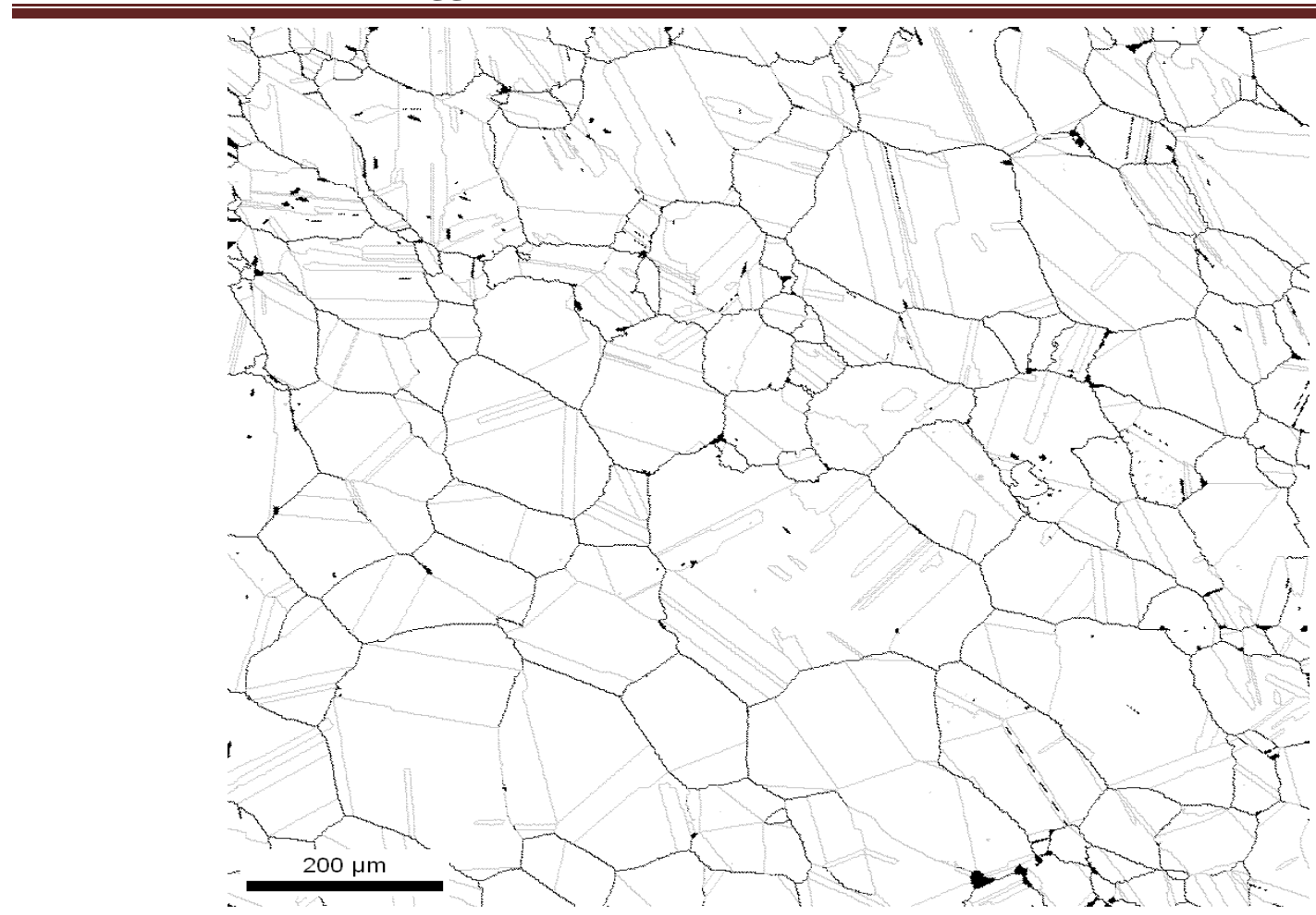

(a)

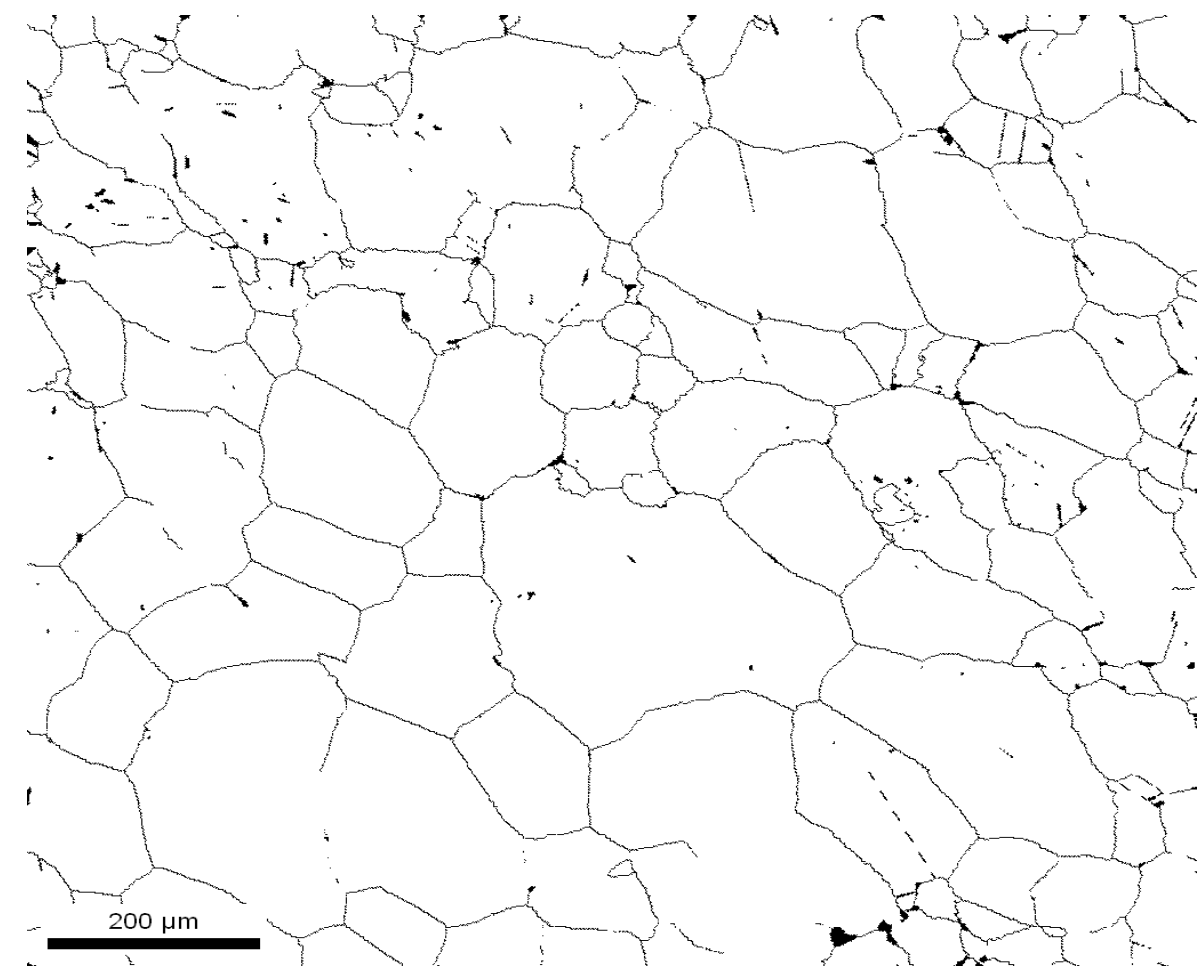

(b)

Fig. 4.102: Grain boundary character distribution of aged Alloy 617. (a) Dark lines are HAGBs and light grey lines are CSL boundaries; (b) HAGB network after removal of CSL boundaries 


\section{Understanding Fundamental Material-Degradation Processes in High Temperature}

Aggressive Chemomechanical Environments
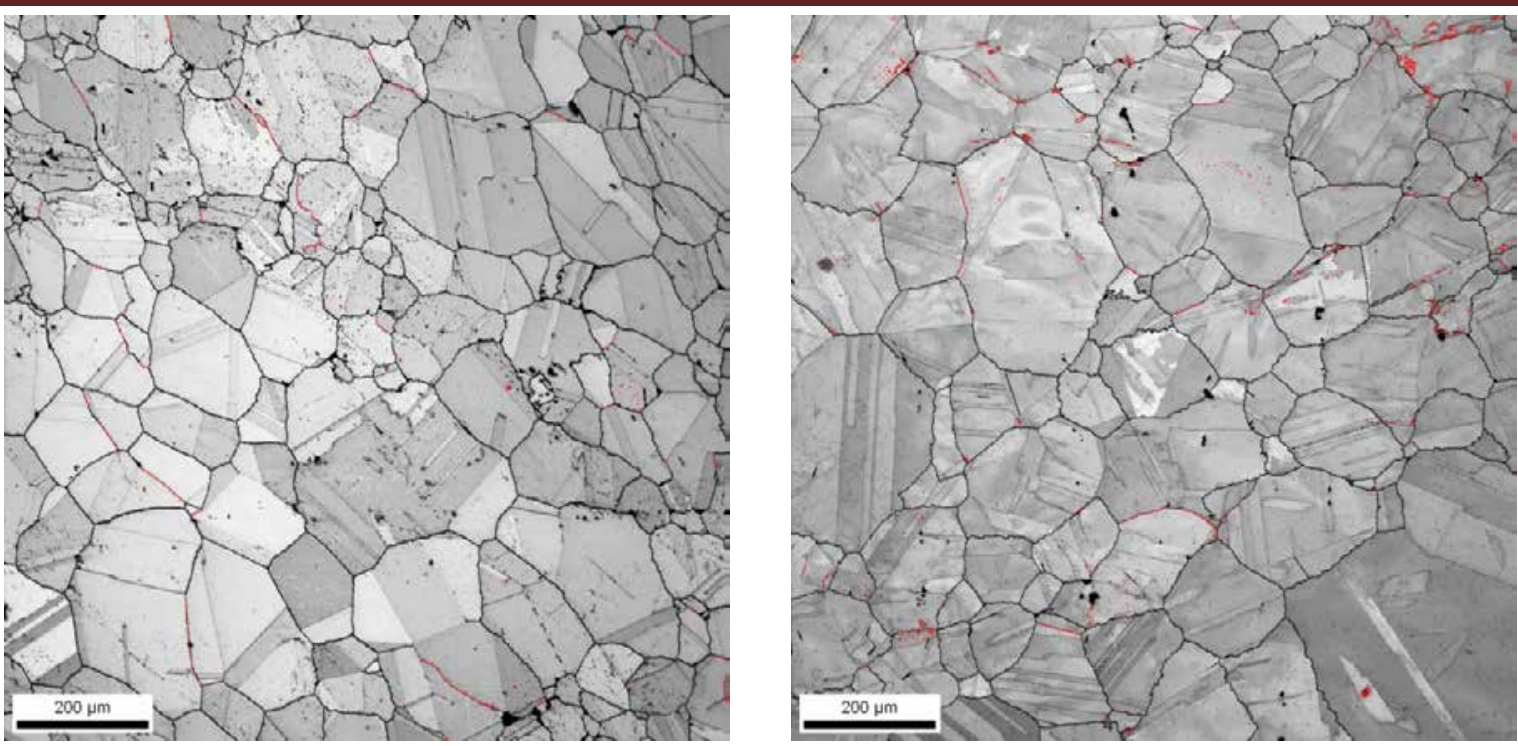

(a)

(b)
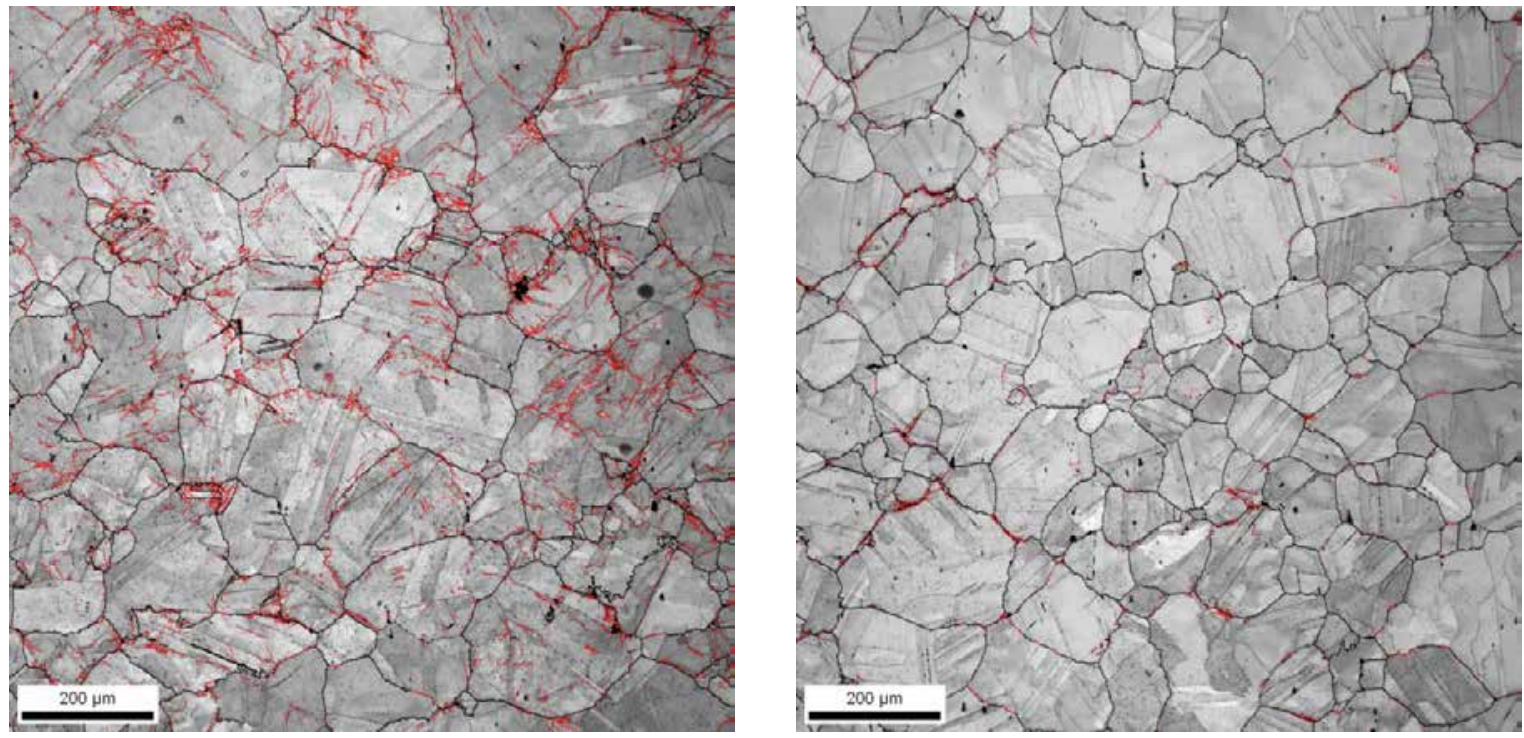

(c)

Fig. 4.103: LAGB distribution highlighted by red lines for different Alloy 617 samples. Test conditions:

(a) aged; (b) $\varepsilon_{\text {tot }}=1.0 \%$; (c) $\varepsilon_{\text {tot }}=1.5 \%$; (d) $\varepsilon_{\text {tot }}=0.5 \%$, dwell time $=3$ minutes; (e) $\varepsilon_{\text {tot }}=1.0 \%$, dwell time $=3$ minutes; (f) $\varepsilon_{\text {tot }}=1.0 \%$, dwell time $=30$ minutes 
Understanding Fundamental Material-Degradation Processes in High Temperature Aggressive Chemomechanical Environments
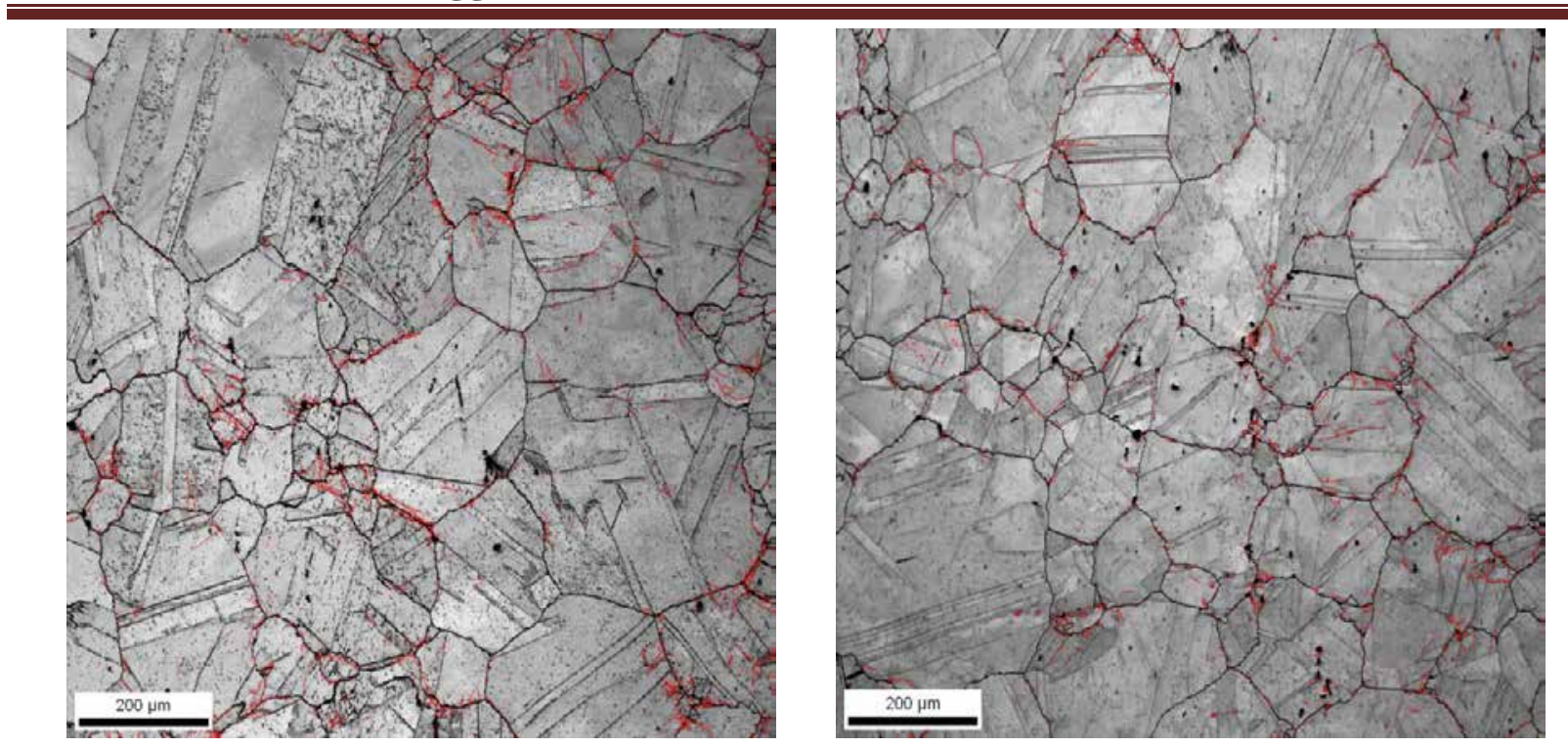

(e)

Fig. 4.103 Cont. 


\section{Understanding Fundamental Material-Degradation Processes in High Temperature}

Aggressive Chemomechanical Environments

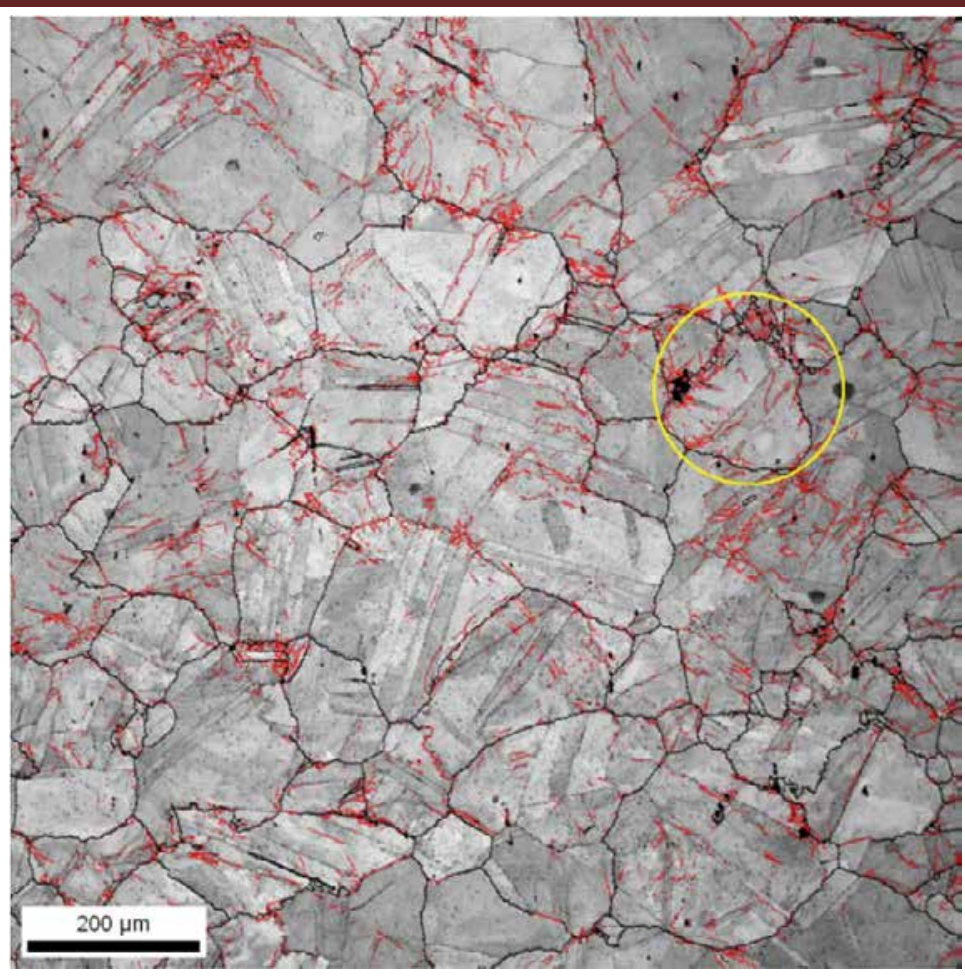

(a)

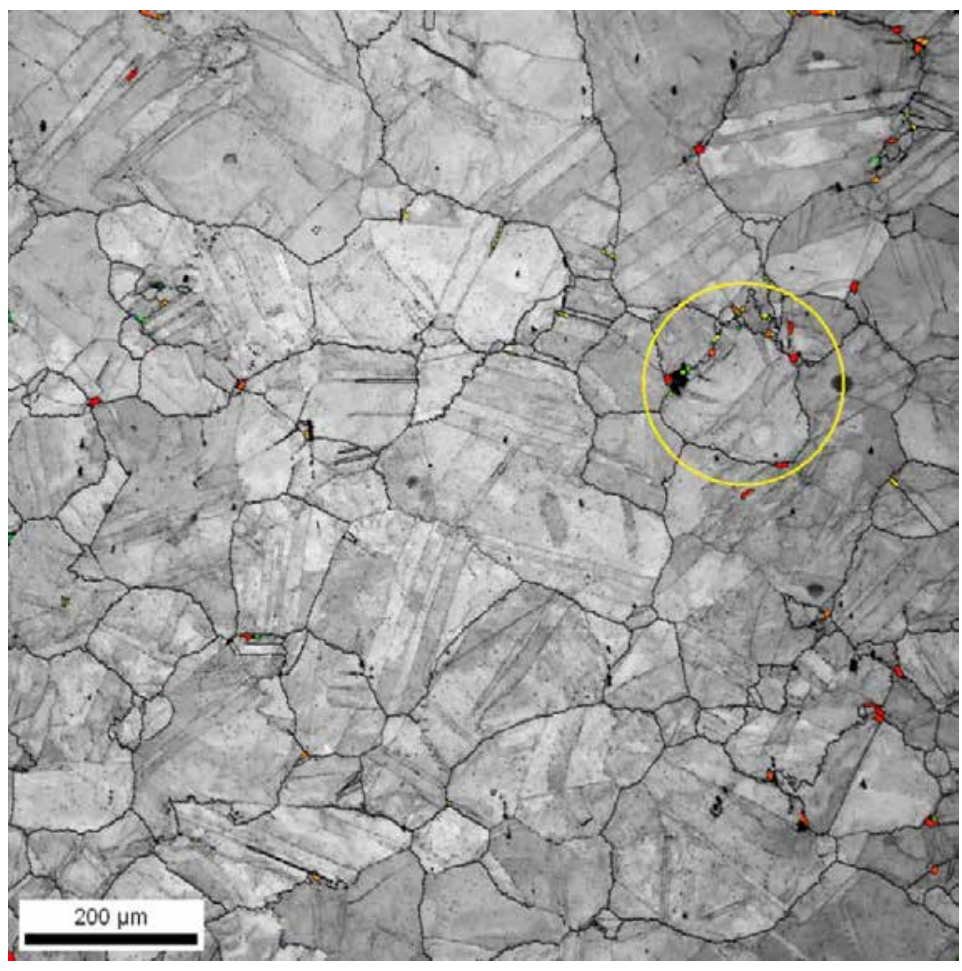

(b)

Fig. 4.104: (a) LAGBs displayed as red lines; (b) grains with grain size $\leq 10 \mu \mathrm{m}$ are highlighted with colors. The yellow circle highlights the correlation of a region rich in LAGBs with a region of DRX. Test condition: $\varepsilon_{\text {tot }}=1.5 \%$, dwell time $=3$ minutes 


\section{Understanding Fundamental Material-Degradation Processes in High Temperature}

Aggressive Chemomechanical Environments
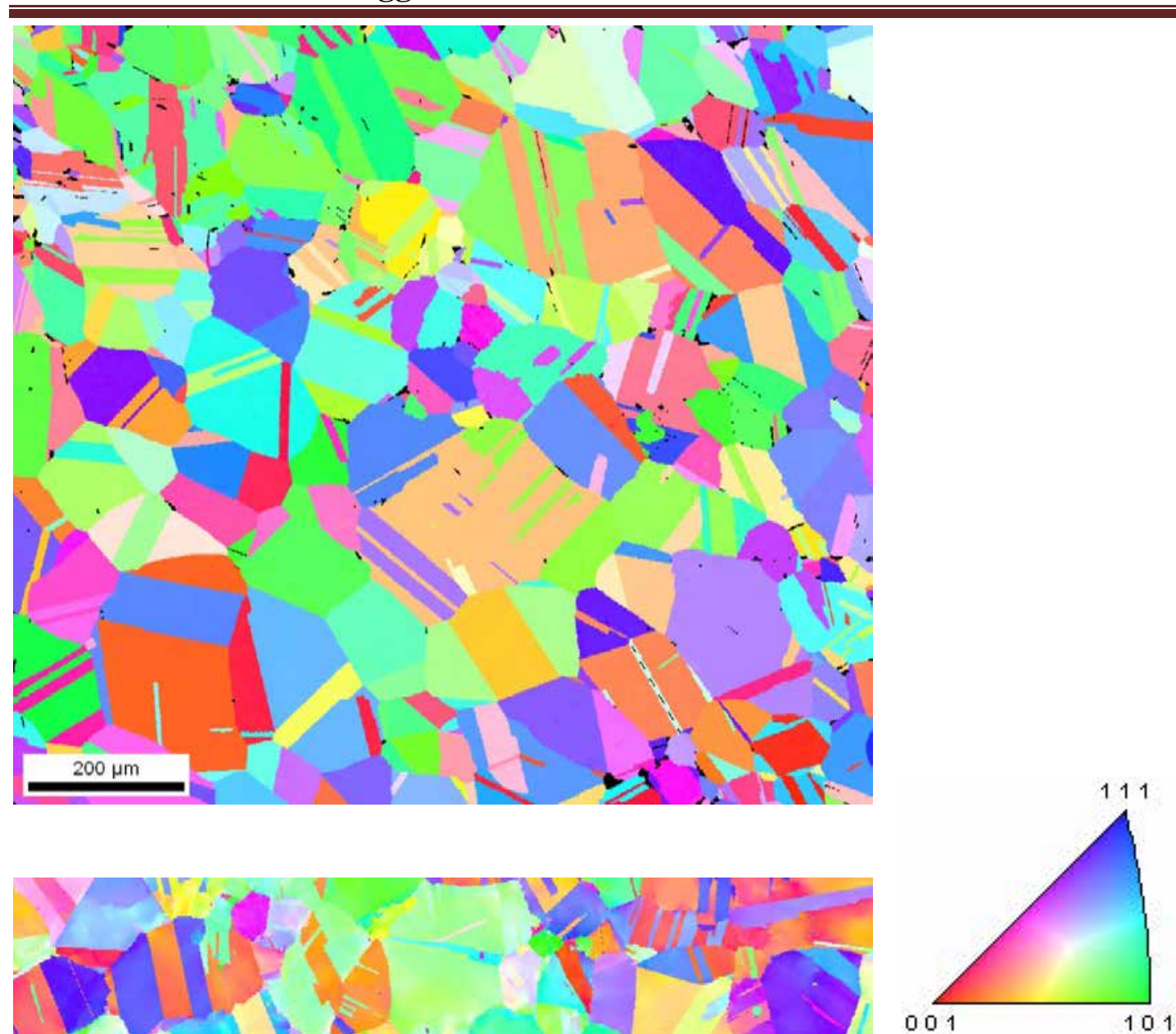

(a)

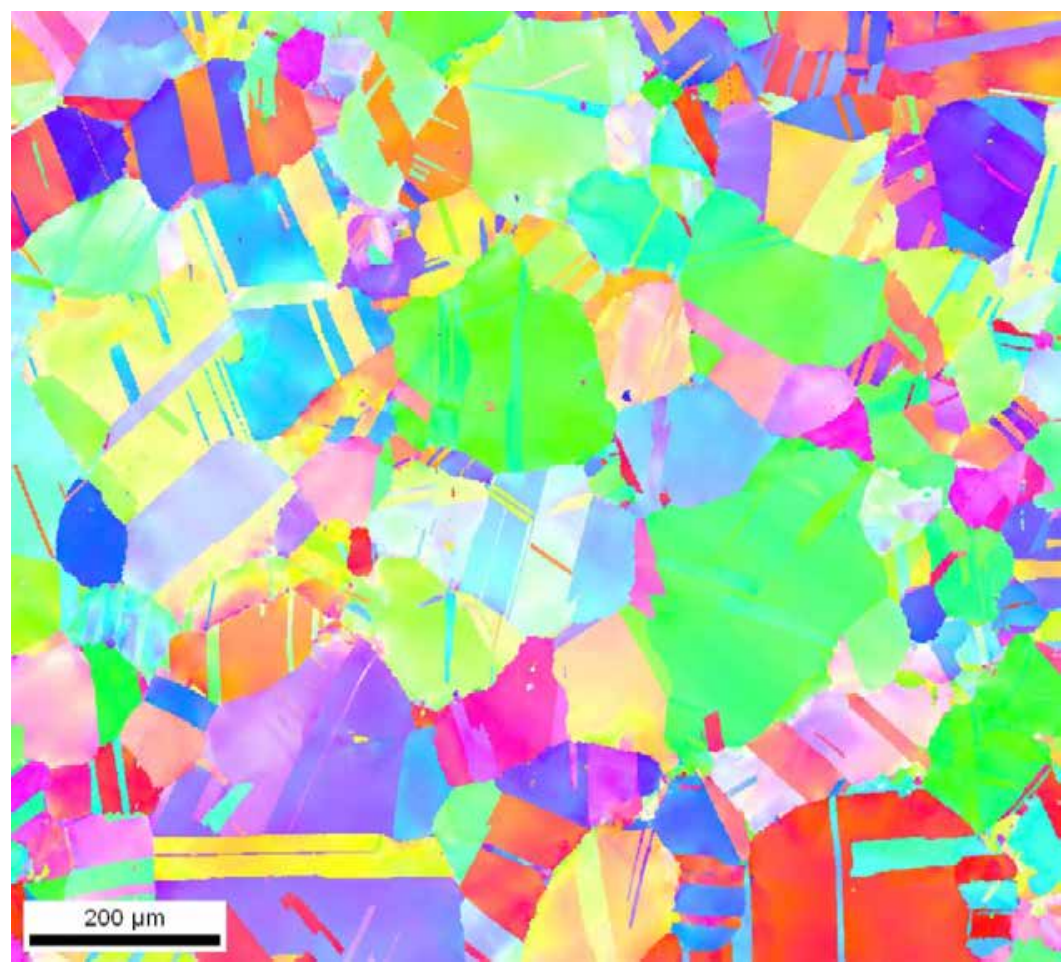

(b)

Fig. 4.105: IPF of Alloy 617 specimens: (a) aged at $850^{\circ} \mathrm{C}$ for 45 minutes; (b) creep-fatigue tested at $1.0 \%$ total strain range with a 10 minute hold time at peak tensile strain. No texture development was observed. 


\section{Understanding Fundamental Material-Degradation Processes in High Temperature Aggressive Chemomechanical Environments}

The same set of EBSD analyses on Alloy 230 were done on a scan area of $730 \mu \mathrm{m} \times 730 \mu \mathrm{m}$ with a step size of $1.2 \mu \mathrm{m}$. The statistical results based on EBSD analysis are listed in Table 4.11. The average grain size for the as-received and aged Alloy 230 specimens was about $40 \mu \mathrm{m}$, about half the grain size of Alloy 617. Mechanical testing, especially at higher total strain ranges and longer dwell times, might slightly reduce the average grain size of the material due to DRX. However, this is not as apparent as for Alloy 617. The average twinning boundary fraction of the Hayes 230 in the as-received condition was about 57\%, which was close to the twinning boundary fraction of Alloy 617. Grain rotation caused the twinning boundary fraction to decrease for the following test conditions: $\varepsilon_{\text {tot }}=1.5 \%$ LCF test, $\varepsilon_{\text {tot }}=1.5 \%$ with a 3 minute dwell time creep-fatigue test, and $\varepsilon_{\text {tot }}=1.0 \%$ with 3-30 minute dwell time creep-fatigue tests. Fig. 4.106 shows two examples of the twinning boundary distribution in Alloy 230. After creep-fatigue testing, many pre-existing parallel annealing twinning boundaries lost their special misorientation character and became random HAGBs.

The black "blocky" spots in Fig. 4.106 and all the following figures derived from EBSD analysis on Alloy 230 signify large carbide precipitates. These spots were different phases from the Ni matrix, and were filled with black color in the OIM analysis software. Before the test, about $70 \%$ of the twinning boundaries in Alloy 230 were coherent twinning boundaries, a value that was slightly larger than the counterpart value for Alloy 617. Once again, regardless of the different test conditions, the fraction of coherent twinning boundaries in the alloy did not change after the test. For illustration purposes, Fig. 4.107 presents the distribution of coherent and incoherent twinning boundaries in two Alloy 230 specimens. After applying the Palumbo criterion, the $\sum \leq 29$ CSL boundary fraction was calculated to be about $60 \%$ for Alloy 230 , which was slightly lower than that of Alloy 617 , but still above the $50 \%$ threshold known to produce a noticeable difference in the intergranular corrosion resistance [4.69].

The CSL boundary calculation for the tested Alloy 230 specimens was omitted for the same reason as for Alloy 617. The grain boundary character distribution of an aged Alloy 230 specimen is shown in Fig. 4.108. Similar to the case of Alloy 617, CSL boundaries can somewhat disrupt the random HAGB network. In addition, a bimodal grain size distribution was seen for Alloy 230. In particular, small grains were found in the region rich in bulky precipitates, while large grains were found in regions containing less bulky precipitates. This phenomenon can be explained by the role of precipitates in restricting grain growth during the solution treatment of the material. Grains surrounded by large numbers of precipitates could not grow as easily as grains with less precipitates during the elevated temperature thermal treatment.

LAGB evolution in Alloy 230 for different test conditions was quite different than that in Alloy 617. Starting with the aged Alloy 230 specimen, Fig. 4.109(a) shows LAGBs existing in the material. After the LCF test with $0.5 \%$ or $1.0 \%$ total strain range, numerous spots enriched with LAGBs spread homogeneously within the material, as shown in Fig. 4.109(b). When the total strain range of the LCF test was increased to $1.5 \%$, LAGBs began to concentrate near original random HAGBs, especially those of smaller grains, as in Fig. 4.109(c). Comparing creep-fatigue tests with 3 minute hold times at peak tensile strain with LCF tests at the same total strain level, such as comparing Fig. 4.109(c) with Fig. 4.109(e), reveals that creep deformation during the strain hold period led to more LAGB formation. Moreover, the morphology of the LAGB distribution changed when a 3 minute dwell time was added to the $1.0 \%$ total strain range LCF test, as seen by comparing Fig. 4.109(b) with Fig. 4.109(d). The LAGBs no longer 


\section{Understanding Fundamental Material-Degradation Processes in High Temperature Aggressive Chemomechanical Environments}

distributed homogeneously as spots spreading within the material, but instead segregated to random HAGBs.

For creep-fatigue tests at $1.0 \%$ total strain range, the total length of the LAGBs increased with the dwell time on peak tensile strain, as shown in Fig. 4.109(d), Fig. 4.109(f), and Table 4.11, since the creep deformation during the strain hold period is related to the length of the strain hold period. In contrast to Alloy 617, the total length of the LAGBs did not decrease during the creep-fatigue test at $1.0 \%$ total strain range with a 30 minute strain hold time. This may be due to the fact that Alloy 230 has a lower stacking fault energy than Alloy 617 (this will be discussed further in the deformation mechanism session of Chapter 5), and hence the dynamic recovery process in Alloy 230 is less efficient than in Alloy 617. Texture development was not observed for all Alloy 230 specimens. Two examples of the grain orientation in Alloy 230 are shown in Fig. 4.110. The grain orientation changed due to the deformation induced by grain rotations after the mechanical testing, and is shown in Fig. 4.110(b). 
Understanding Fundamental Material-Degradation Processes in High Temperature Aggressive Chemomechanical Environments

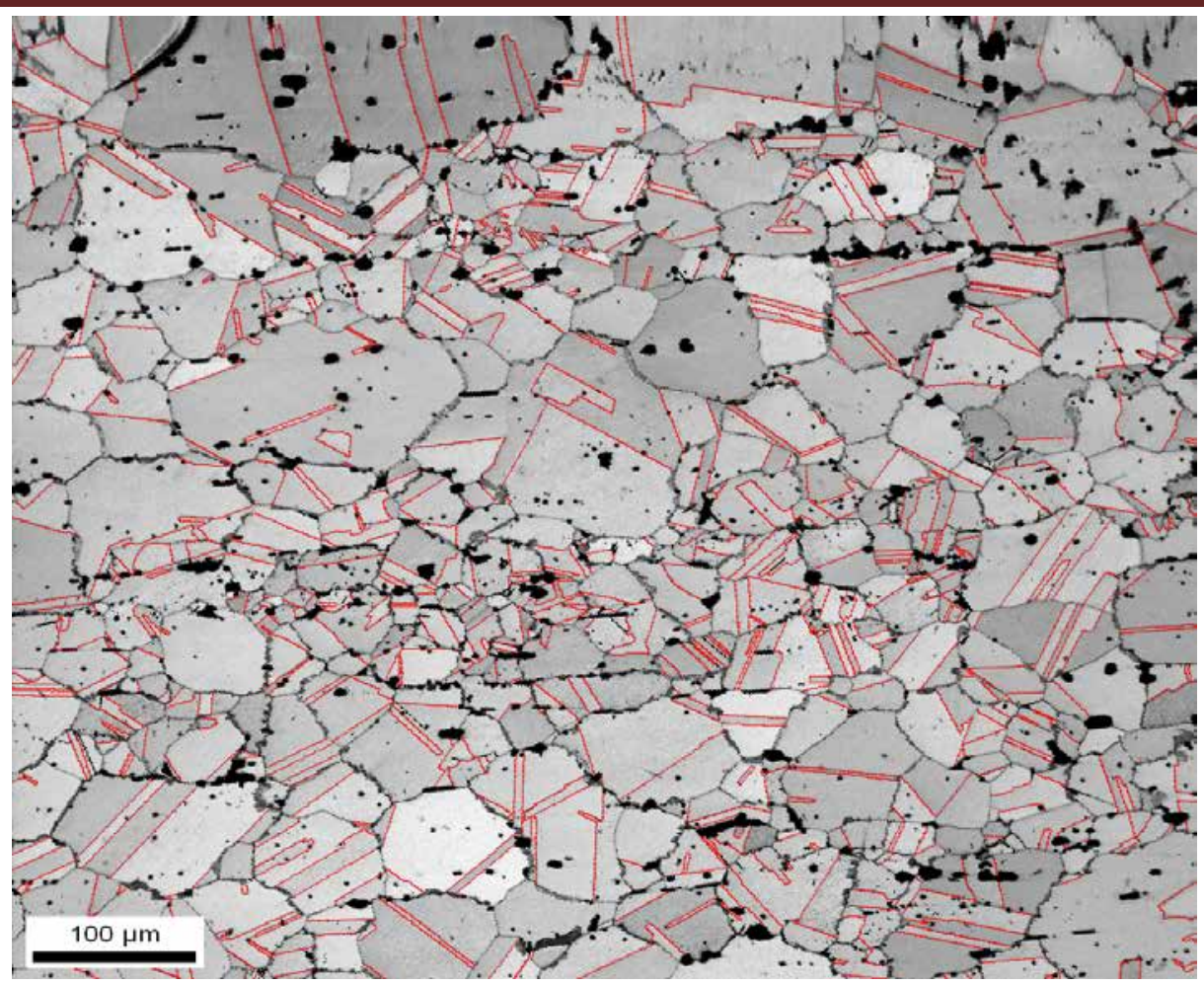

(a)

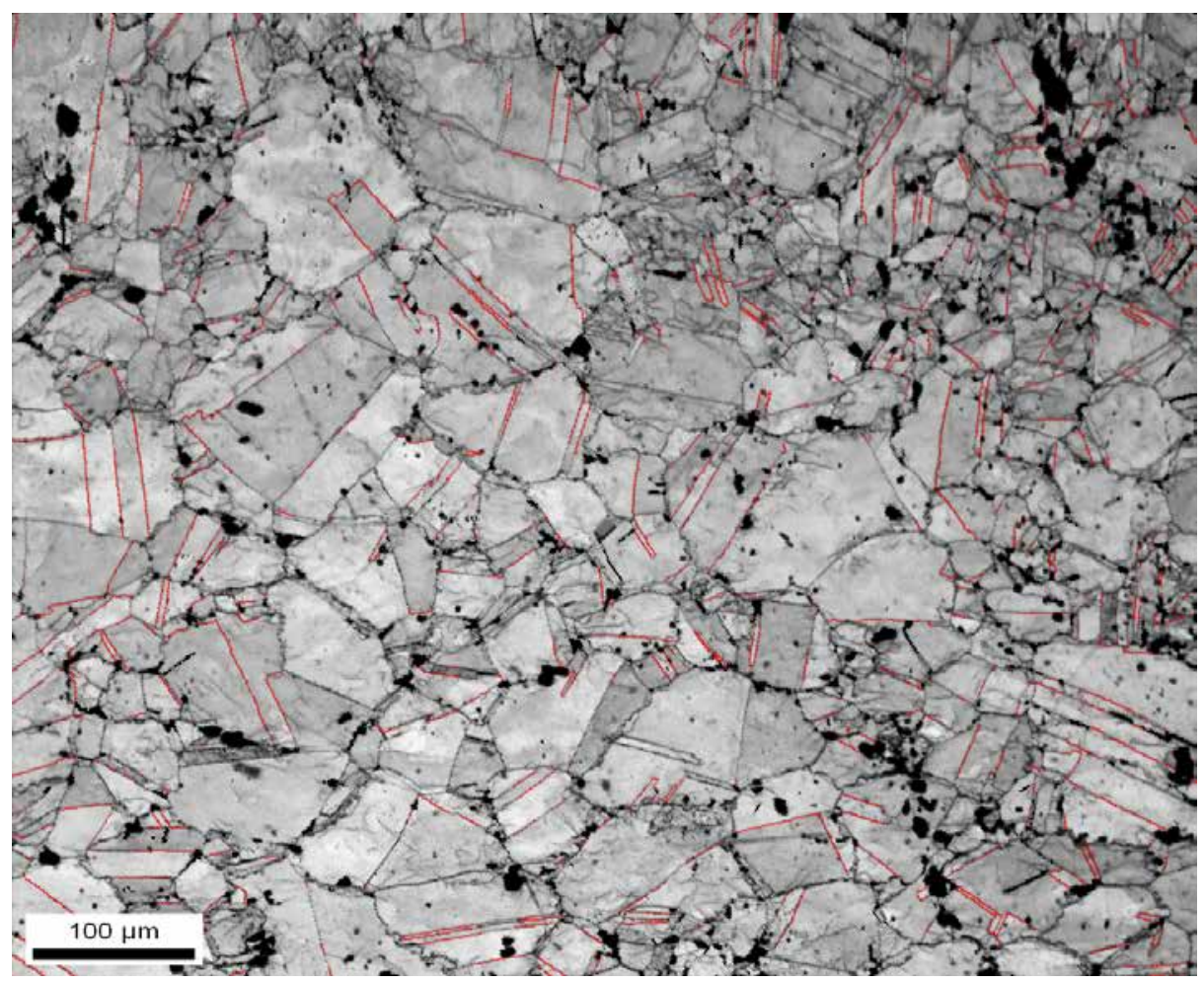

(b)

Fig. 4.106: Twinning boundaries highlighted by red lines in Alloy 230. Test conditions: (a) aged; (b) $\varepsilon_{\text {tot }}=1.5 \%$, dwell time $=3$ minutes 


\section{Understanding Fundamental Material-Degradation Processes in High Temperature}

Aggressive Chemomechanical Environments

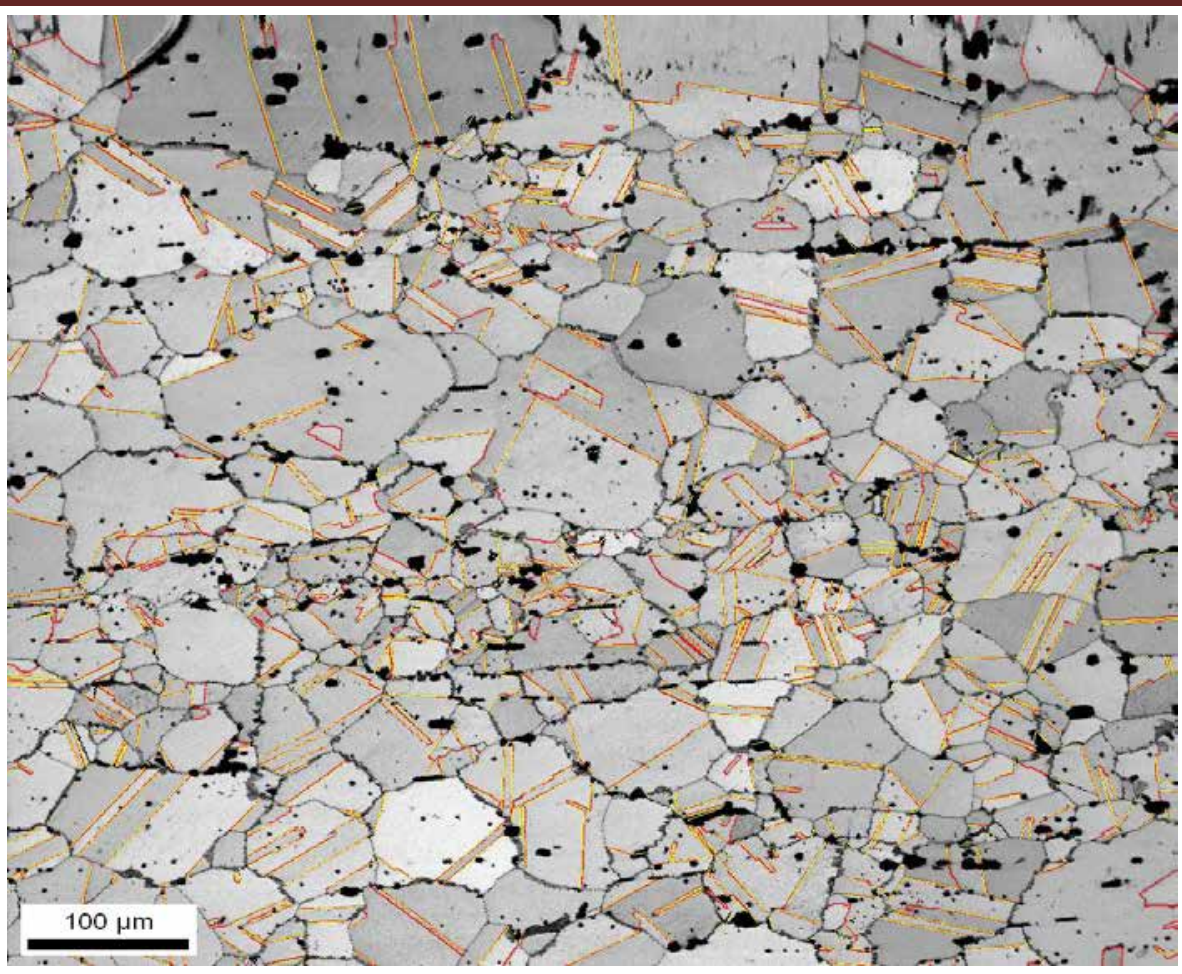

(a)

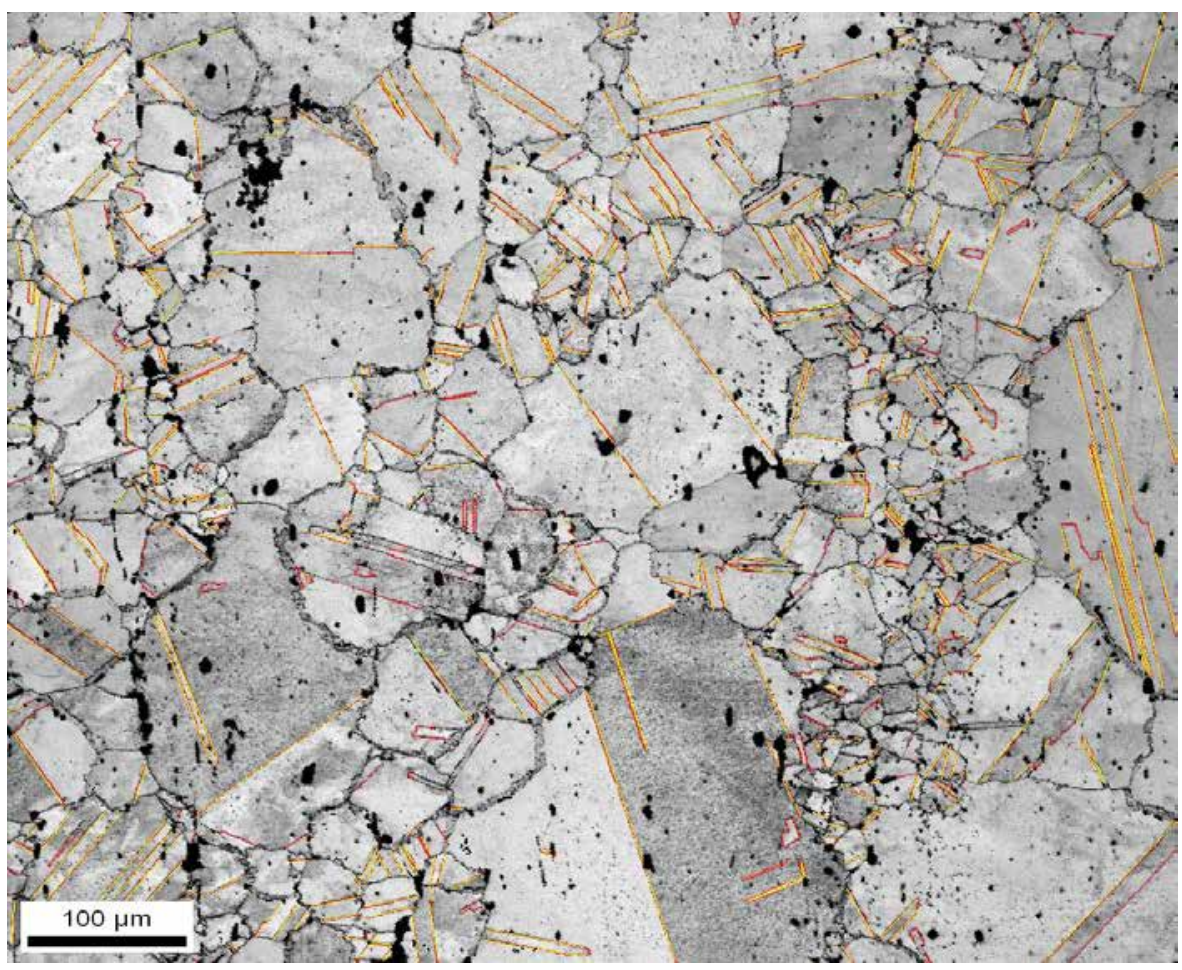

(b)

Fig. 4.107: Yellow lines highlight coherent twinning boundaries and red lines highlight both coherent and incoherent twinning boundaries in Alloy 230. Test conditions: (a) aged; (b) $\varepsilon_{\text {tot }}=1.5 \%$ 


\section{Understanding Fundamental Material-Degradation Processes in High Temperature}

Aggressive Chemomechanical Environments

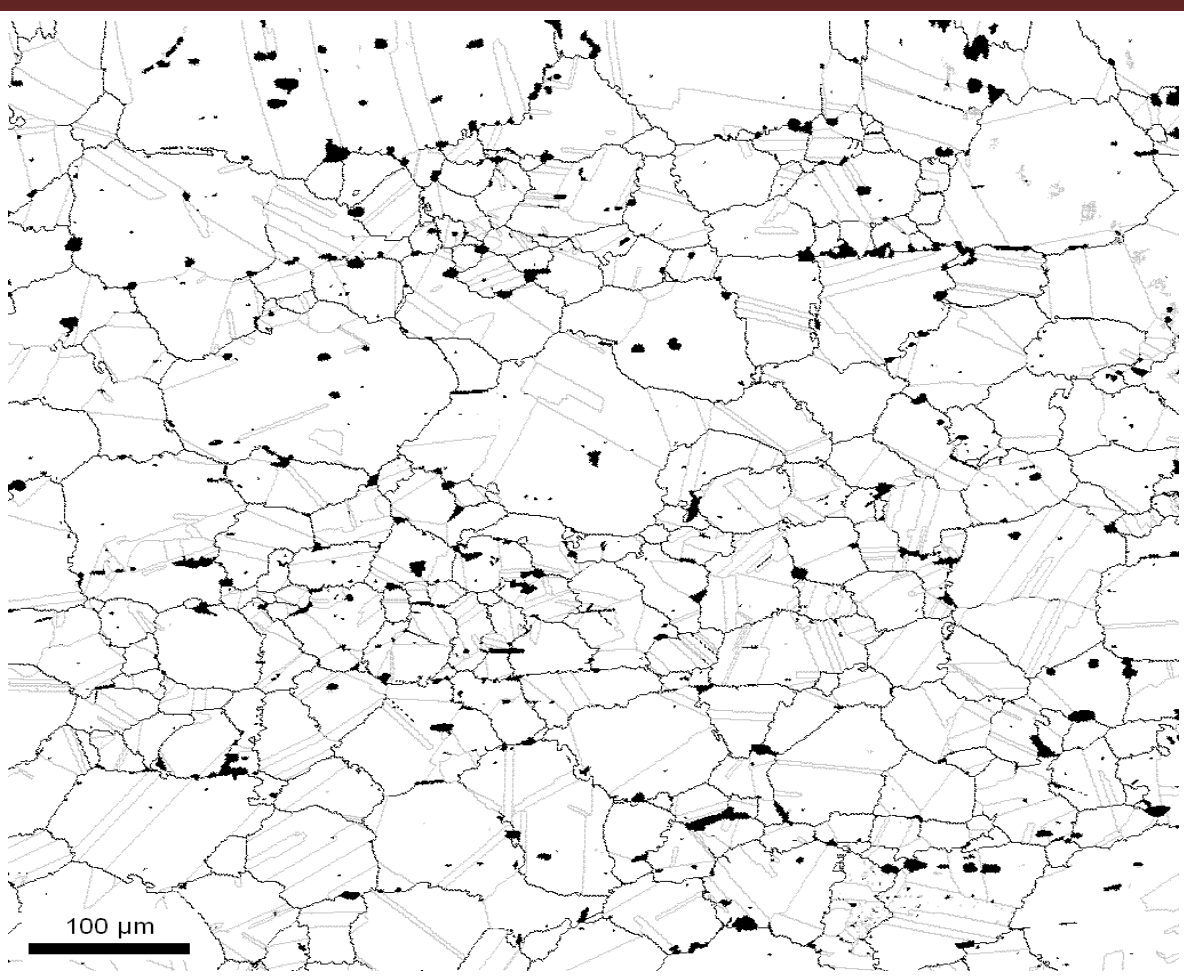

(a)

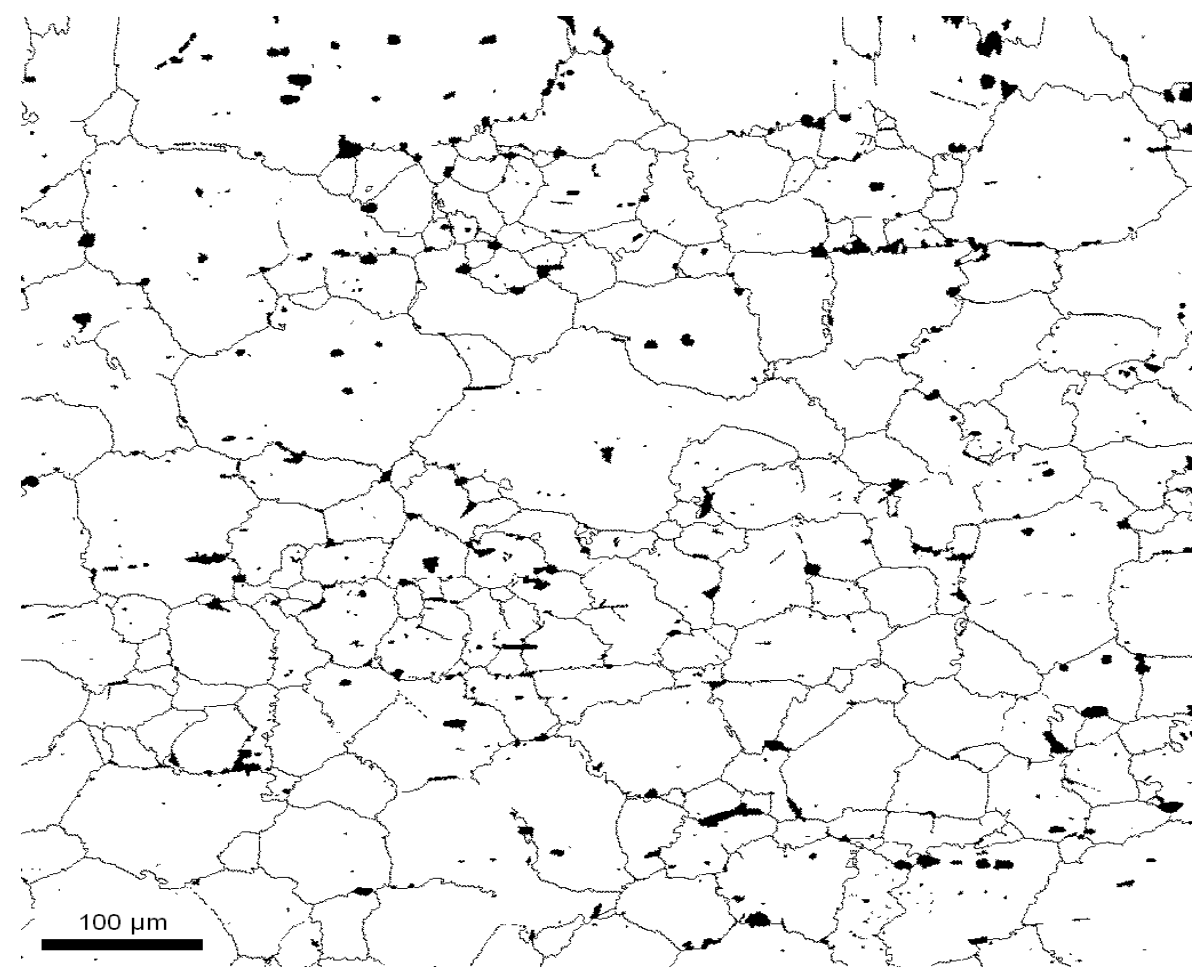

(b)

Fig. 4.108: Grain boundary character distribution of aged Alloy 230. (a) Dark lines are HAGBs, and light grey lines are CSL boundaries; (b) HAGB network after removal of CSL boundaries 


\section{Understanding Fundamental Material-Degradation Processes in High Temperature}

Aggressive Chemomechanical Environments
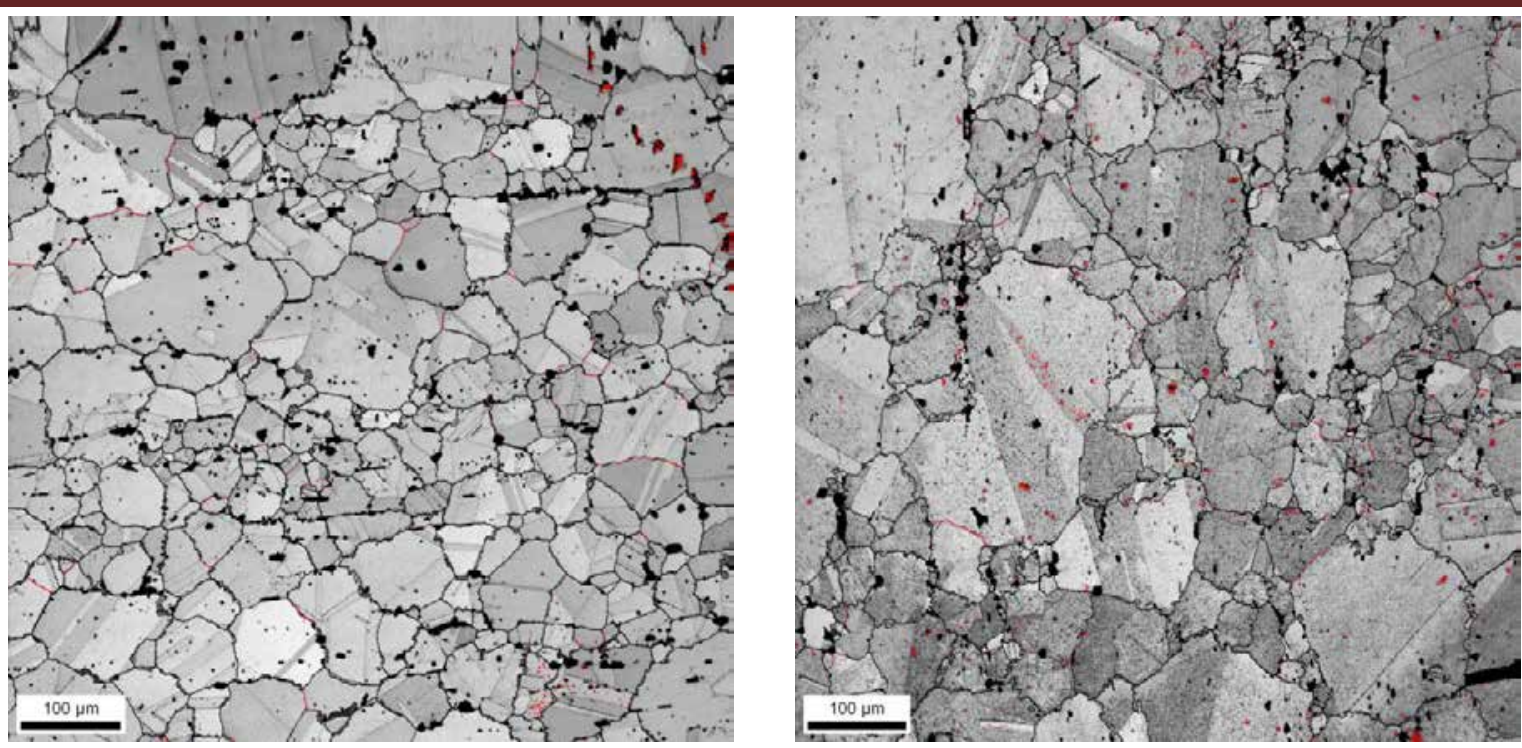

(a)

(b)
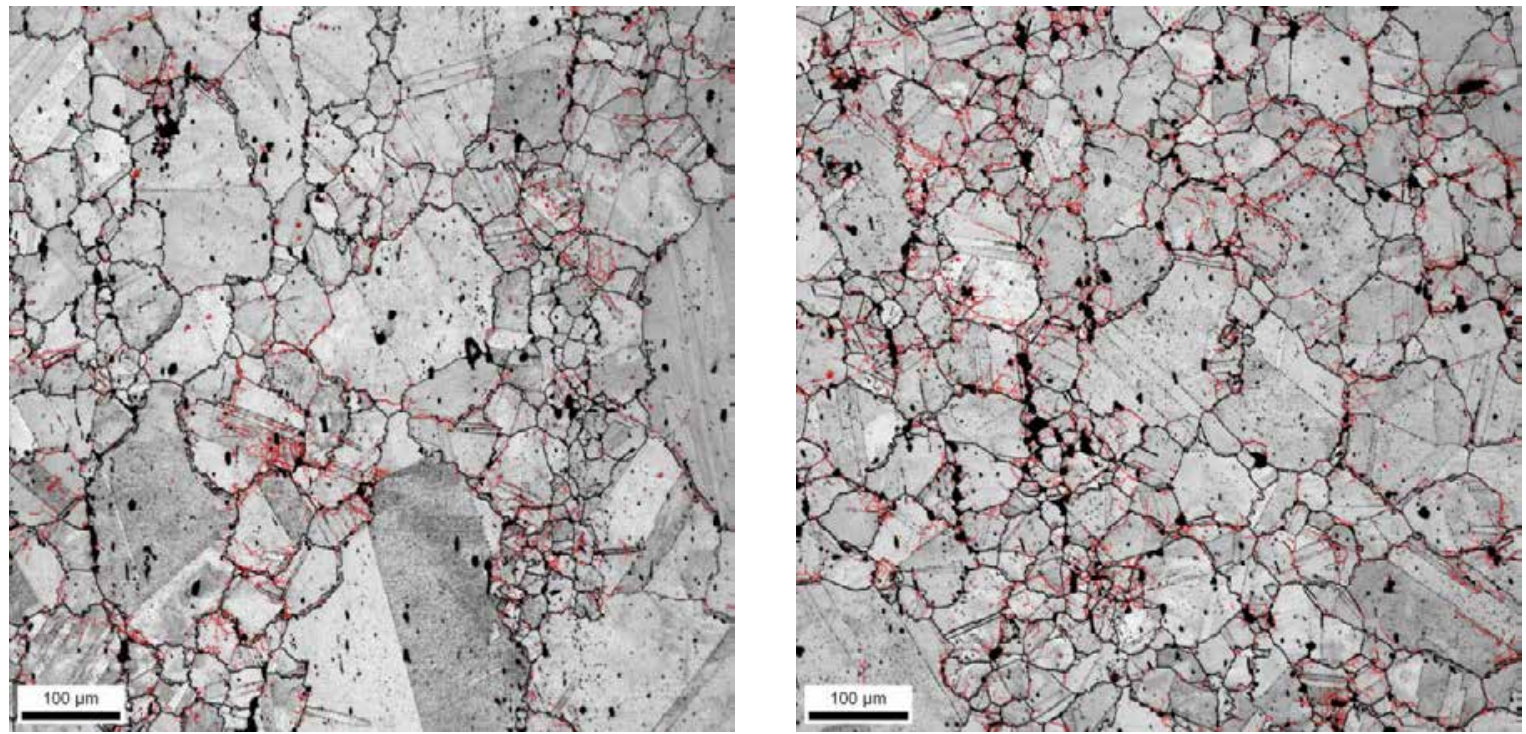

(c)

(d)

Fig. 4.109: LAGB distribution highlighted by red lines for different Alloy 230 samples. Test conditions:

(a) aged; (b) $\varepsilon_{\text {tot }}=1.0 \%$; (c) $\varepsilon_{\text {tot }}=1.5 \%$; (d) $\varepsilon_{\text {tot }}=1.0 \%$, dwell time $=3$ minutes; (e) $\varepsilon_{\text {tot }}=1.5 \%$, dwell time $=3$ minutes; (f) $\varepsilon_{\text {tot }}=1.0 \%$, dwell time $=30$ minutes 
Understanding Fundamental Material-Degradation Processes in High Temperature Aggressive Chemomechanical Environments
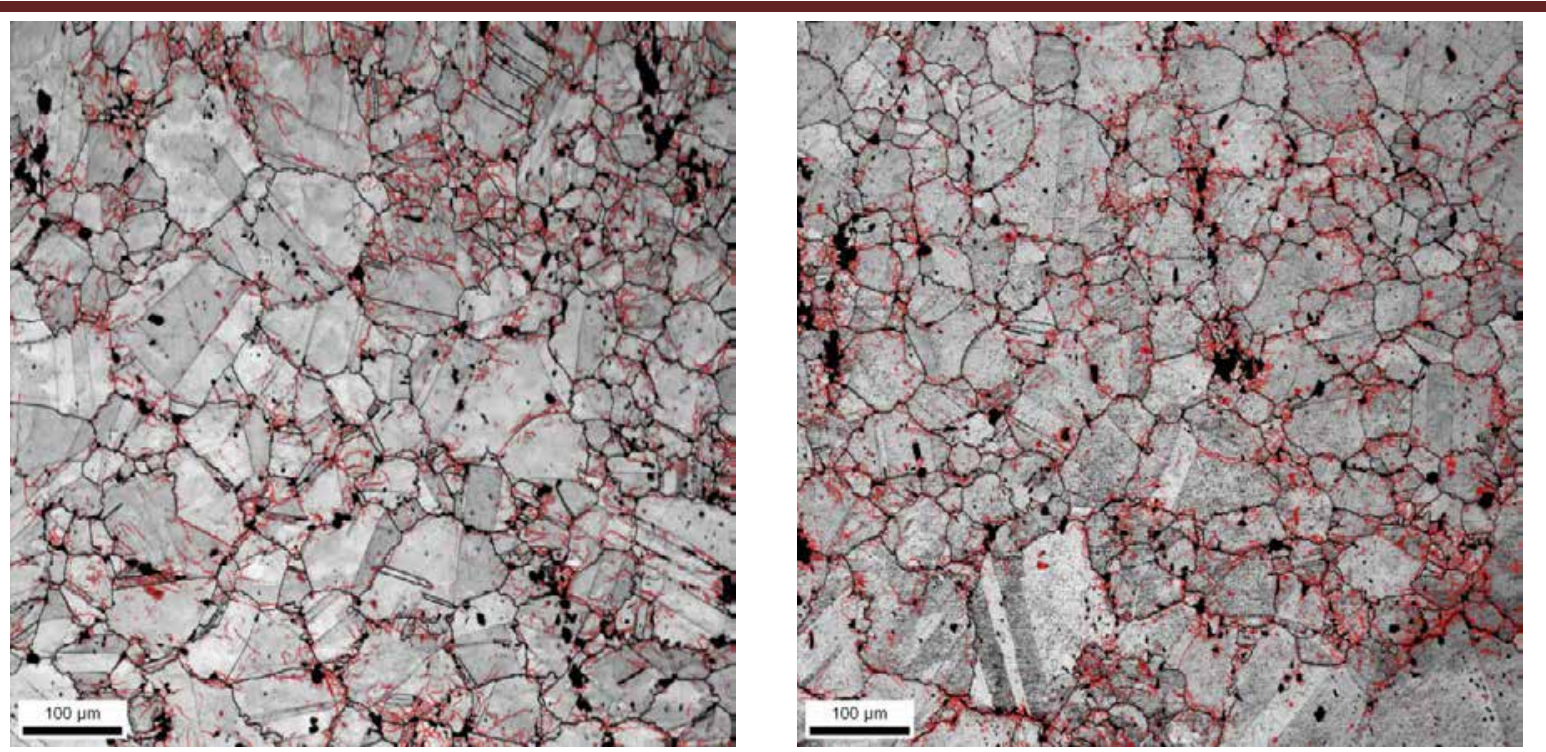

(e)

Fig. 4.109 Cont. 


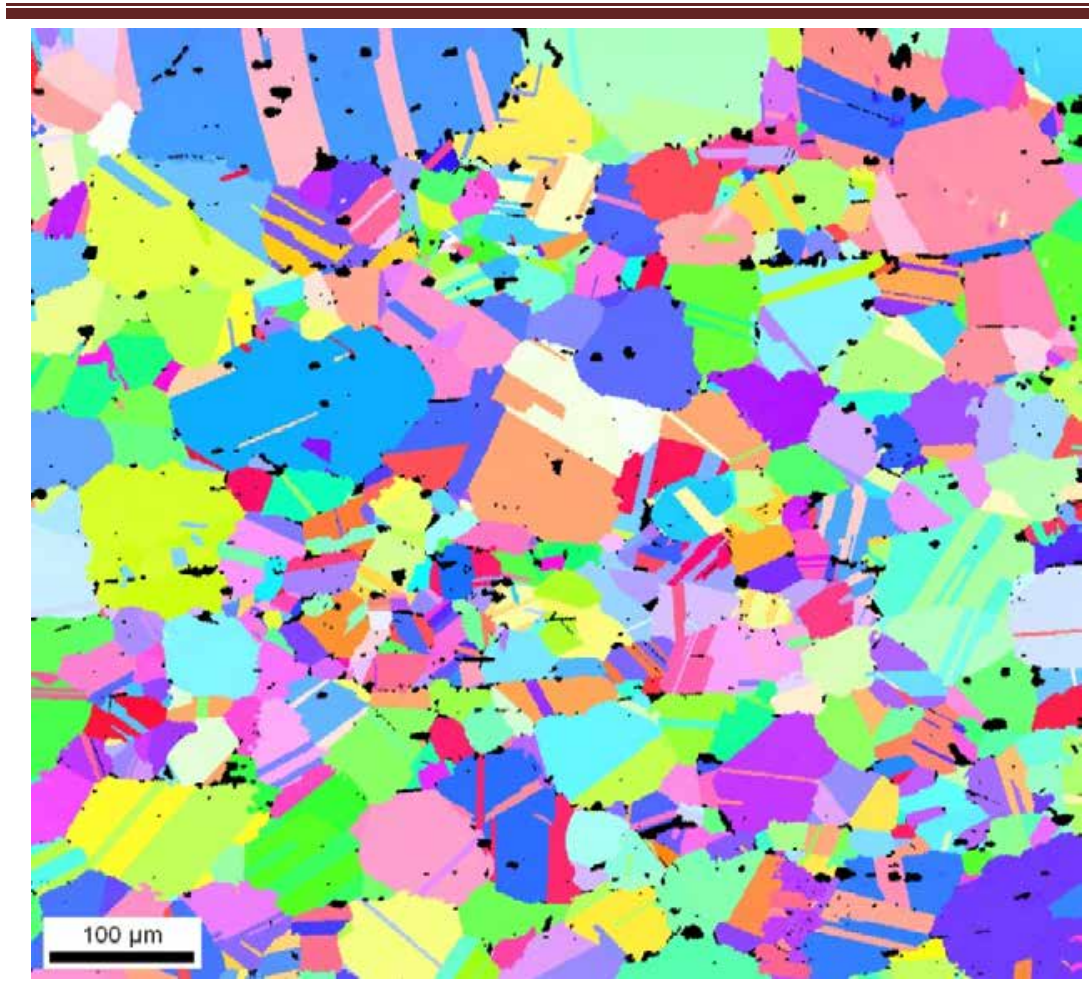

(a)
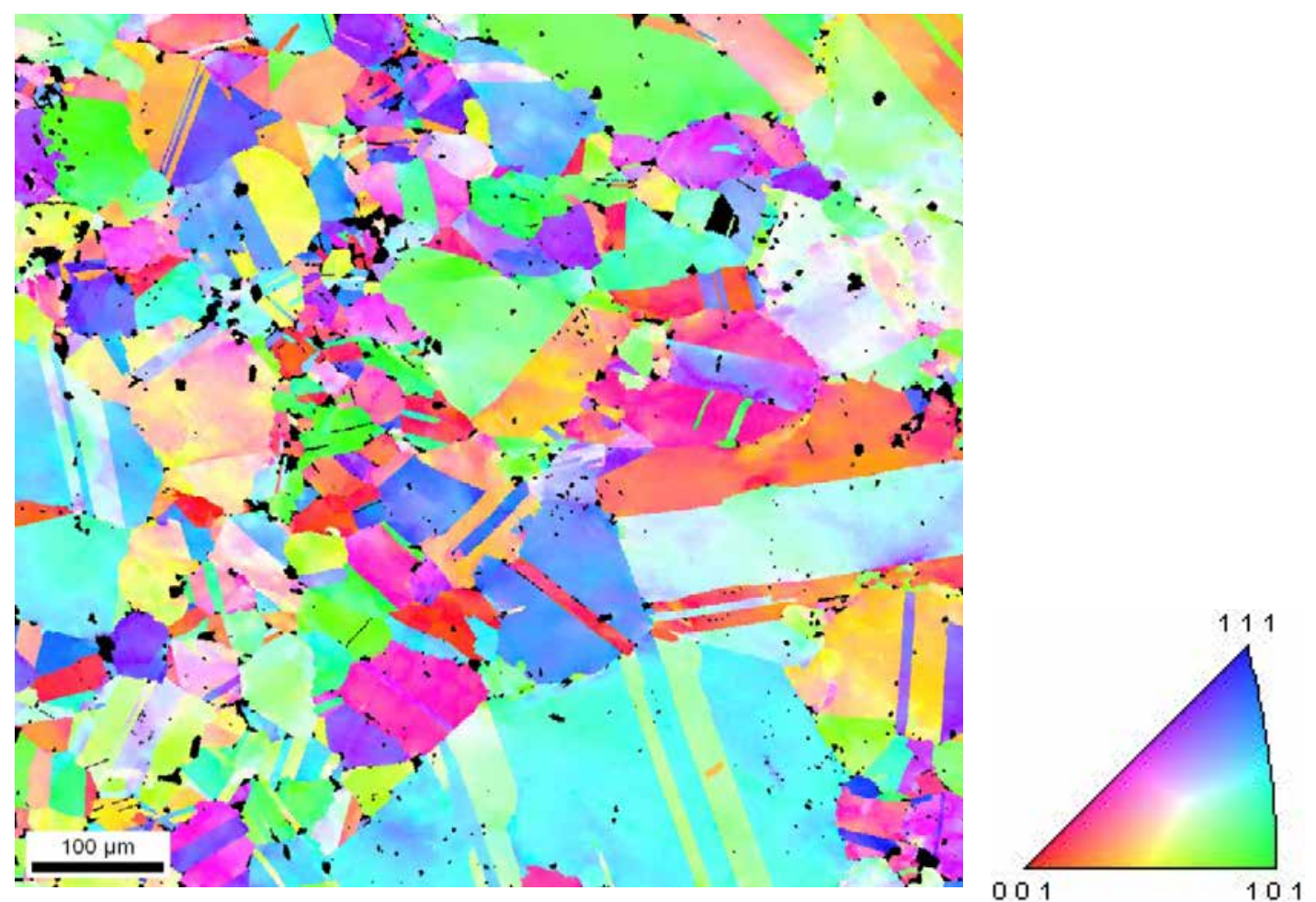

(b)

Fig. 4.110: IPF of Alloy 230 specimens: (a) aged at $850^{\circ} \mathrm{C}$ for 45 minutes; (b) creep-fatigue tested at $1.0 \%$ total strain range with a 10 minute hold time at peak tensile strain. No texture development was observed. 
Understanding Fundamental Material-Degradation Processes in High Temperature Aggressive Chemomechanical Environments

Table 4.11: Statistical EBSD analysis for Alloy 230

\begin{tabular}{|c|c|c|c|c|c|c|c|c|c|c|}
\hline & \multicolumn{10}{|c|}{ Alloy 230} \\
\hline & Raw & Aged & $0.5 \%$ & $1.0 \%$ & $1.5 \%$ & $\begin{array}{c}0.5 \%+3 \\
\min \end{array}$ & $\begin{array}{c}1.0 \%+3 \\
\min \end{array}$ & $\begin{array}{c}1.5 \%+3 \\
\min \end{array}$ & $\begin{array}{c}1.0 \%+10 \\
\min \end{array}$ & $\begin{array}{c}1.0 \%+30 \\
\min \end{array}$ \\
\hline Grain size $(\mu \mathrm{m})^{*}$ & 44.7 & 39.5 & 41.7 & 37.7 & 37.1 & 35.7 & 35.8 & 32.0 & 33.9 & 33.9 \\
\hline Twinning (\%) & 58.9 & 54.2 & 52.3 & 51.8 & 48.8 & 54.4 & 42.2 & 28.2 & 37.0 & 40.2 \\
\hline $\begin{array}{c}\text { Coherent } \\
\text { Twinning (\%) }\end{array}$ & 69.2 & 68.1 & 67.6 & 63.5 & 69.5 & 61.1 & 71.2 & 67.8 & 69.6 & 70.1 \\
\hline CSL fraction $(\%)$ & 64.2 & 57.0 & & & & & N/A & & & \\
\hline LAGB & $\begin{array}{l}3.9 \\
\mathrm{~mm}\end{array}$ & $\begin{array}{l}1.8 \\
\mathrm{~mm}\end{array}$ & $\begin{array}{l}9.2 \\
\mathrm{~mm}\end{array}$ & $\begin{array}{l}5.3 \\
\mathrm{~mm}\end{array}$ & $\begin{array}{l}1.5 \\
\mathrm{~cm}\end{array}$ & $2.7 \mathrm{~cm}$ & $2.5 \mathrm{~cm}$ & $3.9 \mathrm{~cm}$ & $4.2 \mathrm{~cm}$ & $5.0 \mathrm{~cm}$ \\
\hline Texture $(\mathrm{Y} / \mathrm{N})$ & $\mathrm{N}$ & $\mathrm{N}$ & $\mathrm{N}$ & $\mathrm{N}$ & $\mathrm{N}$ & $\mathrm{N}$ & $\mathrm{N}$ & $\mathrm{N}$ & $\mathrm{N}$ & $\mathrm{N}$ \\
\hline
\end{tabular}

*During grain size calculations, coherent twinning boundaries were not counted as GBs since they are generally immobile and not a constituent of the intergranular transport network.

\subsubsection{Correlation of microstructure features with EBSD analysis}

The cracking mode identification in sections 4.9.2 and 4.9.3 was aided by EBSD IPF analysis, since occasionally the SEM images did not have enough contrast to differentiate intergranular cracking from transgranular cracking. For instance, it is difficult to determine the exact cracking mode from the original SEM figure shown in Fig. 4.111(a). However, from the corresponding EBSD IPF analysis shown in Fig. 4.111(b), it is apparent that the cracking mode was transgranular, as the crystal orientation on both sides of any point along the crack path is the same. In contrast, Fig. 4.111(c) illustrates an example of intergranular cracking in which the crystal orientation on both sides of any point along the crack path was different. Table 4.12 summarizes the cracking modes of Alloys 617 and 230 under various test conditions. It is apparent that the introduction of a dwell time at peak tensile strain to LCF tests tends to change the crack initiation mode from transgranular initiation to intergranular initiation, especially for Alloy 617. The longer the dwell time at peak tensile strain, the greater the extent of the intergranular crack propagation in Alloy 617. The results very closely match the changes in the mode of fatigue crack initiation and propagation as a function of the creep component proposed by Gell et al. These results are presented in Table 4.13 [4.47]. Similar changes in the fatigue crack mode due to the creep damage incorporated into the fatigue test were observed in other related works [4.49, 4.35, 4.74, 4.75, 4.76].

Moreover, Alloy 230 exhibited better resistance to intergranular cracking than Alloy 617 under the same creep-fatigue test conditions. The cracking mode results also correlate with creep-fatigue life results, in a sense that intergranular cracking occurs at a faster rate than transgranular cracking, and hence materials that are more subject to intergranular cracking tend to have shorter fatigue lives. 


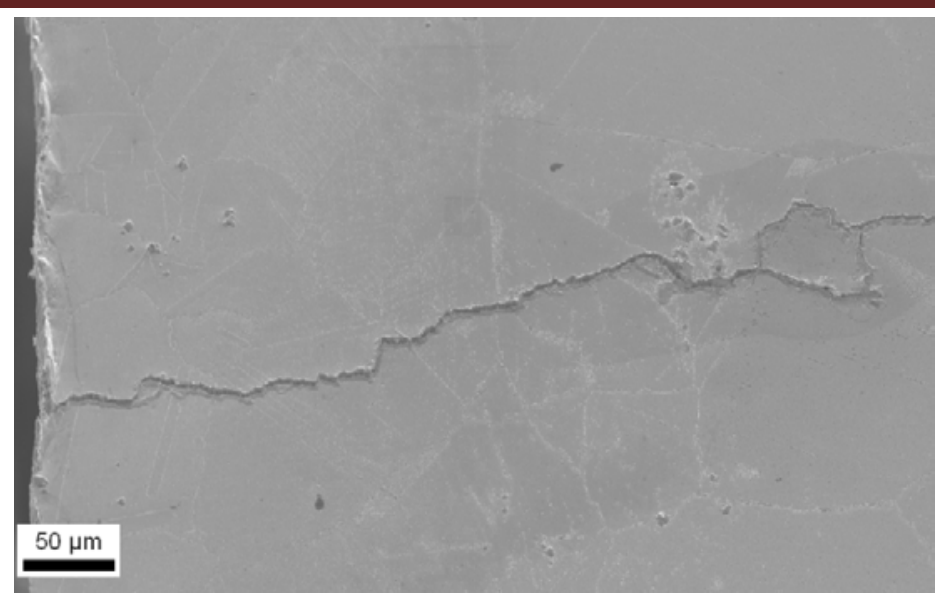

(a)

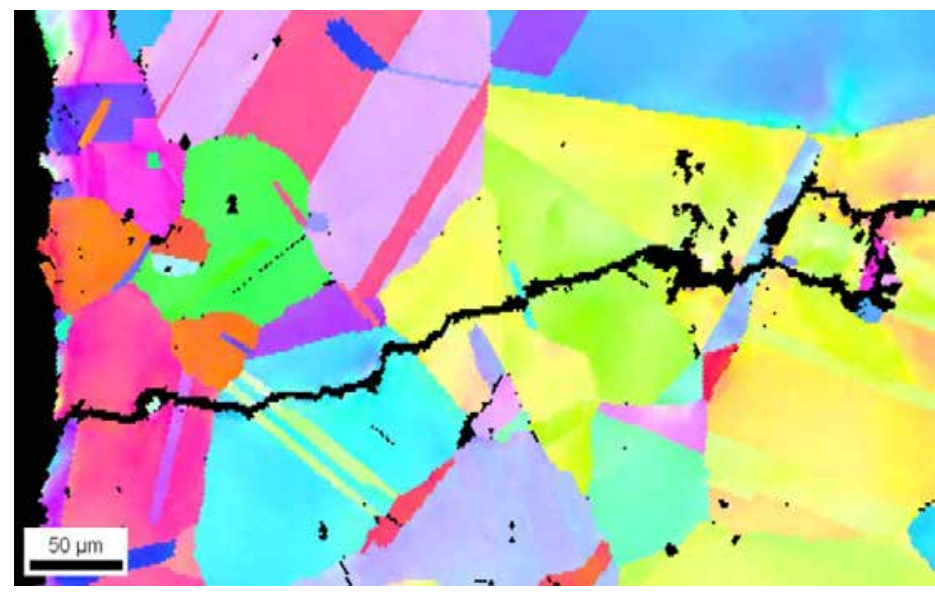

(b)
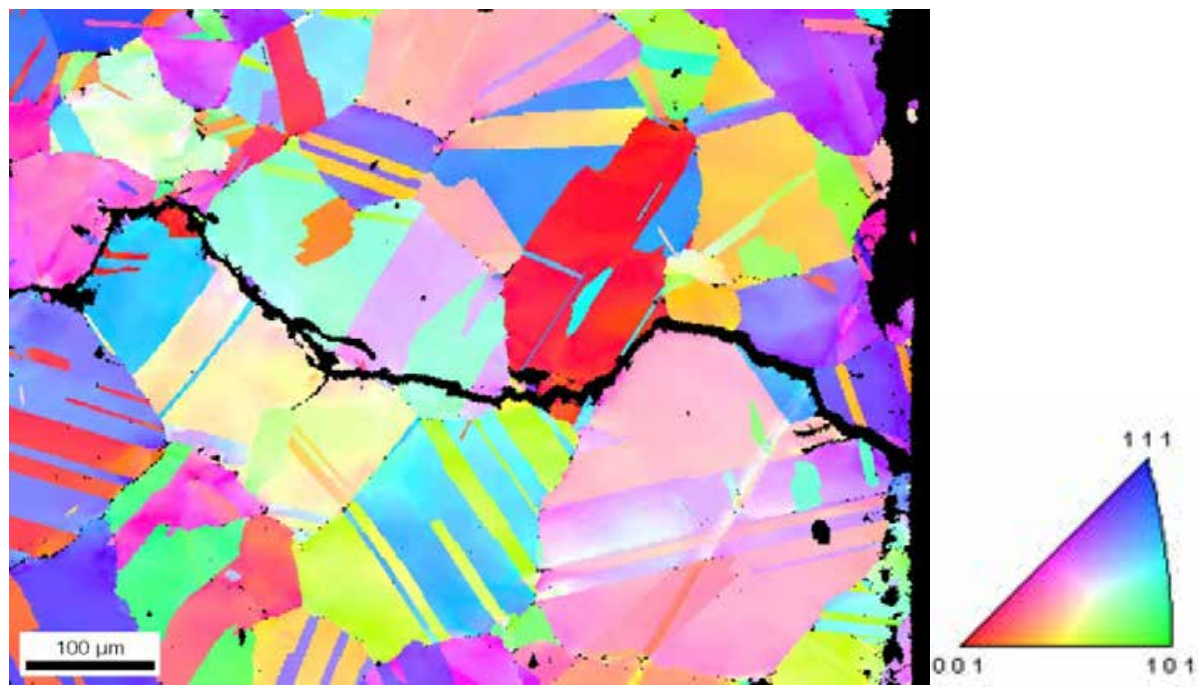

(c)

Fig. 4.111: EBSD IPF clearly distinguishes the (a)\&(b) transgranular cracking of Alloy 617; (c) intergranular cracking of Alloy 617. Test conditions: (a) $\&$ (b) $\varepsilon_{\text {tot }}=1.0 \%$; (c) $\varepsilon_{\text {tot }}=0.5 \%$, dwell time $=$ 3 minutes 


\section{Understanding Fundamental Material-Degradation Processes in High Temperature Aggressive Chemomechanical Environments}

Table 4.12: Crack initiation and propagation modes in Alloy 617 and Alloy 230

\begin{tabular}{|c|c|c|c|c|c|c|c|c|c|}
\hline & & $0.5 \%$ & $1.0 \%$ & $1.5 \%$ & $\begin{array}{c}0.5 \%+3 \\
\min \end{array}$ & $\begin{array}{c}1.0 \%+3 \\
\min \end{array}$ & $\begin{array}{c}1.5 \%+3 \\
\min \end{array}$ & $\begin{array}{c}1.0 \%+10 \\
\min \end{array}$ & $\begin{array}{c}1.0 \%+30 \\
\min \end{array}$ \\
\hline \multirow{2}{*}{$\begin{array}{c}\text { Alloy } \\
617\end{array}$} & Initiation* & $\mathrm{T}$ & $\mathrm{T}$ & $\mathrm{T}$ & $\mathrm{I}$ & $\mathrm{I}$ & $\mathrm{I}$ & $\mathrm{I}$ & $\mathrm{I}$ \\
\cline { 2 - 10 } & Propagation & $\mathrm{T}$ & $\mathrm{T}$ & $\mathrm{T}$ & $\mathrm{I}$ & $\mathrm{T}$ & $\mathrm{T}$ & $\mathrm{I}+\mathrm{T}$ & $\mathrm{I}+\mathrm{T}$ \\
\hline \multirow{2}{*}{$\begin{array}{c}\text { Alloy } \\
230\end{array}$} & Initiation & $\mathrm{T}$ & $\mathrm{T}$ & $\mathrm{T}$ & $\mathrm{T}$ & $\mathrm{T}$ & $\mathrm{I}$ & $\mathrm{T}$ & $\mathrm{I}$ \\
\cline { 2 - 10 } & Propagation & $\mathrm{T}$ & $\mathrm{T}$ & $\mathrm{T}$ & $\mathrm{T}$ & $\mathrm{T}$ & $\mathrm{T}$ & $\mathrm{T}$ & $\mathrm{T}$ \\
\hline
\end{tabular}

*I=intergranular, $\mathrm{T}=$ transgranular

Table 4.13: Changes in the mode of fatigue crack initiation and propagation as creep damage becomes more important (after Ref.[4.47])

1. Transgranular crack initiation and propagation

2. Intergranular crack initiation and Stage I crack propagation

3. Intergranular crack initiation and Stage II crack propagation

4. Intergranular crack initiation and propagation

Random HAGBs with low degrees of atomic matching and high GB energy are typically believed to be subject to intergranular damage [4.77] such as corrosion and void formation. In this study, EBSD scans on areas with GB damages were performed in order to determne if random HAGBs were preferential sites for GB damages. Indeed, oxidation on surface-connected random HAGBs in Alloy 617 was found, as shown in Fig. 4.112. In addition, the intergranular crack initiation and propagation in Alloy 617 tested at $0.5 \%$ total strain range with a 3 minute hold time at maximum tensile strain also followed random HAGBs, as illustrated in Fig. 4.113. Inside the material, the intergranular cracking that was observed after the creep-fatigue tests of Alloy 617 also originated from random HAGBs, as shown in Fig. 4.114(a)\&(b). The aforementioned results highlight the susceptibility of random HAGBs to various GB damaging modes. An optimized grain boundary character distribution with a disrupted random HAGB network is of great importance for improving a material's capability to resist intergranular damaging.

Shown in Fig. 4.114(c) is the strain measurement (from the same area shown in Fig. 4.114(b)), which is based on the Kernel Average Misorientation (KAM) [4.78]. With KAM, the misorientation between a point at the center of the kernel and all points at the perimeter of the kernel were measured. The local misorientation value assigned to the center point was the average of these misorientations. The KAM resulted from lattice rotations caused by dislocations and dislocation arrays in a semi-ordered configuration, and thus was related to the local strain level [4.79]. Strain mapping from Fig. 4.114(c) shows the strain concentration in the vicinity of the random HAGBs, as highlighted in Fig. 4.114(b). 


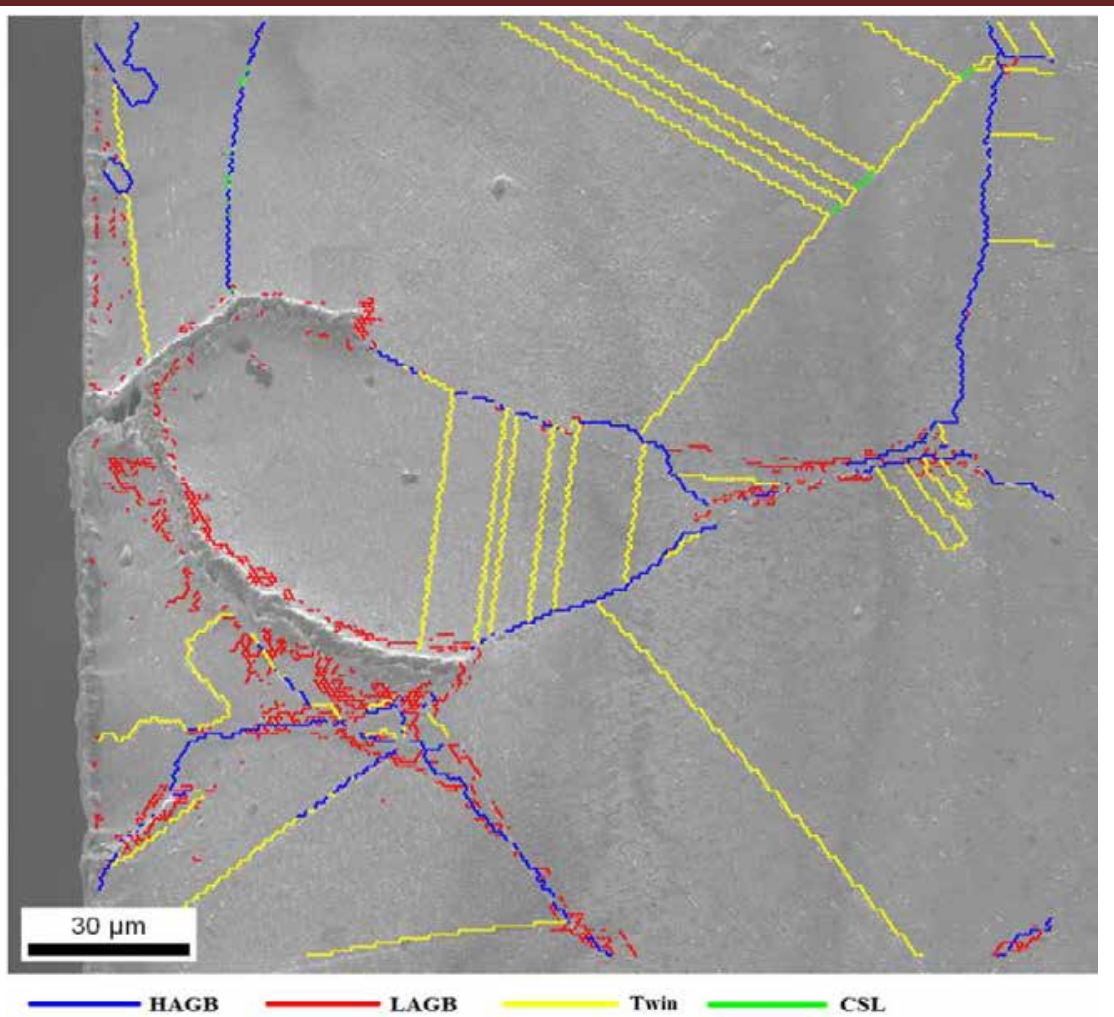

Fig. 4.112: Oxidation along HAGBs in Alloy 617. $\varepsilon_{\text {tot }}=0.5 \%$, dwell time $=3$ minutes
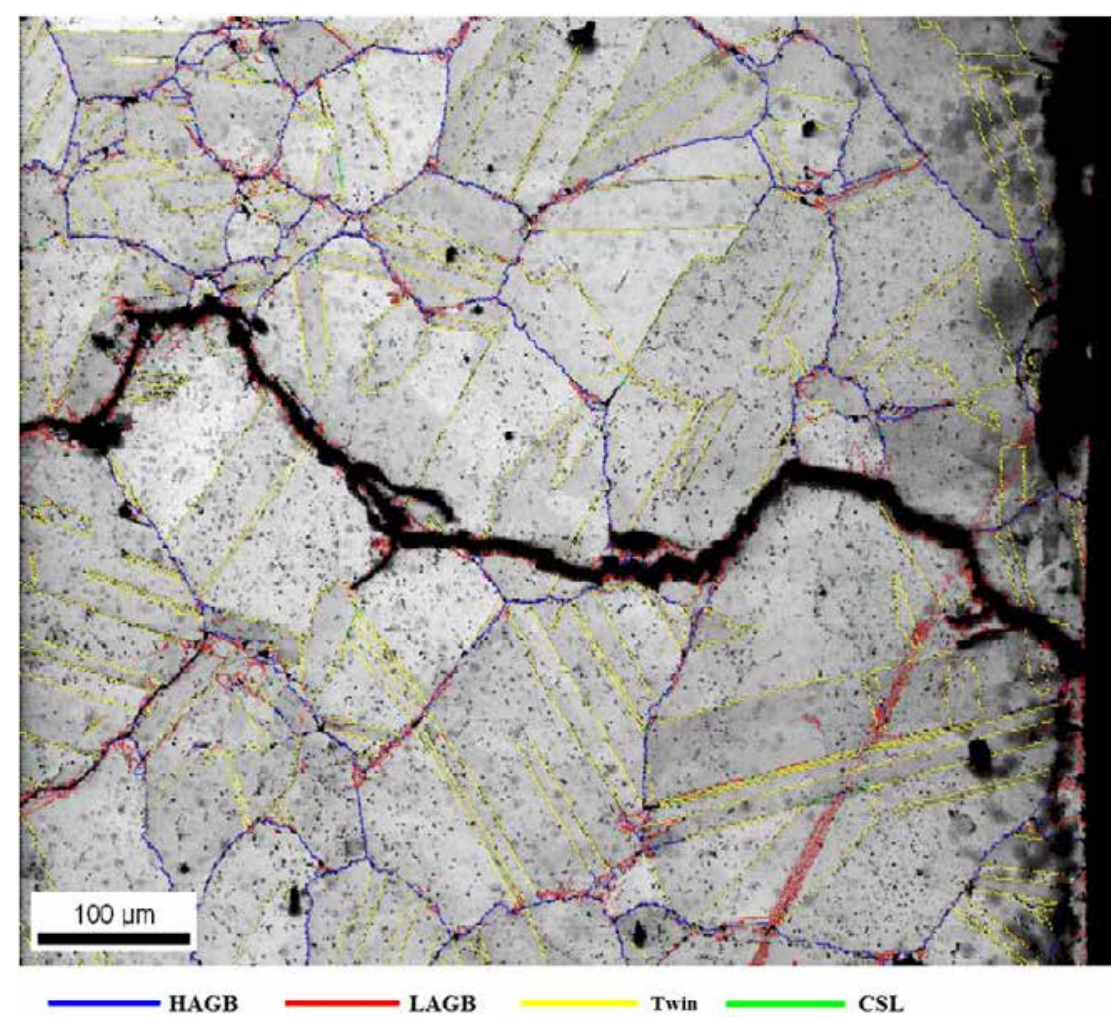

Twin

CSL

Fig. 4.113: Crack initiation and propagation along HAGBs in Alloy 617. $\varepsilon_{\text {tot }}=0.5 \%$, dwell time $=3$ minutes 




(a)

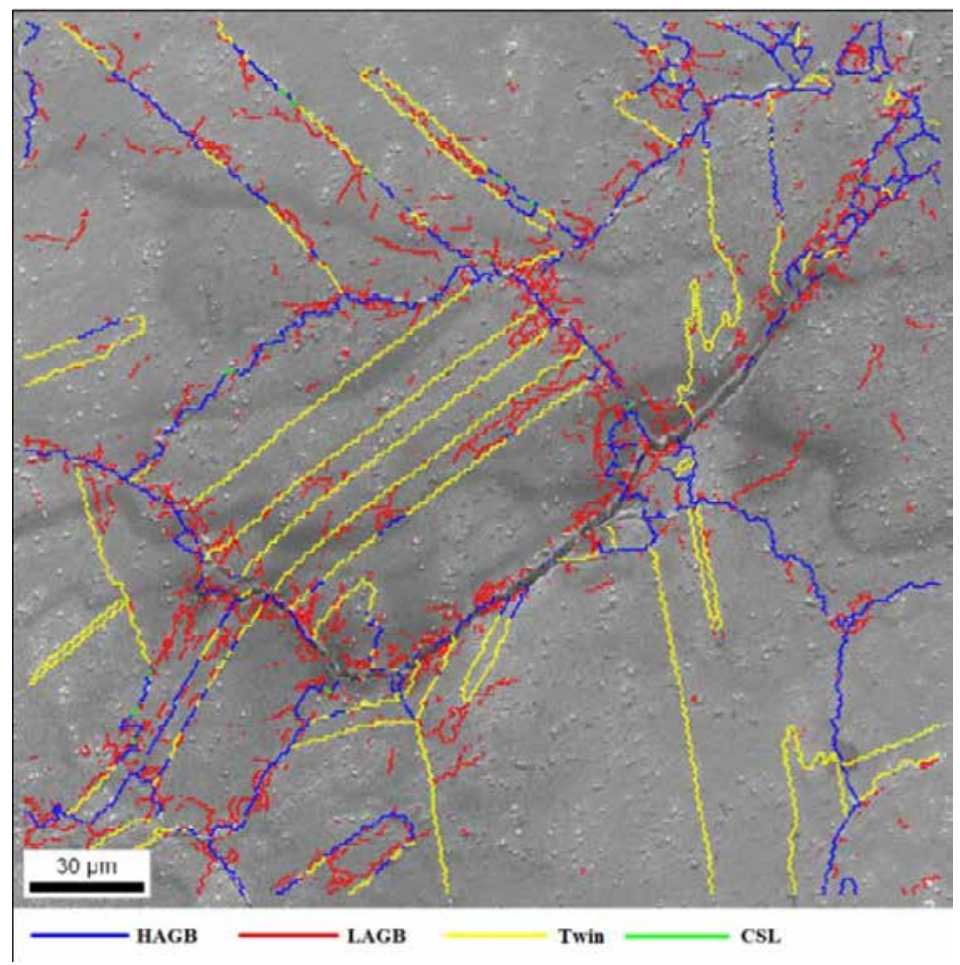

(b)

Fig. 4.114: Interior intergranular cracking on HAGBs of Alloy 617. (a) Original SEM figure; (b) different types of boundaries overlaid on the SEM figure; (c) strain measurements based on KAM. $\varepsilon_{\text {tot }}=1.0 \%$, dwell time $=10$ minutes 


\section{Understanding Fundamental Material-Degradation Processes in High Temperature Aggressive Chemomechanical Environments}

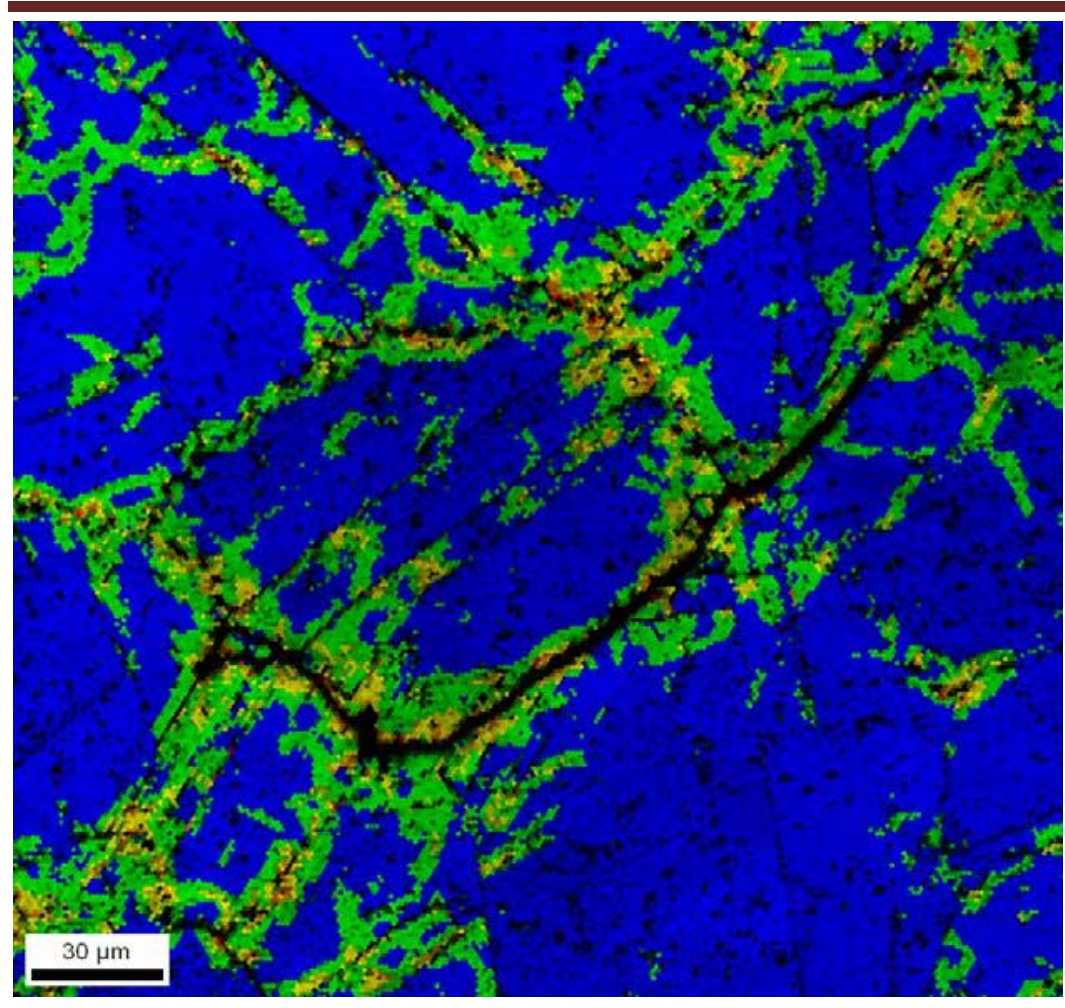

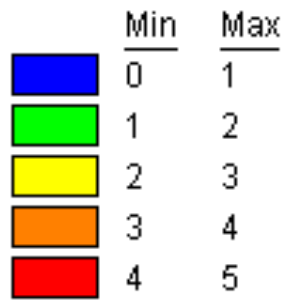

(c)

Fig. 4.114 Cont.

Boundaries containing cellular precipitates in Alloy 230 were studied with EBSD. In Fig. 4.115, it is revealed that a random HAGB existed on the precipitate front. The GB cellular precipitates formed by a mechanism called a discontinuous precipitation reaction, in which a cell composed of a solute-depleted phase (matrix) and the lamellar precipitate forms behind a GB, advancing into a supersaturated matrix [4.61]. Hence, the reaction is a combination of precipitation and GB migration; the supersaturation in the matrix is the driving force for the reaction [4.80, 4.81]. During the discontinuous precipitation reaction, the lamellar precipitate is coherent with the grain, away from which it is growing, and is incoherent with the cell growth front [4.82, 4.83, $4.84,4.85]$. This explains why a random HAGB on the precipitate front was observed, as seen in Fig. 4.115. Under creep-fatigue test conditions, intergranular cracks inside Alloy 230 were found on random HAGBs where cellular precipitates resided, but not on random HAGBs without cellular precipitates. This observation confirms the deleterious effect of $\mathrm{GB}$ cellular $\mathrm{M}_{23} \mathrm{C}_{6}$ precipitates on the mechanical properties of the material.

Fig. 4.116(a)\&(b) illustrate one example of an intergranular crack associated with the GB cellular precipitate. Strain mapping based on KAM, as shown in Fig. 4.116(c), demonstrates an extensive strain accumulation on the cellular precipitate front. On occasion, in Alloy 230, random HAGBs can migrate away from the cellular precipitate and become stripped of GB precipitates. The GBs of this configuration were especially susceptible to intergranular cracking, as shown in Fig. 4.117, due to the lack of GB precipitates that are known to impede GB sliding $[4.86,4.87]$ and the enhanced GB damage due to the creep-fatigue deformation. 


\section{Understanding Fundamental Material-Degradation Processes in High Temperature Aggressive Chemomechanical Environments}

It is worth noting that for the development of GBs denuded of precipitates in Alloy 230, two conditions need to be satisfied: 1) the material must have been subjected to creep-fatigue testing, and 2) cellular $\mathrm{M}_{23} \mathrm{C}_{6}$ carbides must have precipitated on the GB. The first condition highlights the additional driving force for GB migration during creep-fatigue testing. There are several driving forces for GB migration, and in decreasing order of magnitude, they are: chemical, stored deformation energy, GB energy, and others [4.88]. During creep-fatigue testing, the deformation was focused on the GB area. Many dislocations formed near GBs, which eventually led to the strain energy gradient across the GB. Therefore, the strain energy gradient induced by the creep-fatigue test became the additional driving force for GB movement. Cellular $\mathrm{M}_{23} \mathrm{C}_{6}$ carbides on GBs likely do not possess the same ability of general GB precipitates to limit GB migration [4.89], and hence only GBs with cellular precipitates were able to migrate away from cellular precipitates.

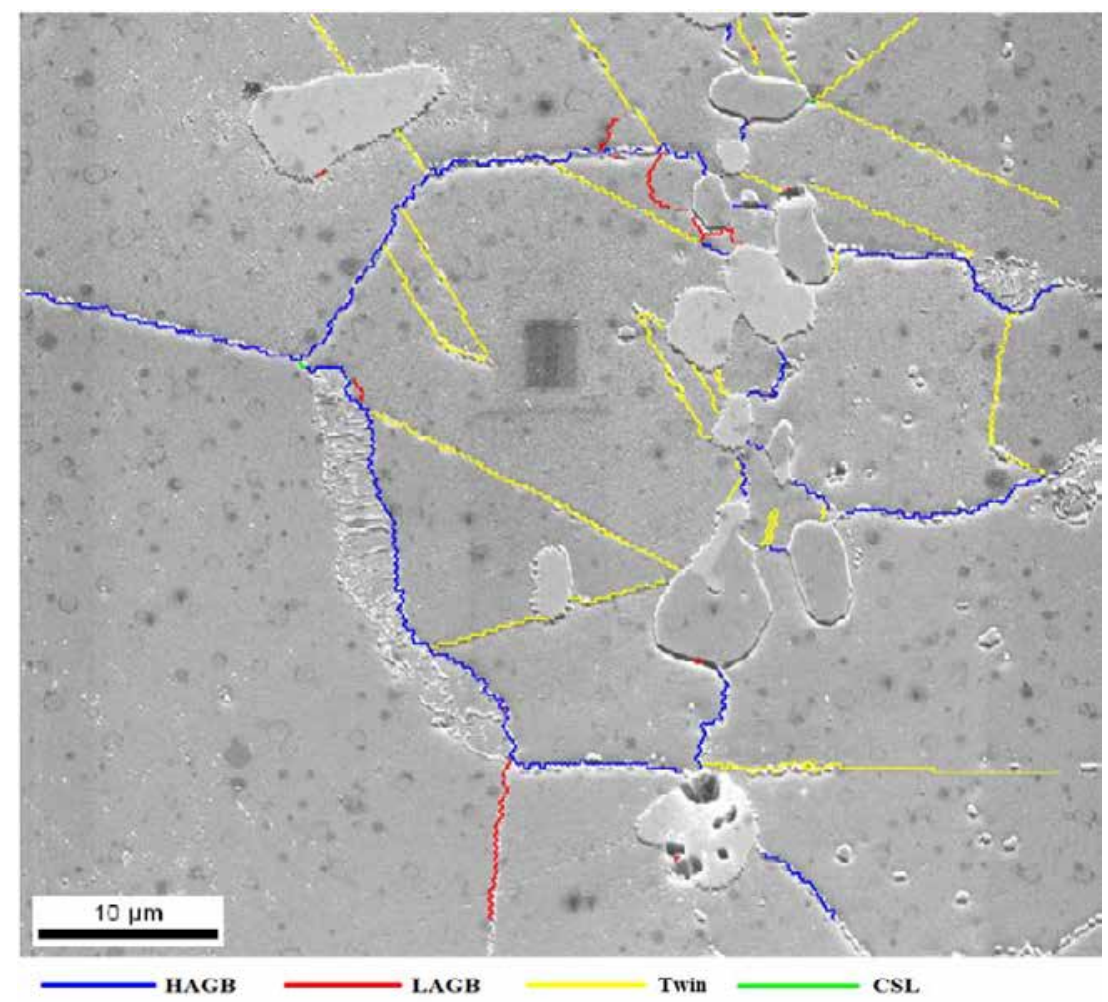

Fig. 4.115: Different boundary types overlaid on the SEM figure indicate the GB migration associated with discontinuous precipitation in Alloy 230. $\varepsilon_{\text {tot }}=1.0 \%$ 


\section{Understanding Fundamental Material-Degradation Processes in High Temperature}

Aggressive Chemomechanical Environments

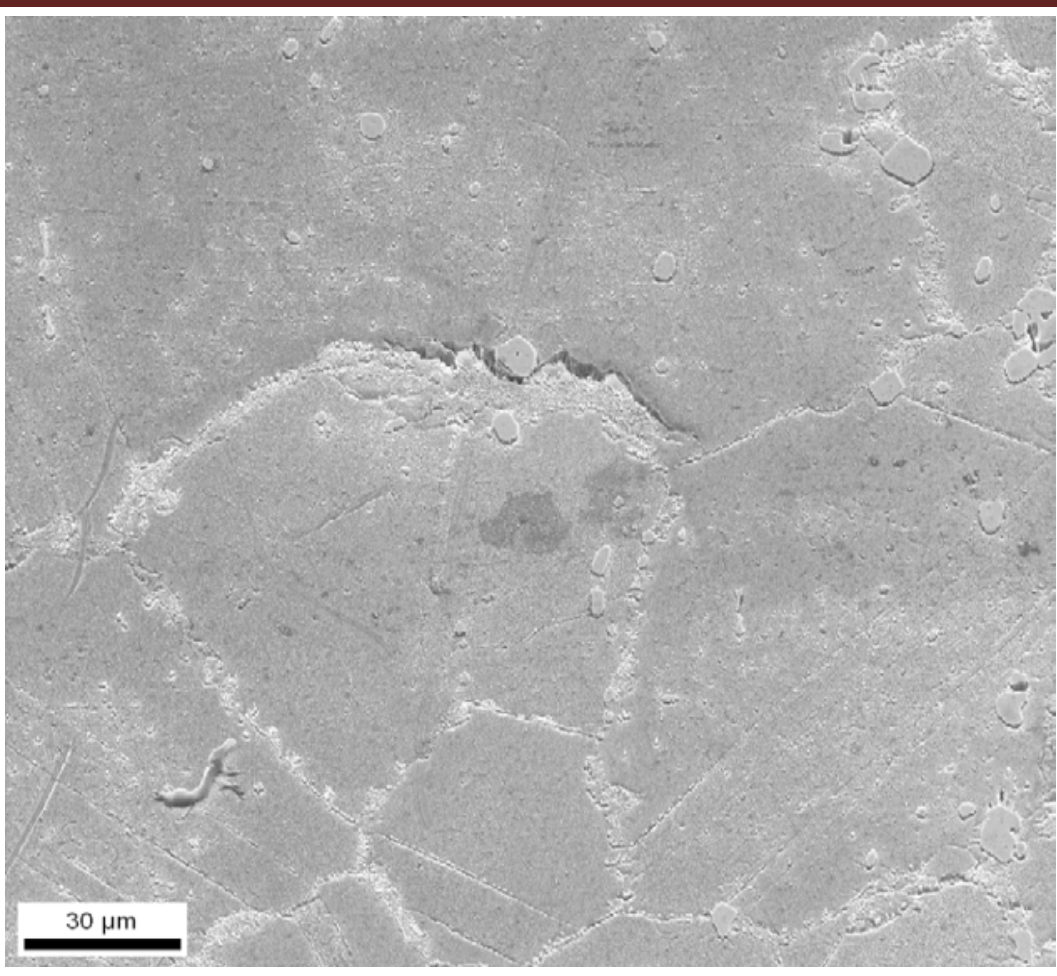

(a)

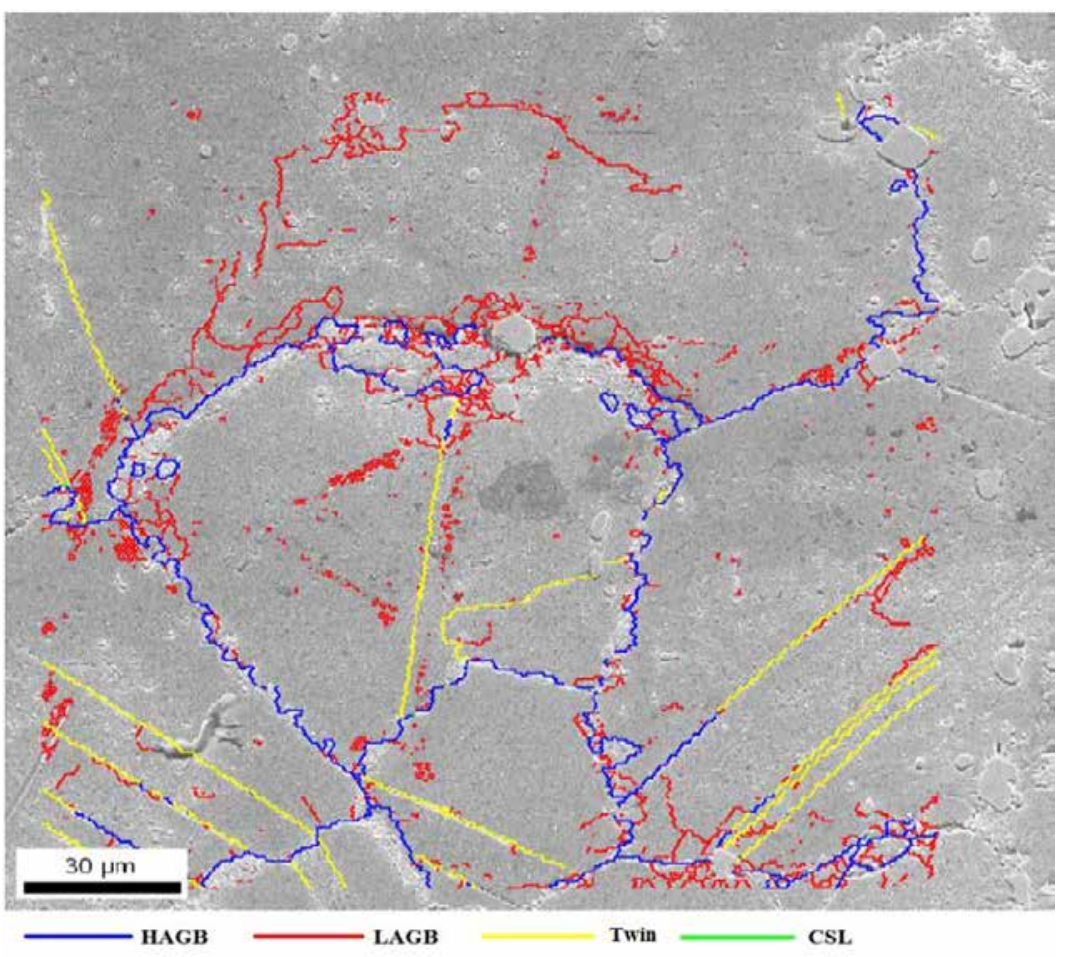

(b)

Fig. 4.116: Interior intergranular cracking on HAGBs in Alloy 230. (a) Original SEM figure; (b) different types of boundaries overlaid on the SEM figure; (c) strain measurements based on KAM. $\varepsilon_{\text {tot }}=1.0 \%$, dwell time $=3$ minutes 
Understanding Fundamental Material-Degradation Processes in High Temperature Aggressive Chemomechanical Environments

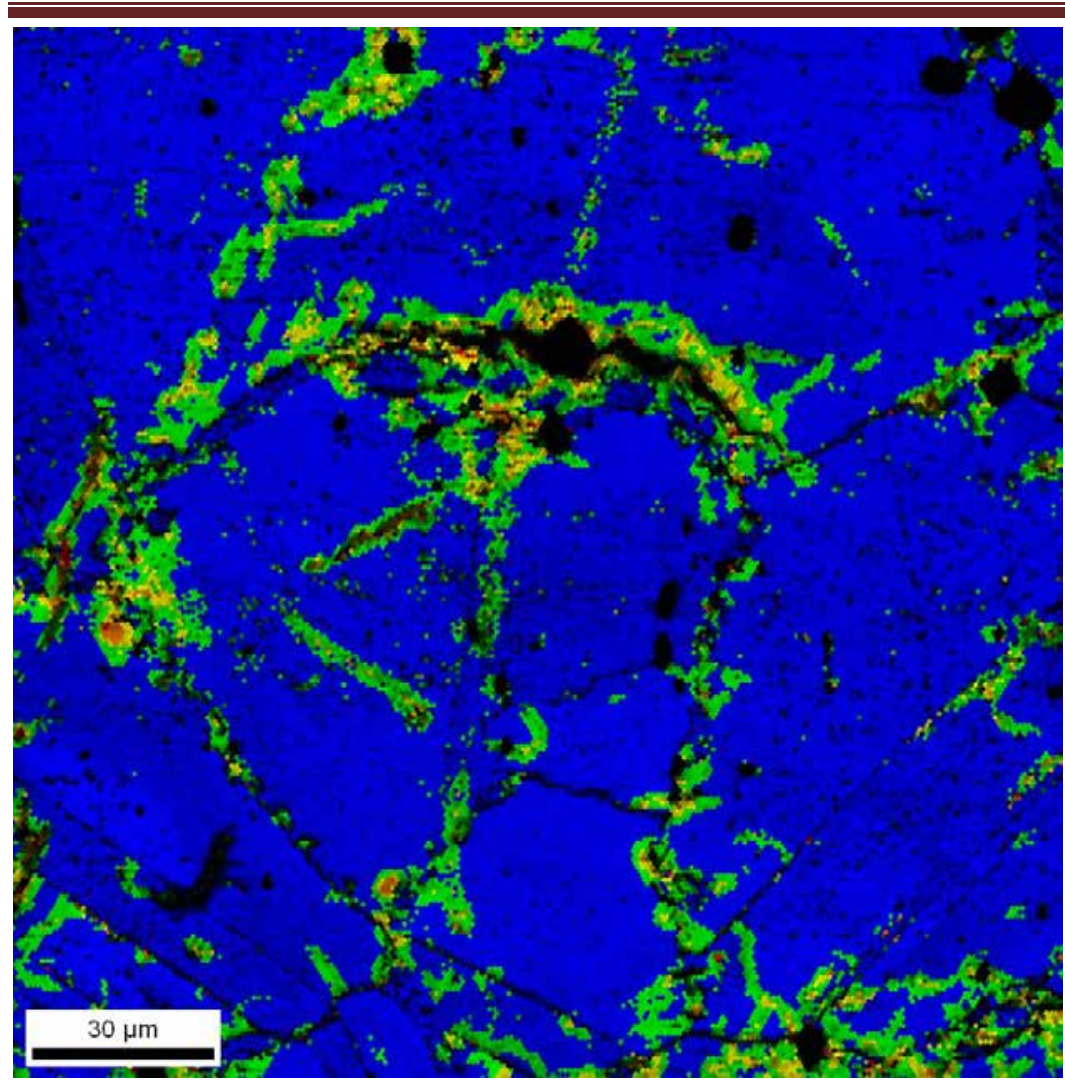

\begin{tabular}{|lll}
\hline & $\frac{\text { Min }}{0}$ & $\frac{\text { Max }}{1}$ \\
\hline$\square$ & 1 & 2 \\
\hline & 2 & 3 \\
\hline$\square$ & 3 & 4 \\
\hline & 4 & 5
\end{tabular}

(c)

Fig. 4.116 Cont. 
Understanding Fundamental Material-Degradation Processes in High Temperature Aggressive Chemomechanical Environments

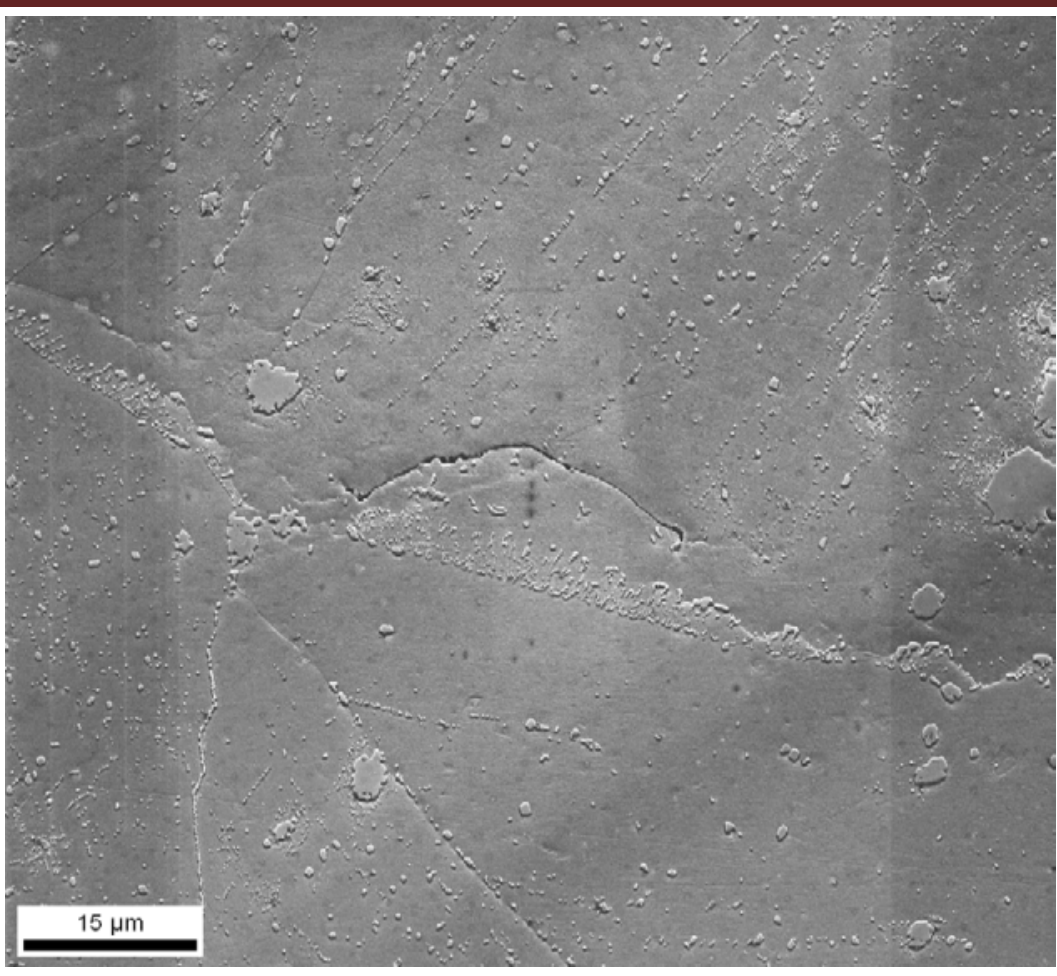

(a)

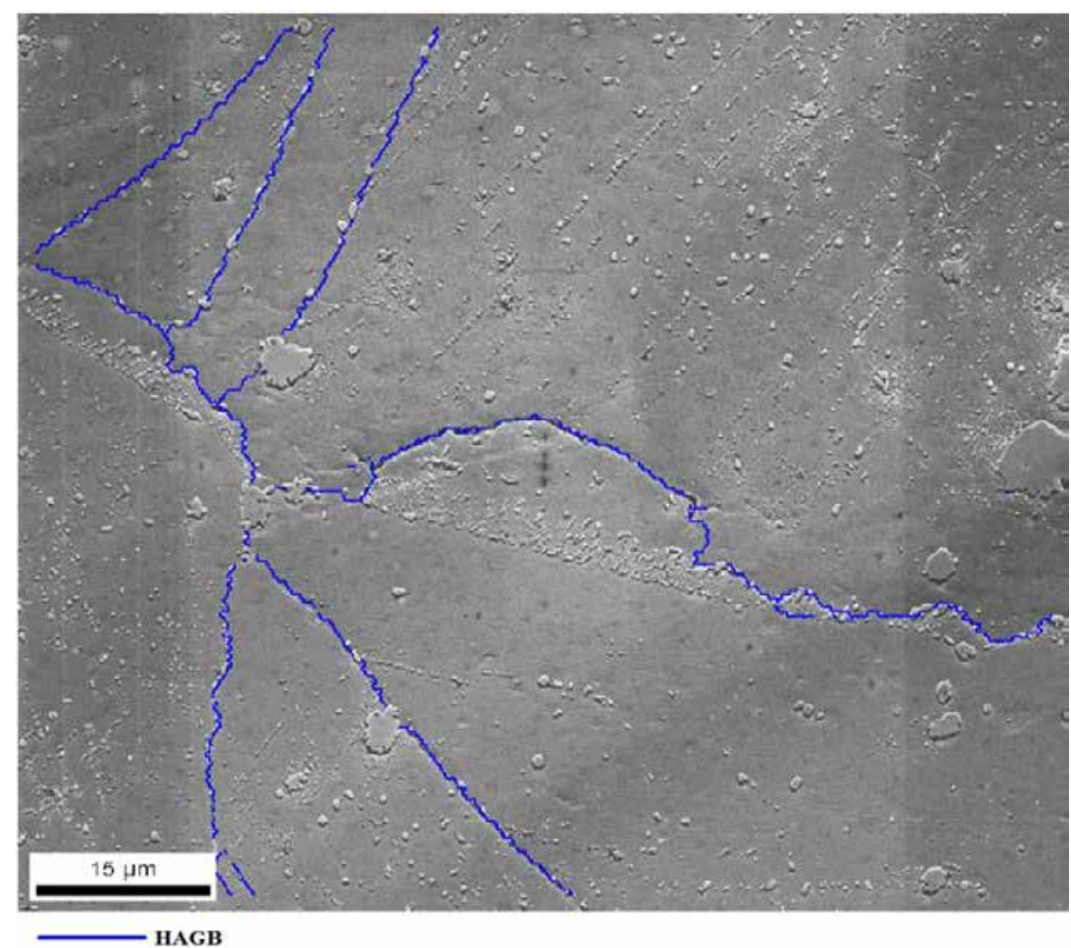

(b)

Fig. 4.117: GBs migrated away from the cellular precipitates and developed intergranular cracking. (a) Original SEM figure; (b) HAGBs overlaid on the original SEM figure. $\varepsilon_{\text {tot }}=1.0 \%$, dwell time $=30$ minutes 


\subsection{Biaxial Thermal Creep Experiment}

\subsubsection{Biaxial creep behavior of Alloy 617}

The biaxial creep behavior of Alloy 617 at $900^{\circ} \mathrm{C}$ is plotted in Fig. 4.118. With increasing applied stress, the rupture time decreases. From the plot, it can be seen that the creep strain development for the stress of $18 \mathrm{MPa}$ is close to the creep strain development at $15 \mathrm{MPa}$. In addition, all the curves exhibit a large tertiary part of creep deformation, while the secondary regime is not distinct.

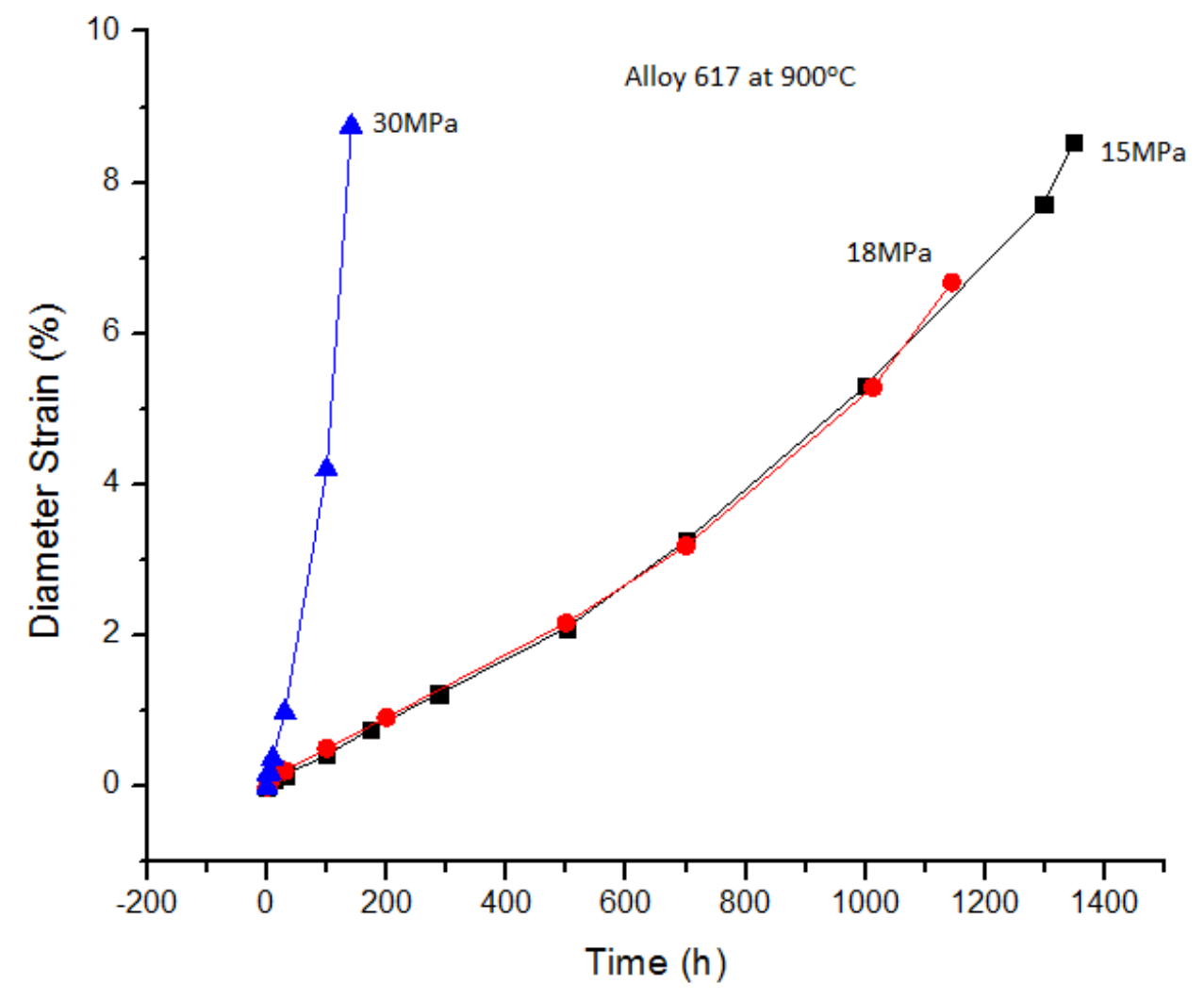

Fig. 4.118: Diameter strain vs. creep exposure time for Alloy 617 at $900^{\circ} \mathrm{C}$

To illustrate the change in creep rate during the test, the creep strain-time curves were differentiated to obtain the creep strain rate using the finite difference formula:

$$
\begin{gathered}
\dot{\varepsilon}\left(\overline{t_{n}}\right)=\frac{\varepsilon_{n+1}-\varepsilon_{n}}{t_{n+1}-t_{n}} \\
\overline{t_{n}}=\frac{t_{n+1}+t_{n}}{2}
\end{gathered}
$$

where $\varepsilon_{n+1}$ and $\varepsilon_{n}$ represent the effective strain at the two consecutive exposure times $t_{n+1}$ and $t_{n}$, respectively. $\dot{\varepsilon}\left(\overline{t_{n}}\right)$ is the creep strain rate, and $\overline{t_{n}}$ is the mean time of the two time intervals. The creep strain rate is plotted as a function of the average strain on a semi-log scale in Fig. 4.119(a)-(c). 


\section{Understanding Fundamental Material-Degradation Processes in High Temperature}

Aggressive Chemomechanical Environments

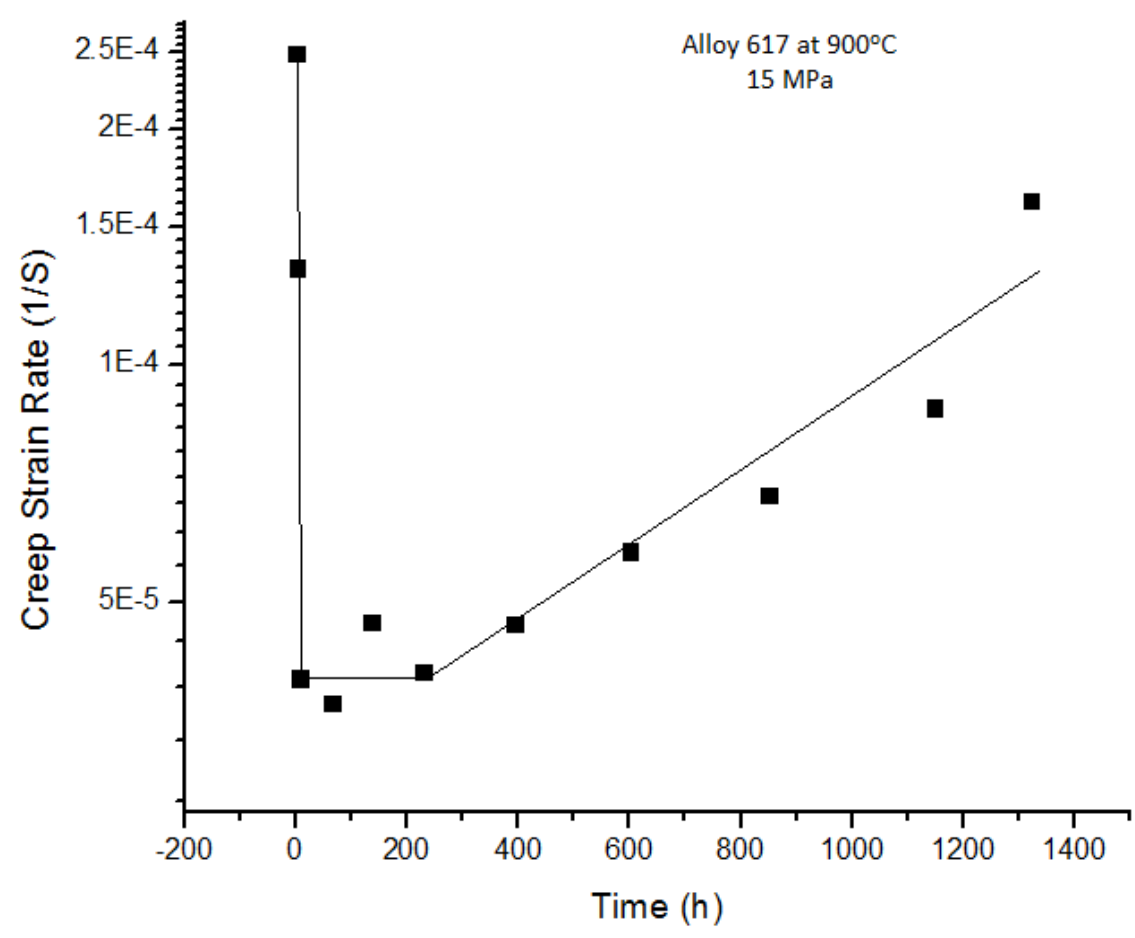

(a)

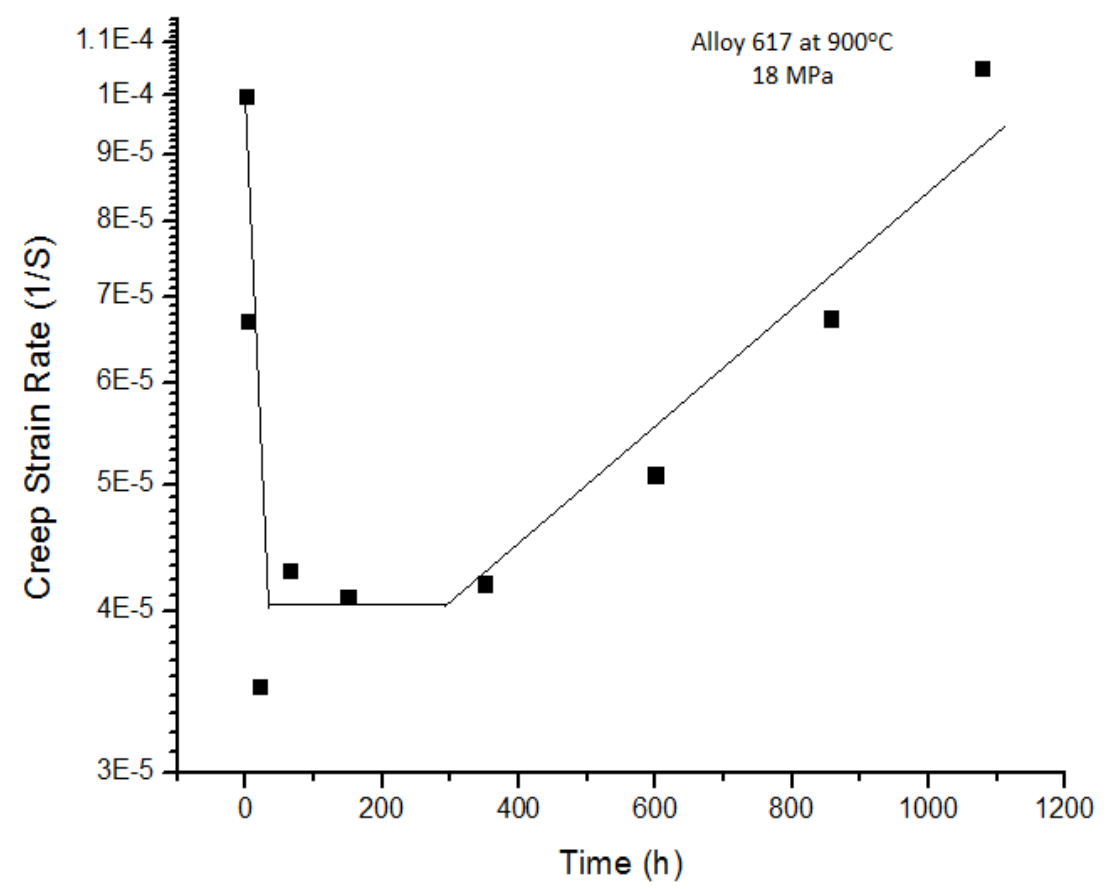

(b)

Fig. 4.119: (a) Creep strain rate for Alloy 617 with an applied stress of $15 \mathrm{MPa}$ at $900^{\circ} \mathrm{C}$; (b) creep strain rate for Alloy 617 with an applied stress of $18 \mathrm{MPa}$ at $900^{\circ} \mathrm{C}$ 


\section{Understanding Fundamental Material-Degradation Processes in High Temperature}

Aggressive Chemomechanical Environments

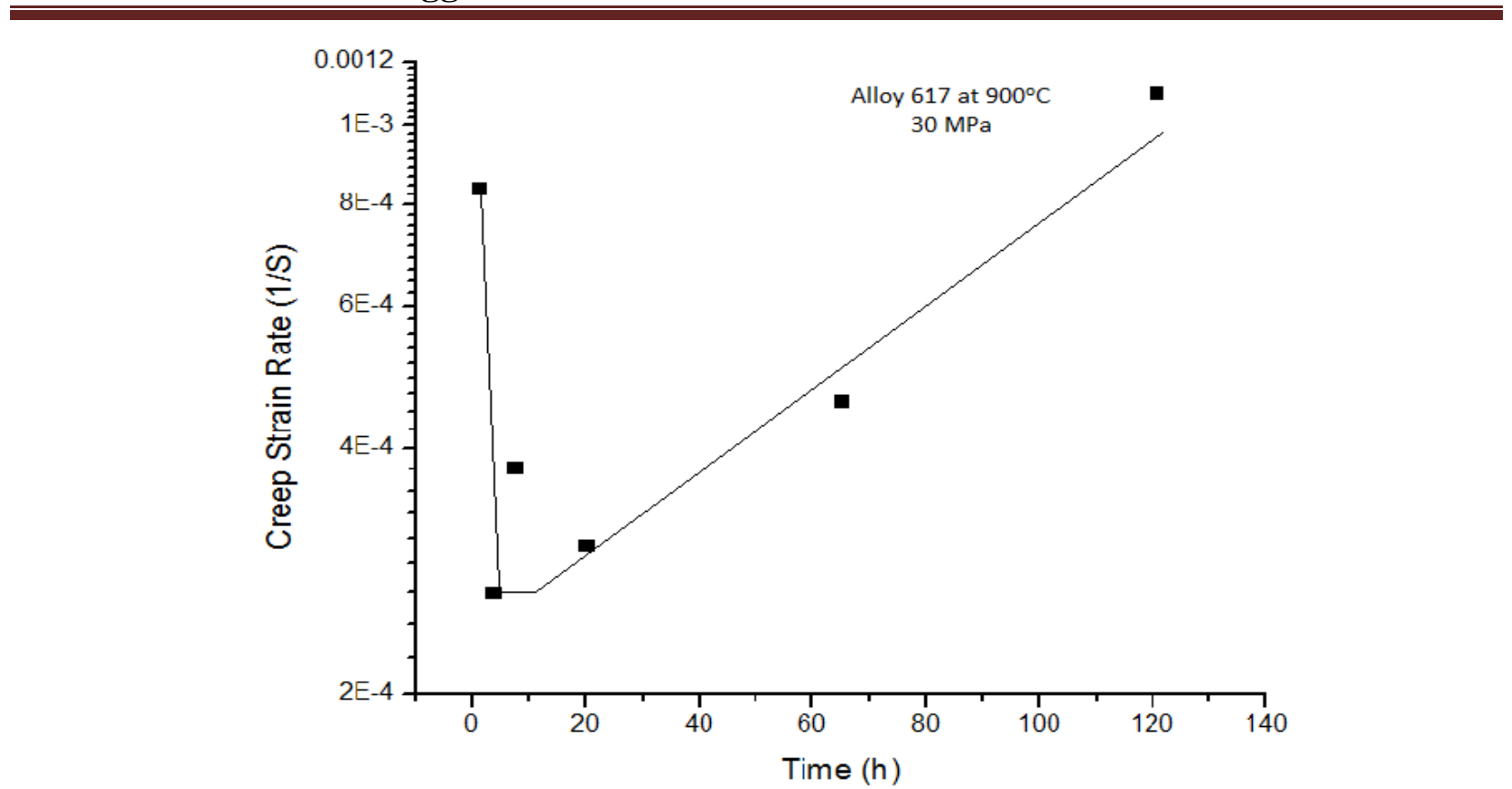

Fig. 4.119 (c) creep strain rate for Alloy 617 with an applied stress of $30 \mathrm{MPa}$ at $900^{\circ} \mathrm{C}$

It should be noted that with increasing applied stress, the steady state creep rate portion decreases. Fig. 4.120(a)-(c) shows the cross-sectional view of SEM images near the fracture surface. Intergranular fracture was observed for all specimens. 
Understanding Fundamental Material-Degradation Processes in High Temperature Aggressive Chemomechanical Environments

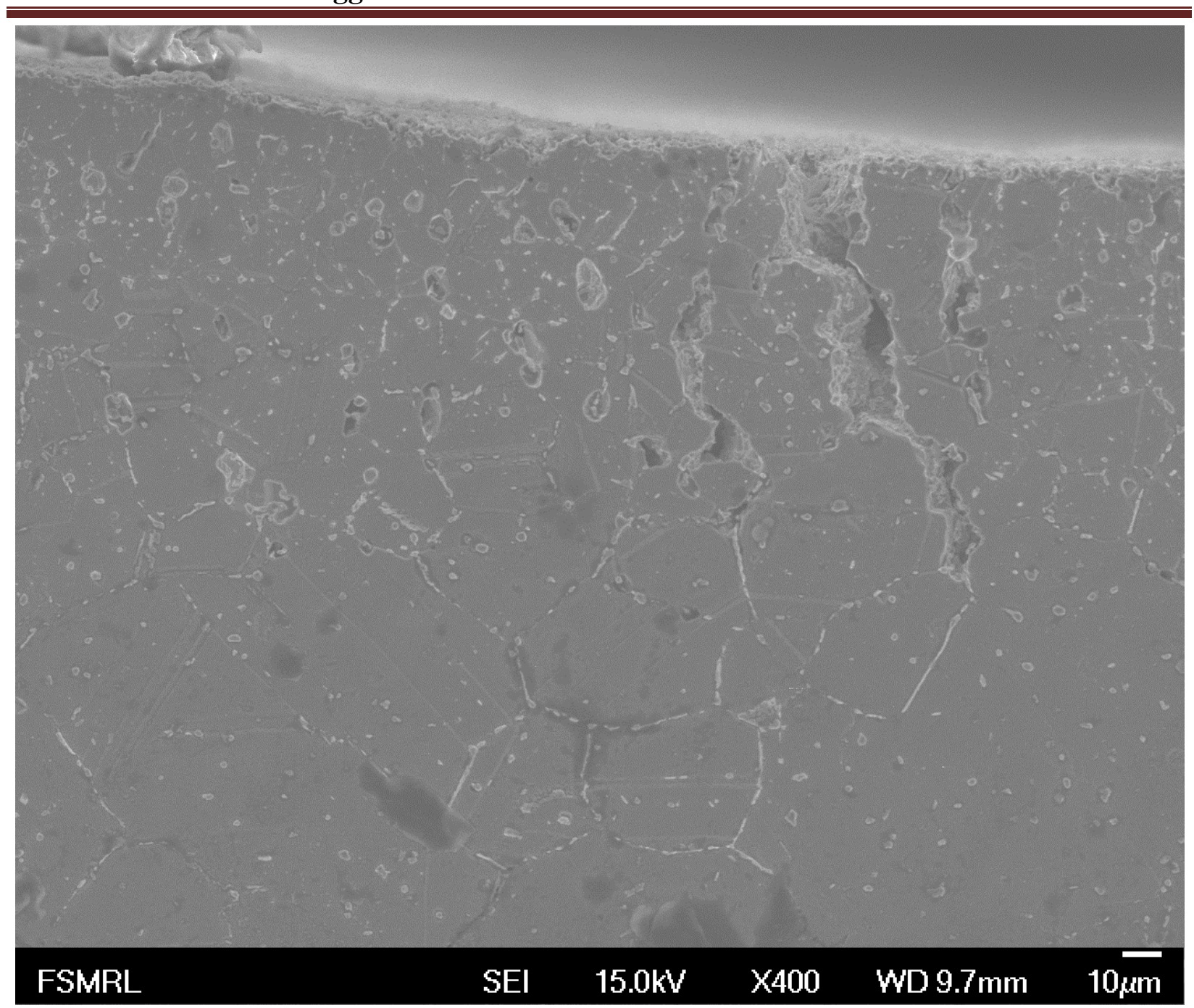

Fig. 4.120: (a) Cross-sectional view of pressurized creep tubes near the fracture surface under the applied stress of $30 \mathrm{MPa}$ at $900^{\circ} \mathrm{C}$ for Alloy 617 
Understanding Fundamental Material-Degradation Processes in High Temperature Aggressive Chemomechanical Environments

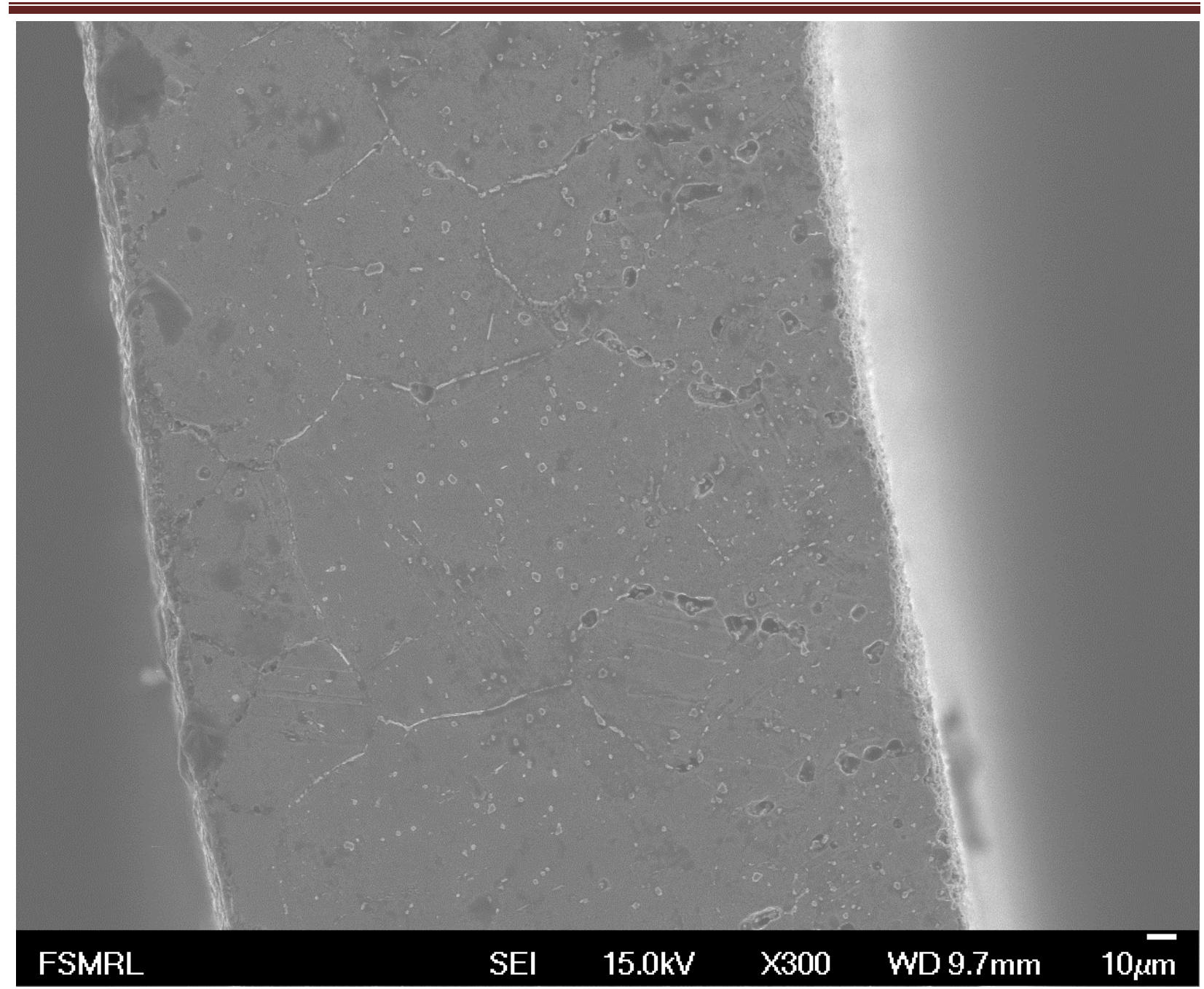

Fig. 4.120: (b) Cross-sectional view of pressurized creep tubes near the fracture surface under the applied stress of $18 \mathrm{MPa}$ at $900^{\circ} \mathrm{C}$ for Alloy 617 


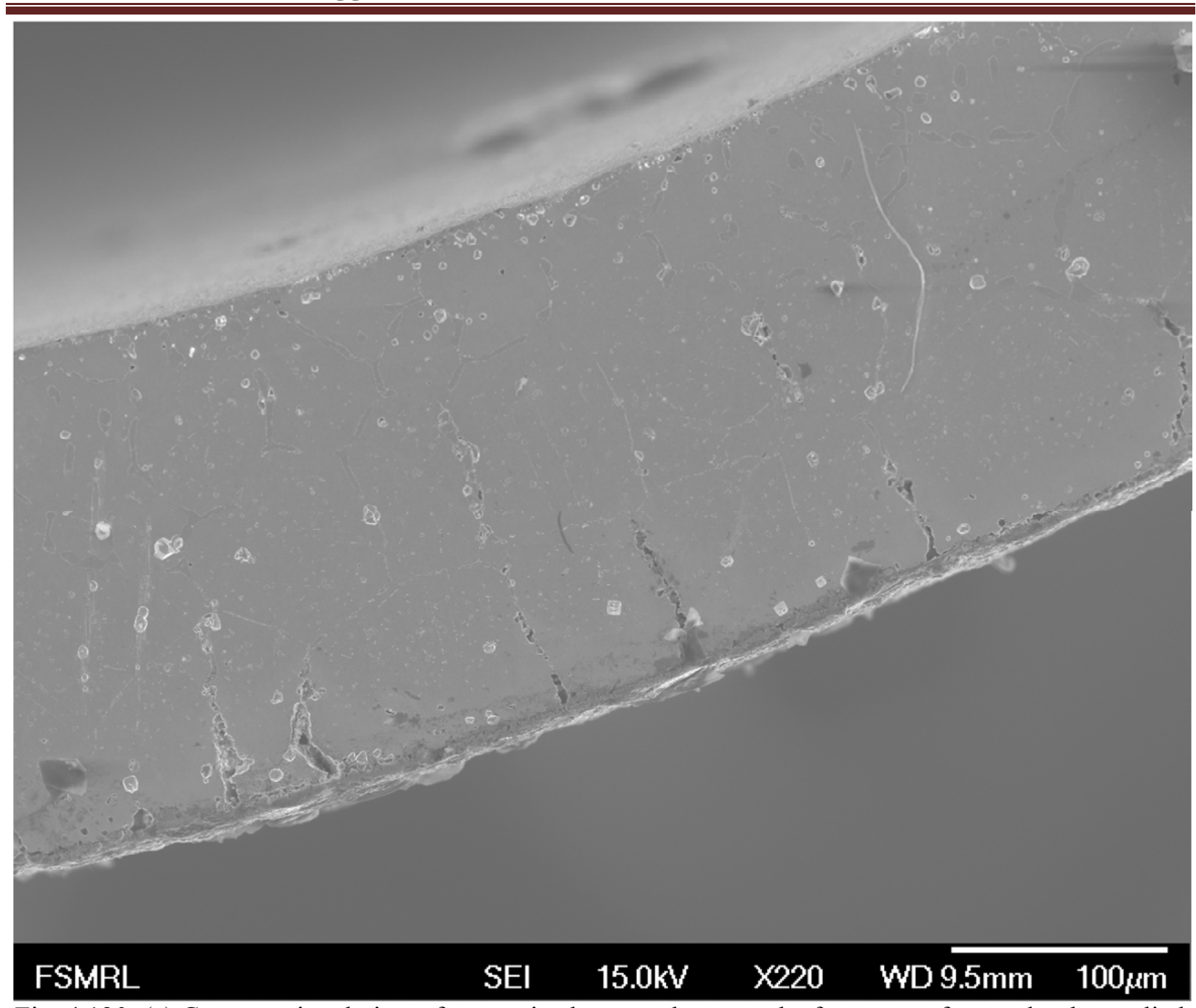

Fig. 4.120: (c) Cross-sectional view of pressurized creep tubes near the fracture surface under the applied stress of $15 \mathrm{MPa}$ at $900^{\circ} \mathrm{C}$ for Alloy 617 


\section{Understanding Fundamental Material-Degradation Processes in High Temperature Aggressive Chemomechanical Environments}

\subsubsection{Biaxial creep behavior of Alloy 230}

The biaxial creep behavior of Alloy 230 at $900^{\circ} \mathrm{C}$ is plotted in Fig. 4.121. As the applied stress is increased, the rupture time decreases. As shown in the plot, all the Alloy 230 creep curves exhibit a large tertiary portion of creep deformation. Even at the lowest stress, the secondary regime is not distinct. The creep strain rate is calculated using equations 4.43 and 4.44 , and is plotted in Fig. 4.122(a)-(d) on a semi-log scale. It should be noted that with an increase in applied stress, the steady state creep rate portion decreases. Fig. 4.123(a)-(d) shows the cross-sectional view of SEM images near the fracture surface. Intergranular fracture was observed for all specimens.

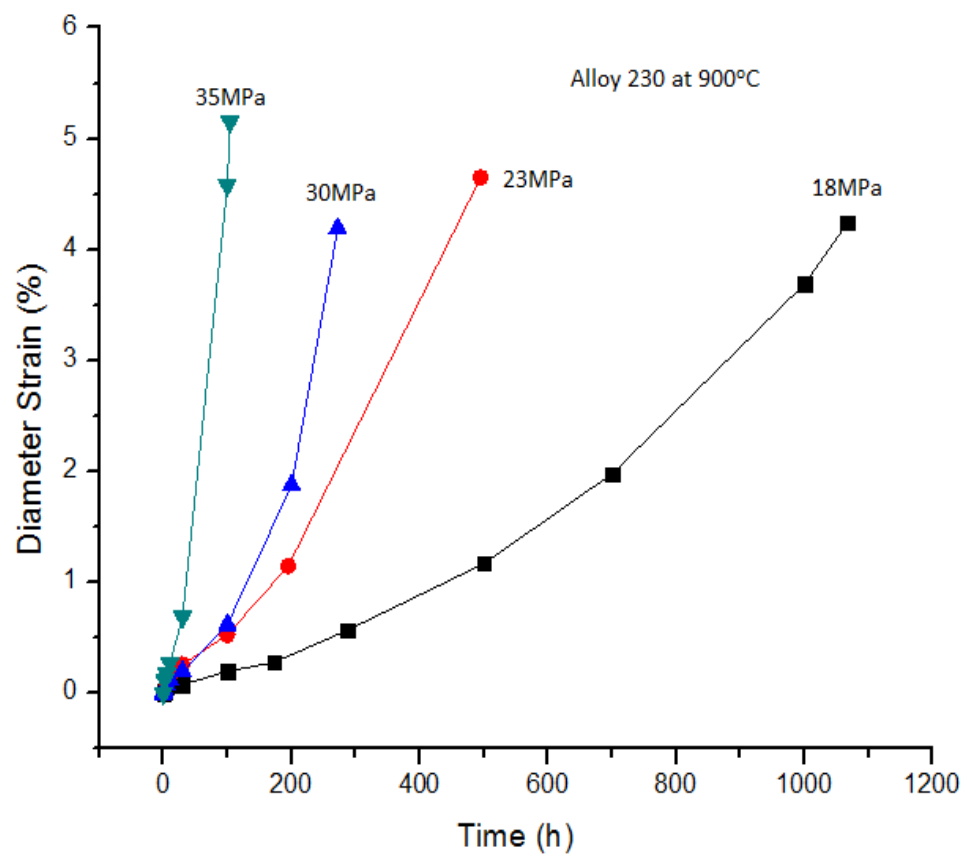

Fig. 4.121: Diameter strain vs. creep exposure time for Alloy 230 at $900^{\circ} \mathrm{C}$ 


\section{Understanding Fundamental Material-Degradation Processes in High Temperature}

Aggressive Chemomechanical Environments

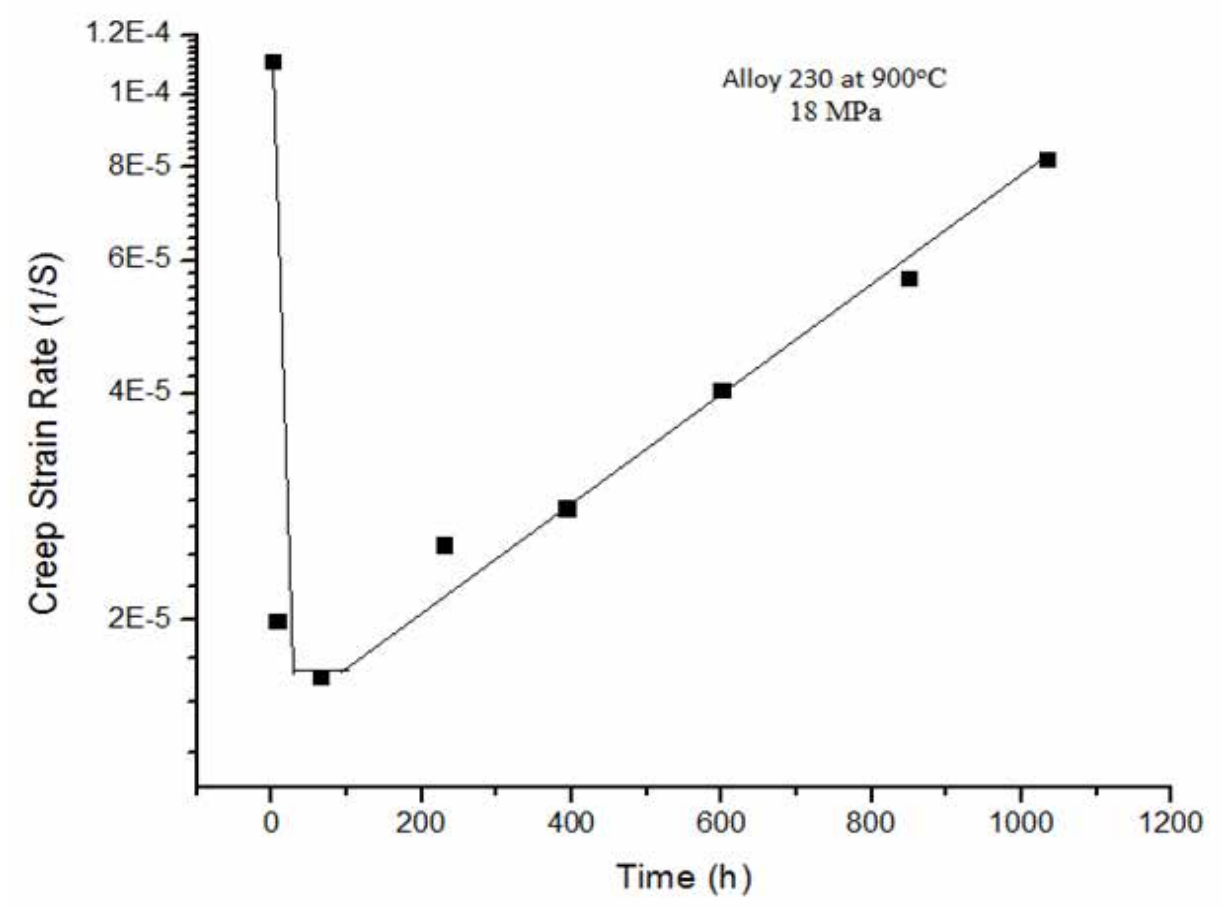

Fig. 4.122: (a) Creep strain rate for Alloy 230 with an applied stress of $18 \mathrm{MPa}$ at $900^{\circ} \mathrm{C}$

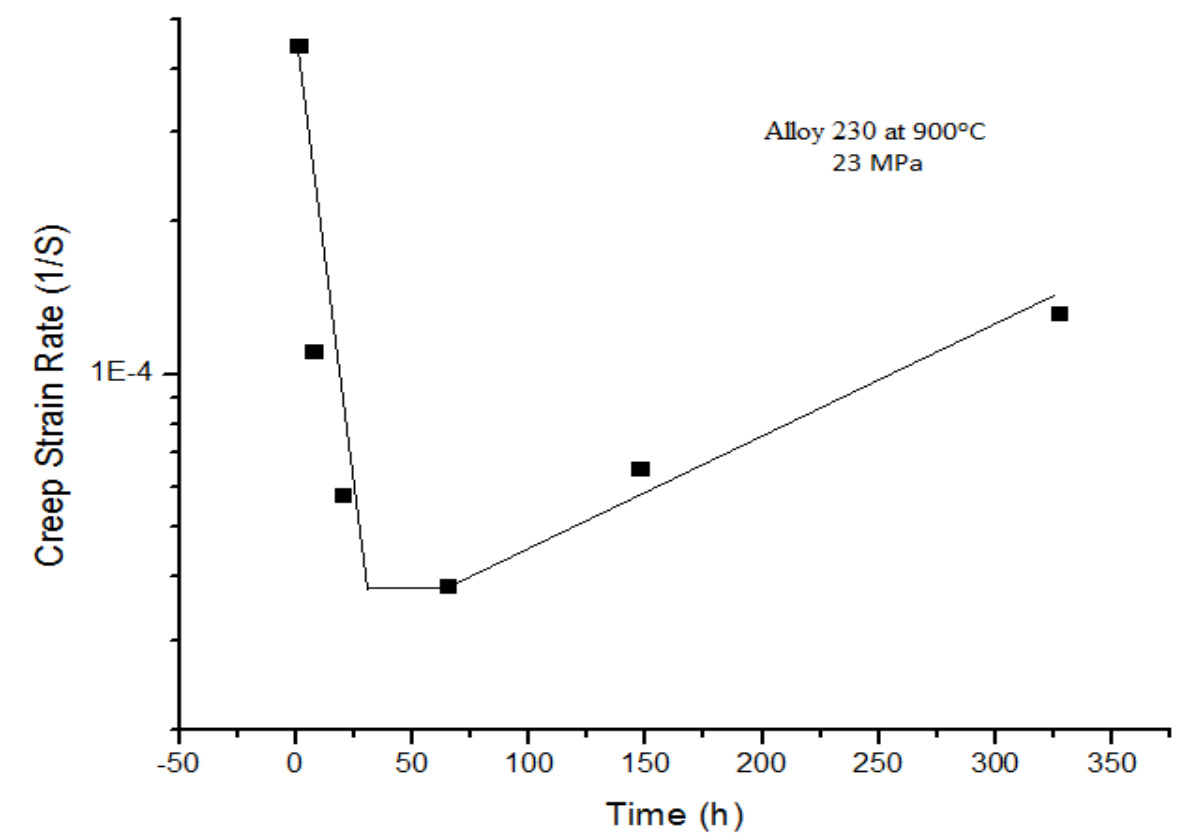

Fig. 4.122: (b) Creep strain rate for Alloy 230 with an applied stress of $23 \mathrm{MPa}$ at $900{ }^{\circ} \mathrm{C}$ 


\section{Understanding Fundamental Material-Degradation Processes in High Temperature}

Aggressive Chemomechanical Environments

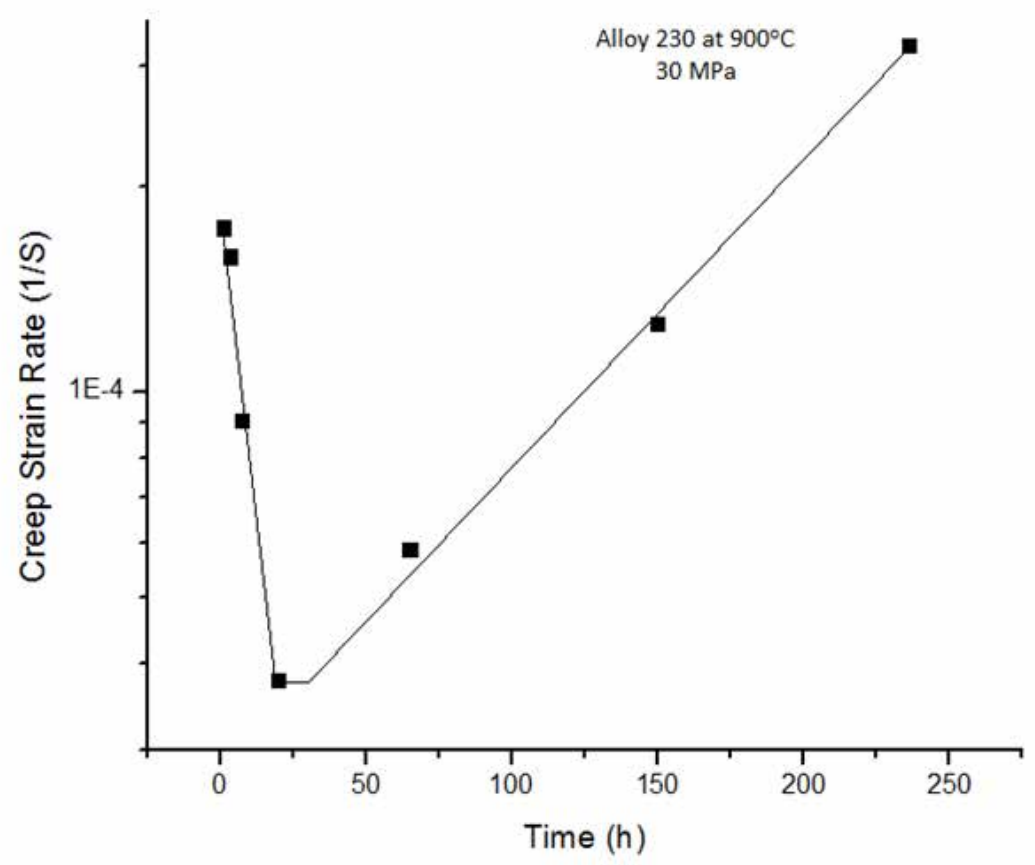

Fig. 4.122: (c) Creep strain rate for Alloy 230 with an applied stress of $30 \mathrm{MPa}$ at $900^{\circ} \mathrm{C}$

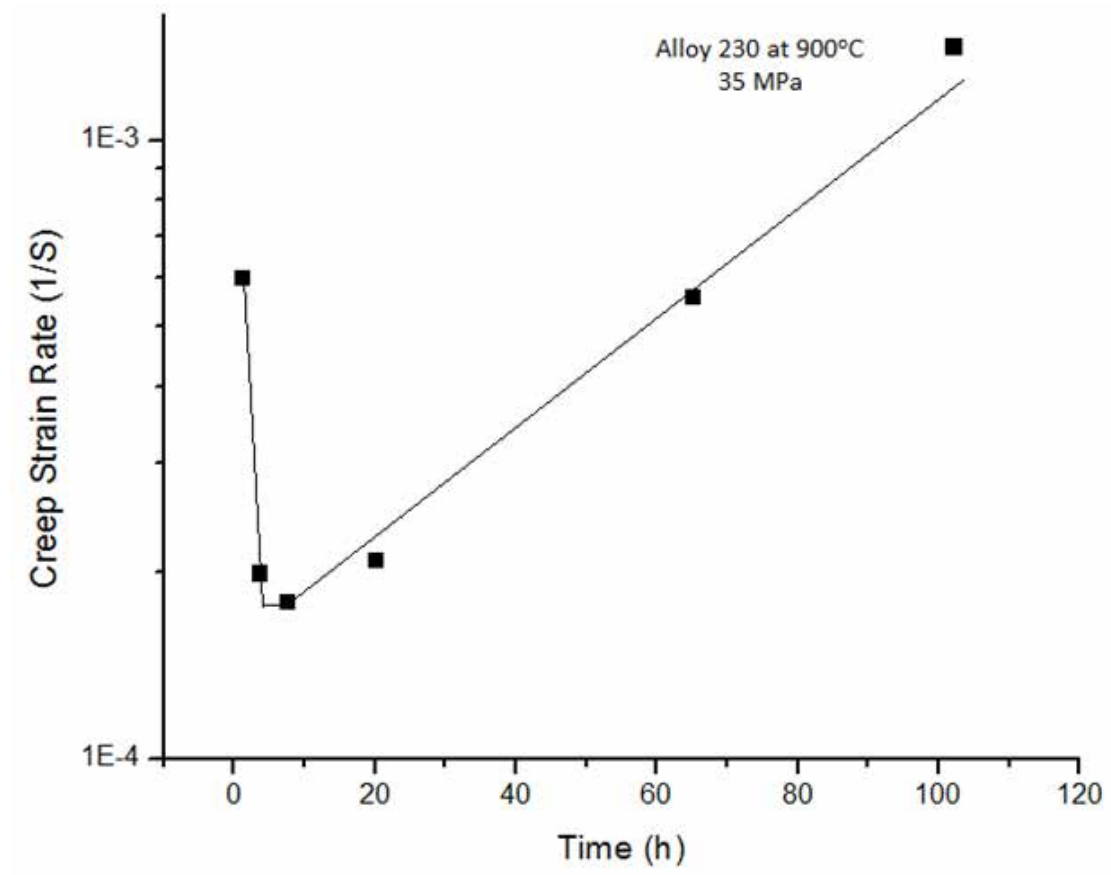

Fig. 4.122: (d) Creep strain rate for Alloy 230 with an applied stress of $35 \mathrm{MPa}$ at $900^{\circ} \mathrm{C}$ 


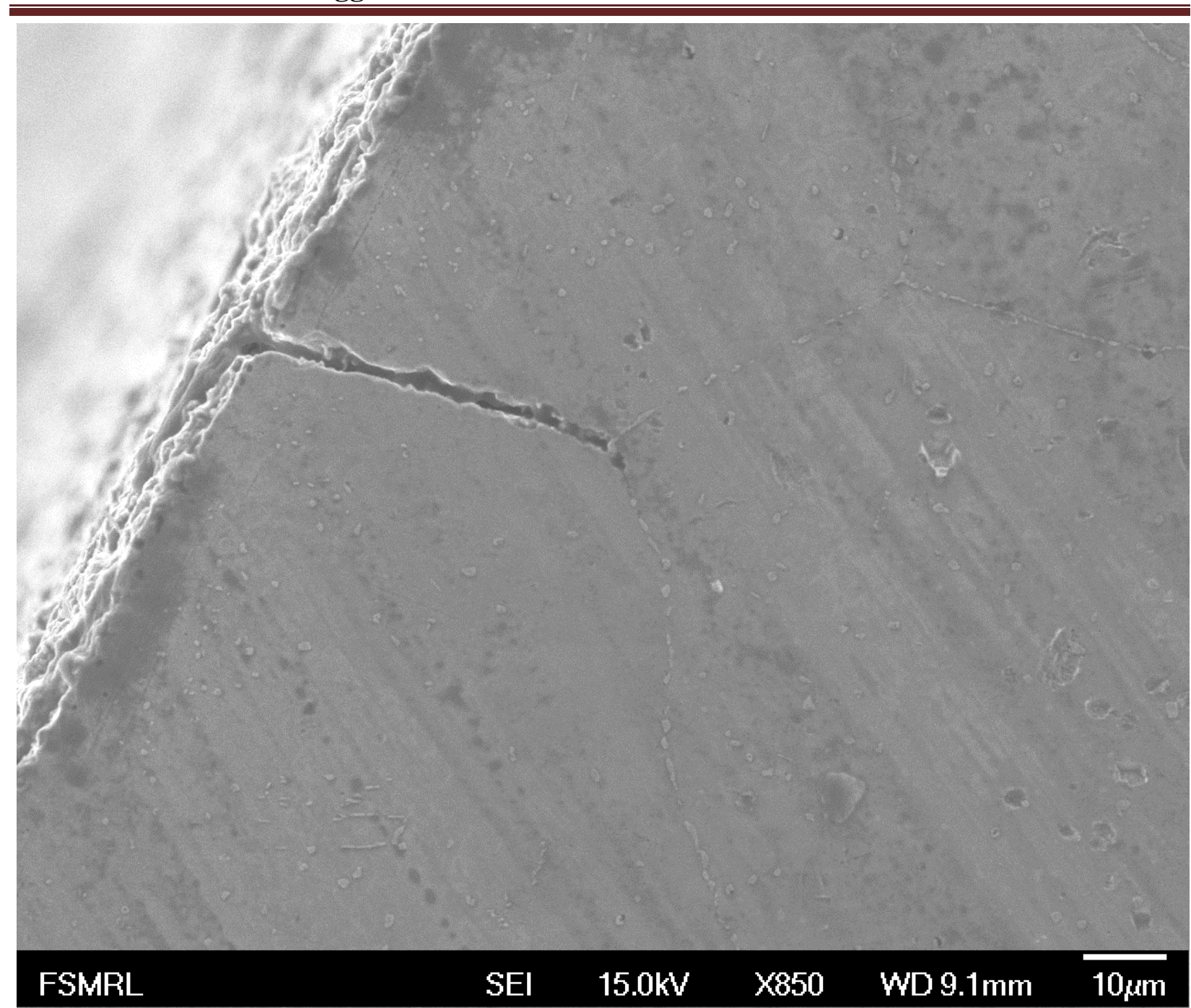

Fig. 4.123: (a) Cross-sectional view of pressurized creep tubes near the fracture surface under the applied stress of $35 \mathrm{MPa}$ at $900^{\circ} \mathrm{C}$ for Alloy 230 


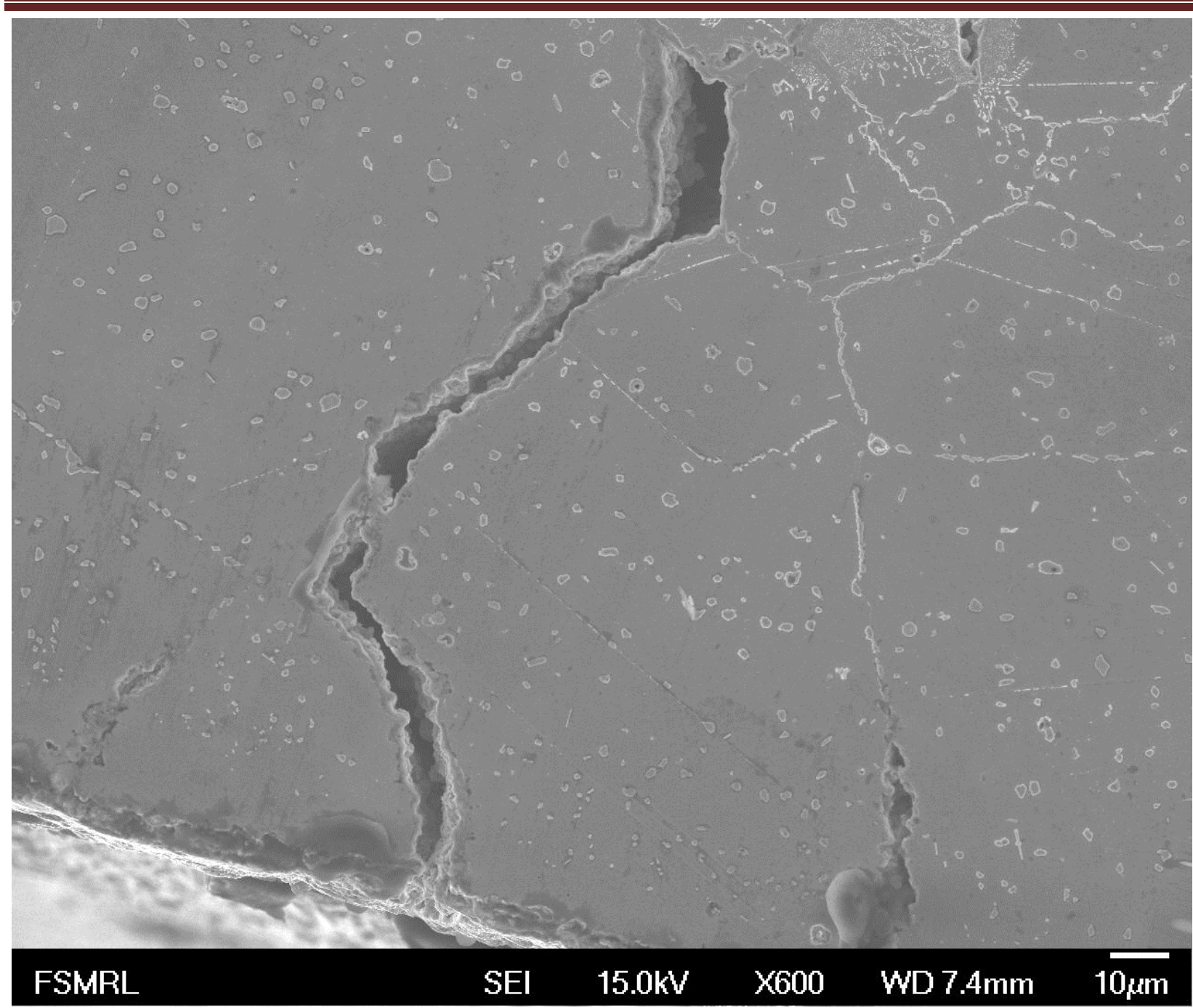

Fig. 4.123: (b) Cross-sectional view of pressurized creep tubes near the fracture surface under the applied stress of $30 \mathrm{MPa}$ at $900^{\circ} \mathrm{C}$ for Alloy 230 
Understanding Fundamental Material-Degradation Processes in High Temperature Aggressive Chemomechanical Environments

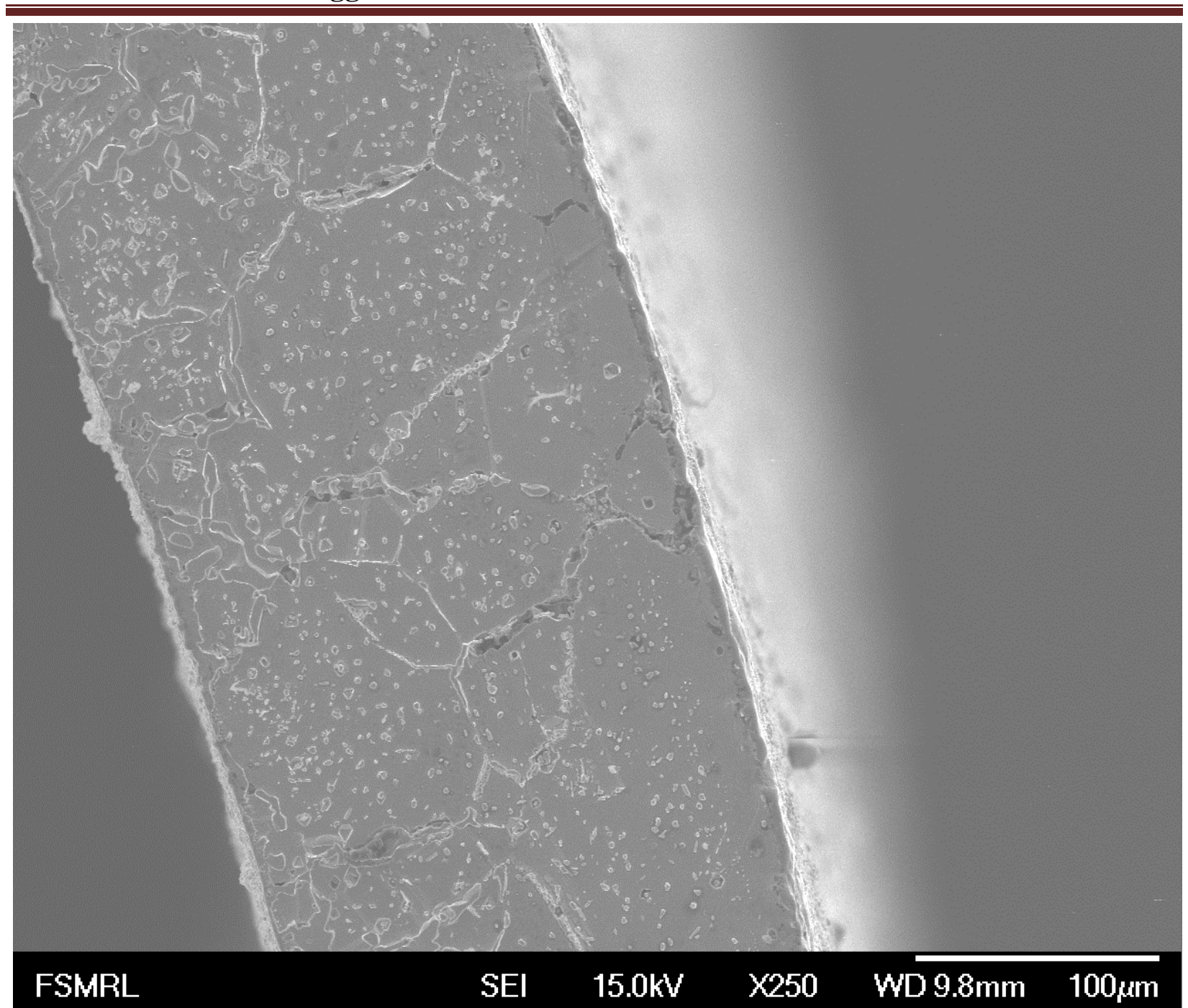

Fig. 4.123: (c) Cross-sectional view of pressurized creep tubes near the fracture surface under the applied stress of $23 \mathrm{MPa}$ at $900^{\circ} \mathrm{C}$ for Alloy 230 


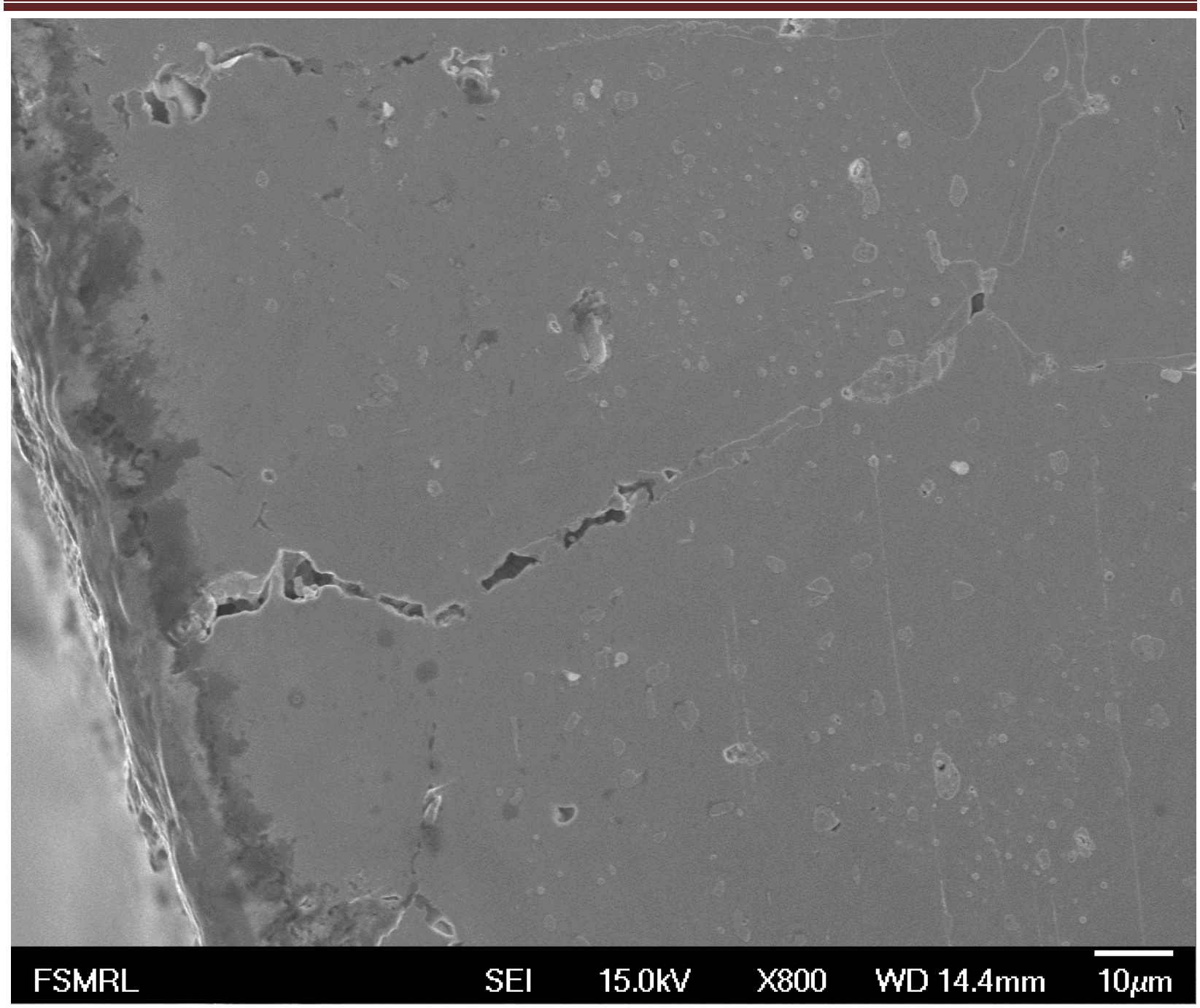

Fig. 4.123: (d) Cross-sectional view of pressurized creep tubes near the fracture surface under the applied stress of $18 \mathrm{MPa}$ at $900^{\circ} \mathrm{C}$ for Alloy 230

\subsection{Biaxial Thermal Creep Simulation}

\subsubsection{Biaxial creep simulation of Alloy 617}

Figs. 4.124 and 4.125 show the fitting curve of $\mathrm{C}(\mathrm{t})$ and $\mathrm{K}(\mathrm{t})$ for Alloy 617 at $950^{\circ} \mathrm{C}$. When fitting the $\mathrm{K}(\mathrm{t})$ curve, the natural $\log$ was taken on both sides of equation 3.6. 
Understanding Fundamental Material-Degradation Processes in High Temperature Aggressive Chemomechanical Environments

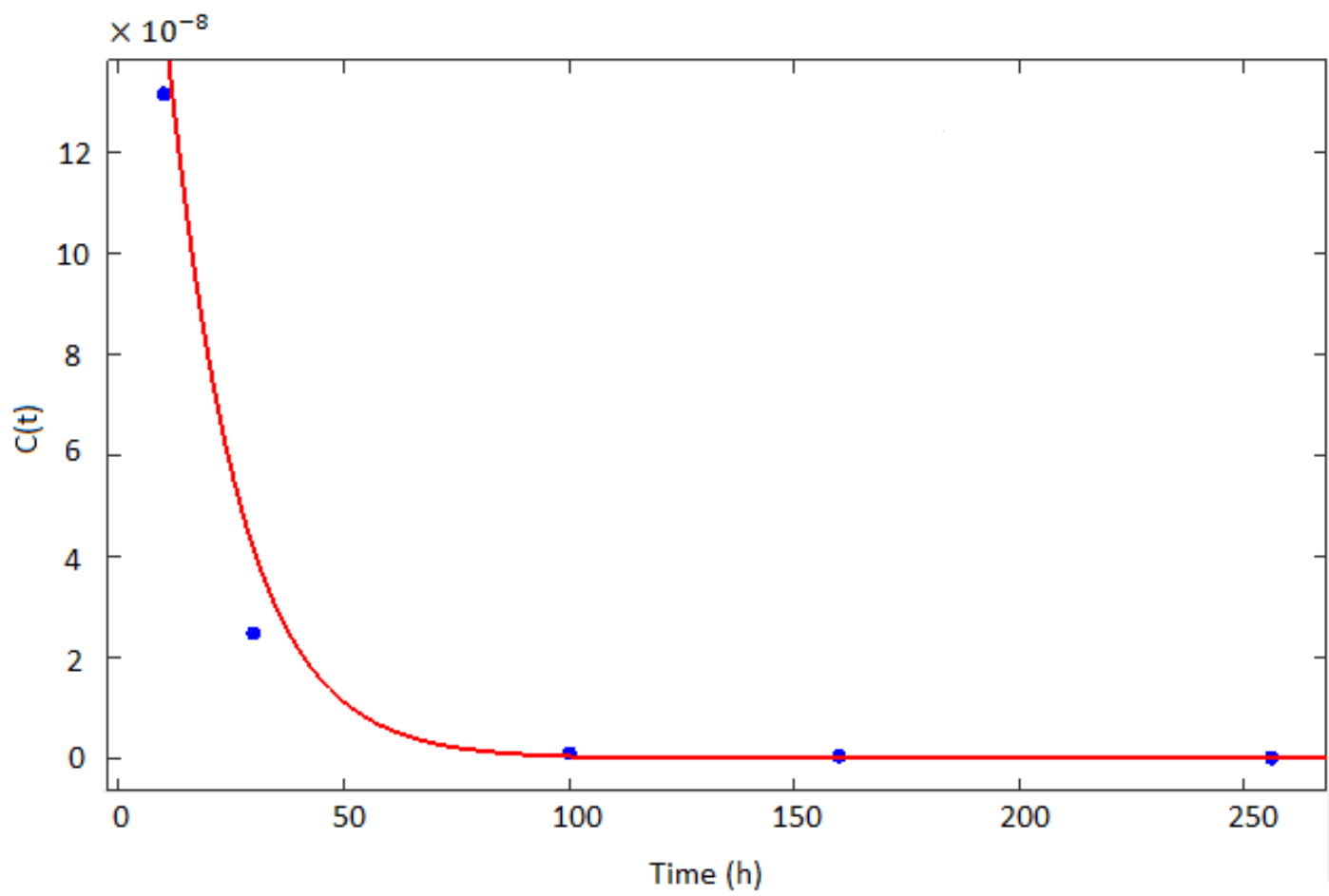

Fig. 4.124: Experimental results and numerical description of $C(t)$

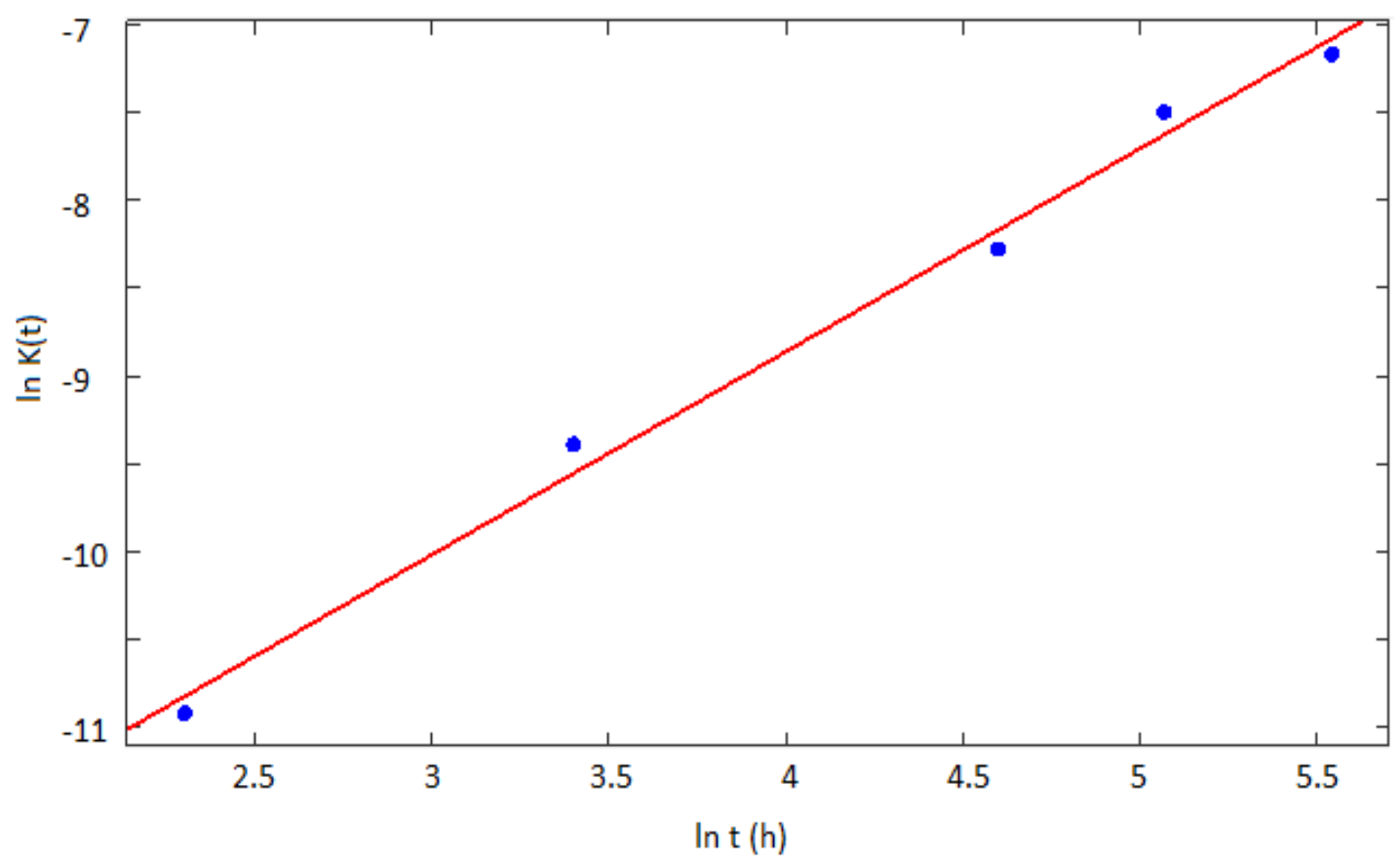

Fig. 4.125: Experimental results and numerical description of $K(t)$ 


\section{Understanding Fundamental Material-Degradation Processes in High Temperature Aggressive Chemomechanical Environments}

From the curve fitting shown above, the values of the constants $A, r, P$, and $m$ for Alloy 617 at $950^{\circ} \mathrm{C}$ are attained. With the same procedure, the corresponding constant values for $900^{\circ} \mathrm{C}$ and $850^{\circ} \mathrm{C}$ are obtained. Table 4.14 lists these values at different temperatures for Alloy 617 . The simulation results for Alloy 617 are plotted in Fig. 4.126(a)-(c).

Table 4.14: Values of equation constants of equation 3.7 for Alloy 617

\begin{tabular}{|c|c|c|c|c|c|}
\hline Temperature $\left(^{\circ} \mathbf{C}\right)$ & $\boldsymbol{A}$ & $\boldsymbol{r}$ & $\boldsymbol{P}$ & $\boldsymbol{m}$ & $\boldsymbol{n}$ \\
\hline $\mathbf{8 5 0}$ & 0.008778 & 0.03115 & $9.261 \mathrm{e}-10$ & 1.401 & 4.1 \\
\hline $\mathbf{9 0 0}$ & 0.01239 & 0.02198 & $1.3219 \mathrm{e}-8$ & 1.413 & 3.714 \\
\hline $\mathbf{9 5 0}$ & $4.0897 \mathrm{e}-7$ & 0.06541 & $1.317 \mathrm{e}-6$ & 1.161 & 3.3 \\
\hline
\end{tabular}

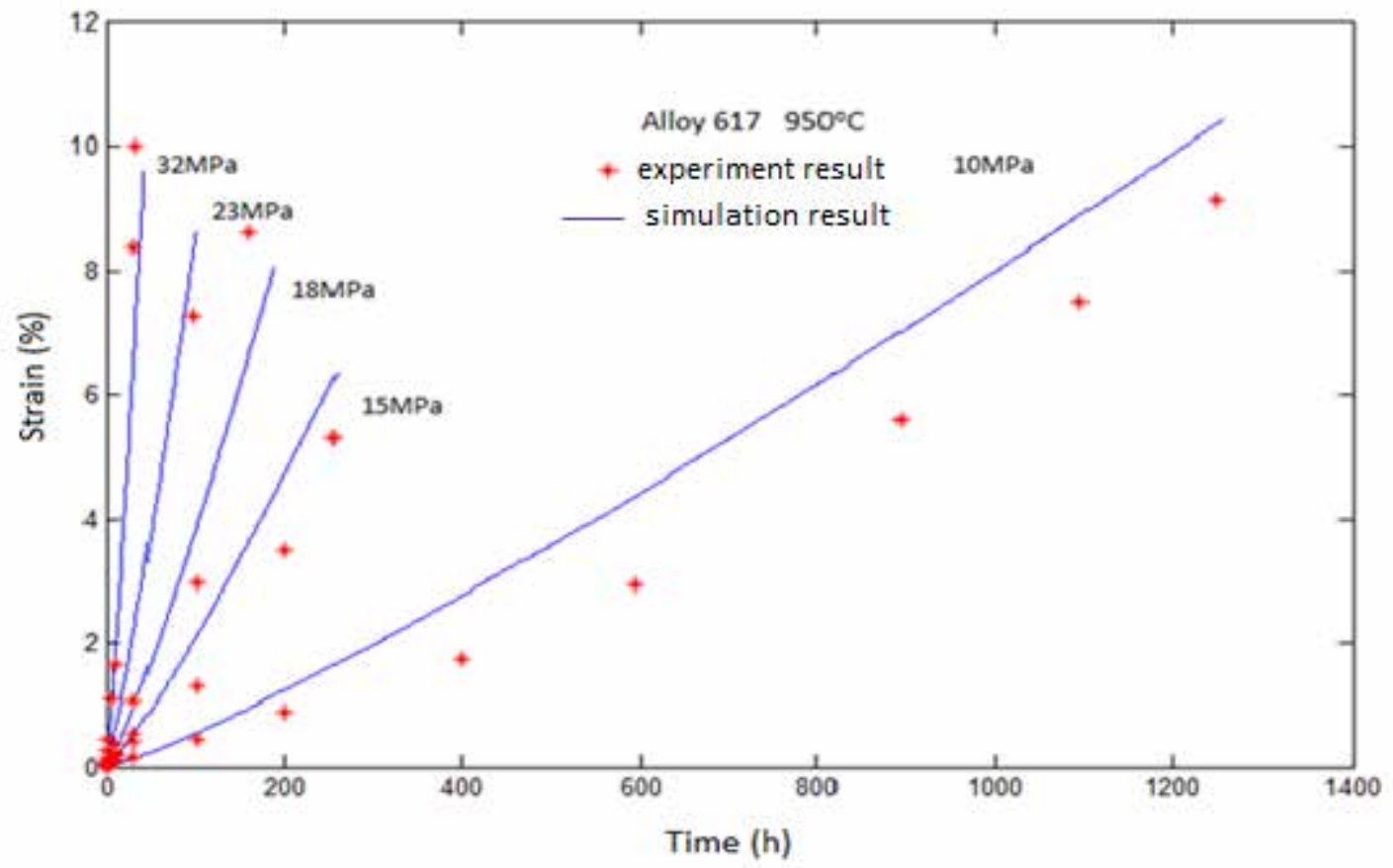

Fig. 4.126: (a) Results of creep experiments and simulations for Alloy 617 at $950^{\circ} \mathrm{C}$. Red stars represent experimental results. 


\section{Understanding Fundamental Material-Degradation Processes in High Temperature Aggressive Chemomechanical Environments}

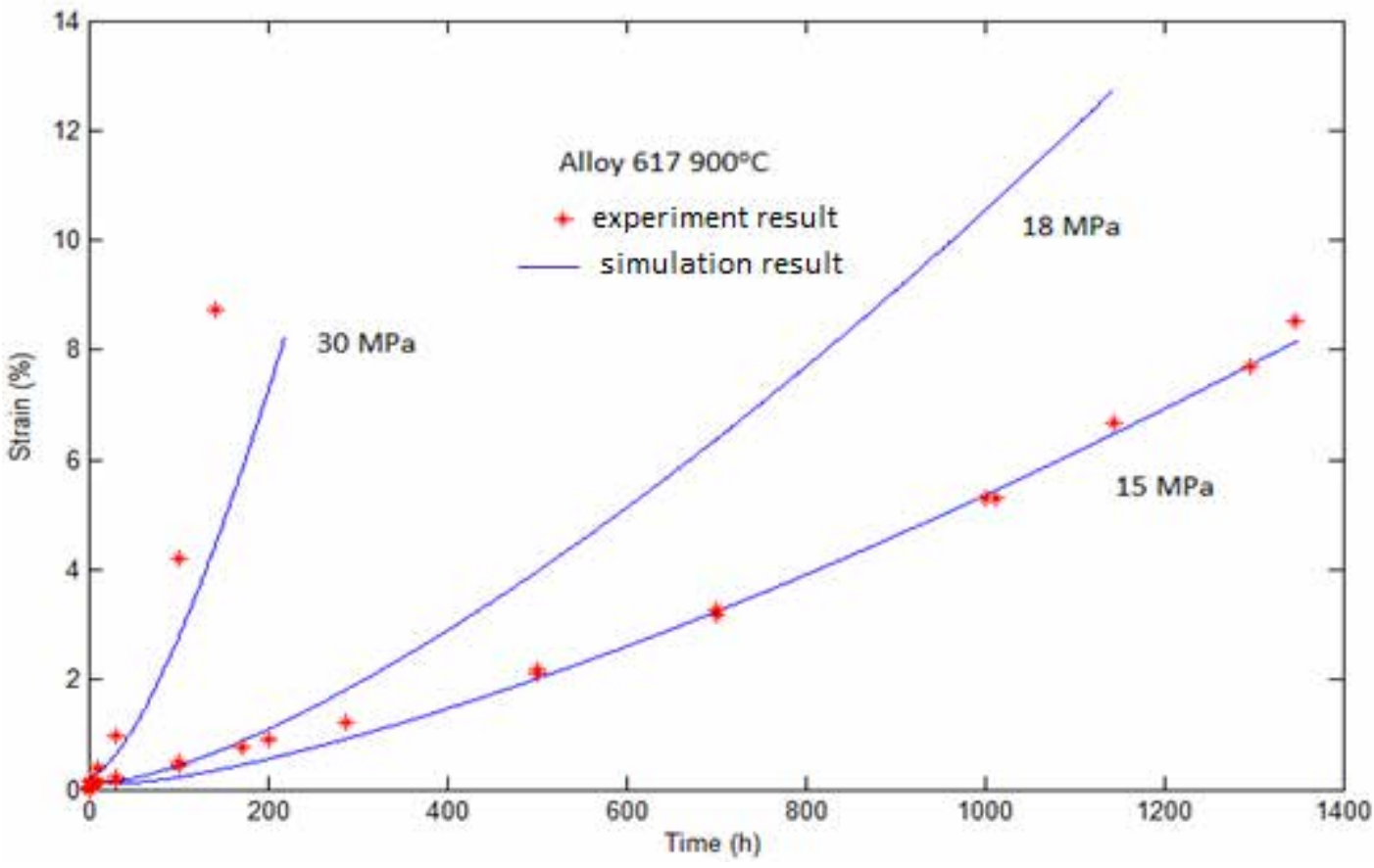

Fig. 4.126: (b) Results of creep experiments and simulations for Alloy 617 at $900^{\circ} \mathrm{C}$. Red stars represent experimental results.

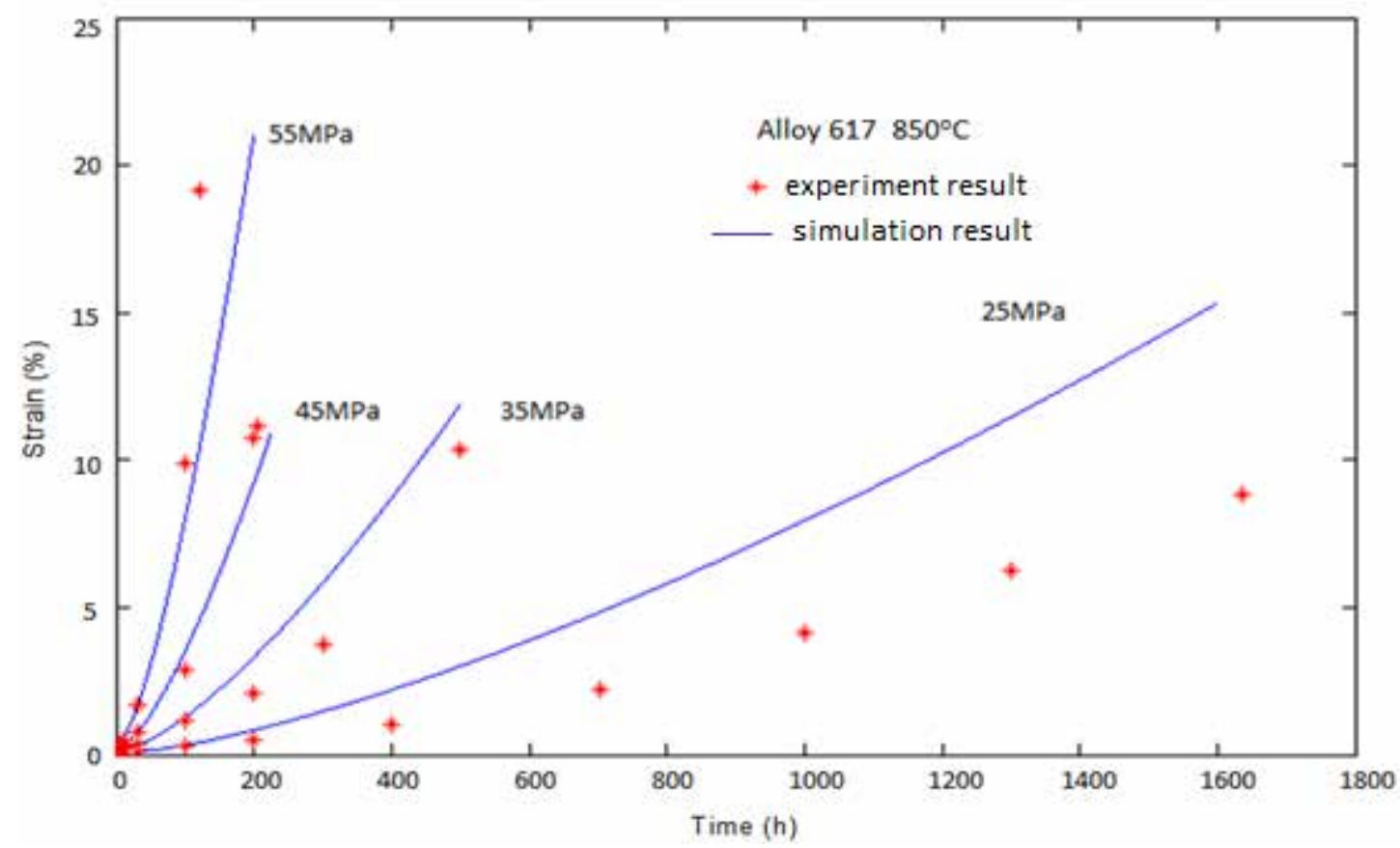

Fig. 4.126: (c) Results of creep experiments and simulations for Alloy 617 at $850^{\circ} \mathrm{C}$. Red stars represent experimental results. 


\section{Understanding Fundamental Material-Degradation Processes in High Temperature Aggressive Chemomechanical Environments}

As illustrated in the plots of Fig. 4.126(a)-(c), in most cases, the simulation is in reasonable agreement with the experimental results at the medium stress level. However, the simulation overestimates the creep development at low stress levels, and underestimates the creep development at high stress levels. The deviation is primarily attributed to the parameter curve fitting, and suggests that more long-term experimental data is required.

\subsubsection{Biaxial creep simulation of Alloy 230}

Figs. 4.124 and 4.125 show the fitting curves $\mathrm{C}(\mathrm{t})$ and $\mathrm{K}(\mathrm{t})$ for Alloy 617 at $950^{\circ} \mathrm{C}$. When fitting the $\mathrm{K}(\mathrm{t})$ curve, the natural $\log$ was taken on both sides of equation 3.6. Using the same method that was used to attain $C(t)$ and $K(t)$ in section 4.12.1, the constants $A, r, P$, and $m$ for Alloy 230 were obtained, and are listed in Table 4.15.

Table 4.15: Values of equation constants of equation 3.7 for Alloy 230

\begin{tabular}{|c|c|c|c|c|c|}
\hline Temperature $\left(^{\circ} \mathbf{C}\right)$ & $\boldsymbol{A}$ & $\boldsymbol{r}$ & $\boldsymbol{P}$ & $\boldsymbol{m}$ & $\boldsymbol{n}$ \\
\hline $\mathbf{8 5 0}$ & 0.008027 & 0.009588 & $8.41 \mathrm{e}-23$ & 1.835 & 11.1 \\
\hline $\mathbf{9 0 0}$ & 0.006461 & 0.007775 & $3.177 \mathrm{e}-10$ & 1.362 & 4.666 \\
\hline $\mathbf{9 5 0}$ & 0.00867 & 0.04153 & $9.54 \mathrm{e}-6$ & 1.152 & 2.0 \\
\hline
\end{tabular}

Fig. 4.127(a)-(c) shows the simulation results of Alloy 230, where the red stars represent the experimental results. As illustrated in the plot, the simulation results are more accurate at high temperatures and high stresses than at low temperatures and low stresses. At temperatures of $900^{\circ} \mathrm{C}$ and $950^{\circ} \mathrm{C}$, the tertiary portion is often underestimated. 


\section{Understanding Fundamental Material-Degradation Processes in High Temperature Aggressive Chemomechanical Environments}

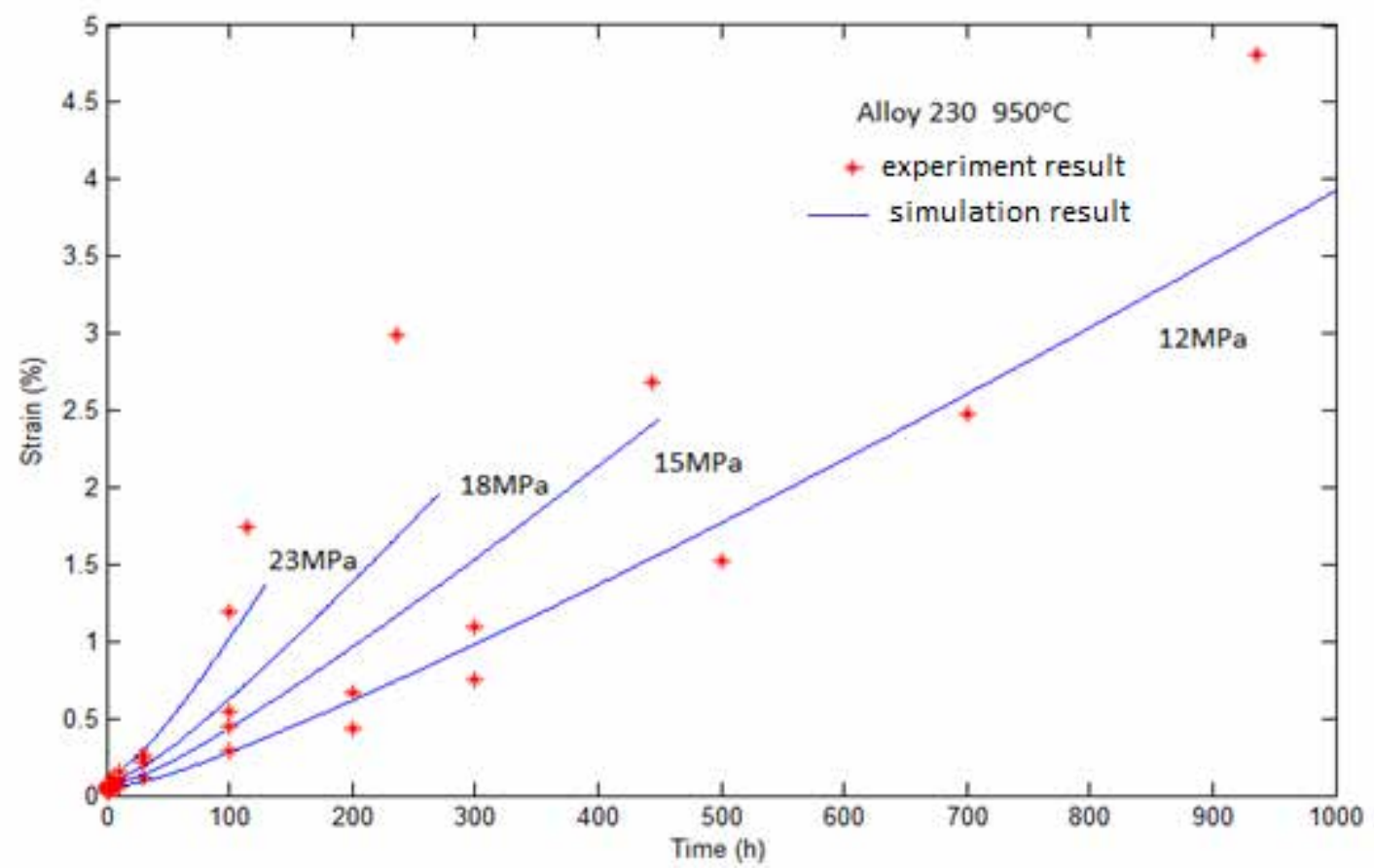

Fig. 4.127: (a) Results of creep experiments and simulations for Alloy 230 at $950^{\circ} \mathrm{C}$

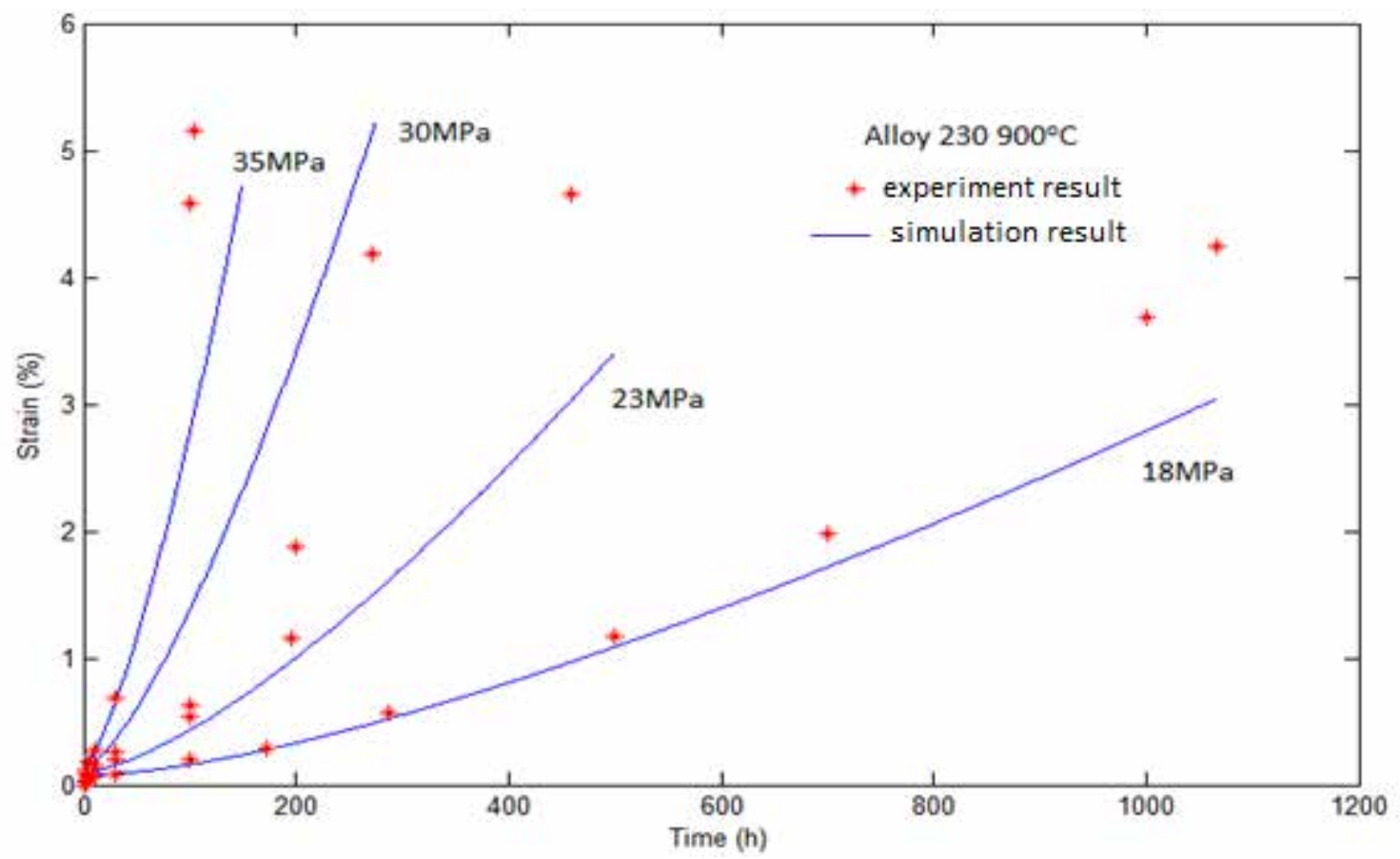

Fig. 4.127: (b) Results of creep experiments and simulations for Alloy 230 at $900^{\circ} \mathrm{C}$ 


\section{Understanding Fundamental Material-Degradation Processes in High Temperature}

Aggressive Chemomechanical Environments

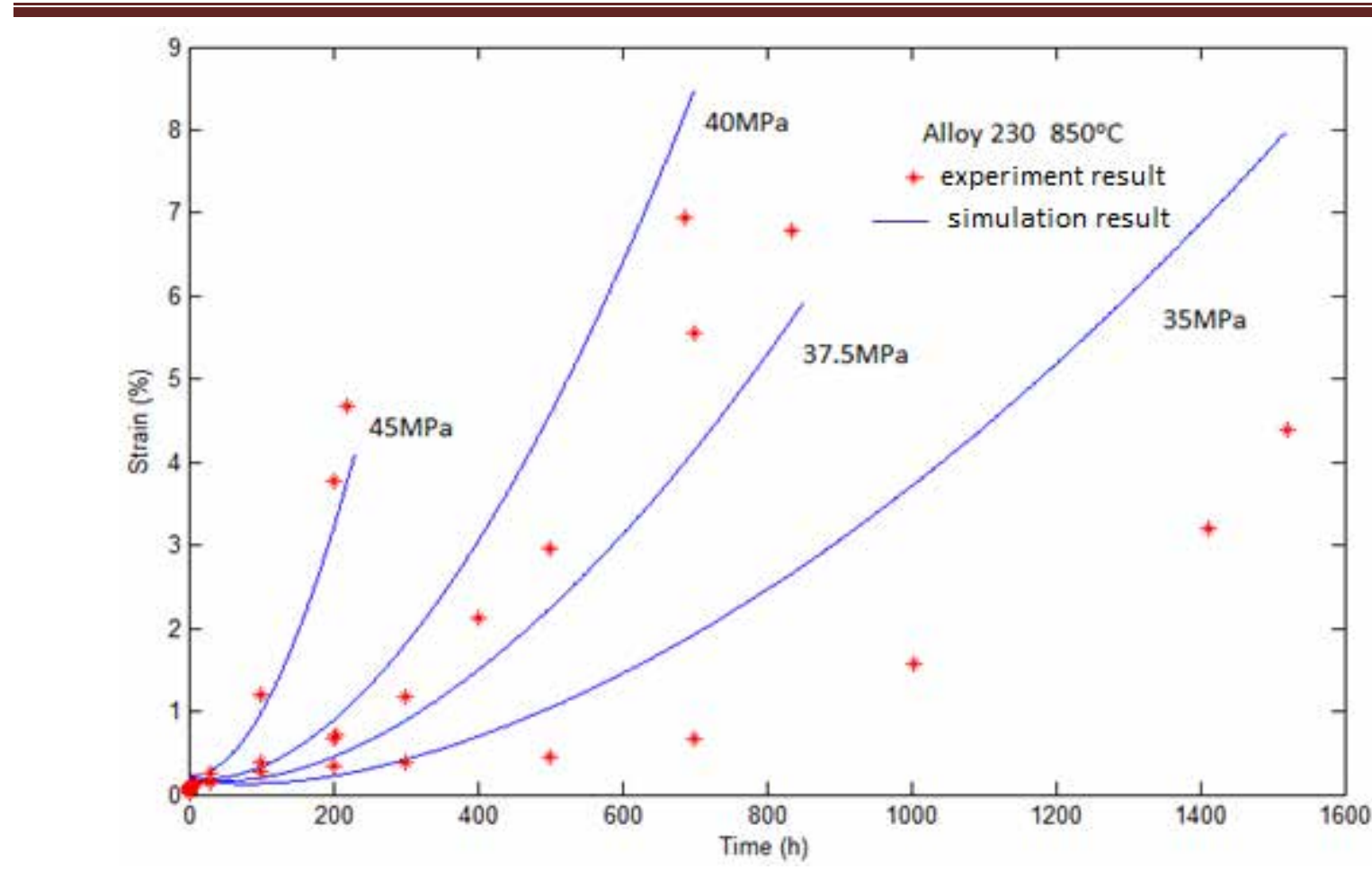

Fig. 4.127: (c) Results of creep experiments and simulations for Alloy 230 at $850^{\circ} \mathrm{C}$

\subsubsection{Finite element analysis for creep behavior}

Fig. 4.128 shows the pressurized creep tube deformed at $32 \mathrm{MPa}$ and $950^{\circ} \mathrm{C}$ after FEM modeling. By using the XY-Data tool, a node in the center region of the deformed model can be selected and its moving magnitude plotted. This moving magnitude can then be compared with the experimental results. The results of this comparison are shown in Fig. 4.129(a)-(f), where the red lines represent the simulation data. 
Understanding Fundamental Material-Degradation Processes in High Temperature Aggressive Chemomechanical Environments

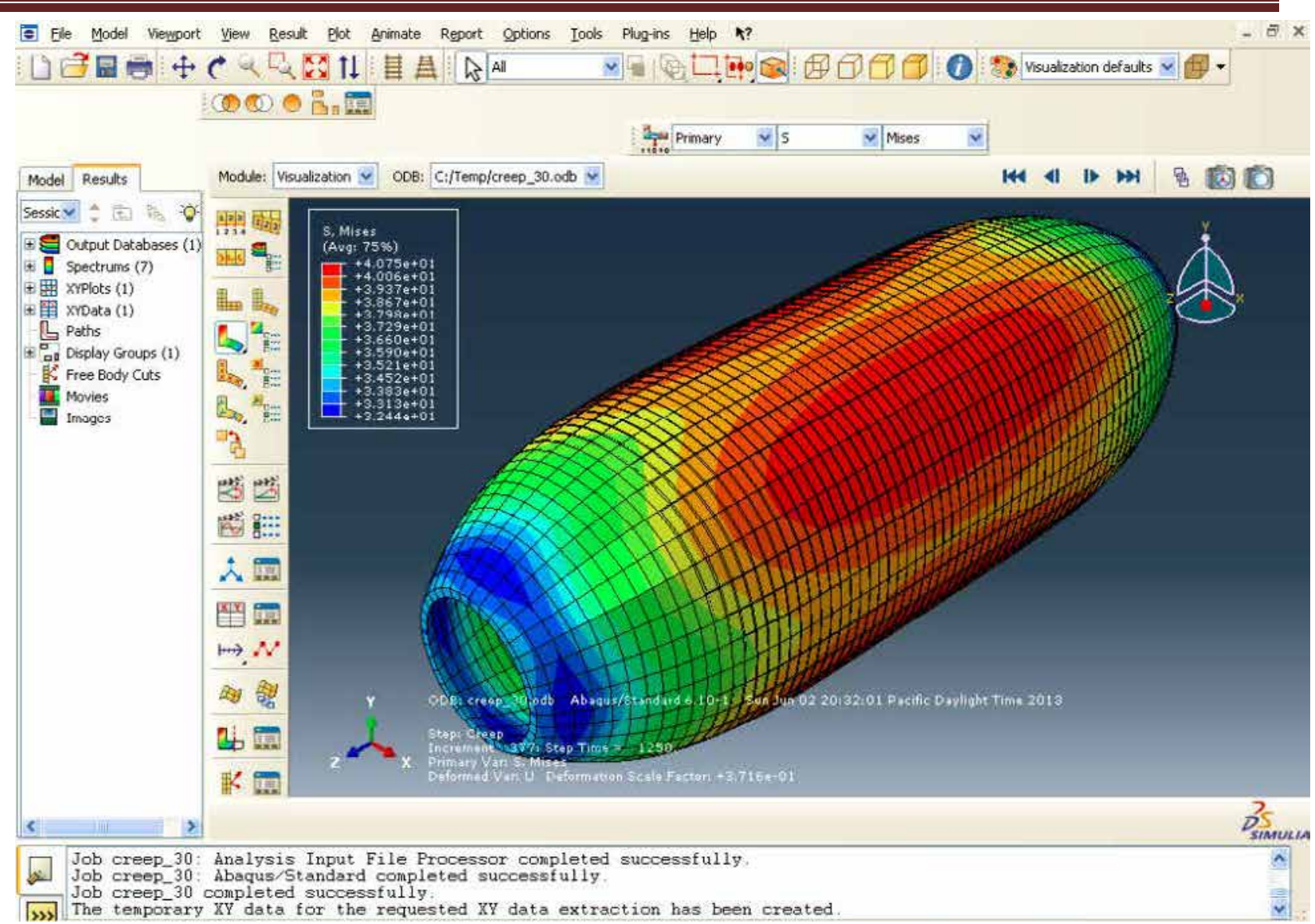

Fig. 4.128: FEM modeling for creep strain development of a pressurized creep tube 


\section{Understanding Fundamental Material-Degradation Processes in High Temperature}

Aggressive Chemomechanical Environments
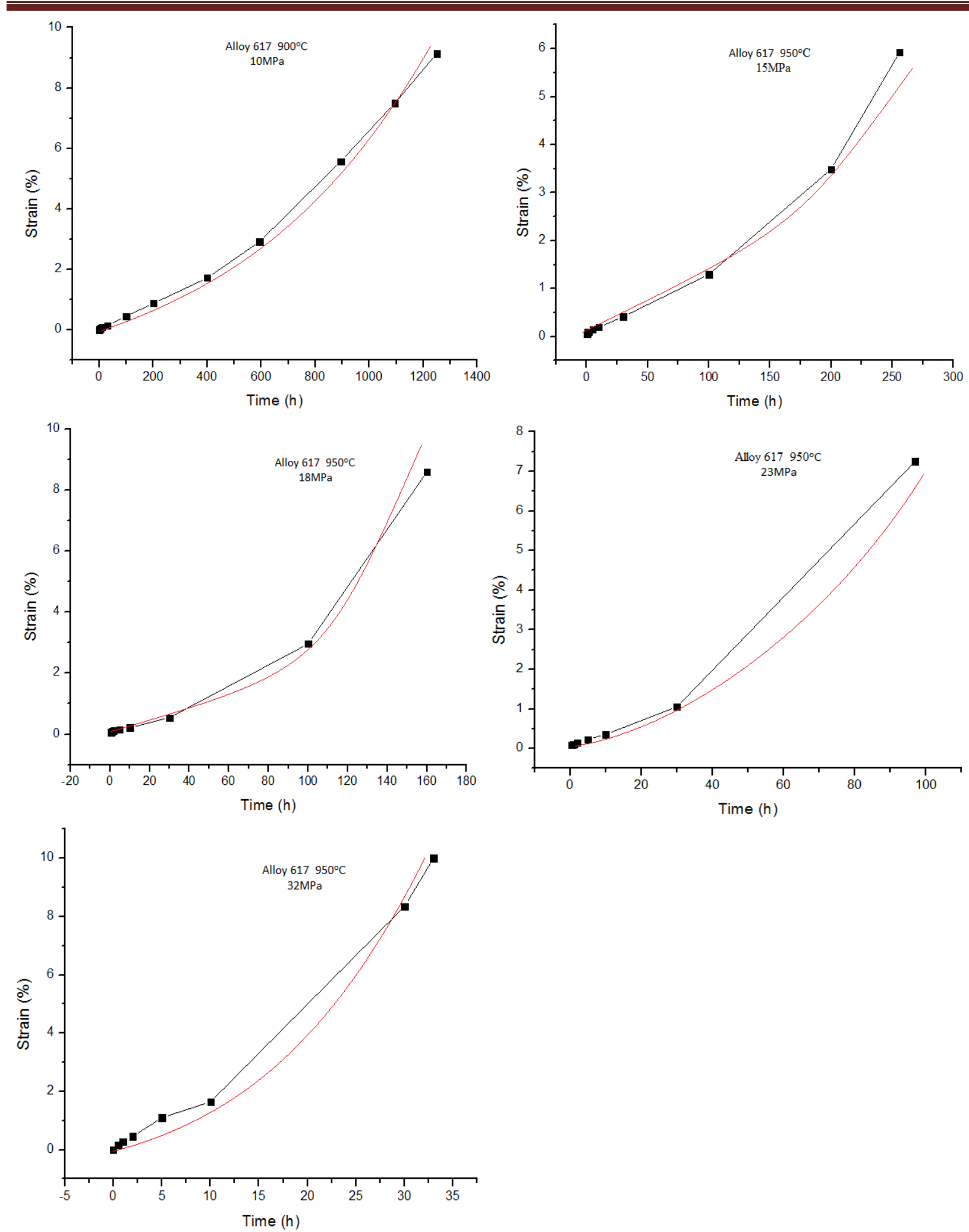

Fig. 4.129: (a) FEM simulation results for Alloy 617 at $950^{\circ} \mathrm{C}$ 


\section{Understanding Fundamental Material-Degradation Processes in High Temperature}

Aggressive Chemomechanical Environments
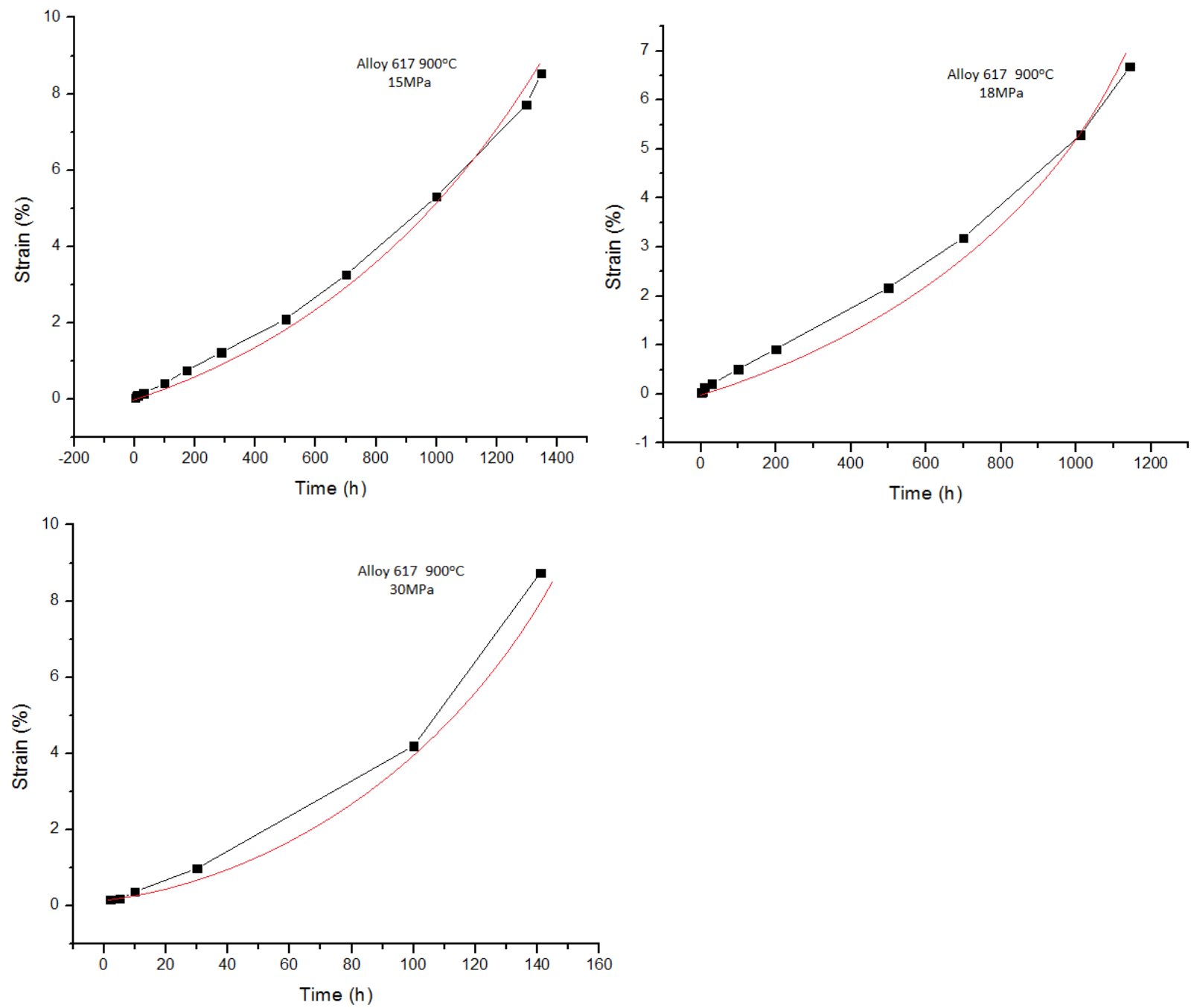

Fig. 4.129: (b) FEM simulation results for Alloy 617 at $900^{\circ} \mathrm{C}$ 


\section{Understanding Fundamental Material-Degradation Processes in High Temperature}

Aggressive Chemomechanical Environments
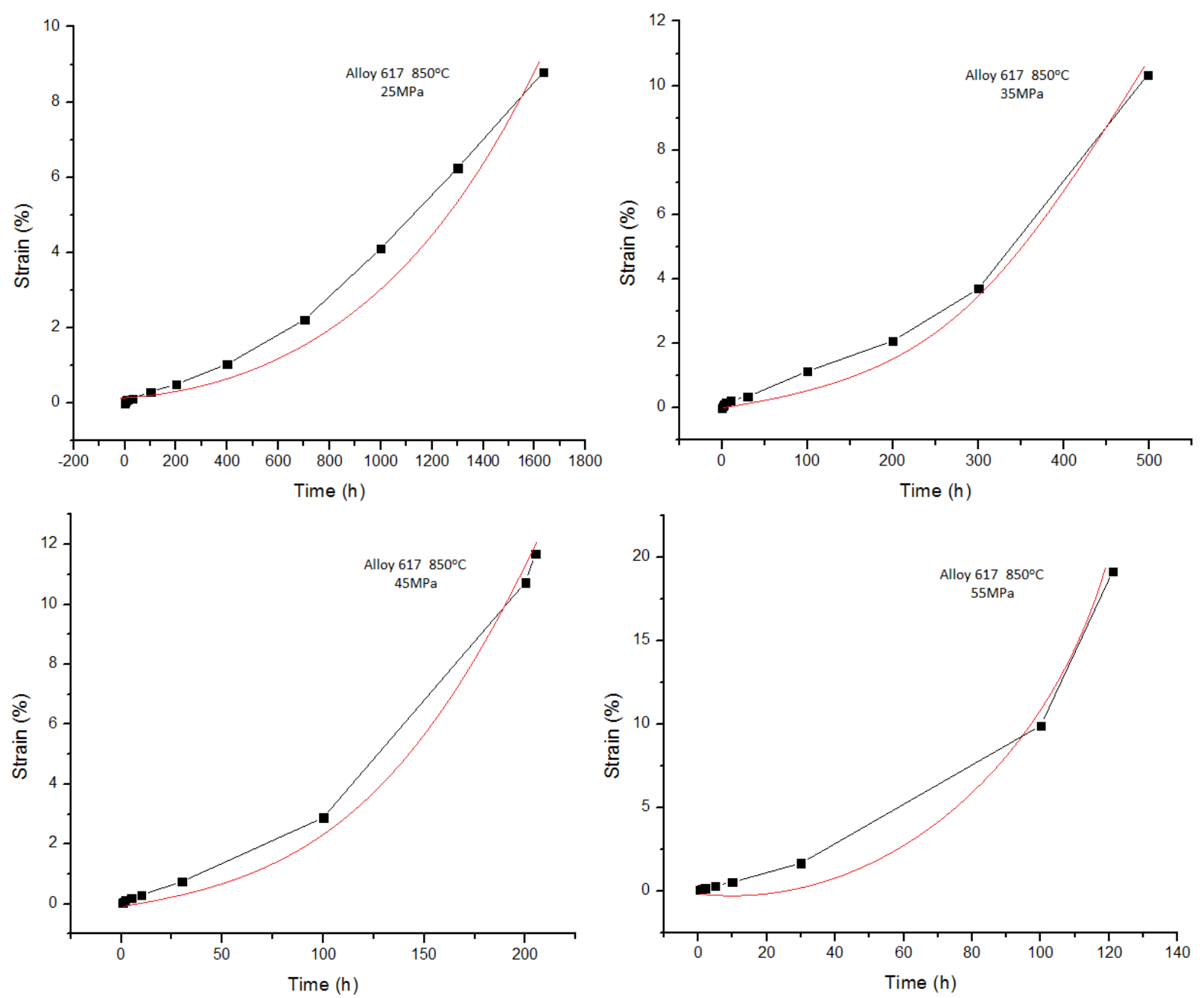

Fig. 4.129: (c) FEM simulation results for Alloy 617 at $850^{\circ} \mathrm{C}$ 


\section{Understanding Fundamental Material-Degradation Processes in High Temperature}

Aggressive Chemomechanical Environments
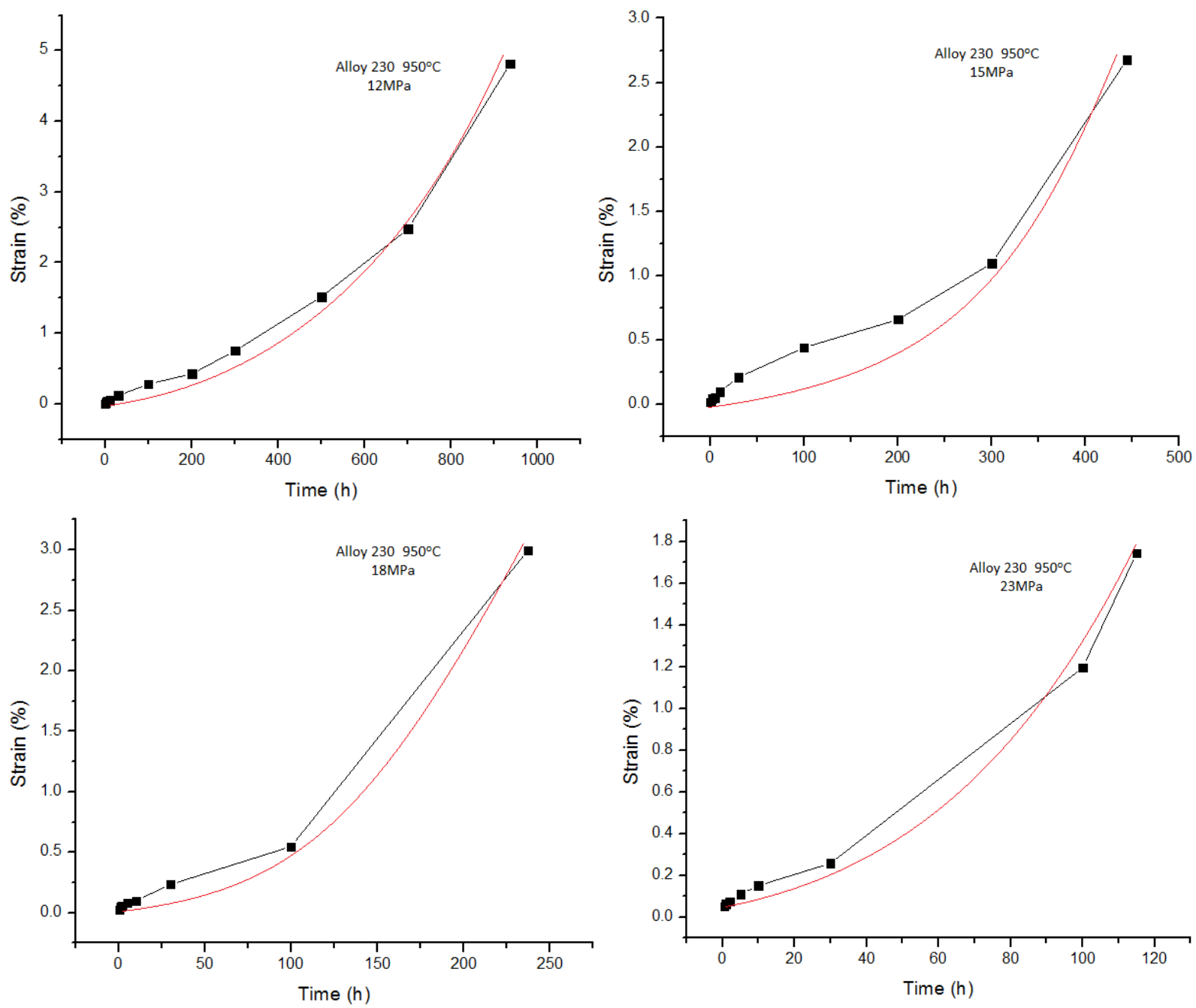

Fig. 4.129: (d) FEM simulation results for Alloy 230 at $950^{\circ} \mathrm{C}$ 


\section{Understanding Fundamental Material-Degradation Processes in High Temperature}

Aggressive Chemomechanical Environments
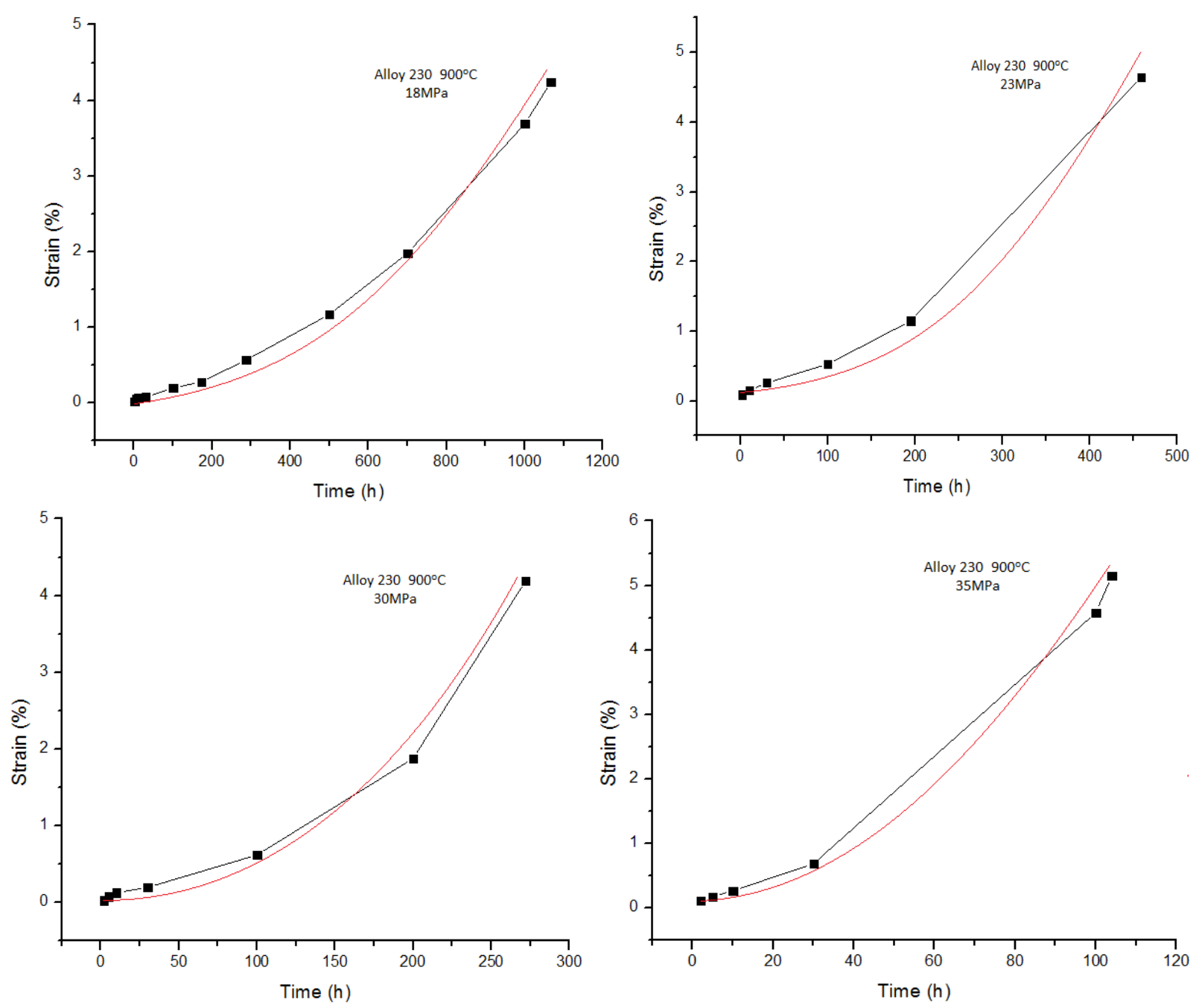

Fig. 4.129: (e) FEM simulation results for Alloy 230 at $900^{\circ} \mathrm{C}$ 


\section{Understanding Fundamental Material-Degradation Processes in High Temperature Aggressive Chemomechanical Environments}
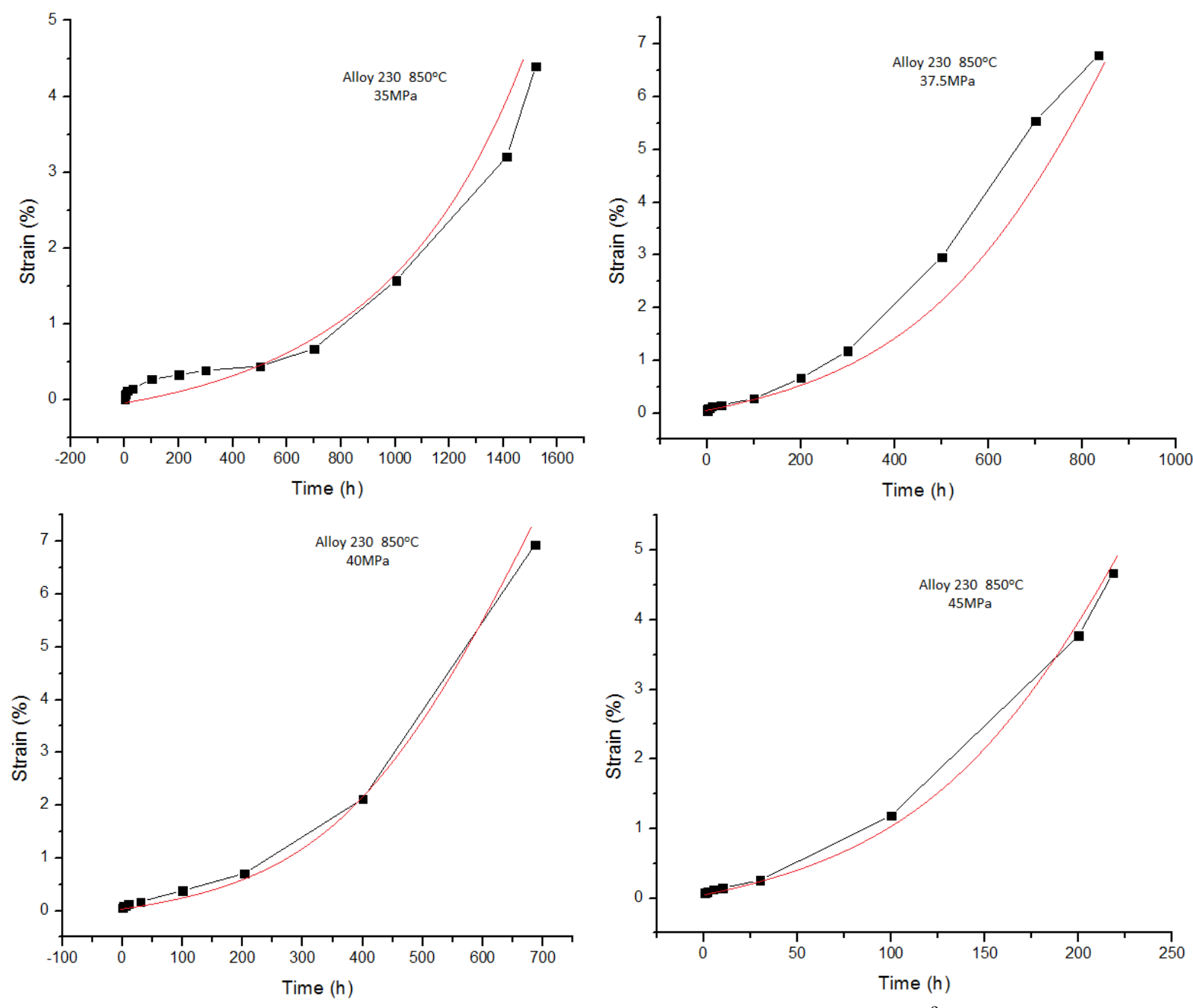

Fig. 4.129: (f) FEM simulation results for Alloy 230 at $850^{\circ} \mathrm{C}$

\subsection{High-temperature Fatigue Crack Growth of Haynes 230}

\subsubsection{Tensile tests}

To determine the mechanical properties of Haynes 230 at both room temperature (RT) and high temperature $(\mathrm{HT})\left(900^{\circ} \mathrm{C}\right)$, a simple uniaxial tension test was performed at each temperature, as shown in Fig. 4.130. Using DIC, the $0.2 \%$ offset yield stress was determined to be $415 \mathrm{MPa}$ for the RT sample and $123 \mathrm{MPa}$ for the HT sample. Another difference between the two stress-strain responses is the continual hardening in the RT experiment and the almost immediate softening in the HT experiment. Observing the DIC strain fields, labeled "a" and "b" in Fig. 4.130, for about $15 \%$ strain, the HT results show far more localization than the RT results. The image of the HT specimen surface near fracture (labeled "c") shows significant damage along the section that fails. Necking and large voids are observed in the entire area shown. The fracture strain of the 


\section{Understanding Fundamental Material-Degradation Processes in High Temperature Aggressive Chemomechanical Environments}

two specimens is quite different, and the lack of damage in the RT sample would be a reasonable explanation for this result.

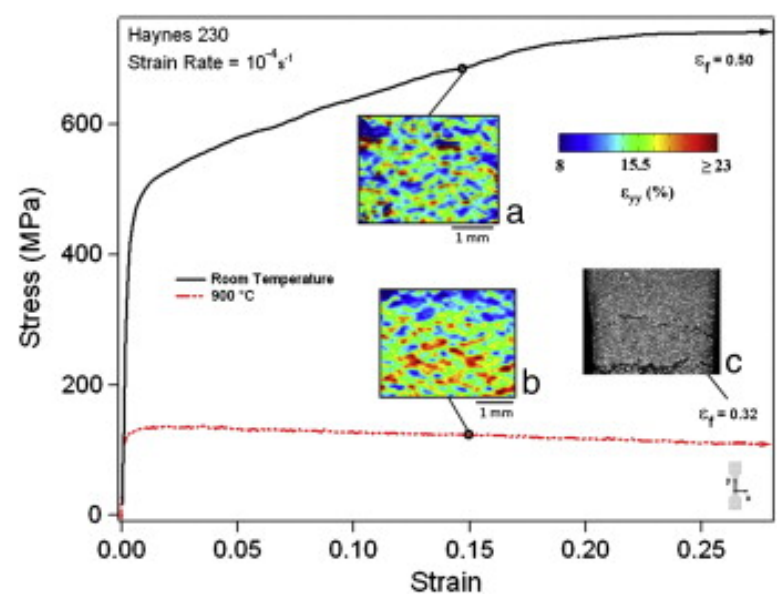

Fig. 4.130: The stress-strain response of Haynes 230 at RT and $900^{\circ} \mathrm{C}$ with fracture strains of $50 \%$ and $32 \%$, respectively. The DIC strain plots at $15 \%$ global strain show the greater localization at $900^{\circ} \mathrm{C}$.

\subsubsection{Fatigue crack growth}

Images were continuously taken during the fatigue crack growth tests in order to use digital image correlation to capture the full field displacements. Crack growth rates were determined by using these images to follow the crack tip advancement. As indicated in Fig. 4.131, both RT and HT fatigue crack growth tests were run. The solid symbols in Fig. 4.131 represent the isotropic stress intensity factor range calculation:

$\Delta K=\Delta \sigma \sqrt{\pi \cdot a} \cdot f(a / w)$

where $\Delta \sigma$ is the stress range, $a$ is the current crack length, and $f(a / w)$ is the geometric correction factor, which can be found in [4.90]. However, equation 4.48 does not account for crack closure. The least-squares regression code explained in [4.91] and the Appendix was used to extract the effective stress intensity factor range from the experiments by considering the full field crack tip displacements, which inherently are effected by crack closure during a fatigue cycle. The effective stress intensity factor results, which consider crack closure, are shown as hollow symbols in Fig. 4.131. The insets included in this Fig. 4.131 show the experimental and regressed vertical displacement contours corresponding to the RT and HT specimens. The Paris law coefficients and exponents for both tests are shown in Table 4.16. The Paris law exponent is $\sim 2$ for both test results, which is in the common range of 2-4 for metals [4.92]. The following form of the Paris law was used to find the effective results that considered crack closure:

$d a /_{d N}=C\left(\Delta K_{e f f}\right)^{m}$

In equation 4.49, $C$ and $m$ are the Paris coefficient and exponent respectively. $\Delta K_{\text {eff }}$ may be expressed as:

$\Delta K_{e f f}=\Delta \sigma_{e f f} \sqrt{\pi \cdot a} \cdot f(a / w)$ 


\section{Understanding Fundamental Material-Degradation Processes in High Temperature Aggressive Chemomechanical Environments}

In equation 4.50, $\Delta \sigma$ eff is the effective stress range (the portion of the loading cycle for which the crack is open), and $a$ is the crack length.

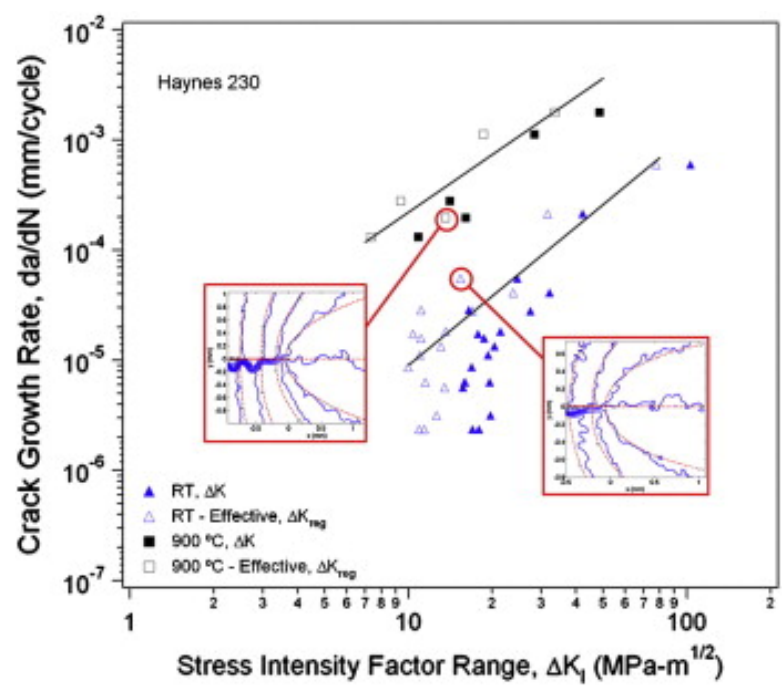

Fig. 4.131: Crack growth rate as a function of the stress intensity factor range for RT and $900^{\circ} \mathrm{C}$ experiments. The inserts are displacement contour plots showing the experimental (blue) and regression (red) vertical displacements in increments of 1 micron, with the black dot indicating the crack tip location.

Table 4.16: Paris law coefficients for Haynes 230 at RT and $900^{\circ} \mathrm{C}$ (with $\mathrm{da} / \mathrm{dN}$ in $\mathrm{mm} /$ cycle and $\Delta K$ in $\mathrm{MPa} \sqrt{\mathrm{m}})$

\begin{tabular}{|c|c|c|}
\hline & $\mathrm{C}$ & $\mathrm{m}$ \\
\hline RT theorhetical & $1.34 \mathrm{E}-08$ & 2.38 \\
\hline RT effective & $7.54 \mathrm{E}-08$ & 2.08 \\
\hline $900^{\circ} \mathrm{C}$ theorhetical & $1.81 \mathrm{E}-06$ & 1.82 \\
\hline $900^{\circ} \mathrm{C}$ effective & $4.20 \mathrm{E}-06$ & 1.74 \\
\hline
\end{tabular}

The crack growth rate for the HT experiment was over an order of magnitude larger than that of the RT experiment, being in the range of $10^{-3}$ to $10^{-2} \mathrm{~mm} /$ cycle. This is an extremely high rate of fatigue crack growth that could lead to failure in a component before the crack is initially detected. Comparing the isotropic and effective stress intensity factor values, the crack closure level for both experiments was an average of $30 \%$ of the load. To show the effectiveness of the regression technique in determining the closure level, two-point digital extensometers, which measure local crack opening levels, were used to find closure values along the crack length for both the RT and HT specimens. The comparison results are provided in Fig. 4.132. The regression technique, also referred to as the full-field method, proved to be efficient in estimating the overall crack closure levels, while the two-point digital extensometers provided insight into the precise crack closure levels at the crack tip. The similarity in the closure levels between the 


\section{Understanding Fundamental Material-Degradation Processes in High Temperature Aggressive Chemomechanical Environments}

two specimens is a surprising result, and provides no conclusion about the faster crack growth rate at HT. Thus, slip irreversibility was investigated.

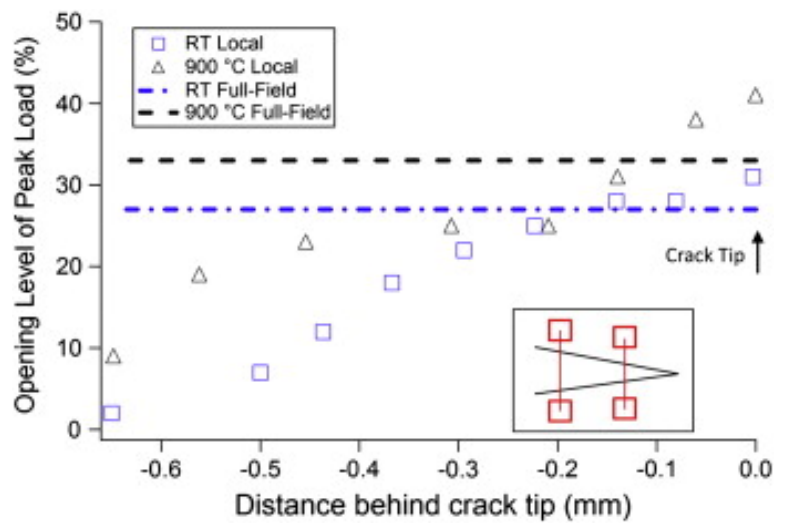

Fig. 4.132: Comparison between the local (digital extensometer) and full-field (regression) crack opening levels for the RT specimen at a crack length of $0.994 \mathrm{~mm}$ and the HT specimen at a crack length of $0.964 \mathrm{~mm}$. Symbols represent the digital extensometer values at various points along the crack, while lines represent the regression-found value for the full-field. The schematic depicts the digital extensometer technique and placement along the crack flanks.

\subsubsection{Slip irreversibility}

With the stress intensity factors known, a plane stress assumption was used to approximate the plastic zone size [4.93]. Shown in Fig. 4.133(a) is the plastic zone for the RT specimen at a crack length of $0.994 \mathrm{~mm}$ at maximum load. Using the effective stress intensity factor of 16.55 $\mathrm{MPa} \sqrt{\mathrm{m}}$, the plastic zone radius was determined to be $0.337 \mathrm{~mm}$. Strains determined from digital image correlation were inserted into the plastic zone, and high values of strain were found throughout the plastic zone. To compare, a HT specimen cycle at a crack length of $0.964 \mathrm{~mm}$ was used, as shown in Fig. 4.133(b). The effective stress intensity factor for this crack length was $9.39 \mathrm{MPa} \sqrt{\mathrm{m}}$, and the corresponding plastic zone radius was found to be $0.664 \mathrm{~mm}$. The strains in the plastic zone show a concentration around the crack tip. Even though the HT specimen had a much smaller effective stress intensity factor range, the lower yield stress caused the plastic zone size to be approximately twice that of the RT specimen at the same crack length. 

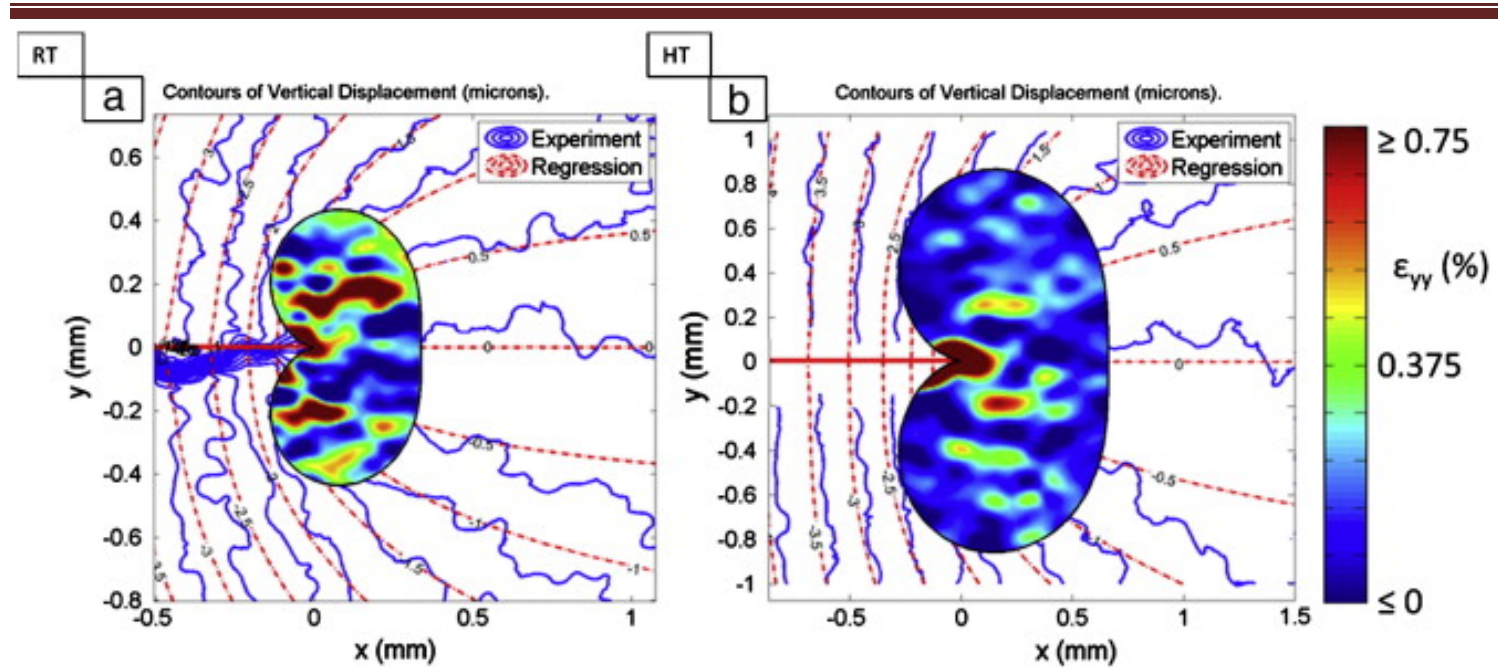

Fig. 4.133: Plots of vertical displacement contours and plastic zones for a selected (a) RT and (b) $900^{\circ} \mathrm{C}$ fatigue crack cycle. Blue contours represent the experimental DIC displacements, and red contours represent the displacement contours (in $\mu \mathrm{m}$ ) determined by least squares regression methods. (a) At a crack length of $0.994 \mathrm{~mm}$, the room temperature specimen had a $\Delta \mathrm{K}$ value of $22.64 \mathrm{MPa} \sqrt{\mathrm{m}}$, a regression-found $\Delta \mathrm{K}_{\text {eff }}$ value of $16.55 \mathrm{MPa} \sqrt{\mathrm{m}}$, and a plastic zone radius of $0.337 \mathrm{~mm}$. (b) At a crack length $0.964 \mathrm{~mm}$, the $900^{\circ} \mathrm{C}$ specimen had a $\Delta \mathrm{K}$ value of $14.08 \mathrm{MPa} \sqrt{\mathrm{m}}$, a regression-found $\Delta \mathrm{K}_{\text {eff }}$ value of $9.39 \mathrm{MPa} \sqrt{\mathrm{m}}$, and a plastic zone radius of $0.666 \mathrm{~mm}$.

Although these plastic zone approximations give much insight into the plasticity caused by the fatigue crack growth, the crack tip slip irreversibility was of great interest due to its correlation with the crack growth rate. Dislocations are emitted from the crack tip during a fatigue cycle, and when they do not fully reverse to the crack tip, strain is accumulated. In order to capture this behavior, measurements of the strain were made at the beginning and end of the cycle. Thus, the difference between points $\mathrm{A}$ and $\mathrm{B}$, as shown in the bottom right of Fig. 4.134, can be considered to be the slip irreversibility for one cycle. To quantitatively measure this at the meso-scale, DIC was utilized. The DIC strain plots in Fig. 4.134 are used as an example of this technique. The two strain plots are one cycle apart, with the image labeled " $\mathrm{A}$ " being recorded at the beginning of the cycle and the image labeled " $\mathrm{B}$ " at the end. The isotropic and effective stress intensity factor ranges were 24.6 $\mathrm{MPa} \sqrt{\mathrm{m}}$ and $15.4 \mathrm{MPa} \sqrt{\mathrm{m}}$, respectively, with a crack closure level of $37 \%$ for the RT example and $13.86 \mathrm{MPa} \sqrt{\mathrm{m}}$ and $9.39 \mathrm{MPa} \sqrt{\mathrm{m}}$, respectively, with a crack closure level of $32 \%$ for the HT example.

Referiing again to Fig. 4.134, the strain plots show strain at the crack tips before the cycle (point " $\mathrm{A}$ ") and then residual strains at the crack tip after the cycle (point "B"). Taking advantage of the digital image correlation resolution, strain measurements in front of the crack at points "A" and "B" were compared, and irreversible strains of $6.35 \times 10^{-4}$ for the RT sample and $5.36 \times 10^{-3}$ for the HT sample were measured. This technique was applied for multiple crack lengths of the RT and HT specimens. As with the two examples provided, the trend of the HT specimen to have an irreversible strain an order of magnitude larger than that of the RT specimen was consistent throughout, as shown in Fig. 4.134. 


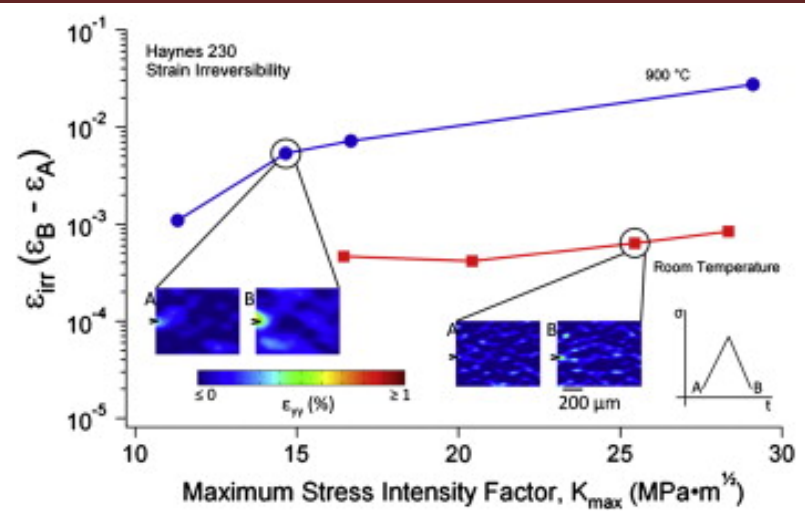

Fig. 4.134: Strain irreversibility as a function of the maximum stress intensity factor during the fatigue crack growth experiments. The schematic shows the two minimum load points (A and B) where the strain measurements were taken. DIC strain plots for both the RT and $900^{\circ} \mathrm{C}$ experiments are shown. The higher strains in the B plots compared to the A plots indicate irreversible strains during the fatigue cycle.

\subsubsection{Scanning electron microscope and transmission electron microscopy analysis}

In order to further understand the microstructural response of the Haynes 230 sample fatigued at $900^{\circ} \mathrm{C}$, images of the fracture surface were taken with a scanning electron microscope (SEM) and the dislocation arrangements were observed with transmission electron microscopy (TEM). The first SEM micrograph, shown in Fig. 4.135(a), shows the classic striations attributed to cyclic fatigue crack growth. This verifies that the external loading was responsible for the propagation of the crack and ultimately the failure of the material. Also evident in this micrograph are the effects of the high temperature damage as it was taken in a region that experienced a high $\Delta \mathrm{K}$. Fig. 4.135(b) provides an example of the smoothness of the surface, which signifies the low amount of oxidation present. This is consistent with the Haynes International, Inc. data and previously completed research [4.94] and [4.95].
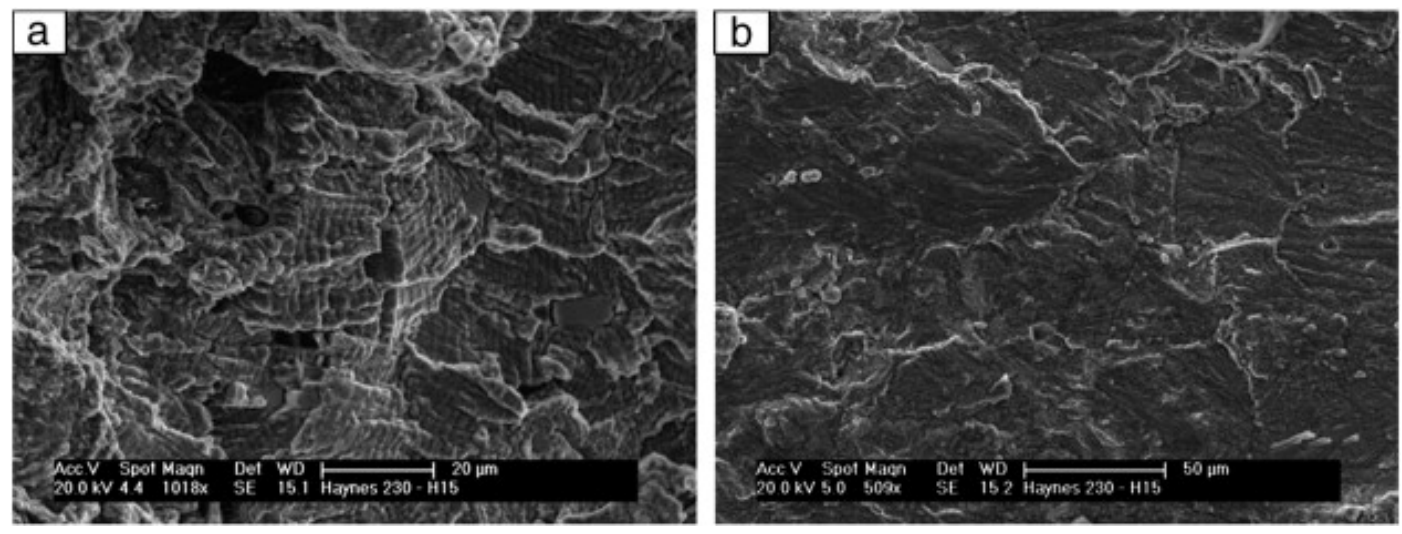

Fig. 4.135: (a) High-magnification SEM micrograph showing the fatigue striations on the $900^{\circ} \mathrm{C}$ specimen;

(b) post-fracture surface of the $900^{\circ} \mathrm{C}$ specimen showing a low level of oxidation and a smooth surface indicative of intragranular fatigue crack growth 


\section{Understanding Fundamental Material-Degradation Processes in High Temperature Aggressive Chemomechanical Environments}

TEM analysis was then performed in order to observe dislocations and any other phenomena present. As shown in Fig. 4.136, planar slip was observed. This is consistent with other solid-solution hardened face-centered cubic (FCC) alloys [4.96] and other nickel-based superalloys fatigued at high frequencies at high temperatures [4.47]. Also of particular interest was the formation of subgrains: several are present in Fig. 4.136. These subgrains were seen throughout the specimen; another example is provided in Fig. 4.137. A large heterogeneity in the microstructure in obvious from this TEM image. On the left, dislocations are present, with nothing to impede their motion. A stacking fault divides the image in half. On the right half of the image, intense dislocation-carbide interactions are observed, providing substantial resistance to dislocation motion. There are two types of carbides present in this image, and they are assumed to be $\mathrm{M}_{6} \mathrm{C}$ and $\mathrm{M}_{23} \mathrm{C}_{6}$. The larger of the two found along the stacking fault is believed to be of the $\mathrm{M}_{6} \mathrm{C}$ type. The $\mathrm{M}_{6} \mathrm{C}$ type undergoes far less interaction with dislocations than the $\mathrm{M}_{23} \mathrm{C}_{6}$ carbides.

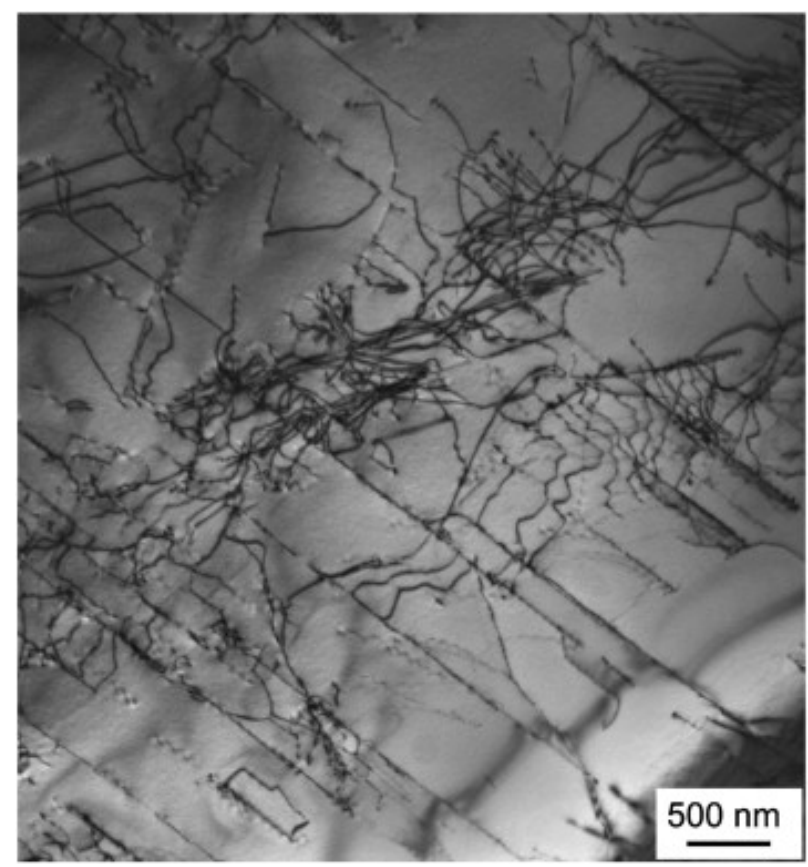

Fig. 4.136: Planar slip in the Haynes 230 material 


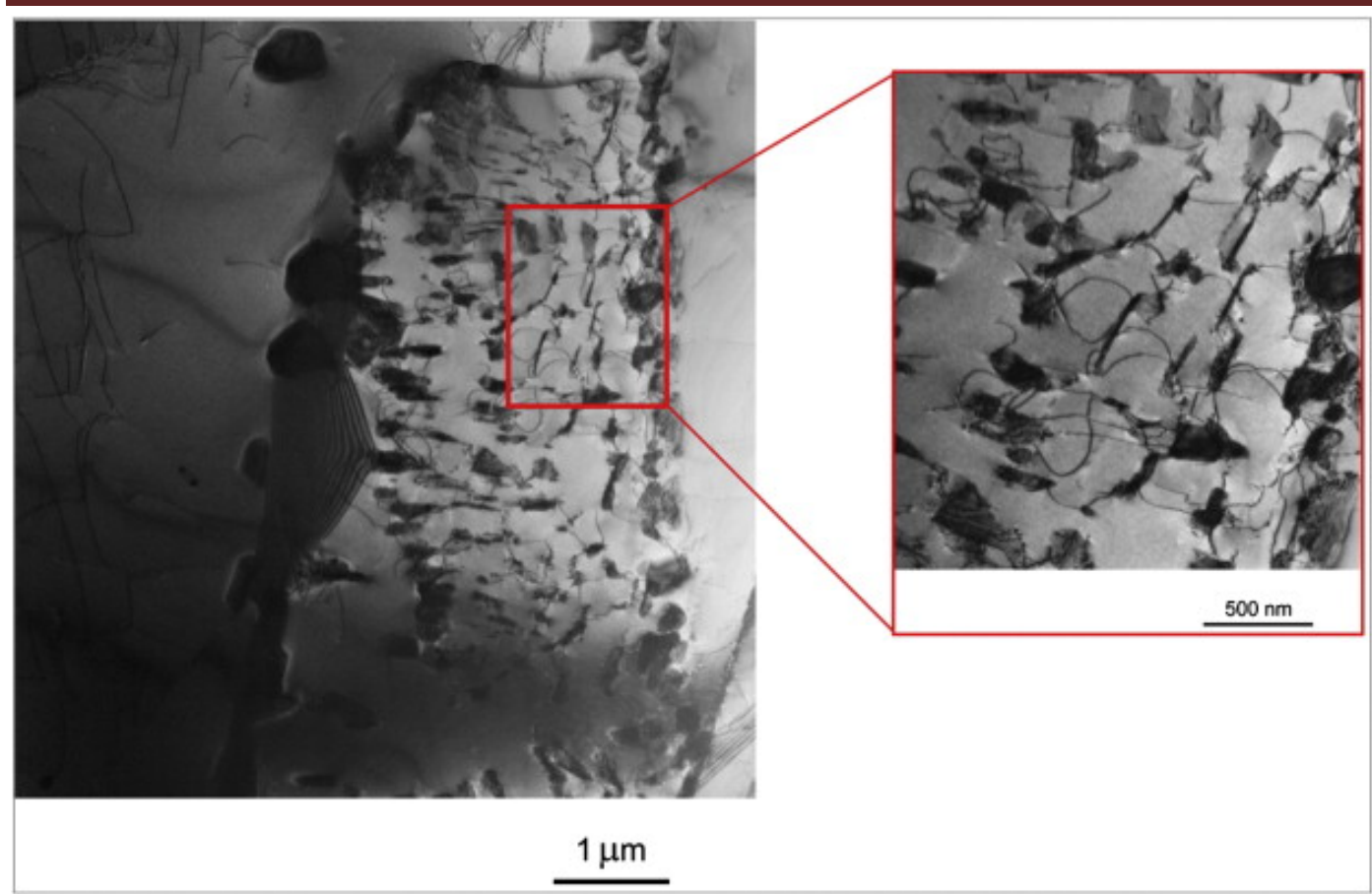

Fig. 4.137: TEM micrograph showing the heterogeneity in Haynes 230. A stacking fault separates areas of low and high carbide density. The cutout shows a dense area of carbide-dislocation interactions.

\subsection{Transpassive Behavior of Ni-based Alloys}

\subsubsection{Polarization curves}

Fig. 4.138 displays both anodic and cathodic polarization curves obtained in $0.1 \mathrm{M} \mathrm{H}_{2} \mathrm{SO}_{4}$ for the various alloy materials considered in this manuscript. Transpassive dissolution of the passive layer on $\mathrm{Cr}$ is sulfate anion-assisted [3.21, 3.22] so the electrode was rotated at $1000 \mathrm{rpm}$ in order to facilitate comparison to the literature. The potential sweep rate $(10 \mathrm{mV} / \mathrm{s})$ is fast for passivating metals, and may not yield a true steady state current density. However, use of this faster scan rate did not yield inconsistencies relative to the literature. 


\section{Understanding Fundamental Material-Degradation Processes in High Temperature Aggressive Chemomechanical Environments}
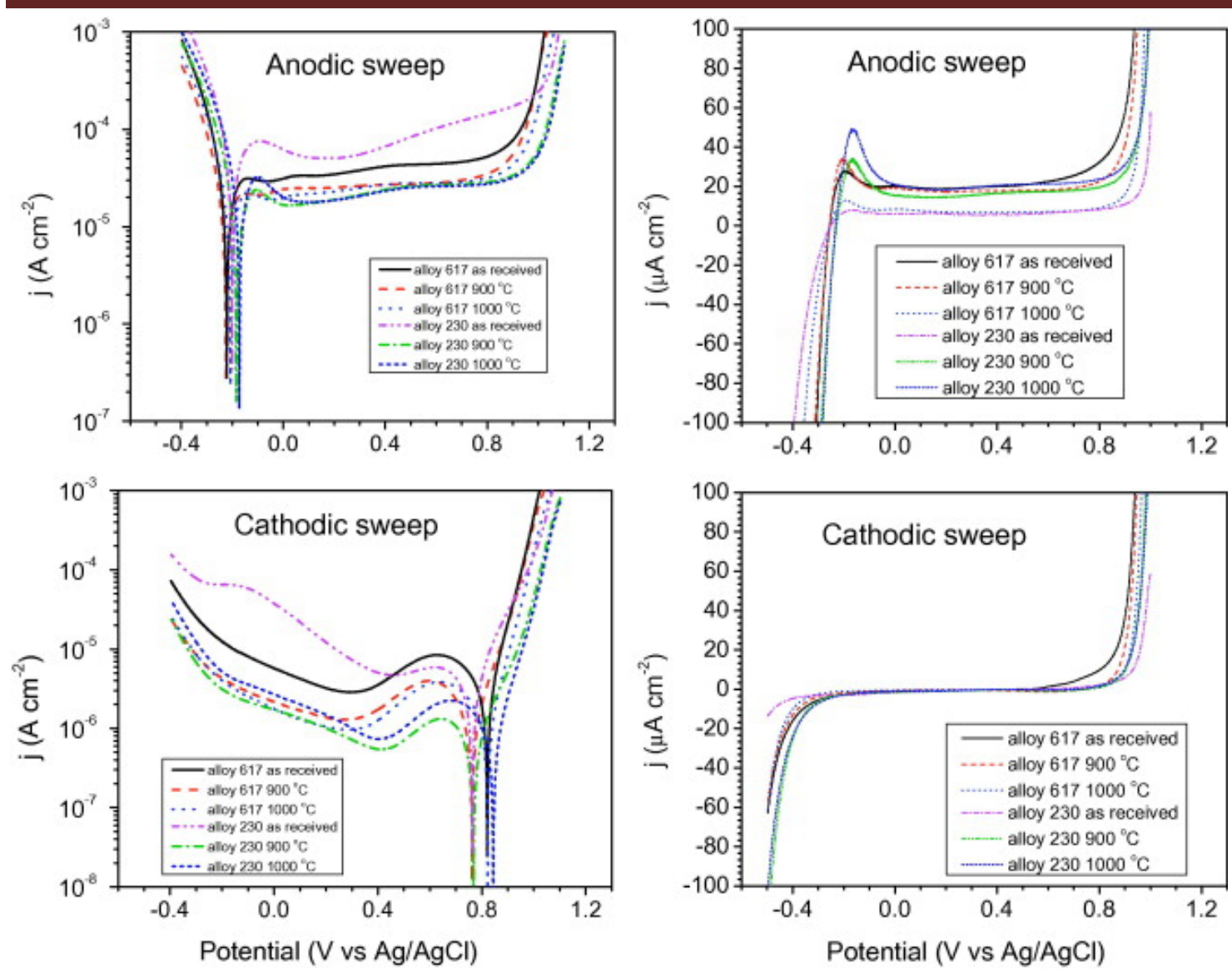

Fig. 4.138: Polarization curves of alloys in $0.1 \mathrm{M} \mathrm{H}_{2} \mathrm{SO}_{4}$ with $10 \mathrm{mV} / \mathrm{s}$ scan rate and $1000 \mathrm{rpm}$ rotation rate. Current is in logarithmic scale on the left and linear scale on the right.

The polarization curves for Alloy 617 with and without heat treatment are provided in Fig. 4.138. The anodic scan shows that the passive region begins at $-0.24 \pm 0.04 \mathrm{~V}$ for all samples, suggesting that both the as-received and heat treated materials will have similar pitting behavior as they share the same critical pitting potential [3.18]. The current density for the samples at 1.0 $\mathrm{V}$ are $1.4 \pm 0.4 \mathrm{~mA} / \mathrm{cm}^{2}$ for the as-received case, $0.70 \pm 0.3 \mathrm{~mA} / \mathrm{cm}^{2}$ for Alloy 617 heat-treated at $900^{\circ} \mathrm{C}$, and $0.74 \pm 0.3 \mathrm{~mA} / \mathrm{cm}^{2}$ for Alloy 617 heat-treated at $1000^{\circ} \mathrm{C}$. Heat treatment causes Alloy 617 to become more corrosion resistant, as is apparent by the reduction in anodic current density. Additionally, the as-received Alloy 617 exhibits an anodic current in the transpassive region roughly 1.6 times larger than that of the heat-treated samples, suggesting that more easily oxidizable material forms on the surface without the heat treatment.

The cathodic potential sweep, also shown in Fig. 4.138, displays a reduced current density for all three samples relative to the anodic sweep, likely originating from the formation of a thicker Cr layer on the anodic sweep. Fig. 4.138 also displays an exponential rise in current at more cathodic potentials for all the samples, with the as-received Alloy 617 exhibiting a higher cathodic current than the heat-treated samples.

The polarization curves for various treatments of Alloy 230 are shown in Fig. 4.138. The onset of the passive region for the heat-treated samples is more positive than that of Alloy 617 at 


\section{Understanding Fundamental Material-Degradation Processes in High Temperature Aggressive Chemomechanical Environments}

$-0.19 \mathrm{~V} \pm 0.04 \mathrm{~V}$ vs. $\mathrm{Ag} / \mathrm{AgCl}$. The current densities for samples at $1.0 \mathrm{~V}$ are $0.28 \pm 0.05$ $\mathrm{mA} / \mathrm{cm}^{2}$ for the as-received Alloy $230,0.08 \pm 0.05 \mathrm{~mA} / \mathrm{cm}^{2}$ for Alloy 230 heat-treated at $900^{\circ} \mathrm{C}$, and $0.07 \pm 0.05 \mathrm{~mA} / \mathrm{cm}^{2}$ for the Alloy 230 heat-treated at $1000^{\circ} \mathrm{C}$. The heat treatment reduces the current density at $1.0 \mathrm{~V}$ vs. the untreated case. The as-received sample has a higher anodic current density than that of the heat treated samples, again suggesting the presence of more easily oxidizable material on the surface prior to heat treatment. The cathodic sweep is similar to the case for Alloy 617, with the as-received specimen having more cathodic current than the heat-treated samples, and all rising exponentially with decreasing potential.

Our observation of increased corrosion resistance with heat treatment has been confirmed in the literature. For example, Kewther et al. demonstrated that the Alloy 617 used in gas turbines for $37,000 \mathrm{~h}$ could become more corrosion-resistant with heat treatment for $1-2 \mathrm{~h}$ at $1174^{\circ} \mathrm{C}$ under inert gas - heat treatment made the Alloy 617 samples $100 \mathrm{mV}$ more noble in a solution containing $0.05 \mathrm{M} \mathrm{H}_{2} \mathrm{SO}_{4}+0.005 \mathrm{M} \mathrm{NaCl}$ [3.18]. Alternatively, heating in air causes carbide precipitates to form alongside grain boundaries and a passive oxide composed of $\mathrm{Cr}_{2} \mathrm{O}_{3}$ to form on top of the bulk alloy, with a thinner $\mathrm{Al}_{2} \mathrm{O}_{3}$ layer underneath. Carbide deposition arising from heating increases the rate of anodic dissolution of $\mathrm{Ni}-\mathrm{Cr}-\mathrm{Mo}$ alloys in boiling $10 \%$ $\mathrm{H}_{2} \mathrm{SO}_{4}$ [3.34]. Segregation of $\mathrm{Cr}$ in the carbide phase also causes embrittlement of the alloy [3.15, $3.18,3.34]$. According to the Pourbaix diagram, in aqueous solution, $\mathrm{Cr}$ (III) should be a dominant species in the passivation layer and the $\mathrm{Cr}(\mathrm{VI})$ species should form at transpassive potentials [4.97].

In regards to electrochemical passivation, in situ XPS measurements of $\mathrm{Ni}-\mathrm{Cr}$ alloys confirm that the passive layer in the double region is composed of $\mathrm{Cr}$ (III) and $\mathrm{Ni}$ (II), and is thin (1-2 nm). Meanwhile, the transpassive region (starting at $1.0 \mathrm{~V}$ vs. SHE) was composed of a mixture of $\mathrm{Cr}(\mathrm{III})$ and $\mathrm{Cr}(\mathrm{VI})$. Jabs et al. showed that $\mathrm{Ni}-\mathrm{Cr}$ alloys with $20 \mathrm{wt} \% \mathrm{Cr}$ develop a hydroxide layer, as evinced by a peak at $577 \mathrm{eV}$ in the XPS after stepping the potential above 0.4 vs. SHE [3.26]. The electrochemical behavior of Alloy 230 in 25-98\% $\mathrm{H}_{2} \mathrm{SO}_{4}$ has been studied with solution temperatures ranging from $25-75^{\circ} \mathrm{C}$ [3.19]. However, the effect of heat aging of the alloy on electrochemistry has not been examined. The delayed onset and reduced current density observed here show that heat treatment causes both alloys to become more resistant to oxidation. In regards to the Mo content, Betova et al. demonstrated that an increased Mo content is associated with an acceleration in the dissolution steps [4.98]. This correlates well with the improved corrosion resistance of Alloy 230 over Alloy 617, since Alloy 230 has a lower Mo content. Overall, our results show that Alloy 230 is more corrosion-resistant than Alloy 617, a result also found in literature.

\subsubsection{Raman spectroscopy}

\section{Spectra of Alloy 617}

In order to determine the species present on both the as-received and heat-treated alloy surfaces, we performed in situ SHINERS on Alloy 617 and Alloy 230 in the anodic and cathodic directions. Fig. 4.139 shows the potential dependent SHINERS obtained from the as-received and heat-treated Alloy 617 specimens in aqueous $0.1 \mathrm{M} \mathrm{H}_{2} \mathrm{SO}_{4}$. Table 4.17 provides a listing of peaks and their assignments. 


\section{Understanding Fundamental Material-Degradation Processes in High Temperature Aggressive Chemomechanical Environments}

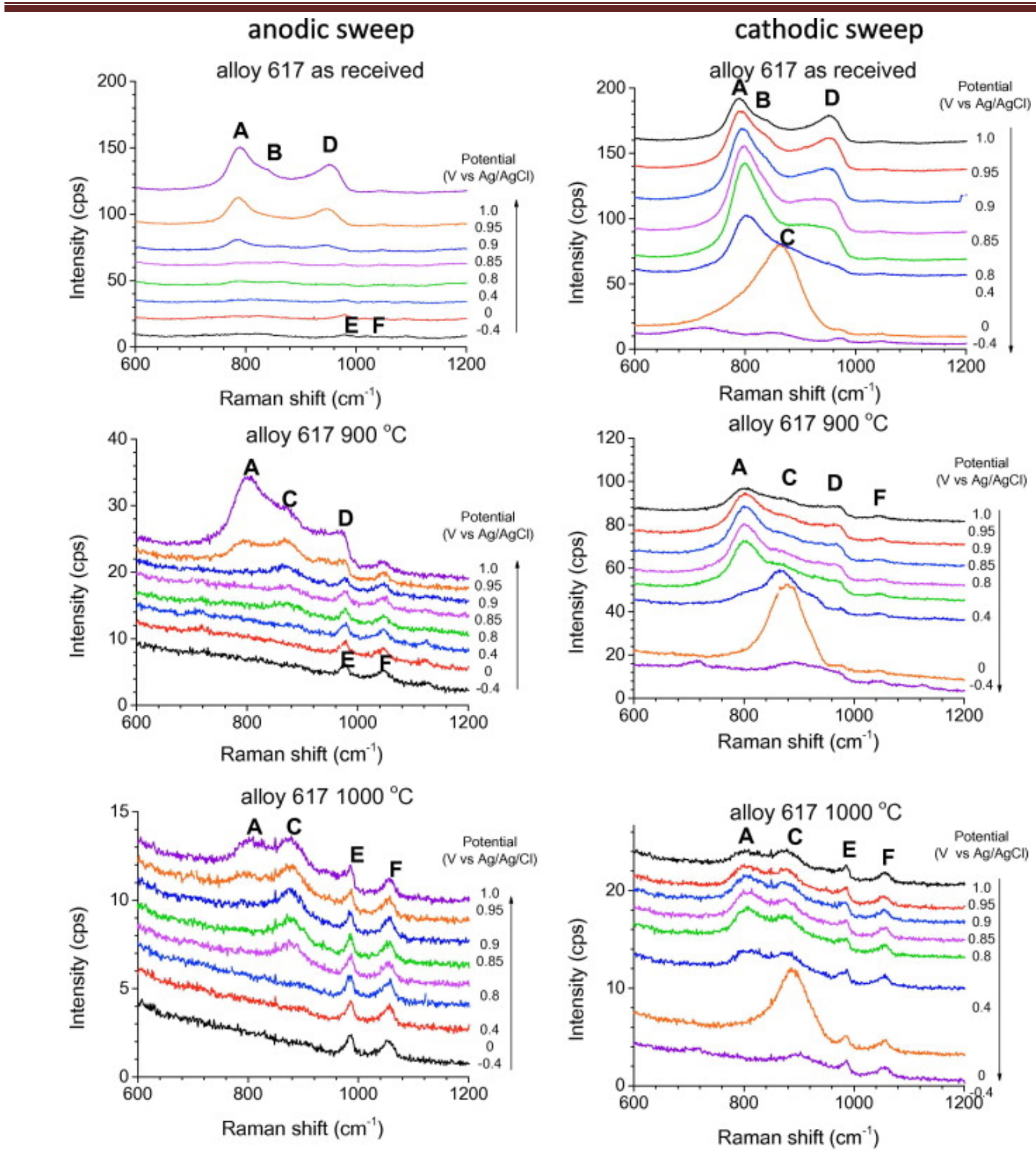

Fig. 4.139: Potential dependent spectra of Ni alloys in $0.1 \mathrm{M} \mathrm{H}_{2} \mathrm{SO}_{4}$. Spectra offset for clarity. 


\section{Understanding Fundamental Material-Degradation Processes in High Temperature}

Aggressive Chemomechanical Environments

Table 4.17: Peaks and assignments for spectra

\begin{tabular}{|l|l|l|l|}
\hline & Peak center $\left(\mathrm{cm}^{-1}\right)$ & \multicolumn{1}{|c|}{ Assignment } & \multicolumn{1}{|c|}{ References } \\
\hline A & 800 & Absorbed $\mathrm{CrO}_{4}{ }^{2-}$ & {$[4.99,4.100]$} \\
\hline B & $810-840$ & $\mathrm{CrO}_{4}{ }^{2-}$ & {$[4.99,4.100]$} \\
\hline C & $850-920$ & Mixed $\mathrm{Cr}(\mathrm{III} / \mathrm{VI})$ oxide, $\mathrm{MoO}_{4}{ }^{2-}$, and $\mathrm{WO}_{4}{ }^{2-}$ & {$[4.101,4.102]$} \\
\hline D & $960-980$ & $\mathrm{Cr}_{2} \mathrm{O}_{7}^{2-}$ & {$[4.99,4.100]$} \\
\hline E & 980 & $\mathrm{SO}_{4}{ }^{2-}$ & {$[4.103]$} \\
\hline F & 1020 & $\mathrm{HSO}_{4}^{-}$ & {$[4.103]$} \\
\hline
\end{tabular}

For the as-received Alloy 617, as the potential is swept from -0.4 to $0.9 \mathrm{~V}$, only peaks from the electrolyte are observed. Specifically, in Fig. 4.139, a peak at $977 \mathrm{~cm}^{-1}$ (peak E) assigned as the symmetric $\mathrm{SO}_{4}{ }^{2-}$ stretch is observed [4.103]. At $0.95 \mathrm{~V}$, a group of peaks with large intensities appear at $783 \mathrm{~cm}^{-1}$ (peak A) and $943 \mathrm{~cm}^{-1}$ (peak D), which are assigned as originating from the symmetric $\mathrm{Cr}-\mathrm{O}$ stretch of adsorbed $\mathrm{CrO}_{4}{ }^{2-}$ and $\mathrm{Cr}_{2} \mathrm{O}_{7}{ }^{2-}$, respectively [4.99] and [4.100]. At $1.0 \mathrm{~V}$, peaks $\mathrm{A}$ and $\mathrm{D}$ increase in intensity and peak A develops a shoulder at $833 \mathrm{~cm}^{-1}$ (peak B, assigned as free $\mathrm{CrO}_{4}{ }^{2-}$ ). On the cathodic sweep, peaks $\mathrm{A}$ and $\mathrm{D}$ continue to increase in intensity until $0.4 \mathrm{~V}$, after which the peaks decrease in intensity. At $0 \mathrm{~V}$, a peak centered at $862 \mathrm{~cm}^{-1}$ (peak C) appears and is assigned a mixed oxide Cr(III/VI) [4.101] and [4.102]. The region in which peak $\mathrm{C}$ occurs is also the region in which the Mo-O symmetric stretch of $\mathrm{MoO}_{4}{ }^{2-}$ would be expected to appear. Indeed, this feature was seen when $\mathrm{MoO}_{4}{ }^{2-}$ was adsorbed onto a $\mathrm{Ag}$ colloid $\left(876 \mathrm{~cm}^{-1}\right)$ [4.99]. As the potential is swept from 0 to $-0.4 \mathrm{~V}$, the intensity of peak $\mathrm{C}$ decreases. Interestingly, there is no feature observed at $898 \mathrm{~cm}^{-1}$ that could be associated with $\mathrm{HCrO}_{4}{ }^{-}[4.101,4.102]$.

For the anodic sweep of the $900^{\circ} \mathrm{C}$ treated Alloy 617 , between potentials of -0.4 to $0.8 \mathrm{~V}$, only peak $\mathrm{E}$, associated with the electrolyte $\mathrm{SO}_{4}{ }^{2-}$, and the broad peak centered at $1020 \mathrm{~cm}^{-1}$ (peak F) assigned to $\mathrm{HSO}_{4}{ }^{-}$, are observed [4.103]. At $0.8 \mathrm{~V}$ a peak at $867 \mathrm{~cm}^{-1}$ (peak C) appears. At $0.9 \mathrm{~V}$ an intense peak at $793 \mathrm{~cm}^{-1}$ (peak A) appears with a shoulder at $970 \mathrm{~cm}^{-1}$ (peak D). All peaks increase in intensity after reaching the switching potential of $1.0 \mathrm{~V}$. On the cathodic sweep, peak $A$ reaches a maximum intensity at about $0.9 \mathrm{~V}$, while the peaks $\mathrm{C}$ and $\mathrm{D}$ reach maximum intensities around $0 \mathrm{~V}$ vs. $\mathrm{Ag} / \mathrm{AgCl}$. Similar to the other two cases, in spectra obtained from the $1000^{\circ} \mathrm{C}$ treated Alloy 617, only electrolyte peaks (E and F) are observed from -0.4 to $0.8 \mathrm{~V}$. At $0.8 \mathrm{~V}$, a peak at $875 \mathrm{~cm}^{-1}$ (peak C), and at $0.95 \mathrm{~V}$, a peak at $800 \mathrm{~cm}^{-1}$ (peak A), appear, while peak D has increased in intensity. At $1.0 \mathrm{~V}$, peak A and peak $\mathrm{C}$ have increased in intensity, while a peak at $960 \mathrm{~cm}^{-1}$ (peak D) has appeared. On the cathodic sweep, peak A reaches its maximum intensity at $0.8 \mathrm{~V}$ while peaks $\mathrm{C}$ and $\mathrm{D}$ reach a maximum between 0.2 and $-0.2 \mathrm{~V}$ before decreasing in intensity.

Fig. 4.140 shows the integrated peak intensity for the Cr species on the Alloy 617 samples relative to their maximum peak intensity. This allows for an easier comparison between the three samples of changes in $\mathrm{Cr}$ speciation with potential. The onset for peak A (associated with adsorbed $\mathrm{CrO}_{4}{ }^{2-}$ ) on the anodic sweep is similar for the three samples. On the cathodic sweep, the maximum intensity for peak $\mathrm{A}$ is reached $0.2 \mathrm{~V}$ earlier for the heat-treated Alloy 617 samples 


\section{Understanding Fundamental Material-Degradation Processes in High Temperature}

Aggressive Chemomechanical Environments

relative to the as-received sample. Conversely, the maximum intensity for peaks C and D are delayed for $0.2 \mathrm{~V}$ for the heat-treated Alloy 617 samples relative to the as-received Alloy 617. Thus, heat treatment controls both the nature of the $\mathrm{Cr}$ overlayer and its potential dependent development. 

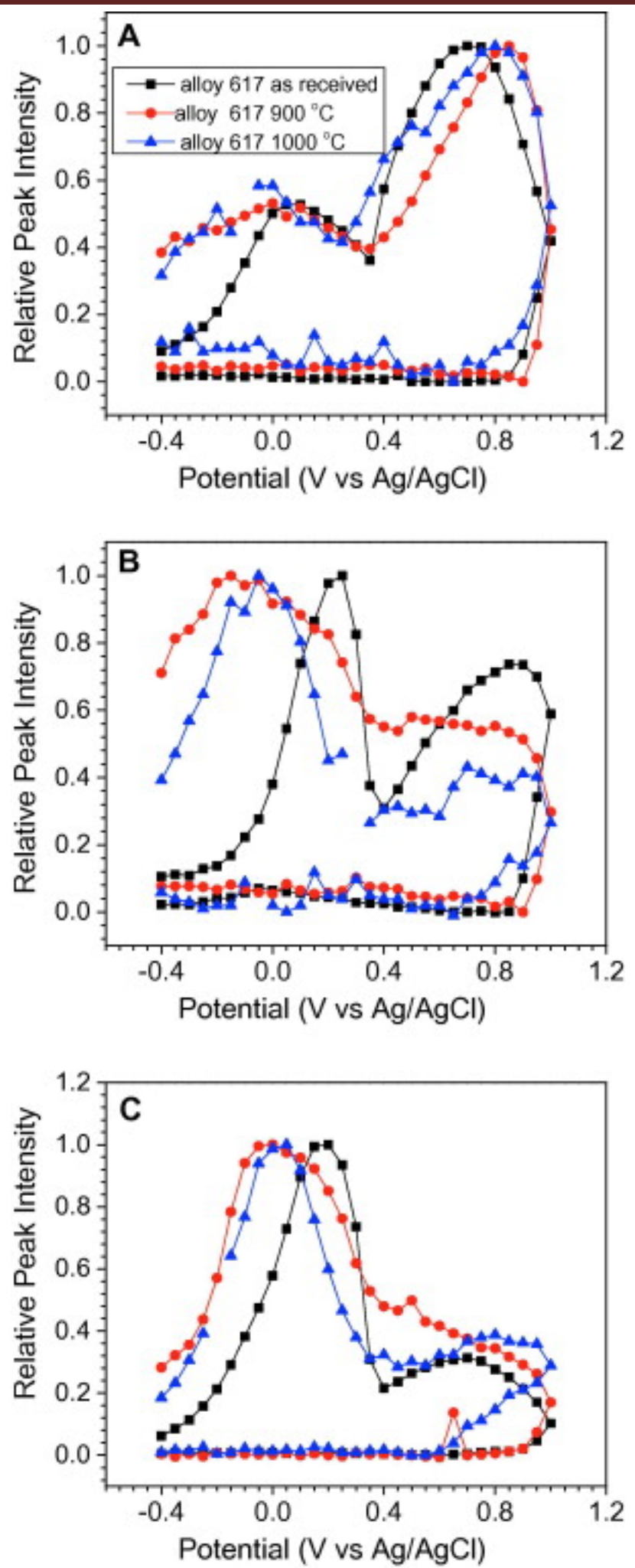

Fig. 4.140: Relative peak intensity vs. potential plot for (a) $\mathrm{CrO}_{4}{ }^{2-}$; (b) mixed oxide; (c) $\mathrm{Cr}_{2} \mathrm{O}_{7}{ }^{2-}$ 


\section{Understanding Fundamental Material-Degradation Processes in High Temperature Aggressive Chemomechanical Environments}

\section{Spectra of Alloy 230}

Fig. 4.141 and Fig. 4.142 present spectra and intensity vs. potential curves, respectively, for the three $\mathrm{Cr}$ species observed from the heat-treated and as-received Alloy 230 samples.

\section{anodic sweep}
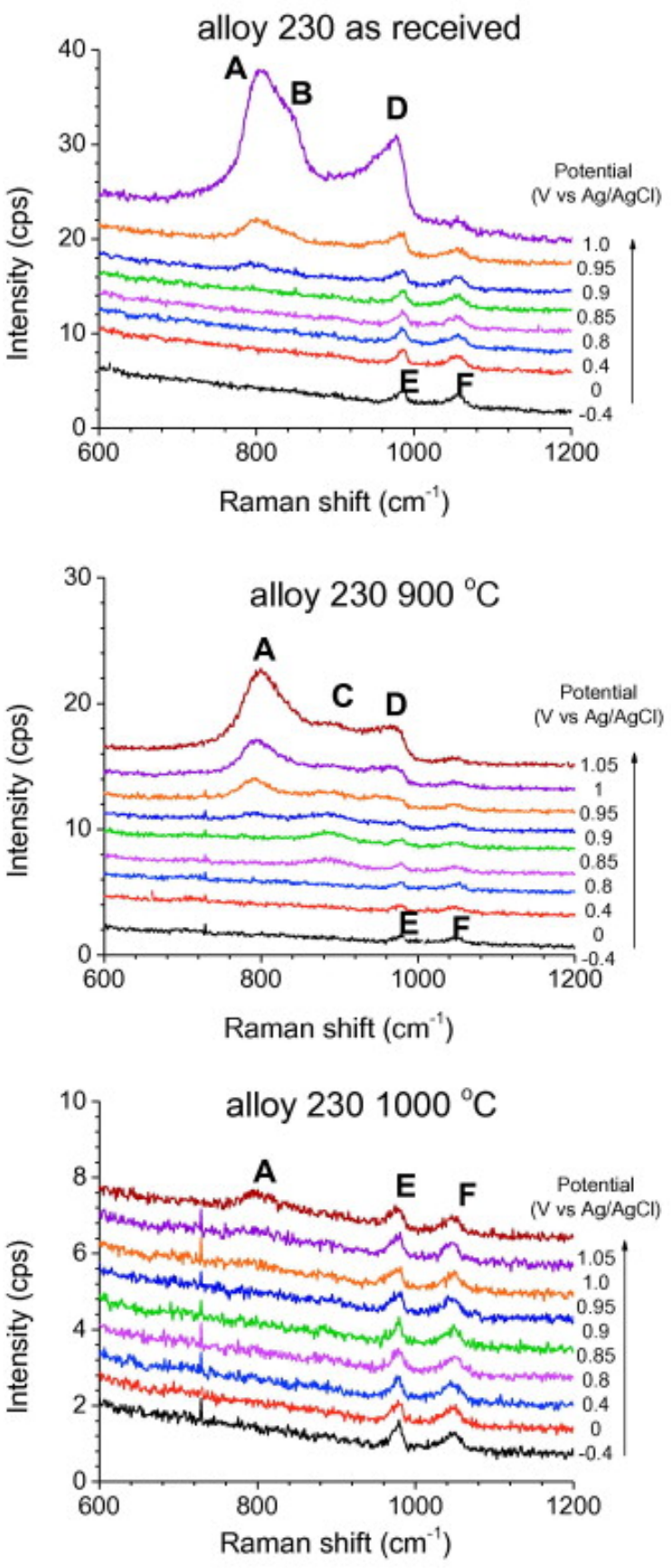

cathodic sweep
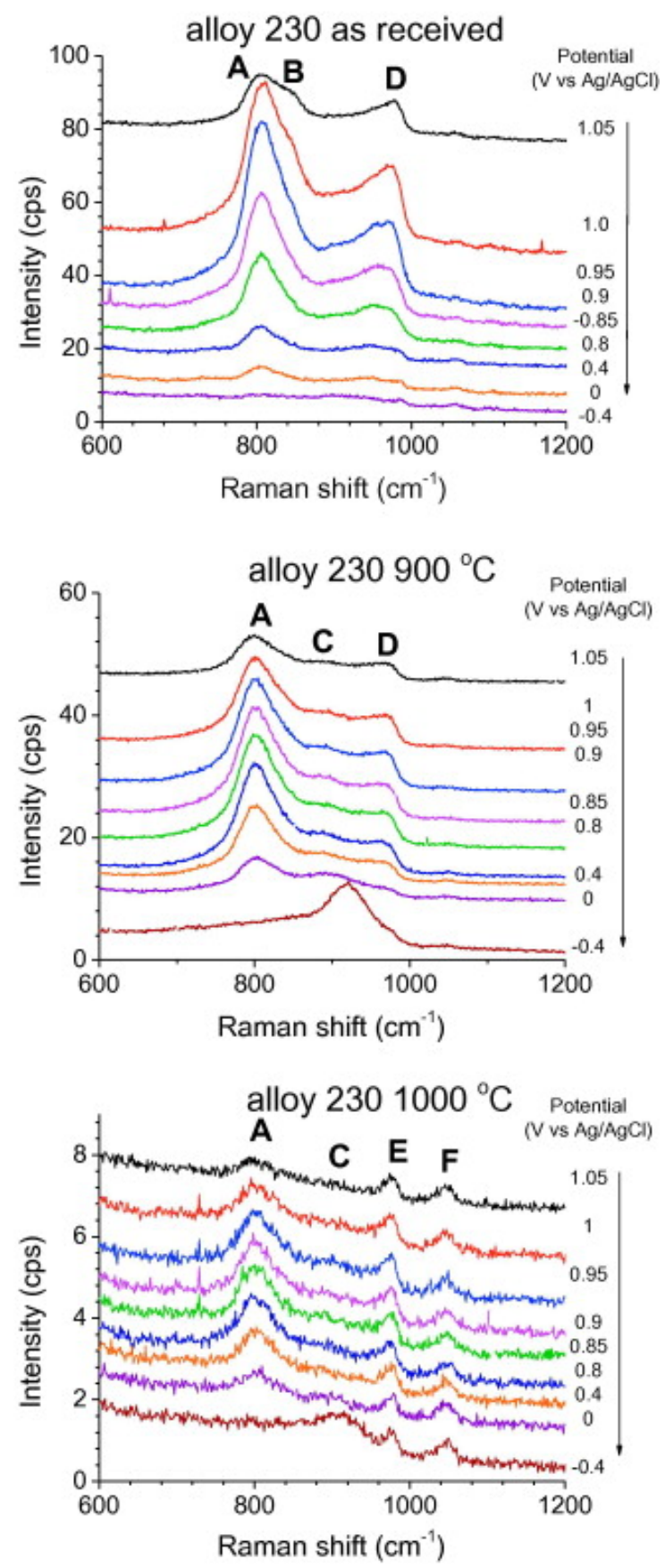

Fig. 4.141: Potential dependent spectra of Alloy 230 in $0.1 \mathrm{M} \mathrm{H}_{2} \mathrm{SO}_{4}$. Spectra offset for clarity. 

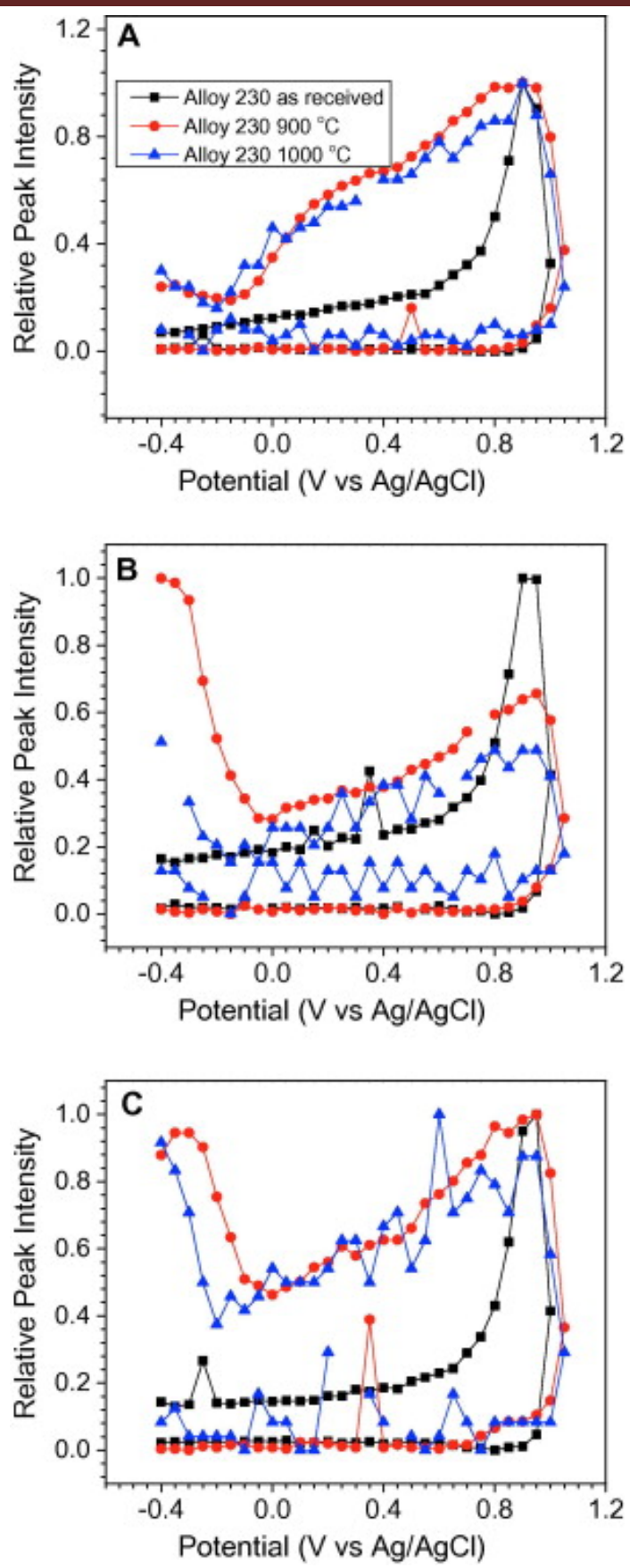

Fig. 4.142: Relative peak intensity vs. potential plot for (a) $\mathrm{CrO}_{4}{ }^{2-}$; (b) mixed oxide; (c) $\mathrm{Cr}_{2} \mathrm{O}_{7}{ }^{2-}$

For the as-received Alloy 230 sample, as the potential is swept from -0.4 to $0.9 \mathrm{~V}$, only electrolyte peaks (E and F) are observed. From 0.85 to $0.9 \mathrm{~V}$, a peak centered at $800 \mathrm{~cm}^{-1}$ (peak A) appears and grows in intensity. At $1.0 \mathrm{~V}$, peak A develops a shoulder at $847 \mathrm{~cm}^{-1}$ (peak B) and a peak at $978 \mathrm{~cm}^{-1}$ appears (peak D). On the cathodic sweep, both A and D increase in intensity as the peak is swept $0.9 \mathrm{~V}$, exhibit a rapid decrease in intensity by $0.8 \mathrm{~V}$, and continue to experience a gradual decline in intensity until the end of the scan at $-0.4 \mathrm{~V}$. 


\section{Understanding Fundamental Material-Degradation Processes in High Temperature Aggressive Chemomechanical Environments}

Similar to the as-received case, only electrolyte peaks (E and F) are observed on the $900^{\circ} \mathrm{C}$ treated Alloy 230 sample as the potential is swept from -0.4 to $0.4 \mathrm{~V}$. At $0.8 \mathrm{~V}$, a broad peak centered at $882 \mathrm{~cm}^{-1}$ (peak C) appears. At $0.95 \mathrm{~V}$, two other peaks appear at $792 \mathrm{~cm}^{-1}$ (peak A) and $975 \mathrm{~cm}^{-1}$ (peak D). All three peaks increase in intensity until a potential of $1.05 \mathrm{~V}$ is reached. On the cathodic sweep, peak A increases in intensity until $0.8 \mathrm{~V}$, then decreases in intensity as the potential is swept to more negative values. Peaks $C$ and $D$ remain at the same intensity until $0 \mathrm{~V}$, and then increase in intensity until $-0.4 \mathrm{~V}$ the end of the cathodic sweep. This spectral region is also where adsorbed $\mathrm{MoO}_{4}{ }^{2-}$ and $\mathrm{WO}_{4}{ }^{2-}\left(876\right.$ and $912 \mathrm{~cm}^{-1}$, respectively) would occur [4.99].

For the $1000^{\circ} \mathrm{C}$ treated Alloy 230 , as the potential is swept from -0.4 to $0.95 \mathrm{~V}$, only the electrolyte peaks (E and F) are observed. At $1.0 \mathrm{~V}$, a peak centered at $795 \mathrm{~cm}^{-1}$ (peak A) appears and grows in intensity at $1.05 \mathrm{~V}$. On the cathodic sweep, peak A increases in intensity, reaches a maximum at $0.8 \mathrm{~V}$, and then slowly decreases in intensity as the potential is swept to more negative values, finally reaching $0 \mathrm{~V}$. After $0 \mathrm{~V}$, a peak at $911 \mathrm{~cm}^{-1}$ (peak C) appears and grows in intensity for the remaining duration of the cathodic sweep. In this region, adsorbed $\mathrm{MoO}_{4}{ }^{2-}$ and $\mathrm{WO}_{4}{ }^{2-}$ (876 and $912 \mathrm{~cm}^{-1}$, respectively) occur [4.99].

As with Alloy 617, the spectra for Alloy 230 samples show no evidence of the presence of $\mathrm{HCrO}_{4}{ }^{-}$. Also, heat-treated samples show a delayed onset of the $\mathrm{CrO}_{4}{ }^{-}$peak and the development of a mixed oxide peak during transpassive dissolution.

The observation that peaks assigned to $\mathrm{Cr}(\mathrm{VI})$ species appear at anodic potentials suggests that $\mathrm{Cr}(\mathrm{VI})$ is the major product formed in the transpassive region, a result which is consistent with the literature [3.20, 3.21, 3.26]. There is no clear evidence for the formation of $\mathrm{Cr}_{2} \mathrm{O}_{3}$, the major product, when either Alloy 230 or Alloy 617 is heated in air [3.15]. However, the mixed $\mathrm{Cr}(\mathrm{III}) / \mathrm{Cr}(\mathrm{VI})$ species is observed on the cathodic sweep. We note that the $\mathrm{Cr}(\mathrm{III})$ species has a smaller Raman scattering cross-section relative to the $\mathrm{Cr}(\mathrm{VI})$ peaks for spectra produced on roughened or colloidal samples of $\mathrm{Ag}, \mathrm{Au}$, and $\mathrm{Cu}$ [4.99], [4.100], and [4.104]. SERS spectra of alloy C22 (a Ni-Cr-Mo alloy) in $\mathrm{HCl}$ displays broad peaks at 430 and $490 \mathrm{~cm}^{-1}$, which can be attributed to an amorphous $\mathrm{Cr}(\mathrm{III})$ species [3.33]. However, we were not able to assign features in this spectral region in our measurements.

The other interesting aspect of the spectra reported above concerns the delayed onset potential related to the formation of peaks associated with $\mathrm{Cr}_{2} \mathrm{O}_{7}{ }^{2-}$ and mixed oxide, and the accelerated potential of the appearance of peak $\mathrm{A}$, associated with $\mathrm{CrO}_{4}{ }^{2-}$ in the heat-treated samples. We suggest that the heat treatment produces more fully-oxidized $\mathrm{Cr}, \mathrm{W}$, and Mo material relative to $\mathrm{Cr}_{2} \mathrm{O}_{7}{ }^{2-}$, the consequences of which are seen in the spectra. Interestingly, the Pourbaix diagram for $\mathrm{Cr}$ shows that $\mathrm{HCrO}_{4}^{-}$, rather than chromate, should be stable in the potential region interrogated (at $\mathrm{pH} 1$ ), so the presence of $\mathrm{CrO}_{4}{ }^{2-}$ suggests a less acidic environment overall. An alternate explanation for the increase in intensity of the mixed oxide peak after the decrease in intensity for the $\mathrm{CrO}_{4}{ }^{2-}$ peak is that the soluble $\mathrm{Cr}$ species diffuse away, leaving behind the less soluble Mo and $\mathrm{W}$ species. Correspondingly, we note that heat treatment leads to an increase in corrosion resistance.

Overall, heat treatment of both Alloy 617 and Alloy 230 increases the relative intensity of the mixed oxide peak, seen in the transpassive region and persisting on the cathodic scan. Alloy 617 exhibits a greater relative peak intensity of the mixed alloy phase to the $\mathrm{CrO}_{4}{ }^{-}$peak on the 


\section{Understanding Fundamental Material-Degradation Processes in High Temperature Aggressive Chemomechanical Environments}

anodic sweep. The $\mathrm{Cr}_{2} \mathrm{O}_{7}{ }^{2-}$ peak reaches a maximum intensity at $0 \mathrm{~V}$ in Alloy 617 and $-0.4 \mathrm{~V}$ in the heat-treated Alloy 230 samples. The as-received Alloy 230 does not develop a mixed oxide phase that increases in intensity at the cathodic potential.

X-ray photoelectron spectroscopy of Alloy 617 and Alloy 230
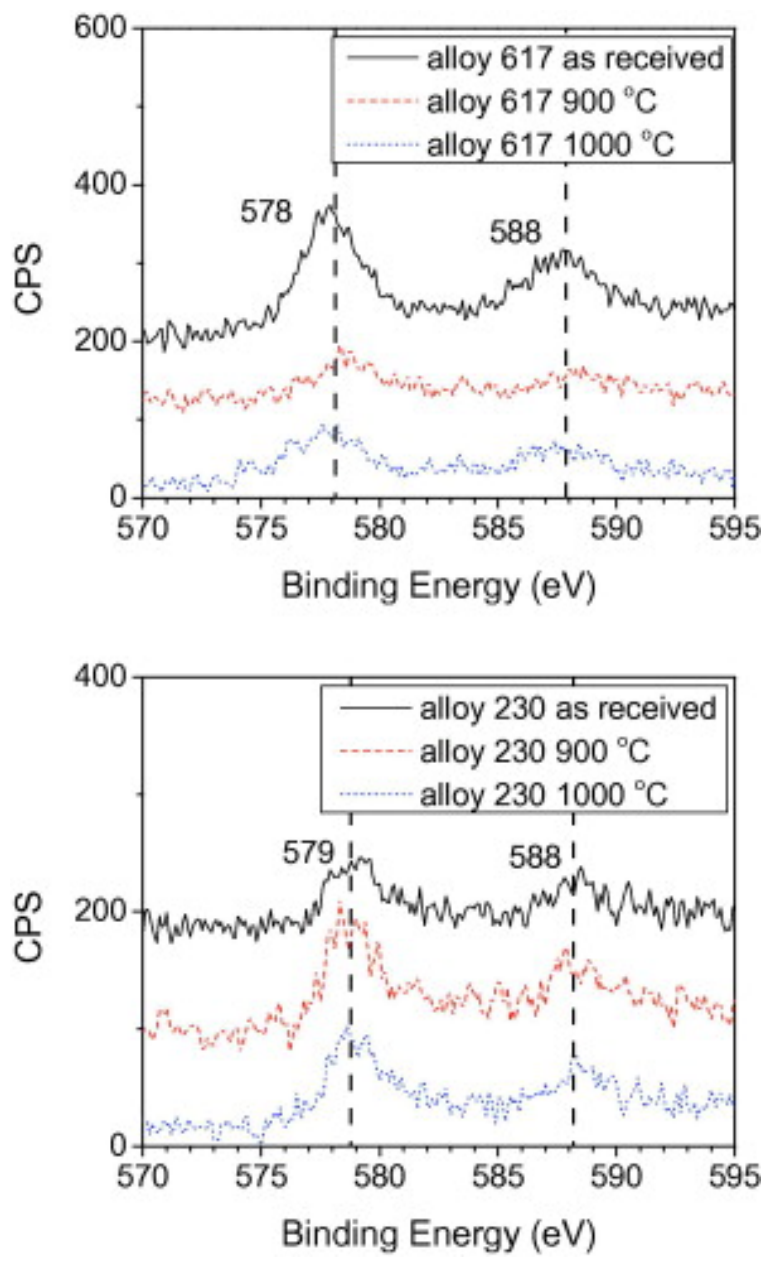

Fig. 4.143: Ex situ XPS of Ni alloys after potential cycling. Spectra are offset for clarity.

Table 4.18 displays a summary of the relative amounts of the most abundant elements in the alloys (Ni, Cr, Mo, Co, and $\mathrm{W}$ ) before and after oxidation, determined from low resolution survey scans, some of which are shown in Fig. 4.144. Samples were oxidized by applying a potential of $1.1 \mathrm{~V}$ vs. $\mathrm{Ag} / \mathrm{AgCl}$ to the Ni sample for 60 seconds. The as-received and heat-treated Alloy 617 samples exhibit the same behavior - the percentage of $\mathrm{Cr}$ increases, $\mathrm{Ni}$ and $\mathrm{Co}$ decrease, and Mo remains the same after electrochemical oxidation. For the Alloy 230 sample, the percentage of $\mathrm{Cr}$ and $\mathrm{W}$ increases, while $\mathrm{Ni}$ and Mo decrease. The as-received Alloy 230 has a higher fraction of $\mathrm{W}$ compared to the heat-treated samples. Heat treatment has a minimal effect 


\section{Understanding Fundamental Material-Degradation Processes in High Temperature Aggressive Chemomechanical Environments}

on the oxidation state and relative amount of $\mathrm{Cr}$ found ex situ for the Ni samples before and after corrosion in $0.1 \mathrm{M} \mathrm{H}_{2} \mathrm{SO}_{4}$.

Table 4.18: Relative atomic \% of five major elements: Ni, Cr, Mo, W, Co before and after oxidizing at $1.1 \mathrm{~V}$ for 60 seconds.

\begin{tabular}{|c|c|c|c|c|c|c|}
\hline & \multicolumn{2}{|c|}{ Alloy 617 as recieved } & \multicolumn{2}{|c|}{ Alloy $617900^{\circ} \mathrm{C}$} & \multicolumn{2}{|c|}{ Alloy $6171000^{\circ} \mathrm{C}$} \\
\hline & Before & After & Before & After & Before & After \\
\hline $\mathrm{Ni}$ & 40 & 11 & 38 & 12 & 48 & 13 \\
\hline $\mathrm{Cr}$ & 26 & 77 & 30 & 76 & 12 & 75 \\
\hline Mo & 13 & 13 & 12 & 11 & 16 & 12 \\
\hline \multirow[t]{3}{*}{ Co } & 20 & 0.1 & 20 & 1 & 23 & -- \\
\hline & \multicolumn{2}{|c|}{ Alloy 230 as received } & \multicolumn{2}{|c|}{ Alloy $230900^{\circ} \mathrm{C}$} & \multicolumn{2}{|c|}{ Alloy $2301000^{\circ} \mathrm{C}$} \\
\hline & Before & After & Before & After & Before & After \\
\hline $\mathrm{Ni}$ & 36 & -- & 45 & 14 & 58 & 14 \\
\hline $\mathrm{Cr}$ & 9 & 29 & 51 & 75 & 35 & 46 \\
\hline Mo & 53 & 4 & 5 & 4 & 3 & -- \\
\hline $\mathrm{W}$ & 2 & 67 & - & 7 & 4 & 40 \\
\hline
\end{tabular}


Understanding Fundamental Material-Degradation Processes in High Temperature Aggressive Chemomechanical Environments
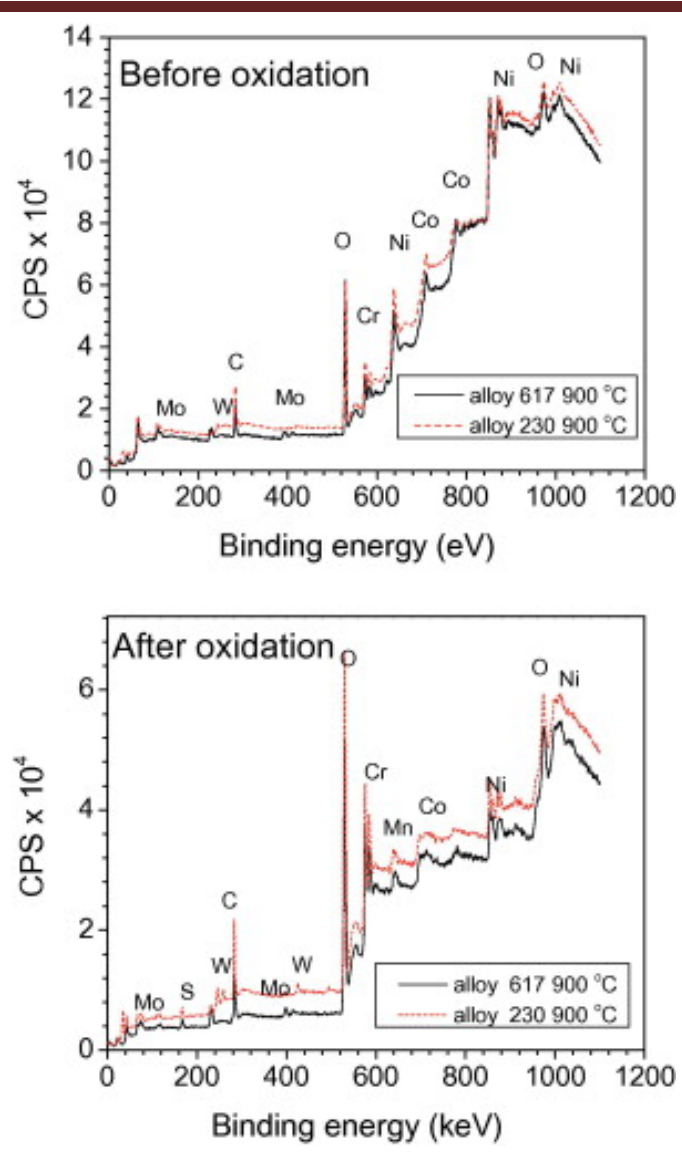

Fig. 4.144: Ex situ XPS survey spectra of heat-treated Ni alloys before and after potential cycling 


\subsection{Effect of Orientation of Alloy 617}

\section{DISCUSSION}

Differences in yield strength in various directions indicates an intrinsic Bauschinger effect in the Alloy 617 plate [5.1]. Previous studies of hot-rolled aluminum alloy plates revealed a similar phenomenon, with little observed change in strength in the rolling and long-transverse directions, but a considerable loss of strength in the short-transverse direction [5.1]. A comparison of the relative strengths of Alloy 617, Alloy MA754 (an oxide dispersion-strengthened nickel-based alloy), and two aluminum alloys is shown in Fig. 5.1. The data for Alloy MA754 is taken from a bar with a transverse section of $90 \times 32 \mathrm{~mm}$ [5.2]. The data for aluminum alloys is taken from plates with thicknesses of about 2 to 2.5 inches [5.1]. The loss of relative yield strength in the short-transverse direction in Alloy 617 was found to be over $15 \%$, which is larger than the values $(<10 \%)$ for MA754 and the aluminum alloys. Hence, the effect of orientation is an important issue in the plate form of Alloy 617, and must be taken into account during engineering design.
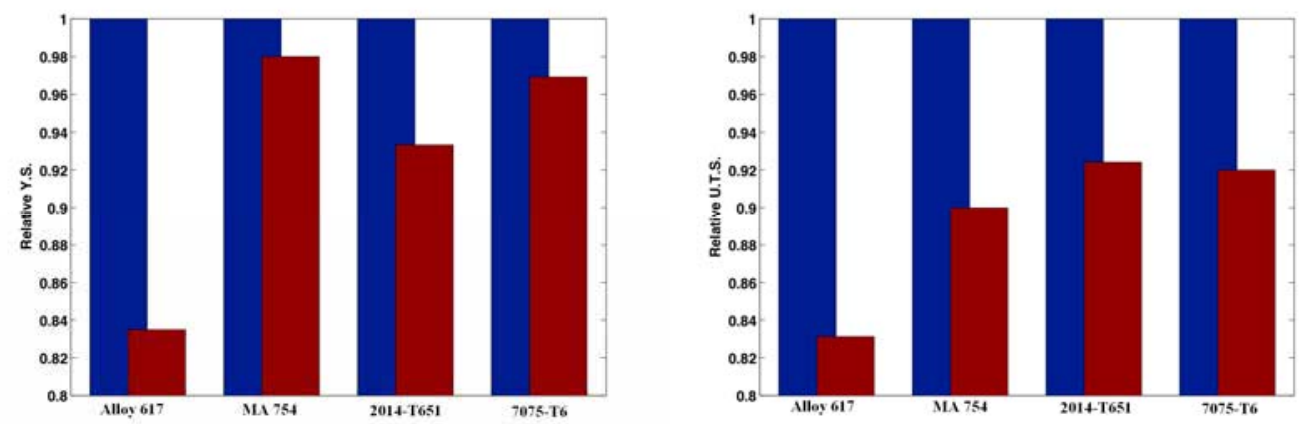

(a)

(b)

Fig. 5.1: Relative strengths of various alloys at room temperature: (a) Y.S.; (b) U.T.S

Two main sources of anisotropic material properties can typically be found in polycrystalline materials [5.3]. The first source is material texture produced by strong deformation (e.g. cold work) that develops a preferred crystal orientation, such as $(110)<112>$ rolling texture $(\alpha$ brass type) in the sheet form of face-centered cubic (FCC) metals [4.4]. The second source is mechanical fibering composed of an alignment of inclusion particles and matrix crystals. Compared to cold rolled products, hot rolled metals and alloys generally have more isotropic mechanical properties. Solid solution annealing after hot work also helps to minimize residual stresses. Therefore, no obvious texture is observed in the as-received Alloy 617 (Fig. 4.13). Instead, significant mechanical fibering is found in the plate form of Alloy 617, which results in the directionality of mechanical properties. During hot rolling, the inclusion particles were generated with asymmetric shapes and spatial distributions. The growth of recrystallized grains was subsequently restricted by inclusion particles, and thus the new grains presented a specific alignment. Additionally, the inclusion particles were not re-dissolved during solid solution annealing. As a result, the microstructure characterized by inclusion and crystal fibers, or mechanical fibering, was preserved after solid solution treatment. Therefore, this microstructure that is composed of carbide bands in the transverse direction can be found extensively in the plate form of Alloy 617 [4.64, 4.54, 4.15, 5.4, 5.5]. 


\section{Understanding Fundamental Material-Degradation Processes in High Temperature Aggressive Chemomechanical Environments}

Mechanical fibering in Alloy 617 also contributes to an anisotropic microstructure that is developed during tensile testing. The high volume fraction of carbide particles impedes the dislocation movement and consequently causes dislocation pileups and a high density of subgrain structures surrounding the particles. Therefore, as seen in strain contouring, the highly strained areas are concentrated on the interphase boundaries (Fig. 4.14(b) and Fig. 4.15(b)). In the short-transverse direction, this phenomenon is amplified due to the steep strain gradient. The region between two inclusion bands can be easily deformed, whereas the area close to inclusion is hardened by grain and interphase boundaries. Hence, the plastic deformation is localized. This can be seen in an aged specimen of Alloy 617, where intergranular carbide chains were disconnected between two carbide bands as a result of localized deformations (Fig. 4.17). However, the situation changes for elevated temperatures. Due to the thermal-enhanced mobility of dislocations and atoms, subgrain boundaries can transform into grain boundaries and develop new grains. The number, size, and distribution of recrystallized grains are dependent on the plastic strain. The highly strained area during plastic deformations, which is also the inclusion-rich area in Alloy 617, is subjected to increased dynamic recrystallization. This explains why the dynamic recrystallized grains are distributed in the same way as the strain contouring at low temperatures (Fig. 4.14(b) and Fig. 4.15(b)).

Another important result of mechanical fibering is the initiation of pre-mature failure by the ligaments of carbides bands in a tension loading. This was found to only occur at temperatures lower than $700^{\circ} \mathrm{C}$. At higher temperatures, fracture was initiated by triple junction cracks from grain boundary sliding. The early rupture of the short-transverse specimens can be observed by its undeveloped texture. During the plastic deformation of Alloy 617 at low temperatures, crystallographic planes tended to rotate to form $<111>$ or $<100>$ textures to minimize easy glide. The amount of crystals oriented parallel to the $\langle 111\rangle$ or $\langle 100\rangle$ direction (texture development) can be used to examine the level of plastic deformations. A fully developed texture in the long-transverse specimen of Alloy 617 indicates that the material experienced a strong deformation during tensile testing (Fig. 4.16(a)). In contrast, the texture was found to be undeveloped in the short-transverse specimen, seen as a relatively smooth intensity distribution in the pole figures (Fig. 4.16(b)).

Finally, the effect of orientation can also be magnified in the creep condition for Alloy 617. Totemeier and Lillo reported that specimens of MA754 have a comparable tensile strength in the transverse direction to the value in the longitudinal direction. However, a markedly lower transverse strength was found in the creep condition [5.2]. Thus, the effect of orientation for the structural materials is important for long-term, high-temperature environments, such as the VHTR, where creep damage will certainly occur. Significant effort should be placed into minimizing the effect of orientation and even eliminating the source of anisotropy in Alloy 617. High temperature annealing is a simple method to remove the inclusion particles. Kihara et al. reported that all of the inclusion particles in Alloy 617 were dissolved following aging at $1200^{\circ} \mathrm{C}$ for 20 hours [4.89]. However, the new grain size for the aged Alloy 617 was $0.36 \mathrm{~mm}$, more than twice the value of the as-received condition. Creep resistance increases as a result of increased grain size. However, the yield strength of the aged alloy likely decreases due to the loss of strengthening by the fine grains. The corresponding loss in yield strength may fail to meet the American Society for Testing and Materials (ASTM) standards [5.6]. Hence, it will be a challenging task to remove inclusion particles from Alloy 617 by high-temperature annealing while still maintaining its strong mechanical properties. 


\section{Understanding Fundamental Material-Degradation Processes in High Temperature Aggressive Chemomechanical Environments}

\subsection{Strain Rate Senstivity Analysis}

The measured strain-rate sensitivities are plotted in Fig. 5.2(a)\&(b) for Alloy 617 and Alloy 230, respectively. Note that the blue points are interpreted from constant flow stresses at high temperatures. For each alloy, the strain-rate sensitivity does not linearly increase with temperature. The value of $m$ is small at low temperatures (RT to $700^{\circ} \mathrm{C}$ ), indicating a strain rate that is independent of flow stress for both alloys. The only exception is a higher value of $m$ that occurs at room temperature for Alloy 230. This temperature independence of the strain-rate sensitivity is attributed to dynamic strain aging. As discussed in section 3.1, dynamic strain aging can cause abnormal work-hardening that can be reflected in irregularities in strain-stress diagrams (the Portevin-LeChatelier effect). Moreover, dynamic strain aging can cause flow stresses to be independent of both strain rate and temperature [4.3]. For the temperature intervals in which dynamic strain aging occurs, there is a maximum degree of work hardening that corresponds to a specific strain rate at one temperature. A specimen deformed at the intermediate strain rate can experience a higher degree of work hardening compared to specimens deformed either at a lower or higher strain rate. This can be seen in the results of tensile testing in Tables 4.2 and 4.3 (or the strain-stress diagrams in APPENDIX A). For some studied temperatures (e.g. $300^{\circ} \mathrm{C}$ for Alloy 230), the flow stress at a strain rate of $0.005 \mathrm{~s}^{-1}$ was found to be even higher than the stresses at a strain rate of $0.001 \mathrm{~s}^{-1}$ and $0.01 \mathrm{~s}^{-1}$. Due to this irregular strain rate independence, the strain-rate sensitivities are measured to be close to zero at temperatures below $800^{\circ} \mathrm{C}$ for both alloys.

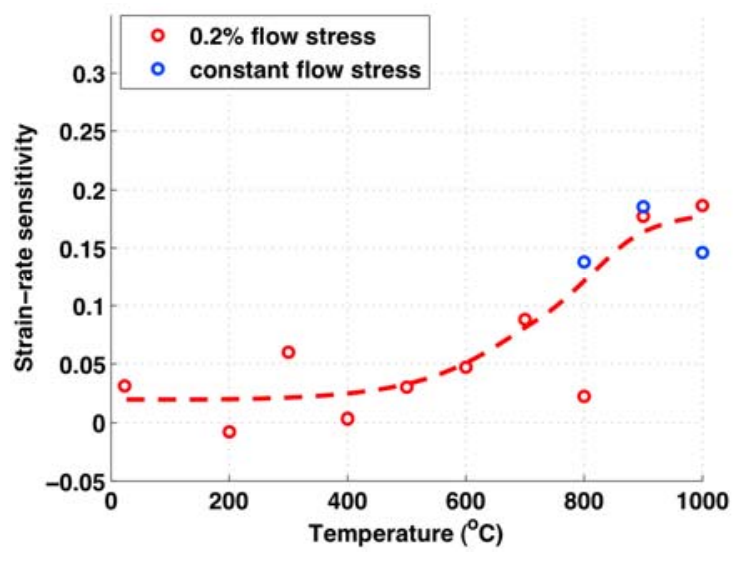

(a)

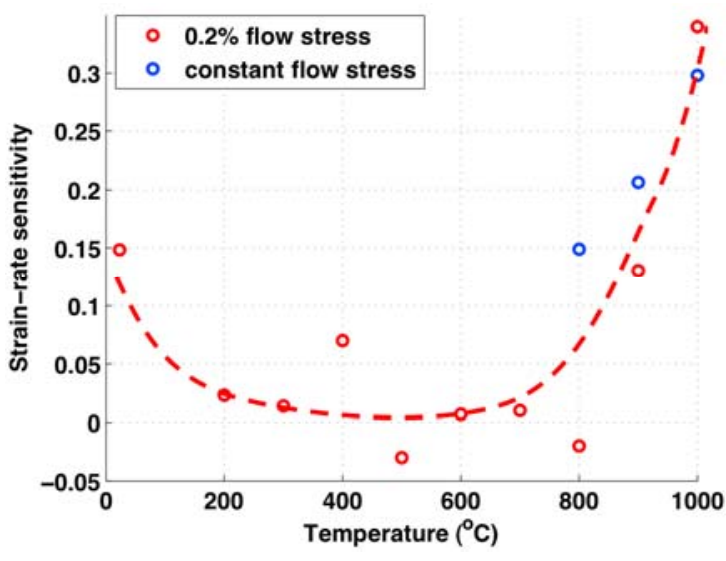

(b)

Fig. 5.2: Strain-rate sensitivity vs. temperature up to $1000^{\circ} \mathrm{C}$ for (a) Alloy 617 and (b) Alloy 230. The blue points are interpreted from constant flow stresses.

The strain-rate sensitivity for $0.2 \%$ flow stress was found to decrease at $800^{\circ} \mathrm{C}$, but increase considerably at 900 and $1000^{\circ} \mathrm{C} .800^{\circ} \mathrm{C}$ is treated as a critical temperature for both Alloy 617 and Alloy 230, not only because it is the equicohesive temperature, but also because carbide precipitation occurs at this temperature (Fig. 4.4(b)). The precipitate hardening can be seen by comparing the strain-stress diagrams at 700 and $800^{\circ} \mathrm{C}$ - the curves in the inelastic regions have a stiffer slope at $800^{\circ} \mathrm{C}$ than at $700^{\circ} \mathrm{C}$ (Fig. 5.3). The flow stress during early yielding is significantly impacted by precipitate hardening. Thus, the influence of the applied strain rate is 


\section{Understanding Fundamental Material-Degradation Processes in High Temperature Aggressive Chemomechanical Environments}

relatively weakened (Fig. 5.3(b)). However, due to higher mobility, dislocations are able to climb over particles when the applied stress is sufficiently large. As a result, the plastic flow becomes stabilized after the alloys experience maximum stress (Fig. 4.22(a) and 4.23(a)).

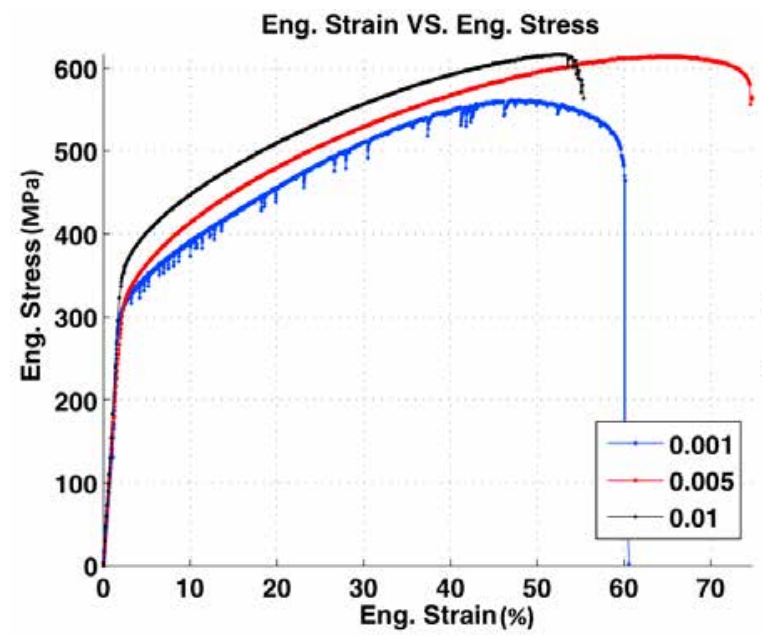

(a)

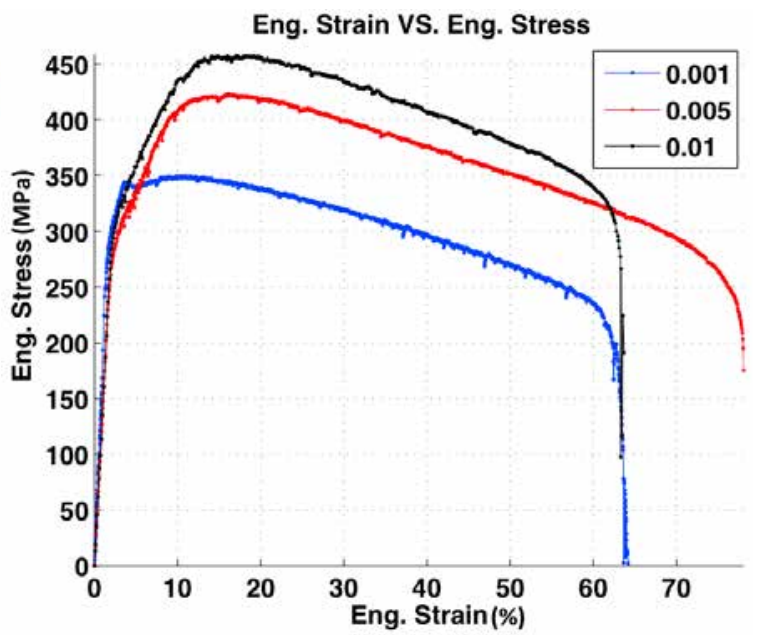

(b)

Fig. 5.3: Engineering strain-stress diagrams for Alloy 617 at (a) $700^{\circ} \mathrm{C}$ and (b) $800^{\circ} \mathrm{C}$

Since the constant flow stresses observed from 800 to $1000^{\circ} \mathrm{C}$ are nearly independent of strain, it is more convenient and accurate to interpret the strain-rate sensitivities from the constant flow stresses. For both alloys, the value of $m$ under constant flow stresses increases with temperature, except at $900^{\circ} \mathrm{C}$ for Alloy 617 (Fig. 5.2). The strain-rate sensitivity is slightly higher at $900^{\circ} \mathrm{C}$ than at $1000^{\circ} \mathrm{C}$. This small difference may be due to a measurement error, but more importantly, it suggests that the strain-rate sensitivity is very close for 900 and $1000^{\circ} \mathrm{C}$ for Alloy 617. From the comparison between Fig. 5.2(a) and (b) (or Tables 4.4 and 4.5), one should note that Alloy 230 is more sensitive to strain rate at high temperatures. Therefore, a small decrease in the loading rate can cause a large reduction in the strength of Alloy 230.

The strain-rate sensitivity has been found maintain a nearly constant value for a wide range of strain rates. This has been found to be true for the 6063 aluminum alloy - there exists a constant strain-rate sensitivity on the $0.2 \%$ flow stress in the strain rate ranging from $2 \times 10^{-2}$ to 2 $\times 10^{-5} \mathrm{~s}^{-1}$ [5.7]. Therefore, it is possible to extend short-term material properties to approximate long-term loading responses by using the strain-rate sensitivity. Based on equation 4.8 , assuming $1 \%$ total elongation according to the ASME limit, the constant flow stresses during a long-term (up to 60 years) loading are calculated and shown in Tables 5.1 and 5.2 for Alloy 617 and Alloy 230 , respectively. The allowable stresses interpolated from the data obtained in the ASME codes are also provided for comparison. If the alloys are considered for use up to 60 years, current allowable stresses for the expected operation temperatures of the VTHR $\left(800\right.$ to $\left.1000^{\circ} \mathrm{C}\right)$ are overestimated, except for the stress for Alloy 617 at $1000^{\circ} \mathrm{C}$. For Alloy 617, the allowable stresses are in fact higher than those for the estimated constant flow stresses for one year. This suggests that the alloy loading at a constant strain rate for one year (strain rate: $\sim 3 \times 10^{-10} \mathrm{~s}^{-1}$ ) can still maintain allowable stresses. However, when loading for a very long time (i.e. 60 years, 


\section{Understanding Fundamental Material-Degradation Processes in High Temperature Aggressive Chemomechanical Environments}

strain rate: $\sim 5 \times 10^{-12} \mathrm{~s}^{-1}$ ), the material cannot withstand the allowable stresses, and instead it will rupture before the end of service life. This prognosis is even worse for Alloy 230 due to its higher value of strain-rate sensitivity. For a one-year estimation, the allowable stress is higher than the constant flow stress for 800 and $900^{\circ} \mathrm{C}$, but it is already lower than the value for $1000^{\circ} \mathrm{C}$. For an estimation of 10 years, the allowable stresses in all studied temperatures were found to be lower than their estimated stresses. The analysis suggests that current ASME allowable stresses for both alloys cannot be directly applied to high-temperature, long-term design. In fact, only a short-term application can use the established codes.

Table 5.1: Estimated constant flow stresses and ASME allowable stresses for Alloy 617

\begin{tabular}{ccccc}
\hline Temperature $\left({ }^{\circ} \mathbf{C}\right)$ & $\mathbf{1}$ Year & $\mathbf{1 0}$ Years & $\mathbf{6 0}$ Years & ASME allowable \\
\hline $\mathbf{8 0 0}$ & $52.5 \mathrm{MPa}$ & $38.2 \mathrm{MPa}$ & $29.8 \mathrm{MPa}$ & $\sim 31 \mathrm{MPa}$ \\
$\mathbf{9 0 0}$ & $13.7 \mathrm{MPa}$ & $9.0 \mathrm{MPa}$ & $6.4 \mathrm{MPa}$ & $\sim 12.3 \mathrm{MPa}$ \\
$\mathbf{1 0 0 0}$ & $14.6 \mathrm{MPa}$ & $10.4 \mathrm{MPa}$ & $8.0 \mathrm{MPa}$ & $\sim 4.8 \mathrm{MPa}$ \\
\hline
\end{tabular}

Table 5.2: Estimated constant flow stresses and ASME allowable stresses for Alloy 230

\begin{tabular}{ccccc}
\hline Temperature $\left({ }^{\circ} \mathbf{C}\right)$ & $\mathbf{1}$ Year & $\mathbf{1 0}$ Years & $\mathbf{6 0}$ Years & ASME allowable \\
\hline $\mathbf{8 0 0}$ & $44.9 \mathrm{MPa}$ & $31.9 \mathrm{MPa}$ & $24.4 \mathrm{MPa}$ & $\sim 32 \mathrm{MPa}$ \\
$\mathbf{9 0 0}$ & $10.4 \mathrm{MPa}$ & $6.4 \mathrm{MPa}$ & $4.4 \mathrm{MPa}$ & $\sim 10.2 \mathrm{MPa}$ \\
$\mathbf{1 0 0 0}$ & $1.3 \mathrm{MPa}$ & $0.6 \mathrm{MPa}$ & $0.4 \mathrm{MPa}$ & $\sim 2.6 \mathrm{MPa}$ \\
\hline
\end{tabular}

In addition to the evaluation of allowable stresses, the rupture stresses in the ASME code were also examined. The rupture stresses for Alloy 617 are developed in the ASME code case N-47-28. The interpolated rupture stresses for 1, 10, and 60 years are shown in Table 5.3. Generally, both estimated flow stresses and documented rupture stresses possess an analogous degradation tendency with time. For 800 and $1000^{\circ} \mathrm{C}$, the ASME rupture stresses are more conservative with lower values compared to the estimated flow stresses. In contrast, the ASME rupture stresses are lower than the estimated flow stresses at $900^{\circ} \mathrm{C}$, which can be attributed to measurement error. In general, the rupture stresses from the ASME code should be sufficiently conservative according to the strain-rate sensitivity analysis. One should note that there is a knockdown factor for rupture stress in the ASME code for engineering design. This design limit stands as "67\% of rupture stress," which has been incorporated in the ASME B\&PV Code, Section III, Subsection NH. Therefore, the permitted stresses for 60 years are only 7.3 and 3.0 $\mathrm{MPa}$ for 900 and $1000^{\circ} \mathrm{C}$ after applying the knockdown factor. These permitted stresses are not adequate for long-term VHTR operation. Further engineering improvement and material development are needed to meet the requirements of the VHTR. 
Understanding Fundamental Material-Degradation Processes in High Temperature Aggressive Chemomechanical Environments

Table 5.3: Rupture stresses for Alloy 617 interpolated from Ref. 10 (ASME code case N-47-28)

\begin{tabular}{cccc}
\hline Temperature $\left({ }^{\circ} \mathbf{C}\right)$ & $\mathbf{1}$ Year & $\mathbf{1 0}$ Years & $\mathbf{6 0}$ Years \\
\hline $\mathbf{8 0 0}$ & $58 \mathrm{MPa}$ & $35 \mathrm{MPa}$ & $26 \mathrm{MPa}$ \\
$\mathbf{9 0 0}$ & $25 \mathrm{MPa}$ & $15 \mathrm{MPa}$ & $11 \mathrm{MPa}$ \\
$\mathbf{1 0 0 0}$ & $11 \mathrm{MPa}$ & $6.8 \mathrm{MPa}$ & $4.5 \mathrm{MPa}$ \\
\hline
\end{tabular}

\subsection{Long-term Thermal Aging of Alloy 617 and Alloy 230}

The aging process for both Alloy 617 and Alloy 230 is characterized by particle nucleation and growth. According to the microstructural development, the aging process can essentially be divided into two stages: 1) the early stage, featuring fine and ordered precipitates within matrix grains and boundaries (Fig. 4.31(a)) and 2) the overaging stage, with depletion of small precipitates within the grains (Figs. 4.35 and 4.36) along with a significant coarsening of intergranular particles. During aging, the favorable nucleation sites for precipitates, determined by a higher free energy (or larger average atomic volume) compared to the host matrix, are interphase boundaries, grain boundaries, and twin boundaries. Thus, the size of the particles in these areas is much larger than the particle size within the host matrix. After an extended aging time, the precipitates diffused and stabilized in the areas with the highest interfacial free energy (e.g. interphase boundaries and high-angle grain boundaries). Both matrix and twin boundaries were found to be free of carbide particles due to their low interfacial free energy. The depletion of carbide particles in the twin boundaries is analogous to the anti-sensitization of low $\sum$ boundaries in steel: the low $\sum$ boundaries show a higher intergranular resistance for $\mathrm{Cr}$ depletion than high-angle grain boundaries [5.8]. The results were also in good agreement with the findings by Schlegel et al. that low $\sum$ boundaries tend to suppress intergranular precipitation in Alloy 617 during creep [4.54].

Whittenberger performed an aging test on both Alloy 617 and Alloy 230 at $820^{\circ} \mathrm{C}$ over 20,000 hours [5.9]. No change in grain size was observed in his experiment. Tan et al. annealed Alloy 617 at 850 and $1000^{\circ} \mathrm{C}$ for 4 and 6 weeks after thermomechanical processing [3.2, 4.6], and found that the grain boundary character distribution (GBCD) in Alloy 617 was preserved after thermal aging. In the current study, it was also found that 3000 hours aging at 900 and $1000^{\circ} \mathrm{C}$ did not influence the grain size distribution or GBCD for both Alloy 617 and Alloy 230. Their exceptional thermal stability is partially attributed to "Zener pinning" by large intrinsic carbides and age-coarsened intergranular particles. These particles inhibit the grain boundary migration and impede grain growth during aging. Hence, the grain boundary characters are preserved.

The maintenance for inexistence of topologically close-packed phases (e.g. sigma $(\sigma)$, $\operatorname{mu}(\mu)$, or chi $(\chi))$ is critical to ensure material strength during service life, since these detrimental phases can cause brittle failure at low temperatures and a loss of strength at high temperatures [4.20]. According to ThermoCalc ${ }^{\circledR}$ modeling, the $\sigma$ phase can precipitate in Alloy 617 if the alloying composition is not well-controlled [4.17]. Nevertheless, no TCP was observed in either alloy at any aging condition. This finding also agrees well with previous aging studies for Alloy 


\section{Understanding Fundamental Material-Degradation Processes in High Temperature Aggressive Chemomechanical Environments}

617 at lower temperatures by $\mathrm{Wu}$ et al. [4.15] and for a similar temperature range by Mankins et al. [3.44], Kihara et al. [4.89], and Kirchhofer et al. [5.10].

The analysis of intergranular particle growth provides insights into the kinetics of precipitation processes in Alloy 617 and Alloy 230. Particle nucleation and growth are essentially controlled by two factors, both of which are temperature-dependent. The first factor is the driving force of particle nucleation, which depends on the content of the second-phase in the equilibrium condition. The second factor lies in the diffusion kinetics of heterogeneous elements (e.g. C, W, or Mo) that form the particles. The diffusion kinetics are directly related to the aging temperature such that a higher temperature promotes the diffusion of heterogeneous atoms, and vice versa. According to the predicted equilibrium phase diagram of Alloy 617 [4.14], the content of particles (including both $\mathrm{M}_{6} \mathrm{C}$ and $\mathrm{M}_{23} \mathrm{C}_{6}$ ) is slightly higher at $1000^{\circ} \mathrm{C}$ than at $900^{\circ} \mathrm{C}$. This suggests a larger driving force at $1000^{\circ} \mathrm{C}$ for particle nucleation in Alloy 617. Moreover, the heterogeneous elements in both alloys obtained a higher diffusibility at $1000^{\circ} \mathrm{C}$. The slower diffusion process and the lower driving force significantly limit the particle growth in the alloys aged at $900^{\circ} \mathrm{C}$. The temperature dependence of particle growth can be seen in 2D (Fig. 4.41) and $3 \mathrm{D}$ (Fig. 4.43) analyses that show growth of larger intergranular particles at $1000^{\circ} \mathrm{C}$ than at $900^{\circ} \mathrm{C}$ during aging.

Intergranular particle growth is also studied through analysis of the particle-coarsening rate $d Y / d t$ (Fig. 4.44). At $900^{\circ} \mathrm{C}$, most of the volume gain (in fraction) of intergranular particles occurs during the early stage of aging with a sharp decline in the coarsening rate. This suggests a dramatic loss of growth momentum. In contrast, the particle coarsening rate increases in the first few hundred hours during aging at $1000^{\circ} \mathrm{C}$ before the decline occurs. During this period of time, the carbide nucleation continues, accompanied by the growth of existing precipitates, until the equilibrium volume fraction is developed. It should be noted that this stage, characterized by a combination of particle nucleation and growth, also occurred for the alloys aging at $900^{\circ} \mathrm{C}$, but within a very short time ( $<<10$ hours) due to a low equilibrium volume fraction of second-phase particles. Therefore, this transient at $900^{\circ} \mathrm{C}$ was not observed in the present study.

Intergranular particle growth is analyzed based on the measured data fitted by the adapted Avarmi equation (equation 4.15). The original form of the Avrami equation is:

$$
X=1-\exp \left(-K t^{n}\right)
$$

In equation 5.1, $X$ is the overall transformed volume fraction of second-phase particles, and $K$ and $n$ are the physical interpretations of the Avrami constants that are known as the Avrami coefficient and the Avrami exponent, respectively. If we assume that the number of intergranular particles does not change with time (i.e. no impingement takes place during aging), then $X$ and $Y$ can be considered to be equal in value. Then, we can refer to the value of $n$ that relates the balance between nucleation and growth, and suggest the mechanism of transformation.

Table 5.4: Results and interpretation of $n$

\begin{tabular}{cccc}
\hline & Alloy 617 & Alloy 230 & Mechanism [5.11] \\
\hline $\mathbf{9 0 0} \mathbf{C}$ & 0.682 & 0.547 & Thickening of large plates $(n \approx 0.5)$ \\
$\mathbf{1 0 0 0}^{\circ} \mathbf{C}$ & 1.27 & 1.34 & Growth of particles of large volume $(n=1-1.5)$ \\
\hline
\end{tabular}




\section{Understanding Fundamental Material-Degradation Processes in High Temperature Aggressive Chemomechanical Environments}

Table 5.4 shows the possible mechanisms of the particle evolution of Alloy 617 and Alloy 230 during aging at $900^{\circ} \mathrm{C}$ and $1000^{\circ} \mathrm{C}$. The "thickening of large plates" at $900^{\circ} \mathrm{C}$ indicates a dominant coarsening process, whereas the "growth of particles of a large volume" suggests a mixed process involving both nucleation and growth [5.11]. The interpretations of $n$ agree well with the previous discussion.

Different heterogeneous elements can attribute to the different kinetics for particle growth in Alloy 617 and Alloy 230. W, as a main alloying element in Alloy 230, is less thermally active than Co and Mo in Alloy 617. The lower thermal activity of $\mathrm{W}$ can be reflected in its much higher melting temperature $\left(T_{m}\right.$ of $\mathrm{W}=3422^{\circ} \mathrm{C}>>T_{m}$ of $\mathrm{Mo}=2623^{\circ} \mathrm{C}$ and $T_{m}$ of $\left.\mathrm{Co}=1495^{\circ} \mathrm{C}\right)$. The diffusion rate of $\mathrm{W}$ in the $\gamma$ matrix is therefore lower than that of Co and Mo, which results in a relatively slower process of precipitate growth in Alloy 230 (Fig. 4.41 and Fig. 4.43). Additionally, the varying diffusion kinetics in the alloys can be reflected by the changes in mechanical properties with aging. This will be discussed further in the following section.

As introduced in section 4.4, the strength development with aging typically involves three strengthening mechanisms: dislocation cutting $\left(\tau_{\text {cut }}\right)$, dislocation looping $\left(\tau_{\text {loop }}\right)$, and solid solution strengthening $\left(\tau_{s s s}\right)$ (Fig. 4.28). Other fundamental material strengthening mechanisms include strain hardening $\left(\tau_{s h}\right)$ and grain-boundary hardening $\left(\tau_{H P}\right.$, where the subscript "HP" denotes the Hall-Petch relationship). Therefore, a comprehensive relation to describe the overall strength $\tau_{t o t}$ of the alloys can be given as:

$\tau_{\text {tot }}=\tau_{0}+\tau_{H P}+\tau_{\text {sh }}+\tau_{\text {sss }}+\tau_{\text {loop }}+\tau_{\text {cut }}$

where $\tau_{0}$ is intrinsic strength of the material without additional strengthening mechanisms. The value of $\tau_{s h}$ is close to the frictional stress for the dislocation movement, also known as the Peierls stress, and can be expressed in terms of the width, $w$, of a dislocation:

$\tau_{0}=G \exp \left(-\frac{2 \pi w}{b}\right)$

Note that the $\mathrm{M}_{23} \mathrm{C}_{6}$ carbides are the main precipitates during the early stage of aging. These particles are non-deformable and partially coherent to the $\gamma$ matrix during aging. Fig. 5.4 shows a SEM image for an Alloy 230 specimen that has been plastic deformed and processed by fine polishing and acid etching. The dislocation looping/bowing around the particles induces a strained area that can be easily etched out. The fine particles within etched pits suggest that the dislocation bowing/looping is the strengthening mechanism for aged alloys (Fig. 5.4). Therefore, the component $\tau_{\text {cut }}$ can be discarded from equation 5.2 because dislocation cutting does not occur. 


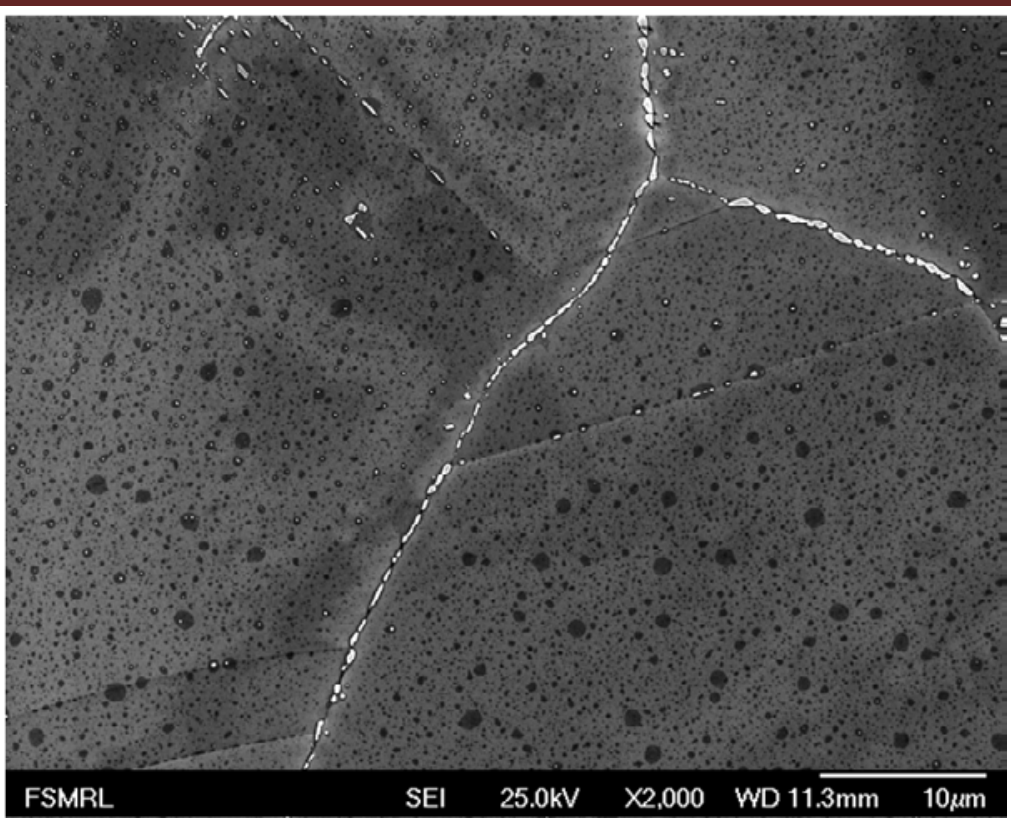

Fig. 5.4: Alloy 230 aged at $1000^{\circ} \mathrm{C}$ for 10 hours

According to microstructural analysis, the grain size does not change with aging, so the strength component of grain-boundary hardening, $\tau_{H P}$, can be considered to be constant during aging. If we assume that the dislocation density does not change significantly with aging, then the strain hardening component, $\tau_{\text {sh }}$, can also be treated as aging-independent. Accordingly, equation 5.2 can be simplified to:

$\tau_{\text {tot }}=\tau_{\text {sss }}+\tau_{\text {loop }}+\tau_{\text {other }}$

$\tau_{\text {other }}$ is composed of all strengthening components that are invariable with aging. Thus, the overall strength development with aging can be analyzed based on the balance of $\tau_{\text {loop }}$ and $\tau_{\text {sss. }}$ In fact, the particle strengthening is much more efficient and stronger than the solid solution strengthening, and controls the overall hardening response during aging. Accordingly, the changes in mechanical properties observed in both alloys with aging can be interpreted. For Alloy 617 aged at $900^{\circ} \mathrm{C}$, nucleation of fine particles results in precipitate strengthening (increasing $\tau_{\text {loop }}$ ) and a loss of solid solution strengthening (decreasing $\tau_{\text {sss }}$ ) in a very short time. The precipitate strengthening is sufficiently large to compensate the loss of solid solution strengthening. As intergranular particles grow at the expense of fine precipitates, the spatial distance between particles ( $L$ in equation 4.12) continues to increase. Therefore, $\tau_{\text {loop }}$ gradually decreases and becomes less effective for the overall strength $\left(\tau_{t o t}\right)$. When the particles finally stabilize in the boundaries, $\tau_{\text {tot }}$ becomes lower than the value before aging due to the loss of solid solution strengthening. The results of hardness and tensile tests show this aging process characterized by short-term hardening and long-term softening (Fig. 4.45(a) and Fig. 4.46). However, this sectional process is not observed in alloys aged at $1000^{\circ} \mathrm{C}$. Due to a much higher diffusion rate, the particles grow rapidly at $1000^{\circ} \mathrm{C}$ compared to $900^{\circ} \mathrm{C}$. This leads to a sharp decline of strength within a short time $(<<10$ hours) because of the loss of fine particles as hardeners. Particle strengthening is not enough to compensate for the loss of strengthening by solute elements. Hence, the softening effect is dominant for Alloy 617 aging at $1000^{\circ} \mathrm{C}$. Fig. 5.5 shows the schematic of the overall aging effect of Alloy 617 at 900 and $1000^{\circ} \mathrm{C}$. 


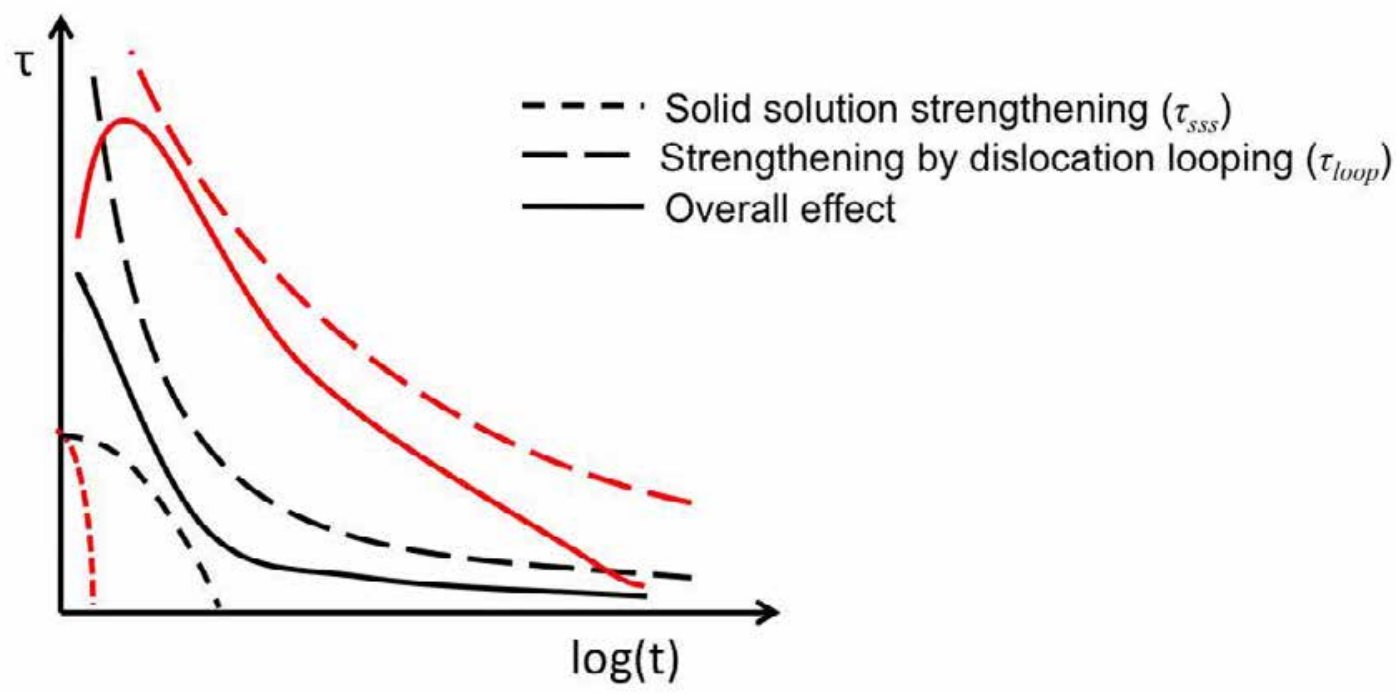

Fig. 5.5: Schematic of the overall aging effect of Alloy 617 at $900^{\circ} \mathrm{C}$ (red) and $1000^{\circ} \mathrm{C}$ (black)

For Alloy 230, the changes in mechanical properties with aging follow a similar developmental trend as is found for Alloy 617. However, as discussed in section 4.4.7, the main heterogeneous element, W, in Alloy 230 has a lower diffusibility than the Mo and Co in Alloy 617. Thus, the precipitate growth in Alloy 230 is slower, and results in a moderate decline of particle strengthening. This explains the observation of a longer hardening effect for Alloy 230 than for Alloy 617 (Figs. 4.45-4.47).

After long-term aging, a loss of strength is not only found in tensile testing at room temperature, but also at high temperatures up to $1000^{\circ} \mathrm{C}$. These strength reductions with thermal aging are analogous to the results of the orientation effect analysis (section 4.2). Both studies suggest that the degradation of tensile properties at high temperatures is comparable to that at low temperatures.

Finally, the measured hardness of Alloy 617 after 300 and 1000 hours of aging is higher than the values reported in ref. 5.12. The aging effect on microstructural evolution and the associated mechanical properties is dependent on chemical composition and the history of heat treatment. From the data summarized in ref. 4.14, the kinetics and magnitude of changes in hardness and tensile properties with aging are different from one temperature to another. Therefore, proper control of chemical compositions and a regulation of heat treatment are both necessary to maintain the design margins of Alloy 617 and Alloy 230. 


\section{Understanding Fundamental Material-Degradation Processes in High Temperature Aggressive Chemomechanical Environments}

\subsection{High-energy X-Ray Diffraction Study of Alloy 230}

The $\gamma$ matrix in the long-transverse and short-transverse samples of Alloy 230 has a similar response as the lattice strain development during tensile loading, although an early failure occurs for the short-transverse specimen. The material's elastic anisotropy is revealed by different responses of $h k l$-specific reflections, which are typical for FCC metals [4.29, 5.13]. Calculated from Kröner's self-consistent method, Young's moduli for different $(h k l)$ reflections provide a sufficient approximation for converting measured lattice strains to internal stresses. Although this analysis only considers one phase, the results agree well with true stresses from the mechanical measurements (Fig. 4.59). The analysis also suggests that the load transferred from the $\gamma$ matrix to the second-phase particles is insignificant in Alloy 230 during deformation at room temperature.

One should take note of the observed Bauschinger effect in the Alloy 230 specimens. From mechanical measurements, the difference in Y.S. between the long- and short-transverse specimens was found to be $\sim 80 \mathrm{MPa}$ (Table 4.7 ). This can be predicted by the measurement of the residual stress in the specimens before tensile loading. The measured lattice strains parallel to the applied stress $\varepsilon_{11}$ are $-1.21 \times 10^{-4}$ and $2.76 \times 10^{-4}$ for the long- and short-transverse specimens, respectively (Fig. 4.60 and Fig. 4.61). Thus, the corresponding difference in residual stress is 83 $\mathrm{MPa}$, which agrees well with the mechanical measurements.

The deformation process of carbide particles in Alloy 230 can be revealed by analyzing the lattice strain responses for the long-transverse specimens. During the alloy's elastic deformation, the lattice strain of the carbide increases linearly with increasing external applied stress. The lattice strain dramatically increases during early yielding until it reaches its maximum value. This process is the result of significant dislocation movements during material yielding and, consequently, a great number of dislocations bow around large, non-deformable particles. However, unlike the sustained loading behavior of the particles in refs. 3.4 and 3.6, the lattice strain of the $\mathrm{M}_{6} \mathrm{C}$ carbide in Alloy 230 cannot increase during plastic deformation. Instead, lattice strain gradually decreases, in contrast to the linear straining of the $\gamma$ matrix. This indicates that the large carbides with low volume fractions cannot maintain large internal stresses and begin to rupture upon early yielding. The continued particle fracture process results in a decreasing loading capacity (therefore reducing the lattice strain) during plastic deformation. The particle's maximum internal stress, achieved during early yielding in the material, can be defined as the critical fracture stress for the $\mathrm{M}_{6} \mathrm{C}$ type carbide in Alloy 230. This is the maximum internal stress that the $\mathrm{M}_{6} \mathrm{C}$ carbide is able to withstand. Particle fracture will initiate once the internal stress is greater than this critical value $(\sim 1344 \mathrm{MPa}$, corresponding to the lattice strain of $\sim 0.0047)$.

Because of the residual stress on the particles as well as the early rupture of the specimen, the loading behavior of the carbide in the short-transverse specimen cannot be divided into multiple stages as can be done with the long-transverse specimen. However, the carbide in both specimens achieved the same value of lattice strain ( 0.0047) after yielding (Fig. 4.60(b)). This confirms the analysis for the critical fracture stress in the long-transverse specimen. Moreover, the analysis of the short-transverse specimen suggests that the critical fracture stress for the $\mathrm{M}_{6} \mathrm{C}$ type carbide in Alloy 230 is independent of loading orientation. In other words, the loading direction on the specimens, either long- or short-transverse, will not change the initial stress of carbide fracture. Note that the $\mathrm{M}_{6} \mathrm{C}$ carbide is lamellar-shaped and embedded in the $\gamma$ matrix (Fig. 4.49). From the mechanical tests on Alloy 230 (also seen in the effect of orientation of 


\section{Understanding Fundamental Material-Degradation Processes in High Temperature Aggressive Chemomechanical Environments}

Alloy 617, section 4.2), the anisotropic geometry of the carbide as well as their distribution in the material are found to have an impact on all tensile properties (Fig. 4.52 and Table 4.7). Nevertheless, the maximum stress that the carbides can withstand is found to be independent of their shape and distribution within the alloy.

Complementary studies using SEM analysis for the ruptured specimens are shown in Fig. 5.6. Similar to the anisotropy of Alloy 617, the early failure of the short-transverse Alloy 230 specimen is caused by the ligament of carbide bands. Most of the carbides still maintain their integrity after the sample rupture (Fig. 5.6(b)). In contrast, almost all the carbides fractured in the ruptured long-transverse specimen (Fig. 5.6(a)). The difference in the carbide integrity explains the observation of different lattice strains (and therefore different internal stresses) when the specimens fail.

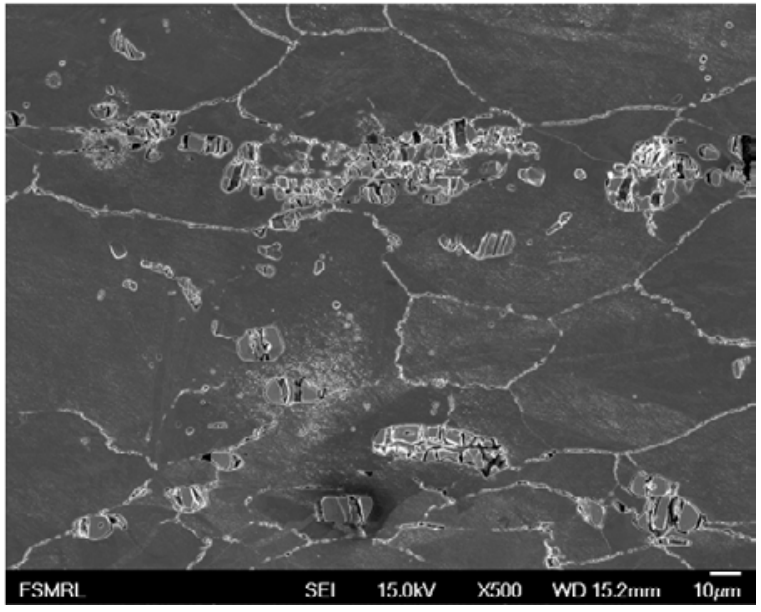

(a)

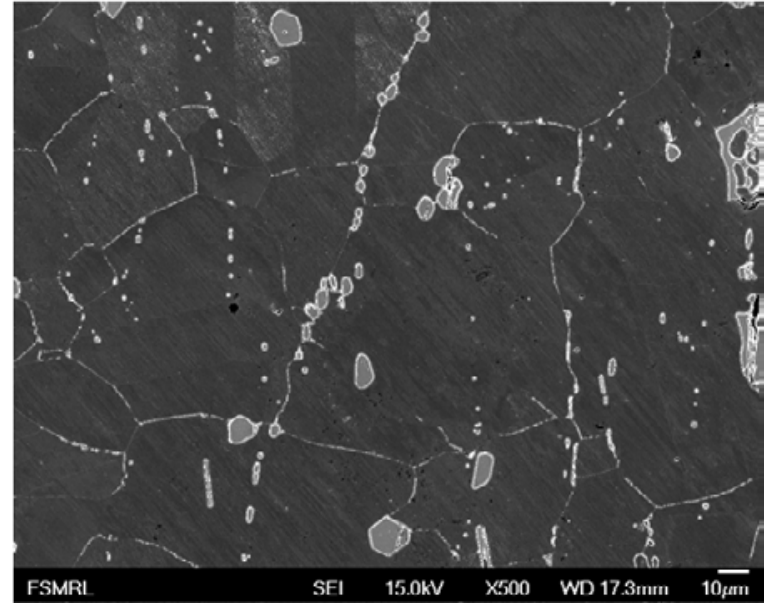

(b)

Fig. 5.6: Carbide fractures in ruptured specimens: (a) long-transverse; (b) short-transverse

Finally, it must be noted that the present study is based on tensile tests conducted at room temperature. Since both alloys are expected to be used at high temperatures, the actual loading behavior for both the $\gamma$ matrix and carbides might differ from that found during tests at low temperatures. The high-energy synchrotron technique can be further applied to study the high-temperature mechanical behavior of Alloy 230 (e.g. creep and creep-fatigue).

\subsection{In-situ Creep Study Using Pressurized Creep Tubes}

From the analysis of diffraction patterns from pressurized creep tubes, a complete creep curve is obtained with a better continuity than the interrupted strain measurements employed in previous studies [4.32]. This method also allows for an assessment of the in-situ microstructure development during different creep stages. First, the range of local strains and the crystalline size can be evaluated by analyzing peak broadening. Since the effective crystalline size remains relatively constant during creep, the peak width development can generally be considered to be 


\section{Understanding Fundamental Material-Degradation Processes in High Temperature Aggressive Chemomechanical Environments}

caused by the local strain or undirected micro distortion [4.25, 5.14, 4.26]. From the analysis, the three-stage peak width development was observed. During the early stage, the FWHM of the (311) reflection decreased and reached a static level in about 60 minutes. This indicates a decrease in dislocation density in the early stages of creep, which is caused by the rearrangement and recombination of dislocations (Fig. 4.64(a)). The result agrees well with the creep study completed by Pyzalla et al, and suggests that small residual stresses may result during the manufacturing of the tube used in the present study [5.14]. The static level of the FWHM lasts for about 2 hours, then increases until the tube fractures. This peak broadening is most likely attributed to the development of associated localized stress concentrations caused by the nucleation, growth, and coalescence of a large volume of creep voids. These results could be used to provide a better division of the secondary and tertiary creep regimes.

The analysis of the diffraction peak shifting also showed that higher internal stresses are developed after 160 minutes, which is close to the moment when the diffraction peak starts broadening. Thus, despite a relatively large data variation due to small applied stresses, the results of lattice strain measurements agree well with the analysis of diffraction peak broadening and indicate that the onset of the accelerated development of creep voids occurs about 180 minutes after the start of the creep test.

This technique, which uses the combination of pressurized creep tubes and high-energy synchrotron X-rays, can be coupled with small angle X-ray scattering (SAXS) or X-ray tomography to more directly observe the initiation of plasticity-induced void formation [3.6]. The multi-scale measurement and X-ray technique crossover can provide an insight into the creep deformation for prospective materials for nuclear energy development. Finally, it should be noted that the specimen configuration in the present study simulates the heat transport and exchange tubing and piping in the NGNP system. This paves the way for using synchrotron $\mathrm{X}$-ray diffraction as a non-destructive method for investigating many components in practical engineering applications.

\subsection{Creep-fatigue Life Prediction}

\subsubsection{Linear damage summation}

In this section, the ability of the linear damage summation to correlate the test data is evaluated. First, the stress-rupture data of Alloy 617 and Alloy 230 at $850^{\circ} \mathrm{C}$ from Refs. $[5.15,5.16,5.17]$ is fitted with the following equation:

$$
\begin{aligned}
\log \left(t_{r}\right)=a+ & b \\
& \times \log (\sigma)
\end{aligned}
$$

where $t_{r}$ is the time to rupture at a stress level $\sigma$. The curve fit and original data are plotted in Fig. 5.7, where it can be seen that the creep-rupture curves are linear on a log-log plot for the input range of stresses. Since $t_{r}$ is a function of $\sigma$, which changes during the stress relaxation period, a constitutive equation is needed to describe the stress relaxation behavior of materials. This is done by fitting the stress relaxation data with Origin, a data analysis software. In the past, several different constitutive equations were proposed to model the stress relaxation behavior 


\section{Understanding Fundamental Material-Degradation Processes in High Temperature} Aggressive Chemomechanical Environments

$[5.18,4.39,4.55,5.19]$. In this study, however, a different equation was used that was proven to match the stress relaxation data with an adjusted $r$ correlation value better than 0.96 :

$$
\begin{gathered}
\sigma=a+b \times e^{-t / t_{1}+c \times e^{-t / t_{2}}+d} \\
\times e^{-t / t_{3}}
\end{gathered}
$$

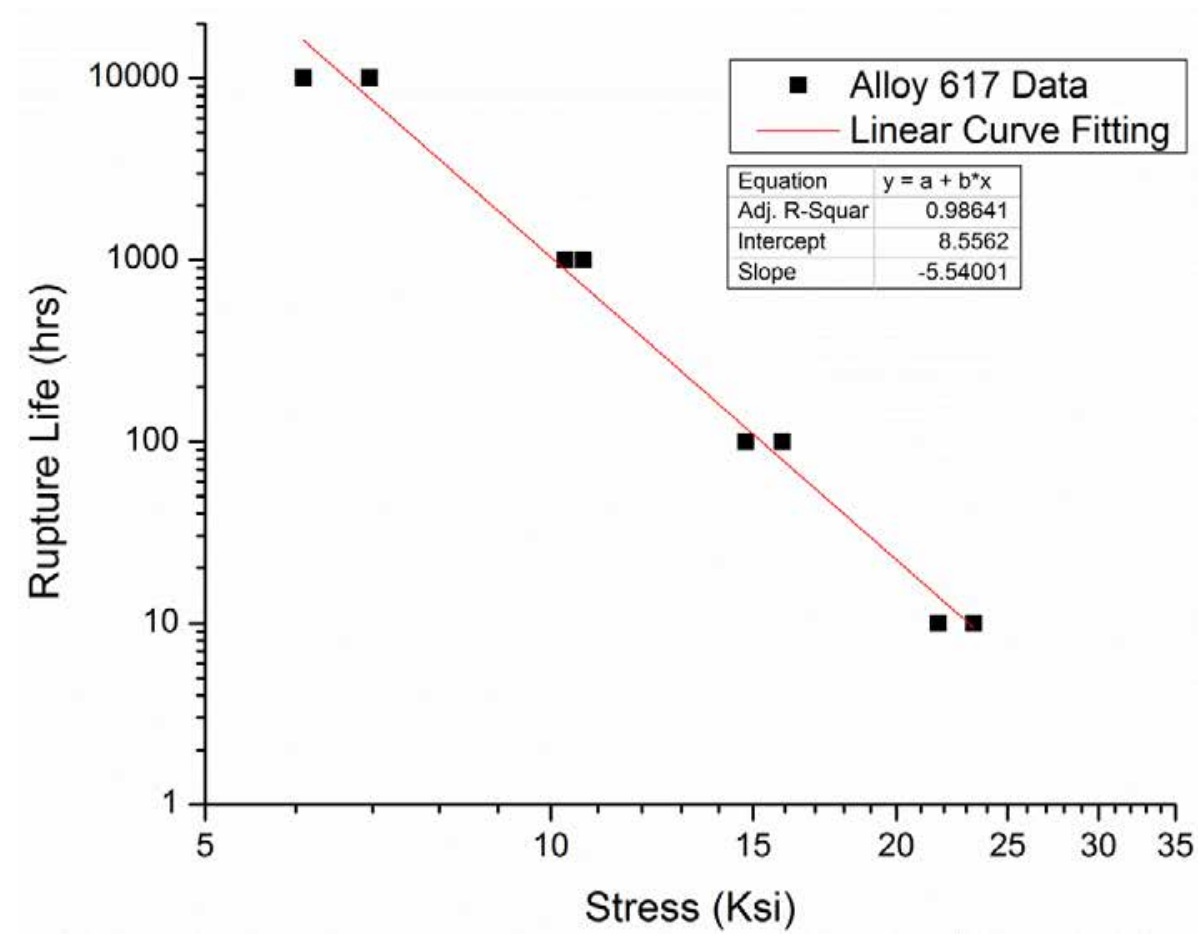

(a) 


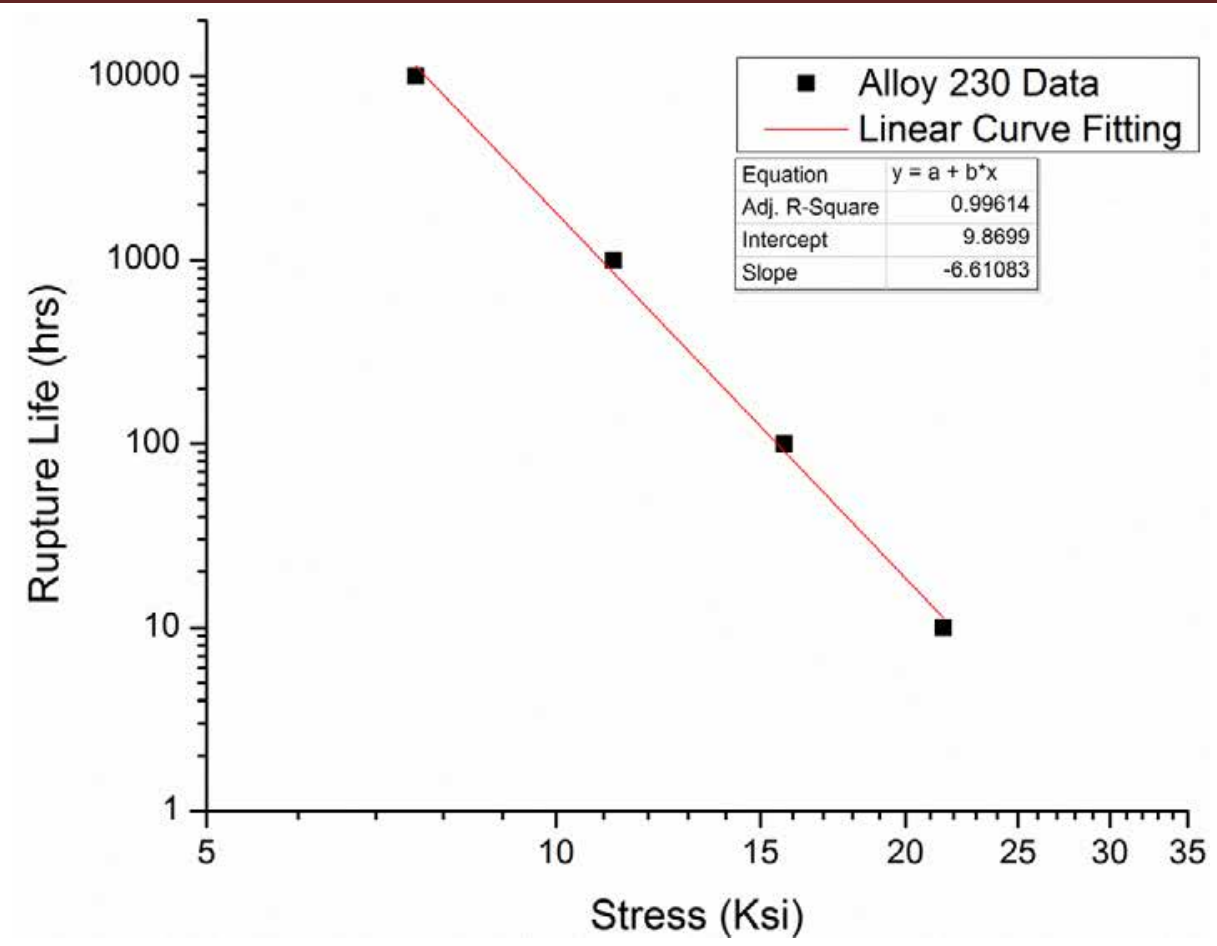

(b)

Fig. 5.7: Stress rupture data and linear curve fitting for (a) Alloy 617; (b) Alloy 230 (after Ref. [5.15, $5.16,5.17])$

For illustration purposes, several fitting curves and original stress relaxation curves are shown in Fig. 5.8. All the fitting curves closely match the original data. The creep damage calculation in linear damage summation requires the summation of the creep damage of each cycle. This is unrealistic, considering the cyclic creep-fatigue life of tested materials, which varies from hundreds to thousands of cycles. Some approximations are needed for the calculation of creep damage. In the work of Campbell, creep-fatigue tests were done on stainless steel 304, and the author calculated the creep damage of the mid-life cycle and assumed the total creep damage equaled the multiplication of the mid-life cycle creep damage by the cyclic creep-fatigue life. Since the cyclic stress curves in Campbell's study were fairly flat over most of the specimen life (which meant that the mid-life stress relaxation curve should be representative of the material relaxation behavior throughout the specimen life), Campbell's presumption should most likely be valid [5.18]. In this study, however, cyclic stress curves, shown in Fig. 4.72, changed during the test. Therefore, the mid-life relaxation curve cannot be considered representative of the material relaxation behavior throughout the specimen life. To balance the complexity and accuracy in the creep damage calculation performed in linear damage summation, the stress relaxation curve at the mid point cycle (of around every $10 \%$ of creep-fatigue life) was sampled and used as the representative of that $10 \%$ creep-fatigue life. Fig. 5.9 schematically illustrates the applied approximation strategy. In such cases, each creep-fatigue test condition would yield ten sets of constant parameters, as per equation 5.6, which are summarized in Table 5.5. Therefore: 


$$
\begin{aligned}
D_{c}=\sum \frac{\Delta t}{t_{r}(\sigma)} & \\
& \approx \sum_{n=1}^{10}\left(\int_{0}^{t_{0}} \frac{d_{t}}{t_{r}(\sigma)} \times N_{10 \%}\right)_{n}
\end{aligned}
$$

In equation $5.7, N_{10 \%}$ is the number of cycles for the $10 \%$ creep-fatigue life of one specific creep-fatigue test condition, $t_{r}(\sigma)$ is the stress rupture time defined in equation 5.5, and $\sigma$ represents stress relaxation data of the green middle point cycle highlighted in Fig. 5.9 that can be described by equation 5.6. The summation over ten signifies the summation of creep damage which occurs during each 10\% creep-fatigue life. In summary, Table 5.6 shows the damage fraction due to the creep and fatigue components as well as the summation of these two, i.e. the total damage. The results are also illustrated in Fig. 5.10. For the same test conditions, roughly the same fraction of fatigue damage was endured by both Alloy 617 and Alloy 230, except for the creep-fatigue test at $0.5 \%$ total strain range with a 3 minute hold time in peak tensile strain and the creep-fatigue tests at $1.0 \%$ total strain range with 10 minute and 30 minute hold times in peak tensile strain, due to greater reduction in the cyclic creep-fatigue life of Alloy 617 . However, the creep damage portion varied greatly for these two materials, with much high creep damage being born by Alloy 230. This is due to the higher flow stress exhibited by Alloy 230 during the stress relaxation period, as shown in Fig. 4.74. In addition, the maximum tensile stress at the onset of the stress relaxation period for both alloys exceeds the maximum stress level of available stress rupture data. Thus, applying equation 5.5 to calculate the stress rupture time at a higher stress level than the original input data requires extrapolation of the available stress rupture data, which in turn may increase the uncertainty in the creep damage calculation results. Nevertheless, existing literatures reveal that sometimes there was no detrimental creep-fatigue interactions or that the creep-fatigue interactions could result in a longer creep or fatigue life $[4.50,5.51,5.20]$. The degree of creep-fatigue interaction can be deduced from the distance between the origin of the coordinates and the data point in Fig. 5.10. A short distance signifies that there is a strong synergic interaction between creep and fatigue. There is no obvious influence of the total strain range on the creep-fatigue interaction in Alloy 617, as the data points corresponding to tests with 3 minute dwell times at different total strain ranges display almost the same distance from the origin. However, this is not true for Alloy 230, as the creep-fatigue test condition of $0.5 \%$ total strain range with a 3 minute hold time yielded the least creep-fatigue interaction. The length of the dwell time at peak tensile strain may play a role in the creep-fatigue interaction, especially for Alloy 617. As shown in Fig. 5.10, for the creep-fatigue tests with $1.0 \%$ total strain range, the longer the dwell period, the closer the data point becomes to the origin.

The linear damage summation failure criterion is represented by a straight black dashed line in Fig. 5.10. Most of the Alloy 617 data matches the line in a plausible way except for the creep-fatigue test condition of $1.0 \%$ total strain range with a 30 minute hold time, at which the linear damage summation failure criterion overestimated the material's capability to sustain the creep-fatigue damage. In regards to Alloy 230, the conventional linear damage summation failure criterion considerably underestimated the material's creep-fatigue life, as all the data points for Alloy 230 were found to be far above the black dashed line. The bilinear blue dot line shown in the same figure is based on the failure criterion of the draft code case for Alloy 617 [5.21]. Although conservative enough for estimating the creep-fatigue life for Alloy 617 and Alloy 230, the draft code failed to fully exploit the available safety margins of these two 


\section{Understanding Fundamental Material-Degradation Processes in High Temperature}

Aggressive Chemomechanical Environments

materials, as all the creep-fatigue damage calculation results were well above the blue dotted line. Therefore, the linear damage summation failure criterion could only predict the creep-fatigue life of Alloy 617 for limited test conditions, and lacked enough accuracy for predicting the creep-fatigue life of Alloy 230. 
Understanding Fundamental Material-Degradation Processes in High Temperature Aggressive Chemomechanical Environments
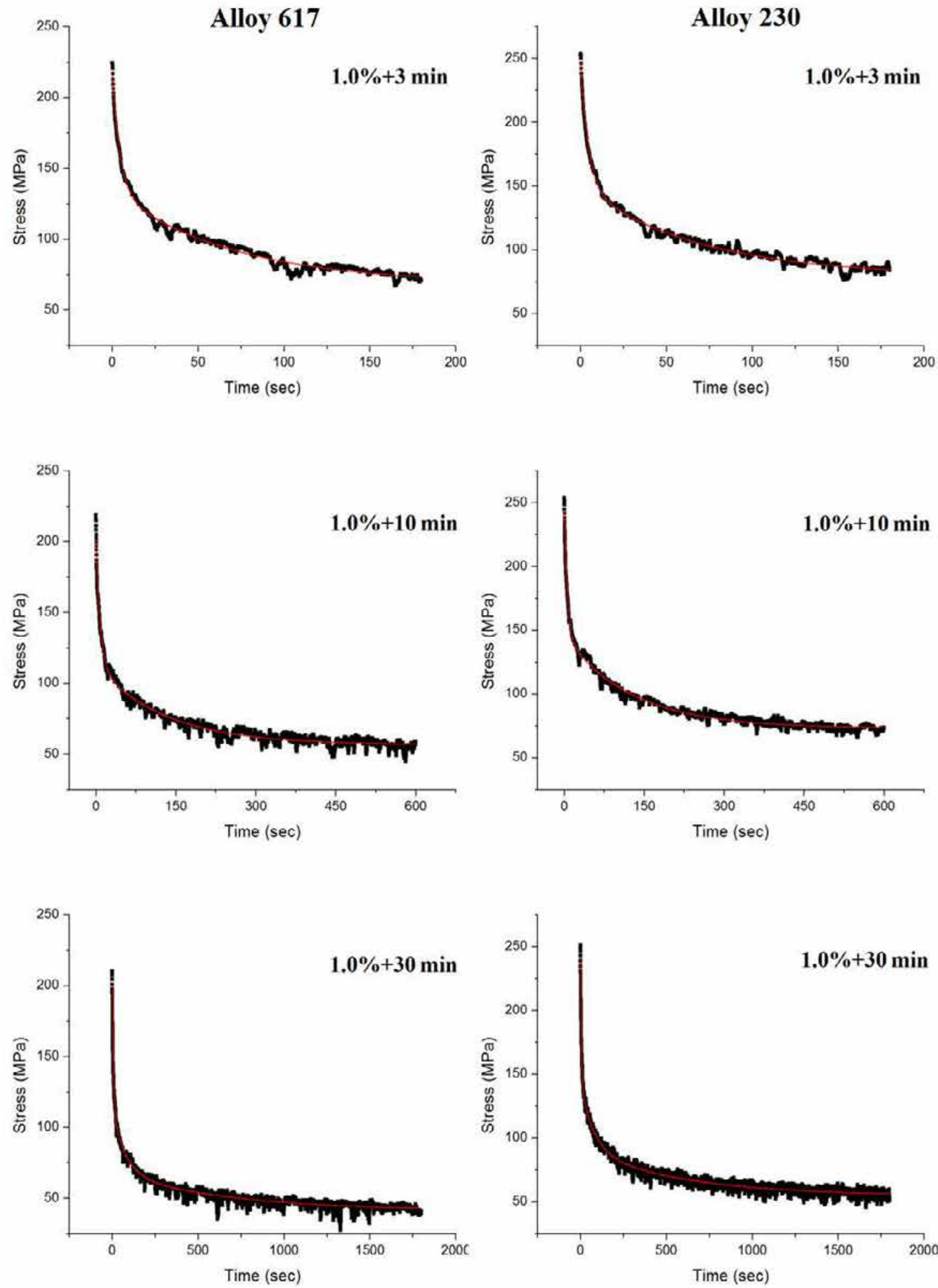

Original data

Fitting curve

Fig. 5.8: Nonlinear curve fitting of the stress relaxation data of Alloy 617 and Alloy 230. Test conditions: $850^{\circ} \mathrm{C}, 1.0 \%$ total strain range, with 3,10 , and 30 minute hold times at peak tensile strain 


\section{Understanding Fundamental Material-Degradation Processes in High Temperature Aggressive Chemomechanical Environments}

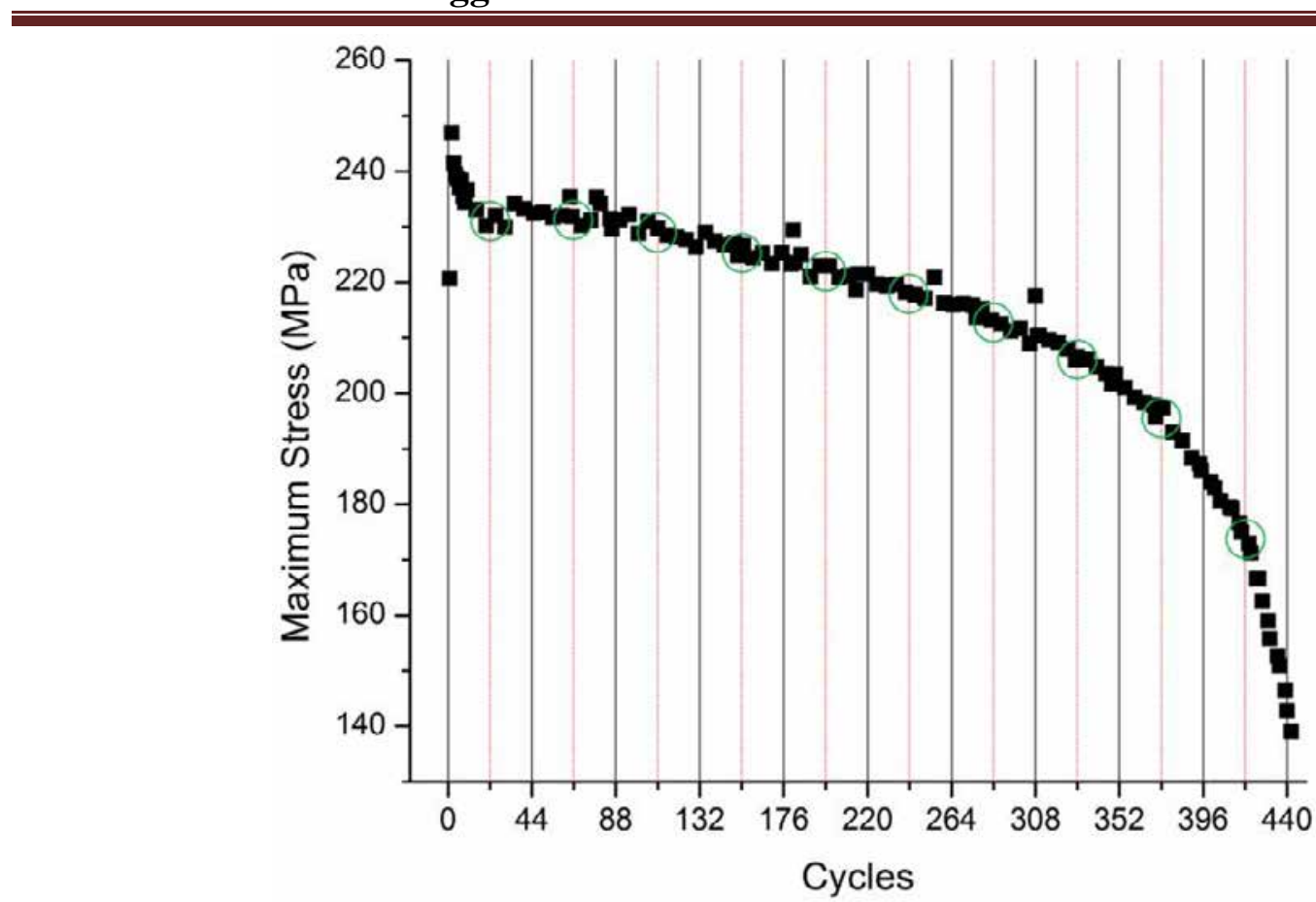

Fig. 5.9: Approximation strategy for creep damage calculation in linear damage summation. The green circles highlight the middle point cycle of each $10 \%$ creep-fatigue life.

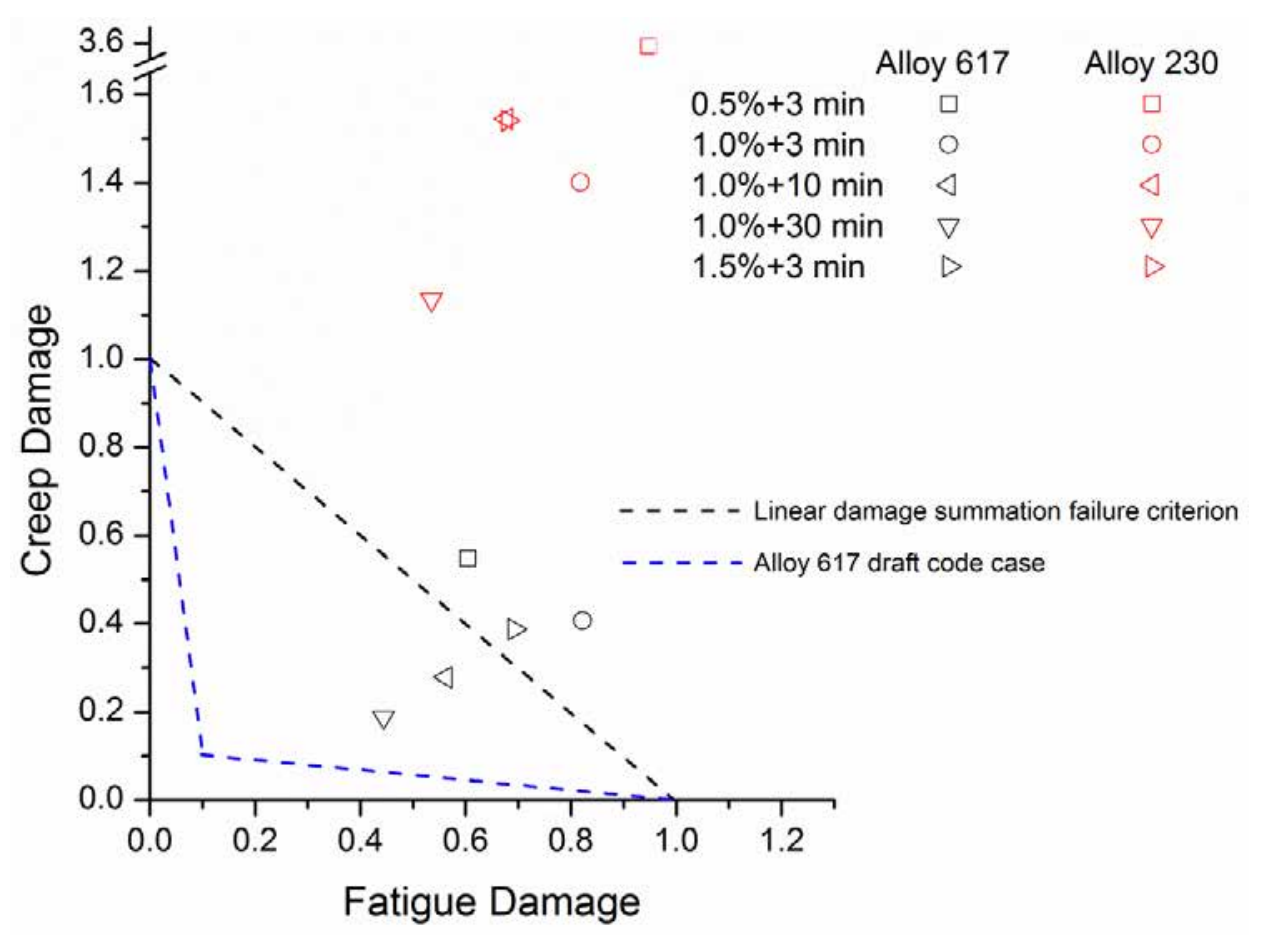

Fig. 5.10: Comparison of creep and fatigue damage endured by Alloy 617 and Alloy 230 based on linear damage summation. The black dashed line shows the ideal failure criterion of linear damage summation, and the bilinear blue dotted line outlines the failure criterion of the draft code case for Alloy 617 . 


\section{Understanding Fundamental Material-Degradation Processes in High Temperature Aggressive Chemomechanical Environments}

Table 5.5: Calculation of constant parameters for the stress relaxation curve

\begin{tabular}{|c|c|c|c|c|c|c|c|c|}
\hline & $\begin{array}{c}\text { Test } \\
\text { condition }\end{array}$ & $\mathrm{a}$ & b & $\mathrm{c}$ & d & $\mathrm{t}_{1}$ & $t_{2}$ & $t_{3}$ \\
\hline & \multirow{10}{*}{$0.5 \%+3 \mathrm{~min}$} & 66.9235 & 76.7032 & 85.2015 & - & 2.48452 & 41.7769 & - \\
\hline & & 58.4056 & 64.7531 & 91.5869 & - & 67.7978 & 5.09041 & - \\
\hline & & 57.6512 & 89.8213 & 66.3139 & - & 4.65815 & 66.8321 & - \\
\hline & & 57.9804 & 62.1852 & 89.0995 & - & 61.5093 & 5.48142 & - \\
\hline & & 40.9412 & 65.9313 & 96.7719 & - & 125.657 & 7.10229 & - \\
\hline & & 57.0533 & 80.5962 & 68.9254 & - & 4.39795 & 51.8995 & - \\
\hline & & 57.3813 & 65.0867 & 76.4287 & - & 49.8716 & 5.39104 & - \\
\hline & & 51.6373 & 84.3034 & 65.8080 & - & 3.97409 & 64.7046 & - \\
\hline & & 54.8547 & 72.5313 & 60.7064 & - & 4.58335 & 46.2101 & - \\
\hline & & 47.4926 & 54.6244 & 66.8704 & - & 54.1940 & 3.77315 & - \\
\hline \multirow{20}{*}{\multicolumn{2}{|c|}{ 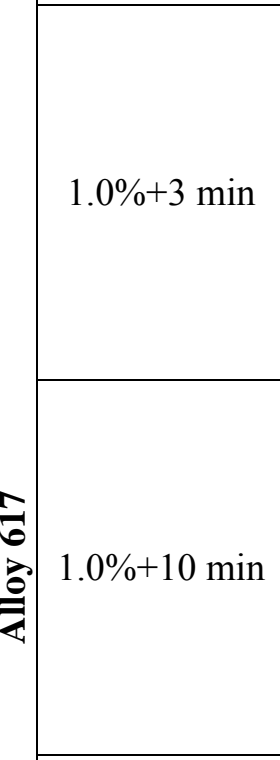 }} & 78.6668 & 84.4461 & 69.8051 & - & 4.23913 & 54.1164 & - \\
\hline & & 76.2370 & 68.0834 & 90.8700 & - & 56.1602 & 3.32007 & - \\
\hline & & 73.5431 & 62.1395 & 90.7977 & - & 63.7531 & 4.52032 & - \\
\hline & & 64.4972 & 56.7269 & 94.8092 & - & 104.757 & 7.45331 & - \\
\hline & & 59.9200 & 92.062 & 63.5167 & - & 6.04264 & 113.685 & - \\
\hline & & 63.8196 & 84.4270 & 64.9594 & - & 5.3908 & 85.6085 & - \\
\hline & & 67.6427 & 53.7650 & 86.6183 & - & 78.7186 & 5.93508 & - \\
\hline & & 62.0595 & 84.1653 & 64.0081 & - & 3.82841 & 80.8989 & - \\
\hline & & 66.3206 & 59.6475 & 74.3624 & - & 52.8718 & 4.23119 & - \\
\hline & & 54.3722 & 54.8441 & 70.4886 & - & 84.7432 & 4.45216 & - \\
\hline & & 54.2226 & 45.7077 & 83.8742 & 41.4074 & 40.8499 & 4.83157 & 318.741 \\
\hline & & 58.0239 & 64.6594 & 68.3579 & 46.4787 & 19.6050 & 1.96069 & 196.294 \\
\hline & & 53.7076 & 68.3724 & 60.5446 & 50.9435 & 19.4713 & 1.68289 & 228.272 \\
\hline & & 57.0560 & 54.6704 & 44.7825 & 65.2532 & 25.0781 & 189.776 & 3.16499 \\
\hline & & -5.4132 & 79.8816 & 58.2972 & 87.3538 & 2118.09 & 62.2330 & 4.7485 \\
\hline & & 51.0236 & 31.0667 & 44.8301 & 80.1799 & 312.611 & 55.713 & 5.55406 \\
\hline & & 51.4786 & 40.5202 & 45.0479 & 76.3305 & 226.798 & 30.1246 & 3.64501 \\
\hline & & 50.8113 & 45.6682 & 52.6051 & 62.9480 & 170.406 & 19.7829 & 2.67519 \\
\hline & & 28.0384 & 51.1107 & 83.0269 & 35.9017 & 68.9421 & 5.31888 & 1271.04 \\
\hline & & 35.5311 & 44.9545 & 31.1413 & 67.3387 & 49.0496 & 484.573 & 4.56132 \\
\hline \multirow{10}{*}{\multicolumn{2}{|c|}{$1.0 \%+30 \mathrm{~min}$}} & 47.7771 & 50.3670 & 31.6897 & 92.2104 & 75.4096 & 599.161 & 5.2833 \\
\hline & & 46.2466 & 49.3954 & 95.4235 & 33.5889 & 67.0065 & 4.90319 & 540.190 \\
\hline & & 44.5568 & 57.7237 & 32.6682 & 84.0884 & 52.2370 & 596.397 & 4.52367 \\
\hline & & 44.4326 & 90.3236 & 52.5029 & 29.3599 & 4.83495 & 64.2234 & 594.207 \\
\hline & & 43.9654 & 30.9434 & 49.6001 & 85.5968 & 521.008 & 58.8115 & 5.32689 \\
\hline & & 41.4042 & 44.0205 & 29.4290 & 86.6871 & 70.8449 & 599.241 & 6.3454 \\
\hline & & 41.5286 & 47.5767 & 26.0768 & 82.1044 & 67.8442 & 603.400 & 6.02395 \\
\hline & & 40.4489 & 44.0031 & 30.7650 & 77.1450 & 52.5647 & 476.965 & 5.86898 \\
\hline & & 38.6112 & 38.1052 & 78.9329 & 26.9764 & 78.3062 & 6.83073 & 574.182 \\
\hline & & 34.8138 & 66.2695 & 5.73506 & 39.6540 & 54.3459 & 26.6125 & 496.650 \\
\hline \multirow{10}{*}{\multicolumn{2}{|c|}{$1.5 \%+3 \mathrm{~min}$}} & 89.4463 & 81.0688 & 80.3851 & - & 65.4790 & 3.66859 & - \\
\hline & & 87.8647 & 86.4286 & 79.2801 & - & 58.2333 & 2.7433 & - \\
\hline & & 86.2982 & 76.4799 & 88.7411 & - & 2.40065 & 55.8717 & - \\
\hline & & 87.4713 & 83.9667 & 75.4658 & - & 49.7735 & 2.66056 & - \\
\hline & & 84.7533 & 74.8692 & 78.3067 & - & 3.33482 & 55.2510 & - \\
\hline & & 78.9089 & 77.5344 & 80.1326 & - & 61.9247 & 3.11235 & - \\
\hline & & 80.4226 & 65.0856 & 80.0888 & - & 3.0984 & 48.7171 & - \\
\hline & & 76.4341 & 78.0664 & 73.4967 & - & 3.07539 & 54.6234 & - \\
\hline & & 70.7537 & 71.4445 & 70.2414 & - & 3.91721 & 65.1359 & - \\
\hline & & 65.3421 & 65.0535 & 65.5611 & - & 62.0513 & 3.39642 & - \\
\hline
\end{tabular}




\section{Understanding Fundamental Material-Degradation Processes in High Temperature Aggressive Chemomechanical Environments}

Table 5.5 Cont.

\begin{tabular}{|c|c|c|c|c|c|c|c|c|}
\hline & & 81.9196 & 69.8288 & 60.9954 & - & 98.1425 & 6.91185 & - \\
\hline & & 81.7505 & 79.4697 & 91.4698 & - & 55.4559 & 4.82476 & - \\
\hline & & 79.2552 & 62.3509 & 98.0993 & - & 68.2895 & 6.19061 & - \\
\hline & & 73.9438 & 91.7915 & 74.0469 & - & 4.42399 & 68.9855 & - \\
\hline & $050+3 \mathrm{~min}$ & 75.2037 & 71.3265 & 92.8428 & - & 59.7608 & 4.69961 & - \\
\hline & $0.5 \%+3 \mathrm{~min}$ & 72.1419 & 98.0900 & 69.6091 & - & 4.2983 & 68.6672 & - \\
\hline & & 66.0366 & 100.851 & 60.5035 & - & 6.72655 & 99.1481 & - \\
\hline & & 73.2587 & 91.5025 & 58.3623 & - & 6.50001 & 69.8591 & - \\
\hline & & 72.1602 & 93.0125 & 60.0198 & - & 5.06739 & 61.1624 & - \\
\hline & & 63.8231 & 77.3887 & 54.7674 & - & 5.80132 & 66.9796 & - \\
\hline & & 85.5442 & 103.009 & 74.2744 & - & 5.59763 & 69.9792 & - \\
\hline & & 84.1548 & 101.259 & 73.7036 & - & 5.01217 & 71.7106 & - \\
\hline & & 82.1777 & 93.5633 & 69.8802 & - & 6.69094 & 74.0286 & - \\
\hline & & 81.4438 & 67.8524 & 101.361 & - & 75.5353 & 5.25381 & - \\
\hline & $10 \%+3 \mathrm{~min}$ & 81.8663 & 93.9937 & 71.5977 & - & 4.66997 & 63.2927 & - \\
\hline & $1.0 \%+3 \mathrm{~min}$ & 75.9759 & 68.5102 & 97.2049 & - & 78.8596 & 5.29095 & - \\
\hline & & 80.8656 & 66.7022 & 89.5577 & - & 56.5050 & 4.65357 & - \\
\hline & & 74.1157 & 86.4657 & 63.9037 & - & 5.147 & 72.8003 & - \\
\hline & & 71.4473 & 58.9718 & 72.7353 & - & 64.8439 & 5.59538 & - \\
\hline & & 63.2805 & 53.3947 & 57.9163 & - & 72.4416 & 6.51392 & - \\
\hline & & 73.9798 & 81.1200 & 68.5116 & 68.0852 & 15.4848 & 2.14631 & 160.453 \\
\hline & & 68.8164 & 52.3892 & 55.6403 & 92.8505 & 31.2478 & 210.635 & 4.43149 \\
\hline & & 54.9525 & 98.9328 & 61.1743 & 48.6016 & 4.31982 & 50.1155 & 505.881 \\
\hline ले & & 67.4785 & 62.5853 & 82.5515 & 54.2358 & 24.5612 & 2.77681 & 203.287 \\
\hline & $10 \%+10 \mathrm{~min}$ & 64.9742 & 92.5370 & 37.1256 & 57.6983 & 4.3884 & 358.303 & 66.4869 \\
\hline & $1.0 \%+10 \mathrm{~min}$ & 4.45273 & 88.5159 & 65.9459 & 90.3921 & 3.71385 & 58.1901 & 1703.37 \\
\hline & & 17.3958 & 72.5786 & 64.2627 & 84.5479 & 1471.10 & 62.1116 & 4.16295 \\
\hline & & -124374 & 61.2806 & 82.5335 & 124460. & 68.2005 & 4.18234 & 3257840 \\
\hline & & -128701 & 84.2354 & 60.3440 & 128780 . & 4.10601 & 82.0631 & 5045570 \\
\hline & & 52.1741 & 75.5146 & 50.6591 & 30.0448 & 3.65194 & 66.1432 & 440.122 \\
\hline & & 57.5019 & 104.352 & 63.3849 & 47.4498 & 5.99265 & 64.2170 & 575.784 \\
\hline & & 56.0041 & 104.557 & 60.2152 & 38.7441 & 5.47326 & 80.3709 & 666.074 \\
\hline & & 57.7212 & 39.9867 & 59.1257 & 97.8587 & 544.907 & 64.6960 & 5.42348 \\
\hline & & 56.3895 & 36.8810 & 54.6678 & 102.457 & 587.059 & 73.9975 & 5.76099 \\
\hline & $0 \min$ & 54.3374 & 33.7266 & 58.5745 & 103.385 & 747.460 & 83.2239 & 5.37909 \\
\hline & $1.0 \% 0^{-}$ & 54.4738 & 99.6585 & 52.3788 & 36.8859 & 5.62583 & 75.3919 & 588.194 \\
\hline & & 54.1966 & 53.9913 & 36.8956 & 94.5415 & 77.6786 & 602.719 & 4.68877 \\
\hline & & 52.6770 & 92.2411 & 54.2239 & 37.4629 & 4.83836 & 74.4125 & 625.213 \\
\hline & & 52.0710 & 54.1218 & 87.8317 & 36.8777 & 70.2683 & 4.54271 & 539.746 \\
\hline & & 47.6386 & 54.5022 & 34.4125 & 80.4864 & 65.6892 & 642.572 & 4.62933 \\
\hline & & 96.4182 & 100.903 & 84.4136 & - & 5.27727 & 67.0094 & - \\
\hline & & 91.3813 & 105.799 & 80.4304 & - & 5.6956 & 81.8214 & - \\
\hline & & 96.9036 & 82.1848 & 98.2492 & - & 62.3612 & 4.93073 & - \\
\hline & & 96.2506 & 80.4252 & 92.4812 & - & 63.4945 & 5.49766 & - \\
\hline & & 93.9585 & 98.9064 & 81.3010 & - & 4.52093 & 66.6817 & - \\
\hline & $1.5 \%+3 \mathrm{~min}$ & 97.2429 & 82.1931 & 93.5802 & - & 60.1521 & 4.49967 & - \\
\hline & & 96.8933 & 89.1726 & 84.9193 & - & 4.30469 & 58.6575 & - \\
\hline & & 93.9592 & 85.2088 & 84.3718 & - & 4.43927 & 58.9906 & - \\
\hline & & 93.0719 & 90.5634 & 80.5209 & - & 4.15076 & 57.4465 & - \\
\hline & & 88.1119 & 80.7591 & 76.5922 & - & 55.1637 & 3.8653 & 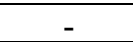 \\
\hline
\end{tabular}


Understanding Fundamental Material-Degradation Processes in High Temperature Aggressive Chemomechanical Environments

Table 5.6 Creep-fatigue damage calculation for Alloy 617 and Alloy 230 based on linear damage summation

\begin{tabular}{|c|c|c|c|c|}
\hline Materials & Test Condition & Creep Damage & Fatigue damage & Total Damage \\
\hline \multirow{4}{*}{ Alloy 617} & $0.5 \%+3 \mathrm{~min}$ & 0.548 & 0.605 & 1.153 \\
\cline { 2 - 5 } & $1.0 \%+3 \mathrm{~min}$ & 0.407 & 0.822 & 1.229 \\
\cline { 2 - 5 } & $1.0 \%+10 \mathrm{~min}$ & 0.278 & 0.562 & 0.840 \\
\cline { 2 - 5 } & $1.0 \%+30 \mathrm{~min}$ & 0.188 & 0.444 & 0.632 \\
\cline { 2 - 5 } & $1.5 \%+3 \mathrm{~min}$ & 0.387 & 0.692 & 1.079 \\
\hline \multirow{5}{*}{ Alloy 230 } & $0.5 \%+3 \mathrm{~min}$ & 3.580 & 0.948 & 4.528 \\
\cline { 2 - 5 } & $1.0 \%+3 \mathrm{~min}$ & 1.401 & 0.818 & 2.219 \\
\cline { 2 - 5 } & $1.0 \%+10 \mathrm{~min}$ & 1.545 & 0.677 & 2.222 \\
\cline { 2 - 5 } & $1.0 \%+30 \mathrm{~min}$ & 1.135 & 0.535 & 1.670 \\
\cline { 2 - 5 } & $1.5 \%+3 \mathrm{~min}$ & 1.541 & 0.680 & 2.221 \\
\hline
\end{tabular}

\subsubsection{Frequency-modified tensile hysteresis energy}

The mid-life LCF test results of both materials were used to fit the cyclic strain hardening exponent $n^{\prime}$ and cyclic strength coefficient $K^{\prime}$ from the equation:

$\frac{\Delta \varepsilon}{2}=\frac{\Delta \sigma}{2 E}+\left(\frac{\Delta \sigma}{2 K^{\prime}}\right)^{\frac{1}{n^{\prime}}}$

Then, calculation of the tensile hysteresis energy per cycle, $\Delta U_{i n}$, was done based on the following equation:

$\Delta U_{\text {in }}=\alpha \cdot \sigma_{T} \cdot \Delta \varepsilon_{\text {in }}$

The results from equation 5.9 were then substituted into the following equation, along with the LCF life data $N_{f}$ to fit the material constants $\beta$ and $C_{1}$ :

$\Delta U_{\text {in }} \cdot\left(N_{f}\right)^{\beta}=C_{1}$

All the original input data, intermediate calculation results, and final fitting results are listed in Table 5.7. Afterwards, creep-fatigue data at $1 \%$ total strain range with 3,10 , and 30 minute hold times were used to calculate the material constants in the following equation:

$\Delta U_{\text {in }} \cdot\left(N_{f} \cdot v^{k-1}\right)^{\beta}=C_{2}$ 


\section{Understanding Fundamental Material-Degradation Processes in High Temperature Aggressive Chemomechanical Environments}

The results are shown in Table 5.8. The last step of creep-fatigue life estimation for the creep-fatigue tests of $0.5 \%$ total strain range with 3 minute hold times and $1.5 \%$ total strain range with 3 minute hold times involved computing $\Delta U_{i n}$ and $v$ at these two conditions, and substituting them into equation 5.11 to yield the estimated creep-fatigue life $N_{f_{\text {estimate }}}$. The comparison between the actual and estimated creep-fatigue life is included in Table 5.9. The frequency-modified tensile hysteresis energy modeling showed promising results, as the difference between the predicted and actual creep-fatigue life was less than $30 \%$. In general, if the fatigue life predicted by a model is within a factor of two of the actual fatigue life, the model is considered to be satisfactory [4.39]. Although more creep-fatigue tests at different experimental conditions, such as temperature, strain rate, total strain range, dwell time, etc., are needed to further evaluate the frequency-modified tensile hysteresis energy method, in this study, this model showed a superior prediction accuracy for the elevated temperature creep-fatigue life of Alloy 617 and Alloy 230.

Table 5.7: Constant fitting based on mid-life LCF data for frequency-modified tensile hysteresis energy modeling

\begin{tabular}{|c|c|c|c|c|c|c|}
\hline & \multicolumn{3}{|c|}{ Alloy 617} & \multicolumn{3}{|c|}{ Alloy 230} \\
\hline$\Delta \varepsilon(\%)$ & 0.5 & 1.0 & 1.5 & 0.5 & 1.0 & 1.5 \\
\hline $\mathrm{E}(\mathrm{GPa})^{*}$ & \multicolumn{3}{|c|}{153} & \multicolumn{3}{|c|}{160.5} \\
\hline$\Delta \sigma(\mathrm{MPa})$ & 465.612 & 485.785 & 491.264 & 549.946 & 569.168 & 600.524 \\
\hline$\sigma_{T}(\mathrm{MPa})$ & 230.575 & 233.812 & 237.027 & 272.232 & 276.579 & 288.827 \\
\hline$\Delta \varepsilon_{\text {in }}(\%)$ & 0.2592 & 0.7697 & 1.2321 & 0.2060 & 0.7000 & 1.1948 \\
\hline$\Delta U_{i n}(\mathrm{MPa})$ & 0.5797 & 1.7458 & 2.8328 & 0.5357 & 1.8492 & 3.2963 \\
\hline$N_{f}$ & 2280 & 755 & 480 & 2420 & 824 & 500 \\
\hline$n^{\prime}$ & \multicolumn{3}{|c|}{0.0309} & \multicolumn{3}{|c|}{0.0469} \\
\hline$\alpha^{* *}$ & \multicolumn{3}{|c|}{0.9700} & \multicolumn{3}{|c|}{0.9552} \\
\hline$K^{\prime}$ & \multicolumn{3}{|c|}{288.5604} & \multicolumn{3}{|c|}{379.9100} \\
\hline$\beta$ & \multicolumn{3}{|c|}{1.0143} & \multicolumn{3}{|c|}{1.1519} \\
\hline$C_{1}$ & \multicolumn{3}{|c|}{1470.5168} & \multicolumn{3}{|c|}{4232.0423} \\
\hline
\end{tabular}

*E is the material's Young's modulus at $850^{\circ} \mathrm{C}$

${ }^{* *} \alpha$ is calculated as $\alpha=1 /\left(1+n^{\prime}\right)$ for both materials 


\section{Understanding Fundamental Material-Degradation Processes in High Temperature Aggressive Chemomechanical Environments}

Table 5.8: Constant fitting based on the mid-life data of creep-fatigue tests at $1.0 \%$ total strain range with varied strain hold times for frequency-modified tensile hysteresis energy modeling

\begin{tabular}{|c|c|c|c|c|c|c|}
\hline & \multicolumn{3}{|c|}{ Alloy 617} & \multicolumn{3}{|c|}{ Alloy 230} \\
\hline $\begin{array}{l}\text { Hold time } \\
\text { (min) }\end{array}$ & 3 & 10 & 30 & 3 & 10 & 30 \\
\hline$\sigma_{T}(\mathrm{MPa})$ & 224.869 & 221.519 & 210.199 & 256.747 & 254.528 & 251.019 \\
\hline$\Delta \varepsilon_{i n}(\%)$ & 0.8233 & 0.8348 & 0.8394 & 0.8021 & 0.8224 & 0.8304 \\
\hline$\Delta U_{i n}(\mathrm{MPa})$ & 1.7958 & 1.7939 & 1.7116 & 1.9671 & 1.9995 & 1.9910 \\
\hline$N_{f}$ & 644 & 440 & 348 & 674 & 558 & 441 \\
\hline$v(1 / s)$ & $3.8462 \mathrm{E}-3$ & $1.4706 \mathrm{E}-3$ & $5.3191 \mathrm{E}-4$ & $3.8462 \mathrm{E}-3$ & $1.4706 \mathrm{E}-3$ & $5.3191 \mathrm{E}-4$ \\
\hline$C_{2}$ & \multicolumn{3}{|c|}{8199.172} & \multicolumn{3}{|c|}{13775.901} \\
\hline$k$ & \multicolumn{3}{|c|}{0.6655} & \multicolumn{3}{|c|}{0.7906} \\
\hline
\end{tabular}

Table 5.9: Comparison of the actual creep-fatigue life with the creep-fatigue life estimation from frequency-modified tensile hysteresis energy modeling

\begin{tabular}{|c|c|c|c|c|}
\hline & \multicolumn{2}{|c|}{ Alloy 617} & \multicolumn{2}{c|}{ Alloy 230} \\
\hline Test condition & $0.5 \%+3 \mathrm{~min}$ & $1.5 \%+3 \mathrm{~min}$ & $0.5 \%+3 \mathrm{~min}$ & $1.5 \%+3 \mathrm{~min}$ \\
\hline$\Delta \varepsilon_{\text {in }}(\%)$ & 0.3640 & 1.2963 & 0.3174 & 1.3083 \\
\hline$\sigma_{T}(\mathrm{MPa})$ & 212.825 & 234.537 & 240.514 & 279.251 \\
\hline$\Delta U_{\text {in }}(\mathrm{MPa})$ & 0.7515 & 2.9492 & 0.7291 & 3.4897 \\
\hline$v(1 / s)$ & $4.5455 \mathrm{E}-3$ & $3.3333 \mathrm{E}-3$ & $4.5455 \mathrm{E}-3$ & $3.3333 \mathrm{E}-3$ \\
\hline$N_{f_{-} \text {actual }}$ & 1380 & 332 & 2295 & 340 \\
\hline$N_{f_{-} \text {estimate }}$ & 1575 & 369 & 1667 & 401 \\
\hline Difference $(\%)^{*}$ & 14.1 & 11.1 & 27.4 & 17.9 \\
\hline
\end{tabular}

$*$ Difference(\%) $=\frac{\left|N_{f_{\text {estimate }}}-N_{f_{\text {actual }}}\right|}{N_{f_{\text {actual }}}} \times 100 \%$ 


\section{Understanding Fundamental Material-Degradation Processes in High Temperature}

Aggressive Chemomechanical Environments

\subsection{Deformation Mechanisms of Creep and Fatigue}

In LCF tests of both materials, as the total strain range increased, deformation would primarily occur near random HAGBs, since the test temperature was above the material's equicohesive temperature and the strength of the GBs was lower than the strength of the grain itself $[4.5,4.65]$. The appearances of LAGBs near original random HAGBs, especially at large total strain ranges, confirmed the localized deformation on random HAGBs, as illustrated in Figs. 4.103 and 4.109. In creep-fatigue tests, due to the peak tensile strain hold period, additional creep damage was introduced to both materials. In order to investigate the exact deformation mechanisms responsible for the creep damage during the strain hold period, the deformation map most relevant to the studied materials (Alloys 617 and 230) was identified, and is shown in Fig. 5.11. The deformation map was derived from a Ni-Cr-TiO2 alloy, which is a Ni-based alloy with a grain size of $200 \mu \mathrm{m}$ that combines solution and dispersion hardening. Alloys 617 and 230 are also solid-solution strengthened, with an additional precipitation strengthening contribution. However, precipitates in Alloys 617 and 230 were coarse, and hence behaved like dispersions [5.22]. Therefore, the deformation map of Ni-Cr-TiO2 should be representative of the deformation behavior in Alloys 617 and 230. Based on the stress relaxation data from the mid-life cycle of each creep-fatigue test, the range of normalized shear stress to which the materials were subject could be calculated. This is presented as the red zone in Fig. 5.11.

During the first few seconds of the strain hold period, the stress level was high and dislocation glide-controlled flow, shown as the plasticity region in Fig. 5.11, was the main mechanism for the material deformation. As the stress relaxation continued, the stress level reduced dramatically, and then decreased relatively slowly for the remaining strain hold time. At low stress levels, the material entered the high-temperature power-law creep region in Fig. 5.11, and the main deformation mechanism was dislocation climb-controlled creep. Due to the high test temperature $(\mathrm{T} / \mathrm{TM} \approx 0.7)$, at the atomic level, the dislocation climb was controlled by the diffusive motion of vacancies to or from the climbing dislocation through the lattice [5.22]. The dislocation climb-controlled creep process during the strain hold period in creep-fatigue testing encouraged dislocation bypassing of precipitate obstacles inside grains. This explains why there were fewer LAGBs pinned down by precipitates in creep-fatigue tested Alloy 230 specimens than in LCF tested Alloy 230 specimens (comparing Fig. 4.109 (b) and (d)).

Another important creep deformation mechanism is called grain boundary sliding. It is generally thought to occur by GB dislocation motion. In Fig. 5.11, there is no region corresponding to grain boundary sliding, since uncertainties exist regarding the suitable constitutive equation for this mechanism [4.5]. However, recent studies have shown that the dislocation creep field can be subdivided, with a grain boundary sliding contribution existing at the lower stress levels [4.5]. Inasmuch as the material stress level was relatively low for most of the strain dwell time, grain boundary sliding was expected to be operational during the stress relaxation period. Indeed, abundant LAGBs formed near original random HAGBs after creep-fatigue tests of Alloy 617 and 230, as seen in Figs. 4.103 and 4.109. In addition, grain boundary sliding processes must be accompanied by other deformation modes in order to maintain grain contiguity [4.5]. There are two major deformation processes that are normally affiliated with grain boundary sliding: 1) diffusional creep at high temperatures, and 2) dislocation creep, involving the glide and climb of lattice dislocations, at lower temperatures and elevated stresses [4.5]. If neither deformation processes are operable, GB decohesion would develop by the formation of a planar array of GB cavities which eventually coalesce to form GB 


\section{Understanding Fundamental Material-Degradation Processes in High Temperature Aggressive Chemomechanical Environments}

cracks. This should explain the occurrence of intergranular cracks inside Alloys 617 and 230 after creep-fatigue tests, as shown in Figs. 4.97 and 4.99.

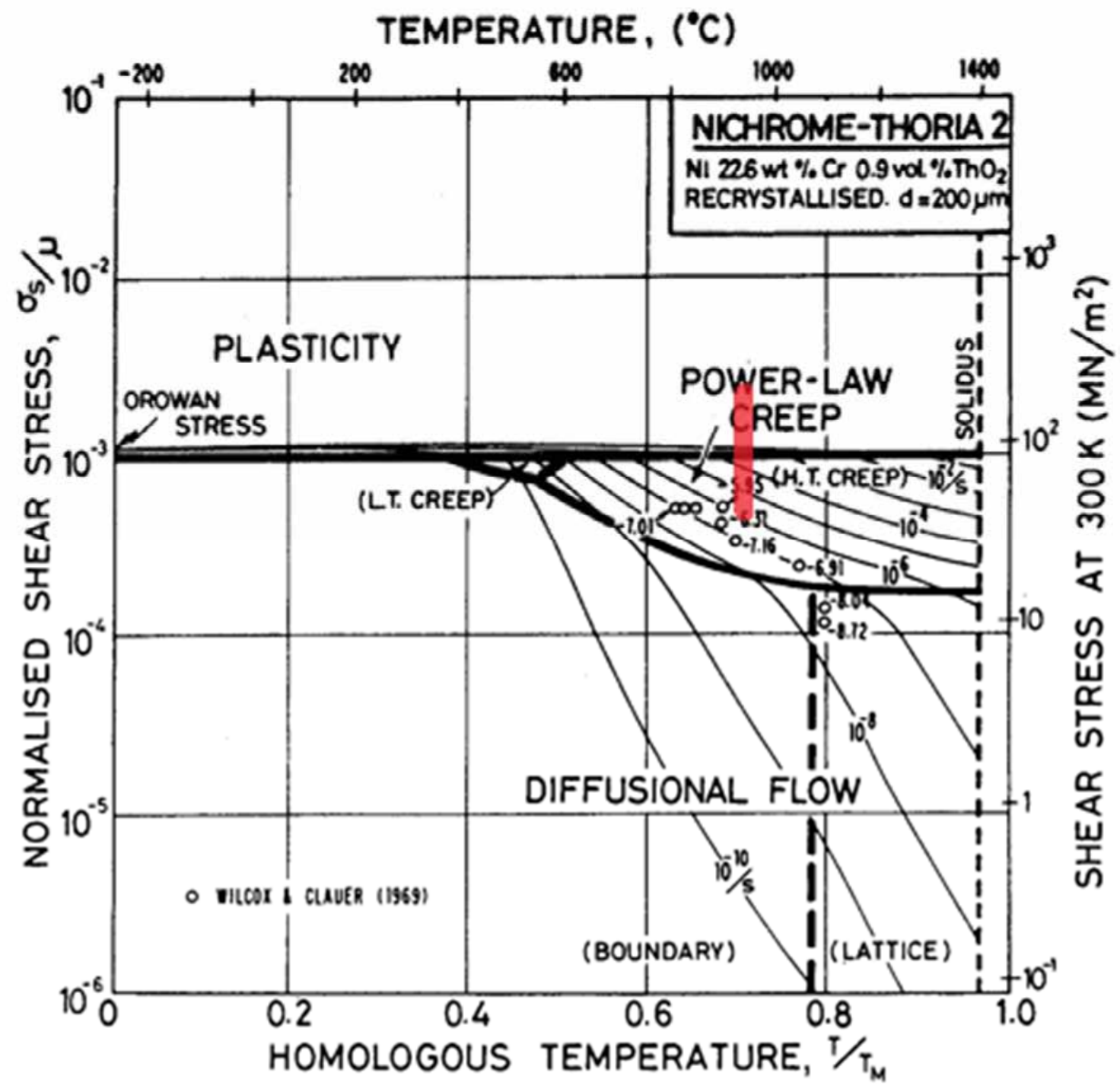

Fig. 5.11: Deformation mechanism map for a Ni-Cr- $\mathrm{ThO}_{2}$ alloy. The red area corresponds to all possible stress and temperature states of Alloy 617 and 230 during the stress relaxation period (after Ref.[5.22])

Despite the similarities in the deformation mechanisms of both materials described in the previous paragraph, some differences in the distribution of LAGBs exist for Alloys 617 and 230, as shown in Figs. 4.103 and 4.109. In Alloy 230, the fraction of precipitates inside the material was higher than that in Alloy 617, based on the 3D SEM montage shown in Fig. 4.76. The major difference in alloying elements for Alloys 617 and 230, namely, Co for Alloy 617 and W for Alloy 230, might cause a relatively lower stacking fault energy in Alloy 230, since the electron vacancy number of $\mathrm{W}$ is much higher than that of $\mathrm{Co}$, and the stacking fault energy of FCC alloys is known to decrease with increasing electron vacancy number of the alloy elements [4.57, 3.45]. Due to the large fraction of carbide precipitates and the reduced dislocation mobility in conjunction with the low stacking fault energy, for LCF tests with smaller total strain ranges, i.e., 


\section{Understanding Fundamental Material-Degradation Processes in High Temperature Aggressive Chemomechanical Environments}

$0.5 \%$ and $1.0 \%$, dislocation motion in the grain was greatly impeded by intragranular precipitates of Alloy 230, and hence many LAGBs formed as numerous small spots throughout the specimen, as seen in Fig. 4.109(b). In contrast, no apparent LAGBs were observed inside the grain at the same test conditions for Alloy 617, as shown in Fig. 4.103(b). This observation most likely occurred because, in Alloy 617, there were fewer intragranular precipitations and the greater amount of mobile dislocations could bypass the precipitate obstacle via cross-slip or climb.

For the LCF test with 1.5\% total strain range, high stress levels assisted the dislocations in bypassing the precipitates, causing less LAGB spots to be observed inside the grain in Alloy 230, as demonstrated in Fig. 4.109(c). It is worth noting that, in Alloy 230, LAGBs preferred to segregate to random HAGBs of small grains rather than to large grains. Two factors might lead to this phenomenon. First, areas composed of small grains were enriched with precipitates which obstructed dislocation movement and caused local plasticity. Second, small grain regions contained more GBs than large grain regions per unit area, and since GBs were not as strong as grains when the test temperature was above the material's equicohesive temperature, more deformation was expected to take place in the small grain region.

\subsection{Influence of Material Properties on LCF and the Creep-fatigue Life}

\subsubsection{Precipitation}

Alloy 230 had a larger volume fraction of carbide precipitates than Alloy 617 in the as-received state. These carbide precipitates in Alloy 230 also appeared to be coarser. The large fraction of precipitates was favorable in pinning down dislocation movement, and the coarse precipitate particles helped to disperse slip, so the material deformed more homogeneously [4.57]. This can be seen when comparing the LAGB distribution in Alloys 617 and 230 at the same test conditions, as shown in Figs. 4.103 and 4.109. For the LCF test with 1.5\% total strain range and for most of the creep-fatigue tests, the LAGB distribution was more localized and segregated to random HAGBs in Alloy 617, while existing inside the grain or around large precipitate particles in Alloy 230. Slip dispersal can promote improved fatigue properties when the fracture is entirely transgranular [4.47]. As presented in Table 4.12, the fracture modes of Alloys 617 and 230 in LCF tests were completely transgranular, as was the crack propagation in both of these alloys in most of the creep-fatigue tests. Hence, the high precipitation volume fraction and the coarse carbide precipitates in Alloy 230 should contribute to the longer LCF and creep-fatigue life of Alloy 230 over Alloy 617.

Cellular precipitates that formed by discontinuous precipitation reactions were found in Alloy 230 after the material was exposed to the elevated temperature of $850^{\circ} \mathrm{C}$. The reaction is of interest because it usually has a significant effect on the mechanical, physical, and chemical properties of many commercial alloys, and may cause such deleterious effects as significant decreases in the high-temperature ductility, rapid initiation of GB cracks, and creep cavitation [4.61]. In fact, in Alloy 230, surface crack growth along GBs with cellular precipitates was observed when tests were done at 1.5\% total strain range (Fig. 4.92 and Fig. 4.98(b)), and material interior intergranular cracking on GBs with cellular precipitates was observed in creep-fatigue tests (Fig. 4.99). Nevertheless, the GB cellular structure of $\mathrm{M}_{23} \mathrm{C}_{6}$ can be avoided 


\section{Understanding Fundamental Material-Degradation Processes in High Temperature Aggressive Chemomechanical Environments}

by appropriate heat treatment and chemistry control, with the result of further improving the mechanical properties of Alloy 230.

\subsubsection{Oxidation resistance}

There is clear evidence from various sources that Alloy 230 shows better oxidation resistance than Alloy 617 at elevated temperatures [5.23, 3.45, 5.24]. For LCF and creep-fatigue tests at high temperatures, time-dependent damage, such as oxidation, cannot be ignored. Existing literatures have also indicated that Alloy 617 has a higher fatigue life in inert environments (helium) than in air during high temperature LCF tests [4.53, 5.25]. Oxidation, like an increased creep component, acts to promote intergranular cracking and can have a variety of influences on the mode and rate of fatigue cracking [4.47]. As a fatigue life is consumed in crack initiation and propagation, the effect of oxidation on fatigue life can be subdivided into the effects on crack initiation and propagation. In the crack initiation stage, random HAGBs were found to be the preferential paths for oxidation due to higher diffusion rates of elements on random HAGBs. At $850^{\circ} \mathrm{C}$, for relatively shorter tests, such as LCF tests with normal or high strain rates, the oxidation on the material surface is negligible, and classic transgranular crack initiation and propagation dominates in both materials, as shown in Table 4.12. However, if GB oxidation is promoted, such as in creep-fatigue tests or LCF tests with low strain rates, fatigue cracks can readily initiate in the GB oxides since most oxides are intrinsically brittle, which leads to early intergranular crack initiation. This is exactly the case for the creep-fatigue tests with 3 minute dwell times at peak tensile strain of Alloy 617, which are also recorded in Table 4.12.

In contrast, cracks still initiated transgranularly in the more oxidation-resistant Alloy 230, even under the 3 minute dwell time creep-fatigue tests (GB embrittlement due to the presence of cellular precipitates may cause intergranular crack initiation in Alloy 230 under the creep-fatigue test with $1.5 \%$ total strain range and a 3 minute strain hold time). In the crack propagation stage, the influence of oxidation on the crack growth rate depends on the relative size of the oxidation-affected zone and the fatigue damage zone in the absence of oxygen (such as fatigue tests in vacuum) per cycle. If the cyclic fatigue damage zone is larger than the oxidation-affected zone per cycle, then the crack propagation mode and rate will be relatively unchanged by oxidation and vice versa [4.47]. For the creep-fatigue test of Alloy 617 at $0.5 \%$ total strain range with a 3 minute strain dwell time, because of the low cyclic stress level in conjunction with the $0.5 \%$ total strain range, the cyclic fatigue damage zone was small, and the increment of crack growth in each cycle might be controlled by the size of the oxidation-affected zone at the crack tip. Oxygen diffusion ahead of the crack tip was the possible cause of the weakening of the GB, which lead to intergranular propagation and resulted in a faster crack growth rate and the largest reduction in fatigue life among all creep-fatigue tests of Alloy 617 [4.48].

This process may be viewed as a similar case of SCC [4.47]. For Alloy 617 under creep-fatigue tests at $1.0 \%$ or $1.5 \%$ total strain range with 3 minute dwell times, the cyclic fatigue damage dominated over the oxidation damage, and hence cracks propagated transgranularly, causing the negative impact of oxidation to be limited. For creep-fatigue tests at longer dwell times, i.e. 10 and 30 minutes, the oxidation-affected zones were larger than their counterparts at shorter dwell times because the materials had more soak time at the elevated temperature. Therefore, in the very early stages of crack growth, cracks propagated intergranularly through the first two or three grains. As cracks advanced into the material, the cyclic fatigue damage zone enlarged due to increases in the stress intensity (a function of crack 


\section{Understanding Fundamental Material-Degradation Processes in High Temperature Aggressive Chemomechanical Environments}

length). After attaining a critical crack depth, the cyclic fatigue damage surpassed the oxidation damage, which caused further growth of the cracks in directions normal to the applied stress rather than the preferential paths of oxygen diffusion, e.g. random HAGBs. This explains the occurrence of a mixed crack propagation mode in Alloy 617 during the creep-fatigue tests with 10 and 30 minute strain hold times. The same rule also applies to Alloy 230 during creep-fatigue tests. However, the oxidation-affected zone should be considerably smaller as a result of the better oxidation resistance of Alloy 230. Therefore, the crack propagation mode was transgranular for all the creep-fatigue test conditions, which may explain the better creep-fatigue performance of Alloy 230 over Alloy 617, especially in the creep-fatigue test at $0.5 \%$ total strain range with a 3 minute strain hold time and in creep-fatigue tests at $1.0 \%$ total strain range with 10 and 30 minute strain hold times.

\subsection{Biaxial Creep Mechanism Analysis}

The creep deformation of Alloy 617 and Alloy 230 shown in Figs. 4.118 and 4.121 indicates that the tertiary creep regime constitutes the major portion of the creep life for all samples tested at $900^{\circ} \mathrm{C}$, which is in accord with Tung's observations at $850^{\circ} \mathrm{C}$ and $950^{\circ} \mathrm{C}$ [5.26]. With increasing applied stress, the time duration of the secondary regime decreases. For example, in Fig. 4.122 (a)-(d), for Alloy 230, the time duration of the secondary regime decreases from a few hundred hours to a few hours with increasing applied stress. Similarly, by comparing the same stress at different temperatures, it can be seen that the time duration of the secondary regime decreases with increasing temperature. The stress exponent can be then calculated by the following equation:

$\dot{\varepsilon}_{s}=A\left(\frac{\sigma}{E}\right)^{n} \exp \left(-\frac{Q}{R T}\right)$

where $A$ is a constant, $n$ is the stress exponent, $\sigma$ is the external stress, $E$ is the elastic modulus of the material, $R$ is the gas constant, and $Q$ is the activation energy characterizing the creep process. The stress exponent for both Alloy 617 and Alloy 230 at $900^{\circ} \mathrm{C}$, calculated by equation 5.12, are shown in Fig. 5.12 and Fig. 5.13, respectively. 


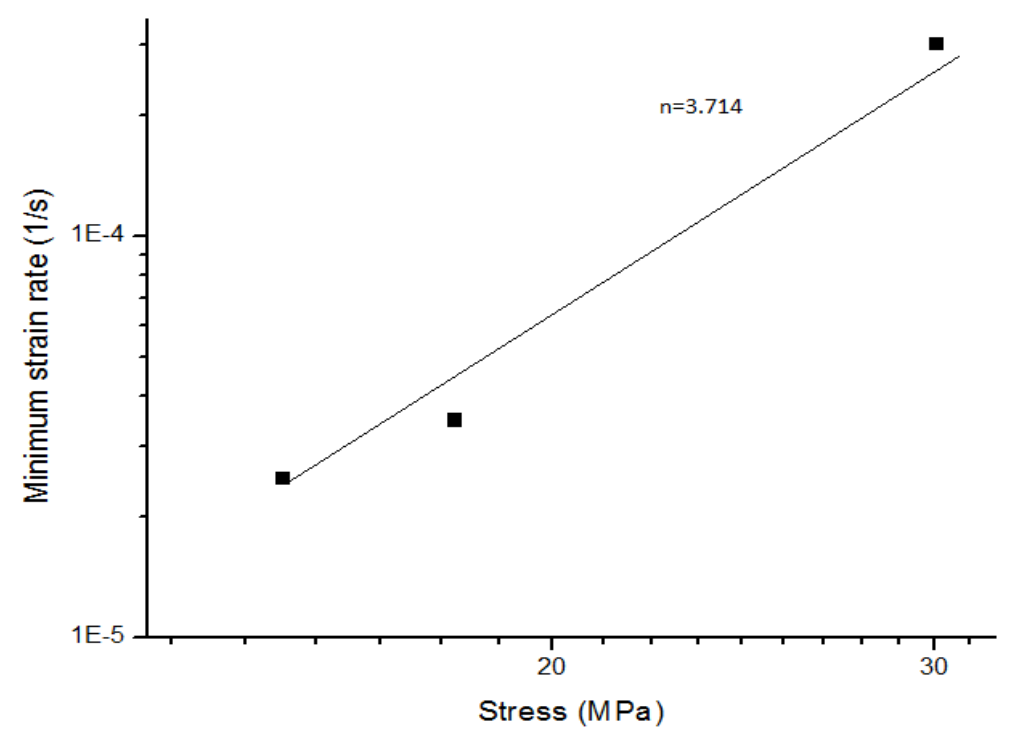

Fig. 5.12: Stress exponent analysis for Alloy 617 at $900^{\circ} \mathrm{C}$

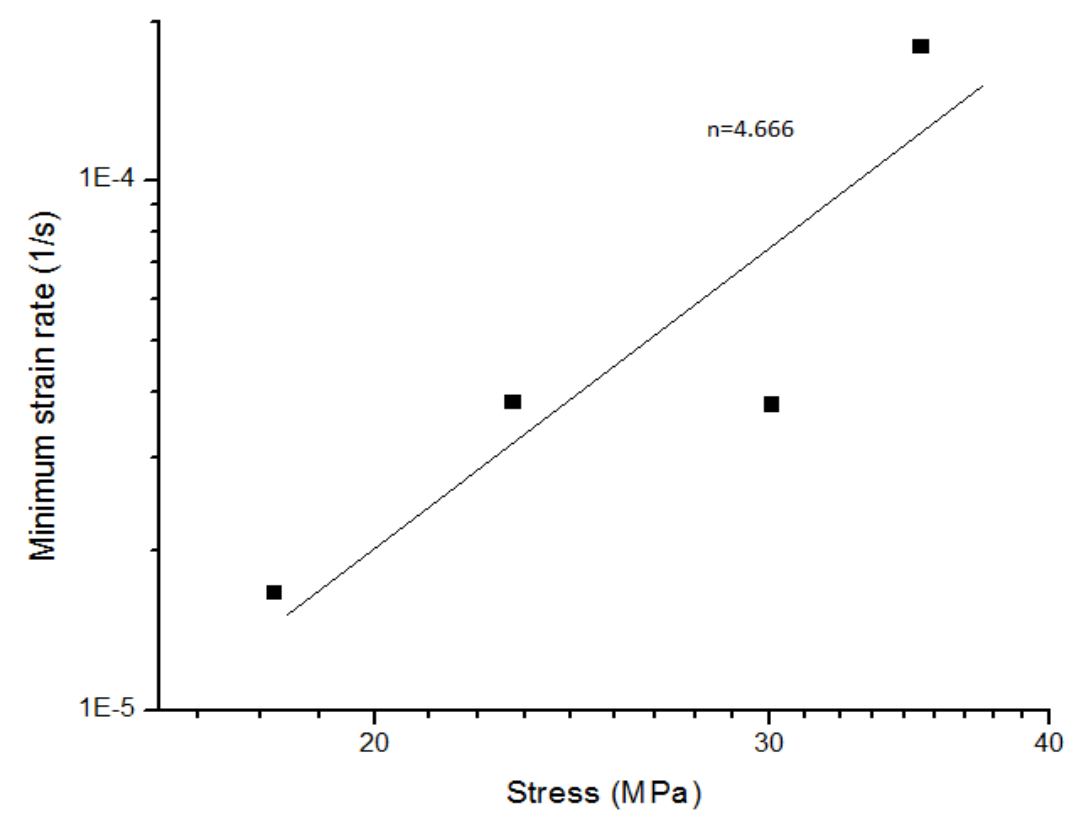

Fig. 5.13: Stress exponent analysis for Alloy 230 at $900^{\circ} \mathrm{C}$

For Alloy 617 at $900^{\circ} \mathrm{C}$, the stress exponent was measured to be 3.714 , indicating that a viscous glide mechanism is the rate controlling step [5.23]. From the previous work by Tung, the stress exponent $n$ was measured to be 4.1 at $850^{\circ} \mathrm{C}$ and 3.3 at $950^{\circ} \mathrm{C}$, so there is no significant change in the stress exponent for these two temperatures. The stress exponent analysis results show that at all three temperatures, Alloy 617 maintains similar creep mechanisms, which can be defined to be a combination of a viscous glide mechanism and a dislocation diffusion mechanism. Although the viscous glide mechanism dominates all three temperatures, the dislocation diffusion becomes more significant as the studied temperature is decreased. 


\section{Understanding Fundamental Material-Degradation Processes in High Temperature Aggressive Chemomechanical Environments}

For Alloy 230 at $900^{\circ} \mathrm{C}$, the stress exponent was measured to be 4.666 , indicating climb-controlled dislocation creep [5.27]. The stress exponent $n$ is measured to be 11.1 for $850^{\circ} \mathrm{C}$ and 2 for $950^{\circ} \mathrm{C}$, which indicates a significant change in the creep mechanism between the two temperatures. It is believed that the high stress exponent is attributed to the disruption of dislocation motion by carbides precipitated within the material. The stress exponent dramatically decreased from $850^{\circ} \mathrm{C}$ to $950^{\circ} \mathrm{C}$ because, as the temperature rises, less carbides are precipitated, so the interaction of dislocation with carbides becomes weakened, and consequently the stress exponent decreases. With increasing temperature, the grain boundary sliding mechanism has a greater impact on the creep life, which further decreases the value of the stress exponent. Previous studies by Tung [5.26] show that grain boundary sliding is not the dominant creep mechanism at $950^{\circ} \mathrm{C}$. The most likely creep mechanism is a combination of dislocation creep and grain boundary sliding. The current study confirmed that the combined creep mechanism is also dominant at $900^{\circ} \mathrm{C}$, while the change in the creep mechanism occurs at $850^{\circ} \mathrm{C}$, where dislocation creep becomes more significant.

Many intergranular creep fractures are observed in the SEM images of Figs. 4.120 and 4.123. These intergranular creep fractures originate at triple junctions of grain boundaries and propagate along grain boundaries perpendicular to the hoop stress along the cross section. Many intergranular voids and cavities can be found at grain boundaries, which suggests that nucleation, growth, and coalescence of intergranular creep voids and cavities are micro-mechanisms leading to creep rupture. Voids and micro cracks always form at grain boundaries, primarily due to impurity segregation to the boundaries and the interaction of grain boundary sliding with geometrical constraints. With increasing temperature and stress, it becomes much easier for the voids to form and grow. This trend will further decrease the connection parts of the grain boundary perpendicular to the hoop stress, which will then cause a further decrease in the steady state creep stage time, which is exactly in accord with the experimental results.

The creep life vs. applied stress for both alloys at all three temperatures is shown in Fig. 5.14. Observation of this figure leads to the conclusion that the creep life of Alloy 617 and Alloy 230 is similar at $950^{\circ} \mathrm{C}$ and $900^{\circ} \mathrm{C}$, whereas Alloy 230 has better creep properties than Alloy 617 at $850^{\circ} \mathrm{C}$. From Fig. 5.14, the similar slopes observed for Alloy 617 at all three temperatures further justify the assumption that a similar creep mechanism is shared, while the distinct slope for Alloy 230 at $850^{\circ} \mathrm{C}$ indicates a different creep mechanism from the other two studied temperatures. 


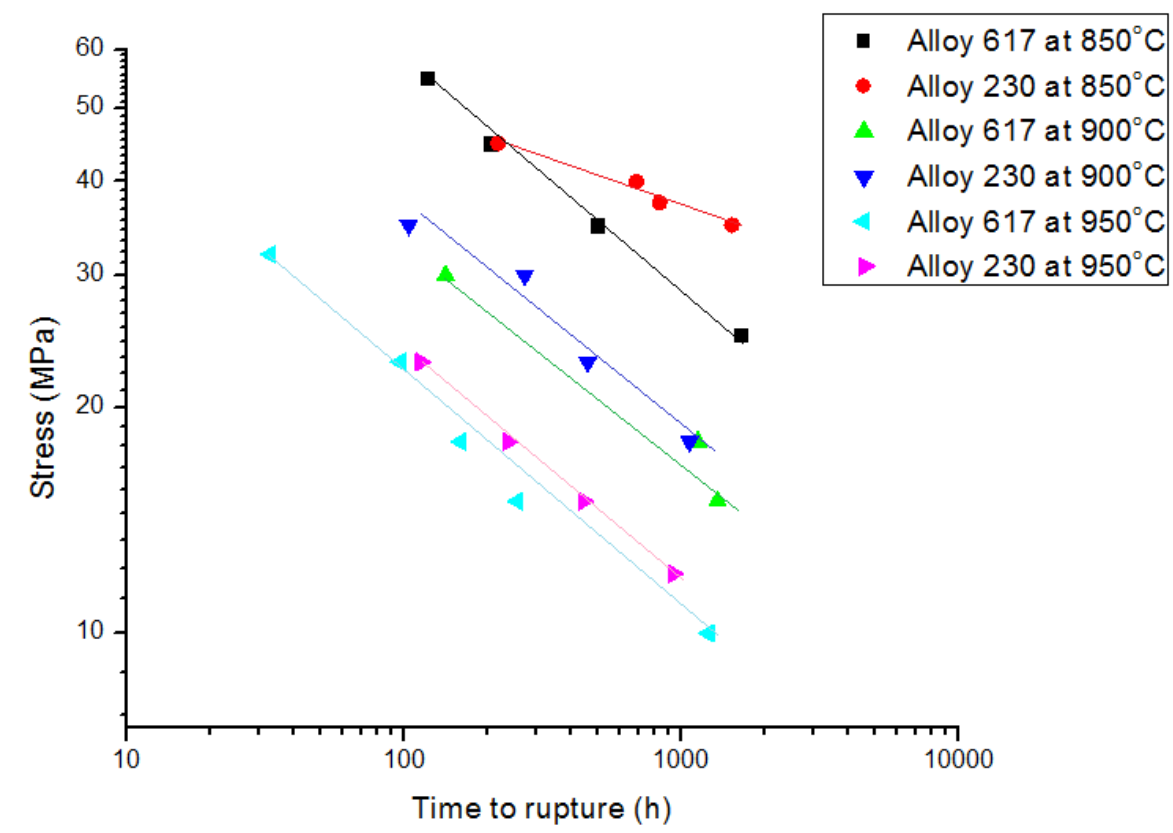

Fig. 5.14: Creep life of Alloy 617 and Alloy 230 at $850^{\circ} \mathrm{C}, 900^{\circ} \mathrm{C}$, and $950^{\circ} \mathrm{C}$

\subsection{Evaluation of the Empirical Relation Predicting Long-term Creep Life}

The Monkman-Grant relation [5.28] provides a correlation between the steady-state creep rate and the creep rupture time, and is described as:

$t_{r} \varepsilon_{m \iota n}^{i}=C_{0}$

where $\alpha$ and $C_{0}$ are material constants, independent of stress and temperature. This relation has been verified for many materials, including pure metals, solid alloys, and more complicated alloy systems. Figs. 5.15 and 5.16 show creep life as a function of the minimum creep rate for Alloy 617 and Alloy 230. 


\section{Understanding Fundamental Material-Degradation Processes in High Temperature}

Aggressive Chemomechanical Environments

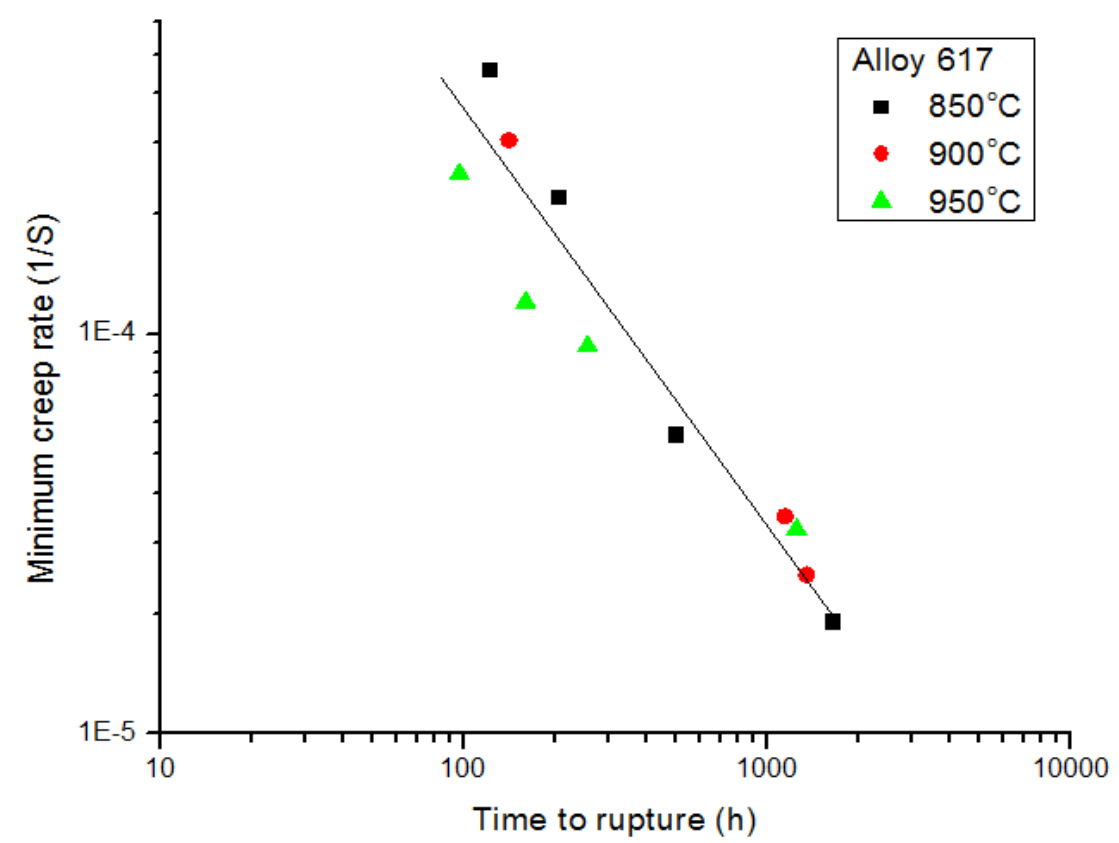

Fig. 5.15: Relationship between minimum creep rate and rupture time for Alloy 617

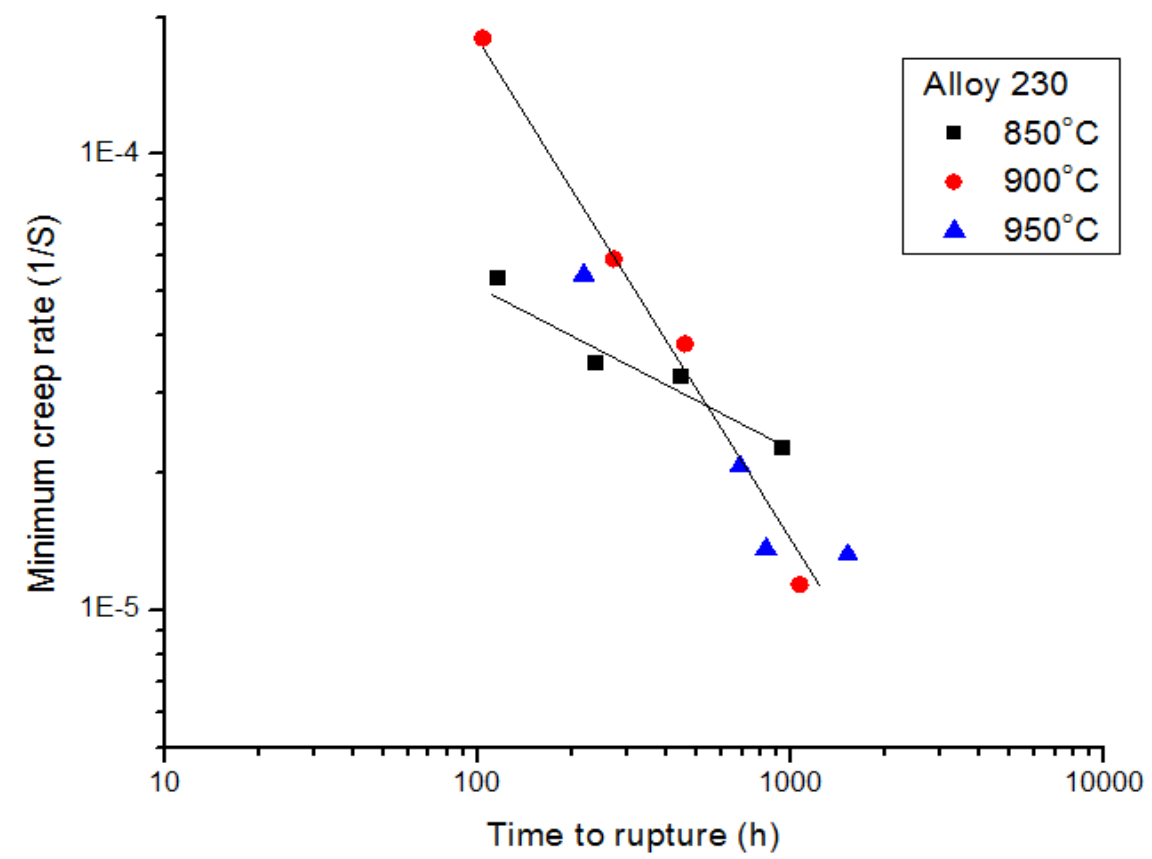

Fig. 5.16: Relationship between minimum creep rate and rupture time for Alloy 230

From Figs. 5.15 and 5.16, it is clear that the Monkman-Grant relation provides an acceptable method for predicting the long-term creep life of Alloy 617 at the studied temperatures. However, for Alloy 230, the material constants seem to be temperature dependent. Temperatures $900^{\circ} \mathrm{C}$ and $950^{\circ} \mathrm{C}$ share one group of constants, while temperature $850^{\circ} \mathrm{C}$ uses another group. This phenomenon may be evidence in support of the hypothesis that the creep mechanism for 


\section{Understanding Fundamental Material-Degradation Processes in High Temperature Aggressive Chemomechanical Environments}

Alloy 230 is similar at $900^{\circ} \mathrm{C}$ and $950^{\circ} \mathrm{C}$, but is different at $850^{\circ} \mathrm{C}$. Tables 5.10 and 5.11 list the values of $\alpha$ and $C_{0}$ for Alloys 617 and 230, respectively.

Table 5.10: Constant values in the Monkman-Grant Equation of Alloy 617

\begin{tabular}{cc}
\hline$\alpha$ & $\boldsymbol{C}_{\mathbf{0}}$ \\
\hline 0.91957 & 0.00258 \\
\hline
\end{tabular}

Table 5.11: Constant values in the Monkman-Grant Equation of Alloy 230

\begin{tabular}{ccc}
\hline Temperature $\left({ }^{\circ} \mathrm{C}\right)$ & $\alpha$ & $\boldsymbol{C}_{\mathbf{0}}$ \\
\hline $900 \& 950$ & 1.5731 & $7.85 \mathrm{e}-8$ \\
850 & 0.6887 & 0.2575 \\
\hline
\end{tabular}

\subsection{Crack Closure Effects of Alloy 230}

As reported in section 4.13.2, crack closure was present for both cases. Crack closure phenomena have been extensively studied beginning with Christensen, who discovered roughness-induced crack closure in 1963 [5.29]. Due to the pure mode I loading on the specimens tested in this study, roughness-induced crack closure was concluded not to be the main crack closure phenomenon. Mode II loading is typically necessary to see roughness effects. By observing the crack faces on the failed specimens, very little deviations and asperities were seen supporting the previous conclusion. Thus, other forms of crack closure had to be considered.

By first taking into account the RT and HT cases together, plasticity-induced crack closure was the most likely form of crack closure present in this study. Elber discovered this phenomenon and found that the residual plasticity stretching from the crack tip across the entire crack face provided a shielding mechanism from the remote loading [5.30, 5.31]. The DIC strain fields showed residual plasticity behind the crack tip and in shear bands extending from the crack tip. From this result it can be gleaned that plasticity-induced crack closure is present and is the dominating form of crack closure. Previously, studies have concluded that load ratio (R) effects are minimized when considering the effective stress intensity factor range [5.32]. Other studies have looked at the crystallography of single crystals and found that the effective stress intensity factor range can also be used to eliminate scatter in fatigue crack growth data [5.33]. The usefulness of taking into account crack closure is evident for removing the dependence on the loading conditions, but does not account for the differences in RT and HT fatigue crack growth rates seen in this investigation.

At high temperatures, the propagation rate of cracks can be several orders of magnitude higher than that at room temperature. This has also been shown to be true in previous fatigue crack growth studies of Haynes 230 [5.34, 4.75]. Under these conditions, the crack tip might experience creep and environmental attack. Oxidation and hold times have been shown to increase the crack growth rate of Haynes 230 at highly elevated temperatures [4.94, 4.95, 4.75]. In order to determine the effects of these high-temperature phenomena, oxide-induced crack closure must be considered in the HT case.

Although this nickel-based superalloy has excellent oxidation resistance and a high frequency was chosen in an effort to minimize environmental effects, the freshly exposed material, due to the crack growth, is still oxidized. Investigations were performed in the 1980s to 


\section{Understanding Fundamental Material-Degradation Processes in High Temperature Aggressive Chemomechanical Environments}

determine the effect that oxides have on fatigue crack growth in steels [5.35], copper [5.36], and nickel-based superalloys [5.37]. Ritchie, Suresh, and Moss commented that the oxide films forming at the crack tip and any fretting of this film due to the roughness-induced crack closure will increase the closure and decrease $\Delta \mathrm{K}$ [5.38]. Although this information sheds light onto the role of crack closure on the fatigue crack growth in this study, both specimens in this current study show the same level of crack closure of about 30\%. Also, an important finding in the literature is that oxide thickness needs to exceed or equal the crack tip opening displacement (CTOD) in order to have an effect. This is not the case for the crack growth condition studied here. Therefore, crack closure provides no explanation for the much faster crack growth rates in the HT case.

\subsection{Slip Irreversibility of Crack of Alloy 230}

The crack tip is the other area of interest for understanding the mechanics of fatigue crack growth. Dislocation interactions with the microstructure features, whether these are grain/twin boundaries or defects, control the slip irreversibility during a fatigue cycle. This occurs when dislocations interact with boundaries and a residual dislocation is left within that boundary [5.39]. Certain types of boundaries can play a significant role in increasing the resistance to fatigue crack growth and have been the focus of "grain boundary engineering." These boundaries are typically described as low-sigma-numbered, as defined in terms of coincidence site lattice theory [5.40]. Of particular interest have been annealing twins, or $\Sigma 3$ boundaries, and studies have shown that a greater number of $\Sigma 3$ boundaries leads to increased fatigue crack growth resistance. The twin boundary acts as a barrier to slip, leading to a dislocation pile-up and a stress field that prevents dislocations from being emitted from the crack tip [5.41]. The microstructure of the Haynes 230 used in this study is described in section 3.7 and the very high amount of $\Sigma 3$ boundaries found in this nickel-based alloy contributes to its good fatigue crack growth resistance (Paris exponent of $\mathrm{m} \approx 2$ ).

As seen in Fig. 4.136, Haynes 230 demonstrated planar slip. Planar slip is commonly associated with solid solution hardened nickel-based superalloys and is encouraged by the inclusion of tungsten, which reduces the stacking fault energy. This characteristic of the alloy leads to decreased slip irreversibility, which reduces the fatigue crack growth rate [5.42]. Mughrabi quantified the cyclic slip irreversibility as the ratio between the irreversible plastic shear slip and the total cumulative plastic shear slip [5.43]. This idea was the basis of the measurements made in section 4.13.3. The results concurred with Mughrabi and showed increasing fatigue crack growth rates with increasing slip irreversibility.

A relationship exists between the slip irreversibilites, crack growth rates, and temperature, as the irreversibilities and growth rates were an order of magnitude higher for the HT case than the RT case. Since the cyclic frequency was high enough to minimize creep effects, the high temperature effects on dislocation motion must be elucidated. Increasing temperature increases the cross-slip of screw dislocations and reduces the reversible slip, since they will no longer return on that glide plane [5.43]. Dislocation climb, vacancies, and the annihilation of dislocations all prevent the return of dislocations to the crack tip [5.44, 5.45]. The addition of this high temperature plastic flow has a direct influence on the slip irreversibility, and therefore directly impacts the fatigue crack growth rate. Since the first observations in 1935, it has been well established that subgrain formation occurs at high temperatures [5.46]. Since subgrains have 


\section{Understanding Fundamental Material-Degradation Processes in High Temperature Aggressive Chemomechanical Environments}

a slightly different orientation compared to the surrounding material, the slip planes will be realigned. This creates a discontinuity of slip [5.47]. Previous research has indicated that the presence of barriers that prohibit the reverse of slip during the unloading portion of a fatigue cycle will increase the amount of slip irreversibility [5.41]. Due to the increased amount of subgrains present at HT compared to RT, it is expected that a much higher amount of slip irreversibility will be found. These effects are evinced by the much higher fatigue crack growth rate of the HT specimens compared to the RT specimens, as shown in Fig. 4.131. This study provided quantitative, experimental evidence that slip irreversibility is able to describe the fatigue crack growth rate differences between experiments with different temperatures. 


\section{Understanding Fundamental Material-Degradation Processes in High Temperature Aggressive Chemomechanical Environments}

\section{CONCLUSIONS}

Nuclear energy is considered to be a promising energy source for the future. If technical issues pertaining to structural materials for next generation reactors can be overcome, nuclear energy can become more economic and reliable, with vast improvements in safety. Currently, material development and characterization of high-temperature materials stands as one of the leading challenges, and perhaps the most limiting factor, in the development and advancement of next generation reactors, particularly the VHTR. In this thesis work, fundamental material studies have been conducted for two of the most promising structural materials for the VHTR: Alloy 617 and Alloy 230. The objective of this work was to investigate the material performance (short-term tensile response and long-term aging development) for a high-temperature environment. In addition, this study employed an advanced measurement technique, high-energy synchrotron radiation, in order to investigate deformation processes (time-independent and time-dependent) during in-situ loading.

\subsection{Alloy properties}

The deformation mechanisms of Alloy 617 and Alloy 230 during tensile loading at temperatures up to $1000^{\circ} \mathrm{C}$ were clarified using experimentation. At temperatures from 300 to $700^{\circ} \mathrm{C}$, the yield strength was found to be temperature independent as a result of additional strain hardening provided by dynamic strain aging. However, higher temperatures $\left(>800^{\circ} \mathrm{C}\right)$ activated additional deformation mechanisms, including dislocation creep and dynamic recrystallization, leading to a significant decrease in strength at $800^{\circ} \mathrm{C}$. Consequently, the fracture mechanisms changed from inclusion particle cracks at temperatures up to $700^{\circ} \mathrm{C}$ to triple junction cracks from 800 to $1000^{\circ} \mathrm{C}$. A small number of recrystallized grains were observed in the highly strained area connected to triple junction cracks during the tensile tests completed at $800^{\circ} \mathrm{C}$. Dynamic recrystallization became predominant at $1000^{\circ} \mathrm{C}$. A large number of fine grains were found to recrystallize in grain boundaries and interphase grain boundaries, particularly in the inclusion-rich area in the necking part of the specimen. So, it was found that high strain, large particles, and small grains promote the formation of new grains. Additionally, EBSD analyses showed that a steady state recrystallized microstructure at $1000^{\circ} \mathrm{C}$ was never achieved, although the tensile strain was larger than 0.5 by the conclusion of testing.

The effect of orientation on the tensile properties of Alloy 617 was also analyzed. Lower material strengths were detected for specimens with non-zero angles of orientation with the long-transverse direction. The yield strength was found to considerably decrease once the loading direction deviated from the long-transverse direction, while the ultimate tensile strength was found to decrease with a linear trend from the long-transverse to the short-transverse direction. This mechanical anisotropy has a comparable impact on Alloy 617 for temperatures up to $1000^{\circ} \mathrm{C}$. The dependency of plastic deformation on loading orientation was analyzed through several microstructural examinations. The results show that mechanical fibering, consisting of an alignment of inclusion particles and matrix crystals, is responsible for the mechanical anisotropy of Alloy 617 with varying performance across the studied temperature range. At low temperatures $\left(<800^{\circ} \mathrm{C}\right)$, Alloy 617 did not deform homogenously as a result of microstructure gradients. The inclusion-rich area, seen as clusters from the rolling plane and bands from the transverse plane, could not be smoothly deformed, and thus the area developed high local strains under tensile load. The steep strain gradient in the short-transverse direction caused early fracture in the material, leading to a much lower tensile strength. At high temperatures $\left(800-1000^{\circ} \mathrm{C}\right)$, the 


\section{Understanding Fundamental Material-Degradation Processes in High Temperature Aggressive Chemomechanical Environments}

highly strained areas caused by dislocation pileups at barriers (e.g. interphase boundaries) were subject to dynamic recrystallization. The orientation dependence of dynamic recrystallization with different dimensions and alignments of recrystallized grains results from the anisotropic spatial distribution of inclusion particles (i.e. mechanical fibering) in Alloy 617. This leads to an orientation dependence of mechanical properties at elevated temperatures.

Strain-rate sensitivity analysis for each alloy was conducted in order to approximate the long-term flow stresses. The strain-rate sensitivities for the $0.2 \%$ flow stress were found to be temperature independent $(m \approx 0)$ at temperatures ranging from $\mathrm{RT}$ to $700^{\circ} \mathrm{C}$ due to dynamic strain aging. At elevated temperatures $\left(800-1000^{\circ} \mathrm{C}\right)$, the strain-rate sensitivity significantly increased $(m>0.1)$. Compared to Alloy 617, Alloy 230 displayed higher strain-rate sensitivities at high temperatures. This leads to lower estimated long-term flow stresses. Results of this analysis have also been used to evaluate current ASME allowable design limits. According to the comparison with the estimated flow stresses, the allowable design stresses for either alloy according to ASME B\&PV Code did not provide adequate degradation estimations for the long-term service life. However, rupture stresses for Alloy 617, developed in ASME code case $\mathrm{N}-47-28$, can generally satisfy the safety margin estimated in the study following the strain-rate sensitivity analysis. Nevertheless, additional material development studies must be completed, since the design parameters for rupture stresses are constrained such that current VHTR conceptual designs cannot satisfy the limits.

\subsection{Thermal aging development}

The thermal degradation processes for Alloy 617 and Alloy 230 were analyzed through long-term, high-temperature aging experiments. A similar microstructure evolution characterized by particle nucleation and growth was observed in each alloy. Fine and semi-coherent $\mathrm{M}_{23} \mathrm{C}_{6}$ precipitates nucleated in the early stage of aging and provided additional particle strengthening for each alloy. With further aging, large intergranular particles continued to grow at the expense of fine, intragranular precipitates. After long-term aging ( $>1000$ hours), these fine precipitates had diffused and coarsened in high angle grain boundaries and interphase boundaries due to their high interfacial free energy. For the same reason, the carbide precipitates located in twin boundaries during the early stage of aging were depleted. Despite significant volume gain, most intergranular particles grown during thermal aging were found to maintain a cube-on-cube orientation with the $\gamma$ matrix after aging for over 1000 hours.

The kinetics of microstructure development were also investigated by dimension measurements and coarsening-rate analysis of intergranular particles. At both 900 and $1000^{\circ} \mathrm{C}$, most volume gain (by fraction) of intergranular particles occurred during the early stage of aging. After this early development, the growth rate decreased with time. The intergranular particle growth and associated growth rate development were controlled by the thermal aging temperature. Due to a lower equilibrium volume fraction of carbides at $900^{\circ} \mathrm{C}$, particle nucleation was completed in a short period of time $(<<10$ hours). Hence, the following microstructural development was dominantly characterized by particle coarsening. In contrast, a mixed process incorporating particle nucleation and growth was observed in the aging process at $1000^{\circ} \mathrm{C}$ due to a higher equilibrium volume fraction of carbides. Additionally, heterogeneous elements (e.g. C, $\mathrm{W}$, or Mo) that form the intergranular particles obtained a higher diffusibility at higher 


\section{Understanding Fundamental Material-Degradation Processes in High Temperature Aggressive Chemomechanical Environments}

temperatures. Therefore, larger intergranular particles were found in specimens aged at $1000^{\circ} \mathrm{C}$ compared to specimens aged at $900^{\circ} \mathrm{C}$.

Results of mechanical tests were in good agreement with microstructure observations. Both the hardness measurements and tensile tests showed a typical aging process characterized by short-term strengthening and long-term softening. Generally, both alloys that were aged at $900^{\circ} \mathrm{C}$ attained higher yield and tensile strengths with a longer hardening time compared to samples aged at $1000^{\circ} \mathrm{C}$. This agrees well with the completed microstructural analysis and suggests a more intense diffusion process at higher temperatures, so that precipitate hardening can only be maintained for a relatively short time period. Alloy 230 exhibited a longer effect of age-hardening compared to Alloy 617. The difference in duration of strengthening can be attributed to the diffusibility of the main heterogeneous elements in the alloys. The diffusibility of W in Alloy 230 is lower compared to Co and Mo in Alloy 617, and this results in a relatively slower process of precipitate growth. The strengthening by fine particles within the $\gamma$ matrix can therefore be maintained for a longer time in Alloy 230. Material softening after long-term aging resulted in a loss of strength in specimens aged at all studied temperatures (RT to $1000^{\circ} \mathrm{C}$ ). Analogous to the analysis on the orientation effect, the long-term aging degradation for high-temperature tensile properties was found to be comparable to the degradation for low-temperatures properties.

During thermal aging of Alloy 617 and Alloy 230, no significant changes in grain boundary character distribution or grain size distribution were observed in either alloy. The exceptional thermal stability for both alloys is partially attributed to "Zener pinning" by large intrinsic carbides and age-coarsened intergranular particles. These particles inhibit the grain boundary migration and impede grain growth during aging. Hence, grain boundary character is preserved. In addition, even though $\sigma$ phase is predicted from the calculated equilibrium phase diagram, this phase is not observed during the aging experiment. This finding agrees with all previous experimental studies and suggests that TCP phases will not be a significant threat for both alloys.

\subsection{Synchrotron radiation study}

With recent developments in synchrotron radiation techniques, various fundamental materials questions that have been difficult to answer using traditional destructive examinations have received solutions, in some cases by using a single X-ray exposure. However, nuclear material research is a relatively new area to implement synchrotron radiation study. In this work, high-energy synchrotron X-ray diffraction was applied to study the tensile response of Alloy 230. The lattice strain response for reflections from the $\gamma$ matrix was found to be nearly linear with the external applied stress. Thus, the lattices strain/internal stress conversion becomes straightforward based on the elastic, self-consistent Kröner's method. The $\gamma$ matrix of Alloy 230 in both the long- and short-transverse directions experienced a similar process of lattice strain development during tensile loading, although the short-transverse specimen possesses a shorter elongation due to an early failure. The Bauschinger effect seen in Alloy 230 specimens can be measured from the difference in residual stresses, which agrees well with results of mechanical testing.

Due to the large particle size and small volume fractions, $\mathrm{M}_{6} \mathrm{C}$ carbides in Alloy 230 have a limited loading capacity. By increasing the external applied stress in the long-transverse specimen, the lattice stain of the carbide decreases as a result of the continued fracture process 


\section{Understanding Fundamental Material-Degradation Processes in High Temperature Aggressive Chemomechanical Environments}

observed during plastic deformation in the material. The maximum internal stress of the particle was achieved during early yielding of the material and can be defined as the critical fracture stress for the $\mathrm{M}_{6} \mathrm{C}$ type carbide in Alloy 230. This critical value was found to be $\sim 1344 \mathrm{MPa}$ corresponding to a lattice strain of $\sim 0.0047$. The analysis on the short-transverse specimen further confirms this conclusion and suggests that the critical fracture stress is independent of loading orientation.

In addition to tensile experiments, a unique analysis technique was developed in this work to characterize the in-situ deformation response of pressurized creep tubes at elevated temperatures. The technique allows the macroscopic creep strain and the microstructural development to be measured simultaneously during a single X-ray exposure. A typical creep curve with identifiable secondary and tertiary creep response was obtained by analyzing the $\mathrm{X}$-ray diffraction patterns. Additionally, in-situ observations of the development of dislocation structures and lattice strain during deformation make it possible to track the development of creep void nucleation, growth, and coalescence. This technique, which uses a combination of pressurized creep tubes and high-energy synchrotron X-rays, can be used with small angle X-ray scattering (SAXS) to more directly observe the initiation of plasticity-induced void formation. Finally, it should be noted that the specimen configuration in the present study simulates the heat transport and exchange tubing and piping in the VHTR system. This study paves the way for using synchrotron X-ray diffraction as a non-destructive method to investigate components in practical engineering applications.

\subsection{Creep-fatigue behavior}

To investigate the major damage mechanism for materials used in the VHTR, LCF and creep-fatigue tests of Alloy 617 and Alloy 230 were conducted in this study. Two creep-fatigue life prediction methods, linear damage summation and frequency-modified tensile hysteresis energy modeling, were evaluated. In addition, various microanalysis techniques were used to study the differences in the damage mechanisms of Alloy 617 and Alloy 230 under LCF and creep-fatigue tests. The results of the experiments and analysis lead to the following conclusions:

1. Under all LCF test conditions, Alloy 230 performed slightly better than Alloy 617.

2. Compared to the LCF results, the cycles to failure for both materials was reduced under creep-fatigue test conditions. Again, Alloy 230 exhibited a longer creep-fatigue life than Alloy 617 , especially for the creep-fatigue tests with the smallest total strain range, i.e. $0.5 \%$, and longer dwell times, i.e. 10 and 30 minutes at $1.0 \%$ total strain range. It was also found that longer hold times at the maximum tensile strain would cause a further reduction in the creep-fatigue lives of both materials.

3. The linear damage summation failure criterion could predict the creep-fatigue life of Alloy 617 for limited test conditions, but considerably underestimated the creep-fatigue life of Alloy 230. In contrast, frequency-modified tensile hysteresis energy modeling showed promising results, as the difference between the predicted and actual creep-fatigue life of Alloy 617 and Alloy 230 was less than $30 \%$.

4. Comparing the crack initiation and propagation mode of Alloy 617 and Alloy 230 under LCF and creep-fatigue tests reveals that creep-fatigue test conditions tended to transform the transgranular cracking mode observed in LCF tests to the intergranular cracking mode, especially for the creep-fatigue tests with longer dwell times at peak tensile strain. Moreover, 


\section{Understanding Fundamental Material-Degradation Processes in High Temperature Aggressive Chemomechanical Environments}

Alloy 230 demonstrated better resistance to intergranular cracking than Alloy 617 under the creep-fatigue deformation. For both materials, additional creep damage in creep-fatigue testing was also manifested by material interior intergranular cracking orientated at an angle of 45 degrees to the axial stress or perpendicular to the axial stress.

5. Because the test temperature $\left(850^{\circ} \mathrm{C}\right)$ was higher than both the materials' equicohesive temperatures, the strength of the grain boundaries was lower than the strength of the grains. Therefore, during the LCF tests, the deformation tended to concentrate in the original random high angle grain boundary region, especially for the higher total strain range LCF tests and for Alloy 617. The localized deformation near random high angle grain boundaries was enhanced for both materials during creep-fatigue tests due to the additional creep deformation and grain boundary sliding process incurred at the strain hold period.

6. The largest fatigue life reduction was observed in the creep-fatigue test of Alloy 617 at $0.5 \%$ total strain range with a 3 minute dwell time at maximum tensile strain. Similar to the case of SCC, oxidation-assisted crack propagation along grain boundaries promoted intergranular cracking of Alloy 617 and resulted in a faster crack growth rate at the above test condition.

7. Based on SEM and EBSD analysis, although there are many similarities between the microstructures of Alloy 617 and Alloy 230, the higher volume fraction of carbide precipitates and better oxidation resistance of Alloy 230 contributed to its dominance in LCF and creep-fatigue life over Alloy 617.

8. Grain boundary cellular precipitation, formed by a discontinuous precipitation reaction in Alloy 230, might have a deleterious effect on the LCF and creep-fatigue properties of Alloy 230 , and can be avoided by appropriate heat treatment and chemistry control to further improve the mechanical properties of Alloy 230.

\subsection{Biaxial creep behavior}

Biaxial thermal creep deformation studies using pressurized creep tube technology were carried out at $900^{\circ} \mathrm{C}$. Based on the results of the experiments, creep modeling was conducted to predict creep behavior at different temperatures and stresses. Creep mechanisms were analyzed at $900^{\circ} \mathrm{C}$ in order to compare the results with the results of the tests performed at $950^{\circ} \mathrm{C}$ and $850^{\circ} \mathrm{C}$. The conclusions are as follows:

1. The creep curves of both Alloy 617 and Alloy 230 show three distinct regimes: the primary, secondary, and tertiary regimes. The tertiary regime constitutes the major portion of the creep life. With increasing temperature and stress, the scope of the secondary regime decreases.

2. The equation $\varepsilon=A \sigma \cosh ^{-1}(1+r t)+P \sigma^{n} t^{m}$ was used to simulate creep strain development for both alloys at temperatures of 850,900 , and $950^{\circ} \mathrm{C}$. The simulation results reasonably agree with the experimental results at the medium stress level, but overestimate at low stress levels and underestimate at high stress levels. The FEM simulation provides a better simulation result.

3. Similar creep mechanisms were found at 850,900 , and $950^{\circ} \mathrm{C}$ for Alloy 617. In contrast, the creep mechanism for Alloy 230 at $850^{\circ} \mathrm{C}$ is different from the ones at 900 and $950^{\circ} \mathrm{C}$. Both Alloy 617 and Alloy 230 have similar creep rupture lives at 900 and $950^{\circ} \mathrm{C}$, while Alloy 230 has a longer creep life compared to Alloy 617 at $850^{\circ} \mathrm{C}$.

4. The Monkman-Grant relation provides an acceptable method for predicting the long-term creep life of Alloy 617. However, the material constants for Alloy 230 seem to be 


\section{Understanding Fundamental Material-Degradation Processes in High Temperature Aggressive Chemomechanical Environments}

temperature-dependent. This can be attributed to the dramatic creep mechanism change at $850^{\circ} \mathrm{C}$.

\subsection{Fatigue crack growth}

The fatigue crack growth of Haynes 230 was studied at room temperature (RT) and high temperature (HT). Analysis focused on the significance of any crack closure and the crack tip slip irreversibility and how these two phenomena are related to the crack growth rates. The conclusions are as follows:

1. The material showed good fatigue crack growth resistance both in the RT and HT tests $(\mathrm{m} \sim 2)$, although the HT fatigue crack grew at an appreciably faster rate. The role of microstructure is manifested through the irreversibility of slip at the crack tip.

2. Crack closure levels of approximately $30 \%$ of the load were determined at both test temperatures. Plasticity-induced crack closure was the main form of crack closure present, and oxide-induced crack closure was not a significant factor in the HT case.

3. Slip irreversibility was quantitatively measured as the difference in accumulated strain per cycle at the crack tip, and it was over an order of magnitude higher in the HT case than the RT case.

4. Dislocations emitted at the crack tip were influenced by high temperature effects, as reflected in the slip irreversibility measurements. Quantitative measurements of slip irreversibility were found to be an accurate method for distinguishing the differences between the crack growth rates at $\mathrm{HT}$ and $\mathrm{RT}$.

\subsection{Corrosion resistance}

We show that there is a correlation between transpassive current density and observation of surface $\mathrm{Cr}$ species in the Raman spectra. Delayed transpassive dissolution is associated with early onset of $\mathrm{Cr}(\mathrm{VI})$ species in spectra. The difference between transpassive current densities from Alloy 617 and Alloy 230 suggests that the two alloys have different corrosion susceptibilities, and indeed Alloy 230 is more corrosion resistant. In both alloys, heat treatment resulted in a delayed onset of the transpassive region, likely because the heat treatment causes more oxophilic elements to become available to the surface. An increase in the relative intensity of the mixed oxide phase occurs with heat treatment [3.18].

Finally, we examined reasons that heat-treated Alloy 230 and Alloy 617 exhibit greater corrosion resistance relative to the as-received materials. The XPS measurements show that oxidation is associated with an increased $\mathrm{Cr}$ content at the surface and speciation of the $\mathrm{Cr}$ to form protective oxides, a process that is well-known to increase the passivity of electrode surfaces $[3.20,3.21,3.22,3.23,3.24,3.25]$. It is also understood that the average grain size grows and the distribution of sizes increases after heating in a furnace [5.39], thus leading to the presence of fewer reactive grain boundaries. There are other possible contributory factors to increased corrosion resistance with the heat treated materials. $\mathrm{Al}_{2} \mathrm{O}_{3}$ is known to form as a consequence of internal oxidation [5.39, 5.40]; this $\mathrm{Al}_{2} \mathrm{O}_{3}$ might contribute to increased corrosion resistance. Formation of a surface layer of $\mathrm{MnCr}_{2} \mathrm{O}_{4}$ (spinel) imparts Alloy 230 with better corrosion resistance than Alloy 617 (as Alloy 230 contains twice as much $\mathrm{Mn}$ ) at $900^{\circ} \mathrm{C}$; however, both undergo spallation and volatilization of the $\mathrm{Cr}_{2} \mathrm{O}_{3}$ layer at $1100^{\circ} \mathrm{C}$ [5.40]. A study 


\section{Understanding Fundamental Material-Degradation Processes in High Temperature}

Aggressive Chemomechanical Environments

involving surface segregation in Haynes 230 showed that while $\mathrm{S}, \mathrm{P}$, and $\mathrm{Si}$ remained at the surface at $875^{\circ} \mathrm{C}$, no change in $\mathrm{Ni}$ or $\mathrm{Cr}$ content was observed [5.42].

Interestingly we found less intense $\mathrm{Cr}$ signals in the Raman spectra from the $1000^{\circ} \mathrm{C}$ annealed sample, which might be due the formation of volatile $\mathrm{CrO}_{3}$ from $\mathrm{Cr}_{2} \mathrm{O}_{3}$ at temperatures above $950^{\circ} \mathrm{C}$ [5.39]. We note, however, that a decrease in $\mathrm{Cr}$ content following electrochemical oxidation was not observed in the XPS. 


\section{Understanding Fundamental Material-Degradation Processes in High Temperature Aggressive Chemomechanical Environments}

APPENDIX A: Stress Strain Diagrams for Alloy 617 and Alloy 230

The engineering strain-stress diagrams for Alloy 617 testing at three strain rates and various temperatures are given in Figs. A.1 through A.10.

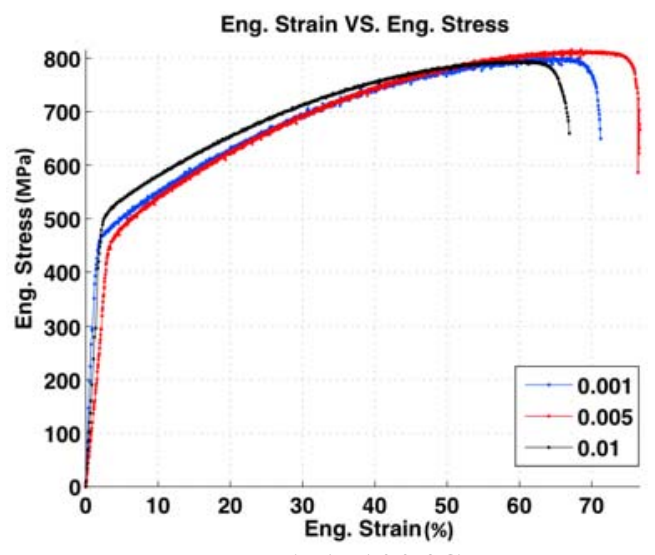

A.1. $100{ }^{\circ} \mathrm{C}$

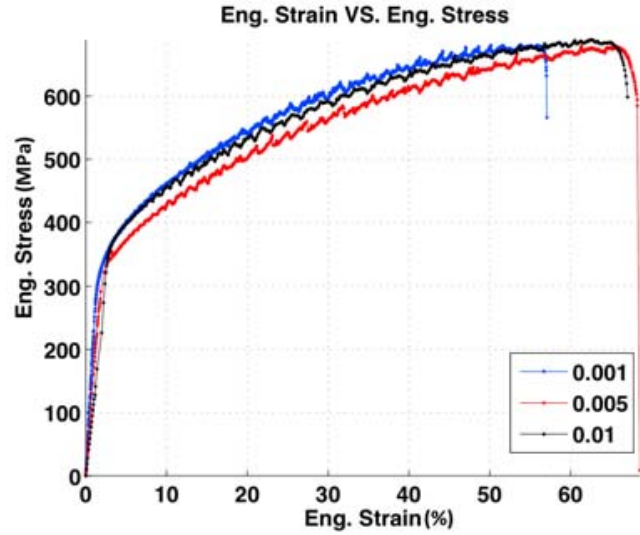

A.3. $300{ }^{\circ} \mathrm{C}$

Eng. Strain VS. Eng. Stress

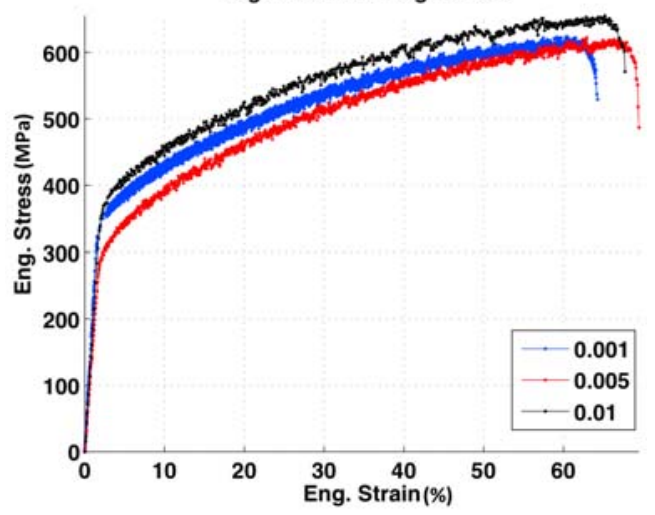

A.5. $500{ }^{\circ} \mathrm{C}$

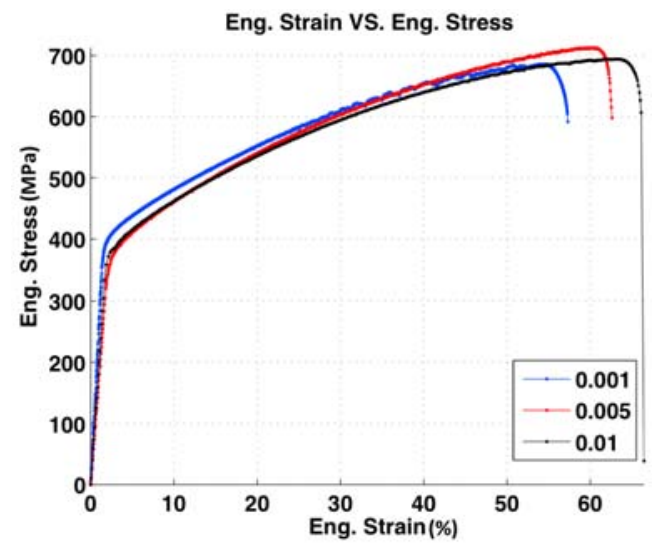

A.2. $200^{\circ} \mathrm{C}$

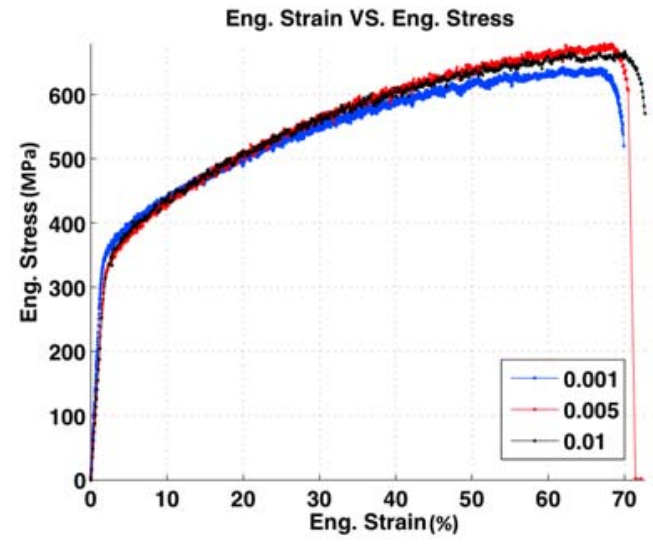

A.4. $400{ }^{\circ} \mathrm{C}$

Eng. Strain VS. Eng. Stress

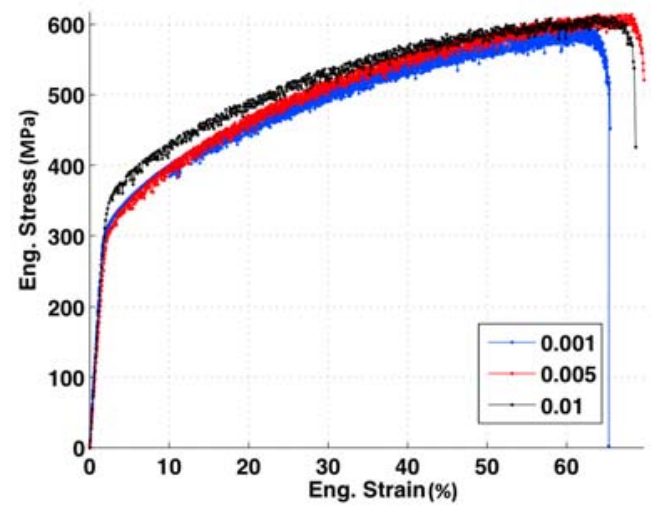

A.6. $600{ }^{\circ} \mathrm{C}$ 


\section{Understanding Fundamental Material-Degradation Processes in High Temperature}

Aggressive Chemomechanical Environments
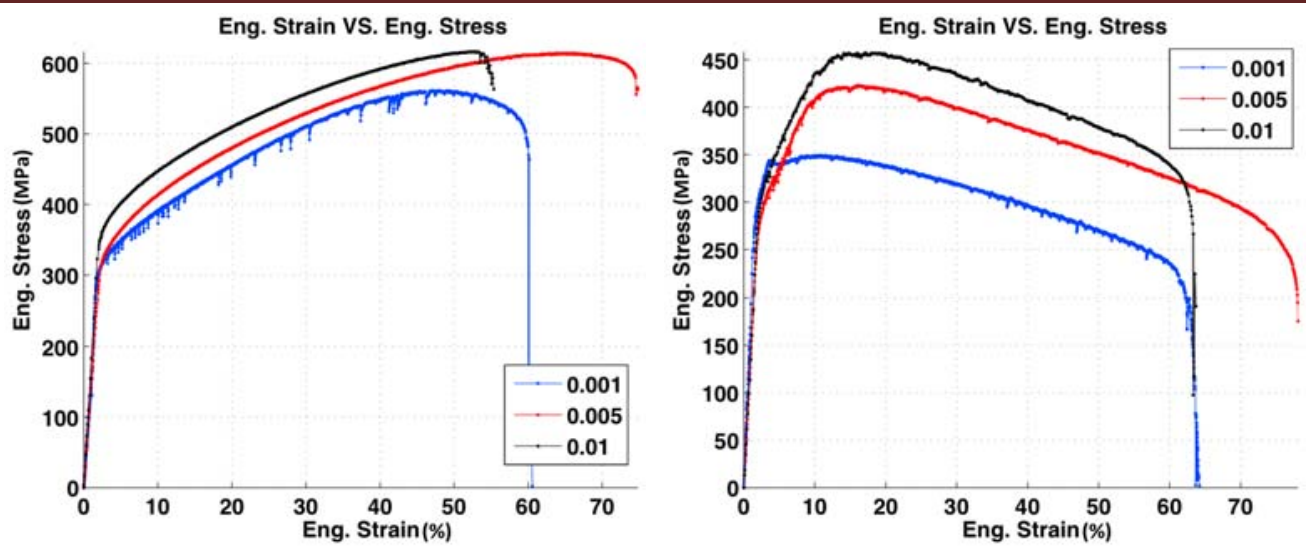

A.7. $700{ }^{\circ} \mathrm{C}$

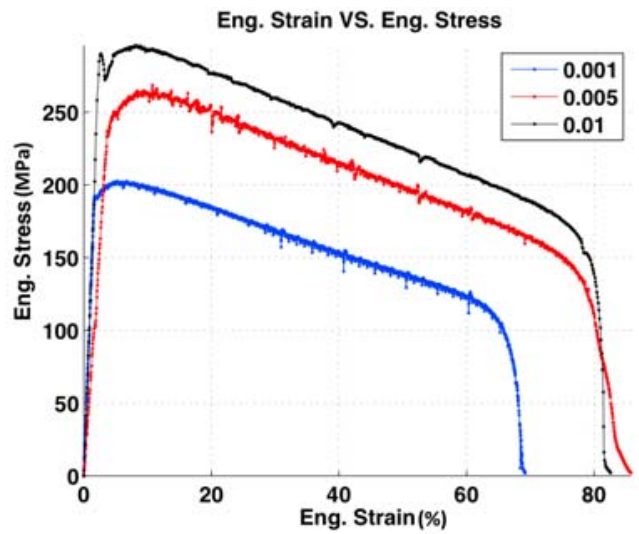

A.9. $900{ }^{\circ} \mathrm{C}$
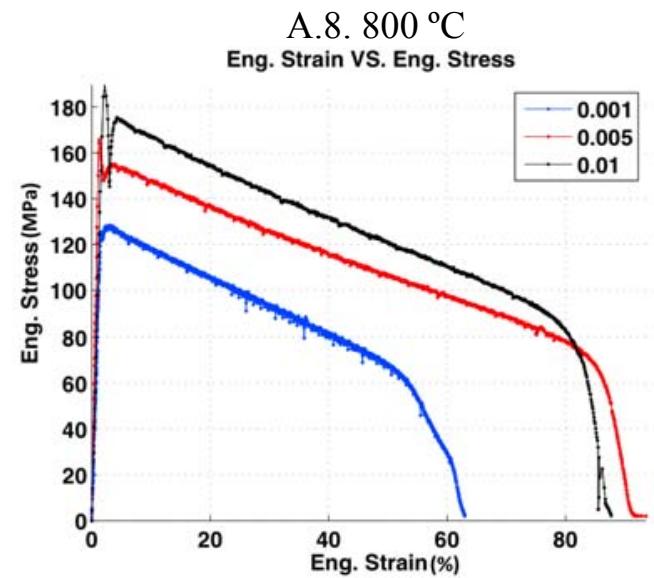

A. $10.1000^{\circ} \mathrm{C}$

The engineering strain-stress diagrams for Alloy 230 testing at three strain rates and various temperatures are given in Figs. A.11 through A.20.

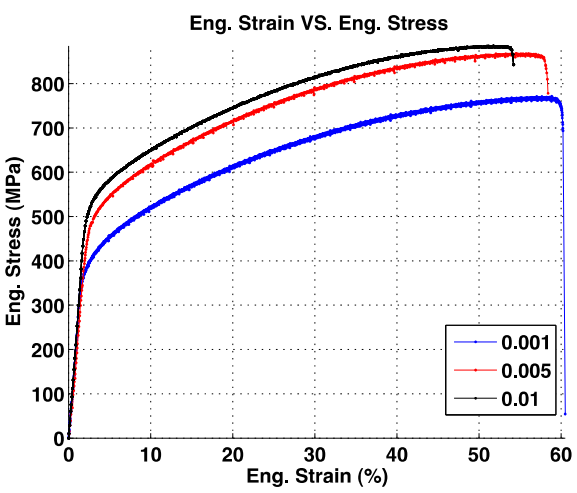

A. $11.100{ }^{\circ} \mathrm{C}$

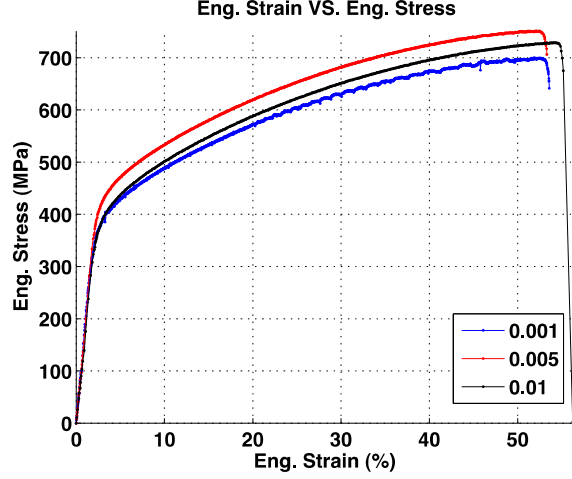

A. $12.200^{\circ} \mathrm{C}$ 


\section{Understanding Fundamental Material-Degradation Processes in High Temperature}

Aggressive Chemomechanical Environments
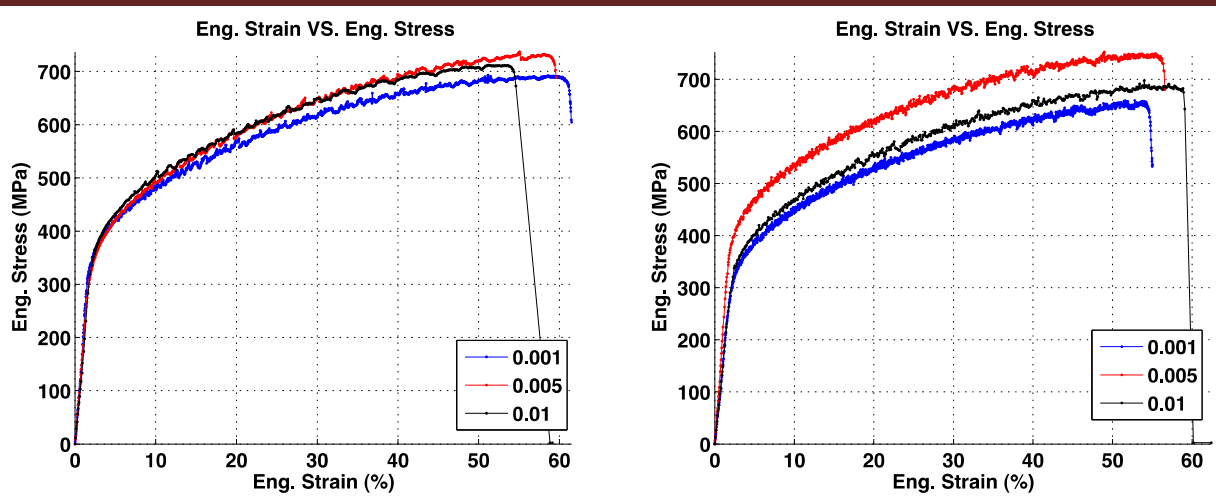

A. $13.300^{\circ} \mathrm{C}$

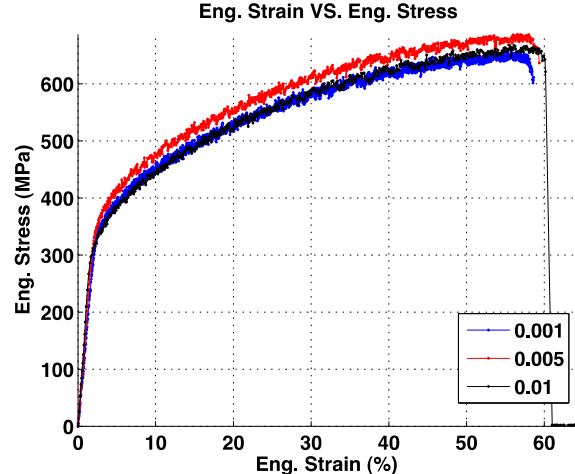

A. $14.400^{\circ} \mathrm{C}$

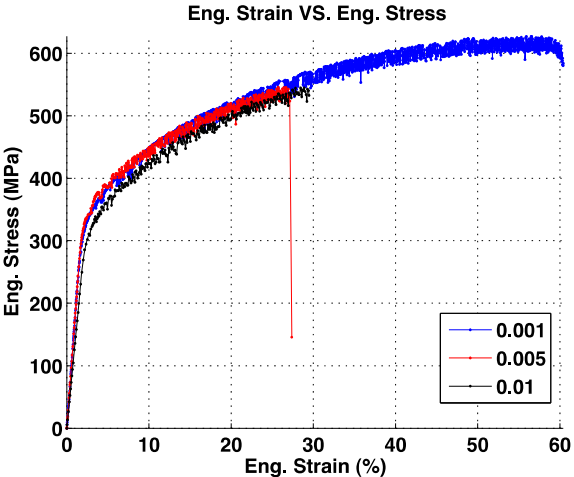

A. $15.500{ }^{\circ} \mathrm{C}$

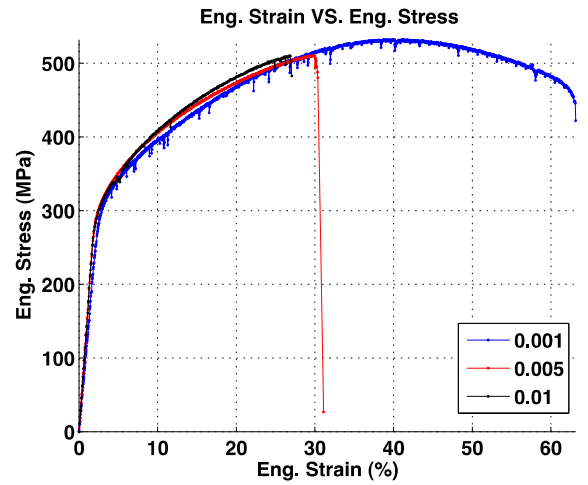

A. $17.700{ }^{\circ} \mathrm{C}$

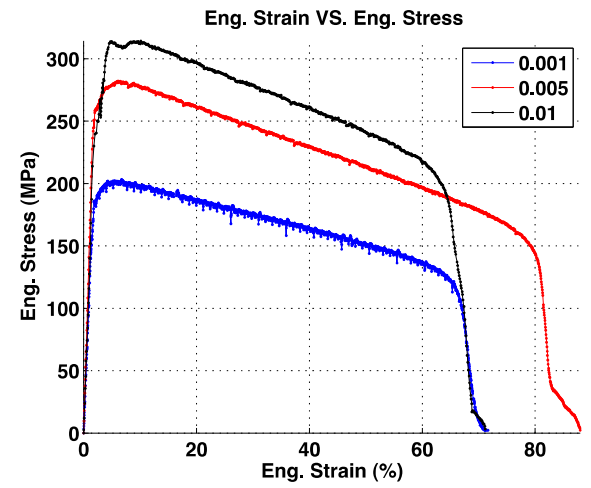

A. $19.900{ }^{\circ} \mathrm{C}$

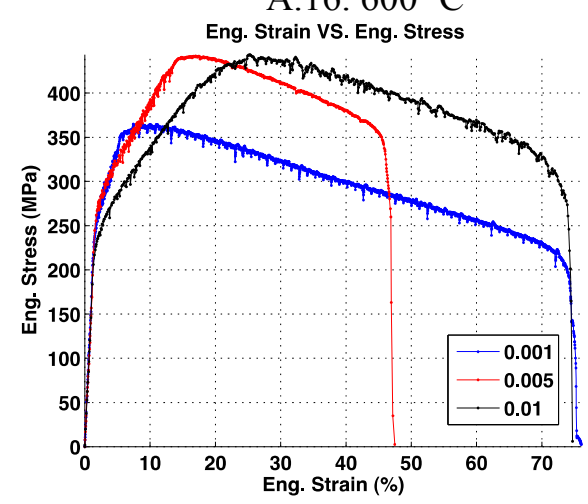

A. $18.800^{\circ} \mathrm{C}$

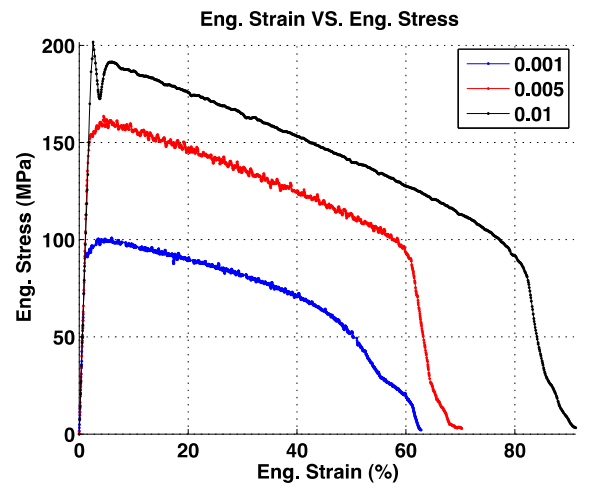

A.20. $1000^{\circ} \mathrm{C}$ 


\section{Understanding Fundamental Material-Degradation Processes in High Temperature Aggressive Chemomechanical Environments}

\section{APPENDIX B: Least Squares Anisotropic Regression}

The least squares anisotropic regression used during this study utilized the vertical DIC displacements to find the effective stress intensity factors. This is an ideal analysis technique since it is directly based on the experimental data. Crack closure effects were inherently included in the analysis since the crack opening displacements were influenced by any crack closure phenomena present. Only the vertical displacements were used since the crack only opened in the tensile, mode I manner.

The vertical displacements as a function of the mode I stress intensity factor, $\mathrm{K}_{\mathrm{I}}, \mathrm{T}$-stress, and rigid body motion for plane stress are:

$$
\mathrm{v}=\frac{K_{1}}{\mu} \sqrt{\frac{r}{2 \pi}} \sin \left(\frac{\theta}{2}\right)\left[\frac{1}{2}\left(\frac{3-v}{1+v}\right)+1-\cos \left(\frac{\theta}{2}\right)\right]-\frac{1}{2 \mu}\left(\frac{v}{1+v}\right) \operatorname{Tr} \sin (\theta)+\operatorname{Ar} \cos (\theta)+B
$$

where $A$ is the rigid body rotation coefficient, $B$ is the rigid body translation coefficient, $T$ is the T-stress term, $r$ is the distance from the crack tip, $\theta$ is the angle from the crack line ahead of the tip, $v$ is Poisson's ratio, and $\mu$ is the shear modulus. Thousands of vertical displacements $\mathrm{v}$, for each level of the load, were known from the DIC correlations at their specific locations given by $r$ and $\theta$. The shear modulus and Poisson's ratio were obtained for the material at each temperature. A least squares regression was then employed to find the four unknowns $\left(K_{1}, T, A\right.$, and $B$ ). Details of a similar least squares approach are given in McNeill et al. [6.1]. 


\section{REFERENCES}

[2.1] Natesan, K., A. Purohit, S. W. Tam, "Materials Behavior in HTGR Environments," Nuclear Regulatory Commission, NUREG/CR-6824 (July 2003)

[2.2] T. McGreevy, D.L.Marriott and P.Carter,"High Temperature Design Methods Development Advances for 617:Status and Plans, ORNL/TM-2005/515, July 31st 2005

[3.1] W. R. Corwin, T. D. Burchell, C. E. Duty, Y. Katoh, J. W. Klett, T. E. McGreevy, R. K. Nanstad, W. Ren, P. L. Rittenhouse, L. L. Snead, R. W. Swindeman, D. F. Wilson, "Next Generation Nuclear Plant Materials Research and Development Program Plan," (Revision 3), INL/EXT-06-11701, 2006

[3.2] L. Tan, K. Sridharan, T.R. Allen, R.K. Nanstad, D.A. McClintock, Microstructure tailoring for property improvements by grain boundary engineering, J Nucl Mater. 374 (2008) $270-280$.

[3.3] M.L. Young, J. DeFouw, J.D. Almer, D.C. Dunand, Load partitioning during compressive loading of a Mg/MgB2 composite, Acta Materialia. 55 (2007) 3467-3478.

[3.4] M.L. Young, J.D. Almer, M.R. Daymond, D.R. Haeffner, D.C. Dunand, Load partitioning between ferrite and cementite during elasto-plastic deformation of an ultrahigh-carbon steel, Acta Materialia. 55 (2007) 1999-2011.

[3.5] J. Almer, U. Lienert, R.L. Peng, C. Schlauer, M. Oden, Strain and texture analysis of coatings using high-energy x-rays, J Appl Phys. 94 (2003) 697-702.

[3.6] X. Pan, X.L. Wu, X. Chen, K. Mo, J. Almer, D.R. Haeffner, J.F. Stubbins, Temperature and particle size effects on flow localization of $9-12 \% \mathrm{Cr}$ ferritic/martensitic steel by in situ X-ray diffraction and small angle scattering, J Nucl Mater. 398 (2010) 220-226.

[3.7] X. Pan, Tensile Fracture Mechansims of Ferritic/Martensitic Structural Materials, in: Department of Nuclear, Plasma and Radiological Engineering, University of Illinois at Urbana-Champaign, Urbana, 2008.

[3.8] ASTM Standard E2714, "Standard test method for creep-fatigue testing," ASTM International, West Conshohocken, PA, 2009, DOI: 10.1520/E2714-09.

[3.9] http://www.ms.ornl.gov/fusionreactor/pdf/june1999/kurtz1.pdf

[3.10]http://www.betalasermike.com/index.php/en/beta-lasermike-products-en/diameter-a-ovalit yen

[3.11] H. Tung, Surface Degradation and Creep of Inconel 617 and Haynes 230 at High Temperature. $\mathrm{PhD}$ thesis, University of Illinois at Urbana-Champaign, 2012

[3.12] Swindeman, R. W. and Pugh, C. E. "Creep Studies on Type 304 Stainless Steel (Heat 8043813) Under Constant and Varying Loads", ORNL-TM 4427, June 1974

[3.13] Sutton M, Orteu J-J, Schreier H. Image Correlation for Shape Motion and Deformation Measurements: Basic Concepts, Theory and Applications. Spring Media; 2009.

[3.14] Sutton MA, Wolters WJ, Peters WH, Ranson WF, McNeill SR. Determination of displacements using an improved digital correlation method. Image Vision Comput $1983 ; 1: 133$. 


\section{Understanding Fundamental Material-Degradation Processes in High Temperature Aggressive Chemomechanical Environments}

[3.15] K. Mo, G. Lovicu, H.-M. Tung, X. Chen, J.F. Stubbins, High temperature aging and corrosion study on alloy 617 and alloy 230, J. Eng. Gas Turbines Power 133 (2011) 052908-052909.

[3.16] X. Vitart, A. Le Duigou, P. Carles, Hydrogen production using the sulfur-iodine cycle coupled to a VHTR: an overview, Energy Convers. Manage. 47 (2006) 2740-2747.

[3.17] N. Ahmed, M.S. Bakare, D.G. McCartney, K.T. Voisey, The effects of microstructural features on the performance gap in corrosion resistance between bulk and HVOF sprayed Inconel 625, Surf. Coat. Technol. 204 (2010) 2294-2301.

[3.18] A. Kewther, M. Hashmi, B. Yilbas, Corrosion properties of Inconel 617 alloy after heat treatment at elevated temperature, J. Mater. Eng. Perform. 10 (2001) 108-113.

[3.19] K. Habib, Electrochemical behavior of a high strength nickel-based alloy in H2SO4, Desalination 93 (1993) 537-544

[3.20] M. Bojinov, G. Fabricius, P. Kinnunen, T. Laitinen, K. Mäkelä, T. Saario, G. Sundholm, The mechanism of transpassive dissolution of $\mathrm{Ni}-\mathrm{Cr}$ alloys in sulphate solutions, Electrochim. Acta 45 (2000) 2791-2802.

[3.21] M. Bojinov, G. Fabricius, P. Kinnunen, T. Laitinen, K. Mäkelä, T. Saario, G. Sundholm, K. Yliniemi, Transpassive dissolution of $\mathrm{Ni}-\mathrm{Cr}$ alloys in sulphate solutions-comparison between a model alloy and two industrial alloys, Electrochim. Acta 47 (2002) 1697-1712.

[3.22] M. Bojinov, A. Galtayries, P. Kinnunen, A. Machet, P. Marcus, Estimation of the parameters of oxide film growth on nickel-based alloys in high-temperature water electrolytes, Electrochim. Acta 52 (2007) 7475-7483.

[3.23] M. Bojinov, T. Tzvetkoff, The influence of solution anion on the mechanism of transpassive dissolution of ferrous-and nickel-based alloys, J. Phys. Chem. B 107 (2003) 5101-5112.

[3.24] M. Keddam, H. Takenouti, N. Yu, Transpassive dissolution of Ni in acidic sulfate media: a kinetic model, J. Electrochem. Soc. 132 (1985) 2561-2566.

[3.25] P. Schmuki, S. Virtanen, A.J. Davenport, C.M. Vitus, Transpassive dissolution of Cr and sputter-deposited $\mathrm{Cr}$ oxides studied by in situ X-ray near-edge spectroscopy, J. Electrochem. Soc. 143 (1996) 3997-4005.

[3.26] T. Jabs, P. Borthen, H.H. Strehblow, X-ray photoelectron spectroscopic examinations of electrochemically formed passive layers on Ni-Cr alloys, J. Electrochem. Soc. 144 (1997) 1231-1243.

[3.27] M. Abdallah, H.E. Megahed, M.M. Elnager, E.M. Mabrouk, D. Radwan, Pitting corrosion of nickel alloys and its inhibition by some dihydrazide derivatives, Bull. Electrochem. 19 (2003) 245-254.

[3.28] P. Delichere, A. Hugot-Le Goff, S. Joiret, Study of thin corrosion films by in situ Raman spectroscopy combined with direct observation of nuclear reactions, Surf. Interface Anal. 12 (1988) 419-423. 


\section{Understanding Fundamental Material-Degradation Processes in High Temperature Aggressive Chemomechanical Environments}

[3.29] D.-J. Kim, H.-C. Kwon, H.P. Kim, Effects of the solution temperature and the pH on the electrochemical properties of the surface oxide films formed on alloy 600, Corros. Sci. 50 (2008) 1221-1227.

[3.30] J.H. Kim, I.S. Hwang, Development of an in situ Raman spectroscopic system for surface oxide films on metals and alloys in high temperature water, Nucl. Eng. Des. 235 (2005) 1029-1040.

[3.31] J.E. Maslar, W.S. Hurst, J.W.J. Bowers, J.H. Hendricks, In situ Raman spectroscopic investigation of stainless steel hydrothermal corrosion, Corrosion 58 (2002) 739-747.

[3.32] T.S. Mintz, T.M. Devine, Influence of surface films on the susceptibility of Inconel 600 to stress corrosion cracking, Key Eng. Mater. 261-263 (2004) 875-884

[3.33] T. Cohen-Hyams, S. Harrington, M. Miyagusuku, F. Wang, T.M. Devine, Influence of temperature and $\mathrm{pH}$ on the surface films formed on $\mathrm{Cr}$ and alloy C22, ECS Trans. 11 (2008) 87-97.

[3.34] F.G. Hodge, Effect of aging on anodic behavior of Ni-Cr-Mo alloys, Corrosion 29 (1973) $375-383$.

[3.35] H. Hirano, H. Takaku, T. Kurosawa, The relationship between the characteristics of oxide film and stress corrosion susceptibility of $\mathrm{Ni}-\mathrm{Cr}-\mathrm{Fe}$ alloy in high temperature water, Corros. Sci. 31 (1990) 557-562.

[3.36] H. Kim, D.B. Mitton, R.M. Latanision, Corrosion behavior of Ni-base alloys in aqueous $\mathrm{HCl}$ solution of $\mathrm{pH} 2$ at high temperature and pressure, Corros. Sci. 52 (2010) 801-809.

[3.37] S. Penttilä, I. Betova, M. Bojinov, P. Kinnunen, A. Toivonen, Estimation of kinetic parameters of the corrosion layer constituents on steels in supercritical water coolant conditions, Corros. Sci. 53 (2011) 4193-4203.

[3.38] R.B. Rebak, Z. Szklarska-Smialowska, The mechanism of stress corrosion cracking of alloy 600 in high temperature water, Corros. Sci. 38 (1996) 971-988.

[3.39] C. Sun, R. Hui, W. Qu, S. Yick, Progress in corrosion resistant materials for supercritical water reactors, Corros. Sci. 51 (2009) 2508-2523.

[3.40] J.-F. Li, S.-B. Li, J.R. Anema, Z.-L. Yang, Y.-F. Huang, Y. Ding, Y.-F. Wu, X.-S. Zhou, D.-Y. Wu, B. Ren, Z.-L. Wang, Z.-Q. Tian, Synthesis and characterization of gold nanoparticles coated with ultrathin and chemically inert dielectric shells for SHINERS applications, Appl. Spectrosc. 65 (2011) 620-626.

[3.41] J.F. Li, Y.F. Huang, Y. Ding, Z.L. Yang, S.B. Li, X.S. Zhou, F.R. Fan, W. Zhang, Z.Y. Zhou, Y. WuDe, B. Ren, Z.L. Wang, Z.Q. Tian, Shell-isolated nanoparticle-enhanced Raman spectroscopy, Nature 464 (2010) 392-395.

[3.42] N.R. Honesty, A.A. Gewirth, Shell-isolated nanoparticle enhanced Raman spectroscopy (SHINERS) investigation of benzotriazole film formation on $\mathrm{Cu}(100), \mathrm{Cu}(111)$, and $\mathrm{Cu}$ (poly), J. Raman Spectrosc. 43 (2012) 46-50.

[3.43] M.E. Biggin, In situ vibrational spectroscopic and electrochemical study of electrodeposition additives on copper surfaces, in: School of Chemical Sciences, University of Illinois at Urbana-Champaign, Champaign, IL, 2001. 
[3.44] W.L. Mankins, J.C. Hosier, T.H. Bassford, Microstructure and Phase-Stability of Inconel Alloy 617, Metall Trans. 5 (1974) 2579-2590.

[3.45] H.M. Tawancy, D.L. Klarstrom, M.F. Rothman, Development of a New Nickel-Base Superalloy, Jom-J Min Met Mat S. 36 (1984) 58-62.

[4.1] A.K. Roy, V. Marthandam, Mechanism of yield strength anomaly of Alloy 617, Mat Sci Eng a-Struct. 517 (2009) 276-280.

[4.2] M.S. Rahman, G. Priyadarshan, K.S. Raja, C. Nesbitt, M. Misra, Characterization of high temperature deformation behavior of INCONEL 617, Mech Mater. 41 (2009) 261-270.

[4.3] R.E. Reed-Hill, R. Abbaschian, Physical Metallurgy Principles, 3rd ed., CL-Engineering, 1991.

[4.4] G.E. Dieter, Mechanical Metallurgy, 3rd ed., McGraw-Hill, 1988.

[4.5] R.W. Hertzberg, Deformation and fracture mechanics of engineering materials, 4th edition, John Wiley \& Sons, New York, 1996.

[4.6] L. Tan, K. Sridharan, T.R. Allen, Effect of thermomechanical processing on grain boundary character distribution of a Ni-based superalloy, J Nucl Mater. 371 (2007) 171-175.

[4.7] F. Montheillet, J.-P. Thomas, Dynamic Recrystallization of Low Stacking Fault Energy Metals, Springer, Netherlands, 2004.

[4.8] T.S. Srivatsan, C.W. Meyers, J.T. Berry, A method for determining the tensile properties and anisotropy of aluminum alloys, Journal of Testing and evaluation. 15 (1987) 196-204.

[4.9] R.E. Mizia, Next Generation Nuclear Plant Intermediate Heat Exchanger Acquisition Strategy, in, Idaho National Laboratory, 2008.

[4.10] K. Mo, G. Lovicu, H.-M. Tung, X. Chen, J. Stubbins, In-plane anisotropy in microstructure and mechanical behavior of Alloy 617 following high temperature aging, in: TMS 2010 Supplemental Proceedings, Seattle, 2010, pp. 491-498.

[4.11] A. Day, P. Trimby, CHANNEL 5 Manual, HKL Technology, 2004.

[4.12] K. Mo, G. Lovicu, H.-M. Tung, X. Chen, J. Stubbins, microstructural evolution of Alloy 617 and Alloy 230 following high temperature aging, in: ASME 2010 Pressure Vessels \& Piping Division / K-PVP Conference, Bellevue, Washington, USA, 2010, 25847.

[4.13] W.J. Ren, R. Swindeman, A Review on Current Status of Alloys 617 and 230 for Gen IV Nuclear Reactor Internals and Heat Exchangers, J Press Vess-T Asme. 131 (2009) 044002.

[4.14] W.J. Ren, R. Swindeman, A Review Paper on Aging Effects in Alloy 617 for Gen IV Nuclear Reactor Applications, J Press Vess-T Asme. 131 (2009) 024002.

[4.15] Q.Y. Wu, H.J. Song, R.W. Swindeman, J.P. Shingledecker, V.K. Vasudevan, Microstructure of long-term aged IN617Ni-base superalloy, Metall Mater Trans A. 39A (2008) 2569-2585.

[4.16] http://cst-www.nrl.navy.mil/lattice/.

[4.17] W. Ren, R. Swindeman, M. Santella, Developing a Nuclear Grade of Alloy 617 for Gen IV Nuclear Energy Systems, in: ICAPP '10, San Diego, CA, USA, 2010. 


\section{Understanding Fundamental Material-Degradation Processes in High Temperature Aggressive Chemomechanical Environments}

[4.18] X. Pan, Tensile Fracture Mechansims of Ferritic/Martensitic Structural Materials, in: Department of Nuclear, Plasma and Radiological Engineering, University of Illinois at Urbana-Champaign, Urbana, 2008.

[4.19] K.H. Kuo, C.L. Jia, Crystallography of M23C6 and M6C Precipitated in a Low-Alloy Steel, Acta Metallurgica. 33 (1985) 991-996.

[4.20] N. Stoloff, Wrought and P/M Superalloys, ASM handbook.

[4.21] M.A. Meyer, K.K. Chawla, Mechanical Behavior of Materials, Cambridge University Press, New York, 2008. [4.22] http://www.haynesintl.com/.

[4.23] M.T. Hutchings, P.J. Withers, T.M. Holden, T. Lorentzen, Introduction to the characterization of residual stress by neutron diffraction, Taylor \& Francis Group, LLC, 2005.

[4.24] M.E. Fitzpatrick, A. Lodini, Analysis of Residual Stress by Diffraction using Neutron and Synchrotron Radiation, Taylor \& Francis Inc., 2003.

[4.25] I.C. Noyan, J.B. Cohen, Residual Stress, Measurement by Diffraction and Interpretation, Springer-Verlag, New York, 1987.

[4.26] V. Hauk, Structural and Residual Stress Analysis by Nondestructive Methods, Elsevier, 1997.

[4.27] J.D. Eshelby, The Determination of the Elastic Field of an Ellipsoidal Inclusion, and Related Problems, Proc R Soc Lon Ser-A. 241 (1957) 376-396.

[4.28] H. Pottebohm, G. Neite, E. Nembach, Elastic Properties (the Stiffness Constants, the Shear Modulus and the Dislocation Line Energy and Tension) of Ni-Al Solid-Solutions and of the Nimonic Alloy Pe16, Mater Sci Eng. 60 (1983) 189-194.

[4.29] B. Clausen, T. Lorentzen, T. Leffers, Self-consistent modelling of the plastic deformation of FCC polycrystals and its implications for diffraction measurements of internal stresses, Acta Materialia. 46 (1998) 3087-3098.

[4.30] Y.F. Li, Y.M. Gao, Z.J. Fan, B. Xiao, Q.W. Yue, T. Min, S.Q. Ma, First-principles study on the stability and mechanical property of eta $\mathrm{M} 3 \mathrm{~W} 3 \mathrm{C}(\mathrm{M}=\mathrm{Fe}, \mathrm{Co}, \mathrm{Ni})$ compounds, Physica B-Condensed Matter. 405 (2010) 1011-1017.

[4.31] L. Mishnaevsky, U. Weber, S. Schmauder, Numerical analysis of the effect of microstructures of particle-reinforced metallic materials on the crack growth and fracture resistance, Int J Fracture. 125 (2004) 33-50.

[4.32] M. Li, T. Nagasaka, D. Hoelzer, M. Grossbeck, S. Zinkle, T. Muroga, K. Fukumoto, H. Matsui, M. Narui, Biaxial thermal creep of two heats of $\mathrm{V} 4 \mathrm{Cr} 4 \mathrm{Ti}$ at 700 and $800^{\circ} \mathrm{C}$ in a liquid lithium environment, J Nucl Mater. 367-370 (2007) 788-793.

[4.33] E.R. Gilbert, L.D. Blackburn, Creep Deformation of 20 Percent Cold-Worked Type 316 Stainless-Steel, J Eng Mater-T Asme. 99 (1977) 168-180.

[4.34] ASTM Standard E2714, "Standard test method for creep-fatigue testing," ASTM International, West Conshohocken, PA, 2009, DOI: 10.1520/E2714-09. 


\section{Understanding Fundamental Material-Degradation Processes in High Temperature Aggressive Chemomechanical Environments}

[4.35] K. Bhanu Sankara Rao, H. Meurer and H. Schuster, "Creep-fatigue interaction of Inconel 617 at $950{ }^{\circ} \mathrm{C}$ in simulated nuclear reactor helium," Materials Science and Engineering A, vol. 104, pp. 37-51, 1988.

[4.36] T. C. Totemeier, "High-temperature creep-fatigue of alloy 617 base metal and weldments," ASME Conf. Proc., vol. 2007, pp. 255-260, 2007.

[4.37] Q. K. Zhang and Z. F. Zhang, "In situ observations on creep fatigue fracture behavior of $\mathrm{Sn}-4 \mathrm{Ag} / \mathrm{Cu}$ solder joints," Acta Materialia, vol. 59, pp. 6017-6028, 2011.

[4.38] G. D. Smith and D. H. Yates, "Optimization of the fatigue properties on Inconel alloy 617," in International Gas Turbine and Aeroengine Congress and Exposition, June 3, 1991 June 6, 1991.

[4.39] L. J. Chen, P. K. Liaw, R. L. McDaniels, G. Y. Wang, K. Liaw, J. W. Blust, S. A. Thompson, P. F. Browning, A. K. Bhattacharya, J. M. Aurrecoechea, R. R. Seeley and D. L. Klarstrom, "Low-cycle fatigue behavior and creep-fatigue life prediction of three superalloys," in Proceedings of a Symposium on Modelling the Performance of Engineering Structural Materials II, November 4, 2001 - November 8, 2001, pp. 101-117.

[4.40] C. Wells and C. Sullivan, "Low-cycle fatigue of Udimet 700 at 1700 F," ASM Transactions Quarterly, vol. 61, pp. 149, 1968.

[4.41] G. R. Halford, B. A. Lerch and M. A. McGraw, Fatigue, Creep Fatigue, and Thermomechanical Fatigue Life Testing, ASM Handbook-vol. 8 Mechanical Testing and Evalution, ASM International, Materials Park, OH, 2000.

[4.42] W. F. Hosford, Mechanical Behavior of Materials, Cambridge; New York: Cambridge University Press, 2010.

[4.43] C. Stocker, M. Zimmermann and H. Christ, "Localized cyclic deformation and corresponding dislocation arrangements of polycrystalline Ni-base superalloys and pure nickel in the VHCF regime," Int. J. Fatigue, vol. 33, pp. 2-9, 2011.

[4.44] L. F. Coffin, "A study of the effects of cyclic thermal stresses on a ductile metal," Trans. ASME, vol. 76, pp. 931-950, 1954.

[4.45] L. F. Coffin and R. P. Wesley, "Apparatus for study of effects of cyclic thermal stresses on ductile metals," Trans. ASME, vol. 76, pp. 923-930, 1954.

[4.46] N. E. Dowling, Mechanical Behavior of Materials: Engineering Methods for Deformation, Fracture and Fatigue, Upper Saddle River, NJ; London: Pearson Education, 2007.

[4.47] M. Gell and G. R. Leverant, "Mechanisms of high-temperature fatigue," Fatigue at Elevated Temperatures, ASTM STP 520, pp. 37-67, 1973.

[4.48] S. R. Bhanu, H. Schiffers, H. Schuster and H. Nickel, "Influence of time and temperature dependent processes on strain controlled low cycle fatigue behavior of alloy 617," Metallurgical Transactions A, vol. 19 A, pp. 359-371, 1988.

[4.49] H. Meurer, G. K. H. Gnirss, G. Raule, H. Schuster, G. Ullrich and W. Mergler, " Investigations on the fatigue behavior of high-temperature alloys for high-temperature gas-cooled reactor components," Nucl. Technol., vol. 66, pp. 315-323, 1984. 


\section{Understanding Fundamental Material-Degradation Processes in High Temperature Aggressive Chemomechanical Environments}

[4.50] G. R. Halford, "Evolution of creep-fatigue life prediction models," in Winter Annual Meeting of the American Society of Mechanical Engineers, December 1, 1991 - December 6, 1991, pp. 43-57.

[4.51] J. Zrnik, J. Semenak, V. Vrchovinsky and P. Wangyao, "Influence of hold period on creep-fatigue deformation behaviour of nickel base superalloy," Materials Science and Engineering A, vol. 319, pp. 637-642, 2001.

[4.52] T. C. Totemeier and H. Tian, "Creep-fatigue-environment interactions in Inconel 617," Materials Science and Engineering A, vol. 468-470, pp. 81-87, 2007.

[4.53] J. P. Strizak, C. R. Brinkman, M. K. Booker and P. L. Rittenhouse, "Influence of temperature, environment, and thermal aging on the continuous cycle fatigue behavior of Hastelloy X and Inconel 617," ORNL/TM-8130, 1982.

[4.54] S. Schlegel, S. Hopkins, E. Young, J. Cole, T. Lillo and M. Frary, "Precipitate redistribution during creep of alloy 617," Metallurgical and Materials Transactions A, vol. 40, pp. 2812-2823, 2009.

[4.55] R. Abbaschian, L. Abbaschian and R. E. Reed-Hill, Physical Metallurgy Principles, Stamford, CT: Cengage Learning, 2009.

[4.56] L. Singhal and J. Martin, "Growth of M23C6 carbide on incoherent twin boundaries in austenite," Acta Metallurgica, vol. 15, pp. 1603, 1967.

[4.57] N. S. Stoloff, C. T. Sims and W. C. Hagel, Superalloys II, New York: Wiley, 1987.

[4.58] J. H. Driver and R. W. K. Honeycombe, "Elevated-temperature fatigue of a Nb-stabilized austenitic stainless steel," Journal of the Iron and Steel Institute, vol. 209, pp. 729-38, 1971.

[4.59] G. R. Leverant, M. Gell and S. W. Hopkins, "The effect of strain rate on the flow stress and dislocation behavior of a precipitation-hardened nickel-base alloy," Materials Science and Engineering, vol. 8, pp. 125-133, 1971.

[4.60] T. S. Jo, J. H. Lim and Y. D. Kim, "Dissociation of Cr-rich M23C6 carbide in alloy 617 by severe plastic deformation," J. Nucl. Mater., vol. 406, pp. 360-364, 2010.

[4.61] D. B. Williams and E. P. Butler, "Grain boundary discontinuous precipitation reactions," International Metals Reviews, vol. 26, pp. 153-183, 1981.

[4.62] M. A. Burke and C. G. Beck, "High temperature low cycle fatigue behavior of the nickel base alloy In-617," Metallurgical Transactions A, vol. 15 A, pp. 661-670, 1984.

[4.63] D. Kim, I. Sah and C. Jang, "Effects of high temperature aging in an impure helium environment on low temperature embrittlement of alloy 617 and Haynes 230," J. Nucl. Mater., vol. 405, pp. 9-16, 2010.

[4.64] T. Lillo, J. Cole, M. Frary and S. Schlegel, "Influence of grain boundary character on creep void formation in alloy 617," Metallurgical and Materials Transactions A, vol. 40, pp. 2803-2811, 2009.

[4.65] K. Mo, Microstructural Evolution and Mechanical Behavior in Nickel Based Alloys for Very high Temperature Reactor. Dissertation, University of Illinois at Urbana-Champaign, 2011 


\section{Understanding Fundamental Material-Degradation Processes in High Temperature Aggressive Chemomechanical Environments}

[4.66] S. Xia, H. Li, T. G. Liu and B. X. Zhou, "Appling grain boundary engineering to Alloy 690 tube for enhancing intergranular corrosion resistance," J. Nucl. Mater., vol. 416, pp. 303-310, 2011.

[4.67] K. A. Unocic, R. W. Hayes, M. J. Mills and G. S. Daehn, "Microstructural features leading to enhanced resistance to grain boundary creep cracking in ALLVAC 718Plus," Metall. Mat. Trans. A, vol. 41, pp. 409-420, 2010.

[4.68] G. Palumbo, K. T. Aust, E. M. Lehockey, U. Erb and P. Lin, "On a more restrictive geometric criterion for special CSL grain boundaries," Scr. Mater., vol. 38, pp. 1685-1690, 1998.

[4.69] D. S. Lee, H. S. Ryoo and S. K. Hwang, "A grain boundary engineering approach to promote special boundaries in Pb-base alloy," Materials Science and Engineering A, vol. 354, pp. 106-111, 2003.

[4.70] T. Biro and P. J. Szabo, "Effect of thermo-mechanical fatigue on the microstructure of steels," in 4th Hungarian Conference on Materials Science, Testing and Informatics, October 12, 2003 - October 14, 2005, pp. 183-188.

[4.71] M. Shimada, H. Kokawa, Z. J. Wang, Y. S. Sato and I. Karibe, "Optimization of grain boundary character distribution for intergranular corrosion resistant 304 stainless steel by twin-induced grain boundary engineering," Acta Materialia, vol. 50, pp. 2331-2341, 2002.

[4.72] R. J. Amodeo and N. M. Ghoniem, "Review of experimental observations and theoretical models of dislocation cells and subgrains," Res Mechanica, vol. 23, pp. 137-160, 1988.

[4.73] F. Montheillet, J. -P. Thomas, "Dynamic Recrystallization of Low Stacking Fault Energy Metals," Metallic Materials with High Structural Efficiency, pp. 357-368, 2004

[4.74] S. Hsu, "Time-dependent crack growth in a heat-resistant alloy Inconel 617," J. Nucl. Sci. Technol., vol. 30, pp. 302-313, 1993.

[4.75] Y. L. Lu, L. J. Chen, P. K. Liaw, G. Y. Wang, C. R. Brooks, S. A. Thompson, J. W. Blust, P. F. Browning, A. K. Bhattacharya, J. M. Aurrecoechea and D. L. Klarstrom, "Effects of temperature and hold time on creep-fatigue crack-growth behavior of Haynes 230 alloy," Materials Science and Engineering A, vol. 429, pp. 1-10, 2006.

[4.76] Y. L. Lu, P. K. Liaw, Y. Sun, G. Y. Wang, S. A. Thompson, J. W. Blust, P. F. Browning, A. K. Bhattacharya, J. M. Aurrecoechea and D. L. Klarstrom, "Hold-time effect on the elevated-temperature crack growth behavior of solid-solution-strengthened superalloys," Acta Materialia, vol. 55, pp. 767-775, 2007.

[4.77] H. Hong, B. Rho and S. Nam, "Correlation of the M23C6 precipitation morphology with grain boundary characteristics in austenitic stainless steel," Materials Science and Engineering A, vol. 318, pp. 285-292, 2001.

[4.78] D. J. Child, G. D. West and R. C. Thomson, "Assessment of surface hardening effects from shot peening on a Ni-based alloy using electron backscatter diffraction techniques," Acta Materialia, vol. 59, pp. 4825-4834, 2011.

[4.79] S. D. Sitzman, "Introduction to EBSD analysis of micro- to nanoscale microstructures in metals and ceramics," in Testing, Reliability, and Application of Micro and Nano-Material Systems II, March 15, 2004 - March 17, 2004, pp. 78-90. 


\section{Understanding Fundamental Material-Degradation Processes in High Temperature Aggressive Chemomechanical Environments}

[4.80] W. C. Hagel and H. J. Beattie, "Cellular and general precipitation during high temperature ageing," Conference on Precipitation Processes in Steels, Special Report 64, Published by Iron and Steel Institute, pp. 98-107, 1958.

[4.81] E. Hornbogen, "Systematics of the cellular precipitation reactions," Metallurgical Transactions, vol. 3, pp. 2717-2727, 1972.

[4.82] J. Oblak and W. Owczarsk, "Cellular recrystallization in a nickel-base superalloy," Transactions of the Metallurgical Society of AIME, vol. 242, pp. 1563, 1968.

[4.83] L. Singhal and J. Martin, "Growth of M23C6 carbide on grain boundaries in an austenitic stainless steel," Transactions of the Metallurgical Society of AIME, vol. 242, pp. 814, 1968.

[4.84] K. N. Melton and J. W. Edington, "Discontinuous reactions in an aluminum-29 at. \% zinc alloy," Acta Metallurgica, vol. 22, pp. 1457-1462, 1974.

[4.85] R. Kossowsky, "Cellular precipitation in Ni-51Cr lamellar eutectic and cast Ni-44Cr alloys," Metallurgical and Materials Transactions B, vol. 1, pp. 1623-1627, 1970.

[4.86] E. Smith and J. T. Barnby, "Crack nucleation in crystalline solids," Metal Science, vol. 1, pp. 56-64, 1967.

[4.87] C. Stöcker, M. Zimmermann and H. Christ, "Effect of precipitation condition, prestrain and temperature on the fatigue behaviour of wrought nickel-based superalloys in the VHCF range," Acta Materialia, vol. 59, pp. 5288-5304, 2011.

[4.88] G. Gottstein and L. S. Shvindlerman, Grain Boundary Migration in Metals: Thermodynamics, Kinetics, Applications, Boca Raton: CRC Press, 1999.

[4.89] S. Kihara, J. B. Newkirk, A. Ohtomo and Y. Saiga, "Morphological changes of carbides during creep and their effects on the creep properties of Inconel 617 at $1000 \mathrm{C}$," Metallurgical Transactions A, vol. 11 A, pp. 1019-1031, 1980.

[4.90] Tada H, Paris PC, Irwin GR. The stress analysis of cracks handbook. Hellertown, Pa: Del Research Corp.; 1973.

[4.91] Carroll J, Efstathiou C, Lambros J, Sehitoglu H, Hauber B, Spottswood S, et al. Investigation of fatigue crack closure using multiscale image correlation experiments. Eng Fract Mech 2009;76:2384.

[4.92] Suresh S. Fatigue of materials. Cambridge, UK: Cambridge University Press; 1991.

[4.93] Anderson TL. Fracture Mechanics: Fundamentals and Applications. second ed.Boca Raton: CRC Press; 1994.

[4.94] Kim D, Jang C, Ryu W. Oxidation Characteristics and Oxide Layer Evolution of Alloy 617 and Haynes 230 at $900{ }^{\circ} \mathrm{C}$ and $1100{ }^{\circ} \mathrm{C}$. Oxid Met 2009;71:271.

[4.95] Kun M, Gianfranco L, Hsiao-Ming T, Xiang C, James FS. High Temperature Aging and Corrosion Study on Alloy 617 and Alloy 230. J Eng Gas Turbines Power 2011;133:052908.

[4.96] Gerold V, Karnthaler HP. On the origin of planar slip in f.c.c. alloys. Acta Metall 1989;37:2177.

[4.97] B. Beverskog, I. Puigdomenech, Revised pourbaix diagrams for chromium at 25-300 C, Corros. Sci. 39 (1997) 43-57. 


\section{Understanding Fundamental Material-Degradation Processes in High Temperature Aggressive Chemomechanical Environments}

[4.98] I. Betova, M. Bojinov, P. Kinnunen, T. Laitinen, P. Pohjanne, T. Saario, M. Vilpas, Transpassive dissolution mechanism of Ni-based alloys in a simulated bleaching solution, J. Electrochem. Soc. 149 (2002) B499-B509.

[4.99] H. Feilchenfeld, O. Siiman, Adsorption and aggregation kinetics and its fractal description for chromate, molybdate, and tungstate ions on colloidal silver from surface Raman spectra, J. Phys. Chem. 90 (1986) 4590-4599.

[4.100] B.L. Hurley, R.L. McCreery, Raman spectroscopy of monolayers formed from chromate corrosion inhibitor on copper surfaces, J. Electrochem. Soc. 150 (2003) B367-B373.

[4.101] J.D. Ramsey, R.L. McCreery, In situ Raman microscopy of chromate effects on corrosion pits in aluminum alloy, J. Electrochem. Soc. 146 (1999) 4076-4081.

[4.102] J.D. Ramsey, L. Xia, M.W. Kendig, R.L. McCreery, Raman spectroscopic analysis of the speciation of dilute chromate solutions, Corros. Sci. 43 (2001) 1557-1572.

[4.103] G.M. Brown, G.A. Hope, In situ spectroscopic evidence for the adsorption of SO42-ions at a copper electrode in sulfuric acid solution, J. Electroanal. Chem. 382 (1995) 179-182.

[4.104] J.J. Hatch, A.A. Gewirth, Potential dependent chromate adsorption on gold, J. Electrochem. Soc. 156 (2009) D497-D502.

[5.1] H.Y. Hunsicker, New Graphic Method for Analysis of Hot Deformation and Effects on Directional Properties, T Metall Soc Aime. 245 (1969) 29.

[5.2] T.C. Totemeier, T.M. Lillo, Effect of orientation on the tensile and creep properties of coarse-grained INCONEL alloy MA754, Metall Mater Trans A. 36A (2005) 785-795.

[5.3] M.A. Meyer, K.K. Chawla, Mechanical Behavior of Materials, Cambridge University Press, New York, 2008.

[5.4] G.F.V. Voort, G.M. Lucas, E.P. Manilova, Metallography and Microstructures of Heat-Resistant Alloys, ASM Handbook. 9 (2004) 820-859.

[5.5] J.K. Benz, J.H. Kim, R.G. Ballinger, Effect of Oxygen Potential on Crack Growth in Alloys for Advanced Energy Systems, J Eng Gas Turb Power. 132 (2010) 102901.

[5.6] ASTM B 168-08, 2008.

[5.7] R. Mignogna, C. Dantonio, R. Maciag, Mukherje.K, Mechanical Behavior of 6063-Aluminum, Metall Trans. 1 (1970) 1771.

[5.8] H.Y. Bi, H. Kokawa, Z.J. Wang, M. Shimada, Y.S. Sato, Suppression of chromium depletion by grain boundary structural change during twin-induced grain boundary engineering of 304 stainless steel, Scripta Mater. 49 (2003) 219-223.

[5.9] J.D. Whittenberger, Effect of Long-Term 1093-K Exposure to Air or Vacuum on the Structure of Several Wrought Superalloys, J Mater Eng Perform. 2 (1993) 745-758.

[5.10] H. Kirchhofer, F. Schubert, H. Nickel, Precipitation Behavior of Ni-Cr-22fe-18mo (Hastelloy-X) and Ni-Cr-22co-12mo (Inconel-617) after Isothermal Aging, Nucl Technol. 66 (1984) 139-148.

[5.11] J.W. Christian, The Theory of Transformations in Metals and Alloys - Part I: Equilibrium and General Kinetic Theory, Pergamon, 1975. 


\section{Understanding Fundamental Material-Degradation Processes in High Temperature Aggressive Chemomechanical Environments}

[5.12] R. Wright, Summary of Studies of Aging and Environmental Effects on Inconel 617 and Haynes 230, INL/EXT-06-11750. (2006) 39.

[5.13] B. Clausen, T. Lorentzen, M.A.M. Bourke, M.R. Daymond, Lattice strain evolution during uniaxial tensile loading of stainless steel, Mat Sci Eng a-Struct. 259 (1999) 17-24.

[5.14] A. Pyzalla, B. Camin, T. Buslaps, M. Di Michiel, H. Kaminski, A. Kottar, A. Pernack, W. Reimers, Simultaneous tomography and diffraction analysis of creep damage, Science. 308 (2005) 92-95.

[5.15] Special Metals Corporation, "Inconel alloy 617, " Paper No. SMC-029, 2005.

[5.16] Haynes International Inc., "Haynes 230 alloy," High-Temperature Alloys Brochure, Paper No. H-3000H, 2007.

[5.17] F. Schubert, U. Bruch, R. Cook, H. Diehl, P. J. Ennis, W. Jakobeit, H. J. Penkalla, E. te Heesen and G. Ullrich, "Creep rupture behavior of candidate materials for nuclear process heat applications," Nucl. Technol., vol. 66, pp. 227-240, 1984.

[5.18] R. D. Campbell, "Creep/fatigue interaction correlation for 304 stainless steel subjected to strain- controlled cycling with hold times at peak strain," vol. 93 Ser B, pp. 887-892, 1971.

[5.19] Richard Wright, "Overview of the NGNP High Temperature Metals Program," Very High Temperature Reactor (VHTR) Research \& Development 4th Annual Technical Review Meeting, Albuquerque, NM, April 26, 2011 - April 28, 2011.

[5.20] T. H. Hyde, L. Xia, A. A. Becker and W. Sun, "Fatigue, creep and creep/fatigue behavior of a nickel base superalloy at $700{ }^{\circ} \mathrm{C}$," Fatigue and Fracture of Engineering Materials and Structures, vol. 20, pp. 1295-1303, 1997.

[5.21] Sam Sham, "ASME Code and Constitutive Modeling," Very High Temperature Reactor (VHTR) Research \& Development 4th Annual Technical Review Meeting, Albuquerque, NM, April 26, 2011 - April 28, 2011.

[5.22] H. J. Frost and M. F. Ashby, Deformation-Mechanism Maps, Oxford Oxfordshire; New York: Pergamon Press, 1982.

[5.23] D. Kim, C. Jang and W. S. Ryu, "Oxidation characteristics and oxide layer evolution of Alloy 617 and Haynes 230 at 900 C and 1100 C," Oxidation Metals, vol. 71, pp. 271-293, 2009.

[5.24] S. K. Srivastava and D. L. Klarstrom, "LCF behavior of several solid solution strengthened alloys used in gas turbine engines," in International Gas Turbine and Aeroengine Congress and Exposition, June 11, 1990 - June 14, 1990, pp. GT80.

[5.25] F. Schubert, "Evaluation of materials for heat exchanging components in advanced helium-cooled reactors," Specialists' meeting on heat exchanging components of gas-cooled reactors Duesseldorf (Germany), April 16, 1984 - April 19, 1984, IWGGCR-9, pp. 309-334.

[5.26] M. D. Matthew, V. Singh, W. Chen and R. P. Wahi, "Life prediction for a nickel base alloy nimonic PE 16 under low cycle fatigue loading at $923 \mathrm{~K}$," Acta Metallurgica Et Materialia, vol. 39, pp. 1507-1513, 1991.

[5.27] A. Schwartz and M. Kumar, Electron Backscatter Diffraction in Materials Science, Springer, 2009. 


\section{Understanding Fundamental Material-Degradation Processes in High Temperature Aggressive Chemomechanical Environments}

[5.28] F.C. Monkman, N. J. Grant: Proc. ASTM, 1956, vol. 56, pp. 593-620.

[5.29] Christensen RH. Fatigue crack growth affected by metal fragments wedged between opening-closing crack surfaces. Appl Mater Res 1963;2:207.

[5.30] Elber W. Fatigue crack closure under cyclic tension. Eng Fract Mech 1970;2:37.

[5.31] Elber W. The Significance of Fatigue Crack Closure, ASTM STP - 486; 1971. p. 230.

[5.32] Staal HU, Elen JD. Crack closure and influence of cycle ratio R on fatigue crack growth in type 304 stainless steel at room temperature. Eng Fract Mech 1979;11:275.

[5.33] Sangid MD, Pataky GJ, Sehitoglu H, Hamilton RF, Maier HJ. High resolution analysis of opening and sliding in fatigue crack growth. Int J Fatigue 2011;37:134.

[5.34] Lee S, Lu Y, Liaw P, Choo H, Thompson S, Blust J, et al. High-temperature tensile-hold crack-growth behavior of Hastelloy X alloy compared to HAYNES 188 and HAYNES 230 alloys. Mech Time-Depend Mater 2008;12:31.

[5.35] Suresh S, Zamiski G, Ritchie D. Oxide-Induced Crack Closure: An Explanation for Near-Threshold Corrosion Fatigue Crack Growth Behavior. Metall Mater Trans A 1981;12:1435.

[5.36] Liaw PK, Leax TR, Williams RS, Peck MG. Influence of oxide-induced crack closure on near-threshold fatigue crack growth behavior. Acta Metall 1982;30:2071.

[5.37] King JE. Surface damage and near-threshold fatigue crack growth in a Ni-base superalloy in vacuum. Fatigue Fract Eng Mater Struct 1982;5:177.

[5.38] Ritchie RO, Suresh S, Moss CM. Near-Threshold Fatigue Crack Growth in 2 1/4 Cr-1Mo Pressure Vessel Steel in Air and Hydrogen. J Eng Mater Technol 1980;102:293.

[5.39] Ezaz T, Sangid MD, Sehitoglu H. Energy barriers associated with slip-twin interactions. Phil Mag 2007;91:1464.

[5.40] Gao Y, Ritchie R, Kumar M, Nalla R. High-cycle fatigue of nickel-based superalloy ME3 at ambient and elevated temperatures: Role of grain-boundary engineering. Metall Mater Trans A 2005;36:3325.

[5.41] Sangid MD, Pataky GJ, Sehitoglu H, Rateick RG, Niendorf T, Maier HJ. Superior fatigue crack growth resistance, irreversibility, and fatigue crack growth microstructure relationship of nanocrystalline alloys. Acta Mater 2011; 59:7340.

[5.42] Mott NF. A theory of the origin of fatigue cracks. Acta Metall 1958;6:195.

[5.43] Mughrabi H. Cyclic Slip Irreversibilities and the Evolution of Fatigue Damage. Metall Mater Trans A 2009;40:1257.

[5.44] Pineau A, Antolovich SD. High temperature fatigue of nickel-base superalloys - A review with special emphasis on deformation modes and oxidation. Eng Fail Anal 2009; 16:2668.

[5.45] Ellyin F. Fatigue Damage, Crack Growth and Life Prediction. Springer; 1996.

[5.46] Jenkins CHM, Mellor GA. Investigation of behaviour of metals under deformation at high temperatures-1. J Iron Steel Inst 1935;132:179. 


\section{Understanding Fundamental Material-Degradation Processes in High Temperature Aggressive Chemomechanical Environments}

[5.47] Manson S. Fatigue: A complex subject-Some simple approximations. Exp Mech 1965;5:193.

[6.1] McNeill SR, Peters WH, Sutton MA. Estimation of stress intensity factor by digital image correlation. Eng Fract Mech 1987;28:101.

[7.1] C.-H. Ma, J.-H. Huang, Haydn Chen, Thin Solid Films, 418 (2002), p. 73

[7.2] J.H. Huang, Y. Kae-Jy, P. Sit and G.-P Yu., Surface and Coatings Technology, 200 (2006), pp. 4291-4299

[7.3] HAYNES International, Inc. "HAYNES 230 Alloy,” Publication No. H-3060D, 2009

[7.4] B. Geddes, H. Leon, X. Huang, (2010). Superalloys: Alloying and Performance. Materials Park, Ohio: ASM International.

[7.5] R. Raj, "Development of Processing Map for Use in Warm-Forming and Hot-forming Process," Metallurgical Transactions A, vol. 12A, pp. 1089-1097, 1981 [7.6] M. S. A. Karunaratne, R.C. Reed, "Interdiffusion of the platinum-group metals in nickel at elevated temperatures," Acta Materialia, vol. 51, pp. 2905-2919, 2003[7.7] J. K. Wright, R. N. Wright (INL), T.-L. Sham (ORNL) "Next Generation Nuclear Plant Steam Generator and Intermediate Heat Exchanger Materials Research and Development Plan," Idaho National Laboratory Plan, Project No. 23747, Document ID PLN-2804, 2010

[7.8] W. Ren, R. W. Swindeman, "A Review Paper on Aging Effects in Alloy 617 for Gen IV Nuclear Reactor Application," Journal of Pressure Vessel Technology, vol. 131, 2009

[7.9] F. Montheillet, "Moving Grain Boundaries during Hot Deformation of Metals: Dynamic Recrystallization," Moving Interfaces in Crystalline Solids, pp. 203-256, 2004

[7.10] W. Ren, R. Swindeman, "A Review on Current Statues of Alloy 617 and Alloy 230 for Gen IV Nuclear Reactor Internals and Heat Exchangers," Journal of Pressure Vessel Technology, Transactions of ASME, vol. 131, no. 4, pp. 0440021-04400215, 1998

[7.11] H.J. Christ, L. Berchtold, H.G. Sockel, Oxid. Met. 26 (1986) 45-76.

[7.12] W.C. Hagel, A.U. Seybolt, J. Electrochem. Soc. 108 (1961) 1146.

[7.13] R.E. Lobnig, H.P. Schmidt, K. Hennesen, H.J. Grabke, Oxid. Met. 37 (1992) 81-93.

[7.14] S.C. Tsai, A.M. Huntz, C. Dolin, Oxid. Met. 37 (1995) 581-596.

[7.15] D.J. Young, High Temperature Oxidation and Corrosion of Metals, Elsevier Ltd., 2008. p.33

[7.16] D. Burgreen, Elements of Thermal Stress Analysis, C.P. Press, New York, 1971. p. 462.

[7.17] C.S. Richard, J. Lu, G. Béranger, F. Decomps, J. Therm. Spray Technol. 4 (4) (1995) 342.

[7.18] M. Schütze, Protective Oxide Scales and Their Breakdown, Wiley, New York, 1997. p. 43.

[7.19] J.F. Shackelford, W. Alexander, CRC Materials Science and Engineering Handbook, 3rd ed., CRC Boca Raton FL, 2001. p. 465.

[7.20] http://www.specialmetals.com/products/inconelalloy617.php.

[7.21] R.W. Hoffman, Thin Solid Films 34 (1976) 185. 
[7.22] W.D. Nix, B.M. Clemens, J. Mater. Res. 14 (1999) 3467-3473.

[7.23] P. Kofstad, K.P. Lillerud, Oxid. Met. 17 (1982) 177-194.

[7.24] A.C.S. Sabioni, B. Lesage, A.M. Huntz, J.C. Pivin, C. Monty, Phil. Mag. A 66 (3) (1992) 333.

[7.25] S.J. Rothman, L.J. Nowicki, G.E. Murch, J. Phys. F. 10 (1980) 383.

[7.26] P. Jian, L. Jian, H. Bing, G. Xie, J. Power Sources 158 (2006) 354-360.

[7.27] A. Ul-Hamid, Anti-Corrosion Methods and Materials, 51 (2004) pp. 216 - 222

[7.28] H.J. Christ, U. Kunecke, K. Meyer and H.G. Sockel, Oxid. Met., 30 (1988), pp. 27-51

[7.29] J. Zurek, D.J. Young, E. Essuman, M. Hansel, H.J. Penkalla, L. Niewolak, W.J. Quadakkers, Mater. Sci. Eng. A 477 (2008) 259-270.

[7.30] T. Norby, J. Phys. IV Coll. C9 3, (1993) 99.

[7.31] T. Norby, Adv. Ceram. 23, (1987) 107.

[7.32] A. Galerie, Y. Wouters, M. Caillet, Mater. Sci. Forum, 369-372 (2001), pp. 237-238.

[7.33] S. Gossé, T. Alpettaz, S. Chatain and C. Guéneau, Journal of Engineering for Gas Turbines and Power, 131 (2009) 062901.

[7.34] H. Li, W. Chen, Corr. Sci. 52 (2010) 2481

[7.35] W.G. Kim, S.N. Yin, G.G. Lee, Y.W. Kim, S.J. Kim, Int. J. Pressure Vessels Piping, 87 (June (6)) (2010), pp. 289-295

[7.36] F. Schubert, H.J. Seehafer, E. Bodmann, Proc. ASME-Conf. Status of Materials Development and Technology for Advanced Nuclear Heat Exchangers, Denver, USA (Oct. 1982)

[7.37] Kassner, M.E., "Fundamentals of Creep in Metals and Alloys", 2009, Elsevier Science; 2nd edition, p.61

[7.38] Kassner, M.E., "Fundamentals of Creep in Metals and Alloys", 2009, Elsevier Science; 2nd edition, p. 125

[7.39] M. Maurizio, A. Giuliano, L.Valentino, Materials Science Forum, 2285 (2010) 638.

[7.40] P.J. Ennis, A. Zielinska-Lipiec, O. Wachter, A. Czyrska-Filemonowicz, Acta Mater. 45 (1997) 4901-4907.

[7.41] M Sakane, H. Tokura., Int J Damage Mech 11 (2002) 247.

[7.42] D.R. Hayhurst, J Mech Phys Solids, 20 (1972) 381390.

[7.43] C.S. Seok, B.K. Bae, J.M. Koo, K.L. Murty, Eng Failure Anal, 13 (2006), p. 389

[7.44] S. Gollapudi, I. Charit, K.L. Murty, Acta Mater. 56 (2008) 2406-2419.

[7.45] J. C. M. Li, "Petch Relation and Grain Boundary Sources," Transactions of the Metallurgical Society of AIME, vol. 227, pp. 239-247, 1963

[7.46] R. W. Hertzberg, (1996). Deformation and Fracture Mechanics of Engineerign Materials (4th ed.), Hoboken, New Jersey: John Wiley \& Sons 
Understanding Fundamental Material-Degradation Processes in High Temperature Aggressive Chemomechanical Environments

[7.47] S. Q. Yuan, H.B. Wu, C.J. Shang, X.L. He, "Formation of two hardening peaks in $\mathrm{Nb}$-containing microalloyed steel during aging," Transaction of Material and Heat Treatment, vol. 26, pp. 69-73, 2005 
This project supported the graduate work of four students. Three students have obtained Ph.D degrees and two students have obtained masters degrees from the University of Illinois resulting from funding in this program.

\begin{tabular}{|c|c|c|c|c|}
\hline Name & Thesis Title & Degree & Year & Position \\
\hline MO, Kun & $\begin{array}{l}\text { Microstructural evolution and } \\
\text { mechanical behavior in nickel } \\
\text { based alloys for very high } \\
\text { temperature reactor }\end{array}$ & Ph.D & 2011 & $\begin{array}{c}\text { Senior Software } \\
\text { Engineer } \\
\text { China Nuclear Power } \\
\text { Technology Research } \\
\text { Institute } \\
\text { Currently, Postdoc } \\
\text { University of Illinois } \\
\end{array}$ \\
\hline $\begin{array}{l}\text { CHEN, } \\
\text { Xiang }\end{array}$ & $\begin{array}{l}\text { High temperature } \\
\text { creep-fatigue behavior of } \\
\text { Alloy } 617 \text { and Alloy } 230\end{array}$ & Ph.D & 2012 & $\begin{array}{l}\text { Postdoc, } \\
\text { Oak Ridge National } \\
\text { Laboratory }\end{array}$ \\
\hline $\begin{array}{c}\text { TUNG, } \\
\text { Hsiao-Ming }\end{array}$ & $\begin{array}{l}\text { Surface degradation and creep } \\
\text { of Inconel } 617 \text { and Haynes } \\
230 \text { at high temperatures }\end{array}$ & Ph.D & 2012 & $\begin{array}{c}\text { Research Scientist } \\
\text { Tsinghua University, } \\
\text { Taiwan }\end{array}$ \\
\hline ZHAO, Yang & $\begin{array}{l}\text { High temperature aging study } \\
\text { on long-term aged Alloy } 617 \\
\text { and Alloy } 230\end{array}$ & M.S. & 2012 & $\begin{array}{l}\text { Graduate Student, } \\
\text { Purdue University }\end{array}$ \\
\hline Lv, Wei & Inconel 617 and Haynes 230 & M.S. & 2012 & \\
\hline
\end{tabular}




\section{Journal Papers}

\section{PUBLICATIONS AND PRESENTATIONS}

1. Kun Mo, Gianfranco Lovicu, Hsiao-Ming Tung, Xiang Chen, James F. Stubbins, "High Temperature Aging and Corrosion Study on Alloy 617 and Alloy 230", Journal of Engineering for Gas Turbines and Power, Vol. 133, paper No. 052908, 2011

2. Kun Mo, Hsiao-Ming Tung, Jonathon Almer, Meimei Li, Xiang Chen, Weiying Chen, Jon B. Hansen, James F. Stubbins, "Synchrotron radiation study on Alloy 617 and Alloy 230 for VHTR application”, Journal of Pressure Vessel Technology, Vol. 135, paper No. 021502,2013

3. Kun Mo, Gianfranco Lovicu, Hsiao-Ming Tung, Xiang Chen, Yinbin Miao Jon B. Hansen, James F. Stubbins, "Effect of orientation on plastic deformations of Alloy 617 for VHTR Applications”, Journal of Nuclear Materials, Volume 443, pp 366-377, 2013

4. Kun Mo, Xiang Chen, Gianfranco Lovicu, Hsiao-Ming Tung, James F. Stubbins, "Mechanism of Plastic Deformation of a Ni-based Superalloy for VHTR Applications", Journal of Nuclear Materials, Vol. 441, pp. 695-703, 2013

5. Kun Mo, Hsiao-Ming Tung, Xiang Chen, Yang Zhao, James F. Stubbins, "Mechanism of Plastic Deformation of a Ni-based Superalloy for VHTR Applications", Nuclear Technology, Vol. 183, pp. 455-463, 2013

6. Xiang Chen, Mikhail A. Sokolov, Sam Sham, Donald L. Erdman III, Jeremy T. Busby, Kun Mo, James F. Stubbins, "Experimental and Modeling Results of Creep-Fatigue Life of Inconel 617 and Haynes 230 at $850^{\circ} \mathrm{C}$ ", Journal of Nuclear Materials, Vol. 432, pp. 94-101, 2013

7. Xiang Chen, Zhiqing Yang, Mikhail A. Sokolov, Donald L. Erdman III, Kun Mo, James F. Stubbins, "Low Cycle Fatigue and Creep-Fatigue Behavior of Ni-based Alloy 230 at $850^{\circ}$ C", Materials Science and Engineering: A, Vol. 108, pp. 152-162, 2013

8. Hsiao-Ming Tung, James F. Stubbins, "Incipient oxidation kinetics and residual stress of the oxide scale grown on Haynes 230 at high temperatures", Materials Science and Engineering: A, Vol. 538, pp. 1-6, 2012

9. Hsiao-Ming Tung, James F. Stubbins, "Incipient corrosion behavior of Haynes 230 under a controlled reducing atmosphere at high temperatures", Journal of Nuclear Materials, Vol. 427, pp. 389-392, 2012

10. Hsiao-Ming Tung, James F. Stubbins, "Incipient oxidation kinetics of Alloy 617 and residual stress of the oxide scale formed in air at temperatures between 850 and $1000^{\circ} \mathrm{C}$ ", Journal of Nuclear Materials, Vol. 424, pp. 23-28, 2012

11. Garrett J. Pataky, Huseyin Sehitoglu, Hans J. Maier, "Very High Temperature Fatigue Crack Growth in Haynes 230", Materials Characterization, Vol. 75, pp. 69-78, 2013

12. Garrett J. Pataky, Huseyin Sehitoglu, Hans J. Maier, "Creep deformation and mechanisms in Haynes 230 at $800{ }^{\circ} \mathrm{C}$ and $900{ }^{\circ} \mathrm{C}$ ", Journal of Nuclear Materials, Vol. 443, pp. 484-490, 2013

13. Garrett J. Pataky, Michael D. Sangid, Huseyin Sehitoglu, Reginald F. Hamilton, Hans J. Maier, Petros Sofronis, "Full field measurements of anisotropic stress intensity factor 


\section{Understanding Fundamental Material-Degradation Processes in High Temperature}

Aggressive Chemomechanical Environments

ranges in fatigue”, Engineering Fracture Mechanics, Volume 94, pp 13-28, 2012

14. Nicole R. Honesty, Andrew A. Gewirth, "Investigating the effect of aging on transpassive behavior of Ni-based alloys in sulfuric acid with shell-isolated nanoparticle enhanced Raman spectroscopy (SHINERS)", Corrosion Science, Vol. 67, pp. 67 - 74, 2013

15. Xiao Pan, Xianglin Wu, Kun Mo, Xiang Chen, Jonathon Almer, Dean R. Haeffner, Jan Ilavsky, James F. Stubbins, "Lattice strain and damage evolution of $9-12 \% \mathrm{Cr}$ ferritic/martensitic steel during in situ tensile test by X-ray diffraction and small angle scattering", Journal of Nuclear Materials, Vol. 407, pp.10-15, 2010

16. Xiao Pan, Xianglin Wu, Xiang Chen, Kun Mo, Jonathon Almer, Dean R. Haeffner, James F. Stubbins, "Temperature and particle size effects on flow localization of $9-12 \% \mathrm{Cr}$ ferritic/martensitic steel by in situ X-ray diffraction and small angle scattering", Journal of Nuclear Materials, Vol. 398, pp. 220-226, 2010

17. Xiang Chen, Zhiqing Yang, Mikhail A. Sokolov, Donald L. Erdman III, Kun Mo, James F. Stubbins, "Effect of Creep and Oxidation on Reduced Fatigue life of Ni-based Alloy 617 at $850^{\circ} \mathrm{C} "$, Journal of Nuclear Materials, Vol. 444, pp. 393-403, 2014

18. Hsiao-Ming Tung, Kun Mo, James F. Stubbins, "Biaxial Thermal Creep of Inconel 617 and Haynes 230 at 850 and $950^{\circ} \mathrm{C}$ ", Journal of Nuclear Materials, Vol. 447, pp. 28-37, 2014

19. Kun Mo, Zhangjian Zhou, Yinbin Miao, Di Yun, Hsiao-Ming Tung, Guangming Zhang, Weiying Chen, Jonathon Almer, and James F. Stubbins, "Synchrotron study on load partitioning between ferrite/martensite and nanoparticles of a 9Cr ODS steel", submitted to Journal of Nuclear Materials

\section{Selected Conference Papers}

1. Kun Mo, Wei Lv, Hsiao-Ming Tung, Di Yun, Yinbin Miao, Kuan-Che Lan, James F. Stubbins, "Biaxial thermal creep of Alloy 617 and Alloy 230 for VHTR applications", Proceedings of the ASME Symposium on Elevated Temperature Application of Materials for Fossil, Nuclear, and Petrochemical Industries, Seattle, WA, 2014, accepted

2. Kun Mo, Hsiao-Ming Tung, Xiang Chen, Yang Zhao, James F. Stubbins, "Design Stress Evaluation based on Strain-Rate Sensitivity Analysis for Nickel Alloys used in the Very-High Temperature Nuclear System”, PHYSOR 2012, Knoxville, TN, 2012

3. Kun Mo, Hsiao-Ming Tung, Jonathon Almer, Meimei Li, Xiang Chen, Weiying Chen, Jon B. Hansen, James F. Stubbins, "Synchrotron radiation study on Alloy 617 and Alloy 230 for VHTR application", Proceedings of the ASME 2011 Pressure Vessels \& Piping Conference, PVP2011-57393, 2011

4. Kun Mo, Xiang Chen, Gianfranco Lovicu, Hsiao-Ming Tung, James F. Stubbins, "Mechanism of Plastic Deformation of a Ni-based Superalloy for VHTR Applications", Transactions of the American Nuclear Society, 2010, paper No. 1309

5. Kun Mo, Gianfranco Lovicu, Hsiao-Ming Tung, Xiang Chen, James F. Stubbins, "In-plane anisotropy in microstructure and mechanical behavior of Alloy 617 following high temperature aging", Proceedings of TMS 2010, Volume 2, pp. 491-498, 2010 


\section{Understanding Fundamental Material-Degradation Processes in High Temperature}

Aggressive Chemomechanical Environments

6. Kun Mo, Gianfranco Lovicu, Hsiao-Ming Tung, Xiang Chen, James F. Stubbins, "High Temperature Aging and Corrosion Study on Alloy 617 and Alloy 230", Proceedings of the 18th International Conference on Nuclear Engineering, ICONE18-30207, 2010

7. Kun Mo, Gianfranco Lovicu, Hsiao-Ming Tung, Xiang Chen, James F. Stubbins, "Microstructural Evolution of Alloy 617 and Alloy 230 following High Temperature Aging", Proceedings of the ASME 2010 Pressure Vessels \& Piping Division, PVP2010-25847, 2010

\section{Selected Presentations}

1. "Mechanism of Plastic Deformation of a Ni-based Superalloy for VHTR Applications", ANS annual meeting, San Diego, CA, July, 2010

2. "Microstructural Evolution of Alloy 617 and Alloy 230 following High Temperature Aging", ASME 2010 Pressure Vessels and Piping Conference (PVP2010), Bellevue, WA, July, 2010

3. "High Temperature Aging and Corrosion Study on Alloy 617 and Alloy 230", the 18th International Conference on Nuclear Engineering, Xi'an, China, June, 2010

4. "In-plane anisotropy in microstructure and mechanical behavior of Alloy 617 following high temperature aging”, TMS 2010, Seattle, WA, February, 2010

5. "Creep Deformation Study on Alloy 617 and Alloy 230 Using Miniature Pressurized Creep Tubes", Workshop on the Role of Synchrotron Radiation in Solving Scientific Challenges in Advanced Nuclear Energy Systems, Argonne National Lab, Argonne, IL, January, 2010

6. "Materials Development for High Temperature Gas-Cooled Nuclear Reactors", IAEA Technical Meeting on "Enhancement of accelerator based real-time methods in development and characterization of materials for energy applications", Argonne National Lab, Argonne, IL, January, 2011

7. "A high energy Synchrotron x-ray study of biaxial thermal creep of AISI 316L steel", TMS 2011, San Diego, California, March, 2011

8. "High Temperature Structural Materials Characterization for HTGR Applications. Part I: Coordinated Experiments and Modeling", Very High Temperature Reactor (VHTR) Research \& Development 4th Annual Technical Review Meeting, Albuquerque, NM, April 26-28, 2011

9. "High Temperature Structural Materials Characterization for HTGR Applications. Part II: Creep-Fatigue Testing and Microanalyses", Very High Temperature Reactor (VHTR) Research \& Development 4th Annual Technical Review Meeting, Albuquerque, NM, April 26-28, 2011

10. "High Temperature Structural Materials Characterization for HTGR Applications. Part III: High Temperature Corrosion Study on Inconel 617”, Very High Temperature Reactor (VHTR) Research \& Development 4th Annual Technical Review Meeting, Albuquerque, NM, April 26-28, 2011 


\section{Understanding Fundamental Material-Degradation Processes in High Temperature}

Aggressive Chemomechanical Environments

11. "Creep-Fatigue Behavior of Alloy 617 and Alloy 230", 5th Very High Temperature Reactor (VHTR) Research \& Development 5th Annual Technical Review Meeting, May 24-26, 2012 Salt Lake City, Utah

12. "A non-destructive synchrotron study of the pressurized creep tube", the ASME Applied Mechanics and Materials Conference, McMAT-2011, Chicago, Jun, 2011

13. "Synchrotron radiation study on Alloy 617 and Alloy 230 for VHTR application", ASME 2011 Pressure Vessels \& Piping Division Conference (PVP2011), Jul, Baltimore, 2011

14. "Design Stress Evaluation based on Strain-Rate Sensitivity Analysis for Nickel Alloys used in the Very-High Temperature Nuclear System", PHYSOR 2012, Knoxville, TN, 2012

15. "High-energy Synchrotron Radiation Study on Anisotropic Loading Behavior of Alloy 230 for VHTR Applications”, TMS 2013, San Antonio, TX, Mar, 2013

16. "Bi-axial Creep Tests for Ni-based Alloy 617 and Alloy 230 for VHTR Part 1: Bi-axial Creep Tests at 850 and $950^{\circ} \mathrm{C}$ " VHTR Technology Development Office 6th Annual Technical Review Meeting 2013, Idaho Falls, ID, May, 2013

17. "Bi-axial Creep Tests for Ni-based Alloy 617 and Alloy 230 for VHTR Part 2: Facility Development, Creep Modeling and Further Bi-axial Creep Tests" VHTR Technology Development Office 6th Annual Technical Review Meeting 2013, Idaho Falls, ID, May, 2013

18. "Synchrotron Assisted Nuclear Material Development for Current and Generation IV Nuclear Systems", Advanced Test Reactor National Scientific User Facility Users Week 2013, Idaho Falls, ID, Jun, 2013 